\title{
Psychological therapies for women who experience intimate partner violence (Review)
}

Hameed M, O'Doherty L, Gilchrist G, Tirado-Muñoz J, Taft A, Chondros P, Feder G, Tan M, Hegarty $\mathrm{K}$

Hameed M, O'Doherty L, Gilchrist G, Tirado-Muñoz J, Taft A, Chondros P, Feder G, Tan M, Hegarty K. Psychological therapies for women who experience intimate partner violence.

Cochrane Database of Systematic Reviews 2020, Issue 7. Art. No.: CD013017. DOI: 10.1002/14651858.CD013017.pub2.

www.cochranelibrary.com 
TABLE OF CONTENTS

HEADER

ABSTRACT

PLAIN LANGUAGE SUMMARY

SUMMARY OF FINDINGS

BACKGROUND

OBJECTIVES

METHODS

RESULTS

Figure 1.

Figure 2.

Figure 3.

Figure 4.

DISCUSSION

AUTHORS' CONCLUSIONS

ACKNOWLEDGEMENTS

REFERENCES

CHARACTERISTICS OF STUDIES

DATA AND ANALYSES

Analysis 1.1. Comparison 1: Intervention vs Control, Outcome 1: Depression (continuous)

Analysis 1.2. Comparison 1: Intervention vs Control, Outcome 2: Depression (categorical)

Analysis 1.3. Comparison 1: Intervention vs Control, Outcome 3: Depression (recruitment setting of participants) ..................

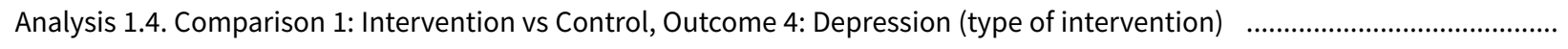

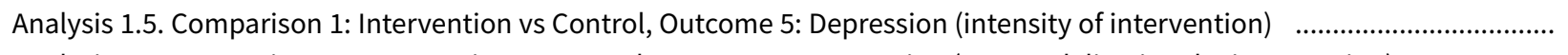

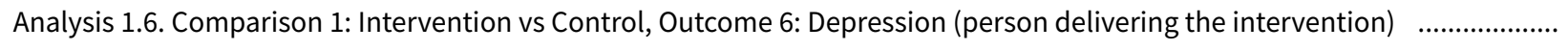

Analysis 1.7. Comparison 1: Intervention vs Control, Outcome 7: Self-efficacy

Analysis 1.8. Comparison 1: Intervention vs Control, Outcome 8: Dropouts from treatment

Analysis 1.9. Comparison 1: Intervention vs Control, Outcome 9: Mental health

Analysis 1.10. Comparison 1: Intervention vs Control, Outcome 10: Anxiety (continuous)

Analysis 1.11. Comparison 1: Intervention vs Control, Outcome 11: Anxiety (categorical)

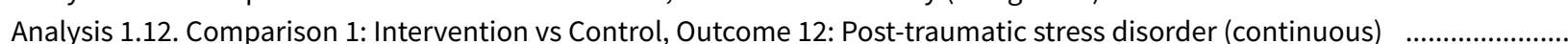

Analysis 1.13. Comparison 1: Intervention vs Control, Outcome 13: Quality of life

Analysis 1.14. Comparison 1: Intervention vs Control, Outcome 14: Re-exposure to IPV (continuous)

Analysis 1.15. Comparison 1: Intervention vs Control, Outcome 15: Re-exposure to IPV (categorical)

Analysis 1.16. Comparison 1: Intervention vs Control, Outcome 16: Safety planning and/or safety behaviour (continuous) .....

Analysis 1.17. Comparison 1: Intervention vs Control, Outcome 17: Safety planning and/or safety behaviour (categorical) .....

Analysis 1.18. Comparison 1: Intervention vs Control, Outcome 18: Use of healthcare and IPV services ..................................

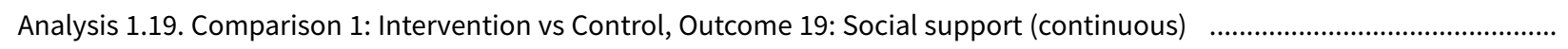

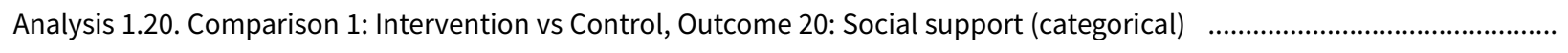
APPENDICES

HISTORY

CONTRIBUTIONS OF AUTHORS

DECLARATIONS OF INTEREST

SOURCES OF SUPPORT

DIFFERENCES BETWEEN PROTOCOL AND REVIEW 
[Intervention Review]

\section{Psychological therapies for women who experience intimate partner violence}

Mohajer Hameed ${ }^{1}$, Lorna O'Doherty², Gail Gilchrist ${ }^{3}$, Judit Tirado-Muñoz ${ }^{4}$, Angela Taft ${ }^{5}$, Patty Chondros ${ }^{1}$, Gene Feder ${ }^{6}$, Melissa Tan ${ }^{1}$, Kelsey Hegarty1,7

1Department of General Practice, The University of Melbourne, Melbourne, Australia. 2Faculty of Health and Life Sciences, Coventry University, Coventry, UK. ${ }^{3}$ National Addiction Centre, Institute of Psychiatry, Psychology and Neuroscience, King's College London, London, UK. ${ }^{4}$ Addiction Research Group, IMIM-Institut Hospital del Mar d'Investigacions Mèdiques, Barcelona, Spain. ${ }^{5}$ The Judith Lumley Centre, La Trobe University, Melbourne, Australia. ${ }^{6}$ Centre for Academic Primary Care, Population Health Sciences, Bristol Medical School, University of Bristol, Bristol, UK. ${ }^{7}$ The Royal Women's Hospital, Victoria, Australia

Contact address: Kelsey Hegarty, k.hegarty@unimelb.edu.au.

Editorial group: Cochrane Common Mental Disorders Group.

Publication status and date: New, published in Issue 7, 2020.

Citation: Hameed M, O'Doherty L, Gilchrist G, Tirado-Muñoz J, Taft A, Chondros P, Feder G, Tan M, Hegarty K. Psychological therapies for women who experience intimate partner violence. Cochrane Database of Systematic Reviews 2020, Issue 7. Art. No.: CD013017. DOI: 10.1002/14651858.CD013017.pub2.

Copyright ( 2020 The Cochrane Collaboration. Published by John Wiley \& Sons, Ltd.

\section{A B S T R A C T}

\section{Background}

Intimate partner violence (IPV) against women is prevalent and strongly associated with mental health problems. Women experiencing IPV attend health services frequently for mental health problems. The World Health Organization recommends that women who have experienced IPV and have a mental health diagnosis should receive evidence-based mental health treatments. However, it is not known if psychological therapies work for women in the context of IPV and whether they cause harm.

\section{Objectives}

To assess the effectiveness of psychological therapies for women who experience IPV on the primary outcomes of depression, self-efficacy and an indicator of harm (dropouts) at six- to 12-months' follow-up, and on secondary outcomes of other mental health symptoms, anxiety, quality of life, re-exposure to IPV, safety planning and behaviours, use of healthcare and IPV services, and social support.

\section{Search methods}

We searched the Cochrane Common Mental Disorders Controlled Trials Register (CCMDCTR), CENTRAL, MEDLINE, Embase, CINAHL, PsycINFO, and three other databases, to the end of October 2019. We also searched international trials registries to identify unpublished or ongoing trials and handsearched selected journals, reference lists of included trials and grey literature.

\section{Selection criteria}

We included randomised controlled trials (RCTs), quasi-RCTs, cluster-RCTs and cross-over trials of psychological therapies with women aged 16 years and older who self-reported recent or lifetime experience of IPV. We included trials if women also experienced coexisting mental health diagnoses or substance abuse issues, or both. Psychological therapies included a wide range of interventions that targeted cognition, motivation and behaviour compared with usual care, no treatment, delayed or minimal interventions. We classified psychological therapies according to Cochrane Common Mental Disorders's psychological therapies list.

\section{Data collection and analysis}

Two review authors extracted data and undertook 'Risk of Bias' assessment. Treatment effects were compared between experimental and comparator interventions at short-term (up to six months post-baseline), medium-term (six to under 12 months, primary outcome time 
point), and long-term follow-up (12 months and above). We used standardised mean difference (SMD) for continuous and odds ratio (OR) for dichotomous outcomes, and used random-effects meta-analysis, due to high heterogeneity across trials.

\section{Main results}

We included 33 psychological trials involving 5517 women randomly assigned to experimental (2798 women, 51\%) and comparator interventions (2719 women, 49\%). Psychological therapies included 11 integrative therapies, nine humanistic therapies, six cognitive behavioural therapy, four third-wave cognitive behavioural therapies and three other psychologically-orientated interventions. There were no trials classified as psychodynamic therapies. Most trials were from high-income countries (19 in USA, three in Iran, two each in Australia and Greece, and one trial each in China, India, Kenya, Nigeria, Pakistan, Spain and UK), among women recruited from healthcare, community, shelter or refuge settings, or a combination of any or all of these. Psychological therapies were mostly delivered face-to-face (28 trials), but varied by length of treatment (two to 50 sessions) and staff delivering therapies (social workers, nurses, psychologists, community health workers, family doctors, researchers). The average sample size was 82 women (14 to 479 ), aged 37 years on average, and $66 \%$ were unemployed. Half of the women were married or living with a partner and just over half of the participants had experienced IPV in the last 12 months (17 trials), $6 \%$ in the past two years (two trials) and $42 \%$ during their lifetime (14 trials).

Whilst 20 trials (61\%) described reliable low-risk random-sampling strategies, only 12 trials (36\%) described reliable procedures to conceal the allocation of participant status.

While 19 trials measured women's depression, only four trials measured depression as a continuous outcome at medium-term follow-up. These showed a probable beneficial effect of psychological therapies in reducing depression (SMD $-0.24,95 \% \mathrm{Cl}-0.47$ to -0.01 ; four trials, 600 women; moderate-certainty evidence). However, for self-efficacy, there may be no evidence of a difference between groups (SMD -0.12, $95 \% \mathrm{Cl}-0.33$ to 0.09 ; one trial with medium-term follow-up data, 346 women; low-certainty evidence). Further, there may be no difference between the number of women who dropped out from the experimental or comparator intervention groups, an indicator of no harm (OR $1.04,95 \% \mathrm{Cl} 0.75$ to 1.44; five trials with medium-term follow-up data, 840 women; low-certainty evidence). Although no trials reported adverse events from psychological therapies or participation in the trial, only one trial measured harm outcomes using a validated scale.

For secondary outcomes, trials measured anxiety only at short-term follow-up, showing that psychological therapies may reduce anxiety symptoms (SMD -0.96, 95\% Cl-1.29 to -0.63; four trials, 158 women; low-certainty evidence). However, within medium-term follow-up, low-certainty evidence revealed that there may be no evidence between groups for the outcomes safety planning (SMD $0.04,95 \% \mathrm{Cl}-0.18$ to 0.25 ; one trial, 337 women), post-traumatic stress disorder (SMD $-0.24,95 \% \mathrm{Cl}-0.54$ to 0.06 ; four trials, 484 women) or re-exposure to any form of IPV (SMD $0.03,95 \% \mathrm{Cl}-0.14$ to 0.2 ; two trials, 547 women).

\section{Authors' conclusions}

There is evidence that for women who experience IPV, psychological therapies probably reduce depression and may reduce anxiety. However, we are uncertain whether psychological therapies improve other outcomes (self-efficacy, post-traumatic stress disorder, reexposure to IPV, safety planning) and there are limited data on harm. Thus, while psychological therapies probably improve emotional health, it is unclear if women's ongoing needs for safety, support and holistic healing from complex trauma are addressed by this approach. There is a need for more interventions focused on trauma approaches and more rigorous trials (with consistent outcomes at similar followup time points), as we were unable to synthesise much of the research.

\section{PLAIN LANGUAGE SUMMARY}

\section{Psychological therapies for women who experience intimate partner violence}

\section{The review question}

Domestic violence (physical, emotional, sexual abuse and controlling behaviour by a partner or ex-partner) is common worldwide and causes long-lasting emotional and physical health problems. Psychological therapies (counselling by trained people) may improve women's mental health and enable them to focus on making safety plans, accessing resources for themselves and their children, and ultimately to escape the domestic violence.

We searched scientific literature worldwide up to the end of October 2019 for trials comparing a group of female domestic violence survivors who received psychological therapy with those who did not, to understand whether such therapies are safe and effective.

\section{Trial characteristics}

Women had been randomly placed in one group (the intervention) or the other (comparison). We found 33 trials involving 5517 women, with an average age of 37 years, and two-thirds of them were unemployed. Half of them were married or living with a partner, and for half of them the domestic violence was in the last 12 months. Psychological therapies were mostly delivered face-to-face but varied by the length of treatment ( 2 to 50 sessions) and the staff who delivered the therapies (social workers, nurses, psychologists, community health workers, family doctors, researchers). Women were invited from healthcare settings, community centres and domestic violence refuges and shelters. Nineteen trials measured women's depression, two assessed self-efficacy (if women believed they were capable of making 
changes in their lives) and all measured dropout from the groups. We used the number of dropouts to measure harmful effects. Most trials followed up on the women within six months of starting the trial.

\section{Key results}

We found evidence that psychological therapies probably reduce depression and may reduce anxiety symptoms for women who have experienced domestic violence (six to 12 months after the therapy). Psychological therapies do not appear to cause any harm. However, we are uncertain whether psychological therapies improve self-efficacy, mental health, quality of life, social support, uptake of healthcare and domestic violence services, safety planning or reduce post-traumatic stress disorder and re-exposure to any form of domestic violence.

Overall, there is a need for more trials with consistent outcomes at similar follow-up time points as we were unable to combine much of the research to give an overall picture. Thus, while women experiencing domestic violence may be helped by psychological therapies to improve their emotional health, which may in turn help their ongoing needs of safety, support and holistic healing from complex trauma, we are uncertain whether psychological therapies improve these aspects of their lives. 


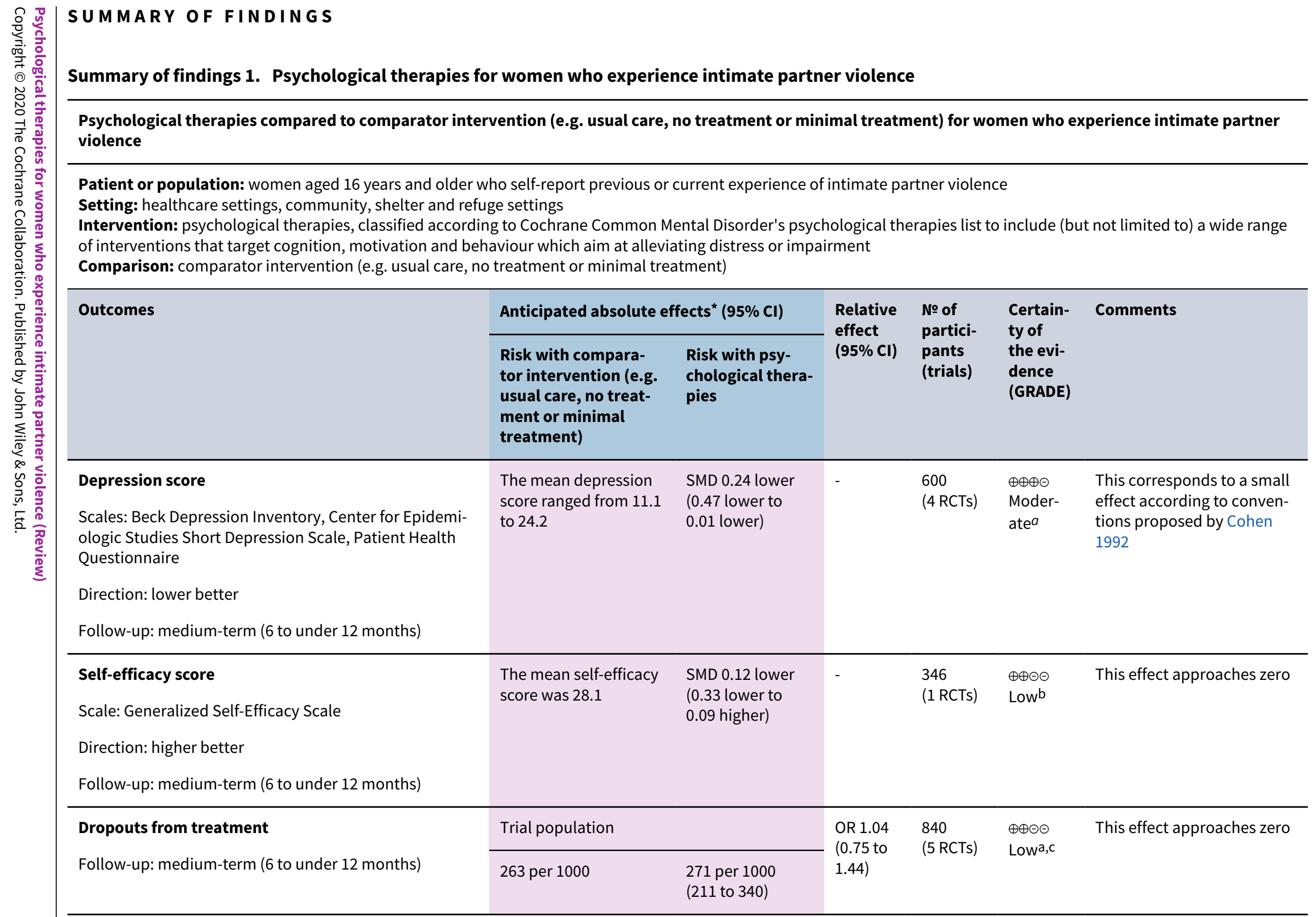




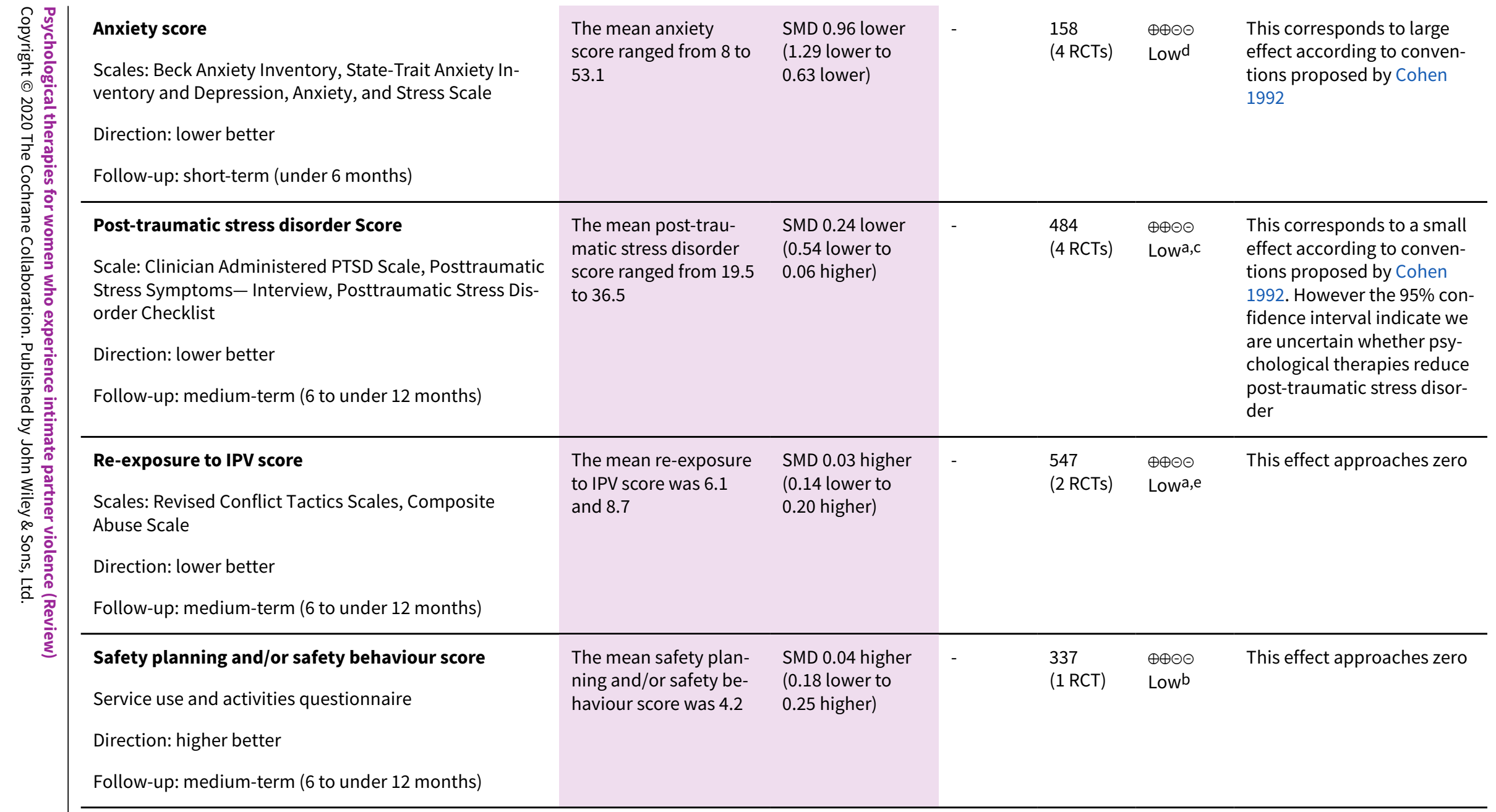

${ }^{*}$ The risk in the intervention group (and its 95\% confidence interval) is based on the assumed risk in the comparison group and the relative effect of the intervention (and its $95 \% \mathrm{Cl}$ ).

Cl: confidence interval; OR: odds ratio

\section{GRADE Working Group grades of evidence}

High certainty: We are very confident that the true effect lies close to that of the estimate of the effect

Moderate certainty: We are moderately confident in the effect estimate: the true effect is likely to be close to the estimate of the effect, but there is a possibility that it is substantially different

Low certainty: Our confidence in the effect estimate is limited: the true effect may be substantially different from the estimate of the effect

Very low certainty: We have very little confidence in the effect estimate: the true effect is likely to be substantially different from the estimate of effect 
aDowngraded one level due to unclear risk of bias in allocation concealment (risk of bias)

bowngraded two level due to very small number of trials / participants (imprecision).

cDowngraded one level due to inconsistency of results across trials (inconsistency).

dDowngraded two level due to high risk of bias in allocation concealment (risk of bias).

eDowngraded one level due to small number of trials / participants (imprecision). 


\section{B A C K G R O U N D}

\section{Description of the condition}

Intimate partner violence (IPV) is a prevalent issue across countries, with one in three women globally reporting violence from an intimate partner at some stage in their lives (World Health Organization 2013a). This review adopts the World Health Organization (WHO) definition of IPV as "any behaviour within an intimate relationship that causes physical, psychological or sexual harm to those in the relationship" (Krug 2002). This includes acts of psychological abuse, such as intimidation and constant belittling, and controlling behaviour such as monitoring movements, isolation from family and friends and restricting access to financial resources, medical care, employment and education (Krug 2002). Compared to men, women experiencing IPV are more likely to suffer from repeated, severe violence from their intimate partners and are also more likely to be murdered by a current or former intimate partner (World Health Organization 2013a). For these reasons, this review focuses on women exposed to IPV, although it is acknowledged that IPV against men is also an important issue that requires further research.

Recent studies have estimated IPV as the leading cause of death, disability and illness for women of childbearing age, mostly as a result of mental health issues associated with IPV (Ayre 2016). Abused women commonly suffer with chronic health problems (Black 2001; World Health Organization 2013a), present very frequently to healthcare services and require a wide range of medical services (Black 2001). The most prevalent mental health problems in women exposed to IPV are depression, anxiety, posttraumatic stress disorder (PTSD) and alcohol use disorders (Howard 2013; Rees 2011; Trevillion 2012; World Health Organization 2013a). Abused women are more than twice as likely to suffer with depression compared with non-abused women (Devries 2013). Women who experience IPV have also been found to be more likely to suffer from PTSD compared with non-abused women (Oram 2013), and more likely to develop dependency on alcohol and illicit substances (Oram 2013). Women exposed to IPV often also suffer from low self-esteem and hopelessness (Papadakaki 2009). There is growing awareness of the wide range of negative mental health symptoms associated with experience of IPV, including the understanding of the term complex trauma (Baird 2019; Courtois 2012; Hermann 1992). Given this high prevalence of mental health problems in women exposed to IPV, there is a potential for psychological therapies to improve women's mental health functioning.

\section{Description of the intervention}

For the purpose of this review, broadly defined, psychological therapies include a wide range of interventions that target cognition, motivation and behaviour which aim at alleviating distress or impairment (see Cochrane Common Mental Disorders' (CCMD) psychological therapies list for definitions). These include:

1. behaviour therapy/behaviour modification (e.g. activity scheduling, psycho-education, problem-focused);

2. cognitive behavioural therapy (CBT; e.g. rational emotive therapy, restructuring, role play);

3. third-wave CBTs (e.g. acceptance and commitment therapy, mindfulness);
4. psychodynamic therapies (e.g. countertransference, transference, object relations);

5. humanistic therapies (e.g. existential therapy, grief work);

6. integrative therapies (e.g. motivational interviewing and interpersonal therapy);

7. systemic therapies (e.g. solution-focused brief therapy, narrative therapy);

8. other psychologically-orientated Interventions (e.g. art therapy, meditation, music therapy).

We did not include therapies that were not aimed at individual women, for example, couples or family therapy, as in our protocol (Tan 2018).

Cognitive behavioural processes, which underlie some of the psychological interventions listed, have slightly different targets for change that can be sub-classified into the following (Dobson 2009):

1. cognitive re-structuring, which focuses on internal underlying beliefs and thoughts with the aim to challenge maladaptive thought patterns;

2. coping skills therapy, which focuses on identification and alteration of cognitions and behaviours that may increase the impact of negative external events. This type of therapy is primarily used for problems that are external to the person, focusing on reducing the consequences of negative external events;

3. problem-solving therapies which combine cognitive restructuring and coping skills therapy to change internal thought patterns and optimise responses to external negative events.

The number of psychological intervention sessions in IPV intervention trials can vary greatly, from one to 20 sessions (Hegarty 2013; Kiely 2010). There is limited evidence from IPV trials about the recommended length of treatment (World Health Organization 2013b). Similarly, there is wide variation across interventions in who delivers the psychological intervention from formally trained mental healthcare workers to social work students (Kiely 2010), to trained healthcare workers and family doctors (Hegarty 2013). Drawing on mental health literature, low-intensity psychological interventions for mild-to-moderate mental health symptoms are recommended at an intensity of at least five up to 12 sessions according to internationally recognised psychological guidelines (National Collaborating Centre Mental Health 2019; NICE 2009). High-intensity psychological interventions, for more severe symptoms, are recommended at an intensity of 16 to 20 sessions (NICE 2009).

All psychologically-orientated interventions at some level aim to reduce stress and promote recovery from the trauma of the IPV. This may enable women to take further actions for safety and well-being for themselves and their children, despite the fact that control of ending the IPV and abusive behaviours is in the hands of their partner or ex-partner. Survivors value the importance of positive therapeutic relationships that can promote a safe and trusting relational experience after being abused that may assist with trauma symptoms (Feder 2006). Further, there is often limited acknowledgment of the complex trauma symptoms women with IPV experience in mental health settings (Warshaw 2013) or the need for trauma informed approaches in evaluations of psychological therapies (Bisson 2013) or training 
of mental health professionals (World Health Organization 2013b). Thus even though this review examines specific psychological therapies, it is important to acknowledge that the staff and organisations delivering psychological therapies need to adhere to the principles of trauma informed care (Warshaw 2013). These include physical and psychological safety, trust, transparency, peer support, collaboration, mutuality, empowerment, voice, choice, and an understanding of cultural, historical and gender issues (World Health Organization 2013b).

\section{How the intervention might work}

In considering individual psychological interventions for IPV, it must first be recognised that women experiencing IPV often have little to no control over their partner's behaviour (Hegarty 2008). Furthermore, there may be limited insight by women into labelling of the behaviours they are experiencing as IPV (Reisenhofer 2013). This is particularly relevant among women with long histories of trauma, poor self-esteem and social isolation as a result of the IPV they have experienced (Papadakaki 2009). Such individualand relationship-level circumstances contribute to the difficult process of decision-making about accessing help or escaping the violence, or both (O'Doherty 2016). In addition, factors in a person's community and the wider social and cultural milieu, including race, immigration status, disability, and sexuality add to the complexity of a woman's journey and need to be considered with respect to responses for women experiencing IPV (Chavis 2008). Decisions such as disclosing abusive behaviour, uptake of safety behaviours and leaving an abusive relationship are therefore much more complex than they may appear, particularly for women still in the relationship where IPV is happening. Further, taking such action by a woman may be impaired by the mental health consequences of exposure to IPV, including depression, anxiety and PTSD. However, psychological therapies alone may not address re-victimisation and women may need advocacy (Rivas 2019) and justice responses for their partners.

In this complicated setting, we consider the role of psychological therapies and their potential for changing perceived support, cognitions, beliefs and behaviours for individual women. We will describe how behaviour therapy, CBT-based interventions, integrative therapies, humanistic therapies and other psychologically-orientated interventions might impact on a woman experiencing IPV. Firstly, cognitive behavioural interventions (e.g. formal CBT, CBT-based techniques, Trauma Focused-CBT, third wave-CBT) are based on the proposition that behaviours are often cognitively mediated (Butler 2006). Because cognitive activity may be monitored and altered, behaviours (for example, seeking help for IPV) may be changed through cognitive changes (Dobson 2009). Addressing certain thinking patterns and beliefs may therefore result in positive changes in symptoms and behaviours that may reduce some of the negative consequences of IPV exposure (Butler 2006). It is important at this stage to recognise that women experiencing IPV often make significant efforts to minimise harm, and certain behaviours and cognitions (such as safety planning) have been associated with harm reduction (Tiwari 2005). These positive cognitions and behaviours provide a good example of important potential targets for psychological therapies. Further, third wave-CBTs, for example, acceptance and commitment therapy and mindfulness CBT, act on changing the individual's relationship to distress and pain through acceptance, being present and committed action (Hayes 2006).
Integrative therapies such as motivational interviewing may be useful in assisting women. Motivational interviewing is based on the Transtheoretical model (Miller 2002), which identifies five stages of change with various strategies identified to facilitate a shift from one stage to the next (Prochaska 1992). A number of authors have however challenged the application of the Transtheoretical model to IPV interventions (Chang 2005; Zink 2004), as women who are abused have minimal control over their partner's behaviour. It is therefore not immediately obvious what the target for change should be (Reisenhofer 2013). For example, a woman might be cognitively and emotionally prepared to leave the relationship, but be constrained by an objective external barrier to change, and may take other actions such as safety behaviours (Chang 2005). Cluss and colleagues have proposed an alternative model, the Psychosocial Readiness Model, to describe the process of change for victims of partner abuse (Cluss 2006). The Psychosocial Readiness Model encompasses external as well as internal factors: awareness that the partner's behaviour is abuse, perceived support from others and self-efficacy/perceived power. Self-efficacy is the ability of women to believe that they can be successful when they take action (Benight 2004). It is a context-specific assessment of competence to perform a specific or range of tasks in a given domain (Bandura 1986). It has recently been proposed that this model could apply to counselling women exposed to IPV using motivational interviewing (Hegarty 2008; Hegarty 2013; Saftlas 2014).

Thirdly, humanistic therapies (e.g. supportive and non-directive therapy) may be helpful for women exposed to IPV. For example supportive counselling in which the therapist empathically engages the recipient, helps them to feel understood and assists with organising their life and solving life problems, also has the potential to improve victims' mental health and ability to take action (de Mello 2005). For women who have decided that the abuse must end, but whose intentions are not translated into action due to perceived external barriers, then supportive interventions and non directive techniques may be helpful, especially for depression (Mynors-Wallis 2000).We know that depression is common in women exposed to IPV (World Health Organization 2013a), and we know from qualitative studies that women who have experienced IPV have identified healthcare clinicians as a source of assistance from whom they would seek support, although there have only been limited studies in mental health settings (Feder 2006). Taken together, these findings support the possibility that humanistic therapies delivered in healthcare settings might be helpful for women exposed to IPV.

Psychodynamic therapies, including brief psychotherapy, group therapy and person centred therapy, might assist women to reflect and understand what is happening in a supportive way. Other psychologically-orientated interventions, for example, insightorientated therapies, art therapy, music therapy, and meditation may be helpful conjuncts for women who have left the relationship to assist them in managing ongoing trauma symptoms. Finally, of the systemic therapies, narrative therapy may also assist with developing an understanding of how their symptoms relate to their narrative of what has happened to them. Couples and family therapy are excluded from this review, as in our protocol (Tan 2018).

This background supports the findings from emerging research as previously outlined, that psychological therapies may positively impact upon the mental health and well-being of women 
experiencing IPV, even without overt diagnoses of mental health conditions (Hegarty 2013; Kiely 2010; Kubany 2004; Nelson 2012).

\section{Why it is important to do this review}

IPV is globally prevalent, with devastating, far-reaching and long-lasting individual and societal consequences (World Health Organization 2013c). It is important to gather and evaluate systematically the evidence about psychological therapies that may help women exposed to IPV, as we have few interventions available that have been shown to help the safety, health and well-being of women and their children. Routine screening of women for IPV in health settings, in the absence of structured intervention, was shown in a Cochrane Review to have no impact upon health outcomes and re-exposure to violence (O'Doherty 2015). Advocacy in the multifaceted form of safety, legal, housing and financial advice, and facilitated access to community resources such as shelters, emergency housing and counselling is another intervention that may be offered to women (Rivas 2015). However, evidence from a Cochrane Review of the effect of advocacy for women exposed to IPV has been equivocal (Rivas 2019). In addition, another Cochrane Review examining the impact of educational and skills-based interventions for relationship violence among adolescents and young adults also found no impact upon episodes, attitudes or behaviours related to relationship violence (Fellmuth 2013). Further research is therefore required to investigate the effectiveness of other interventions.

The effectiveness of cognitive behavioural interventions in the treatment of depression, anxiety and PTSD in general populations has been well demonstrated in Cochrane Reviews (Bisson 2013; Butler 2006; Hunot 2007). Given the prevalence of these specific mental health problems among women experiencing IPV (World Health Organization 2013a), and guidance to offer women experiencing mental health problems and IPV standard treatment by therapists who have an understanding of complex trauma from IPV (World Health Organization 2013b), it is a reasonable next step to examine the impact of psychological therapies generally for women experiencing IPV. Women exposed to IPV are often referred for psychological interventions, but the impact of these interventions remains uncertain. Before psychological interventions can be recommended, it is important to evaluate the helpfulness of these interventions in this population. To our knowledge, the role of psychological therapies with and for women exposed to IPV has never been systematically assessed to the level of a Cochrane Review (Feder 2009; Tirado-Muñoz 2014). This review seeks to address this important knowledge gap and provide practitioners and policymakers with a further evidence base to guide effective responses to IPV for women, whether or not they have been diagnosed with a mental health condition.

\section{OB JECTIVES}

To assess the effectiveness of psychological therapies for women who experience IPV on the primary outcomes of depression, self-efficacy and an indicator of harm (dropouts) at six- to 12months' follow-up, and on secondary outcomes of other mental health symptoms, anxiety, quality of life, re-exposure to IPV, safety planning and behaviours, use of healthcare and IPV services, and social support.

\section{METHODS}

\section{Criteria for considering studies for this review}

\section{Types of studies}

We included randomised controlled trials (RCTs) and quasi-RCTs. We included quasi-RCTs because research in this area is still emerging. Cluster-RCTs and cross-over trials were also eligible for inclusion.

\section{Types of participants}

\section{Participant characteristics}

We included participants identifying as women aged 16 years and older. Where trials involved a subset of eligible participants, we included these trials in the review if the trial included over $50 \%$ eligible participants who had been stratified, randomised and analysed separately.

\section{Condition}

We included women who self-reported recent or past experience of IPV. This included same-sex partners. We included women who experienced sexual violence, but only if this was perpetrated by an intimate partner.

\section{Co-morbidities}

We included women with co-existing mental health diagnoses or substance abuse issues, or both, in addition to having experienced recent or previous IPV.

\section{Setting}

We included women recruited via healthcare, community and shelter or refuge settings. Healthcare settings here are taken to include general practice, antenatal and postnatal services, hospital emergency services, gynaecology services, sexual health clinics, mental health services, community health centres, and drug and alcohol services. Community settings included women's and IPV support organisations, justice settings and refuge facilities.

\section{Types of interventions}

\section{Experimental Intervention}

The experimental intervention consisted of psychological therapies, which are taken here to broadly include a wide range of therapies that target cognition, motivation and behaviour which aim at alleviating distress or impairment. We classified psychological therapies according to the Cochrane Collaboration Depression, Anxiety and Neurosis Group (CCDAN) classification of psychological interventions. These include:

1. behaviour therapy/behaviour modification (e.g. social skills training, behaviour contracting, activity scheduling, exposure therapy and psycho-education);

2. CBT (e.g. problem-solving, rational emotive therapy, role play, restructuring);

3. third-wave CBTs (e.g. acceptance and commitment therapy, mindfulness, meta-cognitive therapy, compassion-focused);

4. psychodynamic therapies (e.g. insight-orientated therapy, countertransference, transference, object relations, psychoanalytic therapy); 
5. humanistic therapies (e.g. existential therapy, expressive therapy, supportive therapy, non-directive therapy);

6. integrative therapies (e.g. motivational interviewing, interpersonal therapy, counselling, eclectic therapy, transtheoretical);

7. systemic therapies (e.g. conjoint therapy, couples, marital or relationship therapy, family therapy);

8. other psychologically-orientated interventions (e.g. art therapy, bibliotherapy, colour therapy, music therapy, psychodrama).

We included trials where interventions involved one or more sessions, where each session consisted of at least 30 minutes. For all interventions, we included trials of any duration or frequency of treatment, as long as treatment met the above stated criteria. In this review, consistent with our protocol (Tan 2018), we excluded systemic therapies such as couple-based therapies, since the focus of the current review is on psychological therapies with women only who experience IPV.

Given the lack of standardised definitions for the training requirements, we did not apply any restrictions to the minimum training requirements for psychological therapy delivery as this would be an arbitrary restriction. Training for the delivery of these interventions was liberal, and included healthcare workers (e.g. medical and allied health workers) and non-healthcare workers (e.g. lay people who have received training). There is a current lack of consensus about consistent minimum requirements for formal psychological therapy training. Using CBT as an example, the Beck Institute specifies important components of CBT in the Cognitive Therapy Scale but does not specify the minimum training required to achieve such competencies (Young 1980). The British Psychological Society compiled a list of core competencies required for CBT delivery in recognition of the disparities between health professions with regard to CBT training, but the minimum training to achieve these competencies similarly was not specified (Roth 2007). Rakovshik and McManus attempted to review the effectiveness of CBT training, but were unable to generate definitive conclusions about the relationship between CBT training and therapist competence (Rakovshik 2010). Furthermore, they were also unable to find a standardised definition of therapist competence, nor were they able to find a standardised method to measure such competence (Rakovshik 2010).

For all interventions, we did not restrict mode of intervention delivery and included face-to-face, any type of digital delivery including telephone, mobile phone and computer-based delivery. Face-to-face and telephone interventions were delivered by either healthcare workers or lay people with specific training in psychological techniques. Mobile phone and computer-based delivery was developed by healthcare workers or by lay people, including IPV organisations. We included both individual and group delivery of the intervention.

\section{Comparator intervention}

Comparator interventions consisted of usual care, no treatment, delayed provision of psychological interventions (also referred to as waiting-list conditions) and minimal interventions such as screening, information provision and referral to community services such as women's shelters.

\section{Special circumstances}

We included trials where psychological therapies were delivered as an adjunct to advocacy or screening for IPV, where the comparator intervention group received advocacy or screening without psychological interventions.

\section{Types of outcome measures}

We included trials that met the above inclusion criteria, regardless of whether they reported on the following outcomes.

\section{Primary outcomes}

1. Depression with outcome measures including the Center for Epidemiologic Studies Depression Scale (CES-D; Radloff 1977), the Patient Health Questionnaire (PHQ; Spitzer 1999), Beck Depression Inventory (BDI; Beck 1961), Hospital Anxiety and Depression Scale (HADS; Bjelland 2002), and Hamilton Depression Rating Scale (HAM-D; Hamilton 1960)

2. Self-efficacy with outcome measures including the General SelfEfficacy Scale (GSE; Schwarzer 1995)

3. Dropouts from treatment

\section{Secondary outcomes}

1. General mental health symptoms with outcome measures including the Short Form 12-item survey (SF12; Ware 1996)

2. Anxiety with outcome measures including Generalised Anxiety Disorder Seven-item Assessment (GAD-7; Kertz 2013; Spitzer 2006), Beck Anxiety Inventory (BAl; Beck 1988), and Hospital Anxiety and Depression Scale (HADS; Bjelland 2002)

3. PTSD with outcome measures including the PTSD checklist (PCL; Blanchard 1996; Weathers 1991), and Short Screening Scale for DSM-IV Post-traumatic Stress Disorder (Breslau 1999)

4. Quality of life with outcome measures including the WHO Quality of Life scale - abbreviated version (WHOQOL-BREF; Skevington 2004) and EuroQol-5 dimension (EQ-5D; Brooks 1996)

5. Re-exposure to IPV including physical, sexual and psychological abuse with outcome measures including the Composite Abuse Scale (CAS; Hegarty 2005), Revised Conflict Tactics Scale (CTS2; Straus 1996) and Women's Experience with Battering (WEB; Smith 1995)

6. Safety planning or safety behaviour and self-care activities, or both, with outcome measures including the Safety Behaviour Checklist (McFarlane 2004)

7. Use of healthcare and IPV services with outcome measures as defined in the individual trials, since we expect these to be trialspecific as healthcare and IPV services vary greatly between different settings and countries

8. Social support with outcome measures including the Oslo 3 Social Support Scale (OSS3; Dalgard 1996), Interpersonal Support Evaluation List (ISEL; Cohen 1983) and Inventory of Socially Supportive Behaviours (ISSB; Barrera 1981)

We collected additional outcome-related information to further aid understanding of included trials. These included information about analysis of any cost-benefit measures, process evaluation, summary of participants' views about the intervention, and adverse events (harms) related to participation. 


\section{Timing of outcome assessment}

We classified short-term follow-up as up to six months after baseline assessment, medium-term follow-up as six to under 12 months, and long-term follow-up as 12 months or more. The primary outcome time frame was medium-term follow-up, but when there were insufficient trials with available data at mediumterm follow-up, we analysed findings at short and/or long-term follow-up. In addition, when trials reported data from more than one follow-up period, we included data from the latest possible follow-up period in the meta-analysis if data were available.

\section{Hierarchy of outcome measures}

In the event that the included trials used more than one scale to measure single outcomes, in selecting a set of data for inclusion in meta-analysis, we gave preference to scales and measures according to the order in which they are listed above.

\section{Search methods for identification of studies}

We identified RCTs of psychological therapies for women experiencing IPV from the specialised register of the Cochrane Common Mental Disorders Group (CCMDCTR), the Cochrane Central Register of Controlled Trials (CENTRAL) and other key bibliographic databases (most recent search, 29 October 2019).

\section{Electronic searches}

An Information Specialist with CCMD ran searches on the following databases using relevant subject headings (controlled vocabularies) and search syntax, appropriate to each resource. The initial search was conducted in June 2018.

1. Cochrane Common Mental Disorders Controlled Trials Register (CCMDCTR) (all available years) (Appendix 1).

2. Cochrane Central Register of Controlled Trials (Issue 5 of 12, May 2018) (Appendix 2).

3. Ovid MEDLINE (1946 to 28 June 2018).

4. Ovid Embase (1974 to 27 June 2018).

5. Ovid PsycINFO (1806 to June Week 3).

6. Ebsco CINAHL (1982 to 28 June 2018).

7. Database of Abstracts of Reviews for Effectiveness (DARE (archived database)) (all years to Issue 2 of 4, April 2015).

8. Web of Science Social Science Citation Index (1900 to 28 June 2018).

9. Proquest Published International Literature on Traumatic Stress (PILOTS) (1871 to 28 June 2018).

10.Clinical Trial Registers: ClinicalTrials.gov and the WHO International Clinical Trials Registry Platform (ICTRP; www.who.int/ictrp/en/) (all years to 28 June 2018).

We applied no restrictions by date, language or publication status.

When we updated the search in October 2019, we were concerned about the specificity of terms for the intervention, so we ran a new search, population only (IPV) on CENTRAL, in the first instance. The search included additional terms for IPV and was backdated (all years) and de-duplicated, as appropriate. After we screened the new search results from CENTRAL, we then completed further update searches on MEDLINE, Embase, PsycINFO and the Web of Science (2018 onwards) (Appendix 3).
1. Cochrane Central Register of Controlled Trials (CENTRAL; Issue 10 of 12, October 2019).

2. Ovid MEDLINE (2018 to 25 October 2019).

3. Ovid PsycINFO (2018 to October Week 32019 ).

4. Web of Science Social Science Citation Index (2018 to 25 October 2019).

In the update, records from Embase and the international trial registers were captured by CENTRAL on the Cochrane Library, CINAHL and PILOTS were dropped (as they did not retrieve any unique citations in the earlier search) and the CCMDCTR and DARE were out of date at this time.

\section{Searching other resources}

\section{Grey literature}

We searched sources of grey literature including dissertations and theses, clinical guidelines and reports from regulatory agencies in a non-systematic manner (where appropriate) including:

1. World Health Organization,

2. Domestic Violence Data Sources.

\section{Reference lists and handsearching}

We checked the reference lists of all included trials and relevant systematic reviews across various journals to identify additional trials missed from the original electronic databases searches (for example, unpublished or in-press citations). We also conducted a forward citation search on the Web of Science.

\section{Correspondence and personal communication with trial authors}

We contacted 135 trial authors and subject experts for information on unpublished or ongoing trials or to request additional trial data ( $88 \%$ response rate). Final email communication occurred on 15 November 2019.

\section{Data collection and analysis}

\section{Selection of studies}

Two review authors ( $\mathrm{MH}$ and $\mathrm{KH}$ ) independently reviewed titles and abstracts of records found using Covidence. Where possible, we resolved any disagreements between the review authors by discussion. We coded abstracts as 'yes' or 'maybe' (eligible or potentially eligible or unclear) or 'no' (do not retrieve). We retrieved full articles of abstracts selected and four of the review authors $(\mathrm{MH}, \mathrm{JT}, \mathrm{LOD}, \mathrm{GG})$ assessed them against the inclusion criteria. We resolved disagreements between review authors by discussion with the senior author $(\mathrm{KH})$. We contacted trial authors as required, to decide whether trials had met the inclusion criteria. We recorded reasons for excluding ineligible trials. As with earlier stages of the trial selection process, in the event of disagreements that could not be resolved by discussion, we consulted the senior author $(\mathrm{KH})$ as mediator. We made final decisions by consensus. We identified and excluded duplicate records and collated multiple reports that related to the same trial so that each trial rather than each report was the unit of interest in the review. We recorded the selection process in sufficient detail to complete a PRISMA flow diagram (Moher 2009), and 'Characteristics of excluded studies' table. 


\section{Data extraction and management}

$\mathrm{MH}$ developed the data collection form in consultation with $\mathrm{KH}$ AT, JTM, LOD and GG (see Appendix 4). We implemented this data collection tool in Covidence to extract trial characteristics and outcome data. We piloted the form on at least one trial in the review. MH, LOD, GG, JTM and AT contributed to data extraction from included trials.

In summary, we extracted the following trial characteristics.

1. Methods (e.g. brief description of trial design and randomisation method, assessment modality, total duration of trial, date and location of trial).

2. Participants (e.g. total number of participants, baseline sociodemographic characteristics including gender and age, trial setting, trial's inclusion and exclusion criteria, number of eligible people recruited and assigned, numbers dropped out and numbers analysed).

3. Interventions (e.g. number of intervention groups, brief description, type of psychological therapy, mode of delivery, frequency and duration of delivery, level of mental health training of person delivering the intervention and the relevant comparator intervention characteristics, attendance).

4. Outcomes (e.g. primary and secondary outcomes, outcome measures used and timing of outcome measurement).

5. Notes: funding for trial, and notable conflicts of interest of trial authors.

We documented in the 'Characteristics of included studies' table if outcome data were not reported in a usable way. We resolved disagreements by consensus or by involving the senior author as mediator $(\mathrm{KH})$. One review author $(\mathrm{MH})$ exported all data from Covidence into the Review Manager 5 (RevMan 5) file (Review Manager 2014). We double-checked that data had been entered correctly by comparing the data presented in the systematic review with the trial reports. A second review author $(\mathrm{KH})$ checked trial characteristics for accuracy against the trial report.

\section{Main comparisons}

Psychological therapies versus usual care, waiting list and minimal interventions.

\section{Assessment of risk of bias in included studies}

\section{Individually-randomised trials}

Review authors MH, JTM, LOD, GG and AT contributed to assessment of risks of bias for each included trial using the domainbased evaluation criteria outlined in the Cochrane Handbook for Systematic Reviews of Interventions (Higgins 2017). Review authors rated the included trials for each of the domains listed below with ratings of 'high risk of bias', 'low risk of bias' or 'unclear risk of bias', and provided the reasons supporting the judgement. We resolved any disagreements by contacting the trial author as required for more information to clarify risk of bias, then by team discussion. In the event of disagreements that could not be resolved by discussion, we consulted the senior author $(\mathrm{KH})$ as mediator.

For individually-randomised trials, we addressed the following domains to assess risk of bias.
1. Random sequence generation (methods used to generate the allocation sequence that would have produced comparable groups).

2. Allocation concealment (methods used to conceal the allocation sequence to determine whether group allocations could have been foreseen in advance).

3. Blinding of participants and personnel (methods used to blind trial participants and personnel from knowledge of which intervention a participant received).

4. Blinding of outcome assessment (methods used to blind outcome assessors from knowledge of which intervention a participant received).

5. Incomplete outcome data (whether participants' attrition and exclusion reasons were adequately reported).

6. Selective outcome reporting (whether trial authors selectively reported certain outcomes and not others).

7. Other sources of bias (concerns about bias not covered by points 1 to 6 above).

We also assessed the following factors that may have an impact on outcomes.

1. Therapist allegiance/conflict of interest (whether the therapist had a vested interest in the provided therapies).

2. Therapist qualifications and training (whether the person delivering the intervention had appropriate training or qualifications, or both).

3. Researcher allegiance/conflict of interest (whether the researcher had a vested interest in the provided therapies).

4. Protection against contamination (methods used to prevent or minimise the possibility that women in the comparator intervention might receive part or all of the intervention).

5. Reliability of outcome measures (use of measures with appropriate psychometric properties).

6. Treatment fidelity (whether the therapy was measured against a manual or scale).

We summarised the 'Risk of Bias' judgements across different trials for each of the domains listed. We considered blinding separately for different key outcomes where necessary (e.g. for unblinded outcome assessment, risk of bias for all-cause mortality may be very different than for a participant-reported pain scale). Where information on risk of bias related to unpublished data or correspondence with a trial author, we noted this in the 'Risk of Bias' table.

When considering treatment effects, we took into account the risk of bias for the trials that contributed to that outcome. We offer a detailed description of these 'Risk of Bias' domains in Appendix 5.

\section{Cluster-RCTs}

We assessed the risk of bias for cluster-RCTs as outlined above for individually-randomised trials. In addition, we addressed the following domains specific to cluster-RCTs in accordance with Section 16.3.2 of the Cochrane Handbook for Systematic Reviews of Interventions (Higgins 2011).

1. Identification and recruitment bias.

2. Baseline imbalance between randomised groups.

3. Loss of clusters. 
4. Cluster consideration in analysis.

\section{Cross-over trials}

If the design is appropriate, in addition to the criteria outlined for individual-RCTs and cluster-RCTs (if the unit of allocation is a cluster, e.g. stepped-wedge cluster-RCT), we will address domains specific to cross-over trials as outlined in Section 16.4.3 of the Cochrane Handbook for Systematic Reviews of interventions (Higgins 2011).

1. whether there is a carry-over treatment effect from one period to the next.

2. whether only first-period data are available.

3. incorrect analysis is performed.

4. comparability of results with those from parallel-group trials.

5. dropout of participants after the first treatment.

6. number of treatments or periods used unclear.

\section{Measures of treatment effect}

\section{Dichotomous outcomes}

We required counts and percentages by trial arm for each trial that reported dichotomous outcomes. Using the summary data, we calculated the pooled odds ratio (OR) and 95\% confidence interval $(\mathrm{Cl})$ across the trials for each outcome. We synthesised results through random-effects meta-analysis, as we expected to observe high heterogeneity across the trials (e.g. use of various scales to measure the same outcome). In trials where data required to calculate the OR were neither available nor obtainable from trial authors, we provided the findings as published by the trial authors, or where possible we used the RevMan calculator (Review Manager 2014), to compute the relevant statistics.

\section{Continuous outcomes}

We required means and standard deviations by trial arm for trials that reported continuous outcomes. We calculated the mean difference (MD) when the pooled trials used the same scale to measure the outcome of interest. We calculated the standardised mean difference (SMD), where the absolute mean difference is divided by the pooled standard deviation, when trials measured the outcome using different measurement scales. We presented data as a scale with a consistent direction of effect (we documented direction of effect at the data collection stage and reversed it when necessary). Where means and standard deviations were not available or obtainable from the trial authors, we provided the findings as published by the trial authors or used RevMan calculator (Review Manager 2014), to compute the relevant data. We used a narrative approach to describe continuous outcome data that did not have a normal distribution and were reported as medians and interquartile ranges in the papers.

\section{Unit of analysis issues}

\section{Cluster-RCTs}

Statistical methods for cluster-RCTs that allowed for the effect of clustering are described in Section 16.3.3 of the Cochrane Handbook for Systematic Reviews of Interventions (Higgins 2011). If trials had not accounted for the effects of clustering in the analysis, we used an approximate analysis, as described in Section 16.3.6 of the Cochrane Handbook for Systematic Reviews of interventions (Higgins 2011), which in addition to summary measures nominated for continuous and binary outcomes above, requires an estimate of the intra-class correlation coefficient (ICC) of the outcome and average cluster size. The ICC quantifies the proportion of the total variability of the outcome attributable to the variability between clusters (Donner 2000). When available, we extracted estimates of the ICC directly from the published papers. If not reported, we contacted the trial authors in an attempt to obtain such data; otherwise we obtained estimates of the ICC from external sources. If we did not obtain the estimates of ICC from the trial data, we conducted a sensitivity analysis using a range of plausible values for the ICC.

\section{Cross-over trials}

We planned to use statistical methods for cross-over trials outlined in sections 16.4.5 and 16.4.6 of the Cochrane Handbook for Systematic Reviews of interventions to incorporate cross-over trials into the meta-analysis (Higgins 2011). We planned to conduct sensitivity analyses when missing data, such as the standard error of the estimated treatment effect or within-individual correlation coefficient, were imputed for the meta-analyses. For cross-over trials where the unit of allocation was the cluster, we planned to use appropriate statistical methods, as outlined for cluster-RCTs, to account for the clustering in the data. For the meta-analysis, we planned to analyse parallel-group and cross-over trials separately and combine them (section 16.4.7 of the Cochrane Handbook for Systematic Reviews of interventions, Higgins 2011).

\section{Trials with multiple treatment groups}

Where a single trial reported multiple trial arms, we included only the relevant arms. We used statistical methods for trials with multiple intervention groups as described in section 16.5.4 of the Cochrane Handbook for Systematic Reviews of Interventions (Higgins 2011). We examined trials to see whether they had accounted for the effects of multiple interventions in their trials. If we conducted meta-analysis, we combined all relevant comparator interventions, and combined all relevant experimental interventions.

\section{Dealing with missing data}

For included trials, we noted attrition rates and dropouts from treatment. We contacted trial authors in an attempt to verify key trial characteristics and obtain missing numerical outcome data where possible. We documented all correspondence with trial authors, and we reported which trial authors responded in the full review.

It was not possible to use analytical methods that handled missing data because we collected only summary data from the trials and did not source individual-level data from the trial authors (Egger 2001). We addressed the potential impact of missing outcome data in the assessment of risk of bias described earlier. If appropriate, we performed a sensitivity analysis to assess the impact of the missing information about the trials on the results of the systematic review, as described in sections 16.2.2 (dichotomous outcomes) and 16.2.3 (continuous outcomes) of the Cochrane Handbook for Systematic Reviews of Interventions (Higgins 2011).

\section{Assessment of heterogeneity}

We assessed consistency of the results across trials using graphical representations (Egger 1997), and quantified them using the 12 statistic (Higgins 2003), which measures the proportion of variation of the estimated treatment effect attributable to heterogeneity 
across trials included in the meta-analysis rather than sampling error. We interpreted the observed value of the 12 statistic using the guide given in section 9.5.2 of the Cochrane Handbook for Systematic Reviews of Interventions and taking into consideration the size and direction of effects and the strength of evidence for heterogeneity using the $\mathrm{P}$ value from the $\mathrm{Chi}^{2}$ test and the $95 \% \mathrm{CI}$ for the $1^{2}$ statistic (Deeks 2017). As outlined in the Cochrane Handbook for Systematic Reviews of Interventions, a guide to interpretation of the $1^{2}$ statistic is as follows: $0 \%$ to $40 \%$ might not be important; $30 \%$ to $60 \%$ may represent moderate heterogeneity; $50 \%$ to $90 \%$ may represent substantial heterogeneity; and $75 \%$ to $100 \%$ represents considerable heterogeneity (Deeks 2017).

Heterogeneity may include:

1. clinical diversity (e.g. types of intervention, intensity of intervention, types of participants, recruitment settings);

2. methodological diversity (e.g. risk of bias); and

3. statistical heterogeneity, which may be the consequence of clinical or methodological diversity, or both, across the trials (Deeks 2017).

Where there was evidence for statistical heterogeneity, we employed strategies as outlined in Chapter 9.5.3 of the Cochrane Handbook for Systematic Reviews of Interventions to identify potential sources of heterogeneity among the results of the trials (Deeks 2017). In particular, we explored differences in the characteristics of the trials or other factors (e.g. subgroup analyses) as possible explanations for heterogeneity in the results and summarised in the narrative summary any differences that we identified.

\section{Assessment of reporting biases}

We constructed funnel plots if there were more than 10 trials, to investigate any association between effect size and trial precision, which is closely related to sample size (Egger 1997). Such an association could be due to publication or related biases, or due to systematic differences between small and large trials. If we identified an association, we further examined the clinical diversity of the trials as a possible explanation. If appropriate, we conducted sensitivity analysis to determine whether assumptions about the effect of the bias would have an impact on the estimated treatment effect and the conclusions of the review.

\section{Data synthesis}

We performed a meta-analysis if there were sufficient data and it was meaningful to pool the data across the trials; for instance, if the treatments, participants and the underlying clinical question were similar enough for pooling to make sense. The decision whether to perform meta-analysis was determined by the comparability of populations, denominators and interventions (clinical heterogeneity), the comparability of the duration of followup (methodological heterogeneity) and comparability of outcomes. We used a random-effects model to analyse the data across the trials. If it was inappropriate to combine the data in a meta-analysis, we reported the effect sizes with $95 \% \mathrm{Cls}$ or standard errors of individual trials, and summarised them using a narrative approach.

\section{Subgroup analysis and investigation of heterogeneity}

We were aware of the limitations of subgroup analyses, but we remained interested in the following topics in relation to the primary outcomes. If we identified sufficient numbers of trials, we planned to perform subgroup analyses for the following:

1. Recruitment setting of participants: healthcare setting, community setting, shelter/refuge setting.

2. Type of intervention: according to CCDAN classification of psychological interventions.

3. Intensity of intervention: up to four sessions, and five or more sessions.

4. Person delivering the intervention: healthcare workers, nonhealthcare workers.

Recruitment setting has been identified for subgroup analysis as there may be differences in severity of IPV exposure between women recruited via healthcare as opposed to community settings or shelter settings. Recruitment setting may also influence the level of receptiveness to psychological therapies. Subgroup analyses of the type and intensity of interventions, as well as the background of the person delivering the intervention, have important practical implications for our review findings and recommendations. We used a simple approach described in Chapter 9.6.3 of the Cochrane Handbook for Systematic Reviews of Interventions to conduct subgroup analysis (Deeks 2017). If there was a sufficient number of trials in the meta-analysis (at least 10), we used meta-regression techniques, as described in Chapter 9.5.4 of the Cochrane Handbook for Systematic Reviews of Interventions instead (Deeks 2017). If we identified a large degree of heterogeneity, we first checked the data for errors. If data were correct, we planned to conduct a sensitivity analysis by excluding certain trials from the existing meta-analysis to assess the influence of the trials on the degree of heterogeneity.

\section{Sensitivity analysis}

Primary analyses were based on available data from all included trials relevant to the comparison of interest. We had planned to perform sensitivity analyses to determine whether conclusions were robust to decisions made during the review process, such as certainty of data, the inclusion or exclusion of trials from meta-analysis or approaches to analyses. In this review, when appropriate, we conducted sensitivity analyses on the basis of risk of bias. We used allocation concealment as a main risk of bias. If trials were rated 'low' risk of bias for allocation concealment, then they were high certainty; if 'unclear' or 'high', we rated them as low certainty.

\section{Summary of findings and assessment of the certainty of the evidence}

We prepared 'Summary of findings' tables to summarise key findings of this review. We selected up to seven of the most important outcomes (including adverse outcomes with dropouts as an indicator) and presented standardised effect size estimates and $95 \% \mathrm{Cls}$, using the GRADE approach to assess the certainty of the body of evidence (GRADE Working Group). We interpreted the magnitude of SMD point estimates using conventions proposed by Cohen 1992, operationally defined as small, medium, and large effects (SMD 0.2, 0.5, and 0.8 respectively).

We used GRADEpro GDT software and followed standard methods as described in the Cochrane Handbook for Systematic Reviews of Interventions (Schünemann 2017), to prepare our 'Summary of findings' table. GRADE provides a system for classifying the 
certainty of an overall body of evidence. These include the following four potential grades of evidence:

1. high certainty: additional research is unlikely to change our confidence in the estimate of a treatment effect;

2. moderate certainty: additional research will impact on our confidence in the estimate and may change this estimate;

3. low certainty: additional research is very likely to change the estimate; and

4. very low certainty: any estimate of a treatment effect is uncertain.

This classification is based on several characteristics that comprised the body of evidence, including trial design, risk of bias, inconsistency, imprecision, indirectness and publication bias.

We decreased grading for the following reasons.

1. Serious $(-1)$ or very serious $(-2)$ trial limitation for risk of bias.

2. Serious $(-1)$ or very serious $(-2)$ inconsistency between trial results.

3. Some $(-1)$ or major $(-2)$ uncertainty about directness.

4. Serious $(-1)$ or very serious $(-2)$ Imprecision of the pooled estimate.

5. Strong suspicion of publication bias and other considerations $(-1)$.

We included the following important outcomes. The primary time frame was medium-term follow-up (six to under 12 months) and continuous outcome.

1. Depression

2. Self-efficacy

3. Dropouts from treatment

4. Anxiety

5. PTSD
6. Re-exposure to IPV
7. Safety planning or safety behaviours, or both

We created 'Summary of findings' tables after we had entered data into RevMan 5 (Review Manager 2014), written up the results and conducted the 'Risk of Bias' assessment. However, we created the 'Summary of findings' table before writing the discussion, abstract and conclusions, to allow the opportunity to consider the impact of the risk of bias in the trials contributing to each outcome upon the mean therapy effect and our confidence in these findings.

\section{RE S U L T S}

\section{Description of studies}

See: Characteristics of included studies: Characteristics of excluded studies; Characteristics of ongoing studies

\section{Results of the search}

Once we had identified and deleted duplicate citations ( $n=2617$ ), the search identified 4835 abstracts relevant to the review. After deletion of irrelevant articles based on titles and abstracts screened by $\mathrm{MH}$ and $\mathrm{KH}$ (inter-rater reliability Kappa $=0.89,95 \% \mathrm{Cl} 0.88$ to 0.91), 198 full-text records were retrieved for further investigation. Four of the review authors (MH, JT, LOD, GG) contributed to screening and assessing the eligibility of these full-text records (inter-rater reliability Kappa $=0.80,95 \% \mathrm{Cl} 0.72$ to 0.89 ), with any disagreements resolved in negotiation with the senior author $\mathrm{KH}$. Examples of reasons for exclusion were that the trial evaluated advocacy, empowerment or safety planning interventions (e.g. Eden 2015; Kaslow 2010; Pallitto 2016; Taft 2009), couple-based therapies (e.g. Fals-Stewart 2002), or focused on psychological therapies but used a convenience sample with no randomisation (e.g. Zust 2000), see Excluded studies for further details. In total, 33 trials (reported in 65 publications) met the inclusion criteria for the current review, see Figure 1. All were available in English and were published in peer-reviewed journals. 
Figure 1. Trial flow diagram

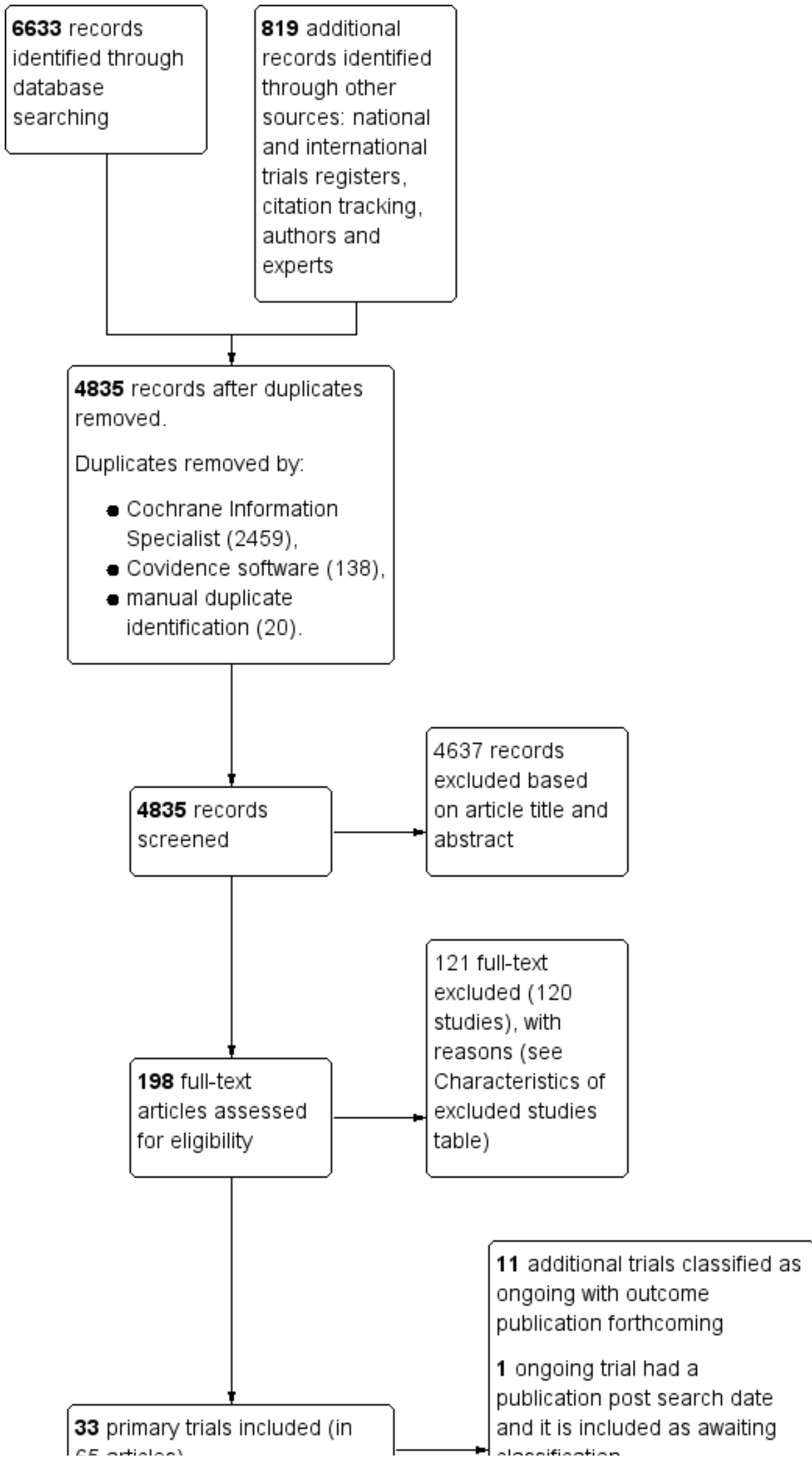


Figure 1. (Continued)

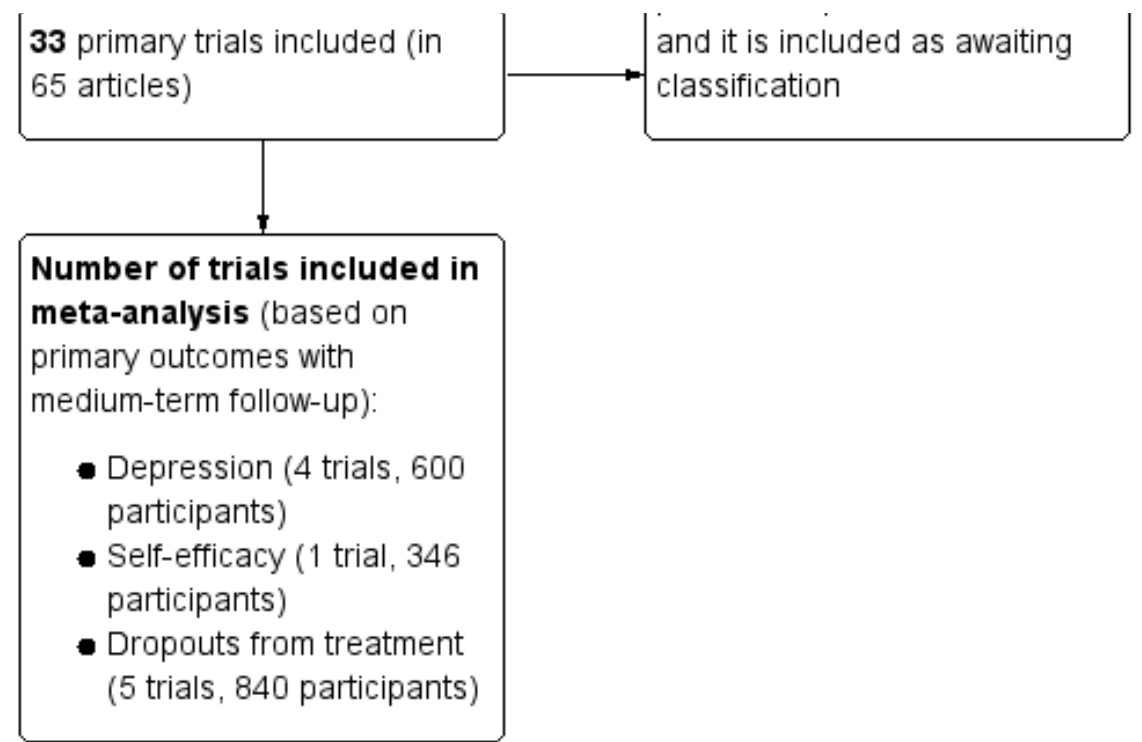

We classified 11 trials as ongoing with outcome publication forthcoming (see Characteristics of ongoing studies). Additionally, one trial was initially classified as ongoing, but the outcome paper was published after our updated search date (Akhtari 2019; see Studies awaiting classification).

\section{Trial characteristics}

\section{Trial designs}

Twenty-five RCTs (Akor 2019; Bowland 2012; Bryant 2017; Cheung 2019; Choo 2016; Cohen 2013; Kiely 2010; Ferrari 2018; Ghahari 2017; Gilbert 2006; Gilbert 2016; Graham-Bermann 2015; Hegarty 2019; Johnson 2011; Kokka 2019; Koopman 2005; Kubany 2004; Michalopoulou 2015; Myers 2015; Orang 2017; Rhodes 2015; Saftlas 2014; Stevens 2015; Tirado-Muñoz 2015; Zlotnick 2019), two feasibility RCTs (Jaffe 2017; Zlotnick 2011), three clusterRCTs (Hegarty 2013; Hirani 2010; Saggurti 2014), and three quasi-experimental RCTs (Franzblau 2008; Hernandez-Ruiz 2005; Taghizadeh 2018), met the criteria for inclusion in this review. All citations in the narrative and quantitative synthesis refer to the main outcome papers. In terms of comparator interventions, most trials $(20,61 \%)$ consisted of usual care or minimal interventions such as screening, information provision and referral to community services such as women's shelters. The remaining trials included psychological therapies in comparison with no treatment (seven trials) or waiting-list/delayed treatment (six trials). In this review, there were no cross-over trials.

\section{Trial location}

Most trials $(19,58 \%)$ were conducted in the USA (Bowland 2012; Choo 2016; Cohen 2013; Franzblau 2008; Gilbert 2006; Gilbert 2016; Graham-Bermann 2015; Hernandez-Ruiz 2005; Jaffe 2017; Johnson 2011; Kiely 2010; Koopman 2005; Kubany 2004; Myers 2015; Rhodes 2015; Saftlas 2014; Stevens 2015; Zlotnick 2011; Zlotnick 2019). Three were conducted in Iran (Ghahari 2017; Orang 2017; Taghizadeh 2018). Two trials were conducted in Australia (Hegarty 2013; Hegarty 2019) and Greece (Kokka 2019; Michalopoulou 2015). Single trials were conducted in China (Cheung 2019), India (Saggurti 2014), Kenya (Bryant 2017), Nigeria (Akor 2019), Pakistan

(Hirani 2010), Spain (Tirado-Muñoz 2015), and the UK (Ferrari 2018). Most trials $(28,85 \%)$ were developed, implemented, evaluated and published over the last decade.

\section{Types of interventions}

We classified the experimental interventions according to CCDAN psychological therapies. These included 11 integrative therapies (Bryant 2017; Choo 2016; Graham-Bermann 2015; Hegarty 2013; Hegarty 2019; Hirani 2010; Rhodes 2015; Saftlas 2014; Stevens 2015; Zlotnick 2011; Zlotnick 2019), nine humanistic therapies (Akor 2019; Franzblau 2008; Gilbert 2016; Kokka 2019; Koopman 2005; Michalopoulou 2015; Orang 2017; Saggurti 2014; Taghizadeh 2018), six CBT (Cohen 2013; Kiely 2010; Ferrari 2018; Gilbert 2006; Johnson 2011; Tirado-Muñoz 2015), four third-wave CBTs (Ghahari 2017; Jaffe 2017; Kubany 2004; Myers 2015), and three other psychologically-orientated Interventions (Bowland 2012; Cheung 2019; Hernandez-Ruiz 2005). We did not classify any trials as psychodynamic therapies in this review. In addition, consistent with our protocol (Tan 2018), we excluded systemic therapies.

\section{Content and main therapeutic techniques}

While we classified the trials according to CCDAN classification of psychological therapies, these included a diverse range of therapies such as narrative exposure therapy (e.g. Orang 2017), traumainformed cognitive therapy (e.g. Kubany 2004), mindfulness-based cognitive therapy (e.g. Ghahari 2017), and spiritually focused psychological intervention (e.g. Bowland 2012). Overall, the intervention models and main therapeutic techniques employed in the trials were heterogeneous. For example, models included trauma recovery and empowerment model (e.g. Bowland 2012), transtheoretical model and psychosocial readiness model (e.g. Hegarty 2013). Some trials were informed by mindfulness-based cognitive theories (e.g. Ghahari 2017), while others were informed by interpersonal psychotherapeutic theories (e.g. Zlotnick 2019). Overall the psychological therapies included a very diverse range of therapeutic techniques such as patient-centred care and active listening, motivational interviewing, psycho-education, cognitive restructuring, social-skills building, problem-solving techniques for validating women's experiences and feelings, and relaxation 
techniques. Most of the trials $(29,88 \%)$ were delivered face-to-face, with the majority being individual (16,55\%), followed by group (11, $38 \%)$, with only a few combining individual and group sessions (2, $7 \%)$. All trials were considered pragmatic interventions, that is, the psychological therapies were tailored to meet the needs and wants of the participating women.

\section{Duration of interventions}

Twenty trials $(61 \%)$ involved five or more psychological sessions (Bowland 2012; Bryant 2017; Cheung 2019; Cohen 2013; Kiely 2010; Ferrari 2018; Ghahari 2017; Gilbert 2006; Graham-Bermann 2015; Hegarty 2013; Hirani 2010; Johnson 2011; Kokka 2019; Kubany 2004; Michalopoulou 2015; Myers 2015; Orang 2017; Saggurti 2014; Stevens 2015; Tirado-Muñoz 2015). Thirteen trials (39\%) involved up to four psychological sessions (Akor 2019; Choo 2016; Franzblau 2008; Gilbert 2016; Hegarty 2019; Hernandez-Ruiz 2005; Jaffe 2017; Koopman 2005; Rhodes 2015; Saftlas 2014; Taghizadeh 2018; Zlotnick 2011; Zlotnick 2019). Overall, the average number of psychological sessions was 9.5 (range two to 50 sessions).

\section{Person delivering the intervention}

In this review, there were 18 trials (55\%) of psychological therapies delivered by healthcare workers including allied health and medical practitioners (Cohen 2013; Kiely 2010; Franzblau 2008; Graham-Bermann 2015; Hegarty 2013; Hirani 2010; Jaffe 2017; Johnson 2011; Koopman 2005; Kubany 2004; Myers 2015; Orang 2017; Rhodes 2015; Saggurti 2014; Stevens 2015; Taghizadeh 2018; Tirado-Muñoz 2015; Zlotnick 2011). Seven psychological trials were delivered by non-healthcare workers including community support workers and women advocates with training in psychological techniques (Bowland 2012; Bryant 2017; Cheung 2019; Ferrari 2018; Gilbert 2006; Hernandez-Ruiz 2005; Saftlas 2014). In addition, three trials were delivered online or led by facilitators, for example, via a website (Choo 2016; Gilbert 2016; Hegarty 2019). In five trials it was unclear about the person (e.g. field co-ordinators) delivering the intervention (Akor 2019; Ghahari 2017; Kokka 2019; Michalopoulou 2015; Zlotnick 2019). Nine trials reported the gender of the therapist or healthcare worker delivering the intervention, with the majority (78\%) being only female (Bowland 2012; Choo 2016; Franzblau 2008; Hernandez-Ruiz 2005; Orang 2017; Stevens 2015; Taghizadeh 2018). Specifically, in Hegarty 2013 and Kubany 2004, 62\% and 84\% respectively, were female therapists.

\section{Funding of trials and conflict of interest}

Twenty-six trials (79\%) reported sponsorship and funding source. These included national institutes and peak funding bodies for health and medical research (e.g. Cheung 2019; Kiely 2010; Ferrari 2018; Hegarty 2013), university-based grants (e.g. Hirani 2010; Taghizadeh 2018), and other sources. Most trials explicitly indicated that these funding bodies did not contribute to the development, design, implementation, evaluation and knowledge dissemination activities. Overall, 23 trials (70\%) provided information about any conflict of interests. Whilst most trials reported no conflicts of interest, including financial relationships with commercial interests, two trials disclosed potential competing interests (Ferrari 2018; Johnson 2011). These included receiving payment for training individuals delivering the psychological intervention, if the psychological trial was approved to be implemented in service settings (Ferrari 2018); and one trial author provided the medication for the treatment of postpartum depression in a separate trial (Johnson 2011). Overall, no other competing interests were disclosed.

\section{Participant characteristics}

A total of 77,006 women aged 16 years and older were approached to take part in the included trials (16 years of age is the minimum age for sexual consent in most countries). Of these, 8297 (11\%) met the main inclusion criteria of women who self-reported previous or recent experience of IPV. Of those eligible, 2343 (28\%) declined to participate; $5954(72 \%)$ signed written informed consent, and 5517 (67\%) fully completed baseline measure(s) and were then randomised into the experimental intervention $(2798,51 \%)$ or comparator interventions $(2719,49 \%)$. The average sample size for the experimental intervention was 82 and ranged from fewer than 10 (e.g. Hirani 2010; Tirado-Muñoz 2015), to more than 200 women participants (e.g. Rhodes 2015; Bryant 2017; Hegarty 2019). Fourteen trials (43\%) reported a priori sample power analysis (Akor 2019; Bowland 2012; Bryant 2017; Cheung 2019; Kiely 2010; Ferrari 2018; Gilbert 2016; Graham-Bermann 2015; Hegarty 2013; Hegarty 2019; Johnson 2011; Rhodes 2015; Saftlas 2014; Zlotnick 2011).

\section{Participants' socio-demographic characteristics}

The age range of the women recruited extended from 16 (e.g. Hegarty 2019) to 83 years (e.g. Bowland 2012). The average age of the total sample was 37 years (range 16 to 83; SD 7.8), with no differences in age between the experimental (mean 36.1; SD 7.5) and comparator interventions (mean 35.9; SD 8.1). The socioeconomic status of women was generally low, with approximately $66 \%$ (3662 women) of the total sample unemployed. None of the trials reported any information about financial dependence or economic support from the intimate partner. In terms of education, the participating women had completed approximately 12.5 years of education (SD 3.2), with around 51\% (2824) having completed secondary education. In terms of relationship status, $54 \%$ (2954) were currently married or living with a partner, whereas approximately $38 \%$ (2111) were divorced, separated, or widowed. More than $90 \%$ of women in four trials were married (Cheung 2019; Orang 2017; Saggurti 2014; Tirado-Muñoz 2015). Fourteen trials reported on the number of dependent children (Ghahari 2017; Gilbert 2006; Graham-Bermann 2015; Hegarty 2013; Hegarty 2019; Hernandez-Ruiz 2005; Hirani 2010; Jaffe 2017; Johnson 2011; Kokka 2019; Koopman 2005; Myers 2015; Rhodes 2015; Saggurti 2014), with an average of two dependent children. Participants' ethnic backgrounds were diverse across trials, and included white, African American, Asian/Pacific Islander, multiracial/other. For example, in one trial (Gilbert 2006), women identified as Latina (59.3\%), African American (15.6\%), and white (20.6\%), whilst some trials involved single ethnic groups such as Chinese (Cheung 2019), Greek (Kokka 2019) and Iranian (Orang 2017).

\section{Participant recruitment settings}

Recruitment settings of participants included healthcare, community, and shelter or refuge settings. Fifteen trials recruited women from healthcare settings such as emergency departments, family doctor clinics, psychiatric clinics, prenatal and family planning clinics, substance use and treatment services (Akor 2019; Bowland 2012; Choo 2016; Cohen 2013; Kiely 2010; Ghahari 2017; Gilbert 2006; Hegarty 2013; Rhodes 2015; Saftlas 2014; Stevens 2015; Taghizadeh 2018; Tirado-Muñoz 2015; Zlotnick 2011; Zlotnick 2019). Nine trials used community settings such as community adult literacy centres and universities (Bryant 2017; Franzblau 2008; 
Gilbert 2016; Hegarty 2019; Hirani 2010; Jaffe 2017; Kokka 2019; Koopman 2005; Saggurti 2014). Four trials recruited women from victim services and shelter settings (Hernandez-Ruiz 2005; Johnson 2011; Kubany 2004; Michalopoulou 2015). Five trials recruited from a combination of either community, health or shelter or refuge settings (Cheung 2019; Ferrari 2018; Graham-Bermann 2015; Myers 2015; Orang 2017).

\section{Participants' experience of IPV, other forms of violence and co- morbidities}

All women included in this review had reported recent or past experiences of any form of IPV. More specifically, included women were screened positive for recent experience of IPV during the previous six months (9 trials, 27\%; Choo 2016; Cohen 2013; Gilbert 2006; Hegarty 2019; Johnson 2011; Orang 2017; Rhodes 2015; Saggurti 2014; Tirado-Muñoz 2015), previous 12-months (8 trials, 24\%; Kiely 2010; Ferrari 2018; Hegarty 2013; Jaffe 2017; Saftlas 2014; Stevens 2015; Zlotnick 2011; Zlotnick 2019), or previous two years (2 trials, 6\%; Cheung 2019; Franzblau 2008). The remaining trials included women who reported ever having experienced IPV at some stage in their lives (14 trials, 42\%; Akor 2019; Bowland 2012; Bryant 2017; Ghahari 2017; Gilbert 2016; Graham-Bermann 2015; Hernandez-Ruiz 2005; Hirani 2010; Kokka 2019; Koopman 2005; Kubany 2004; Michalopoulou 2015; Myers 2015; Taghizadeh 2018). Six trials reported experience of other forms of violence and abuse (Bowland 2012; Cohen 2013; Johnson 2011; Kubany 2004; Rhodes 2015; Zlotnick 2011). For example, most women reported histories of multiple traumas in addition to IPV (Kubany 2004). These included experience of intense fear, helplessness or horror in response to a mean of 9.0 (SD 4.2) types of events listed on the Traumatic Life Events Questionnaire.

\section{Participants' experience of mental health conditions}

Although 19 trials reported on depression scores at baseline, most were means on a scale, which was difficult to translate into diagnoses of depression due to the varied scales used. Hence, it was unclear whether participants met the clinical and diagnostic threshold for mental health conditions such as depression, anxiety or PTSD. Nevertheless, some trials reported on clinical cut-off points for depression, anxiety and PTSD. For example, in Rhodes $2015,86 \%$ of women had scores above the clinical cut-off points for depression and 40\% had PTSD; in Johnson 2011, $61 \%$ of women had major depression; in Hegarty 2013, 50\% had depression and $70 \%$ had anxiety; in Cohen 2013, 77\% of women had PTSD; and in Bryant 2017, 20\% of women reported suicidal intention in the previous month.

\section{Excluded studies}

We excluded 120 studies (121 references) that did not meet the inclusion criteria. In some cases, judgement was difficult, and this led to extended discussion between the review authors to reach a consensus. Overall, decisions for trial exclusions can be described under the headings of (1) women, (2) intervention, and (3) methodological characteristics.

1. Women's characteristics: we excluded trials that recruited women with a history of sexual assaults, rape and sexual aggression that were not by an intimate partner (e.g. Anderson 2010; Bass 2013; Echeburúa 1996; Foa 2005; Leiner 2012; Roemer 2004; Rothbaum 1997). Further, we excluded samples of women who had been sexually abused and traumatised as children (e.g. Fallot 2011; Morrissey 2005; Resick 2012). In Rychtarik 2005, the sample included women from both nonviolent and violent relationships with no separate analyses. We also excluded trials with adolescent participants at ninth grade or with an average age of 13.6 years (e.g. Foshee 2016; Gonzalez Guarda 2015; Shirk 2014).

2. Intervention characteristics: we excluded trials that evaluated advocacy interventions (e.g. Hyman 2002; Taft 2009; Tiwari 2010), or empowerment- or safety-focused interventions (e.g. Bahadir Yilmaz 2018; Constantino 2005; Eden 2015; Kaslow 2010; Pallitto 2016; Taft 2009; Tiwari 2010). In addition, we excluded couple-based therapies (e.g. Brannen 1996; O'Leary 1999; Taft 2017; Zou 2010), and HIV/sexually-transmitted infection (STI) risk reduction interventions with women who experienced IPV (e.g. Carlson 2012; Melendez 2003; Wagman 2015).

3. Methodological characteristics: we excluded trials that compared two types of interventions with no comparator interventions satisfying our criteria. For example, assessing efficacy of CBT group intervention in comparison to a group of women who received CBT-based self-manual (Latif 2017), emotion-focused intervention versus goal-orientated intervention (McWhirter 2011), Eye movement desensitisation reprogramming (EMDR) versus relaxation training with a singlesubject research design (Colosetti 2000), exposure techniques versus communication skills training (Crespo 2010), childparent psychotherapy compared with case management plus individual counselling (Liberman 2005), or forgiveness therapy versus alternative anger validation, interpersonal skill-building intervention (Reed 2006). Further, we excluded trials that used availability sampling with no randomisation. For example, we excluded Tarquinio 2012 because participants were offered the option of choosing EMDR or eclectic therapy.

\section{Risk of bias in included studies}

We assessed methodological quality according to the Cochrane Collaboration's tool for assessing risk of bias (Higgins 2017). Review authors ( $\mathrm{MH}, \mathrm{JT}, \mathrm{LOD}, \mathrm{GG}, \mathrm{AT}$ ) contributed to the assessment of the risk of bias within each included trial based on domains of bias with ratings of 'high' (high risk of bias), 'low' (low risk of bias), and 'unclear' (uncertain risk of bias) using Covidence. Summaries are included in Figure 2 and Figure 3. 
Figure 2. Risk of Bias graph: review authors' judgements about each risk of bias item presented as percentages across all included trials.

Random sequence generation (selection bias)

Allocation concealment (selection bias) Blinding of participants and personnel (performance bias): All outcomes Blinding of outcome assessment (detection bias): All outcomes Incomplete outcome data (attrition bias): All outcomes

Selective reporting (reporting bias)

Other bias
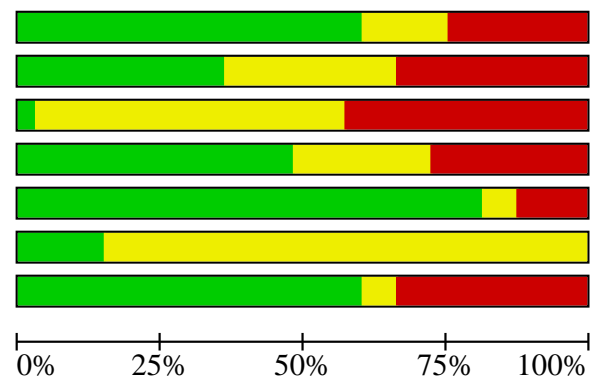
Figure 3.

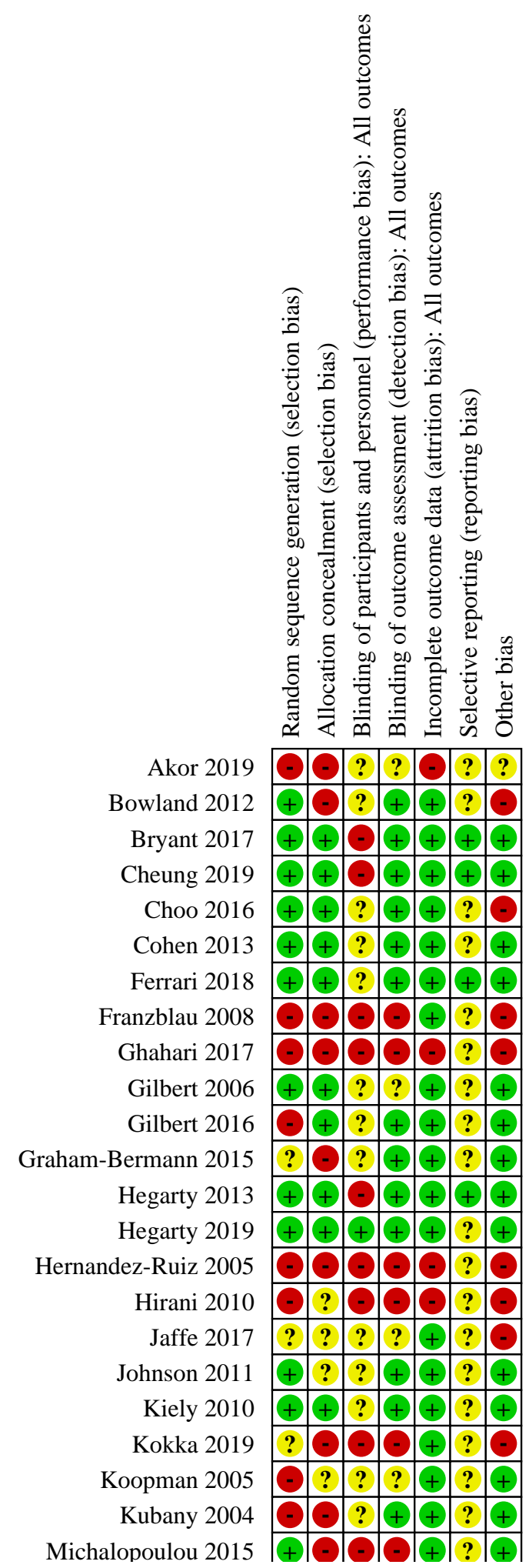


Figure 3. (Continued)

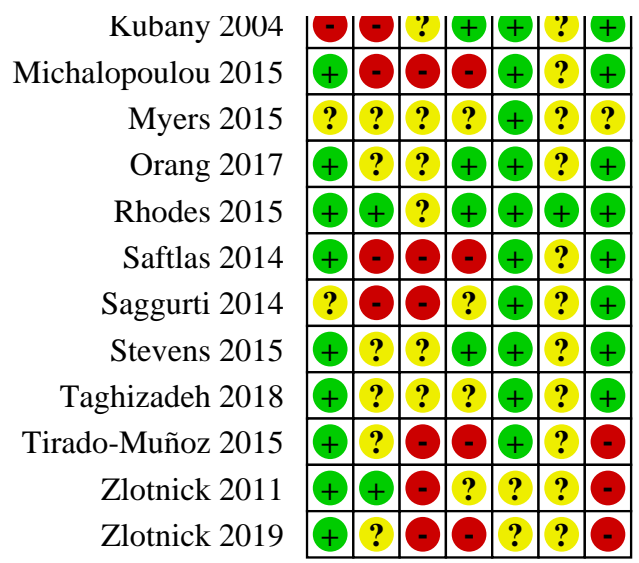

\section{Assessment of risk of bias for individually randomised trials}

\section{Random sequence generation (selection bias)}

All included trials described treatment allocation as random, but only 20 trials described reliable low-risk random sampling strategies, for example, the use of a computer-generated random number table by staff who were independent of the trial (e.g. Bryant 2017; Hegarty 2013; Stevens 2015; Tirado-Muñoz 2015). Whilst eight trials used various methods with a high likelihood of systematic bias, five trials provided insufficient information to enable an assessment of the method used to generate the allocation sequences. These methods included strategies such as consecutive enrolment and allocation (e.g. Kubany 2004), or there was insufficient information and evidence of sequence generation (e.g. Hernandez-Ruiz 2005).

\section{Allocation concealment (selection bias)}

Twelve trials described reliable procedures to conceal the allocation of participant status (Bryant 2017; Cheung 2019; Choo 2016; Cohen 2013; Kiely 2010; Ferrari 2018; Gilbert 2006; Gilbert 2016; Hegarty 2013; Hegarty 2019; Rhodes 2015; Zlotnick 2011); for example, Cheung 2019 used a computer-generated list, recorded by an investigator who was not involved in participant recruitment, and placed in numbered, sequential, sealed, opaque envelopes. Similarly, Ferrari 2018 used a remote, independent, automated telephone randomisation service to conceal allocation. However, in 10 trials allocation concealment was not feasible, due to various logistical constraints such as limited clinic space and staffing (e.g. Saftlas 2014). We rated these trials as high risk of bias. Further, in the remaining 11 trials it was unclear whether allocation concealment was satisfactory.

\section{Blinding of participants and personnel (performance bias)}

Blinding is an important aspect of clinical trials in order to avoid and minimise bias in the design, implementation and evaluation of treatment efficacy. However, in psychological trials, while it is difficult to blind the participants and the clinical staff delivering the therapies, blinding individuals analysing and interpreting the results is feasible. In this review, only one trial (Hegarty 2019), delivered through a website, satisfactorily blinded the participants and the research team until after analysis of the final follow-up data. However, in most trials $(18,55 \%)$ participants and those delivering the psychological therapies were likely to be aware of treatment allocation (Akor 2019; Bowland 2012; Choo 2016; Cohen 2013; Ferrari 2018; Gilbert 2006; Gilbert 2016; GrahamBermann 2015; Jaffe 2017; Johnson 2011; Kiely 2010; Koopman 2005; Kubany 2004; Myers 2015; Orang 2017; Rhodes 2015; Stevens 2015; Taghizadeh 2018). While these trials minimised the possible effect of knowledge of group assignment using various strategies, it remained unclear as to whether all participants as well as the clinical and research personnel remained blinded. Further, in 14 trials $(42 \%)$, due to the nature of the psychological therapies (e.g. professional training in addition to provision of therapy with women), it was not possible to blind the women to their status as treatment or comparator group (Bryant 2017; Cheung 2019; Franzblau 2008; Ghahari 2017; Hegarty 2013; Hernandez-Ruiz 2005; Hirani 2010; Kokka 2019; Michalopoulou 2015; Saftlas 2014; Saggurti 2014; Tirado-Muñoz 2015; Zlotnick 2011; Zlotnick 2019). We rated these trials at high risk of bias, since the expectations of the person delivering the psychological therapy may have contributed to the observed treatment outcome.

\section{Blinding of outcome assessment (detection bias)}

Sixteen trials provided sufficient details of the strategies taken to ensure that blinded assessment of the primary and secondary outcomes and were rated as low risk of bias (Bowland 2012; Bryant 2017; Cheung 2019; Choo 2016; Cohen 2013; Kiely 2010; Ferrari 2018; Gilbert 2016; Graham-Bermann 2015; Hegarty 2013; Hegarty 2019; Johnson 2011; Kubany 2004; Orang 2017; Rhodes 2015; Stevens 2015). Further, some trials explicitly reported that outcome assessors were not blinded to the trial conditions and were rated as high risk of bias (Franzblau 2008; Ghahari 2017; HernandezRuiz 2005; Hirani 2010; Kokka 2019; Michalopoulou 2015; Saftlas 2014; Tirado-Muñoz 2015; Zlotnick 2019). The remaining eight trials provided insufficient information as to whether data collectors were blinded. We rated these as unclear (Akor 2019; Gilbert 2006; Jaffe 2017; Koopman 2005; Myers 2015; Saggurti 2014; Taghizadeh 2018; Zlotnick 2011).

\section{Incomplete outcome data (attrition bias)}

Attrition bias is one of the main limitations threatening the internal validity of the included trials. Overall, the attrition rate was on average $22 \%$ for the experimental and $20 \%$ for the comparator interventions. Most trials $(28,85 \%)$ reasonably described attrition 
and reasons for exclusions of participants (Bowland 2012; Bryant 2017; Cheung 2019; Choo 2016; Cohen 2013; Kokka 2019; Kiely 2010; Ferrari 2018; Franzblau 2008; Gilbert 2006; Gilbert 2016; GrahamBermann 2015; Hegarty 2013; Hegarty 2019; Jaffe 2017; Johnson 2011; Kokka 2019; Koopman 2005; Kubany 2004; Michalopoulou 2015; Myers 2015; Orang 2017; Rhodes 2015; Saftlas 2014; Saggurti 2014; Stevens 2015; Taghizadeh 2018; Tirado-Muñoz 2015). In terms of intent-to-treat analysis, 17 trials used various imputation methods that included all participants, regardless of whether the participant received the psychological therapies. However, in four trials this risk was high (Akor 2019; Ghahari 2017; Hernandez-Ruiz 2005; Hirani 2010) or unclear ( Zlotnick 2011; Zlotnick 2019).

\section{Selective outcome reporting (reporting bias)}

In all trials, outcome measures described in the methods section were also reported in the results sections of the main publication, but this was not sufficient to meet the criteria for low risk for selective outcome reporting. Nevertheless, we rated five trials at low risk of bias, because the primary and secondary outcomes described in the published protocol were also fully reported in the main trial publication (Bryant 2017; Cheung 2019; Ferrari 2018; Hegarty 2013; Rhodes 2015). The remaining trials we rated at unclear risk for selective outcome reporting, due to insufficient evidence from either the main publication or information from trial register sites.

\section{Additional factors that may have an impact on outcomes}

\section{Therapist allegiance/conflict of interest}

In this review, there were four trials where the 'therapist' was also either the author of the trial or conducted the assessment with women in the experimental or comparator interventions, and we rated these at 'high' risk of bias (Bowland 2012; Hernandez-Ruiz 2005; Kubany 2004; Zlotnick 2019). In 11 trials, therapy sessions appeared to have been conducted by independent healthcare or non-healthcare workers who appeared not to have a vested interest in the provided therapies, we rated this as low risk of bias (Cheung 2019; Cohen 2013; Kiely 2010; Ferrari 2018; Gilbert 2006; Graham-Bermann 2015; Hirani 2010; Orang 2017; Rhodes 2015; Stevens 2015; Tirado-Muñoz 2015). Further, four trials delivered the psychological therapies online, for example, via a website, and we rated these as low risk of bias (Choo 2016; Gilbert 2016; Hegarty 2019; Zlotnick 2019). However, for the remaining 14 trials, there were either insufficient details to assess therapist allegiance or unclear conflict of interest when the primary authors also delivered the training (Akor 2019; Bryant 2017; Franzblau 2008; Ghahari 2017; Hegarty 2013; Jaffe 2017; Johnson 2011; Koopman 2005; Michalopoulou 2015; Myers 2015; Saftlas 2014; Saggurti 2014; Taghizadeh 2018; Zlotnick 2011).

\section{Therapist qualifications and training}

Twenty-one trials provided adequate details about who delivered the intervention, therapist qualifications or training prior to delivery of the psychological therapies; we rated these trials at 'low' risk of bias (Bowland 2012; Bryant 2017; Cheung 2019; Cohen 2013; Kiely 2010; Ferrari 2018; Franzblau 2008; Graham-Bermann 2015; Hegarty 2013; Hegarty 2019; Jaffe 2017; Johnson 2011; Kubany 2004; Myers 2015; Orang 2017; Rhodes 2015; Saftlas 2014; Saggurti 2014; Stevens 2015; Taghizadeh 2018; Tirado-Muñoz 2015). We rated the remaining 12 trials at unclear risk of bias because there was insufficient information provided about the nature and level of training or therapists' qualifications (Akor 2019; Choo 2016; Ghahari 2017; Gilbert 2006; Gilbert 2016; Hernandez-Ruiz 2005; Hirani 2010; Kokka 2019; Koopman 2005; Michalopoulou 2015; Zlotnick 2011; Zlotnick 2019).

\section{Researcher allegiance/conflict of interest}

In over half of the trials $(17,52 \%)$, trial authors reported no competing interests (Bryant 2017; Cheung 2019; Choo 2016; Cohen 2013; Kiely 2010; Gilbert 2016; Hegarty 2013; Hegarty 2019; Johnson 2011; Koopman 2005; Myers 2015; Rhodes 2015; Saggurti 2014; Stevens 2015; Taghizadeh 2018; Tirado-Muñoz 2015; Zlotnick 2019). However, in 13 trials this risk remained unclear because of either insufficient details or whether the researchers also conducted the intervention or trained and supervised the individuals who delivered the psychological therapies (Akor 2019; Franzblau 2008; Ghahari 2017; Gilbert 2006; Graham-Bermann 2015; Hirani 2010; Jaffe 2017; Kokka 2019; Kubany 2004; Michalopoulou 2015; Orang 2017; Saftlas 2014; Zlotnick 2011). We rated the remaining three trials at high risk of bias, because the same researcher also delivered the intervention (Bowland 2012; Hernandez-Ruiz 2005), or trial authors explicitly declared competing interests (Ferrari 2018).

\section{Protection against contamination}

Generally, psychological support for or with women who experience IPV occurred within broad routine clinical practice settings such as medical clinics, community health centres, women's shelters and domestic violence services. Further, in terms of psychological clinical trials, women assigned to experimental or comparator interventions may have received some level of psycho-education and support from their interaction with clinical staff, social networks or interaction with other women in the case of group-based interventions. Nevertheless, in this review, 16 trials used reasonable strategies to minimise risk of contamination (Akor 2019; Bowland 2012; Cheung 2019; Choo 2016; Cohen 2013; Kiely 2010; Ferrari 2018; Franzblau 2008; Gilbert 2006; Hegarty 2019; Koopman 2005; Kubany 2004; Rhodes 2015; Stevens 2015; Taghizadeh 2018; Tirado-Muñoz 2015). Strategies to minimise contamination included individual-therapy format, experimental and comparator interventions conducted at dispersed geographical settings, as well as provision of usual care or standard list of social services recourse to all participating women. Three trials used a cluster-RCT methodology, which minimises risk of contamination (Hegarty 2013; Hirani 2010; Saggurti 2014). However, in 10 trials, it was unclear how trial authors viewed or protected against contamination (Bryant 2017; Ghahari 2017; Gilbert 2016; Graham-Bermann 2015; Jaffe 2017; Johnson 2011; Michalopoulou 2015; Orang 2017; Zlotnick 2011; Zlotnick 2019). We rated the remaining four trials at high risk of bias because it was highly likely that individuals from both groups would have interacted with each other and exchanged information about the interventions (Hernandez-Ruiz 2005; Kokka 2019; Myers 2015; Saftlas 2014).

\section{Reliability of outcome measures}

In the review authors' judgement, almost all trials (32, 97\%) included measures that had satisfactory psychometric properties with appropriate references. We rated the remaining trial at unclear risk of bias, because there was insufficient information about the reliability and psychometric properties of the items (Saggurti 2014). 


\section{Treatment fidelity}

Seventeen of the 33 trials reported use of some methodology to evaluate and assess treatment fidelity (Bowland 2012; Bryant 2017; Cheung 2019; Cohen 2013; Ferrari 2018; Gilbert 2006; Graham-Bermann 2015; Hegarty 2019; Johnson 2011; Kubany 2004; Michalopoulou 2015; Myers 2015; Rhodes 2015; Saggurti 2014; Stevens 2015; Tirado-Muñoz 2015; Zlotnick 2019). For example, $10 \%$ of randomly selected psychological sessions were attended by a supervisor, who used a checklist to ensure that relevant treatment elements were provided (e.g. Bryant 2017), or adherence to the treatment manual was discussed with a clinical psychologist on a weekly basis (e.g. Graham-Bermann 2015). However, we rated eight trials at high risk of bias because there was no evidence or information to suggest trial authors had attempted to assess treatment fidelity (Akor 2019; Franzblau 2008; Ghahari 2017; Hernandez-Ruiz 2005; Hirani 2010; Jaffe 2017; Kokka 2019; Taghizadeh 2018). We rated the remaining eight trials at unclear risk of bias because there was insufficient information or the taped sessions were used only for training purposes and not for fidelity assessment (Choo 2016; Kiely 2010; Gilbert 2016; Hegarty 2013; Koopman 2005; Orang 2017; Saftlas 2014; Zlotnick 2011).

\section{Other potential sources of bias}

We also considered other potential sources of bias, not listed above. We rated 20 trials at low risk of other bias (Bryant 2017; Cheung 2019; Cohen 2013; Kiely 2010; Ferrari 2018; Gilbert 2006; Gilbert 2016; Graham-Bermann 2015; Hegarty 2013; Hegarty 2019; Johnson 2011; Koopman 2005; Kubany 2004; Michalopoulou 2015; Orang 2017; Rhodes 2015; Saftlas 2014; Saggurti 2014; Stevens 2015; Taghizadeh 2018). However, we rated 11 trials at high risk of bias because of various issues such as risk of type II errors, baseline differences between groups or small sample sizes (Bowland 2012; Choo 2016; Franzblau 2008; Ghahari 2017; Hernandez-Ruiz 2005; Hirani 2010; Jaffe 2017; Kokka 2019; Tirado-Muñoz 2015; Zlotnick 2011; Zlotnick 2019). We rated the remaining two trials at unclear risk of bias because there was insufficient information about various methodological aspects of the trial such as intervention location, number of participants approached and then recruited, or detailed inclusion and exclusion criteria (Akor 2019; Myers 2015).

\section{Additional assessment of risk of bias for cluster-RCTs}

\section{Cluster consideration in analysis}

Two of the three cluster-RCTs reported appropriate statistical techniques (e.g. intra-class correlation) that accounted for cluster in analysis (Hegarty 2013; Saggurti 2014). However, we rated Hirani 2010 at high risk of bias because there was no evidence to suggest any cluster consideration in the statistical analysis.

\section{Loss of clusters}

We rated all three cluster-RCTs at low risk of bias (Hegarty 2013; Hirani 2010; Saggurti 2014). In terms of Hegarty 2013, in both the experimental and comparator intervention, we excluded only three clusters due to women's non-response or non-participation.

\section{Baseline imbalance between randomised groups}

We rated all three cluster-RCTs at low risk of bias (Hegarty 2013; Hirani 2010; Saggurti 2014). There were no significant differences at baseline between the randomised groups, although in Saggurti

Psychological therapies for women who experience intimate partner violence (Review)

Copyright ( 2020 The Cochrane Collaboration. Published by John Wiley \& Sons, Ltd.
2014, religion differed significantly between groups at baseline $(P$ $=0.03$ ).

\section{Identification and recruitment bias}

We rated all three cluster-RCTs at low risk of bias (Hegarty 2013; Hirani 2010; Saggurti 2014), because identification and selection bias were minimised through various strategies.

\section{Assessment of risk of bias for cross-over trials}

In this review, there were no cross-over trials.

\section{Effects of interventions}

See: Summary of findings 1 Psychological therapies for women who experience intimate partner violence

\section{Primary outcomes}

\section{Depression}

Nineteen trials (58\%) evaluated the effects of the psychological therapies on the primary outcome of depression as a continuous (Bowland 2012; Cheung 2019; Ferrari 2018; Franzblau 2008; Gilbert 2006; Graham-Bermann 2015; Hegarty 2019; Hirani 2010; Johnson 2011; Kokka 2019; Koopman 2005; Kubany 2004; Orang 2017; Saftlas 2014; Stevens 2015; Tirado-Muñoz 2015; Zlotnick 2011) or dichotomous outcome (Kiely 2010; Hegarty 2013). The trials measured depression using the Beck Depression Inventory (trials include Cheung 2019; Franzblau 2008; Graham-Bermann 2015; Hirani 2010; Johnson 2011; Kokka 2019; Koopman 2005; Kubany 2004; Tirado-Muñoz 2015), Center for Epidemiologic Studies Short Depression Scale (Hegarty 2019; Saftlas 2014; Stevens 2015), Patient Health Questionnaire (Ferrari 2018; Orang 2017), Brief Symptom Inventory (Gilbert 2006), Edinburgh Postnatal Depression Scale (Zlotnick 2011), Geriatric Depression Scale (Bowland 2012), Hospital Anxiety and Depression Scale (Hegarty 2013) and Johns Hopkins Depression Scale (Kiely 2010). In all scales, lower scores indicate a better outcome. The trials included CBT (Kiely 2010; Ferrari 2018; Gilbert 2006; Johnson 2011; Tirado-Muñoz 2015), humanistic therapies (Franzblau 2008; Kokka 2019; Koopman 2005; Orang 2017), integrative therapies (Graham-Bermann 2015; Hegarty 2013; Hegarty 2019; Hirani 2010; Saftlas 2014; Stevens 2015; Zlotnick 2011), third-wave CBTs (Kubany 2004) and other psychologically-orientated interventions (Cheung 2019; Bowland 2012). Most trials $(14,74 \%)$ included five or more psychological sessions. Similarly, most therapies $(12,63 \%)$ were delivered by healthcare workers.

While 19 trials provided data about depression, only four trials measured depression as a continuous outcome and two as a dichotomous outcome within our primary time frame of mediumterm follow-up (six to under 12 months). Pooled data showed probable beneficial effect of psychological therapies in reducing depression (continuous outcome: SMD $-0.24,95 \% \mathrm{Cl}-0.47$ to -0.01 ; four trials, 600 women; moderate-certainty evidence, low level of statistical heterogeneity $\mathrm{Chi}^{2}=4.62, \mathrm{P}=0.20, \mathrm{I}^{2}=35 \%$; see Analysis 1.1; and dichotomous outcome: OR $0.68,95 \% \mathrm{Cl} 0.47$ to 0.98; two trials, 528 women; high-certainty evidence, no evidence of statistical heterogeneity $\mathrm{Chi}^{2}=0.00, \mathrm{P}=0.95, \mathrm{I}^{2}=0 \%$; see Analysis 1.2).

In terms of short-term follow-up, results revealed that psychological therapies probably reduce depression (SMD -0.45 , 
$95 \% \mathrm{Cl}-0.67$ to $-0.22 ; 15$ trials, 1247 women; high level of statistical heterogeneity $\mathrm{Chi}^{2}=42.85, \mathrm{P}<0.001, \mathrm{I}^{2}=67 \%$ ), but there may be no evidence of beneficial effect of therapy within long-term followup (SMD $-0.08,95 \% \mathrm{Cl}-0.30$ to 0.14 ; three trials, 503 women; low level of statistical heterogeneity $\mathrm{Chi}^{2}=2.48, \mathrm{P}=0.29, \mathrm{I}^{2}=19 \%$; see Analysis 1.1).
We observed a tendency towards symmetrical funnel plots in Figure 4 , discarding possible publication and reporting biases. In addition, we conducted sensitivity and subgroup analyses.

Figure 4. Funnel plot of comparison: 1 Intervention vs Control, outcome: 1.1 Depression (continuous).

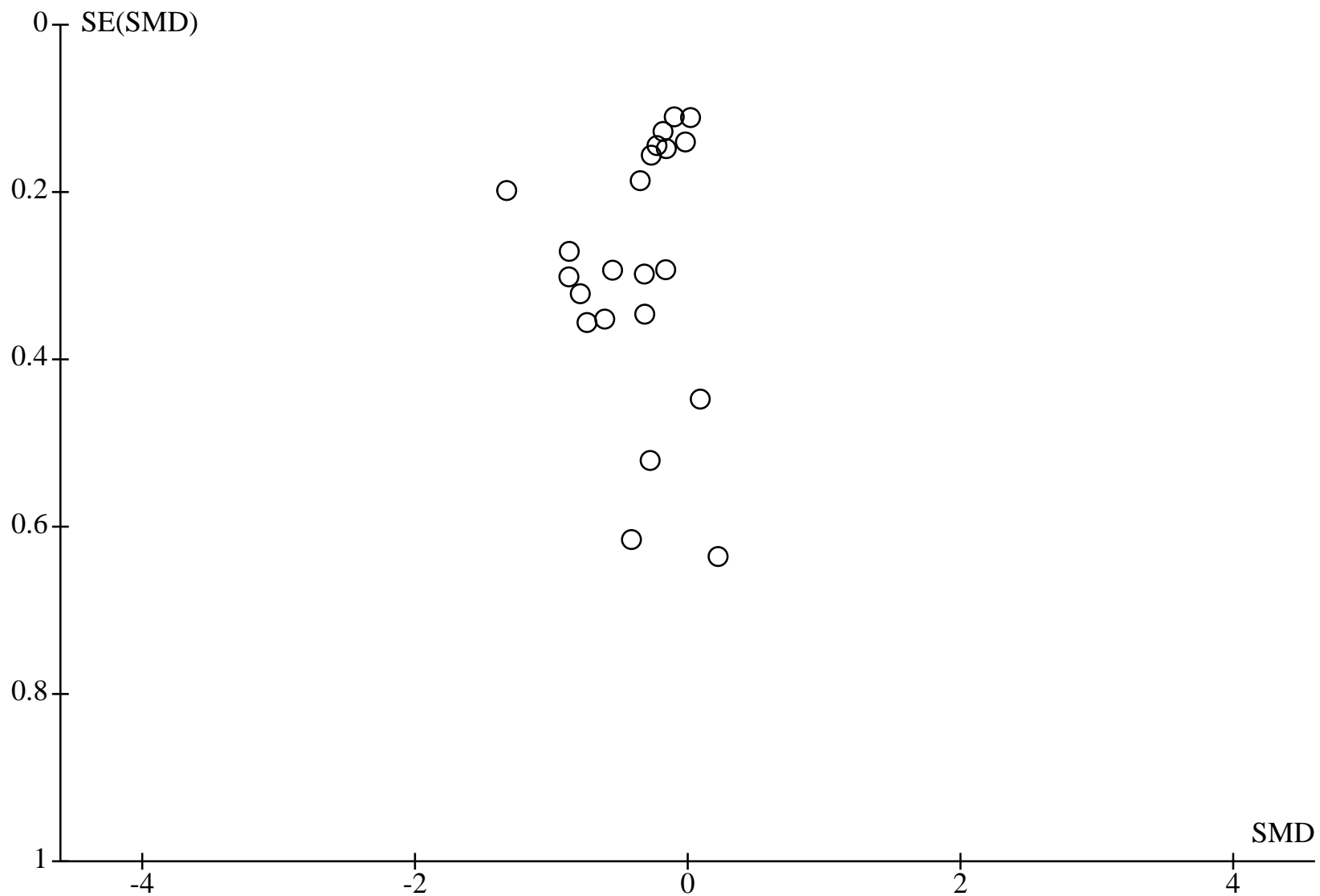

\section{Sensitivity analyses}

We conducted a sensitivity analysis based on allocation concealment (selection bias). Overall, seven trials with low risk of allocation concealment used continuous (Cheung 2019; Ferrari 2018; Gilbert 2006; Hegarty 2019; Zlotnick 2011), or dichotomous outcomes (Hegarty 2013; Kiely 2010), to test the efficacy of psychological therapies in reducing depression.

However, there was an insufficient number of trials with continuous outcome at low risk of bias with available data within medium-term follow-up. Hence, within short-term follow-up, results from three trials with low risk of bias indicated beneficial effects of therapy (SMD $-0.21,95 \% \mathrm{Cl}-0.43$ to 0.00 ), with no evidence of statistical heterogeneity $\left(\mathrm{Chi}^{2}=0.27, \mathrm{P}=0.87, \mathrm{I}^{2}=0 \%\right)$. However, within longterm follow-up, results from two trials with low risk of bias indicated no evidence of beneficial effect of therapy (SMD $-0.10,95 \% \mathrm{Cl}-0.38$ to 0.18$)$, with moderate level of statistical heterogeneity $\left(\mathrm{Chi}^{2}=2.26\right.$, $\left.P=0.13, I^{2}=56 \%\right)$.
In terms of dichotomous outcome, within medium-term follow-up, results indicated that fewer women reported depression following the psychological therapies (OR $0.68,95 \% \mathrm{Cl} 0.47$ to 0.98 ), with no evidence of statistical heterogeneity $\left(\mathrm{Chi}^{2}=0.00, \mathrm{P}=0.95, \mathrm{I}^{2}=0 \%\right)$.

\section{Subgroup analyses}

Results from the prespecified subgroup analyses for depression outcome are included in Analysis 1.3 (recruitment setting), Analysis 1.4 (type of intervention), Analysis 1.5 (intensity of intervention) and Analysis 1.6 (person delivering the intervention). All of the subgroups had insufficient trials with available data at our primary time frame of medium-term follow-up; hence we consistently based analyses on any follow-up time frames, with continuous data.

\section{Recruitment setting of participants}

Trials that evaluated effect of psychological therapies on depression recruited women from healthcare settings (six trials, Bowland 2012; Gilbert 2006; Saftlas 2014; Stevens 2015; TiradoMuñoz 2015; Zlotnick 2011), community (five trials, Franzblau 2008; 
Hegarty 2019; Hirani 2010; Kokka 2019; Koopman 2005), shelter/ refuge (Johnson 2011), and a combination of various settings such as community and shelter/refuge settings (three trials, Ferrari 2018; Graham-Bermann 2015; Kubany 2004), or healthcare and community settings (two trials, Cheung 2019; Orang 2017). There was no evidence of a difference between the subgroups based on recruitment setting of participants (test for subgroup differences: $\left.\mathrm{Chi}^{2}=3.43, \mathrm{df}=3, \mathrm{P}=0.33, \mathrm{I}^{2}=12.5 \%\right)$.

\section{Type of intervention}

There was no evidence of a difference between the subgroups based on type of intervention (test for subgroup differences: $\left.\mathrm{Chi}^{2}=4.93, \mathrm{df}=3, \mathrm{P}=0.18, \mathrm{I}^{2}=39.1 \%\right)$. Results indicate that trials classified as CBT (Ferrari 2018; Gilbert 2006; Johnson 2011; Tirado-Muñoz 2015), humanistic therapies (Franzblau 2008; Kokka 2019; Koopman 2005; Orang 2017), integrative therapies (Graham-Bermann 2015; Hegarty 2019; Hirani 2010; Saftlas 2014; Stevens 2015; Zlotnick 2011), and other psychologically-orientated Interventions (Cheung 2019; Bowland 2012), were probably beneficial in reducing depression.

\section{Intensity of interventions}

Twelve trials tested the effects of five or more psychological sessions in reducing depression in comparison with women receiving usual care (Ferrari 2018; Johnson 2011; Orang 2017; Stevens 2015; Tirado-Muñoz 2015), waiting list (Bowland 2012; Cheung 2019; Graham-Bermann 2015; Hirani 2010; Kokka 2019; Kubany 2004) and minimal intervention (Gilbert 2006). Further, five trials evaluated up to four sessions of psychological therapies with women in comparison with minimal intervention (Hegarty 2019; Koopman 2005; Saftlas 2014), usual care (Zlotnick 2011) and waiting list (Franzblau 2008). There was evidence of a difference between the subgroups based on intensity of intervention (test for subgroup differences: $\left.\mathrm{Chi}^{2}=10.11, \mathrm{df}=1, \mathrm{P}=0.001, \mathrm{I}^{2}=90.1 \%\right)$, with five or more sessions probably reducing depression (SMD -0.49 , $95 \% \mathrm{Cl}-0.73$ to $-0.25 ; 17$ trials, 1729 women).

\section{Person delivering the intervention}

Ten trials of psychological therapies used healthcare workers, including allied health and medical practitioners, to deliver the intervention (Franzblau 2008; Graham-Bermann 2015; Hirani 2010; Johnson 2011; Koopman 2005; Kubany 2004; Orang 2017; Stevens 2015; Tirado-Muñoz 2015; Zlotnick 2011). Five trials used nonhealthcare workers, including community support and women advocacy workers, to deliver the intervention (Bowland 2012; Cheung 2019; Ferrari 2018; Gilbert 2006; Saftlas 2014). There was no evidence of a difference between the subgroups based on the person delivering the intervention (test for subgroup differences: $\mathrm{Chi}^{2}=1.30, \mathrm{df}=1, \mathrm{P}=0.25, \mathrm{I}^{2}=23.0 \%$ ). Healthcare workers and nonhealth care workers delivering the psychological therapies were probably beneficial in reducing depression.

\section{Self-efficacy}

Four trials (12\%) measured and reported self-efficacy as a continuous outcome (Hegarty 2019; Hirani 2010; Kokka 2019; Saftlas 2014). They measured self-efficacy using the General SelfEfficacy Scale (Hegarty 2019; Hirani 2010 ; Kokka 2019), and the Domestic Violence Coping Self-Efficacy Measure (Saftlas 2014), with higher scores indicating a better outcome. We classified these trials as integrative therapies (Hegarty 2019; Hirani 2010; Saftlas 2014), and humanistic therapies (Kokka 2019). The trials recruited women from community (Hegarty 2019; Hirani 2010; Kokka 2019), and healthcare settings (Saftlas 2014). In terms of intervention intensity, Hegarty 2019 and Saftlas 2014 included up to four sessions, and Hirani 2010 and Kokka 2019 had five or more sessions.

There was only one trial with available data within our primary time frame of medium-term follow-up. Results indicated that there may be no evidence of beneficial effect of therapy (SMD $-0.12,95 \% \mathrm{Cl}$ -0.33 to 0.09 ; one trial, 346 women; low-certainty evidence; see Analysis 1.7).

There were insufficient trials available to conduct subgroup analyses.

\section{Dropouts from treatment}

All included trials in this review directly or indirectly reported the number of participants who dropped out from experimental and comparator intervention groups. A total of 5517 women with completed baseline assessment (67\% of those eligible for the trials) were randomly assigned to the experimental $(2798,51 \%)$ or comparator interventions $(2719,49 \%)$. Overall, the dropout rates ranged from $0 \%$ to $55 \%$ and $0 \%$ to $71 \%$ for the experimental and comparator interventions respectively. At medium-term followup, pooled data from five trials showed no evidence to support differences in dropouts between experimental and comparator interventions (SMD 1.04, 95\% $\mathrm{Cl} 0.75$ to 1.44; five trials, 840 women; no evidence of statistical heterogeneity $\mathrm{Chi}^{2}=3.07, \mathrm{P}=0.55, \mathrm{I}^{2}=0 \%$; see Analysis 1.8).

In addition, results showed no evidence of a difference in dropouts between experimental and comparator interventions within shortterm follow-up (SMD 1.21, 95\% Cl 0.98 to $1.48 ; 14$ trials, 3022 women; no evidence of statistical heterogeneity $\mathrm{Chi}^{2}=7.28, \mathrm{P}=$ $0.97, I^{2}=0 \%$, see Analysis 1.8). Similarly, results showed no evidence of a difference in dropouts between experimental and comparator interventions within long-term follow-up (SMD 1.08, 95\% Cl 0.85 to 1.38; six trials, 1655 women; no evidence of statistical heterogeneity $\mathrm{Chi}^{2}=5.62, \mathrm{P}=0.34, \mathrm{I}^{2}=11 \%$; see Analysis 1.8 ).

\section{Secondary outcomes}

\section{Mental health}

Four of the 33 trials (12\%) evaluated the effects of psychological therapies on general measures of mental health as a continuous outcome (Bryant 2017; Ferrari 2018; Hegarty 2013; Orang 2017). Trials measured mental health using a variety of measures including the 12-item General Health Questionnaire (Bryant 2017), Clinical Outcomes in Routine Evaluation-Outcome Measure (Ferrari 2018), Mental Health Status SF-12 (Hegarty 2013), and Perceived Stress Scale (Orang 2017). Lower scores indicate better mental health functioning. These trials included integrative therapies (Bryant 2017; Hegarty 2013), CBT (Ferrari 2018), and humanistic therapies (Orang 2017). All trials included five or more psychological sessions, with women recruited from healthcare settings (Hegarty 2013), community (Bryant 2017), healthcare and community (Orang 2017) and community and shelter/refuge settings (Ferrari 2018). All trials were delivered by a therapist, including two trials by healthcare workers (Hegarty 2013; Orang 2017). 
In terms of our primary time frame of medium-term follow-up results from two trials ( 219 women) showed that there may be no evidence of a difference between groups (SMD $-0.11,95 \% \mathrm{Cl}-0.38$ to 0.16$)$. There was no evidence of statistical heterogeneity $\left(\mathrm{Chi}^{2}=\right.$ $0.03, P=0.87,12=0 \%$; see Analysis 1.9 ). However, results revealed that psychological therapies may improve mental health within short-term follow-up (SMD $-0.34,95 \% \mathrm{Cl}-0.55$ to -0.13 ; two trials, 353 women; no evidence of statistical heterogeneity $\mathrm{Chi}^{2}=0.00, \mathrm{P}$ $=0.95, \mathrm{I}^{2}=0 \%$ ) and long-term follow-up periods (SMD $-0.27,95 \%$ $\mathrm{Cl}-0.48$ to -0.06 ; two trials, 355 women; no evidence of statistical heterogeneity $\mathrm{Chi}^{2}=0.45, \mathrm{P}=0.50, \mathrm{I}^{2}=0 \%$; see Analysis 1.9).

\section{Anxiety}

Six trials measured and reported on anxiety as a continuous (Bowland 2012; Ferrari 2018; Ghahari 2017; Kokka 2019; HernandezRuiz 2005) or dichotomous outcome (Hegarty 2013). Trials measured anxiety using the State-Trait Anxiety Inventory (Ghahari 2017; Hernandez-Ruiz 2005), Depression, Anxiety, and Stress Scale (Kokka 2019), Beck Anxiety Inventory (Bowland 2012), Generalized Anxiety Disorder questionnaire (Ferrari 2018) and Hospital Anxiety and Depression Scale (Hegarty 2013). We classified these psychological trials as CBT (Ferrari 2018), third-wave CBTs (Ghahari 2017), humanistic therapies (Kokka 2019), integrative therapies (Hegarty 2013), and other psychologically-orientated interventions (Bowland 2012; Hernandez-Ruiz 2005). Intervention intensity included up to four sessions with women recruited from a shelter setting (Hernandez-Ruiz 2005), and five or more sessions with women recruited from healthcare settings (Hegarty 2013; Bowland 2012; Ghahari 2017), community (Kokka 2019), or community and shelter/refuge settings (Ferrari 2018). While all six interventions were delivered by a therapist, only one included healthcare workers (e.g. general practitioners, Hegarty 2013).

There were no trials with available continuous data within our primary time frame of medium-term follow-up. However, results indicated that psychological therapies probably reduce anxiety at short-term follow-up (SMD $-0.96,95 \% \mathrm{Cl}-1.29$ to -0.63 ; four trials, 158 women; no evidence of statistical heterogeneity $\mathrm{Chi}^{2}=0.66, \mathrm{P}=$ $0.88, I^{2}=0 \%$ ). In terms of long-term follow up, results from one trial showed no beneficial effect of therapy (SMD $-0.20,95 \% \mathrm{Cl}-0.51$ to 0.10; one trial, 166 women; see Analysis 1.10).

In terms of anxiety as a dichotomous outcome, one trial (Hegarty 2013), showed that fewer women experienced anxiety if they received the integrative therapy, but the $95 \% \mathrm{Cl}$ crossed the line of no therapy effect at medium-term (OR $0.82,95 \% \mathrm{Cl} 0.45$ to $1.49 ; 192$ women) and at long-term follow-up (OR $0.87,95 \% \mathrm{Cl} 0.48$ to 1.57 ; 195 women; see Analysis 1.11).

\section{Post-traumatic stress disorder}

Ten trials measured and reported PTSD as a continuous outcome (Bryant 2017; Cohen 2013; Ferrari 2018; Graham-Bermann 2015; Johnson 2011; Koopman 2005; Kubany 2004; Orang 2017; Stevens 2015; Zlotnick 2011). Trials measured this outcome using the Clinician Administered PTSD Scale (Cohen 2013; Johnson 2011; Kubany 2004), Post-traumatic Stress Disorder Checklist (Stevens 2015; Bryant 2017; Koopman 2005), Longitudinal Interval Follow-up Examination (Zlotnick 2011), Post-traumatic Stress Symptoms - Interview (Orang 2017), PTSD Symptom Scale (Ferrari 2018) and Post-traumatic Stress Scale for Domestic Violence (Graham-Bermann 2015). The trials evaluated the efficacy of CBT
(Cohen 2013; Ferrari 2018; Johnson 2011), humanistic therapies ((Koopman 2005; Orang 2017), Integrative Therapies (Bryant 2017; Graham-Bermann 2015; Stevens 2015; Zlotnick 2011), and thirdwave CBTs (Kubany 2004). Most of these trials (8, 80\%) included five or more sessions delivered by a therapist.

In terms of our primary time frame of medium-term follow-up, pooled results from four trials $(n=484)$ showed that there may be no evidence of a difference between groups (SMD -0.24, 95\% $\mathrm{Cl}-0.54$ to 0.06 ), with a moderate level of statistical heterogeneity (Chi $\left.{ }^{2}=6.57, \mathrm{P}=0.09, \mathrm{I}^{2}=54 \%\right)$. In terms of short and long-term follow-up, results indicated no beneficial effect of therapy (see Analysis 1.12).

\section{Quality of life}

Four trials assessed quality of life (Ferrari 2018; Hegarty 2013; Rhodes 2015; Tirado-Muñoz 2015). Trials measured this outcome using the Short Form-12 (Ferrari 2018), WHO Quality of Life-BREF (Hegarty 2013), Quality of Life Scale (Rhodes 2015), and a visual analogue scale ranking from 0 (lowest quality of life/health) to 100 (highest quality of life/health (Tirado-Muñoz 2015).

Results indicate that there may be no evidence of a difference between groups at medium-term follow-up (SMD 0.10, 95\% Cl -0.07 to 0.27 ; two trials, 557 women; no evidence of statistical heterogeneity $\mathrm{Chi}^{2}=0.02, \mathrm{P}=0.88, \mathrm{I}^{2}=0 \%$; see Analysis 1.13). Similarly, results showed no beneficial effect of therapy at short (SMD 0.16, $95 \% \mathrm{Cl}-0.04$ to 0.36 ; two trials, 382 women; no evidence of statistical heterogeneity $\mathrm{Chi}^{2}=0.00, \mathrm{P}=0.98, \mathrm{I}^{2}=0 \%$ ) or long-term follow-up (SMD 0.11, $95 \% \mathrm{Cl}-0.04$ to 0.25 ; four trials, 699 women; no evidence of statistical heterogeneity $\mathrm{Chi}^{2}=2.33, \mathrm{P}=0.51, \mathrm{I}^{2}=0 \%$ ).

\section{Re-exposure to IPV}

Sixteen trials (49\%) reported data on re-exposure to IPV as a continuous (Ferrari 2018; Hegarty 2019; Jaffe 2017; Kokka 2019; Rhodes 2015; Stevens 2015; Tirado-Muñoz 2015; Zlotnick 2011; Zlotnick 2019) or dichotomous outcome (Gilbert 2006; Gilbert 2016; Hegarty 2013; Hirani 2010; Kiely 2010; Saggurti 2014; Taghizadeh 2018). Trials measured this outcome using the Revised Conflict Tactics Scale (Gilbert 2006; Gilbert 2016; Jaffe 2017; Rhodes 2015; Zlotnick 2011), Conflict Tactics Scale (Kiely 2010; Taghizadeh 2018), Composite Abuse Scale (Ferrari 2018; Hegarty 2013; Hegarty 2019; Stevens 2015; Zlotnick 2019), Psychological Maltreatment of Women Inventory (Tirado-Muñoz 2015), Women Abuse Screening Tool (Kokka 2019), and non-standardised questionnaire (Hirani 2010) or single items such as "Have you and your husband had an argument or fight where he physically or sexually hurt you in the past 3 months?" (physical/sexual violence); and "Was there any coercion or pressure on you to have sex the last time you had sex with your husband?" (sexual coercion) (Saggurti 2014).

These trials evaluated four types of psychological therapies, including CBT (Kiely 2010; Ferrari 2018; Gilbert 2006; TiradoMuñoz 2015), humanistic therapies (Gilbert 2016; Kokka 2019; Saggurti 2014; Taghizadeh 2018), integrative therapies (Hegarty 2013; Hegarty 2019; Hirani 2010; Rhodes 2015; Stevens 2015; Zlotnick 2011; Zlotnick 2019) and third-wave CBTs (Jaffe 2017). Ten of the 16 trials (62\%) included five or more sessions of psychological therapy with women recruited from healthcare settings (Kiely 2010; Gilbert 2006; Hegarty 2013; Stevens 2015; Tirado-Muñoz 2015), community (Hirani 2010; Hegarty 2019; Kokka 2019; Saggurti 2014), and community and/or shelter/refuge settings (Ferrari 2018). 
While 16 trials reported on re-exposure to any form of IPV, only three trials had IPV as an outcome within our primary time frame of medium-term follow-up (six to under 12 months). Results indicated that there may be no evidence of a difference between groups in re-exposure to any form of IPV within medium-term followup (continuous: SMD $0.03,95 \% \mathrm{Cl}-0.14$ to 0.20 ; two trials, 547 women; moderate-certainty evidence; no evidence of statistical heterogeneity $\mathrm{Chi}^{2}=0.07, \mathrm{P}=0.79, \mathrm{I}^{2}=0 \%$; see Analysis 1.14; and dichotomous: OR $1.29,95 \% \mathrm{Cl} 0.55$ to 3.01 ; one trial, 186 women; high-certainty evidence; see Analysis 1.15).

In terms of short and long-term follow-up, results indicated no beneficial effect of therapy in reducing re-exposure to any form of IPV.

\section{Safety planning or safety behaviour, or both}

Three trials reported safety planning or safety behaviour, or both as a dichotomous (Hegarty 2013; Saftlas 2014) or a continuous outcome (Hegarty 2019). Trials measured this outcome using the Safety Promoting Behaviour Checklist (Hegarty 2013; Hegarty 2019), and stage-of-readiness-to-change adapted tool (Saftlas 2014). These trials evaluated integrative therapies delivered faceto-face (Hegarty 2013; Saftlas 2014), or through a website (Hegarty 2019). They recruited women from healthcare (Hegarty 2013; Saftlas 2014), or community settings (Hegarty 2019).

In terms of continuous outcomes within medium-term follow-up, results from Hegarty 2019 indicated that there may be no evidence of a difference between groups (SMD $0.04,95 \% \mathrm{Cl}-0.18$ to 0.25 ; one trial, 337 women; see Analysis 1.16). Similarly, as a dichotomous outcome, in Hegarty 2013 trial, higher numbers of women reported safety planning or safety behaviours, or both, but the $95 \% \mathrm{Cl}$ crossed the line of no beneficial effect of therapy (OR 1.25, 95\% Cl 0.68 to 2.27; one trial, 191 women; see Analysis 1.17).

\section{Use of healthcare and IPV services}

Two trials reported use of healthcare and IPV services as a dichotomous outcome (Hegarty 2013; Rhodes 2015). Both trials evaluated integrative therapies delivered by healthcare workers, with women recruited from healthcare settings. However, only one trial with available data within medium-term follow-up indicated there may be no evidence to support a beneficial effect of therapy in increasing use of healthcare or IPV services (OR 1.21, 95\% CI 0.44 to 3.33; one trial, 364 women; see Analysis 1.18). In terms of short and long-term follow-up, results indicated no beneficial effect of therapy in increasing use of healthcare and/or IPV services.

\section{Social support}

Three trials measured and reported social support as a continuous (Johnson 2011; Stevens 2015) or a dichotomous outcome (Rhodes 2015). Trials measured this outcome using different scales such as the Inventory of Socially Supportive Behaviors (Johnson 2011), Social Provisions Scale (Stevens 2015), and a single-item question "Do you have someone to talk to about any problem?" (Rhodes 2015). We classified the trials as CBT using five or more sessions (Johnson 2011), and integrative therapies using up to four sessions (Rhodes 2015), and five or more sessions (Stevens 2015). All three trials were delivered by a healthcare worker. While one trial recruited women from shelters (Johnson 2011), the other two trials used healthcare settings (Rhodes 2015; Stevens 2015).
Both Johnson 2011 and Stevens 2015 reported social support as a continuous outcome at short- and medium-term follow-up. Results indicated that there may be no evidence of a difference between groups at medium-term follow-up (SMD $0.05,95 \% \mathrm{Cl}-0.21$ to 0.31 ; two trials, 235 women; no evidence of statistical heterogeneity $\mathrm{Chi}^{2}$ $=0.04, P=0.84, I^{2}=0 \%$ ). Similarly, these trials found no evidence of a difference between groups at short-term follow-up (see Analysis 1.19).

In terms of dichotomous outcome, while women who received the experimental intervention (Rhodes 2015) reported higher social support, the $95 \% \mathrm{Cls}$ showed that there may be no evidence of a difference between groups at medium-term follow-up (OR 1.24, $95 \% \mathrm{Cl} 0.68$ to 2.27 ; one trial, 364 women). Similarly, this trial found no evidence of a difference between groups at short or long-term follow-up (see Analysis 1.20).

\section{Women's satisfaction with and views about the psychological therapies}

Twelve trials (36\%) explored women's views of benefits and difficulties experienced in the psychological trials using quantitative and qualitative methodologies, or both (Cheung 2019; Choo 2016; Ferrari 2018; Hegarty 2013; Hegarty 2019; Jaffe 2017; Johnson 2011; Kokka 2019; Kubany 2004; Saggurti 2014; Tirado-Muñoz 2015; Zlotnick 2019). In one trial, the mean overall satisfaction score on the Client Satisfaction Questionnaire (CSQ-8) was $27.7(95 \% \mathrm{Cl} 26.3$ to 29.1$)$ out of a possible 32, with higher scores indicating higher satisfaction (Choo 2016). Similarly, using the same measure (CSQ-8), Kubany 2004 found that the percentage of participants who were maximally satisfied with the psychological intervention was $64 \%$ immediately post-treatment, $67 \%$ at three-month follow-up, and $72 \%$ at six-month follow-up. In two trials, most women agreed that they were glad they had participated (Cheung 2019; Hegarty 2013), and the mean score on how supported they felt by the experimental intervention was higher than in the comparator intervention (Hegarty 2013).

\section{Adverse events and harm related to participation}

Twelve trials monitored and recorded adverse events (harm) related to participation throughout the psychological therapies (Bryant 2017; Cheung 2019; Ferrari 2018; Gilbert 2006; Gilbert 2016; Hegarty 2013; Johnson 2011; Michalopoulou 2015; Rhodes 2015; Saftlas 2014; Stevens 2015; Zlotnick 2019). Of these, eight trials reported no adverse events or harms (Bryant 2017; Cheung 2019; Gilbert 2006; Gilbert 2016; Michalopoulou 2015; Rhodes 2015; Saftlas 2014; Stevens 2015). In Johnson 2011 there were seven hospitalisations (five medical, two substance-related) and four lifethreatening traumatic experiences (two abuse-related) reported over the course of the six-month follow-up period, although these were classified as unrelated to the trial. Similarly, in a trial recruiting women from community, shelter and refuge settings in two UK cities, 22 women (15 in the experimental group, seven in the comparator intervention group) reported a total of 32 serious adverse events (Ferrari 2018). Examples of these adverse events included six cases of attempted suicide, seven cases related to pregnancy or miscarriage, and three cases of injuries from physical assault. Trial authors reported that there was insufficient information to determine whether these harms were specifically related to trial participation. Further, in a trial that recruited perinatal women seeking mental health treatment at an urban hospital-based behavioural health clinic, there were a total of 
22 serious adverse events that were classified as unrelated to participation in the psychological trial (Zlotnick 2019). However, only one trial measured benefits and harms using a validated measure (adapted version of Consequences of Screening Tool; Valpied 2019); and did not detect any significant harms. Most respondents agreed that they were glad they had participated in the project $(145,87.3 \%)$ and there were no differences between groups on the harm-benefit visual analogue scale used as part of harm assessment (experimental mean 77.0 (SD 20.5); comparator mean 73.7 (SD 18.9); MD $4.4(95 \% \mathrm{Cl}-0.8$ to $9.6 ; \mathrm{P}=0.092))$.

\section{Cost-benefit measures}

Three trials reported cost-effectiveness analysis and economic evaluation in their published protocols (Ferrari 2018; Hegarty 2013; Hegarty 2019), but the main outcome papers did not report on any economic evaluation.

\section{Process evaluation}

Most of the included trials $(30,91 \%)$ did not investigate mechanismrelated processes (or at least at the time of searches had not published a process evaluation), i.e. related studies measuring how the psychological interventions might have worked and under what circumstances and in which contexts. One trial deliberately did not measure process evaluation because of the priority of measuring effectiveness of the intervention and limiting the assessment burden on participants (Bryant 2017). Ferrari 2018 conducted a nested qualitative trial (Evans 2018), to explore women's experiences with the psychological therapy offered as part of the trial. They revealed that women valued the educational, psychological and emotional elements of the intervention, but that adherence was affected by women's 'psychological readiness' to engage, as well as practical issues such as housing insecurity, legal proceedings, availability of child care, and breaks in the continuity of professional care (Evans 2018). However, there was unclear information available on the pathway to change. A follow-up process evaluation of Hegarty 2013 using qualitative methods found that favourable doctor communication was strongly associated with engaging women and uptake of the psychological intervention (O'Doherty 2016). In common with Evans 2018, uptake of the psychological intervention was promoted by a concurrent belief that the partner's behaviour was problematic (even if it had not been fully named as abuse). An unintended effect of the research detected by the subsequent interviews was its influence on women's awareness of the abuse, shaping help-seeking cognitions and increasing openness to seeking help. Other key themes promoting uptake of the intervention were primary care or family practice offering a discreet option for care and opportunities created by the research itself to access an intervention. On the other hand, there was fear of disclosure occurring within the time-pressured environment of general practice, and participants largely perceived the role of family doctors as addressing a person's medical problems rather IPV-related issues. This evaluation answered questions related to circumstances and context. A further sub-trial of Hegarty 2013 used path analysis to test mediation effects from assignment to outcome and reported an effect on depression via self-efficacy, doctor support and safety enquiry (Valpied 2019).

\section{DISCUSSION}

Overall, there is very limited examination of psychological therapies for women with intimate partner violence (IPV) in the literature, considering the high association of IPV and mental health problems globally (World Health Organization 2013a). Criticism of psychological approaches for survivors of IPV suggest that psychological therapies may cause harm by blaming or pathologising the survivor (Humphreys 2003; Humphreys 2004). Further, trauma informed approaches suggest that concentrating on symptom reduction (what is wrong with you?) as an intervention instead of exploring past trauma (what has happened to you?) might cause harm when the psychological model is applied to the social problem of IPV (Quadara 2015; Warshaw 2013).

\section{Summary of main results}

Our review aimed to assess the effectiveness of psychological therapies for women who self-reported previous or recent experience of any form of IPV, primarily on depression, self-efficacy and any indicators of harm. Our review includes 33 trials, involving 5517 women randomly assigned to the experimental $(2798,51 \%)$ and comparator interventions (2719, 49\%). We classified the trials according to CCDAN psychological therapies, involving up to four sessions or five or more sessions, delivered by healthcare or non-healthcare workers trained in psychological techniques. We classified follow-up periods as short-term (within six months postbaseline), medium-term (six to 12 months) and long-term (more than 12 months), with medium-term as our primary time frame. The overall certainty of the body of evidence was low to moderate, mainly due to heterogeneity or risk of bias, or both.

For our primary outcomes, evidence showed that for women who experience IPV, psychological therapies probably reduce depression. However, there is limited available evidence of beneficial effects of therapy in enhancing self-efficacy. In addition, whilst there was no evidence of group differences in attrition and dropout rates, there were limited data on harm. Our subgroup analyses indicated that there may be no evidence of a difference between groups on depression, based on type of intervention, recruitment settings of participants or the person delivering the intervention. However, longer duration of psychological therapies (five or more sessions) showed a probable beneficial effect in reducing depression outcome than fewer sessions.

For our secondary outcomes, there was evidence that for women who experience IPV, psychological therapies may reduce anxiety symptoms. However, there is limited available evidence of whether psychological therapies improve general mental health symptoms, quality of life, social support, uptake of healthcare and IPV services and safety planning, or reduce PTSD and re-exposure to any form of IPV. Overall, we did not find evidence that psychological therapies had a negative or harmful effect.

\section{Overall completeness and applicability of evidence}

Most trials were conducted in English-speaking, high-income countries (e.g. USA, Australia, UK), which limits applicability to lowand middle-income settings and for non-English speakers. Some trials used culturally-adapted measures or local-language versions of well-established measures (e.g. Orang 2017), or translated and adapted interventions to meet the needs of the local women (e.g. Tirado-Muñoz 2015). Furthermore, participants' ethnic 
backgrounds were diverse across the trials. The nature of IPV, social and healthcare responses to IPV, as well as the ability to deliver and receptivity to psychological therapies, differ across countries and limit the generalisability of these findings.

We classified psychological therapies in this review according to CCDAN criteria, including CBT (six trials), third-wave CBTs (four trials), humanistic therapies (nine trials), integrative therapies (11 trials) and other psychologically-orientated interventions (three trials). We identified no eligible trials of psychodynamic therapies and only one trial had a specific trauma focus (Kubany 2004). There was clinical and statistical heterogeneity across the trials for certain outcomes (e.g. depression). Whilst a reasonable number of trials (19) provided data on the primary outcome of depression, only four trials reported data about self-efficacy (Hegarty 2019; Hirani 2010; Kokka 2019; Saftlas 2014), and only two trials reported data about the use of healthcare and IPV services (Hegarty 2013; Rhodes 2015). Further, the outcome of PTSD, which is highly associated with IPV, was addressed in only 10 trials. The small number of trials for some outcomes meant that there was insufficient evidence for us to conduct subgroup and sensitivity analyses, so that our understanding of heterogeneity across the trials remains incomplete.

\section{Quality of the evidence}

The quality of trials ranged from low to high, mainly due to risks of bias. Except for blinding of participants and personnel, we assessed only five trials as having low risk of bias in all the criteria that we considered (Bryant 2017; Cheung 2019; Ferrari 2018; Hegarty 2019; Rhodes 2015). For random sequence generation, we rated 12 trials at high or unclear risk of bias because there were insufficient details or evidence about whether trial authors used reliable methods of sequence generation. We rated 21 trials at high or unclear risk because it was not clear if they maintained allocation concealment. Attrition rates and loss at follow-ups were similar between women in the experimental and comparator interventions, and this was not a major concern for most trials. When considering the body of evidence in terms of GRADE for primary outcomes, we rated the certainty of evidence as moderate for depression and low for selfefficacy. For secondary outcomes, the certainty of evidence was mainly low due to risks of bias, inconsistency and imprecision.

\section{Potential biases in the review process}

Risk of introducing potential bias is likely to have occurred at any stage of the review process and we used various strategies to minimise this risk. Two review authors assessed eligibility based on title and abstract, and four review authors assessed eligibility based on full texts. We believe that our search process identified all of the published trials of psychological therapies as defined in this review and published up to the search date. Two review authors made decisions about inclusion or exclusion of trials and any changes made to the protocol were made by discussion and with the involvement of all review authors. Four review authors contributed to data extraction and 'Risk of Bias' assessment. One review author exported data from Covidence to Review Manager 2014 and a second review author checked them. However, assessing risk of bias, for example, requires individual judgement about the impact of bias on outcomes, so it is possible that a different review team may not have agreed with all of our assessments.

\section{Agreements and disagreements with other studies or reviews}

We identified 32 previous reviews that used various methodologies to review a diverse range of domestic violence interventions and prevention programmes (e.g. screening, advocacy, safety and empowerment programmes, primary care, secondary and tertiary interventions, family therapies) for women who have experienced IPV. Of these, only two peer-reviewed systematic reviews assessed the efficacy of certain types of psychological therapies. The first review identified 19 studies (all RCTs) and assessed the efficacy of CBT and advocacy interventions in relation to one main outcome of IPV (Tirado-Muñoz 2014). The second review identified 21 studies (including RCTs and non-RCTs) and assessed the efficacy of short-term mental health interventions for survivors of IPV in relation to several outcomes including IPV and depression (Arroyo 2017). Our review represents a rigorous extension of existing work by synthesising the findings from a larger number of trials and assessing the effectiveness of psychological therapies on a range of primary and secondary outcomes. Overall, based on our statistical analysis and conclusions, we agree with the Arroyo 2017 review, that psychological therapies in general are beneficial in reducing some psychological symptoms compared to comparator conditions (e.g. depression). Furthermore, and similar to the Tirado-Muñoz 2014 review, we produced inconsistent findings about the beneficial effect of psychological therapies in reducing re-exposure to IPV. Overall, our review has extended the evidence available for the efficacy of various types of psychological therapies for women who experience IPV, although limited in conclusions around trauma-focused psychological therapies.

\section{AUTHORS' CONCLUSIONS}

\section{Implications for practice}

Overall, the body of evidence supports trained healthcare and other workers offering and delivering psychological therapies with women who have experienced any form of intimate partner violence (IPV), as they probably reduce depression and may reduce anxiety symptoms. Specifically, in terms of depression outcome, while five or more sessions may be more beneficial than fewer sessions, there was no evidence of a difference between recruitment settings of participants, types of psychological therapies, or the person delivering the intervention. However, clinicians should know that this review found uncertain evidence about the beneficial effect of psychological therapies on other outcomes (self-efficacy, mental health, post-traumatic stress disorder, quality of life, social support, uptake of healthcare and IPV services, re-exposure to IPV, or safety planning). Thus, while psychological therapies for women with IPV may reduce depression and anxiety, their ongoing needs (e.g. reduction to exposure of IPV, safety, support and holistic healing from complex trauma) may not be addressed by this approach. Clinicians and other workers need to identify women's own goals and needs and offer combined strategies that may include advocacy (Rivas 2015), and traumainformed approaches (Warshaw 2013), either through working in a team or seeking training on promoting safety and recovery for women in the context of IPV. Workers need to understand that women may be at different stages on their pathway to safety and healing from the trauma of IPV (García-Moreno 2015). Tailoring their responses to each individual woman is required in addition to delivering a particular psychological therapy as many women need 
to feel safe from the threat of severe violence and death to be able to engage in therapy.

\section{Implications for research}

The evidence base supports that future psychological trials explore women's and practitioners' views and experiences of what they see as appropriate outcomes (O'Doherty 2014), and what they would want from any psychological therapy to improve our understanding of how to assist women's healing and recovery. Holistic healing and recovery is not just reduction in psychological symptoms; there was insufficient evidence to look at a broader concept of improvement in women's lives. We need more trials that distinguish the psychological needs of those recently or currently experiencing IPV from those who have past experiences. We need research that investigates psychological therapies and advocacy (information, safety planning) as combined interventions, either delivered by trained individuals or by a multidisciplinary team. We need research that tests trauma-informed approaches at an individual and system level.

The clinically 'active ingredients' of the reviewed therapies warrant further investigation through process evaluation, e.g. effect of therapeutic relationship. While 17 of the 33 included trials reported the use of some methods to assess treatment fidelity and adherence to treatment protocol or manual, we are unclear about which specific elements and components of the psychological therapies are important and relevant for the participating women.
We considered the overall certainty of the body of evidence to be low to moderate, due to some trials having weak designs and methodologies. Overall, there is a need for more trials with consistent eligibility criteria and outcomes at similar follow-up time points, as we were unable to synthesise much of the research.

\section{ACKNOWLEDGEMENTS}

The review authors thank the Cochrane Common Mental Disorders' (CCMD) editorial team for providing guidance during the completion of the review. We developed search strategies in collaboration with CCMD's Information Specialist. We would also like to thank Jim Harley for administrative support.

The review authors and the CCMD Editorial Team are grateful to the following peer reviewers for their time and comments: Alyson Huntley, Nuala Livingstone, and Katherine Thomas. They would also like to thank Cochrane Copy Edit Support for the team's help.

CRG funding acknowledgement: the National Institute for Health Research (NIHR) is the largest single funder of the CCMD Group.

Disclaimer: the views and opinions expressed herein are those of the review authors and do not necessarily reflect those of the NIHR, the National Health Service or the Department of Health and Social Care. 


\section{R E F E R E N C E S}

\section{References to studies included in this review}

Akor 2019 \{published data only\}

Akor BO, Moses LA, Baamlong ND, Shedul LL, Haruna AS, Abu JM, et al. Effect of counselling on the family function of intimate partner violence victims attending antenatal clinic in a tertiary hospital in North Central Nigeria. South African Family Practice 2019;61(2):69-74.

\section{Bowland 2012 \{published data only\}}

Bowland S, Edmond T, Fallot RD. Evaluation of a spiritually focused intervention with older trauma survivors. Social Work 2012;57(1):73-82.

\section{Bryant 2017 \{published data only\}}

* Bryant RA, Schafer A, Dawson KS, Anjuri D, Mulili C, Ndogoni L, et al. Effectiveness of a brief behavioural intervention on psychological distress among women with a history of gender-based violence in urban Kenya: a randomised clinical trial. PIOS Medicine 2017;14(8):e1002371. [DOI: 10.1371/ journal.pmed.1002371]

Sijbrandij M, Bryant RA, Schafer A, Dawson KS, Anjuri D, Ndogoni $L$, et al. Problem Management Plus (PM+) in the treatment of common mental disorders in women affected by gender-based violence and urban adversity in Kenya; study protocol for a randomized controlled trial.. International Journal of Mental Health Systems 2016;10(1):44.

\section{Cheung 2019 \{published data only\}}

* Cheung DS, Deng W, Tsao SW, Ho RT, Chan CL, Fong DY, et al. Effect of a qigong intervention on telomerase activity and mental health in Chinese women survivors of intimate partner violence: a randomized clinical trial. JAMA Network Open 2019;2(1):e186967.

Tiwari A, Chan CL, Ho RT, Tsao GS, Deng W, Hong AW, et al. Effect of a qigong intervention program on telomerase activity and psychological stress in abused Chinese women: a randomized, wait-list controlled trial. BMC Complementary and Alternative Medicine 2014;14(1):300.

Tiwari A, Cheung DS, Deng W, Fong DY, Tsao SW. Effect of a qigong intervention on telomerase activity of Chinese women survivors of intimate partner violence: a single-blind, waitlist, randomised controlled trial. Lancet 2017;390:S23.

\section{Choo 2016 \{published data only\}}

Choo EK, Tapé C, Glerum KM, Mello MJ, Zlotnick C, Guthrie KM. "That's Where the Arguments Come in": a qualitative analysis of booster sessions following a brief intervention for drug use and intimate partner violence in the emergency department. Substance Abuse: Research and Treatment 2016;10:77-87. [DOI: 10.4137/SART.S33388]

* Choo EK, Zlotnick C, Strong DR, Squires DD, Tapé C, Mello MJ. BSAFER: a web-based intervention for drug use and intimate partner violence demonstrates feasibility and acceptability among women in the emergency department. Substance Abuse 2016;37(3):441-9.
Cohen 2013 \{published data only\}

* Cohen LR, Field C, Campbell AN, Hien DA. Intimate partner violence outcomes in women with PTSD and substance use: a secondary analysis of NIDA Clinical Trials Network "Women and Trauma" Multi-site Study. Addictive Behaviors 2013;38(7):2325-32. [DOI: 10.1016/j.addbeh.2013.03.006]

Hien DA, Campbell AN, Killeen T, Hu MC, Hansen C, Jiang H, et al. The impact of trauma-focused group therapy upon HIV sexual risk behaviors in the NIDA clinical trials network "women and trauma" multi-site study. AIDS and Behavior 2010;14(2):421-30.

Hien DA, Wells EA, Jiang H, Suarez-Morales L, Campbell AN, Cohen LR, et al. Multisite randomized trial of behavioral interventions for women with co-occurring PTSD and substance use disorders. Journal of Consulting and Clinical Psychology 2009;77(4):607-19. [DOI: 10.1037/a0016227]

\section{Ferrari 2018 \{published data only\} $\mathbf{5 8 5 6 1 1 7 0}$}

Brierley G, Agnew-Davies R, Bailey J, Evans M, Fackrell M, Ferrari G, et al. Psychological advocacy toward healing (PATH): study protocol for a randomized controlled trial. Trials 2013;14:221.

Evans M, Malpass A, Agnew-Davies R, Feder G. Women's experiences of a randomised controlled trial of a specialist psychological advocacy intervention following domestic violence: a nested qualitative study. PLOS One 2018;13(11):e0193077.

* Ferrari G, Feder G, Agnew-Davies R, Bailey JE, Hollinghurst S, Howard L, et al. Psychological advocacy towards healing (PATH): a randomized controlled trial of a psychological intervention in a domestic violence service setting. PLOS One 2018;13(11):e0205485.

\section{Franzblau 2008 \{published data only\}}

Franzblau SH, Echevarria S, Smith M, Van Cantfort TE. A preliminary investigation of the effects of giving testimony and learning yogic breathing techniques on battered women's feelings of depression. Journal of Interpersonal Violence 2008;23(12):1800-08.

\section{Ghahari 2017 \{published data only\}}

Ghahari S, Khademolreza N, Poya FS, Ghasemnejad S, Gheitarani B, Pirmoradi MR. Effectiveness of mindfulness techniques in decreasing anxiety and depression in women victims of spouse abuse. Asian Journal of Pharmaceutical Research and Health Care 2017;9(1):28-33.

\section{Gilbert 2006 \{published data only\}}

Gilbert L, El-Bassel N, Manuel J, Wu E, Go H, Golder S, et al. An integrated relapse prevention and relationship safety intervention for women on methadone: testing short-term effects on intimate partner violence and substance use. Violence and Victims 2006;21(5):657-72. 
Gilbert 2016 \{published data only\}

El-Bassel N, Gilbert L, Goddard-Eckrich D, Chang M, Wu E, Hunt T, et al. Efficacy of a group-based multimedia HIV prevention intervention for drug-involved women under community supervision: project WORTH. PLOS One 2014;9(11):e111528.

* Gilbert L, Goddard-Eckrich D, Hunt T, Ma X, Chang M, Rowe J, et al. Efficacy of a computerized intervention on HIV and intimate partner violence among substance-using women in community corrections: a randomized controlled trial. American Journal of Public Health 2016;106(7):1278-86.

\section{Graham-Bermann 2015 \{published data only\}}

Clark HM, Grogan-Kaylor A, Galano MM, Stein SF, MontalvoLiendo N, Graham-Bermann S. Reducing intimate partner violence among Latinas through the Moms' Empowerment Program: an efficacy trial. Journal of Family Violence 2018;33(4):257-68.

Clark HM, Grogan-Kaylor AC, Galano MM, Stein SF, GrahamBermann SA. Moms' Empowerment Program participation associated with improved physical health among Latinas experiencing intimate partner violence. Revista Panamericana de Salud Pública 2018;42(e39):1-7.

Graham-Bermann SA, Miller LE. Intervention to reduce traumatic stress following intimate partner violence: an efficacy trial of the Moms' Empowerment Program (MEP). Psychodynamic Psychiatry 2013;41(2):329-49.

* Graham-Bermann SA, Miller-Graff L. Community-based intervention for women exposed to intimate partner violence: a randomized control trial. Journal of Family Psychology 2015;29(4):537-47. [DOI: 10.1037/fam0000091]

Howell KH, Miller LE, Lilly MM, Burlaka V, Grogan-Kaylor AC, Graham-Bermann SA. Strengthening positive parenting through intervention: evaluating the Moms' Empowerment Program for women experiencing intimate partner violence. Journal of Interpersonal Violence 2015;30(2):232-52.

Miller LE, Howell KH, Graham-Bermann SA. The effect of an evidence-based intervention on women's exposure to intimate partner violence. American Journal of Orthopsychiatry 2014;84(4):321-28

\section{Hegarty 2013 \{published data only\}}

* Hegarty K, O'Doherty L, Taft A, Chondros P, Brown S, Valpied J, et al. Screening and counselling in the primary care setting for women who have experienced intimate partner violence (WEAVE): a cluster randomised controlled trial. Lancet 2013;382(9888):249-58. [DOI: 10.1016/s0140-6736(13)60052-5]

Hegarty KL, Gunn JM, O'Doherty LJ, Taft A, Chondros P, Feder G, et al. Women's evaluation of abuse and violence care in general practice: a cluster randomised controlled trial (weave). BMC Public Health 2010;10(1):2. [DOI: 10.1186/1471-2458-10-2]

Hegarty KL, O'Doherty LJ, Gunn J, Pierce D, Taft AJ. A brief counselling intervention by health professionals utilising the 'readiness to change' concept for women experiencing intimate partner abuse: the weave project. Journal of Family Studies 2008;14(2-3):376-88

O'Doherty L, Taket A, Valpied J, Hegarty K. Receiving care for intimate partner violence in primary care: barriers and enablers for women participating in the weave randomised controlled trial. Social Science \& Medicine 2016;160:35-42.

Reisenhofer SA, Hegarty K, Valpied J, Watson LF, Davey MA, Taft A. Longitudinal changes in self-efficacy, mental health, abuse, and stages of change, for women fearful of a partner: findings from a primary care trial (WEAVE). Journal of Interpersonal Violence 2019;34(2):337-65.

Valpied J, Cini A, O'Doherty L, Taket A, Hegarty K. "Sometimes cathartic. Sometimes quite raw": Benefit and harm in an intimate partner violence trial.. Aggression and Violent Behavior 2014;19(6):673-85. [DOI: 10.1016/j.avb.2014.09.005]

Valpied J, Hegarty K, Brown S, O'Doherty L. The role of selfefficacy and doctor support in mediating improved depression outcomes following counselling by family doctors for intimate partner violence. Family Practice 2020;37(2):255-62. [DOI: 10.1093/fampra/cmz067]

\section{Hegarty 2019 \{published data only\}}

Hegarty K, Tarzia L, Murray E, Valpied J, Humphreys C, Taft A, et al. Protocol for a randomised controlled trial of a web-based healthy relationship tool and safety decision aid for women experiencing domestic violence (I-DECIDE). BMC Public Health 2015;15(1):1-9.

Hegarty K, Tarzia L, Valpied J, Murray E, Humphreys C, Taft A, et al. An online healthy relationship tool and safety decision aid for women experiencing intimate partner violence (IDECIDE): a randomised controlled trial. Lancet Public Health 2019;4(6):e301-10.

Tarzia L, Murray E, Humphreys C, Glass N, Taft A, Valpied J, et al. I-DECIDE: an online intervention drawing on the psychosocial readiness model for women experiencing domestic violence. Women's Health Issues 2016;26(2):208-16.

\section{Hernandez-Ruiz 2005 \{published data only\}}

Hernández-Ruiz E. Effect of music therapy on the anxiety levels and sleep patterns of abused women in shelters. Journal of Music Therapy 2005;42(2):140-58.

\section{Hirani 2010 \{published data only\}}

Hirani S, Karmaliani R, McFarlane J, Asad N, Madhani F, Shehzad S. Testing a community derived intervention to promote women's health: preliminary results of a 3-arm randomized controlled trial in Karachi, Pakistan. Southern Online Journal of Nursing Research 2010;10(3):1.

\section{Jaffe 2017 \{published data only\}}

Jaffe AE, Schreier A, DiLillo D. Feasibility and initial evaluation of Project PEACE: an intervention for college students at risk for dating violence. Partner Abuse 2017;8(3):291-314.

\section{Johnson 2011 \{published data only\}}

Johnson DM, Johnson NL, Perez SK, Palmieri PA, Zlotnick C. Comparison of adding treatment of PTSD during and after 
shelter stay to standard care in residents of battered women's shelters: results of a randomized clinical trial. Journal of Traumatic Stress 2016;29(4):365-73.

* Johnson DM, Zlotnick C, Perez S. Cognitive behavioral treatment of PTSD in residents of battered women's shelters: Results of a randomized clinical trial. Journal of Consulting and Clinical Psychology 2011;79(4):542-51.

Johnson DM, Zlotnick C. A cognitive-behavioral treatment for battered women with PTSD in shelters: findings from a pilot study. Journal of Traumatic Stress 2006;19(4):559-64.

\section{Kiely 2010 \{published data only\}}

El-Khorazaty MN, Johnson AA, Kiely M, El-Mohandes AA Subramanian S, Laryea HA, et al. Recruitment and retention of low-income minority women in a behavioral intervention to reduce smoking, depression, and intimate partner violence during pregnancy. BMC Public Health 2007;7:233.

El-Mohandes AA, Kiely M, Gantz MG, El-Khorazaty MN. Very preterm birth is reduced in women receiving an integrated behavioral intervention: a randomized controlled trial. Maternal and Child Health Journal 2011;15(1):19-28.

El-Mohandes AA, Kiely M, Joseph JG, Subramanian S, Johnson AA, Blake SM, et al. An intervention to improve postpartum outcomes in African-American mothers: a randomized controlled trial. Obstetrics and Gynecology 2008;112(3):611-20

Joseph JG, El-Mohandes AA, Kiely M, El-Khorazaty MN, Gantz MG, Johnson AA, et al. Reducing psychosocial and behavioral pregnancy risk factors: results of a randomized clinical trial among high-risk pregnant African American women. American Journal of Public Health 2009;99(6):1053-61.

* Kiely M, El-Mohandes AA, El-Khorazaty MN, Gantz MG. An integrated intervention to reduce intimate partner violence in pregnancy: a randomized trial. Obstetrics and Gynecology 2010;115(2 Pt 1):273-83. [DOI: 10.1097/AOG.0b013e3181cbd482]

\section{Kokka 2019 \{published data only\}}

Kokka A, Mikelatou M, Fouka G, Varvogli L, Chrousos GP, Darviri C. Stress management and health promotion in a sample of women with intimate partner violence: a randomized controlled trial. Journal of Interpersonal Violence 2019;34(10):2034-55.

\section{Koopman 2005 \{published data only\}}

Koopman C, Ismailji T, Holmes D, Classen CC, Palesh O, Wales T. The effects of expressive writing on pain, depression and posttraumatic stress disorder symptoms in survivors of intimate partner violence. Journal of Health Psychology 2005;10(2):211-21. [DOI: 10.1177/1359105305049769]

\section{Kubany 2004 \{published data only\}}

* Kubany ES, Hill EE, Owens JA, lannce-Spencer C, McCaig MA Tremayne KJ, et al. Cognitive trauma therapy for battered women with PTSD (CTT-BW). Journal of Consulting and Clinical Psychology 2004;72(1):3-18.
Kubany ES, Hill EE, Owens JA. Cognitive trauma therapy for battered women with PTSD: preliminary findings. Journal of Traumatic Stress 2003;16(1):81-91.

Kubany ES, Watson SB. Cognitive trauma therapy for formerly battered women with PTSD: conceptual bases and treatment outlines. Cognitive and Behavioral Practice 2002;9(2):111-27.

\section{Michalopoulou 2015 \{published data only\}}

Michalopoulou E, Tzamalouka G, Chrousos GP, Darviri C. Stress management and intimate partner violence: a randomized controlled trial. Journal of Family Violence 2015;30(6):795-802.

\section{Myers 2015 \{published data only\}}

Myers US, Browne KC, Norman SB. Treatment engagement: female survivors of intimate partner violence in treatment for PTSD and alcohol use disorder. Journal of Dual Diagnosis 2015;11(3-4):238-47.

\section{Orang 2017 \{published data only\}}

Orang T, Ayoughi S, Moran JK, Ghaffari H, Mostafavi S, Rasoulian M, et al. The efficacy of narrative exposure therapy in a sample of Iranian women exposed to ongoing intimate partner violence-a randomized controlled trial. Clinical Psychology \& Psychotherapy 2017;25(6):827-41.

\section{Rhodes 2015 \{published data only\}}

* Rhodes KV, Rodgers M, Sommers M, Hanlon A, Chittams J, Doyle A, et al. Brief motivational intervention for intimate partner violence and heavy drinking in the emergency department: a randomized clinical trial. JAMA 2015;314(5):466-77.

Rhodes KV, Rodgers M, Sommers M, Hanlon A, Crits-Christoph P. The Social Health Intervention Project (SHIP): protocol for a randomized controlled clinical trial assessing the effectiveness of a brief motivational intervention for problem drinking and intimate partner violence in an urban emergency department. BMC Emergency Medicine 2014;14(1):10.

\section{Saftlas 2014 \{published data only\}}

Saftlas AF, Harland KK, Wallis AB, Cavanaugh J, Dickey P, PeekAsa C. Motivational interviewing and intimate partner violence: a randomized trial. Annals of Epidemiology 2014;24(2):144-50.

\section{Saggurti 2014 \{published data only\}}

Raj A, Saggurti N, Battala M, Nair S, Dasgupta A, Naik DD, et al. Randomized controlled trial to test the RHANI Wives HIV intervention for women in India at risk for HIV from husbands. AIDS and Behavior 2013;17(9):3066-80.

* Saggurti N, Nair S, Silverman JG, Naik DD, Battala M, Dasgupta A, et al. Impact of the RHANI Wives intervention on marital conflict and sexual coercion. International Journal of Gynaecology \& Obstetrics 2014;126(1):18-22.

\section{Stevens 2015 \{published data only\}}

Stevens J, Scribano PV, Marshall J, Nadkarni R, Hayes J, Kelleher KJ. A trial of telephone support services to prevent further intimate partner violence. Violence Against Women 2015;21(12):1528-47. 
Taghizadeh 2018 \{published data only\}

Taghizadeh Z, Pourbakhtiar M, Ghasemzadeh S, Azimi K, Mehran A. The effect of training problem-solving skills for pregnant women experiencing intimate partner violence: a randomized control trial. Pan African Medical Journal 2018;30(79):1-11.

Tirado-Muñoz 2015 \{published data only\}

Tirado-Muñoz J, Gilchrist G, Lligoña E, Gilbert L, Torrens M. A group intervention to reduce intimate partner violence among female drug users. Results from a randomized controlled pilot trial in a community substance-abuse center. Adicciones 2015;27(3):168-78.

\section{Zlotnick 2011 \{published data only\}}

Zlotnick C, Capezza NM, Parker D. An interpersonally based intervention for low-income pregnant women with intimate partner violence: a pilot study. Archives of Women's Mental Health 2011;14(1):55-65.

\section{Zlotnick 2019 \{published data only\}}

Zlotnick C, Wernette GT, Raker CA. A randomized controlled trial of a computer-based brief intervention for victimized perinatal women seeking mental health treatment. Archives of Women's Mental Health 2019;22(3):315-25.

\section{References to studies excluded from this review}

\section{Anderson 2010 \{published data only\}}

Anderson T, Fende Guajardo J, Luthra R, Edwards KM. Effects of clinician-assisted emotional disclosure for sexual assault survivors: a pilot study. Journal of Interpersonal Violence 2010;25(6):1113-31.

\section{Arinero 2004 \{published data only\}}

Arinero M, Crespo, M. Evaluación de la eficacia de un programa de tratamiento cognitivo-conductual para mujeres víctimas de maltrato doméstico: un estudio piloto. Psicología Conductual 2004;12(2):233-49.

\section{Bahadir Yilmaz 2018 \{published data only\}}

Bahadir-Yilmaz E, Öz F. The effectiveness of empowerment program on increasing self-esteem, learned resourcefulness, and coping ways in women exposed to domestic violence. Issues in Mental Health Nursing 2018;39(2):135-41.

\section{Bahia 2018 \{published data only\}}

Bahia HK. Effects of the relationship check up on early adults' romantic relationship adjustment and substance use: a pilot study. Counseling Psychology and Human Services Theses and Dissertations 2017;7(B(E)):1-173.

\section{Bass 2013 \{published data only\}}

Bass JK, Annan J, Mclvor Murray S, Kaysen D, Griffiths S, Cetinoglu T, et al. Controlled trial of psychotherapy for Congolese survivors of sexual violence. New England Journal of Medicine 2013;368(23):2182-91.
Braithwaite 2014 \{published data only\}

Braithwaite SR, Fincham FD. Computer-based prevention of intimate partner violence in marriage. Behaviour Research and Therapy 2014;54:12-21.

Brannen 1996 \{published data only\}

Brannen SJ, Rubin A. Comparing the effectiveness of genderspecific and couples groups in a court-mandated spouse abuse treatment program. Research on Social Work Practice 1996;6(4):405-24.

Calderón 2008 \{published data only\}

Calderón SH, Gilbert P, Jackson R, Kohn MA, Gerbert B. Cueing prenatal providers effects on discussions of intimate partner violence. American Journal of Preventive Medicine 2008;34(2):134-7.

Carlson 2012 \{published data only\}

Carlson CE, Chen J, Chang M, Batsukh A, Toivgoo A, Riedel M, et al. Reducing intimate and paying partner violence against women who exchange sex in Mongolia: results from a randomized clinical trial. Journal of Interpersonal Violence 2012;27(10):1911-31.

\section{Chermack 2017 \{published data only\}}

Chermack ST, Bonar EE, Ilgen MA, Walton MA, Cunningham RM, Booth BM, et al. Developing an integrated violence prevention for men and women in treatment for substance use disorders. Journal of Interpersonal Violence 2017;32(4):581-603.

\section{Chronister 2006 \{published data only\}}

Chronister KM, McWhirter EH. An experimental examination of two career interventions for battered women. Journal of Counseling Psychology 2006;53(2):151-64.

\section{Clark 2014 \{published data only\}}

Clark CJ, Lewis-Dmello A, Anders D, Parsons A, Nguyen-Feng V, Henn L, et al. Trauma-sensitive yoga as an adjunct mental health treatment in group therapy for survivors of domestic violence: a feasibility study. Complementary Therapies in Clinical Practice 2014;20(3):152-8.

\section{Cogan 2003 \{published data only\}}

Cogan R, Porcerelli JH. Psychoanalytic psychotherapy with people in abusive relationships: treatment outcome. Journal of Aggression, Maltreatment \& Trauma 2003;7(1-2):29-46.

\section{Cohen 2006 \{published data only\}}

Cohen LR, Hien DA. Treatment outcomes for women with substance abuse and PTSD who have experienced complex trauma. Psychiatric Services 2006;57(1):100-6.

\section{Colosetti 2000 \{published data only\}}

Colosetti SD, Thyer BA. The relative effectiveness of EMDR versus relaxation training with battered women prisoners. Behavior Modification 2000;24(5):719-39.

Constantino 2005 \{published data only\}

Constantino R, Kim Y, Crane PA. Effects of a social support intervention on health outcomes in residents of a domestic 
violence shelter: a pilot study. Issues in Mental Health Nursing 2005;26(6):575-90.

\section{Cort 2014 \{published data only\}}

Cort NA, Cerulli C, Poleshuck EL, Bellenger KM, Xia Y, Tu X, et al. Interpersonal psychotherapy for depressed women with histories of intimate partner violence. Psychological Trauma: Theory, Research, Practice, and Policy 2014;6(6):700-7.

\section{Crespo 2010 \{published data only\}}

Crespo M, Arinero M. Assessment of the efficacy of a psychological treatment for women victims of violence by their intimate male partner. Spanish Journal of Psychology 2010;13(2):849-63.

\section{Cruz-AlmanzaMa 2006 \{published data only\}}

Cruz-Almanza M, Gaona-Márquez L, Sánchez-Sosa JJ. Empowering women abused by their problem drinker spouses: effects of a cognitive-behavioral intervention. Salud Mental 2006;29(5):25-31.

\section{Dutton 2013 \{published data only\}}

Dutton MA, Bermudez D, Matas A, Majid H, Myers NL. Mindfulness-based stress reduction for low-income, predominantly African American women with PTSD and a history of intimate partner violence. Cognitive and Behavioral Practice 2013;20(1):23-32.

\section{Echeburúa 1996 \{published data only\}}

Echeburúa E, De Corral P, Sarasua B, Zubizarreta I. Treatment of acute posttraumatic stress disorder in rape victims: an experimental study. Journal of Anxiety Disorders 1996;10(3):185-99.

\section{Echeburúa 1997 \{published data only\}}

Echeburúa E, De Corral P, Zubizarreta I, Sarasua B. Psychological treatment of chronic posttraumatic stress disorder in victims of sexual aggression. Behavior Modification 1997;21(4):433-56.

\section{Echeburúa 2014 \{published data only\}}

Echeburúa E, Sarasua B, Zubizarreta I. Individual versus individual and group therapy regarding a cognitive-behavioral treatment for battered women in a community setting. Journal of Interpersonal Violence 2014;29(10):1783-801.

\section{Eden 2015 \{published data only\}}

Eden KB, Perrin NA, Hanson GC, Messing JT, Bloom TL, Campbell JC, et al. Use of online safety decision aid by abused women: effect on decisional conflict in a randomized controlled trial. American Journal of Preventive Medicine 2015;48(4):372-83.

\section{Faker 2016 \{published data only\}}

Faker KH, Davoodabadi H, Zakeri A, Janbozorgi M, Dastani M. Comparison of the personality characteristics, physical and psychological abuse in spousal abused applicants in divorced and normal women. Research in Medicine 2016;40(3):155-60.

\section{Fallot 2011 \{published data only\}}

Fallot RD, McHugo GJ, Harris M, Xie H. The trauma recovery and empowerment model: a quasi-experimental effectiveness study. Journal of Dual Diagnosis 2011;7(1-2):74-89.
Fals-Stewart 2002 \{published data only\}

Fals-Stewart W, Kashdan TB, O'Farrell TJ, Birchler GR. Behavioral couples therapy for drug-abusing patients: effects on partner violence. Journal of Substance Abuse Treatment 2002;22(2):87-96.

\section{Fals-Stewart 2006 \{published data only\}}

Fals-Stewart W, Birchler GR, Kelley ML. Learning sobriety together: a randomized clinical trial examining behavioral couples therapy with alcoholic female patients. Journal of Consulting and Clinical Psychology 2006;74(3):579-91.

\section{Feinberg 2016 \{published data only\}}

Feinberg ME, Jones DE, Hostetler ML, Roettger ME, Paul IM, Ehrenthal DB. Couple-focused prevention at the transition to parenthood, a randomized trial: effects on coparenting, parenting, family violence, and parent and child adjustment. Prevention Science 2016;17(6):751-64.

\section{Florsheim 2011 \{published data only\}}

Florsheim P, McArthur L, Hudak C, Heavin S, Burrow-Sanchez J. The Young Parenthood Program: preventing intimate partner violence between adolescent mothers and young fathers. Journal of Couple \& Relationship Therapy 2011;10(2):117-34.

\section{Foa 1995 \{published data only\}}

Foa EB, Hearst-Ikeda D, Perry KJ. Evaluation of a brief cognitivebehavioral program for the prevention of chronic PTSD in recent assault victims. Journal of Consulting and Clinical Psychology 1995;63(6):948-55

\section{Foa 2005 \{published data only\}}

Foa EB, Hembree EA, Cahill SP, Rauch SA, Riggs DS, Feeny NC, et al. Randomized trial of prolonged exposure for posttraumatic stress disorder with and without cognitive restructuring: outcome at academic and community clinics. Journal of Consulting and Clinical Psychology 2005;73(5):953-64.

Foshee 2000 \{published data only\} Foshee VA, Bauman KE, Greene WF, Koch GG, Linder GF, MacDougall JE. The Safe Dates program: 1-year follow-up results. American Journal of Public Health 2000;90(10):1619-22.

\section{Foshee 2016 \{published data only\}}

Foshee VA, Benefield T, Chen MS, Reyes LM, Dixon KS, Ennett ST, et al. The effects of the Moms and Teens for Safe Dates program on dating abuse: a conditional process analysis. Prevention Science 2016;17(3):357-66.

\section{Gallas 2009 \{published data only\}}

Gallas C, Bindeballe N, Gass P, Dressing H. Implementation of a structured group program for stalking victims: a pilot project. Psychotherapeut 2009;54(3):199-204.

\section{Galovski 2009 \{published data only\}}

Galovski TE, Monson C, Bruce SE, Resick PA. Does cognitivebehavioral therapy for PTSD improve perceived health and sleep impairment? Journal of Traumatic Stress 2009;22(3):197-204. 


\section{Gonzalez Guarda 2015 \{published data only\}}

Gonzalez-Guarda RM, Guerra JE, Cummings AA, Pino K, Becerra MM. Examining the preliminary efficacy of a dating violence prevention program for Hispanic adolescents. Journal of School Nursing 2015;31(6):411-21.

Green 2006 \{published data only\}

Green BL, Krupnick JL, Chung J, Siddique J, Krause ED, Revicki D, et al. Impact of PTSD comorbidity on one-year outcomes in a depression trial. Journal of Clinical Psychology 2006;62(7):815-35.

\section{Grip 2011 \{published data only\}}

Grip K, Almqvist K, Broberg AG. Effects of a group-based intervention on psychological health and perceived parenting capacity among mothers exposed to intimate partner violence (IPV): a preliminary study. Smith College Studies in Social Work 2011;81(1):81-100.

\section{Hansen 2014 \{published data only\}}

Hansen NB, Eriksen SB, Elklit A. Effects of an intervention program for female victims of intimate partner violence on psychological symptoms and perceived social support. European Journal of Psychotraumatology 2014;5(1):24797.

\section{Hanson 1993 \{published data only\}}

Hanson KA, Gidycz CA. Evaluation of a sexual assault prevention program. Journal of Consulting and Clinical Psychology 1993;61(6):1046-52.

\section{Harper 2014 \{published data only\}}

Harper Q, Worthington EL Jr, Griffin BJ, Lavelock CR, Hook JN, Vrana SR, et al. Efficacy of a workbook to promote forgiveness: a randomized controlled trial with university students. Journal of Clinical Psychology 2014;70(12):1158-69.

\section{Hayes 2015 \{published data only\}}

Hayes MA, Gallagher MW, Gilbert KS, Creech SK, DeCandia CJ, Beach CA, et al. Targeting relational aggression in veterans: the Strength at Home Friends and Family Intervention. Journal of Clinical Psychiatry 2015;76(6):E774-8.

\section{Hembree 2004 \{published data only\}}

Hembree EA, Cahill SP, Foa EB. Impact of personality disorders on treatment outcome for female assault survivors with chronic posttraumatic stress disorder. Journal of Personality Disorders 2004;18(1):117-27.

\section{Heshmati 2016 \{published data only\}}

Heshmati R, Khaleghkhah A, Jafari E, Marandi M. Effectiveness of self-regulation couple therapy intervention on marital satisfaction and partner abuse (non-physical). Mediterranean Journal of Clinical Psychology 2016;4(1):1-12.

\section{Hesser 2017 \{published data only\}}

Hesser H, Axelsson S, Bäcke V, Engstrand J, Gustafsson T, Holmgren E, et al. Preventing intimate partner violence via the Internet: a randomized controlled trial of emotionregulation and conflict-management training for individuals with aggression problems. Clinical Psychology \& Psychotherapy 2017;24(5):1163-77.

\section{Hoekenga 2010 \{published data only\}}

Hoekenga PH, Thewissen V, Bos AE, Willemse-van Son AH. Effectiveness of a body-oriented intervention on body experience and PTSD symptoms. Psychologie \& Gezondheid 2010;38(5):236-47.

\section{Howard 2003 \{published data only\}}

Howard A, Riger S, Campbell R, Wasco S. Counseling services for battered women: a comparison of outcomes for physical and sexual assault survivors. Journal of Interpersonal Violence 2003;18(7):717-34.

Hughes 2010 \{published data only\} Hughes MJ, Rasmussen LA. The utility of motivational interviewing in domestic violence shelters: a qualitative exploration. Journal of Aggression, Maltreatment \& Trauma 2010;19(3):300-22.

\section{Hyman 2002 \{published data only\}}

Hyman KB. Impact of emergency department intimate partner violence (IPV) advocacy: a longitudinal, randomized trial. Dissertation Abstracts International: Section B: The Sciences and Engineering 2002;62(9-B):4220.

Ironson 2002 \{published data only\}

Ironson G, Freund B, Strauss JL, Williams J. Comparison of two treatments for traumatic stress: a community-based study of EMDR and prolonged exposure. Journal of Clinical Psychology 2002;58(1):113-28.

Iverson 2011 \{published data only\}

Iverson KM, Gradus JL, Resick PA, Suvak MK, Smith KF, Monson CM. Cognitive-behavioral therapy for PTSD and depression symptoms reduces risk for future intimate partner violence among interpersonal trauma survivors. Journal of Consulting and Clinical Psychology 2011;79(2):193-202.

\section{Jack 2012 \{published data only\}}

Jack SM, Ford-Gilboe M, Wathen CN, Davidov DM, McNaughton DB, Coben JH, et al. Development of a nurse home visitation intervention for intimate partner violence. BMC Health Services Research 2012;12(1):1952.

\section{Jones 2013 \{published data only\}}

Jones DL, Kashy D, Villar-Loubet OM, Cook R, Weiss SM. The impact of substance use, sexual trauma, and intimate partner violence on sexual risk intervention outcomes in couples: a randomized trial. Annals of Behavioral Medicine 2013;45(3):318-28.

\section{Kaslow 2010 \{published data only\}}

Kaslow NJ, Leiner AS, Reviere S, Jackson E, Bethea K, Bhaju J, et al. Suicidal, abused African American women's response to a culturally informed intervention. Journal of Consulting and Clinical Psychology 2010;78(4):449.

\section{Katz 2008 \{published data only\}}

Katz KS, Blake SM, Milligan RA, Sharps PW, White DB, Rodan MF, et al. The design, implementation and acceptability of an integrated intervention to address multiple behavioral and 
psychosocial risk factors among pregnant African American women. BMC Pregnancy and Childbirth 2008;8(1):22.

\section{Kelly 2016 \{published data only\}}

Kelly A, Garland EL. Trauma-Informed mindfulness-based stress reduction for female survivors of interpersonal violence: results From a stage I RCT. Journal of Clinical Psychology 2016;72(4):311-28.

\section{Kenyon 2016 \{published data only\}}

Kenyon S, Jolly K, Hemming K, Ingram L, Gale N, Dann SA, et al. Evaluation of Lay Support in Pregnant women with Social risk (ELSIPS): a randomised controlled trial. BMC Pregnancy and Childbirth 2011;12(1):11.

\section{Kingston 2013 \{published data only\}}

Kingston D, Austin MP, Hegadoren K, McDonald S, Lasiuk G, McDonald S, et al. Study protocol for a randomized, controlled, superiority trial comparing the clinical and cost-effectiveness of integrated online mental health assessment-referralcare in pregnancy to usual prenatal care on prenatal and postnatal mental health and infant health and development: the Integrated Maternal Psychosocial Assessment to Care Trial (IMPACT). Trials 2013;15(1):72.

\section{Kraanen 2013 \{published data only\}}

Kraanen FL, Vedel E, Scholing A, Emmelkamp PM. The comparative effectiveness of Integrated treatment for Substance abuse and Partner violence (I-StoP) and substance abuse treatment alone: a randomized controlled trial. BMC Psychiatry 2013;13(1):189.

\section{Krupnick 2008 \{published data only\}}

Krupnick JL, Green BL, Stockton P, Miranda J, Krause E, Mete M. Group interpersonal psychotherapy for low-income women with posttraumatic stress disorder. Psychotherapy Research 2008;18(5):497-507.

\section{Labrador 2006 \{published data only\}}

Javier Labrador F, Fernandez-Velasco MD, Rincon PP. Efficacy of a brief individual treatment program for the posttraumatic stress disorder in women victims of domestic violence. International Journal of Clinical and Health Psychology 2006;6(3):527-47.

\section{Latif 2017 \{published data only\}}

Latif M, Khanam SJ. Effectiveness of cognitive behaviour therapy in reducing anxiety, depression and violence in women affected by intimate partner violence: a randomized controlled trial from a low-income country. Journal of Postgraduate Medical Institute (Peshawar-Pakistan) 2017;31(4):425-31.

\section{Launius 1987 \{published data only\}}

Launius MH, Jensen BL. Interpersonal problem-solving skills in battered, counseling, and control women. Journal of Family Violence 1987;2(2):151-62.

\section{Lee 2015 \{published data only\}}

Lee MY, Zaharlick A, Akers D. Impact of meditation on mental health outcomes of female trauma survivors of interpersonal violence with co-occurring disorders: a randomized controlled trial. Journal of Interpersonal Violence 2017;32(14):2139-65.

Leiner 2012 \{published data only\}

Leiner AS, Kearns MC, Jackson JL, Astin MC, Rothbaum BO. Avoidant coping and treatment outcome in rape-related posttraumatic stress disorder. Journal of Consulting and Clinical Psychology 2012;80(2):317-21.

\section{Liberman 2005 \{published data only\}}

Lieberman AF, Ippen CG, Van Horn P. Child-parent psychotherapy: 6-month follow-up of a randomized controlled trial.. Journal of the American Academy of Child \& Adolescent Psychiatry 2006;45(8):913-8.

* Lieberman AF, Van Horn P, Ippen CG. Toward evidence-based treatment: child-parent psychotherapy with preschoolers exposed to marital violence. Journal of the American Academy of Child \& Adolescent Psychiatry 2005;44(12):1241-8.

\section{Liu 2013 \{published data only\}}

Liu S, Dore MM, Amrani-Cohen I. Treating the effects of interpersonal violence: a comparison of two group models. Social Work with Groups 2013;36(1):59-72.

Lynch 2012 \{published data only\}

Lynch SM, Heath NM, Mathews KC, Cepeda GJ. Seeking safety: an intervention for trauma-exposed incarcerated women? Journal of Trauma \& Dissociation 2012;13(1):88-101.

\section{Mancoske 1994 \{published data only\}}

Mancoske RJ, Standifer D, Cauley C. The effectiveness of brief counseling services for battered women. Research on Social Work Practice 1994;4(1):53-63.

\section{Matud 2016 \{published data only\}}

Matud PM, Padilla V, Medina L, Fortes D. Efficacy of an intervention program for battered women. Terapia Psicologica 2016;34(3):199-208.

McBride 2002 \{published data only\}

McBride DL. Groups for abused women: treatment outcome. Dissertation Abstracts International Section A: Humanities and Social Sciences 2002;62(12-A):4075.

\section{McWhirter 2011 \{published data only\}}

McWhirter PT. Differential therapeutic outcomes of communitybased group interventions for women and children exposed to intimate partner violence. Journal of Interpersonal Violence 2011;26(12):2457-82.

\section{Mejdoubi 2013 \{published data only\}}

Mejdoubi J, Van den Heijkant SC, van Leerdam FJ, Heymans MW, Hirasing RA, Crijnen AA. Effect of nurse home visits vs. usual care on reducing intimate partner violence in young high-risk pregnant women: a randomized controlled trial. PLOS One 2013;8(10):e78185.

\section{Melendez 2003 \{published data only\}}

Melendez RM, Hoffman S, Exner T, Leu CS, Ehrhardt AA. Intimate partner violence and safer sex negotiation: effects 
of a gender-specific intervention. Archives of Sexual Behavior 2003;32(6):499-511.

\section{Miller 2011 \{published data only\}}

Miller E, Decker MR, McCauley HL, Tancredi DJ, Levenson RR, Waldman J, et al. A family planning clinic partner violence intervention to reduce risk associated with reproductive coercion. Contraception 2011;83(3):274-80.

Miller 2016 \{published data only\}

Miller E, Tancredi DJ, Decker MR, McCauley HL, Jones KA, Anderson $\mathrm{H}$, et al. A family planning clinic-based intervention to address reproductive coercion: a cluster randomized controlled trial. Contraception 2016;94(1):58-67.

\section{Morrissey 2005 \{published data only\}}

Morrissey JP, Jackson EW, Ellis AR, Amaro H, Brown VB, Najavits LM. Twelve-month outcomes of trauma-informed interventions for women with co-occurring disorders. Psychiatric Services 2005;56(10):1213-22.

\section{Ngo 2018 \{published data only\}}

Ngo QM, Eisman AB, Walton MA, Kusunoki Y, Chermack ST, Singh V, et al. Emergency department alcohol intervention: effects on dating violence and depression. Pediatrics 2018;142(1):e20173525.

\section{Nguyen Feng 2016 \{published data only\}}

Nguyen-Feng VN, Frazier PA, Greer CS, Meredith L, Howard K, Paulsen J. Testing the efficacy of three brief web-based interventions for reducing distress among interpersonal violence survivors. Translational Issues in Psychological Science 2016;2(4):439-48.

\section{Nishith 2005 \{published data only\}}

Nishith P, Nixon RD, Resick PA. Resolution of trauma-related guilt following treatment of PTSD in female rape victims: a result of cognitive processing therapy targeting comorbid depression? Journal of Affective Disorders 2005;86(2-3):259-65.

\section{Norton 1997 \{published data only\}}

Norton MA. The effects of direct versus Socratic cognitive therapies on battered women with posttraumatic stress disorder (PTSD). Dissertation Abstracts International Section A: Humanities and Social Sciences 1997;58(3-A):0753.

O'Leary 1999 \{published data only\} O'Leary KD, Heyman RE, Neidig PH. Treatment of wife abuse: a comparison of gender-specific and conjoint approaches. Behavior Therapy 1999;30(3):475-505.

\section{Orengo Aguayo 2017 \{published data only\}}

Orengo-Aguayo RE. Implementation of an acceptance and commitment therapy skills group with incarcerated domestic violence offenders: a feasibility pilot study. Dissertation Abstracts International: Section B: The Sciences and Engineering 2017;78(3-B(E)):1-158.

\section{Overbeek 2013 \{published data only\}}

Overbeek MM, De Schipper JC, Lamers-Winkelman F, Schuengel C. Effectiveness of specific factors in community- based intervention for child-witnesses of interparental violence: a randomized trial. Child Abuse \& Neglect 2013;37(12):1202-14.

Pallitto 2016 \{published data only\}

Pallitto C, García-Moreno C, Stöeckl H, Hatcher A, MacPhail C, Mokoatle $\mathrm{K}$, et al. Testing a counselling intervention in antenatal care for women experiencing partner violence: a study protocol for a randomized controlled trial in Johannesburg, South Africa. BMC Health Services Research 2016;16(1):630.

Parcesepe 2016 \{published data only\}

Parcesepe AM, L Engle KL, Martin SL, Green S, Sinkele W, Suchindran $\mathrm{C}$, et al. The impact of an alcohol harm reduction intervention on interpersonal violence and engagement in sex work among female sex workers in Mombasa, Kenya: results from a randomized controlled trial. Drug \& Alcohol Dependence 2016;161:21-8.

Parker 1999 \{published data only\} Parker B, McFarlane J, Soeken K, Silva C, Reel S. Testing an intervention to prevent further abuse to pregnant women. Research in Nursing \& Health 1999;22(1):59-66.

Rasmussen 2008 \{published data only\}

Rasmussen LA, Hughes MJ, Murray CA. Applying motivational interviewing in a domestic violence shelter: a pilot study evaluating the training of shelter staff. Journal of Aggression, Maltreatment \& Trauma 2008;17(3):296-317.

Reed 2006 \{published data only\}

Reed GL, Enright RD. The effects of forgiveness therapy on depression, anxiety, and posttraumatic stress for women after spousal emotional abuse. Journal of Consulting and Clinical Psychology 2006;74(5):920-9.

Resick 2008 \{published data only\}

Resick PA, Galovski TE, Uhlmansiek MO, Scher CD, Clum GA Young-Xu Y. A randomized clinical trial to dismantle components of cognitive processing therapy for posttraumatic stress disorder in female victims of interpersonal violence. Journal of Consulting and Clinical Psychology 2008;76(2):243-58.

Resick 2012 \{published data only\}

Resick PA, Williams LF, Suvak MK, Monson CM, Gradus JL. Long-term outcomes of cognitive-behavioral treatments for posttraumatic stress disorder among female rape survivors. Journal of Consulting and Clinical Psychology 2012;80(2):201-10.

Resnick 2007 \{published data only\}

Resnick H, Acierno R, Waldrop AE, King L, King D, Danielson C, et al. Randomized controlled evaluation of an early intervention to prevent post-rape psychopathology. Behaviour Research and Therapy 2007;45(10):2432-47.

Roemer 2004 \{published data only\}

Roemer L, Salters K. A preliminary study of the effects of directed suppression of rape-related material among rape survivors using unobtrusive measures. Behavioural and Cognitive Psychotherapy 2004;32(2):149-64. 
Rosmalen-Nooijens 2017 \{published data only\}

Rosmalen-Nooijens K, Wong SL, Prins J, Lagro-Janssen T. Young People, Adult Worries: randomized controlled trial and feasibility study of the internet-based self-support method "Feel the ViBe" for adolescents and young adults exposed to family violence. Journal of Medical Internet Research 2017;19(6):e204.

\section{Ross 2013 \{published data only\}}

Ross AR. Impact of psychoeducational advocacy training as compared to psychoeducational support group as an empowering tool for female survivors of domestic violence. Dissertation Abstracts International: Section B: The Sciences and Engineering 2013;74(4-B(E)):1-134.

Rothbaum 1997 \{published data only\}

Rothbaum BO. A controlled study of eye movement desensitization and reprocessing in the treatment of posttraumatic stress disordered sexual assault victims. Bulletin of the Menninger Clinic 1997;61(3):317-34.

Rothbaum 2001 \{published data only\}

Rothbaum BO, Astin M. Prolonged exposure versus EMDR for PTSD rape victims. PA Resick (Chair), Three clinical trials for the treatment of PTSD: outcome and dissemination. Symposium presented at the 35th Annual Convention for the Association for the Advancement of Behavior Therapy, Philadelphia 2001.

\section{Rotheram-Borus 2015 \{published data only\}}

Rotheram-Borus MJ, Tomlinson M, Le Roux I, Stein JA. Alcohol use, partner violence, and depression: a cluster randomized controlled trial among urban South African mothers over 3 years. American Journal of Preventive Medicine 2015;49(5):715-25

\section{Rychtarik 2005 \{published data only\}}

Rychtarik RG, McGillicuddy NB. Coping skills training and 12step facilitation for women whose partner has alcoholism: effects on depression, the partner's drinking, and partner physical violence. Journal of Consulting and Clinical Psychology 2005;73(2):249.

\section{Sanci 2015 \{published data only\}}

Sanci L, Chondros P, Sawyer S, Pirkis J, Ozer E, Hegarty K, et al. Responding to young people's health risks in primary care: a cluster randomised trial of training clinicians in screening and motivational interviewing. PLOS One 2015;10(9):e0137581.

\section{Santandreu 2014 \{published data only\}}

Santandreu M, Ferrer VA. Effectiveness of cognitive behavioral treatment for posttraumatic stress disorder on women victims of intimate partner violence. Behavioral Psychology-Psicologia Conductual 2014;22(2):239-56.

\section{Schumm 2018 \{published data only\}}

Schumm JA, O'Farrell TJ, Murphy MM, Muchowski P. Partner violence among drug-abusing women receiving behavioral couples therapy versus individually-based therapy. Journal of Substance Abuse Treatment 2018;92:1-10.
Sharps 2008 \{published data only\}

Sharps PW, Campbell J, Baty ML, Walker KS, Bair-Merritt MH. Current evidence on perinatal home visiting and intimate partner violence. Journal of Obstetric, Gynecologic, and Neonatal Nursing 2008;37(4):480-91.

\section{Sharps 2016 \{published data only\}}

Sharps PW, Bullock LF, Campbell JC, Alhusen JL, Ghazarian SR, Bhandari SS, et al. Domestic violence enhanced perinatal home visits: the DOVE randomized clinical trial. Journal of Women's Health 2016;25(11):1129-38.

Shirk 2014 \{published data only\}

Shirk SR, DePrince AP, Crisostomo PS, Labus J. Cognitive behavioral therapy for depressed adolescents exposed to interpersonal trauma: an initial effectiveness trial. Psychotherapy 2014;51(1):167-79.

\section{Stith 2004 \{published data only\}}

Stith SM, Rosen H, McCollum EE, Thomsen CJ. Treating intimate partner violence within intact couple relationships: outcomes of multi-couple versus individual couple therapy. Journal of Marital and Family Therapy 2004;30(3):305-18.

Taft 2009 \{published data only\}

Taft AJ, Small R, Hegarty KL, Lumley J, Watson LF, Gold L. MOSAIC (MOthers' Advocates In the Community): protocol and sample description of a cluster randomised trial of mentor mother support to reduce intimate partner violence among pregnant or recent mothers. BMC Public Health 2009;9(1):159.

Taft 2016 \{published data only\}

Taft CT, Creech SK, Gallagher MW, Macdonald A, Murphy CM, Monson CM. Strength at Home Couples program to prevent military partner violence: a randomized controlled trial. Journal of Consulting and Clinical Psychology 2016;84(11):935-45.

\section{Taft 2017 \{published data only\}}

Taft A, Colombini M. Healthcare system responses to intimate partner violence in low and middle-income countries: evidence is growing and the challenges become clearer. BMC Medicine 2017;15(1):127.

\section{Tarquinio 2012 \{published data only\}}

Tarquinio C, Brennstuhl MJ, Rydberg JA, Schmitt A, Mouda F, Lourel M, et al. Eye movement desensitization and reprocessing (EMDR) therapy in the treatment of victims of domestic violence: a pilot study. Revue Européenne de Psychologie Appliquée/European Review of Applied Psychology 2012;62(4):205-12.

Tiwari 2010 \{published data only\}

* Tiwari AF, Salili F, Chan RY, Chan EK, Tang D. Effectiveness of an empowerment intervention in abused Chinese women. Hong Kong Medical Journal 2010;16(Suppl 3):25-8.

\section{Tiwari 2012 \{published data only\}}

Tiwari A, Yuk H, Pang P, Fong DY, Yuen F, Humphreys J, et al. Telephone intervention to improve the mental health of community-dwelling women abused by their intimate partners: 
a randomised controlled trial. Hong Kong Medical Journal 2012;18(Suppl 6):14-7.

\section{Uchendu 2017 \{published data only\}}

PACTR201801002909246 (Uchendu T). The effect of a counseling Intervention on risk of IPV in pregnant women in Nigeria. apps.who.int/trialsearch/Trial2.aspx? TrialID=PACTR201801002909246 (first received 24 December 2017).

Wagman 2015 \{published data only\}

Wagman JA, Gray RH, Campbell JC, Thoma M, Ndyanabo A, Ssekasanvu J, et al. Effectiveness of an integrated intimate partner violence and HIV prevention intervention in Rakai, Uganda: analysis of an intervention in an existing cluster randomised cohort. Lancet Global Health 2015;3(1):e23-33.

\section{Walls 1985 \{published data only\}}

Walls NR. Assessment of rape survivors' adaptation to assault and a comparison of two types of group therapy for rape survivors. Dissertation Abstracts International 1985;46(5B):1703-4.

\section{Weir 2009 \{published data only\}}

Weir BW, O'Brien K, Bard RS, Casciato CJ, Maher JE, Dent CW, et al. Reducing HIV and partner violence risk among women with criminal justice system involvement: a randomized controlled trial of two motivational interviewing-based interventions. AIDS and Behavior 2009;13(3):509-22.

\section{Zarling 2015 \{published data only\}}

Zarling A, Lawrence E, Marchman J. A randomized controlled trial of acceptance and commitment therapy for aggressive behavior. Journal of Consulting and Clinical Psychology 2015;83(1):199-212.

\section{Zou 2010 \{published data only\}}

Zou SH, Zhang YL, Zhang Y, Liu N. Impact of psychological intervention in the newly-married couples on the occurrence to abused wives. Chinese Journal of Clinical Psychology 2010;18(4):530-4.

\section{Zust 2000 \{published data only\}}

Zust BL. Effect of cognitive therapy on depression in rural, battered women. Archives of Psychiatric Nursing 2000;14(2):51-63.

\section{References to studies awaiting assessment}

\section{Akhtari 2019 \{published and unpublished data\}}

Akhtari A, Ranjkesh F, Asadzandi M, Olfati F. The impact of spiritual counseling on domestic violence in pregnant women: a clinical trial. Journal of Urmia Nursing and Midwifery Faculty 2019;175(5):1-10. [unmf.umsu.ac.ir/article-1-3768-en.pdf]

\section{References to ongoing studies}

\section{CTRI/2019/01/017009 \{published and unpublished data\}}

CTRI/2019/01/017009. Impact of behavioral intervention package on the health status of married abused pregnant women, LN Hospital, New Delhi [A randomised controlled trial of the Impact of Behavioral Intervention Package on the health status of married abused pregnant women aged 15-45 years attending antenatal clinic of LNJP hospital, New Delhi]. ctri.nic.in/clinicaltrials [CTRI/2019/01/017009] (first received 9 January 2019).

IRCT20151103024866N11 \{published and unpublished data\} IRCT20151103024866N11. Investigating the effectiveness of Gestalt counselling on the self-esteem and domestic violence against pregnant women refereed to Kerman health centres. en.irct.ir/trial/31236 (first received 20 August 2018).

IRCT2017040628352N4 \{published and unpublished data\} IRCT2017040628352N4. The effect of solution focused counselling on violence rate and quality of life of pregnant women at risk of domestic violence. en.irct.ir/trial/23026 (first received 20 August 2017).

\section{IRCT20171223038002N1 \{published and unpublished data\}} IRCT20171223038002N1. Effect of group-based problem solving on domestic violence in patients with bipolar disorder type i [Effect of group-based problem solving on domestic violence in patients with bipolar disorder in type I in Ebn'e Sina Hospital in Mashhad]. en.irct.ir/trial/28665 (first received 9 May 2018).

IRCT20180204038609N1 \{published and unpublished data\} IRCT20180204038609N1. The effect of group-based cognitivebehavioral therapy on self-esteem of women with domestic violence. en.irct.ir/trial/29656 (first received 8 June 2018).

NCT03484390 \{published and unpublished data\} NCT03484390. Effects of mindfulness on PTSD [Effects of mindfulness on PTSD: a community-based clinical trial among trauma survivors]. clinicaltrials.gov/show/nct03484390 (first received 30 March 2018).

\section{NCT03813901 \{published and unpublished data\}}

NCT03813901. Impact of 'Samalochana' program on women who have experienced intimate partner violence (IPV) [Advaita Vedanta and counselling for women who have experienced intimate partner violence: impact on women's health]. clinicaltrials.gov/ct2/show/NCT03813901 (first received 23 January 2019).

\section{NCT04068662 \{published and unpublished data\}}

NCT04068662. Intervention for IPV-exposed pregnant women [Intervening during the prenatal period with women exposed to intimate partner violence to improve maternal functioning and infant adjustment]. clinicaltrials.gov/show/NCT04068662 (first received 28 August 2019).

\section{NL6450 \{published and unpublished data\}}

NL6450. The effectiveness of "De Nieuwe Toekomst", a Dutch intervention for victims of intimate partner violence [Effect study on "The Nieuwe Toekomst" (A new future) among female victims of intimate partner violence: a mixed method design]. trialregister.nl/trial/6450 (first received 28 July 2017). [NTR6628] 
Sapkota 2019 \{published and unpublished data\}

Sapkota D, Baird K, Saito A, Rijal P, Pokharel R, Anderson D. Counselling-based psychosocial intervention to improve the mental health of abused pregnant women: a protocol for randomised controlled feasibility trial in a tertiary hospital in eastern Nepal. BMJ Open 2019;9(4):e027436.

\section{Tol 2017 \{published data only\}65771265}

Tol WA, Greene MC, Likindikoki S, Misinzo L, Ventevogel P, Bonz AG, et al. An integrated intervention to reduce intimate partner violence and psychological distress with refugees in low-resource settings: study protocol for the Nguvu cluster randomized trial. BMC Psychiatry 2017;17(1):1-13.

\section{Additional references}

\section{Arroyo 2017}

Arroyo K, Lundahl B, Butters R, Vanderloo M, Wood DS. Shortterm interventions for survivors of intimate partner violence: a systematic review and meta-analysis. Trauma, Violence \& Abuse 2017;18(2):155-71. [DOI: 10.1177/1524838015602736]

\section{Ayre 2016}

Ayre J, Lum On M, Webster K, Gourley M, Moon L. Examination of the burden of disease of intimate partner violence against women in 2011: Final report. (ANROWS Horizons, 06/2016). Sydney: ANROWS 2016.

\section{Baird 2019}

Baird SL, Alaggia R, Jenney A. "Like Opening Up Old Wounds": Conceptualizing intersectional trauma among survivors of intimate partner violence. Journal of Interpersonal Violence 2019 May 16 [Epub ahead of print]:886260519848788. [DOI: 10.1177/0886260519848788]

\section{Bandura 1986}

Bandura A. Social Foundations of Thought and Action: a Social Cognitive Theory. New Jersey: Prentice-Hall, 1986.

\section{Barrera 1981}

Barrera M, Sandler IM, Ramsay TB. Preliminary development of a scale of social support: Studies on college students. American Journal of Community Psychology 1981;9:435-46.

\section{Beck 1961}

Beck AT, Ward CH, Mendelson M, Mock J, Erbaugh J. An inventory for measuring depression. Archives of General Psychiatry 1961;4:561-71.

\section{Beck 1988}

Beck AT, Epstein N, Brown G, Steer RA. An inventory for measuring clinical anxiety: psychometric properties. Journal of Consulting and Clinical Psychology 1988;56(6):893-7.

\section{Benight 2004}

Benight CC, Bandura A. Social cognitive theory of posttraumatic recovery: the role of perceived self-efficacy. Behaviour Research \& Therapy 2004;42(10):1129-48.

\section{Bisson 2013}

Bisson JI, Roberts NP, Andrew M, Cooper R, Lewis C. Psychological therapies for chronic posttraumatic stress disorder (PTSD) in adults. Cochrane Database of Systematic Reviews 2013, Issue 12. [DOI: 10.1002/14651858.CD003388.pub4]

\section{Bjelland 2002}

Bjelland I, Dahl AA, Haug TT, Neckelmann D. The validity of the Hospital Anxiety and Depression Scale: an updated literature review. Journal of Psychosomatic Research 2002;52(2):69-77.

\section{Black 2001}

Black CM. Intimate partner violence and adverse health consequences: implications for clinicians. American Journal of Lifestyle Medicine 2001;5:428-39.

\section{Blanchard 1996}

Blanchard EB, Jones-Alexander J, Buckley TC, Forneris CA. Psychometric properties of the PTSD Checklist (PCL). Behaviour Research and Therapy 1996;34(8):669-73.

\section{Breslau 1999}

Breslau N, Peterson E, Kessler R, Schultz L. Short screening scale for DSM-IV posttraumatic stress disorder. American Journal of Psychiatry 1999;156(6):908-11.

\section{Brooks 1996}

Brooks, R. EuroQol: the current state of play. Health Policy 1996;37(1):53-72.

\section{Butler 2006}

Butler AC, Chapman JE, Forman EM, Beck AT. The empirical status of cognitive-behavioral therapy: a review of metaanalyses. Clinical Psychology Review 2006;26(1):17-31.

\section{Chang 2005}

Chang JC, Cluss PA, Ranieri L, Hawker L, Buranosky R, Dado D, et al. Health care interventions for intimate partner violence: what women want. Womens Health Issues 2005;15(1):21-30.

\section{Chavis 2008}

Chavis, A, Hill M. Integrating multiple intersecting identities: a multicultural conceptualisation of the power and control wheel. Women \& Therapy 2008;32(1):121-49.

\section{Cluss 2006}

Cluss $P$. The process of change for victims of intimate partner violence: support for a psychosocial readiness model. Womens Health Issues 2006;16(5):262-74.

\section{Cohen 1983}

Cohen S, Hoberman HM. Positive events and social supports as buffers of life change stress. Journals of Applied Social Psychology 1983;13(2):99-125.

\section{Cohen 1992}

Cohen J. A power primer. Psychological Bulletin 1992;112(1):155. 


\section{Courtois 2012}

Courtois CA, Ford JD. Treatment of Complex Trauma: A Sequenced, Relationship-based Approach. 1st edition. New York: Guilford Press, 2012.

\section{Covidence [Computer program]}

Veritas Health Innovation Covidence [Covidence systematic review software]. Version accessed 1 August 2018. Melbourne, Australia: Veritas Health Innovation, 2015.Available at www.covidence.org.

\section{Dalgard 1996}

Dalbard OS. Community health profile as tool for psychiatric prevention. In: Trent DR, Reed C, editors(s). Promotion of Mental Health. Vol. 5. Avebury: Aldershot, 1996.

\section{de Mello 2005}

de Mello M R, de Jesus Marl J, Bacaitchuk J, Verdell H, Neugebauer R. A systematic review of research findings on the efficacy of interpersonal therapy for depressive disorders. European Archives of Psychiatry and Clinical Neuroscience 2005;255(2):75-82.

\section{Deeks 2017}

Deeks JJ, Higgins JP, Altman DG editor(s) on behalf of the Cochrane Statistical Methods Group. Chapter 9: Analysing data and undertaking meta-analyses. In: Higgins JP, Churchill R, Chandler J, Cumpston MS, editor(s). Cochrane Handbook for Systematic Reviews of Interventions version 5.2.0 (updated June 2017), Cochrane, 2017. Available from www.training.cochrane.org/handbook.

\section{Devries 2013}

Devries KM, Mak JY, Bacchus LJ, Child JC, Falder G, Petzold M, et al. Intimate partner violence and incident depressive symptoms and suicide attempts: a systematic review of longitudinal studies. PLOS Medicine 2013;10:e1001439.

\section{Dobson 2009}

Dobson KS. Handbook of Cognitive-Behavioral Therapies. New York: Guilford Press, 2009.

\section{Donner 2000}

Donner A, Klar N. Design and Analysis of Cluster Randomization Trials in Health Research. 1st edition. New York: Oxford University Press Inc, 2000.

\section{Egger 1997}

Egger M, Davey-Smith G, Schneider M, Minder C. Bias in meta-analysis detected by a simple graphical test. BMJ 1997;315(7109):629-34

\section{Egger 2001}

Egger M, Davey Smith G, Altman DG. Systematic Review in Health Care: Meta-analysis in Context. 2nd edition. London: BMJ Books, 2001.

\section{Evans 2018}

Evans M, Malpass A, Agnew-Davies R, Feder G. Women's experiences of a randomised controlled trial of a specialist psychological advocacy intervention following domestic violence: a nested qualitative study. PLOS One 2018;13(11):e0193077.

\section{Feder 2006}

Feder G, Hutson M, Ramsay J, Taket AR. Women exposed to intimate partner violence: expectations and experiences when they encounter health care professionals: a metaanalysis of qualitative studies. Archives of Internal Medicine 2006;166(1):22-37.

\section{Feder 2009}

Feder G, Ramsay J, Dunne D, Rose M, Arsene C, Norman R, et al. How far does screening women for domestic (partner) violence in different health-care settings meet criteria for a screening programme? Systematic review of nine UK National Screening Committee criteria. Health Technology Assessment 2009;13(16):137-347. [DOI: 10.3310/hta13160]

\section{Fellmuth 2013}

Fellmuth GL, Heffernan C, Nurse J, Habibula S, Sethi D. Educational and skills-based interventions for preventing relationship and dating violence in adolescents and young adults. Cochrane Database of Systematic Reviews 2013, Issue 6. [DOI: 10.1002/14651858.CD004534.pub3]

\section{García-Moreno 2015}

García-Moreno C, Hegarty K, d'Oliveira AF, Koziol-McLain J, Colombini M, Feder $\mathrm{G}$. The health-systems response to violence against women. Lancet 2015;385(9977):1567-79.

\section{GRADE Working Group}

Schünemann H, Brożek J, Guyatt G, Oxman A, editor(s). Handbook for grading the quality of evidence and the strength of recommendations using the GRADE approach (updated October 2013). GRADE Working Group, 2013. Available from gdt.guidelinedevelopment.org/app/handbook/handbook.html.

\section{GRADEpro GDT [Computer program]}

McMaster University (developed by Evidence Prime, Inc.) GRADEpro GDT [GRADEpro Guideline Development Tool [Software]]. Version accessed 29 October 2019. Hamilton (ON): McMaster University (developed by Evidence Prime, Inc.), 2015.Available at gradepro.org.

\section{Hamilton 1960}

Hamilton M. A rating scale for depression. Journal of Neurology, Neurosurgery and Psychiatry 1960;23(1):56-62.

\section{Hayes 2006}

Hayes SC, Luoma JB, Bond PW, Masuda A, Lillis J. Acceptance and commitment therapy: model, processes and outcomes. Behaviour Research and Therapy 2006;44(1):1-25.

\section{Hegarty 2005}

Hegarty K, Bush R, Sheehan M. The composite abuse scale: further development and assessment of reliability and validity of a multidimensional partner abuse measure in clinical settings. Violence and Victims 2005;20(5):529-47. 


\section{Hegarty 2008}

Hegarty K, O'Doherty L, Gunn J, Pierce D, Taft A. A brief counselling intervention by health professionals utilising the 'readiness to change' concept for women experiencing intimate partner abuse: the weave project. Journal of Family Studies 2008;14(2-3):376-88.

\section{Hermann 1992}

Hermann JL. Trauma and Recovery: The Aftermath of Violence. 1st edition. New York: Basic Books, 1992.

\section{Higgins 2003}

Higgins JP, Thompson SG, Deeks JJ, Altman DG. Measuring inconsistency in meta-analyses. BMJ 2003;327(7414):557-60.

\section{Higgins 2011}

Higgins JP, Deeks JJ, Altman DG, editor(s). Chapter 16: Special topics in statistics. In: Higgins JP, Green S, editor(s). Cochrane Handbook for Systematic Reviews of Interventions. Version 5.1.0 (updated March 2011). The Cochrane Collaboration, 2011. Available from training.cochrane.org/handbook/PDF/v5.2/ chapter-16.

\section{Higgins 2017}

Higgins JP, Altman DG, Sterne JA, editor(s). Chapter 8: Assessing risk of bias in included studies. In: Higgins JP, Churchill R, Chandler J, Cumpston MS, editor(s). Cochrane Handbook for Systematic Reviews of Interventions Version 5.2.0 (updated June 2017). The Cochrane Collaboration, 2017. Available from www.training.cochrane.org/handbook.

\section{Humphreys 2003}

Humphreys C, Thiara R. Mental health and domestic violence: 'I call it symptoms of abuse'. British Journal of Social Work 2003;33(2):209-26.

\section{Humphreys 2004}

Humphreys C, Joseph S. Domestic violence and the politics of trauma. Women's Studies International Forum 2004;27(5-6):559-70.

\section{Hunot 2007}

Hunot V, Churchill R, Teixeira V, Silva de Lima M. Psychological therapies for generalised anxiety disorder. Cochrane Database of Systematic Reviews 2007, Issue 1. [DOI: 10.1002/14651858.CD001848.pub4]

\section{Kertz 2013}

Kertz S, Bigda-Peyton J, Bjorgvinsson T. Validity of the generalized anxiety disorder-7 scale in an acute psychiatric sample. Clinical Psychology \& Psychotherapy 2013;20(5):456-464.

\section{Krug 2002}

Krug EG, Dahlberg LL, Mercy JA, Zwi AB, Loranzo R. World report on violence and health. www.who.int/ violence_injury_prevention/violence/world_report/en/ full_en.pdf 2002.

\section{McFarlane 2004}

McFarlane J, Malecha A, Gist J, Watson K, Batten E, Hall I, et al. Increasing the safety-promoting behaviors of abused women. American Journal of Nursing 2004;104(3):40-50.

\section{Miller 2002}

Miller WR, Rollncik S. Motivational Interviewing: Preparing People for Change. 2nd edition. New York: Guilford Press, 2002.

\section{Moher 2009}

Moher D, Liberati A, Tetzlaff J, Altman DG. The PRISMA group. Preferred reporting items for systematic reviews and meta-analyses: The PRISMA statement. PLOS Medicine 2009;6(7):e1000097.

\section{Mynors-Wallis 2000}

Mynors-Wallis LM, Gath DH, Day A, Baker F. Randomised controlled trial of problem solving treatment, antidepressant medication, and combined treatment for major depression in primary care. BMJ 2000;320(7226):26-30.

\section{National Collaborating Centre Mental Health 2019}

National Collaborating Centre for Mental Health. Clinical Guideline NG116. Post-traumatic stress disorder (PTSD): The management of PTSD in adults and children in primary and secondary care. www.nice.org.uk/guidance/ng116 5 December 2018.

\section{Nelson 2012}

Nelson HD, Bougatsos C, Blazina I. Screening women for intimate partner violence: a systematic review to update the U.S. Preventative Services Task Force recommendation. Annals of Internal Medicine 2012;156(11):796-808.

\section{NICE 2009}

National Institute for Health and Clinical Excellence. NICE Clinical Guidelines 90: Depression - The treatment and management of depression in adults (partial update of NICE clinical guideline 23). www.nice.org.uk/guidance/cg90 October 2009.

\section{O'Doherty 2014}

O'Doherty LJ, MacMillan H, Feder G, Taft A, Taket A, Hegarty K. Selecting outcomes for intimate partner violence intervention trials: Overview and recommendations. Aggression and Violent Behavior 2014;19(6):663-72.

\section{O'Doherty 2015}

O'Doherty L, Hegarty K, Ramsay J, Davidson LL, Feder G, Taft A Screening women for intimate partner violence in healthcare settings. Cochrane Database of Systematic Reviews 2015, Issue 7. [DOI: 10.1002/14651858.CD007007.pub3]

\section{O'Doherty 2016}

O'Doherty L, Taket A, Valpied J, Hegarty K. Receiving care for intimate partner violence in primary care: barriers and enablers for women participating in the weave randomised controlled trial. Social Science \& Medicine 2016;160:35-42. 


\section{Oram 2013}

Oram S, Trevillion K, Feder G, Howard LM. Prevalence of experiences of domestic violence among psychiatric patients: systematic review. British Journal of Psychiatry 2013;202(2):94-99.

\section{Papadakaki 2009}

Papadakaki M, Tzamalouka G, Chatzifotiou S, Chliaoutakis J. Seeking for risk factors of Intimate Partner Violence (IPV) in a Greek national sample: the role of self-esteem. Journal of Interpersonal Violence 2009;24(5):732-750.

\section{Prochaska 1992}

Prochaska JO, DiClemente CC, Norcross JC. In search of how people change. Applications to addictive behaviors. American Psychologist 1992;47(9):1102-14.

\section{Quadara 2015}

Quadara A. Implementing trauma-informed systems of care in health settings: The WITH study: State of knowledge paper. ANROWS Landscapes, 10/2015. Sydney, NSW: ANROWS 2015:1-44.

\section{Radloff 1977}

Radloff LS. The CES-D scale: a self-report depression scale for research in the general population. Applied Psychological Measurement 1977;1(3):385-401.

\section{Rakovshik 2010}

Rakovshik SG, McManus F. Establishing evidence-based training in cognitive behavioral therapy: a review of current empirical findings and theoretical guidance. Clinical Psychology Review 2010;30(5):496-516.

\section{Rees 2011}

Rees S, Silove D, Chey T, Ivancic L, Steel Z, Creamer M, et al. Lifetime prevalence of gender-based violence in women and the relationship with mental disorders and psychosocial function. JAMA 2011;306(5):513-21.

\section{Reisenhofer 2013}

Reisenhofer S, Taft A. Women's journey to safety-The Transtheoretical model in clinical practice when working with women experiencing Intimate Partner Violence: a scientific review and clinical guidance. Patient Education and Counseling 2013;93(3):536-48.

\section{Review Manager 2014 [Computer program]}

Nordic Cochrane Centre, The Cochrane Collaboration Review Manager 5 (RevMan 5). Version 5.3. Copenhagen: Nordic Cochrane Centre, The Cochrane Collaboration, 2014.

\section{Rivas 2015}

Rivas C, Ramsay J, Sadowski L, Davidson LL, Dunne D, Eldridge $S$, et al. Advocacy interventions to reduce or eliminate violence and promote the physical and psychosocial wellbeing of women who experience intimate partner abuse. Cochrane Database of Systematic Reviews 2015, Issue 12. [DOI: 10.1002/14651858.CD005043]

\section{Rivas 2019}

Rivas C, Vigurs C, Cameron J, Yeo L. A realist review of which advocacy interventions work for which abused women under what circumstances. Cochrane Database of Systematic Reviews 2019, Issue 6. [DOI: 10.1002/14651858.CD013135]

\section{Roth 2007}

Roth AD, Pilling S. The competencies required to deliver effective cognitive and behavioural therapy for people with depression and with anxiety disorders. pdfs.semanticscholar.org/17ec/102ba06b05d392d9735359d424df9dacf09c ga $=2.50305317 .2015194633 .1589382151-1749534597.1589382151$ 2007.

\section{Schwarzer 1995}

Schwarzer R, Jerusalem M. Generalized self-efficacy scale. In: Weinman J, Wright S, Johnston M, editors(s). Measures in Health Psychology: A User's Portfolio. Causal and Control Beliefs. Windsor: NFER-NELSON, 1995:35-7.

\section{Schünemann 2017}

Schünemann HJ, Oxman AD, Higgins JP, Vist GE, Glasziou P, Akl E, et al, on behalf of the Cochrane GRADEing Methods Group and the Cochrane Statistical Methods Group. Chapter 11: Completing 'Summary of findings' tables and grading the confidence in or quality of the evidence. In: Higgins JP, Churchill R, Chandler J, Cumpston MS, editor(s),.Cochrane Handbook for Systematic Reviews of Interventions version 5.2.0 (updated June 2017). The Cochrane Collaboration, 2017. Available from www.training.cochrane.org/handbook.

\section{Skevington 2004}

Skevington SM, Lotfy M, O'Connell KA. The World Health Organization's WHOQOL-BREF quality of life assessment: psychometric properties and results of the international field trial. A report from the WHOQOL group. Quality of Life Research March 2004;13(2):299-310.

\section{Smith 1995}

Smith PH, Earp JA, DeVellis R. Measuring battering: development of the Women's Experience with Battering (WEB) Scale. Womens Health 1995;1(4):273-88.

\section{Spitzer 1999}

Spitzer RL, Kroenke K, Williams JB. Validation and utility of a self-report version of PRIME-MD. JAMA 1999;282(18):1737-44.

\section{Spitzer 2006}

Spitzer RL, Kroenke K, Williams JB, Löwe B. A brief measure for assessing generalized anxiety disorder: the GAD-7. Archives of Internal Medicine 2006;166(10):1092.

\section{Straus 1996}

Straus MA, Hamby SL, Boney-McCoy S, Sugarman DB. The revised conflict tactics scale (CTS2): development and preliminary psychometric data. Journal of Family issues 1996;17(3):283.

\section{Tan 2018}

Tan M, O'Doherty L, Gilchrist G, Taft A, Feder G, Tirado Muñoz J, et al. Psychological therapies for women who experience 
intimate partner violence. Cochrane Database of Systematic Reviews 2018, Issue 5. [DOI: 10.1002/14651858.CD013017]

\section{Tirado-Muñoz 2014}

Tirado-Muñoz J, Gilchrist G, Farré M, Hegarty K, Torrens M. The efficacy of cognitive behavioural therapy and advocacy interventions for women who have experienced intimate partner violence: a systematic review and meta-analysis. Annals of Medicine 2014;46(8):567-86.

\section{Tiwari 2005}

Tiwari A, Leung WC, Leung TW, Humphreys J, Parker B, Ho PC. A randomised controlled trial of empowerment training for Chinese abused pregnant women in Hong Kong. British Journal of Obstetrics and Gynaecology 2005;112:1249-56.

\section{Trevillion 2012}

Trevillion K, Oram S, Feder G, Howard LM. Experiences of domestic violence and mental disorders: a systematic review and meta-analysis. PLOS One 2012;7(12):e51740.

\section{Valpied 2019}

Valpied J, Hegarty K, Brown S, O'Doherty L. Self-efficacy and doctor support as mediators of depression outcomes following counselling by family doctors for intimate partner violence. Family Practice 2020;37(2):255-62. [DOI: 10.1093/fampra/ cmz067]

\section{Ware 1996}

Ware J Jr, Kosinski M, Keller SD. A 12-Item Short-Form Health Survey: construction of scales and preliminary tests of reliability and validity. Medical Care 1996;34(3):220-33.

\section{Warshaw 2013}

Warshaw C, Sullivan C, Rivera E. A systematic review of trauma-focused interventions for domestic violence survivors. www.nationalcenterdvtraumamh.org/wp-content/ uploads/2013/03/NCDVTMH_EBPLitReview2013.pdf 2013:1-27.

\section{Weathers 1991}

Weathers FW, Juska JA, Keane TM. The PTSD checklist - civilian version (PCL-C). Boston National Center for PTSD, Boston Veterans Affairs Medical Center 1991.

\section{World Health Organization 2013a}

World Health Organization, Department of Reproductive Health and Research, London School of Hygiene and Tropical Medicine, South African Medical Research Council. Global and regional estimates of violence against women: Prevalence and health effects of intimate partner violence and non-partner sexual violence. www.who.int/reproductivehealth/publications/ violence/9789241564625/en/ 2013.

\section{World Health Organization 2013b}

World Health Organization. Responding to intimate partner violence and sexual violence against women. WHO clinical and policy guidelines. www.who.int/reproductivehealth/ publications/violence/9789241548595/en/ 2013.

\section{World Health Organization 2013c}

World Health Organization. Violence against women: a 'global health problem of epidemic proportions'. www.lshtm.ac.uk/ newsevents/news/2013/gender_violence_report.html 2013.

\section{Young 1980}

Young J, Beck AT. Cognitive Therapy Scale Rating Manual. Beck Institute for Cognitive Behavior Therapy 1980.

\section{Zink 2004}

Zink T, Elder N, Jacobson J, Klostermann B. Medical management of intimate partner violence considering the stages of change: precontemplation and contemplation. Annals of Family Medicine 2004;2(3):231-9.

* Indicates the major publication for the study

\section{CHARACTERISTICS OF STUDIES}

Characteristics of included studies [ordered by study ID]

Akor 2019

\section{Study characteristics}

Methods

Trial design: $\mathrm{RCT}$

Trial grouping: parallel group

Randomisation method: victims were allocated into a control and intervention arm by simple balloting of 36 per arm

Primary outcomes: family function measured by SCORE-15 index of family function

Secondary outcomes: NR

Timing of outcome measurements: pre- and post-intervention (the family functions of both the intervention and control group were reassessed at the end, approximately 6 weeks from the first assessment) 
Types of participants: all pregnant victims of IPV $<34$ weeks who consented to the trial were included.

Total duration of trial: approximately 6 weeks

Funding for trial: NR

Conflict of interest of trial authors: trial authors did not report any potential Col

Type of analysis: descriptive statistics, $\mathrm{Chi}^{2}$ and t-test

Assessment modality (e.g. face-to-face, telephone, online): face-to-face

ITT analyses: NR

Participants

\section{Baseline characteristics}

Experimental intervention

- Age: $20-34$ years age group 34 (94.4\%)

- Ethnicity: NR (trial was conducted in North Central Nigeria)

- Employment: NR (although in terms of occupation 19 (52.8\%) were unskilled

- Education: 26 (72.2\%) with tertiary eduction

- Relationship status: 35 (97.2\%) married

- Gender (\% women): 100

- Dependent children: NR

- Number analysed (data analysis at post-treatment stage): NR

- Number of participants eligible assigned: 72

- Number of participants dropped out (by final follow-up): NR

- Comorbidities: NR

- Experience of IPV: all participating women had experienced IPV as measured by AAS

- Experience of other types of violence and abuse: NR

- Remuneration: NR

- Financial dependence (on partner): NR

Comparator intervention

- Age: $20-34$ years age group 28 (77.8\%)

- Ethnicity: NR (trial was conducted in North Central Nigeria)

- Employment: NR (although in terms of occupation 22 (61.1\%) were unskilled

- Education: 17 (47.2\%) with tertiary eduction

- Relationship status: 34 (94.4\%) married

- Gender (\% women): 100

- Dependent children: NR

- Number analysed (data analysis at post-treatment stage): NR

- Number of participants eligible assigned: 72

- Number of participants dropped out (by final follow-up): NR

- Comorbidities: NR

- Experience of IPV: all participating women had experienced IPV as measured by AAS

- Experience of other types of violence and abuse: NR

- Remuneration: NR

- Financial dependence (on partner): NR

Total sample

- Age: $20-34$ years age group $62(86.1 \%)$

- Ethnicity: NR (trial was conducted in North Central Nigeria)

- Employment: NR (although in terms of occupation 41 (56.9\%) were unskilled 
Akor 2019 (Continued)

- Education: 43 (59.7\%) with tertiary eduction

- Relationship status: 69 (95.8\%) married

- Gender (\% women): 100

- Dependent children: NR

- Number analysed (data analysis at post-treatment stage): NR

- Number of participants eligible assigned: 72

- Number of participants dropped out (by final follow-up): NR

- Comorbidities: NR

- Experience of IPV: all participating women had experienced IPV as measured by AAS

- Experience of other types of violence and abuse: NR

- Remuneration: NR

- Financial dependence (on partner): NR

Included criteria: all pregnant victims of IPV $<34$ weeks who consented to the trial were included.

Excluded criteria: pregnant women with no intimate partners, e.g. those who were raped by persons they had no relationship with, were excluded from the trial.

Baseline differences: there were no differences between the groups at pre-test. There was evidence that randomisation procedure was successful.

Total number of participants within all groups: 72

Number of eligible people recruited: NR

Sample power calculation: power analysis and sample size determination

Number of eligible people consented: 72

Total number of participants approached: NR

Number ineligible: NR

Number eligible: 72

Recruitment setting of participants (e.g. healthcare setting, community setting, shelter setting): healthcare setting

Number declined to participate: NR

\section{Intervention characteristics}

Experimental intervention

- Brief description (rationale, main components): counselling was done using the SOS-DoC frame work (S-offer support and assess safety; O-discuss options; S-validate patient's strengths; Do-document observations, assessment, and plans; $\mathrm{C}$-offer continuity).

- Type of intervention: short-term intensive counselling sessions

- Mode of delivery (how): one-on-one face-to-face

- Frequency and duration of delivery (when and how much): 3 counselling sessions at 2-weekly intervals

- Level of mental health training of person delivering the intervention: NR

- Fidelity assessment: NR

- Intervention model: counselling was done using the SOS-DoC frame work (S-offer support and assess safety; O-discuss options; S-validate patient's strengths; Do-document observations, assessment, and plans; C-offer continuity)

- Person delivering the intervention (who provided the intervention): therapist, self-directed or combined: NR

- Intervention location (where): the trial was conducted at the ANC of the University of Abuja Teaching Hospital (UATH), Gwagwalada, North Central Nigeria 
Akor 2019 (Continued)

- Tailoring of intervention (personalised, titrated or adapted): the framework was individualized for each victim and depending on what was reported as the likely cause of the IPV suggestions were made to resolve such problems

- Intervention modifications (during the trial): NR

- Main techniques of intervention: it combines 2 therapeutic counselling techniques, nondirective counselling, which aims at encouraging the client to discuss her/his problems with the counsellor who, through listening, affirms the patient's worth and allows her/him to take time to express their thoughts; and problem-solving therapy, which involves systematically teaching generic skills in active problem solving to reduce stress and enhance self-efficacy

- Intervention manual: NR

- CCDAN psychological therapies: integrative therapies

- Intervention attendance: NR

- Intensity of intervention: up to 4 sessions

- Person delivering the intervention (healthcare workers, non healthcare workers): unclear

- Gender of therapist: NR

Comparator intervention

- Brief description (rationale, main components): waiting list

- Type of intervention: waiting list

Family function
- Outcome type: continuous outcome
- Reporting: fully reported
- Scale: SCORE-15
- Direction: lower is better
Dropouts from treatment
- Outcome type: dichotomous outcome
- Direction: lower is better

Identification

Sponsorship source: NR

Country: Nigeria

Setting: Health

Comments: the trial was conducted at the ANC of the University of Abuja Teaching Hospital (UATH), Gwagwalada, North Central Nigeria

Trial authors: BO Akor, LA Moses, ND Baamlong, LL Shedul, AS Haruna, JM Abu, OU Chira, NR Ripiye \& RA Abdulkareem

Institution: University of Abuja Teaching Hospital, Gwagwalada, Nigeria

Email: blessingaj@yahoo.com

Address: Department of Family Medicine, University of Abuja Teaching Hospital, Gwagwalada, Nigeria

Title (main 'outcome' publication): Effect of counselling on the family function of intimate partner violence victims attending ANC in a tertiary hospital in North Central Nigeria

Registered trial protocol ID: NR 
Adverse events (harm) related to participation: NR

\section{Risk of bias}

\begin{tabular}{lll}
\hline Bias & Authors' judgement & Support for judgement \\
\hline $\begin{array}{l}\text { Random sequence genera- } \\
\text { tion (selection bias) }\end{array}$ & High risk & $\begin{array}{l}\text { Judgement comment: "systematic sampling was used to recruit } 72 \text { IPV victims } \\
\text { who were randomised into } 2 \text { arms of } 36 \text { each by simple balloting". No further } \\
\text { evidence provided about random sequence generation. }\end{array}$
\end{tabular}

Allocation concealment $\quad$ High risk
(selection bias)
(selection bias) Judgement comment: "the victims were then allocated into a control and in-
tervention arm by simple balloting of 36 per arm". No further evidence provid-
ed about allocation concealment.

Blinding of participants and personnel (perforUnclear risk

mance bias)

All outcomes

Judgement comment: there is insufficient information to assess blinding of participants and personnel.

\begin{tabular}{lll}
\hline Blinding of outcome as- & Unclear risk & Judgement comment: "a research assistant, who did not know which arm the \\
sessment (detection bias) & victims belonged to, assessed their family function at the beginning of the tri- \\
All outcomes & al". However, it is not clear as to who and how post-assessment was conduct- \\
& ed.
\end{tabular}

\begin{tabular}{lll}
\hline $\begin{array}{l}\text { Incomplete outcome data } \\
\text { (attrition bias) } \\
\text { All outcomes }\end{array}$ & High risk & $\begin{array}{l}\text { Judgement comment: there is not sufficient information about attrition, exclu- } \\
\text { sion or dropouts at different stages of the trial. }\end{array}$ \\
\hline $\begin{array}{l}\text { Selective reporting (re- } \\
\text { porting bias) }\end{array}$ & Unclear risk & $\begin{array}{l}\text { Judgement comment: all outcomes described in the methods section were ad- } \\
\text { equately collected, analysed and reported in the results section of the main } \\
\text { published trial. However, there was insufficient or unclear evidence from ei- } \\
\text { ther the trial protocol or trial registry sites to assess this domain. }\end{array}$ \\
\hline Other bias & Unclear risk & $\begin{array}{l}\text { Judgement comment: the published trial outcome paper had insufficient in- } \\
\text { formation about various aspects related to the trial, including recruitment, re- } \\
\text { tention rate, as well as limitations of the trial. }\end{array}$ \\
\hline
\end{tabular}

Bowland 2012

\section{Study characteristics}

Methods

Trial design: RCT

Trial grouping: parallel group

Randomisation method: women were paired on scores from a spiritual distress scale and then randomised into treatment or control groups using a random number table.

Primary outcomes: the outcome variables of post-traumatic stress, depression, anxiety, and somatic symptoms were measured using instruments with established validity and reliability.

\section{Secondary outcomes: NR}

Timing of outcome measurements: baseline (prior to the beginning of the intervention); post-treatment (after 11 weeks at the end of intervention); follow-up (3-months from post-treatment) 
Types of participants: sample was composed of adult female community-dwelling survivors (age $\geq 55$ years) who lived in St. Louis, Missouri. Ads were placed in a senior newspaper, and a poster and flyers were circulated at a university hospital. Most participants came from the hospital research volunteer list $(n=28)$

Total duration of trial: a total of 3 experimental groups were held sequentially beginning in July 2006 and ending in January 2008. 3 groups also completed the control condition during the same period. Recruitment for this project ran from February 2006-July 2007

\section{Funding for trial: NR}

\section{Conflict of interest of trial authors: NR}

Type of analysis: MANOVAs, t-tests, and regression analyses

Assessment modality (e.g. face-to-face, telephone, online): face-to-face or by phone

ITT analyses: NR

\section{Participants}

\section{Baseline characteristics}

Experimental intervention

- Age: $M=60.33$ years, $S D=4.6$

- Ethnicity: NR

- Employment: NR

- Education: completed college (13,48\%)

- Relationship status: 11 (50\%) partnered

- Gender (\% women): 100

- Dependent children: NR

- Number analysed (data analysis at post-treatment stage): 21

- Number of participants eligible assigned: 21

- Number of participants dropped out (by final follow-up):

- Comorbidities: NR

- Experience of IPV: NR

- Experience of other types of violence and abuse:

- Remuneration: NR

- Financial dependence (on partner): NR

Comparator intervention

- Age: $M=62.32$ years, $S D=7.7$

- Ethnicity: NR

- Employment: NR

- Education: completed college (14, 52\%)

- Relationship status: 11 (50\%) partnered

- Gender (\% women): 100

- Dependent children: NR

- Number analysed (data analysis at post-treatment stage): 22

- Number of participants eligible assigned: 22

- Number of participants dropped out (by final follow-up): NR

- Comorbidities: NR

- Experience of IPV: 17 (77.3\%)

- Experience of other types of violence and abuse:

- Remuneration: NR

- Financial dependence (on partner): NR

Total sample 
- Age: $M=61.3$ years, with a range of $55-83$ years

- Ethnicity: 36 (84\%) were white, 6 (14\%) were African American, and 1 was Eastern Indian

- Employment: $65 \%$ had incomes of < USD 50,000

- Education: the majority of the sample $(n=27)$ held an associate's or bachelor's degree

- Relationship status: 23 women were partnered and 20 were single at the time of the trial

- Gender (\% women): 100

- Dependent children: NR

- Number analysed (data analysis at post-treatment stage): 36

- Number of participants eligible assigned: 43

- Number of participants dropped out (by final follow-up): 7

- Comorbidities: NR

- Experience of IPV: women reported domestic violence and sexual assault beginning in young adulthood. Approximately $33 \%$ also disclosed chronic emotional abuse in their current primary relationship. Women reported domestic violence and sexual assault beginning in young adulthood

- Experience of other types of violence and abuse: 42 of 43 women reported they had been exposed to multiple types of violence and abuse.

- Remuneration: NR

- Financial dependence (on partner): NR

Included criteria: during the initial phone screening, participants in the trial indicated they had current trauma-related distress. None of the women, however, was in crisis due to current physical or sexual violence. Eligibility criteria for the trial specified that participants have a history in the Christian tradition. This was used to increase the homogeneity of this small sample, because subgroup analysis was not possible. Participants were not in psychotherapy, did not have significant cognitive impairments, and were not actively suicidal or psychotic. Women who did not meet these criteria were referred to other community resources.

Excluded criteria: women who were attending psychotherapy, had significant cognitive impairments, and/or were actively suicidal or psychotic were excluded. Women who did not meet the inclusion criteria were referred to other community resources.

Baseline differences: there were no differences between the groups at pre-test. There was evidence that randomisation procedure was successful.

Total number of participants within all groups: 43

Number of eligible people recruited: 44

Sample power calculation: a power analysis revealed that a sample size of 48 , assuming an effect size of 1.0 , would provide power of approximately $80 \%$ to detect a relationship between the intervention and the mental health outcomes

Number of eligible people consented: 44

Total number of participants approached: 129

Number ineligible: 60

Number eligible: 69

Recruitment setting of participants (e.g. healthcare setting, community setting, shelter setting): healthcare setting (most participants came from the hospital research volunteer list)

Number declined to participate: NR

Experimental intervention

- Brief description (rationale, main components): "practitioners and trauma survivors from an agency serving clients with co-occurring disorders developed a brief group intervention ( $1.5 \mathrm{~h} / \mathrm{session})$ that 
was used in the present trial. The intervention was chosen because it is a manualised psycho-educational, cognitive restructuring, and skill-building approach to addressing spiritual struggles in recovery (Fallot \& The Spirituality Workgroup, 2001-2004). The model is an offshoot of the Trauma Recovery and Empowerment Model (TREM) (Harris \& The Community Connections Trauma Work Group, 1998). The feminist Social Work perspective was used to raise questions, for instance, about endurance and sacrifice of self for others. Alternative viewpoints to patriarchal interpretations of scripture and religious life were sometimes introduced".

- Type of intervention: spiritual intervention (psycho-educational, cognitive restructuring, and skillbuilding)

- Mode of delivery (how): group-based face-to-face

- Frequency and duration of delivery (when and how much): total of 11 weekly sessions, $1.5 \mathrm{~h} /$ session

- Level of mental health training of person delivering the intervention: all of the facilitators in this trial had training in theology and pastoral care

- Fidelity assessment: to assess leader fidelity to the group treatment model, an independent evaluator rated videotaped sessions randomly selected from each of the 6 groups. The mean adherence score for 18 sessions was $83.3 \%$ (SD $=19.40$; range $=40 \%-100 \%)$.

- Intervention model: manualised psycho-educational, cognitive restructuring, and skill-building approach to addressing spiritual struggles in recovery; offshoot of the Trauma Recovery and Empowerment Model (TREM) (manualised psycho-educational, cognitive restructuring, and skill-building approach to addressing spiritual struggles in recovery)

- Person delivering the intervention (who provided the intervention): therapist, self-directed or combined: therapist (the first author, who conducted the intervention, was trained using the TREM model)

- Intervention location (where): NR

- Tailoring of intervention (personalised, titrated or adapted): NR

- Intervention modifications (during the trial): NR

- Main techniques of intervention: psycho-educational, cognitive restructuring, and skill-building. During session 1, participants discussed their spiritual histories in relation to their traumatic experiences. In session 2, spiritual gifts, such as discernment, forgiveness, and trust, were identified as qualities participants had or needed in their lives. In session 3, a spiritual recovery action plan was developed after participants discussed research literature about the value of positive spiritual coping for health. The harm of negative coping, such as viewing God as punishing or abandoning, or being unable to forgive oneself for abuse, was also discussed. Women named spiritual needs and places of struggle in their spiritual lives. Different psychological struggles often connected with trauma, including anger, fear and powerlessness, guilt and shame, despair, and loneliness, were explored in sessions 4 through 8 as challenges with potential spiritual solutions. Experiences within organised religious traditions were also discussed frequently. Sessions 9 through 11 focused on recovery strategies, such as prayer, music, poetry, visiting a new congregation when the old one was not meeting spiritual needs, reading a new translation of sacred text, and joining or creating a spiritual support group

- Intervention manual: the intervention was chosen because it is a manualised psycho-educational, cognitive restructuring, and skill-building approach to addressing spiritual struggles in recovery

- CCDAN psychological therapies: other psychologically-orientated interventions

- Intervention attendance: NR

- Intensity of intervention: $\geq 5$ sessions

- Person delivering the intervention (healthcare workers, non healthcare workers): non-healthcare workers

- Gender of therapist: female

Comparator intervention

- Brief description (rationale, main components): waiting list

- Type of intervention: waiting list

\section{Depression}

- Outcome type: continuous outcome

- Reporting: fully reported

- Scale: GDS 
Bowland 2012 (Continued)

- Range: 0-30

- Direction: lower is better

\section{Anxiety}

- Outcome type: continuous outcome

- Reporting: fully reported

- Scale: BAI

- Range: 0-63

- Direction: lower is better

\section{Dropouts from treatment}

- Outcome type: dichotomous outcome

- Reporting: fully reported

- Direction: lower is better

Sponsorship source: NR

Country: USA

Setting: community

Comments: community-dwelling women survivors of interpersonal trauma (including IPV victims)

Trial authors: Sharon Bowland, Tonya Edmond, and Roger D. Fallot

Institution: Washington University St. Louis, Missouri

Email: aron.bowland@louisville.edu

Address: Washington University St. Louis, Missouri

Title (main 'outcome' publication): Evaluation of a spiritually focused intervention with older trauma survivors

Registered trial protocol ID: NR

Process evaluation: NR

Summary of participants' views about the intervention: NR

Adverse events (harm) related to participation: NR

\section{Risk of bias}

\begin{tabular}{lll}
\hline Bias & Authors' judgement & Support for judgement \\
\hline $\begin{array}{l}\text { Random sequence genera- } \\
\text { tion (selection bias) }\end{array}$ & Low risk & $\begin{array}{l}\text { Judgement comment: "women were paired on scores from a spiritual distress } \\
\text { scale (SDS) and then randomised into treatment or control groups using a ran- } \\
\text { dom number table". }\end{array}$ \\
\hline $\begin{array}{l}\text { Allocation concealment } \\
\text { (selection bias) }\end{array}$ & High risk & $\begin{array}{l}\text { Judgement comment: A total of } 3 \text { experimental groups were held sequential- } \\
\text { ly beginning in July 2006 and ending in January 2008. Three groups also com- } \\
\text { pleted the control condition during the same period. Subsequently, the con- } \\
\text { trol participants were offered the opportunity to enter treatment. There is no } \\
\text { evidence of allocation concealment. }\end{array}$ \\
\hline
\end{tabular}


Bowland 2012 (Continued)

Blinding of participants Unclear risk Judgement comment: "the first author, who conducted the intervention, was and personnel (perfortrained using the TREM model. Further, the testers (Ph.D. students) were blind mance bias)

All outcomes to the treatment conditions of the participants". However, insufficient information provided to adequately assess the blinding of participants and other personnel.

\begin{tabular}{|c|c|c|}
\hline $\begin{array}{l}\text { Blinding of outcome as- } \\
\text { sessment (detection bias) } \\
\text { All outcomes }\end{array}$ & Low risk & $\begin{array}{l}\text { Judgement comment: "the testers were blind to the treatment conditions of } \\
\text { the participants. Testing was conducted by } 5 \text { PhD students with clinical experi- } \\
\text { ence with trauma survivors". }\end{array}$ \\
\hline
\end{tabular}

$\begin{array}{ll}\begin{array}{l}\text { Incomplete outcome data } \\ \text { (attrition bias) }\end{array} & \text { Low risk } \\ \text { All outcomes } & \begin{array}{l}\text { Judgement comment: attrition and exclusions of participants were reasonably } \\ \text { described. The outcome variables of post-traumatic stress, depression, anxi- } \\ \text { ety, and somatic symptoms were fully measured and reported. }\end{array}\end{array}$

$\begin{array}{ll}\begin{array}{l}\text { Selective reporting (re- } \\ \text { porting bias) }\end{array} & \begin{array}{l}\text { Judgement comment: all outcomes described in the methods section were ad- } \\ \text { equately collected, analysed and reported in the results section of the main } \\ \text { published trial. However, there was insufficient or unclear evidence from ei- } \\ \text { ther the trial protocol or trial registry sites to assess this domain. }\end{array}\end{array}$

Other bias High risk

\begin{abstract}
Judgement comment: "limitations in this trial must be acknowledged. Recruitment, screening, and intervention were all completed by Sharon Bowland. Ideally, investigators would not deliver the intervention, to reduce the potential for social desirability bias. Limited funds for advertising, having only 1 person available to conduct recruitment, and extensive preliminary processes led to a slow qualification timeline. Most likely, a larger sample would have resulted in a more accurate measurement of the treatment effects. The fidelity check on the intervention was performed by 1 evaluator, so interrater reliability was not assessed. Finally, social desirability was not measured and cannot be ruled out as a motivator for participants to improve. Finally, some participants had multiple trauma exposures, including childhood abuse, domestic violence, and sexual assault and the data analysis did not differentiate or adjust the analysis for the type of trauma exposure". Overall, these add toward high risk for other sources of bias.
\end{abstract}

\section{Study characteristics}

Trial design: $\mathrm{RCT}$

Trialgrouping: parallel group

Types of participants: women with a history of gender-based violence

Conflict of interest of trial authors: trial authors have declared that no competing interests exist

Funding for trial: trial was supported by Grand Challenges Canada \#0368-04(www. grandchallenges.ca/) to JU \& AS, World Vision Canada (www.worldvision.ca/) to JU, and World Vision Australia (www.worldvision.com.au/ home-east-Africa) to AS.

Primary outcomes: primary outcome was the GHQ-12, which indexes psychological distress, including anxiety and depression

Secondary outcomes: PCL; WHODAS (WHO Disability Adjustment Schedule): PSYCHLOPS (Psychological Outcome Profiles) and LEC scores

Randomisation method: participants were randomly allocated (on a 1:1 ratio) to either a 5-week course of problem management or comparator intervention. Randomisation was conducted at the Uni- 
Bryant 2017 (Continued)

versity of New South Wales, Australia, by staff who were independent of the trial using computerised software that generated random number sequences

Timing of outcome measurements: baseline, post-treatment and 3-month follow-up

Total duration of trial: 15 April 2015-16 January 2016

Assessment modality (e.g. face-to-face, telephone, online): face-to-face

Type of analysis: hierarchical linear models,

ITT analyses: yes

Participants

Experimental intervention

- Age: $M=35.2$ years, $S D=14.1$

- Gender (\% women): 100

- Ethnicity: adult women in urban Kenya (not reported)

- Employment: working: 104 (49.8\%)

- Education: $\mathrm{M}=8.7$ years, $\mathrm{SD}=3.6$

- Relationship status: single 25 (12.0\%); married 122 (58.4\%); divorced/separated 42 (20.1\%); widowed $20(9.5 \%)$

- Dependent children: NR

- Number analysed (data analyses at post-treatment stage): 156

- Number of participants eligible assigned: 209

- Number of participants dropped out (by final follow-up): 53

- Comorbidities: suicidal intention in past month: 50 (23.9\%)

- Experience of IPV: 153 (73.2\%)

- Experience of other types of violence and abuse: physical assault 155 (74.2\%); sexual assault 59 (28.2\%)

- Remuneration: participants were reimbursed KES 300 (approximately USD 3) for each assessment

Comparator intervention

- Age: $\mathrm{M}=35.9$ years, $\mathrm{SD}=12.7$

- Gender (\% women): 100

- Ethnicity: adult women in urban Kenya (not reported)

- Employment: working: 108 (50.9\%)

- Education: 8.2 years (4.2)

- Relationship status: single 30 (14.1\%); married 119 (56.1\%); divorced/separated 45 (21.2\%); widowed $18(8.5 \%)$

- Dependent children: NR

- Number analysed (data analyses at post-treatment stage): 163

- Number of participants eligible assigned: 212

- Number of participants dropped out (by final follow-up): 49

- Comorbidities: suicidal intention in past month: 35 (16.5\%)

- Experience of IPV: 152 (71.7\%)

- Experience of other types of violence and abuse: physical assault 153 (72.2\%); sexual assault 72 (34.0\%)

- Remuneration: participants were reimbursed KSh 300 (approximately US\$3) for each assessment

Total sample:

- Age: NR

- Gender (\% women): 100

- Ethnicity: NR 
- Employment: NR

- Education: NR

- Relationship status: NR

- Dependent children: NR

- Number analysed (data analyses at post-treatment stage): 319

- Number of participants eligible assigned: 421

- Number of participants dropped out (by final follow-up): 102

- Comorbidities: NR

- Experience of IPV: NR

- Experience of other types of violence and abuse: NR

- Remuneration: participants were reimbursed KES 300 (approximately USD 3) for each assessment

Included criteria: inclusion criterion of Gender-Based-Violence was endorsement of any (prior or current) experience of interpersonal violence on either the LEC (25) or the WHO Violence Against Women Instrument (WHO-VAW (26)), which were administered at baseline assessment

Excluded criteria: exclusion criteria included (a) imminent plans of suicide, (b) psychotic disorders, or (c) severe cognitive impairment. Assessors referred any cases of threat of harm or self-harm to local services.

Pretreatment: planned comparisons of women in the PM+ and EUC groups indicated that these 2 groups did not differ on any pretreatment factors.

Total number of participants: 421

Number of eligible people recruited: 451

Sample power calculation: power calculations indicated a minimum sample size of 133 participants/group using the test for paired means (power $=0.95$, alpha $=0.05,2$-sided)

Number of eligible people consented: 421

Total number of participants within all groups: 421

Total number of participants approached: 1393

Recruitment setting of participants (e.g. healthcare setting, community setting, shelter setting): community

Number eligible: 451

Number ineligible: 942

Experimental intervention

- Brief description: WHO developed a brief psychological intervention, termed Problem Management Plus (PM+), comprising strategies that people without qualifications or experience in mental health can be trained to deliver to reduce common mental disorders following adversity

- Type of intervention: problem management

- Mode of delivery (how): face-to-face

- Frequency and duration of delivery (when and how much): 5, weekly, 90-minute individual sessions

- Level of mental health training of person delivering the intervention: $\mathrm{CHWs}$ with no prior training or experience in mental healthcare

- Fidelity assessment: the fidelity checks indicated that $\mathrm{CHWs}$ adhered to the protocol by addressing the requisite $\mathrm{PM}+$ components in the appropriate sessions, including stress reduction $(91.39 \%)$, problem solving (94.25\%), behavioural activation (83.25\%), and accessing social support (92.82\%).

- Intervention model: problem management psychological intervention

- Intervention attendance: 126 (61\%) completed all 5 sessions 
- CCDAN psychological therapies: integrative therapies

- Intervention location (where): the PM+ sessions were provided in the participants' home, unless they preferred to do them in an alternate location for safety or privacy reasons

- Person delivering the intervention (who provided the intervention): therapist, self-directed or combined: therapist (lay CHWs)

- Tailoring of intervention (personalised, titrated or adapted):

- Intervention modification (during the trial): prior to the trial, the translation and cultural adaptation of $\mathrm{PM}+$ was reviewed in 2 workshops with experts on $\mathrm{PM}+$, translators, and $\mathrm{CHWs}$ to ensure that the assessment tools and intervention were appropriate in the Nairobi context. The measures and intervention were adapted for cultural appropriateness in terms of language, metaphors, content, concepts, goals, methods, and context

- Main techniques of intervention: PM+ commenced with an introduction to the programme, MI, psycho education, and stress management (session 1); problem-solving strategies focused on specific problems nominated by the participant and review of stress management strategies (session 2); behavioural activation and review of problem-solving and stress management (session 3); strengthening social supports and review of stress management, problem-solving, behavioural activation, and social supports (session 4); and reinforcement of all strategies and relapse prevention education (session 5).

- Intervention manual: participants allocated to PM+ were offered 5, weekly, 90-minute individual sessions (the full English and Swahili versions of the manual are available at www.who.int/mental_health/emergencies/problem_management_plus/en/).

- Intensity of intervention: $\geq 5$ sessions

- Person delivering the intervention: non-healthcare workers

- Gender of therapist: NR

Comparator intervention

- Brief description: enhanced usual care (non-specific counselling)

- Type of intervention: participants allocated to EUC were referred to primary healthcare centres, where nurses provided non-specific counselling. The nurses providing EUC did not follow a specific manual, they could use the strategies and number of sessions they deemed appropriate, and each nurse could use their judgement on rescheduling missed appointments with participants; there was continuity of the same nurse and same clinic for each woman in EUC

$\begin{array}{ll}\text { Outcomes } & \text { Mental health } \\ \text { - Outcome type: continuous outcome } \\ \text { - Reporting: fully reported } \\ \text { - Scale: GHQ-12 } \\ \text { - Range: 0-36 } \\ \text { PTSD } \\ \text { - Outcome type: continuous outcome } \\ \text { - Reporting: fully reported } \\ \text { - Scale: PCL } \\ \text { - Range: } 0-80 \\ \text { - Direction: lower is better }\end{array}$

\section{Dropouts from treatment}

- Outcome type: dichotomous outcome

- Reporting: fully reported

- Direction: lower is better

Identification

Sponsorship source: trial was supported by Grand Challenges Canada \#0368-04 (www. grandchallenges.ca/) to JU \& AS, World Vision Canada (www.worldvision.ca/) to JU, and World Vision Australia (www.worldvision.com.au/ home-east-Africa) to AS. 
Bryant 2017 (Continued)

\section{Country: Kenya}

Setting: community (participants' home)

Comments: participants were recruited by interviewing 1 woman from 1 of every 10 households in peri-urban areas in Nairobi

Trialauthors: Richard A. Bryant, Alison Schafer, Katie S. Dawson, Dorothy Anjuri, Caroline Mulili, Lincoln Ndogoni, Phiona Koyiet, Marit Sijbrandij, Jeannette Ulate, Melissa Harper Shehadeh, Dusan HadziPavlovic, Mark van Ommeren

\section{Institution:}

Email: r.bryant@unsw.edu.au

Address: School of Psychology, University of New South Wales, Sydney, New South Wales, Australia

Trial title (main outcome publication): Effectiveness of a brief behavioural intervention on psychological distress among women with a history of gender-based violence in urban Kenya: a randomised clinical trial

Registered trial protocol ID: ACTRN12614001291673

Notes Analysis of any cost/benefit measures: NR

Process evaluation: NR. We did not measure mechanism-related processes because of the priority of indexing the effectiveness of PM+ and limiting the assessment burden on participants.

\section{Summary of participants' views about the intervention: NR}

Adverse events (harm) related to participation: adverse reactions were monitored and recorded throughout screening and the intervention. Indications of psychiatric crisis (e.g. imminent suicidal risk as defined by suicidal plan) or need for acute protection were referred to the local advisory board, and referral to appropriate services was made (including local hospitals providing psychiatric care). This programme was safe insofar as it did not cause adverse outcomes, and could be used by women who may be experiencing current IPV, in combination with relevant protective interventions.

\section{Risk of bias}

\begin{tabular}{lll}
\hline Bias & Authors' judgement & Support for judgement \\
\hline $\begin{array}{l}\text { Random sequence genera- } \\
\text { tion (selection bias) }\end{array}$ & Low risk & $\begin{array}{l}\text { Judgement comment: "randomisation was conducted at the University of New } \\
\text { South Wales, Australia, by staff who were independent of the trial using com- } \\
\text { puterised software that generated random number sequences". }\end{array}$
\end{tabular}

$\begin{array}{ll}\begin{array}{l}\text { Allocation concealment } \\ \text { (selection bias) }\end{array} & \text { Low risk } \\ & \begin{array}{l}\text { Judgement comment: "participants were randomly allocated (on a 1:1 ra- } \\ \text { tio) to either a 5-week course of PM+ or EUC, by staff who were independent } \\ \text { of the trial using computerised software that generated random number se- } \\ \text { quences". }\end{array}\end{array}$

\begin{tabular}{lll}
\hline $\begin{array}{l}\text { Blinding of participants } \\
\text { and personnel (perfor- } \\
\text { mance bias) } \\
\text { All outcomes }\end{array}$ & High risk & $\begin{array}{l}\text { Judgement comment: "participants were aware of treatment allocation, but } \\
\text { research assessors were blinded". }\end{array}$ \\
\hline $\begin{array}{l}\text { Blinding of outcome as- } \\
\text { sessment (detection bias) }\end{array}$ & Low risk & $\begin{array}{l}\text { Judgement comment: "assessors were blind to treatment condition, and } \\
\text { bll outcomes }\end{array}$ \\
& $\begin{array}{l}\text { ly from CHWs. Fidelity of masking was measured by having assessors guess } \\
\text { the condition of each participant at the end of each assessment. Assessors } \\
\text { correctly guessed the condition of participants at a chance rate at both post- } \\
\text { treatment }(50.6 \%) \text { and follow-up }(47.5 \%) \text {, indicating that blindness was main- } \\
\text { tained". }\end{array}$
\end{tabular}


Bryant 2017 (Continued)

Incomplete outcome data Low risk Judgement comment: "319 (75.77\%) completed the 3-month follow-up. The (attrition bias) level of attrition was within the $30 \%$ margin with which the power analysis was

All outcomes calculated. Sensitivity analyses indicated that the results were not biased by the attrition of the sample".

Selective reporting (re- Low risk
porting bias)

Judgement comment: all outcome measures mentioned in the methods section were collected, analysed and fully reported in the results section. This was also evident from the published protocol.

Other bias Low risk Judgement comment: none

Cheung 2019

\section{Study characteristics}

Methods

Trial design: RCT

Trial grouping: parallel group

Assessment modality (e.g. face-to-face, telephone, online): face-to-face (including self-reports and biomarker assessment, blood sample)

Conflict of interest of trial authors: Dr Fong reported receiving grants from the Health and Medical Research Fund during the conduct of the trial. Prof Tiwari reported receiving grants from the Research Council of the Hong Kong Special Administrative Region (SAR) Government during the conduct of the trial and grants from the Research Council of the Hong Kong SAR Government outside the submitted work. No other disclosures were reported.

Funding for trial: trial was funded by grant 11121361 from the Health and Medical Research Fund of the Hong Kong SAR Government Food and Health Bureau

Primary outcomes: primary outcome measure was telomerase activity in peripheral blood mononuclear cells

Randomisation method: eligible participants were randomised to either the intervention or the waiting list control group, assigned at a 1:1 ratio by block randomisations with randomly selected block sizes of 4, 6, and 8 . The list was computer-generated, recorded by an investigator (DSTC.) who was not involved in subject recruitment, and placed in numbered, sequential, sealed, opaque envelopes. The envelopes were kept by a centre staff person who was not involved in the trial; thus, the randomisations was centrally controlled to avoid any bias in selection

Secondary outcomes: secondary outcome measures included levels of pro inflammatory cytokines (tumour necrosis factor (TNF) and interleukin (IL) 6) in peripheral blood plasma, depressive symptoms, perceived stress, and perceived coping.

Timing of outcome measurements: data were collected at baseline, after training (after 6 weeks (i.e. on completion of the 6-week group training)), and after the intervention (after 22 weeks (i.e. on completion of the entire Qigong intervention)).

Total duration of trial: 12 March 2014-26 May 2016

Type of analysis: $\mathrm{Chi}^{2}$ test and Mann-Whitney test, mixed-effects models

Types of participants: Chinese women were eligible to participate if they were $\geq 18$ years, willing to undertake the Qigong intervention, available for all the data collection points, and receptive to random allocation and had survived IPV in the preceding 2 years

Intention to Treat: yes

Participants

Baseline characteristics

Psychological therapies for women who experience intimate partner violence (Review) Copyright $\odot 2020$ The Cochrane Collaboration. Published by John Wiley \& Sons, Ltd. 
- Age: $\mathrm{M}=42.0$ years, $\mathrm{SD}=8.7$

- Ethnicity: place of birth: Hong Kong 27 (20\%); Mainland China 109 (80\%)

- Employment: employed 34 (25\%)

- Education: $\leq 6$ years 25 (19\%); 7-13 years 101 (75\%); tertiary 9 (7\%)

- Relationship status: married or cohabiting 124 (91\%); single 4 (3\%); divorced 8 (6\%)

- Gender (\% women): 100

- Dependent children: $\leq 1$ Child 44 (32\%)

- Number analysed (data analysis at post-treatment stage): 120

- Number of participants eligible assigned: 136

- Number of participants dropped out (by final follow-up): 16

- Comorbidities: chronic illness 22 (16\%)

- Experience of IPV: NR

- Experience of other types of violence and abuse: NR

- Remuneration: finally, we will provide an incentive to the participants in the form of food coupons to show our appreciation of their time and efforts.

- Financial dependence (on partner): NR

Comparator intervention

- Age: $M=41.5$ years, $S D=9.3$

- Ethnicity: place of birth: Hong Kong 29 (21\%); Mainland China 106 (79\%)

- Employment: employed $21(16 \%)$

- Education: $\leq 6$ years 15 (11\%); 7-13 years 111 (83\%); tertiary 8 (6\%)

- Relationship status: married or cohabiting 128 (95\%); single 2 (1\%); divorced 5 (4\%)

- Gender (\% women): 100

- Dependent children: $\leq 1$ Child 50 (37\%)

- Number analysed (data analysis at post-treatment stage): 127

- Number of participants eligible assigned: 135

- Number of participants dropped out (by final follow-up): 8

- Comorbidities: chronic illness 22 (16\%)

- Experience of IPV: NR

- Experience of other types of violence and abuse: NR

- Remuneration: finally, we will provide an incentive to the participants in the form of food coupons to show our appreciation of their time and efforts.

- Financial dependence (on partner): NR

Total sample

- Age: $M=42.0$ years, $S D=8.8$

- Ethnicity: Chinese women who have experienced IPV

- Employment: NR

- Education: NR

- Relationship status: mean (SD) number of years of marriage was 21.3 (23.4) in the intervention group and $20.2(21.2)$ in the waiting list control group

- Gender (\% women): 100

- Dependent children: NR

- Number analysed (data analysis at post-treatment stage): 247

- Number of participants eligible assigned: 271

- Number of participants dropped out (by final follow-up): 24

- Comorbidities: NR

- Experience of IPV: Chinese women $(\mathrm{N}=271)$ who survived IPV in the past 2 years recruited from a community centre in Hong Kong, China 
- Experience of other types of violence and abuse: NR

- Remuneration: finally, we will provide an incentive to the participants in the form of food coupons to show our appreciation of their time and efforts.

- Financial dependence (on partner): not clearly reported, however it has been mentioned that 93 (69\%) of women within each intervention and control group experience financial hardship.

Included criteria: Chinese women were eligible to participate if they were $\geq 18$ years, willing to undertake the Qigong intervention, available for all the data collection points, and receptive to random allocation and had survived IPV in the preceding 2 years

Excluded criteria: women were excluded if they had participated in Qigong training or another mindbody intervention within the previous 6 months or had serious medical conditions that might limit their participation in Qigong. They were also excluded if they had psychiatric disorders, used medication or a psychological intervention for stress, or were abused by someone who was not their intimate partner based on their self-reports

Pretreatment: at baseline, there were no statistically significant differences in the participants' socio-demographic characteristics or outcome measures between the intervention and waiting list control groups.

Number eligible: 271

Number ineligible: 1270

Number of eligible people consented: 271

Number of eligible people recruited: 271

Recruitment setting of participants (e.g. healthcare setting, community setting, shelter setting): participants were recruited between 12 March 2014 and 25 October 2015, from a large community centre that provides diversified health and social services for users of all age groups in 3 districts in Hong Kong, covering a population of approximately 830,000 individuals

Sample power calculation: to detect at least $0.075-\mathrm{U}$ differences between the 2 groups with $80 \%$ power and at most a $5 \%$ chance of committing a false-positive error, we needed 113 participants/group. Allowing for attrition of $5 \%$ ( based on a previous clinical trial held in the same community where the present trial was conducted), we rounded up the sample size to at least 240 participants in total.

Total number of participants approached: 1611

Total number of participants within all groups: 271

Number declined to participate: 70

\section{Intervention characteristics}

Experimental intervention

- Brief description (rationale, main components): 1 of the various forms of Qigong is Baduanjin, meaning " 8 pieces of silken brocade" in Mandarin. It consists of 8 movements that are performed in a smooth and graceful manner, hence the name. The movements, combined with breathing and meditation, exercise the mind and body for healing. Baduanjin was selected as the intervention because it has been standardised by the Chinese Health Qigong Association and is easy to perform. The intervention comprises (i) group learning and practice: a 2-h Qigong exercise training session provided by a Qigong master twice a week for 6 consecutive weeks(total, $24 \mathrm{~h}$ ), (ii) weekly group follow-up: a 1$\mathrm{h}$ Qigong exercise session provided by a Qigong master once a week for 4 consecutive months (total, $16 \mathrm{~h}$ ) after the group learning and practice, and (iii) self practice: a 30-minute Qigong exercise session performed by each individual participant once a day for the whole intervention period (total, $63 \mathrm{~h}$ ). Each participant will perform a total of $103 \mathrm{~h}$ of Qigong during the 5.5-month intervention period. For each of the 2-h Qigong exercise training sessions during the 6 -week group learning and practice periods, the activities will include (i) a brief introduction to the basic theories of traditional Chinese medicine and the physiology of mind-body connections, (ii) gentle movement or body stretching in 
a standing posture to facilitate a harmonious flow of qi along the energy channels, and (iii) Qigong exercise training delivered by an experienced Qigong master.

- Type of intervention: Qigong intervention (a type of moving meditation)

- Mode of delivery (how): face-to-face (group sessions and self-exercises)

- Frequency and duration of delivery (when and how much): the intervention lasted 22 weeks and was composed of (1) group training (12 2-h Qigong sessions for 6 weeks (twice weekly)); (2) weekly group follow-up (16 one-h Qigong follow-up practice sessions from week 7 to week 22 (once weekly)); and (3) self-practice (participants were encouraged to practice Qigong $30 \mathrm{~min} /$ day for the entire intervention period (22 weeks)).

- Level of mental health training of person delivering the intervention: the sessions were delivered by a certified Qigong master with $>20$ years of experience in teaching Qigong, who was provided with standardised content of training and follow-up sessions

- Fidelity assessment: a research assistant was assigned to each session to monitor the attendance of participants, their adherence to the intervention, and the Qigong master's use of standardised elements. To monitor the self-practice of Qigong in the intervention group, each participant entered the details of duration and frequency of Qigong undertaken every day into a record card, which was submitted to the research assistant weekly.

- Intervention model: traditional Chinese medicine and the physiology of mind-body connections

- Person delivering the intervention (who provided the intervention): therapist, self-directed or combined: therapist (Qigong master)

- Intervention location (where): NR

- Tailoring of intervention (personalised, titrated or adapted): NR

- Intervention modifications (during the trial): NR

- Main techniques of intervention: the activities will include (i) a brief introduction to the basic theories of traditional Chinese medicine and the physiology of mind-body connections, (ii) gentle movement or body stretching in a standing posture to facilitate a harmonious flow of qi along the energy channels, and (iii) Qigong exercise training delivered by an experienced Qigong master

- Intervention manual: Baduanjin was selected as the intervention because it has been standardised by the Chinese Health Qigong Association 16 and is easy to perform

- Intervention attendance: women in the intervention group who completed the trial $(n=120)$ self-practiced Qigong for a mean (SD) 103.3 (138.2) min/week throughout the intervention. Most of them (89 $(74.2 \%)$ ) attended $\geq 80 \%$ of the training sessions.

- CCDAN psychological therapies: other psychologically-orientated interventions

- Intensity of intervention: $\geq 5$ sessions

- Person delivering the intervention: non-healthcare workers

- Gender of therapist: NR

Comparator intervention

- Brief description (rationale, main components): participants in the waiting list control group could choose to attend monthly health education sessions unrelated to Qigong and given by a registered nurse after 6 weeks on the waiting list and to receive post-intervention Qigong training after data collection was completed (after 22 weeks).

- Type of intervention: health education session

Outcomes

- Outcome type: continuous outcome

- Reporting: fully reported

- Scale: BDIII

- Range: 0-63

- Direction: lower is better

\section{Dropouts from treatment}

- Outcome type: dichotomous outcome

- Reporting: fully reported 
Sponsorship source: trial was funded by grant 11121361 from the Health and Medical Research Fund of the Hong Kong SAR Government Food and Health Bureau

Country: China

Setting: community

Comments: a single-blind randomised clinical trial among Chinese women ( $N=271)$ who survived IPV in the past 2 years recruited from a community centre in Hong Kong, China

Trialauthors: Denise Shuk Ting Cheung; Wen Deng; Sai-Wah Tsao; Rainbow Tin Hung Ho; Cecilia LaiWan Chan; Daniel Yee Tak Fong; Pui Hing Chau, Athena Wai Lin Hong; Helina Yin King Yuk Fung; Joyce Lai Chong Ma; Agnes F. Y. Tiwari

Institution: School of Nursing, Li Ka Shing Faculty of Medicine, University of Hong Kong

Email: wdeng@hku.hk

Address: School of Nursing, Li Ka Shing Faculty of Medicine, University of Hong Kong, 21 Sassoon Rd, Hong Kong, China

Title (main 'outcome' publication): Effect of a Qigong intervention on telomerase activity and mental health in Chinese women survivors of intimate partner violence a randomized clinical trial

Registered trial protocol ID: NCT02060123

Notes Analysis of any cost/benefit measures: NR

Process evaluation: NR

Summary of participants' views about the intervention: all intervention participants rated the degree of liking Qigong as 'like it very much' or 'like it'

Adverse events (harm) related to participation: there were no reports of adverse events or harm arising from participation in the trial.

\section{Risk of bias}

\begin{tabular}{lll}
\hline Bias & Authors' judgement & Support for judgement \\
\hline $\begin{array}{l}\text { Random sequence genera- } \\
\text { tion (selection bias) }\end{array}$ & Low risk & $\begin{array}{l}\text { Judgement comment: "eligible participants were randomised to either the } \\
\text { intervention or the waiting list control group, assigned at a 1:1 ratio by block } \\
\text { randomisation with randomly selected block sizes of 4, 6, and 8. The list was } \\
\text { computer-generated, recorded by an investigator (D.S.T.C.) who was not in- } \\
\text { volved in subject recruitment, and placed in numbered, sequential, sealed, } \\
\text { opaque envelopes". }\end{array}$ \\
& \\
& \\
\hline
\end{tabular}

\begin{tabular}{ll}
$\begin{array}{l}\text { Allocation concealment } \\
\text { (selection bias) }\end{array}$ & $\begin{array}{l}\text { Judgement comment: "the list was computer-generated, recorded by an in- } \\
\text { vestigator who was not involved in subject recruitment, and placed in num- } \\
\text { bered, sequential, sealed, opaque envelopes. The envelopes were kept by a } \\
\text { centre staff person who was not involved in the trial; thus, the randomisation } \\
\text { was centrally controlled to avoid any bias in selection" }\end{array}$ \\
\hline
\end{tabular}

$\begin{array}{ll}\begin{array}{l}\text { Blinding of participants } \\ \text { and personnel (perfor- }\end{array} & \text { Jigh risk } \\ \text { mance bias) } & \text { tered data were not involved in the trial design, did not know the trial hy- } \\ \text { All outcomes } & \text { potheses, and were blinded to group assignment. The nurse who collected } \\ & \text { the blood and the laboratory technicians who processed the blood samples } \\ \text { did not know which group the participant and blood sample belonged to and } \\ \text { were blinded to the group assignment. Participants were not blinded to group } \\ \text { allocation. To minimise the possible effects from knowledge of group assign- }\end{array}$



they had to be randomised into 2 groups with different sequences for receiving Qigong training because of limited class size".

\begin{tabular}{|c|c|c|}
\hline $\begin{array}{l}\text { Blinding of outcome as- } \\
\text { sessment (detection bias) } \\
\text { All outcomes }\end{array}$ & Low risk & $\begin{array}{l}\text { Judgement comment: outcome assessors and research assistants who en- } \\
\text { tered data were not involved in the trial design, did not know the trial hy- } \\
\text { potheses, and were blinded to group assignment }\end{array}$ \\
\hline
\end{tabular}

\begin{tabular}{lll}
\hline $\begin{array}{l}\text { Incomplete outcome data } \\
\text { (attrition bias) } \\
\text { All outcomes }\end{array}$ & Low risk & Judgement comment: attrition and exclusions were adequately reported. \\
\hline $\begin{array}{l}\text { Selective reporting (re- } \\
\text { porting bias) }\end{array}$ & Low risk & $\begin{array}{l}\text { Judgement comment: the primary and secondary measurements in the trial } \\
\text { protocol were adequately reported in the trial outcome trial. }\end{array}$ \\
\hline Other bias & Low risk & Judgement comment: none \\
\hline
\end{tabular}

\section{Choo 2016}

\section{Study characteristics}

Trial design: RCT
Trial grouping: parallel group
Conflict of interest of trial authors: NR
Funding for trial: this work was supported by NIH/NIDA grant K23DA031881. NIDA provided support
for conducting the trial but did not play any role in the design and conduct of the trial; collection, man-
agement, analysis, and interpretation of the data; preparation, review, or approval of the manuscript;
or decision to submit the manuscript for publication.

Primary outcomes: primary clinical outcome measure was past-month drug use, measured by a modified version of the Timeline Followback (TLFB).

Randomisation method: participants launched the program themselves, completed more detailed assessments of drug use and IPV, and then were automatically randomised to intervention or control groups by the software

Secondary outcomes: secondary outcomes included readiness and confidence for changing drug use and the CAS

Timing of outcome measurements: participants completed these measures at baseline and 1- and 3month follow-ups, with the exception of readiness and confidence rulers, which were administered immediately pre- and post-intervention and after the 2-week telephone booster.

Total duration of trial: NR

Types of participants: a screening survey identified women with recent drug use and IPV in the ED.

Assessment modality (e.g. face-to-face, telephone, online): NR

ITT analysis: NR

Types of analysis: descriptive summaries (median, mean, proportions) and associated confidence intervals

Participants

\section{Baseline characteristics}

Experimental intervention 
Choo 2016 (Continued)

- Age: median 26, $($ range $=19-48)$

- Ethnicity: Hispanic/Latino $20 \%$

- Employment: NR

- Education: high school or more $50.0 \%$

- Marital status: single never married $65.0 \%$

- Children: with at least 1 child $60.0 \%$

- Experience of IPV CAS scores (median, range): median $8(0,44)$

- Number analysed (data analysis at post-treatment stage): 14

- Number of participants eligible assigned: 21

- Number of participants dropped out (by final follow-up): 7

- Comorbidities: NR

- Experience of IPV: NR

- Experience of other types of violence and abuse: NR

- Remuneration: participants were given gift cards to a popular pharmacy chain for participation at baseline (USD 25), at the booster (USD 20), and at each of the 2 follow-up assessments (USD 25 each), for a potential total of USD 95

- Financial dependence (on partner): NR

Comparator intervention

- Age: median $=24.5($ range $=19-53)$

- Ethnicity: Hispanic/ Latino $36.8 \%$

- Employment: NR

- Education: high school or more $23.1 \%$

- Marital status: single never married $63.2 \%$

- Children: with at least 1 child $47.4 \%$

- Experience of IPV CAS scores (median, range): median $8(0,41)$

- Number analysed (data analysis at post-treatment stage): 15

- Number of participants eligible assigned: 19

- Number of participants dropped out (by final follow-up): 4

- Comorbidities: NR

- Experience of IPV: NR

- Experience of other types of violence and abuse: NR

- Remuneration: participants were given gift cards to a popular pharmacy chain for participation at baseline (USD 25), at the booster (USD 20), and at each of the 2 follow-up assessments (USD 25 each), for a potential total of USD 95

- Financial dependence (on partner): NR

Total sample

- Age: NR

- Ethnicity: NR

- Employment: NR

- Education: NR

- Marital status: NR

- Children: NR

- Experience of IPV CAS scores (median, range): NR

- Number analysed (data analysis at post-treatment stage): 29

- Number of participants eligible assigned: 40

- Number of participants dropped out (by final follow-up): 11

- Comorbidities: the most common drugs used were marijuana (88\%) and cocaine (30\%)

- Experience of IPV: $45 \%$ of participants reported intimate partner physical abuse and $33 \%$ severe combined intimate partner physical and sexual abuse

- Experience of other types of violence and abuse 
- Remuneration: participants were given gift cards to a popular pharmacy chain for participation at baseline (USD 25), at the booster (USD 20), and at each of the 2 follow-up assessments (USD 25/each), for a potential total of USD 95

- Financial dependence (on partner): NR

Included criteria: research assistants (RAs) used a random number generator to select a random sample of rooms. Within this subset of women, screening took place in 2 steps. First, RAs reviewed the electronic medical record and identified those determined to be English-speaking adult women aged 18-59; those failing to meet these criteria were excluded. Second, RAs invited the remaining, potentially eligible participants to complete a 'Women's Health Survey', self-administered on an iPad (Apple,Inc.). Those who completed the screen and reported both drug use and IPV in the past 3 months were eligible for the trial

Excluded criteria: we excluded those who were critically ill (triaged to the critical care area of the ED), had suicidal ideation or psychosis, were combative or in police custody, or were treated in a supervised setting for alcohol or drug dependence

Baseline differences: trial reported no significant differences between groups at baseline.

Sample power calculation: not reported

Total number of participants: 40

Number ineligible: 4857

Number eligible: 68

Number of eligible people consented: 40

Recruitment setting of participants (e.g. healthcare setting, community setting, shelter setting): healthcare setting (ED)

Number of eligible people recruited: 68

Total number of participants approached: 5419

Total number of participants within all groups: 40

Number declined to participate: 198

Interventions Intervention characteristics

Experimental intervention

- Brief description: 'BSAFER' (Brief Intervention for Substance Use and Partner Abuse for Females in the Emergency Room is a brief Web-based program, designed to be taken on a small tablet-style laptop computer and to be completed in the room during a single ED visit. The main components of the intervention include automated feedback, identification of core values, empowerment, goal setting, social supports, advice, referrals, and summary. The intervention included a booster session conducted by phone within 2 weeks after the initial ED visit.

- Type of intervention: psychoeducation

- Mode of delivery (how): computer-based program and telephone booster

- Frequency and duration of delivery (when and how much): the average time for completion of the active components of the BSAFER Web program (excluding assessments) was $15 \mathrm{~min}$. 30 participants $(75 \%)$ completed the 2-week telephone booster; the average time to complete the BSAFER booster was 20 min

- Level of mental health training of person delivering the intervention: NR

- Fidelity assessment: NR

- Person delivering the intervention (who provided the intervention): therapist, self-directed or combined: combined

- Intervention location (where): private research office in the ED

- Tailoring of intervention (personalised, titrated or adapted): NR 
- Intervention modification (during the trial): NR

- Main techniques of intervention: automated feedback, Identification of core values, empowerment, goal setting, social supports, advice, referrals and summary, followed with a booster telephone session

- Intervention manual: there is insufficient information about the use of manual. BSAFER was created using an intervention design software, Computerized Intervention Authoring Software (CIAS; Interva, Inc).24 The program uses a female parrot avatar ('Polly'), which addresses the participant by name, serves as a guide and narrator for the program, and reads all content aloud, allowing low-literacy participants to complete the program. The intervention included a booster session conducted by phone within 2 weeks after the initial ED visit

- Intervention attendance: 20 (95\%) completed all portions of the BSAFER Web program,

- CCDAN psychological therapies: integrative therapies

- Intensity of intervention: up to 4 sessions

- Person delivering the intervention: web-based with facilitators

- Gender of therapist: female

Comparator intervention

- Brief description: the control arm received a time-matched web-based program on home fire safety, including videos from the National Fire Protection Association. This topic was selected as it seemed likely to be viewed as acceptable and appropriate content to receive in a healthcare setting, yet unlikely to have an effect on trial outcomes

- Type of intervention: no treatment

Outcomes

\section{Dropouts from treatment}

- Outcome type: dichotomous outcome

- Reporting: fully reported

- Direction: lower is better

Identification

Sponsorship source: this work was supported by NIH/NIDA grant K23DA031881. NIDA provided support for conducting the trial but did not play any role in the design and conduct of the trial; collection, management, analysis, and interpretation of the data; preparation, review, or approval of the manuscript; or decision to submit the manuscript for publication.

Country: USA

Setting: $E D$

Comments: clinical setting

Trialauthors: Esther K. Choo, Caron Zlotnick, David R. Strong, Daniel D. Squires, Chantal Tapé \& Michael J. Mello

Institution: Center for Policy and Research in Emergency Medicine, Department of Emergency Medicine, Oregon Health \& Science University

Email: chooe@ohsu.edu

Address: Center for Policy and Research in Emergency Medicine, Department of Emergency Medicine, Oregon Health \& Science University, 3181 SW Sam Jackson Park Road, 11D52 HRC, Portland, OR 97239, USA

Trial title (main outcome publication): BSAFER: a web-based intervention for drug use and intimate partner violence demonstrates feasibility and acceptability among women in the emergency department

Registered trial protocol ID: NCT01709552 
Choo 2016 (Continued)

\section{Process evaluation: NR}

Summary of participants' views about the intervention: in the intervention group, mean overall usability score (SUS) for the BSAFER Web program was $83.5(95 \% \mathrm{Cl} 78.1$ to 88.9$)$ out of a possible 100 . The mean overall satisfaction score (CSQ-8) was 27.7 ( $95 \% \mathrm{Cl} 26.3$ to 29.1 ) out of a possible 32. Intervention arm participants' agreement with statements about the consistency of the BSAFER web-based program and the telephone booster with specific principles or qualities of MI are shown in Tables 3 and 4, respectively. Overall, participants in both arms scored both the BSAFER program and the booster high for all aspects of consistency with MI, with the intervention scoring higher on several individual components

Adverse events (harm) related to participation: NR

\section{Risk of bias}

\begin{tabular}{|c|c|c|}
\hline Bias & Authors' judgement & Support for judgement \\
\hline $\begin{array}{l}\text { Random sequence genera- } \\
\text { tion (selection bias) }\end{array}$ & Low risk & $\begin{array}{l}\text { Judgement comment: "research assistants (RAs) used a random number gen- } \\
\text { erator to select a random sample of rooms. Participants launched the program } \\
\text { themselves, completed more detailed assessments of drug use and IPV, and } \\
\text { then were automatically randomised to intervention or control groups by the } \\
\text { software". }\end{array}$ \\
\hline $\begin{array}{l}\text { Allocation concealment } \\
\text { (selection bias) }\end{array}$ & Low risk & $\begin{array}{l}\text { Judgement comment: "participants were automatically randomised to inter- } \\
\text { vention or control groups by the software" }\end{array}$ \\
\hline $\begin{array}{l}\text { Blinding of participants } \\
\text { and personnel (perfor- } \\
\text { mance bias) } \\
\text { All outcomes }\end{array}$ & Unclear risk & $\begin{array}{l}\text { Judgement comment: there is insufficient information about the blinding of } \\
\text { participants and personnel. }\end{array}$ \\
\hline $\begin{array}{l}\text { Blinding of outcome as- } \\
\text { sessment (detection bias) } \\
\text { All outcomes }\end{array}$ & Low risk & $\begin{array}{l}\text { Judgement comment: "participants launched the web-site program them- } \\
\text { selves, completed more detailed assessments of drug use and IPV, and then } \\
\text { were automatically randomised to intervention or control groups by the soft- } \\
\text { ware". }\end{array}$ \\
\hline $\begin{array}{l}\text { Incomplete outcome data } \\
\text { (attrition bias) } \\
\text { All outcomes }\end{array}$ & Low risk & $\begin{array}{l}\text { Judgement comment: clinical measures/outcomes completed; although } 20 \\
(95 \%) \text { completed all portions of the BSAFER web program, and } 19(100 \%) \text { com- } \\
\text { pleted all portions of the control web program, there was participant attrition } \\
\text { at } 1 \text { and } 3 \text { months' follow-up }\end{array}$ \\
\hline $\begin{array}{l}\text { Selective reporting (re- } \\
\text { porting bias) }\end{array}$ & Unclear risk & $\begin{array}{l}\text { Judgement comment: all outcomes described in the methods section were ad- } \\
\text { equately collected, analysed and reported in the results section of the main } \\
\text { published trial. However, there was insufficient or unclear evidence from ei- } \\
\text { ther the trial protocol or trial registry sites to assess this domain. }\end{array}$ \\
\hline Other bias & High risk & $\begin{array}{l}\text { Judgement comment: as a trial for feasibility and acceptability, this was, by } \\
\text { design, a small sample at a single clinical setting; further, out of convenience, } \\
\text { it only enrolled English-speaking women }\end{array}$ \\
\hline
\end{tabular}

Cohen 2013

\section{Study characteristics}

\begin{tabular}{ll}
\hline Methods & Trial design: $\mathrm{RCT}$ \\
& Trial grouping: parallel group
\end{tabular}


Conflict of interest of trial authors: all authors declare that they have no conflicts of interest.

Funding for trial: research reported in this article was supported by a grant from the NIDA Clinical Trials Network (CTN): U10 DA13035 (Edward Nunes, PI); the NIDA CTN publication committee reviewed a draft of this publication and provided comments.

Primary outcomes: IPV; trauma and PTSD; substance use

Randomisation method: each participant was randomised into 1 of 2 12-session group interventions using 1 blocked randomisation list known only to the trial statistician

Secondary outcomes: not reported

Timing of outcome measurements: participants completed brief, weekly assessments during treatment to collect substance use and PTSD symptom data and were reassessed at 1-week, 3-, 6-, and 12months post-treatment

Total duration of trial: a total of 353 women were recruited via brochures, flyers, and advertisements, as well as through referrals by treatment programme staff, and randomised over 21 months in 2004 and 2005

Type of analysis: $\mathrm{T}$-tests were used for continuous variables and $\mathrm{Chi}^{2}$ tests for dichotomous variables. The bivariate association of risk factors and outcome was determined using logistic regression and controlling for socio-demographic variables.

Types of participants: community-based outpatient treatment programmes

ITT analyses: yes

Participants

\section{Baseline characteristics}

Experimental intervention

- Age: $M=39.3$ years, $S D=9.5$

- Ethnicity: African American/Black (33.0\%); white (47.16\%); Latina (3.98\%); multiracial (15.34\%); other $(0.6 \%)$

- Employment: employed (40.3\%); unemployed (54.6\%); student/retired/disabled (5.1\%)

- Education: 12.7 (2.3) years

- Marital status: married (14.8\%); single (37.5\%); divorced/separated (47.7\%)

- Gender (\% women): $100 \%$

- Dependent children: NR

- Number analysed (data analysis at post-treatment): 140

- Number of participants eligible assigned: 176

- Number of participants dropped out (at final follow-up): 65

- Comorbidities: PTSD (76.7\%); substance use disorder (8.5\%)

- Experience of IPV: adult physical abuse (83.4\%)

- Experience of other types of violence and abuse

- Remuneration: participants were compensated with cash or vouchers valued at USD 20 for the completion of the screening and USD 20 for the completion of the baseline assessments. For the follow-up compensation, participants received USD 20 in cash or vouchers for completion of the 1-week posttreatment follow-up, USD 30 for the 3-month follow-up, USD 40 for the 6-month follow-up, and USD 50 for the 12-month follow-up assessments. In addition, they received USD 10 for completion of weekly treatment assessments. These amounts varied by site, depending on local research trial comparability.

- Financial dependence (on partner): NR

Comparator intervention

- Age: $M=39.0$ years, $S D=9.1$ 
- Ethnicity: African American/Black (35.0\%); white (44.1\%); Latina (9.0\%); multiracial (11.3\%); other (0.6\%)

- Employment: employed (40.1\%); unemployed (55.4\%); student/retired/disabled (4.5\%)

- Education: 12.4 (2.6) years

- Marital status: married (20.3\%); single (36.2\%); divorced/separated (43.5\%\%)

- Gender (\% women): $100 \%$

- Dependent children: NR

- Number analysed (data analysis at post-treatment): 149

- Number of participants eligible assigned: 177

- Number of participants dropped out (at final follow-up): 73

- Comorbidities: PTSD (84.2\%); substance use disorder (9.0\%)

- Experience of IPV: adult physical abuse (86.2\%)

- Experience of other types of violence and abuse

- Remuneration: participants were compensated with cash or vouchers valued at USD 20 for the completion of the screening and USD 20 for the completion of the baseline assessments. For the follow-up compensation, participants received USD 20 in cash or vouchers for completion of the 1-week posttreatment follow-up, USD 30 for the 3-month follow-up, USD 40 for the 6-month follow-up, and USD 50 for the 12-month follow-up assessments. In addition, they received USD 10 for completion of weekly treatment assessments. These amounts varied by site, depending on local research trial comparability.

- Financial dependence (on partner): NR

Total sample

- Age: $M=39.2$ years, $S D=9.3$

- Ethnicity: African American/Black (34.0\%); white (45.6\%); Latina (6.5\%); multiracial (13.3\%); other $(0.6 \%)$

- Employment: employed (40.2\%); unemployed (55.0\%); student/retired/disabled (4.8\%)

- Education: 12.5 (2.4) years

- Marital status: married (17.6\%); single (36.8\%); divorced/separated (17.6\%)

- Gender (\% women):

- Dependent children: NR

- Number analysed (data analysis at post-treatment): 289

- Number of participants eligible assigned: 353

- Number of participants dropped out (at final follow-up): 138

- Comorbidities: PTSD (80.4\%); substance use disorder (8.8\%)

- Experience of IPV: almost all participants reported prior exposure to physical or sexual assault (96\%) and 1 in 10 reported physical or sexual assault in the prior 30 days

- Experience of other types of violence and abuse: a summary of lifetime exposure to traumatic events revealed that the majority of participants had experienced physical abuse (84.8\%) or sexual abuse (67.6\%) during adulthood

- Remuneration: participants were compensated with cash or vouchers valued at USD 20 for the completion of the screening and USD 20 for the completion of the baseline assessments. For the follow-up compensation, participants received USD 20 in cash or vouchers for completion of the 1-week posttreatment follow-up, USD 30 for the 3-month follow-up, USD 40 for the 6-month follow-up, and USD 50 for the 12-month follow-up assessments. In addition, they received USD 10 for completion of weekly treatment assessments. These amounts varied by site, depending on local research trial comparability.

- Financial dependence (on partner): NR specifically, but reported that half of the participants received the majority of their economic support from someone else

Included criteria: eligible participants had at least 1 lifetime traumatic event and met DSM-IV criteria for either full or sub-threshold PTSD in the past 30 days. Sub-threshold PTSD differs from full PTSD in that participants could meet either criterion $\mathrm{C}$ (avoidance of trauma reminders and emotional numbing) or criterion $\mathrm{D}$ (hyper-arousal), but not both as in the full diagnostic requirement. Participants also had to be between the ages of 18 and 65 , have used alcohol or an illicit substance within the prior 6 - 
months, and meet current drug or alcohol abuse/dependence criteria, and be enrolled in the participating treatment programme.

Excluded criteria: women were excluded if they had impaired mental cognition, significant risk of suicidal/homicidal behavior, history of schizophrenia-spectrum diagnosis, or active psychosis.

Pretreatment: no significant differences were found between participants who reported IPV and those who did not report IPV during follow-up on demographic characteristics including age, race/ethnicity, marital status, education, employment or economic dependence.

Number eligible: 370

Number ineligible: 1593

Number of eligible people consented: 353

Number of eligible people recruited: 370

Recruitment setting of participants (e.g. healthcare setting, community setting, shelter setting): community-based outpatient treatment programmes

Sample power calculation: NR

Total number of participants approached: 1963

Total number of participants within all groups: 353

Number declined to participate: 17

Interventions Intervention characteristics

Experimental intervention

- Brief description (rationale, main components): Seeking Safety treatment is a structured CBT with both safety/trauma and substance use components integrated into each session

- Type of intervention: cognitive- behavioral treatment

- Mode of delivery (how): group-based sessions

- Frequency and duration of delivery (when and how much): treatment consisted of $2 \times 90$-min sessions/week for 6 weeks: 12 sessions of Seeking Safety (mean completion 6.2 sessions)

- Level of mental health training of person delivering the intervention: counsellors and supervisors attended a comparable, centralised 3-day workshop on their respective interventions; supervisors received an additional half-day of training focused on how to carry out trial supervision. Following training, counsellors and supervisors were certified in the interventions after successfully completing a training group of at least 4 sessions

- Fidelity assessment: during trial implementation, all intervention sessions were videotaped and a proportion of tapes were rated by the supervisors. Counsellors also met weekly with supervisors. In order to ensure competency on an ongoing basis, supervisors had weekly conference calls with lead team experts. The lead team experts co-rated a randomly selected quarter of the counsellor session tapes reviewed by the supervisor to monitor ratings reliability on adherence measures. Internal consistency and interrater reliability for both interventions were good to excellent.

- Intervention model: cognitive-behavioral strategies

- Person delivering the intervention (who provided the intervention): therapist, self-directed or combined: counsellors

- Intervention location (where): community-based outpatient treatment programmes

- Tailoring of intervention (personalised, titrated or adapted): NR

- Intervention modifications (during the trial): NR

- Main techniques of intervention: each session covered a different topic as follows: safety, taking back power from PTSD, when substances are in control, honesty, setting boundaries in relationships, compassion, healing from anger, creating meaning, integrating the split self, taking good care of oneself, red and green flags, and detaching from emotional pain (grounding). 
- Intervention manual: Seeking Safety Treatment is a short-term manualised therapy, using cognitive-behavioral strategies to reduce substance use and the negative impact of trauma exposure

- Intervention attendance: $6-12$ sessions $(n=103,58 \%) ; 1-5$ sessions $(n=37,21 \%) ; 0$ sessions $(n=36$, 20\%)

- CCDAN psychological therapies: CBT

- Intensity of intervention: $\geq 5$ sessions

- Person delivering the intervention: healthcare workers

- Gender of therapist: NR

Comparator intervention

- Brief description (rationale, main components): Women's Health Education (WHE) is a psychoeducational, manualised health curriculum focused on topics such as understanding the female body, human sexual behaviour, pregnancy and childbirth, sexually transmitted diseases, HIV, and AIDS.

- Type of intervention: psychoeducational (minimal intervention)

PTSD
- Outcomes $\quad$ Outcome type: continuous outcome
- Reporting: fully reported
- Scale: Clinician Administered PTSD Scale (CAPS)
Dropouts from treatment
- Outcome type: dichotomous outcome
- Reporting: fully reported
- Direction: lower is better

Identification

Sponsorship source: research reported in this article was supported by a grant from the NIDA Clinical Trials Network (CTN): U10 DA13035 (Edward Nunes, PI); the NIDA CTN publication committee reviewed a draft of this publication and provided comments.

Country: USA

Setting: community-based outpatient treatment programmes

Comments: this multi-site trial consisted of 7 community-based outpatient treatment programmes in diverse locations across the USA

Trialauthors: Lisa R. Cohen, Craig Field, Aimee N.C. Campbell, Denise A. Hien

Institution: Counseling and Psychological Services, Health Services at Columbia, 2920 Broadway, Mail Code 2606, New York, NY 10027, United States

Email: Ic2130@columbia.edu

Address: corresponding author at: Counseling and Psychological Services, Health Services at Columbia, 2920 Broadway, Mail Code 2606, New York, NY 10027, United States. Tel.: +1 2128542878.

Trial title (main outcome publication): Intimate partner violence outcomes in women with PTSD and substance use: a secondary analysis of NIDA Clinical Trials Network "Women and Trauma" multi-site trial

Registered trial protocol ID: NCT00078156

Notes

Analysis of any cost/benefit measures: NR

Process evaluation: NR

Summary of participants' views about the intervention: NR 
Cohen 2013 (Continued)

Adverse events (harm) related to participation: NR

\section{Risk of bias}

\begin{tabular}{lll}
\hline Bias & Authors' judgement & Support for judgement \\
\hline $\begin{array}{l}\text { Random sequence genera- } \\
\text { tion (selection bias) }\end{array}$ & Low risk & $\begin{array}{l}\text { Judgement comment: "a statistician generated } 1 \text { blocked randomisation list } \\
\text { (block size known only to the statistician) for the entire trial". }\end{array}$ \\
\hline $\begin{array}{l}\text { Allocation concealment } \\
\text { (selection bias) }\end{array}$ & Low risk & $\begin{array}{l}\text { Judgement comment: "each community-based substance abuse treatment } \\
\text { programme received sets of } 60 \text { sealed, tamper-evident, security envelopes, } \\
\text { containing } 1 \text { randomisation number and the corresponding treatment assign- } \\
\text { ment". }\end{array}$ \\
\hline $\begin{array}{l}\text { Blinding of participants } \\
\text { and personnel (perfor- } \\
\text { mance bias) }\end{array}$ & Unclear risk & $\begin{array}{l}\text { Judgement comment: "independent assessors who remained unaware of ran- } \\
\text { domisation assignment performed all baseline and post-treatment assess- } \\
\text { ments. After completion of the baseline assessment, eligible participants were } \\
\text { randomised to Seeking Safety or WHE. However, it is not clear whether partic- } \\
\text { ipants were blinded and also if the counsellors and supervisors were blinded } \\
\text { about which intervention they were randomised to deliver (e.g. Safety Seeking } \\
\text { psychological treatment or treatment as usual)". }\end{array}$ \\
\hline
\end{tabular}

\begin{tabular}{lll}
\hline $\begin{array}{l}\text { Blinding of outcome as- } \\
\text { sessment (detection bias) }\end{array}$ & Low risk & Judgement comment: "following the intervention phase of the trial, assess- \\
All outcomes & ments were conducted by the independent assessor, who was unaware of \\
& randomisation assignment, at 1-week, 3-month, 6-month, and 12-month fol- \\
low-ups".
\end{tabular}

\begin{tabular}{|c|c|c|}
\hline $\begin{array}{l}\text { Incomplete outcome data } \\
\text { (attrition bias) }\end{array}$ & Low risk & $\begin{array}{l}\text { Judgement comment: attrition and exclusions adequately reported. An ITT } \\
\text { sample was used in all analyses. }\end{array}$ \\
\hline
\end{tabular}

All outcomes sample was used in all analyses.

$\begin{array}{ll}\begin{array}{l}\text { Selective reporting (re- } \\ \text { porting bias) }\end{array} & \begin{array}{l}\text { Judgement comment: all outcomes described in the methods section were ad- } \\ \text { equately collected, analysed and reported in the results section of the main } \\ \text { published trial. However, there was insufficient or unclear evidence from ei- } \\ \text { ther the trial protocol or trial registry sites to assess this domain. }\end{array}\end{array}$

Other bias Low risk Judgement comment: none

Ferrari 2018

\section{Study characteristics}

Trial design: RCT
Trial grouping: parallel group
Assessment modality (e.g. face-to-face, telephone, online): all questionnaires (self-reports) were ei-
ther hand-delivered or sent in the post
Conflict of interest of trial authors: the authors of this manuscript have the following competing in-
terests: If the PATH intervention was implemented in service settings, Davies would receive payment
for training and supervising specialist psychological advocates. Domestic Violence Training Ltd provid-
ed support in the form of salary to author AD.
Funding for trial: this paper presents independent research funded by the UK NIHR under its Pro-
gramme Grants for Applied Research scheme (RP-PG-0108-10084) www.nihr.ac.uk/funding-and-sup-
port/funding-for research-studies/how-to-apply-for-funding/. This research was supported by the NIHR
Biomedical Research Centre at University Hospitals Bristol NHS Foundation Trust and the University of


Ferrari 2018 (Continued)

Bristol. Authors on the programme grant application: GeF, TJP, SH, RAD, DS. Domestic Violence Training Ltd provided support in the form of salary to author AD.

Primary outcomes: CORE-OM, PHQ-9

Randomisation method: on completion, the participant was randomised to either receiving the PATH intervention or to the control group through a remote independent automated telephone randomisation service provided by the Bristol Randomised Trials Collaboration (www.bristol.ac.uk/social-community-medicine/centres/brtc/)

Secondary outcomes: GAD7, PSS, SF-12, CAS, PHQ-9, CORE-OM,

Timing of outcome measurements: baseline, 4, 8 and 12 months

Total duration of trial: enrolment April 2011-May 2013

Type of analysis: linear regression analyses and logistic regression analyses

Types of participants: women aged $\geq 16$ years accessing DVA services in 2 UK cities

ITT analyses: yes

Participants

\section{Baseline characteristics}

Experimental intervention

- Age: $\mathrm{M}=33$ years, $\mathrm{SD}=11$

- Ethnicity: white British or other white background 106/126 (84\%)

- Employment: not in formal employment (excluding retirees and students) 90/115 (78\%)

- Education: who completed secondary education 96/116 (83\%)

- Relationship status: currently in a relationship 26/127 (20\%); perpetrator is a current partner 29/118 (25\%)

- Gender (\% women): 100

- Dependent children: is parent $97 / 125$ (78\%) and has child < 4 years living with her $49 / 130(38 \%)$

- Number analysed (data analysis at post-treatment stage): 84

- Number of participants eligible assigned: 131

- Number of participants dropped out (by final follow-up): 46

- Comorbidities: hazardous drinking (Audit-C $\geq 3$ ) 70/126 (56\%); smoked cannabis in past 12 months 36/124 (29\%)

- Experience of IPV: total abuse (total score CAS $\geq 3$ ) 124/129 (96\%)

- Experience of other types of violence and abuse: witnessed DVA as a child 66/129 (51\%) or abused as a child 65/129 (50\%)

- Remuneration: participants were given shopping vouchers for questionnaire completion

- Financial dependence (on partner): NR

Comparator intervention

- Age: $\mathrm{M}=34$ years, $\mathrm{SD}=10$

- Ethnicity: white British or other white background 113/127 (89\%)

- Employment: not in formal employment (excluding retirees and students) 93/121 (77\%)

- Education: who completed secondary education 97/117 (83\%)

- Relationship status: currently in a relationship 25/123 (20\%); perpetrator is a current partner 26/118 $(22 \%)$

- Gender (\% women): 100

- Dependent children: is parent $109 / 129$ (84\%) and has child < 4 years living with her $47 / 130$ (36\%)

- Number analysed (data analysis at post-treatment stage): 83

- Number of participants eligible assigned: 132

- Number of participants dropped out (by final follow-up): 47 
- Comorbidities: hazardous drinking (Audit-C $\geq 3$ ) 65/125 (52\%); smoked cannabis in past 12 months 28/121 (23\%)

- Experience of IPV: total abuse (total score CAS $\geq 3$ ) 124/129 (96\%)

- Experience of other types of violence and abuse: witnessed DVA as a child $67 / 128$ (52\%) or abused as a child 64/128 (50\%)

- Remuneration: participants were given shopping vouchers for questionnaire completion

- Financial dependence (on partner): NR

Total sample

- Age: NR

- Ethnicity: NR

- Employment: NR

- Education: NR

- Relationship status: NR

- Gender (\% women): 100

- Dependent children: NR

- Number analysed (data analysis at post-treatment stage): 165

- Number of participants eligible assigned: 263

- Number of participants dropped out (by final follow-up): 93

- Comorbidities: NR

- Experience of IPV: NR

- Experience of other types of violence and abuse: NR

- Remuneration: participants were given shopping vouchers for questionnaire completion

- Financial dependence (on partner): NR

Included criteria: eligible participants were women aged $\geq 16$ years experiencing DVA, which had led them to seek help from one of the recruiting sites.

Excluded criteria: having a psychotic illness, severe drug or alcohol problem; being unable to read English; currently attending counselling, CBT or other psychological treatments

Pretreatment: the SPA group and advocacy alone group had similar characteristics at baseline (Tables 1 and 2, with more details available elsewhere (45))

Number eligible: 767

Number ineligible: 203

Number of eligible people consented: 263

Number of eligible people recruited: 263

Recruitment setting of participants (e.g. healthcare setting, community setting, shelter setting): community and shelter/refuge

Sample power calculation: an effect size of 0.4-0.5 is consistent with those detected in studies of psychological interventions using the CORE-OM as an outcome measure (34) and is consistent with findings for CBT interventions on PHQ-9 and other measures of depression (35). 200 participants gives a power of $96 \%$ to detect a difference of 0.5 on the CORE-O (a "reliable change index" (36)), corresponding to an effect size of 0.5 , and $81 \%$ power to detect an effect size of 0.4 . Assuming an attrition of $20 \%$, we aimed to recruit 250 women

Total number of participants approached: 1274

Total number of participants within all groups: 263

Number declined to participate: 988 


\section{Experimental intervention}

- Brief description (rationale, main components): specialist psychological advocacy (SPA). Specialist psychological advocates (SPAs) received a 25-day manualised training programme (available from authors) developed by Agnew-Davies. The training addressed the psychological impacts of DVA on women and developed therapeutic skills specifically tailored for this client group. SPAs were trained to work with common presenting problems within a single session model, using a session structure based on the work of Daldrup and colleagues. Topics included post-traumatic stress, depression, anxiety, low self-esteem, unresolved anger and managing loss. SPAs were also provided with handouts and self-help resources that could be used with their clients

- Type of intervention: psycho-education (cognitive behavioural psychological techniques)

- Mode of delivery (how): individualised face-to-face sessions

- Frequency and duration of delivery (when and how much): participants in the intervention arm were assigned a SPA with the aim of receiving eight 1:1 SPA sessions (of $1 \mathrm{~h}$ duration) that alternated with regular advocacy sessions, meeting either weekly or fortnightly with a further 2 'booster' sessions, one and 3 months later

- Level of mental health training of person delivering the intervention: specialist psychological advocates (SPAs) received a 25-day manualised training programme (available from authors) developed by Agnew-Davies

- Fidelity assessment: assessed by listening to a stratified random sample of audio files. A fidelity scale (available from the trial authors) was developed to measure adherence of SPAs to the PATH model. The scale was adapted from a revised version of the Cognitive Therapy Scale (CTS-R), a widely used measure of competence in CBT, and was used to rate the content of the audiotapes

- Intervention model: psychological advocacy

- Person delivering the intervention (who provided the intervention): therapist, self-directed or combined: specialist psychological advocates

- Intervention location (where): a woman potentially interested in participating was referred to a researcher who met with her at a safe location. However, specific details of intervention location were not reported

- Tailoring of intervention (personalised, titrated or adapted): during SPA sessions the advocates used a variety of primarily cognitive behavioural psychological techniques focusing within any 1 session on a specific presenting problem, such as hyper-arousal, sleep difficulties or parenting problems

- Intervention modifications (during the trial): NR

- Main techniques of intervention: during SPA sessions the advocates used a variety of primarily cognitive behavioural psychological techniques focusing within any 1 session on a specific presenting problem, such as hyper-arousal, sleep difficulties or parenting problems. The SPA aimed to empower the participant to apply therapeutic strategies such as relaxation, challenging thoughts or goal setting, to promote recovery from each problem. In addition to the SPA sessions, women in the intervention group received usual care from their advocate

- Intervention manual: SPAs received a 25-day manualised training programme (available from trial authors) developed by Agnew-Davies. The training addressed the psychological impacts of DVA on women and developed therapeutic skills specifically tailored for this client group. SPAs were trained to work with common presenting problems within a single session model, using a session structure based on the work of Daldrup and colleagues

- Intervention attendance: of the 120/130 participants in the SPA group for whom we have information on adherence, $54(45 \%)$ attended $<4$ sessions, and 66 (55\%) attended $\geq 4$, which were-specified as reflecting adequate adherence. These data show that the average number of advocacy sessions was 5.8 (SD 6.6) for control group participants $(N=124)$, and 7.2 (SD 6.8) for SPA clients ( $N=95)$

- CCDAN psychological therapies: CBT

- Intensity of intervention: $\geq 5$ sessions

- Person delivering the intervention: non-healthcare workers

- Gender of therapist: NR

Comparator intervention

- Brief description (rationale, main components): usual care. Participants in the advocacy alone group had access to the usual DVA agency support and advocacy, including safety planning, assistance with 
Ferrari 2018 (Continued)

health and social issues housing problems, budgeting and debt, and legal proceedings. They did not receive SPA sessions and their advocate did not receive specialist training in psychological methods.

- Type of intervention: usual care

\section{Outcomes}

\section{Depression}

- Outcome type: continuous outcome

- Reporting: fully reported

- Scale: PHQ

- Direction: lower is better

\section{Anxiety}

- Outcome type: continuous outcome

- Reporting: fully reported

- Scale: GAD questionnaire

- Direction: lower is better

\section{Mental health}

- Outcome type: continuous outcome

- Reporting: fully reported

- Scale: CORE-OM

- Direction: lower is better

PTSD

- Outcome type: continuous outcome

- Reporting: fully reported

- Scale: PSS

- Direction: lower is better

\section{Re-exposure to IPV}

- Outcome type: ContinuousOutcome

- Reporting: fully reported

- Scale: CAS

- Direction: lower is better

\section{Quality of life}

- Outcome type: continuous outcome

- Reporting: fully reported

- Scale: SF-12

- Direction: higher is better

\section{Dropouts from treatment}

- Outcome type: dichotomous outcome

- Reporting: fully reported

- Direction: lower is better

Sponsorship source: this paper presents independent research funded by the UK NIHR under its Programme Grants for Applied Research scheme (RP-PG-0108-10084) www.nihr.ac.uk/funding-and-support/funding-for research-studies/how-to-apply-for-funding/. This research was supported by the NIHR Biomedical Research Centre at University Hospitals Bristol NHS Foundation Trust and the University of Bristol. Authors on the programme grant application: GeF, TJP, SH, RAD, DS. Domestic Violence Training Ltd provided support in the form of salary to author AD. 
Setting: specialist DVA agencies

Comments: women aged $\geq 16$ years accessing DVA services

Trialauthors: Giulia Ferrari, Gene Feder, Roxane Agnew-Davies, Jayne E. Bailey, Sandra Hollinghurst, Louise Howard, Emma Howarth, Lynnmarie Sardinha, Debbie Sharp, Tim J. Peters

Institution: Centre for Academic Primary Care, Bristol Medical School, University of Bristol, Bristol, United Kingdom

Email: gene.feder@bristol.ac.uk

Address: Department of Global Health and Development, Faculty of Public Health and Policy, London School of Hygiene \& Tropical Medicine, London United Kingdom

Title (main 'outcome' publication): Psychological advocacy towards healing (PATH): a randomised controlled trial of a psychological intervention in a domestic violence service setting

Registered trial protocol ID: ISRCTN58561170

Notes Analysis of any cost/benefit measures: "The cost-effectiveness analysis will be reported in a separate paper".

Process evaluation: a qualitative trial nested within the PATH trial was conducted with the aim to compare the experiences of women receiving a psychological intervention with women receiving usual advocacy in a RCT (PATH: Psychological Advocacy Towards Healing), to illuminate the trial results by exploring women's experiences of benefits and difficulties.

Summary of participants views about the intervention: women valued the educational, psychological and emotional elements of the intervention, they felt safe to explore repressed emotions for the first time and experienced a reduction in self-blame, improved sense of identity and greater selfesteem. They also incorporated new skills and self-help techniques to enable sustainable change. Women receiving usual advocacy reported un-met needs for psychological and emotional support. Adherence was affected by women's psychological 'readiness' to engage, the competing demands of practical issues such as housing insecurity, legal proceedings or the availability of child care, and breaks in the continuity of professional care.

Adverse events (harm) related to participation: 22 women (15 in the SPA group, 7 in the advocacy alone group) reported a total of 32 SAEs (Table 4). For 3 women (4 events) in the intervention group, it was unclear from the information available whether the SAEs were related to trial participation. These events were reported to the NHS Research Ethics Committee (REC) that had approved the trial, following advice from the chair of our trial's independent Data Monitoring \& Ethics Committee. The REC judged that no changes to the conduct of the intervention or trial were necessary. None of the remaining 28 SAEs (19 women) were related to trial participation. Most of the SAEs were hospital admission due to physical or mental ill health. This included 6 cases of attempted suicide, 7 cases related to pregnancy or miscarriage, 3 cases of injuries from physical assault, 10 cases of chest or abdominal pain and 2 planned admissions. Planned admissions and injuries from physical assault were only found in the intervention arm. All other non-miscellaneous categories occurred with a similar frequency in both arms.

\section{Risk of bias}

\begin{tabular}{lll}
\hline Bias & Authors' judgement & Support for judgement \\
\hline $\begin{array}{ll}\text { Random sequence genera- } \\
\text { tion (selection bias) }\end{array}$ & Low risk & Judgement comment: "on completion, the participant was randomised to ei- \\
& & ther receiving the PATH intervention or to the control group through a remote \\
& independent automated telephone randomisation service provided by the \\
& Bristol Randomised Trials Collaboration (www.bristol.ac.uk/social-communi- \\
& ty-medicine/centres/brtc/)". \\
\hline
\end{tabular}

$\begin{array}{ll}\begin{array}{l}\text { Allocation concealment } \\ \text { (selection bias) }\end{array} & \text { Low risk } \\ & \begin{array}{l}\text { Judgement comment: "randomisation was stratified by urban area and } \\ \text { whether women received support from the refuge/safe house- or communi- }\end{array}\end{array}$



until the moment of randomisation".

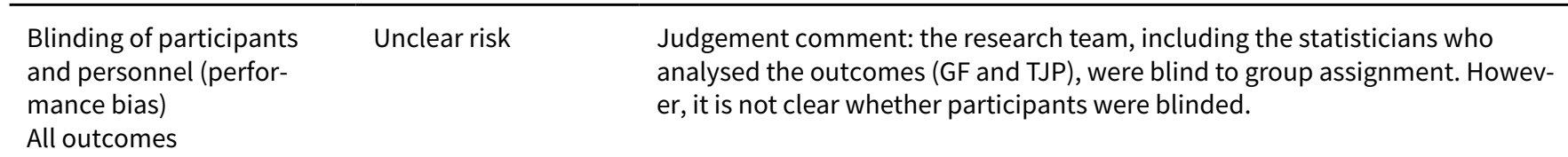

\begin{tabular}{|c|c|c|}
\hline $\begin{array}{l}\text { Blinding of outcome as- } \\
\text { sessment (detection bias) }\end{array}$ & Low risk & $\begin{array}{l}\text { Judgement comment: "over the course of the trial women were asked to self- } \\
\text { complete } 3 \text { further questionnaire booklets } 4,8 \text { and } 12 \text { months post-randomi- }\end{array}$ \\
\hline
\end{tabular}

\begin{tabular}{ll}
\hline $\begin{array}{l}\text { Incomplete outcome data } \\
\text { (attrition bias) }\end{array}$ & Jow risk \\
All outcomes & lected, analysed and fully reported. "64\% of participants were retained for the \\
& full 12-month follow-up period to the end of the trial, although we had primary \\
& outcome data from any follow-up time point available for $78 \%$ of participants \\
& $(78 \%$ SPA group, $77 \%$ advocacy alone group)".
\end{tabular}

\begin{tabular}{|c|c|c|}
\hline $\begin{array}{l}\text { Selective reporting (re- } \\
\text { porting bias) }\end{array}$ & Low risk & $\begin{array}{l}\text { Judgement comment: all primary and secondary outcome measures were col- } \\
\text { lected, analysed and fully reported. This was consistent with the published tri- } \\
\text { al protocol. }\end{array}$ \\
\hline Other bias & Low risk & $\begin{array}{l}\text { Judgement comment: this was a well-designed trial with minimum risk of bias. } \\
\text { Strengths of PATH include: recruitment of a sizeable proportion of the eligible } \\
\text { women experiencing abuse and seeking help; successful collaboration with } \\
\text { agencies in the DVA sector to deliver a psychological intervention with reason- } \\
\text { able fidelity; relatively complete data collection from participants retained in } \\
\text { the trial; outcome data from at least one time point after randomisation from } \\
74 \% \text { of participants; and a nested longitudinal qualitative trial }\end{array}$ \\
\hline
\end{tabular}

\section{Study characteristics}

Methods

Trial design: quasi-experimental; q 2 (race) $\times 4$ (treatment conditions) pre- and post-test design

Trial grouping: parallel group

Assessment modality (e.g. face-to-face, telephone, online): face-to-face

\section{Conflict of interest of trial authors: NR}

Funding for trial: this experiment was supported by Grant P20 MD001089 from the National Center of Minority Health and Health Disparities, NIH. Its contents are solely the responsibility of the trial authors and do not necessarily represent the official view of the National Institutes of Health

Primary outcomes: depression assessed using BDI-II

Randomisation method: within race, participants were randomly assigned to 1 of 4 conditions: testimony, yogic breathing, testimony and yogic breathing, and control

Secondary outcomes: none

Timing of outcome measurements: baseline and post-treatment

Total duration of trial: the experiment took 4 consecutive days to complete

Type of analysis: 1-way analysis of covariance and all testimonies were taped for later transcription, coding, and qualitative analysis. Mean differences and t-test 
Types of participants: African American and European American abused women. A total of 40 women between the ages of 18 and 45, who self-identified as either African American $(n=20)$ or European American ( $n=20$ ) and self-identified as verbally, emotionally, physically, and/or sexually abused by a man with whom they had been intimate within the past 2 years, were invited to participate. The participants were found through advertisements in the local newspaper, placement of flyers on the inside of bathroom stall doors in the court house, at the local state university and community college, and in various bars and other locations

ITT analyses: NR

Participants

\section{Baseline characteristics}

Experimental intervention

- Age: NR

- Ethnicity: NR

- Employment: NR

- Education: NR

- Relationship status: NR

- Gender (\% women): 100

- Dependent children: NR

- Number analysed (data analysis at post-treatment stage): 10

- Number of participants eligible assigned: 10

- Number of participants dropped out (by final follow-up): 0

- Comorbidities: NR

- Experience of IPV: NR

- Experience of other types of violence and abuse: NR

- Remuneration: USD 100

- Financial dependence (on partner): NR

Comparator intervention

- Age: NR

- Ethnicity: NR

- Employment: NR

- Education: NR

- Relationship status: NR

- Gender (\% women): 100

- Dependent children: NR

- Number analysed (data analysis at post-treatment stage): 10

- Number of participants eligible assigned: 10

- Number of participants dropped out (by final follow-up): 0

- Comorbidities: NR

- Experience of IPV: NR

- Experience of other types of violence and abuse: NR

- Remuneration: USD 100

- Financial dependence (on partner): NR

Total sample

- Age: 18-45 years

- Ethnicity: African American ( $n=20,50 \%)$ or European American ( $n=20,50 \%)$

- Employment: NR

- Education: NR

- Relationship status: NR

- Gender (\% women): NR 
- Dependent children: NR

- Number analysed (data analysis at post-treatment stage): 40

- Number of participants eligible assigned: 40

- Number of participants dropped out (by final follow-up): all of the women participated for all 4 days, and not a single participant withdrew from this trial

- Comorbidities: NR

- Experience of IPV: a total of 40 women between the ages of $18-45$, who self-identified as either African American $(n=20)$ or European American $(n=20)$ and self-identified as verbally, emotionally, physically, and/or sexually abused by a man with whom they have been intimate within the past 2 years, were invited to participate

- Experience of other types of violence and abuse: NR

- Remuneration: each participant received USD 100 (1/3 on the 1 st day, $1 / 3$ on the 3 rd day, and $1 / 3$ on the last day)

- Financial dependence (on partner): NR

Included criteria: a total of 40 women between the ages of 18-45, who self-identified as either African American $(n=20)$ or European American $(n=20)$ and self-identified as verbally, emotionally, physically, and/or sexually abused by a man with whom they had been intimate within the past 2 years, were invited to participate.

\section{Excluded criteria: NR}

Pretreatment: NR; group differences in terms of key socio-demographic variables were not reported

Number eligible: 40

Number ineligible: NR

Number of eligible people consented: 40

Number of eligible people recruited: 40

Recruitment setting of participants (e.g. healthcare setting, community setting, shelter setting): community

Sample power calculation: NR

Total number of participants approached: 40

Total number of participants within all groups: 40

Number declined to participate: NR

\section{Intervention characteristics}

Experimental intervention

- Brief description (rationale, main components): testimony brings awareness of and reflection on the violence in abused women's lives, moving them toward self-empowerment

- Type of intervention: giving testimony

- Mode of delivery (how): face-to-face

- Frequency and duration of delivery (when and how much): the experiment took 4 consecutive days to complete. On the first day, the assistant administered the BDI-II, along with a demographic page, to the participant. The second and third days for the testimony condition consisted of taking the participant's testimony for $45 \mathrm{~min}$ each day, for a total of $1.5 \mathrm{~h}$ of intervention. On the 4 th consecutive day, the assistant administered the BDI-II to the participant

- Level of mental health training of person delivering the intervention: women research assistants were matched by race with the participants' race and trained to take the participants' testimonies. All testimonies were taped for later transcription, coding, and qualitative analysis. A master's-level female student was trained to teach the yogic breathing protocol (the yogic breathing protocol is available at 
our web site, www.uncfsu.edu/psychology/Web\&Pages/YBIPV.htm) by the primary investigator, who is a registered yoga teacher.

- Fidelity assessment: NR

- Intervention model: giving testimony

- Person delivering the intervention (who provided the intervention): therapist, self-directed or combined: women research assistants

- Intervention location (where): testimonies were taken in a small room in a discrete location on the third floor of the campus library

- Tailoring of intervention (personalised, titrated or adapted): women research assistants were matched by race with the participants' race and trained to take the participants' testimonies

- Intervention modifications (during the trial): NR

- Main techniques of intervention: having women give testimony about their abuse

- Intervention manual: women research assistants were matched by race with the participants' race and trained to take the participants' testimonies. All testimonies were taped for later transcription, coding, and qualitative analysis

- Intervention attendance: all of the women participated for all 4 days, and not a single participant withdrew from this trial

- CCDAN psychological therapies: other psychologically-orientated interventions

- Intensity of intervention: up to 4 sessions

- Person delivering the intervention: healthcare workers

- Gender of therapist: female

Comparator intervention

- Brief description (rationale, main components): each participant in the control group met with the assistant on the 1st and 4th days, during which time she filled out the BDI-II. At the end of the trial, the controls were offered the opportunity to testify about their experience with spousal abuse or to be trained in yogic breathing. However, none of the participants in the control group chose to participate in either condition.

- Type of intervention: assessment only

Outcomes

\section{Depression}

- Outcome type: continuous outcome

- Reporting: fully reported

- Scale: BDI-II

- Direction: lower is better

\section{Dropouts from treatment}

- Outcome type: dichotomous outcome

- Reporting: fully reported

- Direction: lower is better

Identification

Sponsorship source: this experiment was supported by Grant P20 MD001089 from the National Center of Minority Health and Health Disparities, National Institutes of Health

Country: USA

Setting: community

Comments: African American and European American abused women

Trialauthors: Susan H. Franzblau Sonia Echevarria Michelle Smith Thomas E. Van Cantfort

Institution: Fayetteville State University

Email: sfranzblau@uncfsu.edu 
Address: Department of Psychology, Fayetteville State University, 1200 Murchison Rd., Fayetteville, NC 28301; phone: 910-672-1574

Title (main 'outcome' publication): A preliminary investigation of the effects of giving testimony and learning yogic breathing techniques on battered women's feelings of depression

Registered trial protocol ID: NR

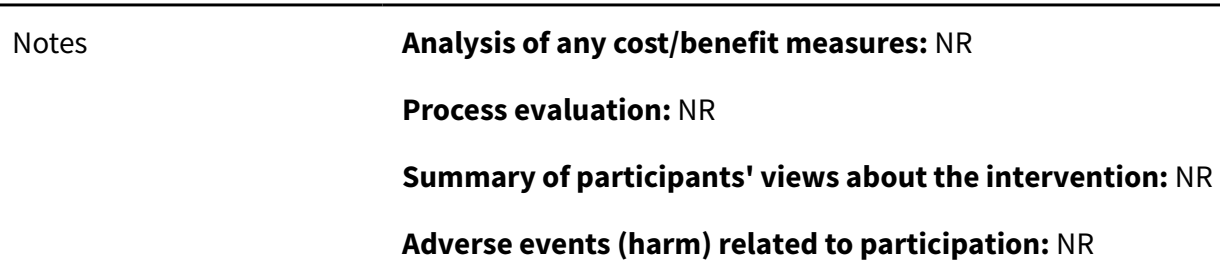

Risk of bias

\begin{tabular}{|c|c|c|}
\hline Bias & Authors' judgement & Support for judgement \\
\hline $\begin{array}{l}\text { Random sequence genera- } \\
\text { tion (selection bias) }\end{array}$ & High risk & $\begin{array}{l}\text { Judgement comment: "the participants were found through advertisements in } \\
\text { the local newspaper, placement of flyers on the inside of bathroom stall doors } \\
\text { in the court house, at the local state university and community college, and in } \\
\text { various bars and other locations. Within race, participants were randomly as- } \\
\text { signed to } 1 \text { of } 4 \text { conditions: testimony, yogic breathing, testimony and yogic } \\
\text { breathing, and control". No further information provided about how partici- } \\
\text { pants were randomised. }\end{array}$ \\
\hline
\end{tabular}

\begin{tabular}{ll}
\hline $\begin{array}{l}\text { Allocation concealment } \\
\text { (selection bias) }\end{array}$ & High risk \\
& $\begin{array}{l}1 \text { of } 4 \text { conditions: testimony, yogic breathing, testimony and yogic breathing, } \\
\text { and control". No further information reported. }\end{array}$
\end{tabular}

Blinding of participants High risk and personnel (perforJudgement comment: "women research assistants were matched by race with mance bias) the participants' race and trained to take the participants' testimonies. All testimonies were taped for later transcription, coding, and qualitative analysis. A All outcomes master's-level female student was trained to teach the yogic breathing protocol. On the first day, the assistant administered the BDI-II, along with a demographic page, to the participant". No further information provided.

\begin{tabular}{|c|c|c|}
\hline $\begin{array}{l}\text { Blinding of outcome as- } \\
\text { sessment (detection bias) } \\
\text { All outcomes }\end{array}$ & High risk & $\begin{array}{l}\text { Judgement comment: "on the first day, the assistant administered the BDI- } \\
\text { II, along with a demographic page, to the participant. On the 4th consecutive } \\
\text { day, the assistant administered the BDI-II to the participant. After the BDI-II } \\
\text { was completed, the assistant debriefed the participant". No further informa- } \\
\text { tion reported. }\end{array}$ \\
\hline
\end{tabular}

\begin{tabular}{ll}
\hline $\begin{array}{l}\text { Incomplete outcome data } \\
\text { (attrition bias) }\end{array}$ & Low risk \\
All outcomes & $\begin{array}{l}\text { Judgement comment: attrition and exclusion was adequately described. } \\
\text { Women's feelings of depression were fully collected, analysed and reported as } \\
\text { an outcome variable. }\end{array}$ \\
\hline
\end{tabular}

$\begin{array}{ll}\begin{array}{l}\text { Selective reporting (re- } \\ \text { porting bias) }\end{array} & \begin{array}{l}\text { Judgement comment: all outcomes described in the methods section were ad- } \\ \text { equately collected, analysed and reported in the results section of the main } \\ \text { published trial. However, there was insufficient or unclear evidence from ei- } \\ \text { ther the trial protocol or trial registry sites to assess this domain. }\end{array}\end{array}$

Other bias High risk

\begin{abstract}
Judgement comment: despite the fact that this experiment was innovative and challenging, particularly to traditional therapeutic techniques, there were several methodological limitations that may contribute to various types of biases (e.g. no information about randomisation method, lack of fidelity assessment, unclear blindness to group assignment and outcome assessors, small sample size).
\end{abstract}




\section{Study characteristics}

Trial design: RCT
Trial grouping: parallel group
Conflict of interest of trial authors: NR
Funding for trial: NR
Primary outcomes: depression and anxiety
Randomisation method: NR
Secondary outcomes: NR
Triallocation: Tehran (psychiatric clinics)
Timing of outcome measurements: baseline and post-treatment
Total duration of trial: NR
Types of participants: women victims of domestic violence referred to several psychiatric clinics in
Tehran for treatment by 2015
Assessment modality (e.g. face-to-face, telephone, online): face-to-face
Type of analysis: descriptive statistics, ANOVA
ITT analyses: NR

\section{Participants}

\section{Baseline characteristics}

Experimental intervention

- Age: NR

- Gender (\% women): 100

- Ethnicity: women victims of domestic violence in Tehran

- Employment: NR

- Education: NR

- Relationship status: NR

- Dependent children: NR

- Number analysed (data analysis at post-treatment stage): NR

- Number of participants eligible assigned: 15

- Number of participants dropped out (by final follow-up): NR

- Comorbidities: NR

- Experience of IPV: NR

- Experience of other types of violence and abuse: NR

- Remuneration: NR

- Financial dependence (on partner): NR

Comparator intervention

- Age: NR

- Gender (\% women): 100

- Ethnicity: women victims of domestic violence in Tehran

- Employment: NR

- Education: NR 
- Relationship status: NR

- Dependent children: NR

- Number analysed (data analysis at post-treatment stage): NR

- Number of participants eligible assigned: 15

- Number of participants dropped out (by final follow-up): NR

- Comorbidities: NR

- Experience of IPV: NR

- Experience of other types of violence and abuse: NR

- Remuneration: NR

- Financial dependence (on partner): NR

Total sample

- Age: 20-25 years

- Gender (\% women): 100

- Ethnicity: women victims of domestic violence in Tehran

- Employment: about $76.6 \%$ of women were housewives and others were employed

- Education: most of the participants had a diploma degree (60\%)

- Relationship status:years was passed from their marriage (46.6\%)

- Dependent children: had 2 children (56.6\%)

- Number analysed (data analysis at post-treatment stage): NR

- Number of participants eligible assigned: 30

- Number of participants dropped out (by final follow-up): NR

- Comorbidities: NR

- Experience of IPV: statistical population consists of women victims of domestic violence in Tehran referred to several psychiatric clinics in Tehran for treatment by 2015

- Experience of other types of violence and abuse: NR

- Remuneration: NR

- Financial dependence (on partner): NR

Included criteria: women victims of domestic violence in Tehran referred to several psychiatric clinics in Tehran for treatment by 2015, presenting with high scores in depression and anxiety inventories

Excluded criteria: none reported

Pretreatment: NR

Sample power calculation: NR

Total number of participants: 30

Number ineligible: NR

Number eligible: NR

Number of eligible people consented: NR

Recruitment setting of participants (e.g. healthcare setting, community setting, shelter setting): healthcare setting (psychiatric clinics)

Number of eligible people recruited: NR

Total number of participants approached: NR

Total number of participants within all groups: 30

Number declined to participate: NR 


\section{Experimental intervention}

- Brief description (rationale, main components): treatment package of the present trial is implemented based on mindfulness-based cognitive therapy protocol for generalised anxiety disorder in the form of 8 sessions with $45 \mathrm{~min} / \mathrm{session}$

- Type of intervention: mindfulness-based cognitive therapy

- Mode of delivery: face-to-face

- Level of mental health training of person delivering the intervention: NR

- Fidelity assessment: NR

- Intervention model: mindfulness-based cognitive therapy

- Mode of delivery (how): face-to-face

- Frequency and duration of delivery (when and how much):

- Person delivering the intervention (who provided the intervention): therapist, self-directed or combined: therapist

- Intervention location (where):

- Tailoring of intervention (personalised, titrated or adapted):

- Intervention modification (during the trial):

- Main techniques of intervention: behavior-emotion-thought-feeling cycle

- Intervention manual: NR

- Intervention attendance: NR

- CCDAN psychological therapies: third-wave CBTs

- Intensity of intervention: $\geq 5$ sessions

- Person delivering the intervention: unclear

- Gender of therapist: NR

Comparator intervention

- Brief description (rationale, main components): waiting list

- Type of intervention: waiting list

\section{Outcomes}

\section{Anxiety}

- Outcome type: continuous outcome

- Reporting: fully reported

- Scale: Spielberger's State-Trait Anxiety Inventory (STAI)

- Range: 20-80

- Direction: lower is better

\section{Dropouts from treatment}

- Outcome type: dichotomous outcome

- Reporting: fully reported

- Direction: lower is better

\section{Sponsorship source: NR}

Country: Iran

Setting: psychiatric clinics

Comments: several psychiatric clinics in Tehran

Trialauthors: Shahrbanoo Ghahari, Nooshin Khademolreza, Fatemeh Sadeghi Poya, Siamak Ghasemnejad, Bahram Gheitarani and Mohammad Reza Pirmoradi

Institution: Department of Clinical Psychology, Iran University of Medical Sciences (IUMS), Center of Excellence in Psychiatry, School of Behavioral Sciences and Mental Health, Tehran, Iran 
Email: mr.pirmoradi@chmail.ir

Address: Department of Clinical Psychology, Iran University of Medical Sciences (IUMS), Center of Excellence in Psychiatry, School of Behavioral Sciences and Mental Health, Tehran, Iran

Trial title (main outcome publication): Effectiveness of Mindfulness Techniques in Decreasing Anxiety and Depression in Women Victims of Spouse Abuse

Registered trial protocol ID: NR

Analysis of any cost/benefit measures: NR
Process evaluation: NR
Summary of participants' views about the intervention: NR
Adverse events (harm) related to participation: NR

Risk of bias

\begin{tabular}{|c|c|c|}
\hline Bias & Authors' judgement & Support for judgement \\
\hline $\begin{array}{l}\text { Random sequence genera- } \\
\text { tion (selection bias) }\end{array}$ & High risk & $\begin{array}{l}\text { Judgement comment: statistical sample consists of } 30 \text { women selected ran- } \\
\text { domly, who were placed in } 2 \text { groups. No further information provided about } \\
\text { sequence generation }\end{array}$ \\
\hline $\begin{array}{l}\text { Allocation concealment } \\
\text { (selection bias) }\end{array}$ & High risk & $\begin{array}{l}\text { Judgement comment: there is insufficient information provided about alloca- } \\
\text { tion concealment. The statistical sample consists of } 30 \text { women selected ran- } \\
\text { domly, who were placed in } 2 \text { groups. }\end{array}$ \\
\hline $\begin{array}{l}\text { Blinding of participants } \\
\text { and personnel (perfor- } \\
\text { mance bias) } \\
\text { All outcomes }\end{array}$ & High risk & $\begin{array}{l}\text { Judgement comment: there is insufficient information about the blinding of } \\
\text { participants and personnel }\end{array}$ \\
\hline $\begin{array}{l}\text { Blinding of outcome as- } \\
\text { sessment (detection bias) } \\
\text { All outcomes }\end{array}$ & High risk & $\begin{array}{l}\text { Judgement comment: there are no details about blinding of outcome asses- } \\
\text { sors. }\end{array}$ \\
\hline $\begin{array}{l}\text { Incomplete outcome data } \\
\text { (attrition bias) } \\
\text { All outcomes }\end{array}$ & High risk & $\begin{array}{l}\text { Judgement comment: the number of participants with completed outcome } \\
\text { data is not reported. Attrition and exclusions were not adequately reported. }\end{array}$ \\
\hline $\begin{array}{l}\text { Selective reporting (re- } \\
\text { porting bias) }\end{array}$ & Unclear risk & $\begin{array}{l}\text { Judgement comment: all outcomes described in the methods section were ad- } \\
\text { equately collected, analysed and reported in the results section of the main } \\
\text { published trial. However, there was insufficient or unclear evidence from ei- } \\
\text { ther the trial protocol or trial registry sites to assess this domain. }\end{array}$ \\
\hline Other bias & High risk & $\begin{array}{l}\text { Judgement comment: there is insufficient information about various domains, } \\
\text { including participant recruitment, research ethics approval, and appropriates } \\
\text { of data analysis techniques }\end{array}$ \\
\hline
\end{tabular}

\section{Gilbert 2006}

\section{Study characteristics}

Methods


Trial grouping: parallel group

Assessment modality (e.g. face-to-face, telephone, online): face-to-face

Conflict of interest of trial authors: NR

Funding for trial: NR

Primary outcomes: Revised CTS Drug and Alcohol Use Behavior Questionnaire, the depression subscale of the BSI, the PCL-C, Sexual Risk Behavior Questionnaire

Randomisation method: of the 40 eligible women, 34 were randomly assigned to either the 12 -session relapse prevention and relationship safety (RPRS) condition $(n=16)$ or the 1-session informational control (IC) $(n=18)$ using allocation procedures, which were designed to minimise bias in participant assignment to conditions in RCTs

\section{Secondary outcomes: NR}

Timing of outcome measurements: baseline and 3-month post-intervention follow-up assessment

Total duration of trial: between June 2003 and February 2004

Type of analysis: participants' change scores (i.e. post-pre) for each outcome variable were dichotomised into improved score versus no improvement or worse score

Types of participants: average age of participants was 41.8 years $(S D=6.6)$. More than half of the participants (59.3\%) were Latina, 15.6\% were African American, and one-fifth were White (20.6\%). Slightly more than half (54.5\%) had graduated from high school. One-fifth $(21.8 \%)$ were currently employed. Almost one-third (32.3\%) of the women identified their primary intimate sexual partner as a common-law husband, $29.4 \%$ as a boyfriend, $20.6 \%$ as a husband, and $8.8 \%$ as a common-law wife or girlfriend. The average length of relationships was 9.4 years $(S D=7.9)$. More than a third $(37.5 \%)$ had a child or children under the age of 18 .

ITT analyses: NR

Participants

\section{Baseline characteristics}

Experimental intervention

- Age: NR

- Ethnicity: NR

- Employment: NR

- Education: NR

- Relationship status: NR

- Gender (\% women): NR

- Dependent children: NR

- Number analysed (data analysis at post-treatment stage): 16

- Number of participants eligible assigned: 16

- Number of participants dropped out (by final follow-up): NR

- Comorbidities: NR

- Experience of IPV: NR

- Experience of other types of violence and abuse: NR

- Remuneration: participants received USD 5 for completing the brief screening interview, USD 25 for completing the baseline interview, and USD 30 for completing the follow-up assessment, which was conducted 3 months after the last session of RPRS for all participants in each condition. We provided USD 25 to participants at the end of each RPRS session and the IC session for transportation costs, any child-care costs, and financial compensation

Comparator intervention

- Age: NR 
- Ethnicity: NR

- Employment: NR

- Education: NR

- Relationship status: NR

- Gender (\% women): NR

- Dependent children: NR

- Number analysed (data analysis at post-treatment stage): 18

- Number of participants eligible assigned: 18

- Number of participants dropped out (by final follow-up): NR

- Comorbidities: NR

- Experience of IPV: NR

- Experience of other types of violence and abuse: NR

- Remuneration: participants received USD 5 for completing the brief screening interview, USD 25 for completing the baseline interview, and USD 30 for completing the follow-up assessment, which was conducted 3 months after the last session of RPRS for all participants in each condition. We provided USD 25 to participants at the end of each RPRS session and the IC session for transportation costs, any child-care costs, and financial compensation

Total sample

- Age: 41.8 years (6.6)

- Ethnicity: Latina: 59.3\%; African American: 15.6\%; white: 20.6\%

- Employment: $21.8 \%$ employed

- Education: $54.5 \%$ completed high school

- Relationship status: almost one-third (32.3\%) of the women identified their primary intimate sexual partner as a common-law husband, $29.4 \%$ as a boyfriend, $20.6 \%$ as a husband, and $8.8 \%$ as a common-law wife or girlfriend

- Gender (\% women): 100

- Dependent children: > a third (37.5\%) had a child or children under the age of 18

- Number analysed (data analysis at post-treatment stage): 34

- Number of participants eligible assigned: 40

- Number of participants dropped out (by final follow-up): 6

- Comorbidities: NR

- Experience of IPV: NR

- Experience of other types of violence and abuse: NR

- Remuneration: participants received USD 5 for completing the brief screening interview, USD 25 for completing the baseline interview, and USD 30 for completing the follow-up assessment, which was conducted 3 months after the last session of RPRS for all participants in each condition. We provided USD 25 to participants at the end of each RPRS session and the IC session for transportation costs, any child-care costs, and financial compensation

Included criteria: eligibility criteria included women who (1) were aged $\geq 18$; (2) were currently enrolled in as an outpatient; (3) reported using any illicit drug in the past 90 days; and (4) reported physical aggression, sexual coercion, injury-related abuse, or severe psychological IPV by an intimate partner in the past 90 days.

Excluded criteria: women were excluded if they (1) had a cognitive impairment that would prevent comprehension of the assessment or intervention, evaluated using the Mini Mental Health Status Exam and Quick Test for Intellectual Functioning, or (2) did not speak and understand English at a conversational level.

Pretreatment: groups did not differ significantly on any of the socio-demographic variables or the IPV, drug use, depression, PTSD, and sexual HIV risk outcome variables.

\section{Number eligible: 40}

Number ineligible: 56 


\section{Number of eligible people consented: 40}

Number of eligible people recruited: 40

Recruitment setting of participants (e.g. healthcare setting, community setting, shelter setting): healthcare setting

\section{Sample power calculation: NR}

Total number of participants approached: 96

Total number of participants within all groups: 34

Number declined to participate: NR

Interventions

\section{Intervention characteristics}

Experimental intervention

- Brief description (rationale, main components): the RPRS allows facilitators to employ strategies derived from social cognitive and empowerment theories to enable participants to avoid IPV and drug use. It relies on group and individual processes of behavior change. The RPRS is tailored to the realities of low-income, African American, and Latina women and focuses on the enhancement of positive evaluations of self-worth, ethnic pride, and risk avoidance as an investment in the future of their communities. The sessions include traditional and contemporary African American and Latina references that further enhance cultural specificity and pride. The cultural content primarily targets African American or Latina women; however, the content is also relevant to other women who share common experiences as low-income, urban, drug-involved women experiencing IPV. Materials and exercises incorporate social cognitive skill building (i.e. coping strategies, boundary setting, negotiation and communication skills) and involve games, brainstorming, role-playing, and small-group discussions that build group cohesion.

- Type of intervention: RPRS

- Mode of delivery (how): face-to-face

- Frequency and duration of delivery (when and how much): RPRS intervention consisted of $11 \times 2-h$ group sessions and 1 individual session designed to promote relationship safety and reduce drug use. The RPRS sessions were conducted twice weekly for 6 weeks.

- Level of mental health training of person delivering the intervention: NR

- Fidelity assessment: all RPRS intervention sessions for both cohorts were digitally recorded and rated by a quality-assurance monitor. This monitor completed a facilitator adherence and competence rating form for each session for both cohorts. The rating form consisted of 37 items rating facilitator skill and adherence to content on a 5-point Likert scale on the delivery of didactic content, modelling and role-playing skills, reinforcement of positive behaviours, showing positive regard for participants, group cohesion, problem solving, and goal setting.

- Intervention model: RPRS

- Person delivering the intervention (who provided the intervention): therapist, self-directed or combined: therapist

- Intervention location (where): NR

- Tailoring of intervention (personalised, titrated or adapted): NR

- Intervention modifications (during the trial): NR

- Main techniques of intervention: materials and exercises incorporate social cognitive skill building (i.e. coping strategies, boundary setting, negotiation and communication skills) and involve games, brainstorming, role playing,and small-group discussions that build group cohesion

- Intervention manual: NR

- Intervention attendance: women attended a mean of $11 / 12$ sessions, half of the women attended all 12 sessions, and the remaining half attended between 9 and 11 sessions.

- CCDAN psychological therapies: CBT

- Intensity of intervention: $\geq 5$ sessions

- Person delivering the intervention: unclear

- Gender of therapist: NR 


\section{Comparator intervention}

- Brief description (rationale, main components): the informational session provided to IC participants consisted of a 1-h didactic presentation of a wide range of local community services (i.e. employment services, job training, housing, domestic violence programmes, legal services, mental health services, low-cost dental services) that women in MMTPs can access, tips on help-seeking, and a comprehensive directory of local IPV-related services. The informational session and directory provided a range of referral sources for addressing issues related to IPV (i.e. shelters, legal services, family and criminal court information on how to obtain an order of protection). Such referrals represent the highest standard of care for addressing IPV in the host MMTPs for this trial

- Type of intervention: IC

\section{Outcomes}

\section{Depression}

- Outcome type: continuous outcome

- Reporting: fully reported

- Scale: BSI

- Direction: lower is better

PTSD

- Outcome type: adverse event (dichotomous)

- Reporting: fully reported

- Scale: PCL-C

- Direction: lower is better

\section{Re-exposure to IPV}

- Outcome type: adverse event (dichotomous)

- Reporting: fully reported

- Scale: CTS-2

- Direction: lower is better

\section{Dropouts from treatment}

- Outcome type: dichotomous outcome

- Reporting: fully reported

- Direction: lower is better

Setting: healthcare setting

Comments: women on methadone who met IPV and drug use criteria

Trialauthors: Louisa Gilbert, Nabila El-Bassel, Jennifer Manuel, Elwin Wu, Hyun Go, Seana Golder, Randy Seewald, Glorice Sanders

Institution: Social Intervention Group, Columbia University, New York, NY

Email: NR

\section{Address: NR}

Title (main 'outcome' publication): an Integrated relapse prevention and relationship safety intervention for women on methadone: testing short-term effects on intimate partner violence and substance use

\section{Registered trial protocol ID: NR}


Gilbert 2006 (Continued)

Notes

\section{Analysis of any cost/benefit measures: NR}

Process evaluation: NR

Summary of participants' views about the intervention: NR

Adverse events (harm) related to participation: prior to conducting the trial, the investigators generated a list of potential adverse events that might occur in this intervention trial based on previous similar studies (see 'Methods'). None of these potential adverse or other adverse events were detected by research assistants, facilitators, the quality assurance monitor, trial investigators, or the MMTP staff.

\section{Risk of bias}

Bias Authors' judgement Support for judgement

Random sequence genera- Low risk tion (selection bias)

Judgement comment: "after all baseline interviews were completed for each cohort, an investigator generated a list of random numbers using Statistical Package for the Social Sciences (SPSS) to construct an allocation sequence that would assign each woman to the RPRS or IC with equal probability"

\begin{tabular}{ll}
\hline $\begin{array}{l}\text { Allocation concealment } \\
\text { (selection bias) }\end{array}$ & Judgement comment: "of the 40 eligible women, 34 were randomly assigned \\
& to either the 12 -session RPRS condition $(\mathrm{n}=16)$ or the 1 -session IC $(\mathrm{n}=18)$ us- \\
ing allocation procedures, which were designed to minimise bias in partici- & pant assignment to conditions in RCTs. Random assignment to the RPRS and \\
IC conditions was effective".
\end{tabular}

\begin{tabular}{|c|c|c|}
\hline $\begin{array}{l}\text { Blinding of participants } \\
\text { and personnel (perfor- } \\
\text { mance bias) } \\
\text { All outcomes }\end{array}$ & Unclear risk & $\begin{array}{l}\text { Judgement comment: "after all baseline interviews were completed for each } \\
\text { cohort, an investigator generated a list of random numbers using Statistical } \\
\text { Package for the Social Sciences (SPSS) to construct an allocation sequence } \\
\text { that would assign each woman to the RPRS or IC with equal probability". How- } \\
\text { ever, no further information provided about blinding personnel and members } \\
\text { of the research team and/or individuals delivering the intervention. }\end{array}$ \\
\hline
\end{tabular}

\begin{tabular}{|c|c|c|}
\hline $\begin{array}{l}\text { Blinding of outcome as- } \\
\text { sessment (detection bias) } \\
\text { All outcomes }\end{array}$ & Unclear risk & $\begin{array}{l}\text { Judgement comment: the baseline and 3-month post-intervention follow-up } \\
\text { assessment were clearly articulated, however, no further information reported } \\
\text { about how and who conducted the assessment. }\end{array}$ \\
\hline
\end{tabular}

\begin{tabular}{lll}
\hline $\begin{array}{l}\text { Incomplete outcome data } \\
\text { (attrition bias) } \\
\text { All outcomes }\end{array}$ & Low risk & $\begin{array}{l}\text { Judgement comment: attrition and exclusions were adequately described. } \\
\text { The retention rate was high, with } 31 \text { women (91\%) completing the 3-month } \\
\text { follow-up interviews. }\end{array}$ \\
\hline $\begin{array}{l}\text { Selective reporting (re- } \\
\text { porting bias) }\end{array}$ & Unclear risk & $\begin{array}{l}\text { Judgement comment: all outcomes described in the methods section were ad- } \\
\text { equately collected, analysed and reported in the results section of the main } \\
\text { published trial. However, there was insufficient or unclear evidence from ei- } \\
\text { ther the trial protocol or trial registry sites to assess this domain. }\end{array}$
\end{tabular}

\begin{tabular}{ll}
\hline Other bias Low risk Judgement comment: none \\
\hline
\end{tabular}

\section{Gilbert 2016}

\section{Study characteristics}

$\begin{array}{ll}\text { Methods } & \text { Trial design: } \mathrm{RCT} \\ & \text { Trial grouping: parallel group }\end{array}$


Assessment modality (e.g. face-to-face, telephone, online): computer-assisted self-interview (selfreports and biological assays)

Conflict of interest of trial authors: none declared

Funding for trial: trial was funded by the National Institute on Drug Abuse to Nabila El Bassel (R01DA025878).

Primary outcomes: IPV victimisation outcomes; current and past substance use

Randomisation method: computer-generated randomisation algorithm was designed to balance the number of women/trial arm via an adaptive, biased-coin procedure

Secondary outcomes: NR

Timing of outcome measurements: baseline, 6-month follow-up, 12 month follow-up

Total duration of trial: RCT was conducted in New York City between November 2009 and January 2012

Type of analysis: logistic regression models

Types of participants: women using substances in multiple community correction sites

ITT analyses: yes

Participants

\section{Baseline characteristics}

Experimental intervention

- Age: $\mathrm{M}=40.5$ years, $\mathrm{SD}=10.9$

- Ethnicity: Black 73 (70.9\%); Latina 15 (14.6\%); other 15 (14.6\%)

- Employment: employment 9 (8.7\%)

- Education: high school or general equivalency diploma 55 (53.4\%)

- Relationship status: single 66 (64.1\%); married 19 (18.4\%); divorced/separated/widowed 18 (17.5\%)

- Gender (\% women): 100

- Dependent children: NR

- Number analysed (data analysis at post-treatment stage): 91

- Number of participants eligible assigned: 103

- Number of participants dropped out (by final follow-up): 12

- Comorbidities: ever used any illicit drug 102 (99.0\%)

- Experience of IPV: any physical IPV 61 (59.2\%); any injurious IPV 59 (57.3\%); any sexual IPV 50 (48.5\%); severe physical IPV 55 (53.4\%); severe injurious IPV 53 (51.5\%); severe sexual IPV 40 (38.8\%)

- Experience of other types of violence and abuse: NR

- Remuneration: USD 265

- Financial dependence (on partner): NR

Comparator intervention

- Age: $\mathrm{M}=42.1$ years, $\mathrm{SD}=9.7$

- Ethnicity: Black 68 (66.7\%); Latina 15 (14.7\%); other 19 (18.6\%)

- Employment: employment 9 (8.8\%)

- Education: high school or general equivalency diploma 55 (53.9\%)

- Relationship status: single 66 (64.7\%); married 18 (17.6\%); divorced/separated/widowed 18 (17.6\%)

- Gender (\% women): 100

- Dependent children: NR

- Number analysed (data analysis at post-treatment stage): 94

- Number of participants eligible assigned: 102

- Number of participants dropped out (by final follow-up): 8 
- Comorbidities: Ever used any illicit drug 99 (97.1\%)

- Experience of IPV: any physical IPV 58 (56.9\%); any injurious IPV 51 (50.0\%); any sexual IPV 54 (52.9\%); severe physical IPV 53 (52.0\%); severe injurious IPV 44 (43.1\%); severe sexual IPV 40 (39.2\%)

- Experience of other types of violence and abuse: NR

- Remuneration: USD 265

- Financial dependence (on partner): NR

Total sample

- Age: $\mathrm{M}=41.5$ years, $\mathrm{SD}=10.5$

- Ethnicity: Black 208 (68.0\%); Latina 47 (15.4\%); other 51 (16.7\%)

- Employment: employment 25 (8.2\%)

- Education: high school or general equivalency diploma 176 (57.5\%)

- Relationship status: single 202 (66.0\%); married 49 (16.0\%); divorced/separated/widowed 55 (18.0\%)

- Gender (\% women): 100

- Dependent children: NR

- Number analysed (data analysis at post-treatment stage): 278

- Number of participants eligible assigned: 306

- Number of participants dropped out (by final follow-up): 28

- Comorbidities: ever used any illicit drug 300 (98.0\%)

- Experience of IPV: any physical IPV 185 (60.5\%); any injurious IPV 177 (57.8\%); any sexual IPV 166 (54.2\%); severe physical IPV 170 (55.6\%); severe injurious IPV 151 (49.3\%); severe sexual IPV 117 $(38.2 \%)$

- Experience of other types of violence and abuse: NR

- Remuneration: participants were reimbursed for completing assessments and intervention sessions up to a maximum of USD 265.

- Financial dependence (on partner): NR

Included criteria: eligible women reported: being aged $\geq 18$ years; being mandated to community corrections (i.e. probation, parole, community court, drug treatment court or an alternative-to-incarceration programme) in the past 90 days; using illicit drugs, binge drinking, or attending a substance abuse treatment programme in the past 90 days; engaging in unprotected vaginal or anal intercourse within the past 90 days; and having at least 1 other HIV risk factor.

Excluded criteria: potential participants were considered ineligible if they were unable to complete the informed consent process due to a psychiatric or cognitive impairment, unable to speak English, or if the they were actively trying to become pregnant. Women with pregnancy intentions were excluded due to the intervention's emphasis on condom use. Women who did not have an address where they could receive mail, lived more than 90 min from New York City, or planned to move more than 90 min outside of New York City were also excluded

Pretreatment: we did not find significant differences in any of the characteristics by trial condition

Number eligible: 449

Number ineligible: 655

Number of eligible people consented: 337

Number of eligible people recruited: 449

Recruitment setting of participants (e.g. healthcare setting, community setting, shelter setting): community corrections sites

Sample power calculation: we estimated that with a sample of 112 women/arm, the trial would have $80 \%$ statistical power, assuming alpha $=.05$, 2-sided hypothesis testing, no covariance adjustment, ICCs of.05 for the primary behavioural outcomes, and the following minimum effect sizes: an absolute increase in proportion of condom-protected sex of 15 percentage points, and an absolute decrease of five acts of unprotected sex (a relative decrease of $20 \%$ ). Power analyses used high estimates for ICCS and did not account for covariance adjustment to remain conservative 


\section{Total number of participants approached: 1104}

Total number of participants within all groups: 306

Number declined to participate: NR

Interventions

\section{Intervention characteristics}

Experimental intervention

- Brief description (rationale, main components): the intervention was informed by social cognitive learning theory, which focuses on observation, modelling, and skill rehearsal through role play and feedback from group members. Empowerment theory also guided a strengths-based approach to build collective efficacy of women to negotiate safe relationships and counter stigma that they face as women in community corrections.

- Type of intervention: psycho-education (IPV prevention)

- Mode of delivery (how): computerised

- Frequency and duration of delivery (when and how much): computerised WORTH also consisted of 4 weekly group sessions lasting 90-120 min, led by 2 facilitators

- Level of mental health training of person delivering the intervention: facilitators in the multimedia arm played a less active role than in the traditional WORTH arm, requiring lower levels of training and supervision.

- Fidelity assessment: the discussion section reports that "high fidelity of implementing intervention conditions confirmed by quality assurance, and blind assessment of outcomes".

- Intervention model: social cognitive learning theory and empowerment theory

- Person delivering the intervention (who provided the intervention): therapist, self-directed or combined: multimedia

- Intervention location (where): laptop (Interventions were conducted at a community research site)

- Tailoring of intervention (personalised, titrated or adapted): during each session, participants used individual laptops to independently view video vignettes of 4 fictional role models to promote identification and emotional engagement. Computerised self-paced modules covered the same IPV screening, prevention, and service referral activities that were conducted in the traditional WORTH arm. Some activities (e.g. safety plan and IPV service referrals) were recorded in an electronic log that was printed for participants.

- Intervention modifications (during the trial): for this trial, minor modifications to WORTH were made to make it more contextually relevant for substance-using women in community corrections. However, no further information provided about intervention modifications during the trial

- Main techniques of intervention: during each session, participants used individual laptops to independently view video vignettes of 4 fictional role models to promote identification and emotional engagement. Computerised self-paced modules covered the same IPV screening, prevention, and service referral activities that were conducted in the traditional WORTH arm. Some activities (e.g. safety plan and IPV service referrals) were recorded in an electronic log that was printed for participants.

- Intervention manual: protocol S1 Trial Protocol

- Intervention attendance: NR

- CCDAN psychological therapies: humanistic therapies

- Intensity of intervention: up to 4 sessions

- Person delivering the intervention: computerised with facilitators

- Gender of therapist: unclear

Comparator intervention

- Brief description (rationale, main components): the Wellness Promotion control arm also consisted of 4 weekly group sessions lasting between 90 and $120 \mathrm{~min}$, designed to control for modality and dosage. Core components included activities such as maintaining a healthy diet, promoting fitness, addressing tobacco use, learning stress-reduction exercises. None of the Wellness Promotion activities focused on IPV prevention

- Type of intervention: minimal intervention 
Gilbert 2016 (Continued)

Re-exposure to IPV
- Outcomes $\quad$ - Reporting: fully reported
- Scale: Revised CTS
- Direction: lower is better
Dropouts from treatment
- Outcome type: dichotomous outcome
- Reporting: fully reported
- Direction: lower is better

Identification

Sponsorship source: trial was funded by the National Institute on Drug Abuse to Nabila El-Bassel (R01DA025878).

Country: USA

Setting: community correction sites

Comments: research assistants actively recruited and screened 1104 women from multiple community correction sites by handing out flyers and inviting women to be screened

Trialauthors: Louisa Gilbert, PhD, Dawn Goddard-Eckrich, MS, Timothy Hunt, MS, Xin Ma, MS, Mingway Chang, PhD, Jessica Rowe, MDes, Tara McCrimmon, MPH, Karen Johnson, PhD, Sharun Goodwin, BS, Maria Almonte, MSW, and Stacey A. Shaw, PhD

Institution: Social Intervention Group, Columbia University, New York, NY

Email: Ig123@columbia.edu

Address: Social Intervention Group, Columbia University School of Social Work, 1255 Amsterdam Ave, Room 832, New York, NY 10027

Title (main 'outcome' publication): Efficacy of a computerized intervention on HIV and intimate partner violence among substance-using women in community corrections: a randomized controlled trial

Registered trial protocol ID: NR

Notes

Analysis of any cost/benefit measures: NR

Process evaluation: NR

Summary of participants' views about the intervention: NR

Adverse events (harm) related to participation: no adverse events were detected by trial staff or through quality assurance procedures.

\section{Risk of bias}

Bias Authors' judgement Support for judgement

Random sequence genera- High risk tion (selection bias)

Judgement comment: there is no evidence of sequence generation. Although women were not recruited randomly, we were able to access a key population of women with high levels of HIV risk, targeting those in most need of prevention services. "Findings may not be generalisable to all women under community supervision due to non-random sampling procedures. However there was a random allocation of participants into trial arms".

\begin{tabular}{ll}
\hline $\begin{array}{l}\text { Allocation concealment } \\
\text { (selection bias) }\end{array}$ & Low risk \\
& $\begin{array}{l}\text { Judgement comment: "we randomly assigned groups of 4-9 women to re- } \\
\text { ceive } 1 \text { of the } 3 \text { conditions. The computer-generated randomisation algorithm } \\
\text { was designed to balance the number of women/trial arm via an adaptive, bi- }\end{array}$ \\
\hline
\end{tabular}

Psychological therapies for women who experience intimate partner violence (Review)

Copyright @ 2020 The Cochrane Collaboration. Published by John Wiley \& Sons, Ltd. 
Gilbert 2016 (Continued)

ased-coin procedure. The investigator who designed the randomisation program was not involved in conducting the trial, but was involved in the statistical analysis".

$\begin{array}{ll}\begin{array}{l}\text { Blinding of participants } \\ \text { and personnel (perfor- }\end{array} & \text { Unclear risk } \\ \text { mance bias) } & \begin{array}{l}\text { Judgement comment: "the investigator who designed the randomisation pro- } \\ \text { gram was not involved in conducting the trial, but was involved in the statisti- } \\ \text { All outcomes }\end{array} \\ & \begin{array}{l}\text { nal analysis. Investigators were masked to treatment assignment until the fi- } \\ \text { locked in September } 2013 \text { and the trial arms were unmasked". However, it is } \\ \text { not clear whether participants were blinded. }\end{array}\end{array}$

\begin{tabular}{|c|c|c|}
\hline $\begin{array}{l}\text { Blinding of outcome as- } \\
\text { sessment (detection bias) } \\
\text { All outcomes }\end{array}$ & Low risk & $\begin{array}{l}\text { Judgement comment: "trained recruitment and retention staff engaged par- } \\
\text { ticipants, who were assessed with repeated measures at baseline, as well as } \\
\text { 3-month, } 6 \text {-month, and } 12 \text {-month post-treatment". Further, the trial reports } \\
\text { that investigators were masked to treatment assignment until the final 12- } \\
\text { month follow-up assessment. In the discussion section, it is reported that trial } \\
\text { strengths included blind assessment of outcomes. }\end{array}$ \\
\hline
\end{tabular}

Incomplete outcome data Low risk
(attrition bias)

All outcomes

\begin{abstract}
Judgement comment: attrition and exclusions adequately described. "Of randomised participants, 267 completed the 3-month follow-up assessment (i.e. $87 \%$ retention rate); 277 completed the 6 -month (91\%); and 278 completed the 12 - month assessment (91\%). The retention rates at each follow-up, which were $\geq 87 \%$ for all 3 follow-up assessments, did not significantly differ by condition".
\end{abstract}

\begin{tabular}{lll}
\hline $\begin{array}{l}\text { Selective reporting (re- } \\
\text { porting bias) }\end{array}$ & Unclear risk & $\begin{array}{l}\text { Judgement comment: all outcomes described in the methods section were ad- } \\
\text { equately collected, analysed and reported in the results section of the main } \\
\text { published trial. However, there was insufficient or unclear evidence from ei- } \\
\text { ther the trial protocol or trial registry sites to assess this domain. }\end{array}$ \\
\hline Other bias & Low risk & Judgement comment: none. \\
\hline
\end{tabular}

\section{Study characteristics}

Methods

Trial design: $\mathrm{RCT}$

Trial grouping: parallel group

Assessment modality (e.g. face-to-face, telephone, online): face-to-face

Conflict of interest of trial authors: NR

Funding for trial: this research was funded by National Injury Prevention Center, Centers for Disease Control Grant \#510499

Primary outcomes: IPV; BDI; The Anxiety and Parental Child rearing Styles Scale

Randomisation method: sequential assignment procedure

Secondary outcomes: NR

Timing of outcome measurements: data were collected at baseline, 10 weeks later (Time 1 and postintervention) and at 8-month follow-up (Time 2). However, all control group (CG) families were offered the opportunity to participate in the intervention at the end of the 10-week period and thus, were not interviewed a third time.

Total duration of trial: NR 
Graham-Bermann 2015 (Continued)

Type of analysis: hierarchical linear modelling

Types of participants: women who participated in the trial were recruited through flyers and newspaper advertisements, at social service agencies and through shelters for abused women in five urban locations in Michigan.

ITT analyses: NR

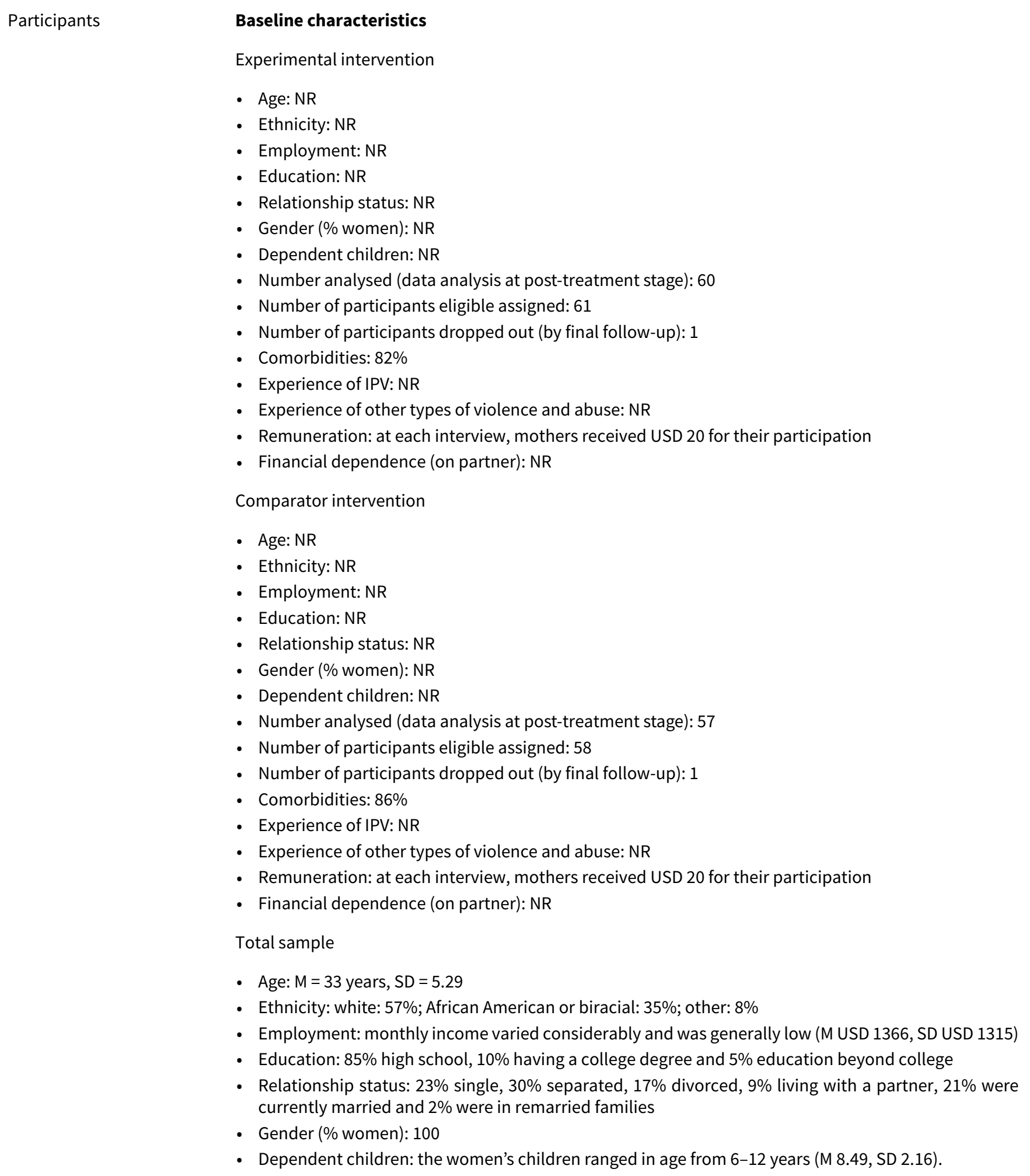


- Number analysed (data analysis at post-treatment stage): 173

- Number of participants eligible assigned: 181

- Number of participants dropped out (by final follow-up): 8

- Comorbidities: NR

- Experience of IPV: the women's self-reports on their history of IPV indicated that the average length of their violent relationship was 10 years (M 125.13 months, SD 71.73), with a mean of 1.70 violent partners (SD 1.23) in their lifetime. Control tactics $M=95.46, S D=79.01$; physical threats $M=45.72$, $S D=48.13$; sexual violence $M=37.38, S D=59.72$; physical violence: mild $M=18.89, S D=30.86$; severe $M=11.13, S D=19.08$

- Experience of other types of violence and abuse: NR

- Remuneration: at each interview, mothers received USD 20 for their participation

- Financial dependence (on partner): NR

Included criteria: trial criteria included women who had physical conflict in their relationship with an intimate partner during the past year and with children between the ages of 6 and 12 .

Excluded criteria: NR

Pretreatment: mother and child $(\mathrm{M}+\mathrm{C})$ and child only $(\mathrm{CO})$ did not differ from CG participants on ethnicity, income, maternal age or marital status, education, child age or gender, or exposure to IPV, and MC did not differ from CO participants on the same variables.

Number eligible: 218

Number ineligible: 3

Number of eligible people consented: 181

Number of eligible people recruited: 218

Recruitment setting of participants (e.g. healthcare setting, community setting, shelter setting): community

Sample power calculation: based on 0.8 power to detect a significant difference (calculated in G-Power, $P=0.05, d=0.25$, for repeated measures, within-between interaction), approximately 60 participants were required for each trial condition

Total number of participants approached: 221

Total number of participants within all groups: 181

Number declined to participate: 14

Interventions Intervention characteristics

Experimental intervention

- Brief description (rationale, main components): the Moms' Empowerment Program (MEP; Graham-Bermann, 1994/2011) was designed to address the needs of abused women using group therapy with an interpersonal relationship focus. Based in part on Sullivan's (1953) interpersonal theory, the MEP emphasises the whole person and explores strengths and abilities that can be used to compensate for biopsychosocial dysfunction. Rather than focus on psychopathology, interpersonal relationships are the nexus of the MEP treatment. Given the women's histories of violence and abuse, and for many, dysfunction in their family of origin, the group was designed to provide a venue for exploring relationship issues, including parent-child relationships, expectations derived from their family of origin, and social support.

- Type of intervention: MEP. A community-based therapeutic group intervention. Group therapy with an interpersonal relationship focus; safety planning and communication with children

- Mode of delivery (how): group face-to-face

- Frequency and duration of delivery (when and how much): 10-week programme

- Level of mental health training of person delivering the intervention: MEP group therapists are community service providers, such as therapists at local mental health clinics, or graduate students in 
clinical psychology and social work at the University of Michigan, as was the case for the present trial. Therapists receive intensive training in clinical work with women exposed to IPV, including identifying and treating symptoms of traumatic stress, as well as ethical issues in working with at-risk populations. All therapists follow a training manual that describes session topics, the research evidence for relevant issues, and example process notes. In the present trial therapists received weekly supervision by the first author where process notes were reviewed and treatment adherence discussed and evaluated.

- Fidelity assessment: group therapists received intensive training in group therapy, ethics, and clinical work with at-risk populations. They are supervised weekly by a licensed clinical psychologist, where process notes, session plans and adherence to the training manual are reviewed and evaluated

- Intervention model: interpersonal theory (mother-child)

- Person delivering the intervention (who provided the intervention): therapist, self-directed or combined: therapist

- Intervention location (where): each community provided a setting for the MEP, such as rooms in an existing mental health clinic, a shelter outreach centre, or an education setting

- Tailoring of intervention (personalised, titrated or adapted): NR

- Intervention modifications (during the trial): NR

- Main techniques of intervention: each MEP intervention group consisted of 6-8 women and 2 trained co-leaders, or therapists, who provided support and feedback, as well as educational materials.

- Intervention manual: Both programmes were manualised

- Intervention attendance: attendance ranged from 5-10 sessions (M 7.35, SD1.50)

- CCDAN psychological therapies: integrative therapies

- Intensity of intervention: $\geq 5$ sessions

- Person delivering the intervention: healthcare workers

- Gender of therapist: NR

Comparator intervention

- Brief description (rationale, main components): no treatment

- Type of intervention: no treatment

\section{Outcomes}

\section{Depression}

- Outcome type: continuous outcome

- Reporting: fully reported

- Scale: BDI

- Direction: lower is better

\section{Dropouts from treatment}

- Outcome type: dichotomous outcome

- Reporting: fully reported

- Direction: lower is better

Identification

Sponsorship source: this research was funded by National Injury Prevention Center, Centers for Disease Control Grant \#510499

Country: USA

Setting: community

Comments: women who participated in the trial were recruited through flyers and newspaper advertisements, at social service agencies and through shelters for abused women in 5 urban locations in Michigan

Trialauthors: Sandra A. Graham-Bermann, Laura Miller-Graff

Institution: Department of Psychology, University of Michigan 
Email: andragb@umich.edu

Address: Department of Psychology, University of Michigan, 530 Church Street, Ann Arbor, MI 48109-1043.

Title (main 'outcome' publication): Community-based intervention for women exposed to intimate partner violence: a randomized control trial

Registered trial protocol ID: NR

Analysis of any cost/benefit measures: NR
Process evaluation: NR
Summary of participants' views about the intervention: NR
Adverse events (harm) related to participation: NR

Risk of bias

\begin{tabular}{lll}
\hline Bias & Authors' judgement & Support for judgement \\
\hline $\begin{array}{l}\text { Random sequence genera- } \\
\text { tion (selection bias) }\end{array}$ & Unclear risk & $\begin{array}{l}\text { Judgement comment: "following consent, a sequential randomised control } \\
\text { assignment procedure allocated participants to } 3 \text { conditions: mother-plus- } \\
\text { child received intervention (M C), child-only received intervention (CO), and a } \\
\text { waiting list comparison group (CG)". However, no further information provided } \\
\text { about the nature of randomisation methods and/or sequence generation. }\end{array}$ \\
& &
\end{tabular}

Allocation concealment High risk (selection bias)
Judgement comment: "a sequential assignment procedure was used to assign women and children to the 3 conditions. That is, the first 7 women and children were assigned to the MC condition by the project co-ordinator who did not provide either intervention or evaluation. The next 7 children were assigned to the $\mathrm{CO}$ condition, and the following 7 were assigned to the comparison group (CG) condition where the women and children were put on a waiting list for treatment but did not participate in either intervention".

\begin{tabular}{|c|c|c|}
\hline $\begin{array}{l}\text { Blinding of participants } \\
\text { and personnel (perfor- } \\
\text { mance bias) } \\
\text { All outcomes }\end{array}$ & Unclear risk & $\begin{array}{l}\text { Judgement comment: "women gave their informed consent and were inter- } \\
\text { viewed by researchers blind to group assignment". However, it is unclear if } \\
\text { participants were blinded to their group assignment. }\end{array}$ \\
\hline $\begin{array}{l}\text { Blinding of outcome as- } \\
\text { sessment (detection bias) } \\
\text { All outcomes }\end{array}$ & Low risk & $\begin{array}{l}\text { Judgement comment: "women gave their informed consent and were inter- } \\
\text { viewed by researchers blind to group assignment". }\end{array}$ \\
\hline $\begin{array}{l}\text { Incomplete outcome data } \\
\text { (attrition bias) } \\
\text { All outcomes }\end{array}$ & Low risk & $\begin{array}{l}\text { Judgement comment: attrition and exclusions were adequately described. } \\
\text { "40 children and mothers (18\%) who were interviewed at baseline did not con- } \\
\text { tinue participation in the trial. Of these, } 2 \text { were dropped for unrepresentative } \\
\text { high income, } 3 \text { moved out of state, } 12 \text { lost housing and contact with the tri- } \\
\text { al, } 3 \text { lost child custody, } 14 \text { declined the second interview once contacted, } 1 \\
\text { child developed cancer, and } 1 \text { was injured in a fire. } 4 \text { children moved to foster } \\
\text { care and were not permitted to continue in the trial. Of the } 123 \text { children and } \\
\text { their mothers in the intervention programme, } 7(5.7 \%) \text { were not interviewed } \\
\text { a third time ( } 1 \text { from each intervention condition stayed in the programme but } \\
\text { refused the interviews, and } 5 \text { dropped out after the intervention- } 4 \text { of whom } \\
\text { were from the co group)". }\end{array}$ \\
\hline
\end{tabular}

\begin{tabular}{|c|c|c|}
\hline $\begin{array}{l}\text { Selective reporting (re- } \\
\text { porting bias) }\end{array}$ & Unclear risk & $\begin{array}{l}\text { Judgement comment: all outcomes described in the methods section were ad- } \\
\text { equately collected, analysed and reported in the results section of the main }\end{array}$ \\
\hline
\end{tabular}




Other bias Low risk Judgement comment: none.

Hegarty 2013

Study characteristics

Methods

Participants
Trial design: cluster-RCT

Trial grouping: parallel group

Assessment modality (e.g. face-to-face, telephone, online): data collected from women by postal questionnaire at the screening stage and at 3 further points over the duration of the project.

Conflict of interest of trial authors: We declare that we have no conflicts of interest.

Funding for trial: Australian National Health and Medical Research Council

Primary outcomes: quality of life, mental health status, safety planning, safety behaviours

Randomisation method: a statistician who was otherwise not involved in the trial follow-up generated a random allocation sequence in Stata,24 stratified by location of each doctor's practice (urban vs rural), with random permuted block sizes of 2 and 4 within each stratum (appendix).

Secondary outcomes: HADS depression and anxiety score; enquiry from doctor about woman's safety; enquiry from doctor about child's safety; comfort to discuss fear

Timing of outcome measurements: baseline, and at 6 and 12 months following the invitation to the intervention

Total duration of trial: doctors were recruited 31 January 2008-18 January 2010; doctors and their female patients were randomised between 22 September 2008-18 June 2010. 6-month data collection occurred from 26 August 2009-24 June 2011, and 12-month data collection from 18 March 2010-24 November 2011.

Type of analysis: descriptive statistics, linear mixed-effects model, marginal logistic regression, multivariable regression analysis, multiple imputation, ITT and complete case analysis

Types of participants: women (16-50 years) seen by their GPs in participating family clinics

ITT analyses: yes

\section{Baseline characteristics}

Experimental intervention

- Age: $M=37 \cdot 9$ years, $S D=8 \cdot 8$

- Ethnicity: NR

- Employment: unemployed 32 (27\%)

- Education: year 12 schooling not completed $51(38 \%)$

- Relationship status: married 33 (25\%); separated or divorced 51 (38\%); never married 50 (37\%); lives with a partner $66(48 \%)$

- Gender (\% women): 100

- Dependent children: 73 (53\%)

- Number analysed (data analysis at post-treatment stage): 96

- Number of participants eligible assigned: 137

- Number of participants dropped out (at final follow-up): 41 
- Comorbidities: NR

- Experience of IPV: positive for abuse on CAS (total score $\geq 7$ ) 101 (75\%)

- Experience of other types of violence and abuse: NR

- Remuneration: NR for women participants

- Financial dependence (on partner): NR

Comparator intervention

- Age: $M=39 \cdot 1$ years, $S D=7 \cdot 3$

- Ethnicity: NR

- Employment: unemployed 41 (33\%)

- Education: year 12 schooling not completed $63(47 \%)$

- Relationship status: married 50 (37\%); separated or divorced 48 (36\%); never married 36 (27\%); lives with a partner $78(58 \%)$

- Gender (\% women): 100

- Dependent children: 86 (64\%)

- Number analysed (data analysis at post-treatment stage): 100

- Number of participants eligible assigned: 135

- Number of participants dropped out (at final follow-up): 35

- Comorbidities: NR

- Experience of IPV: positive for abuse on CAS (total score $\geq 7) 93(71 \%)$

- Experience of other types of violence and abuse: NR

- Remuneration: NR for women participants

- Financial dependence (on partner): NR

Total sample

- Age: $M=38 \cdot 5$ years, $S D=8 \cdot 1$

- Ethnicity: English not first language $15(6 \%)$

- Employment: unemployed 73 (30\%)

- Education: year 12 schooling not completed 114 (42\%)

- Relationship status: married 83 (31\%); separated or divorced 99 (37\%); never married 86 (31\%); lives with a partner $144(53 \%)$

- Gender (\% women): 100

- Dependent children: 159 (59\%)

- Number analysed (data analysis at post-treatment stage): 196

- Number of participants eligible assigned: 272

- Number of participants dropped out (at final follow-up): 76

- Comorbidities: NR

- Experience of IPV: positive for abuse on CAS (total score $\geq 7$ ) 194 (73\%)

- Experience of other types of violence and abuse:

- Remuneration: practices are reimbursed at a rate of USD 500 for time involved in generating patient lists

- Financial dependence (on partner): NR

Included criteria: doctors were eligible for inclusion if they worked $\geq 3$ sessions/week, used electronic records, and if $70 \%$ or more of their patients spoke English. For every doctor recruited, women (aged 16-50 years) who had visited the doctor in the past 12 months were mailed a brief survey from the practice (done by researchers). Women who screened positive for fear of their partner and provided contact details were eligible for the trial and were invited to participate by telephone by researchers.

Excluded criteria: if women patients had misinterpreted the fear item, had experienced fear but not in the past 12 months, had insufficient English language skills, or were no longer seeing the trial doctor. We excluded otherwise eligible doctors if no women were enrolled from the practice. We did not send a health and lifestyle survey to women for whom we had no address or if their doctor anticipated difficulties in responding because of cognitive impairment or poor English-language skills. 
Pretreatment: baseline characteristics of doctors and women were much the same between the intervention and control groups, as were the response rates to the 6-month and 12-month follow-up surveys. Scores for both primary and secondary outcomes were also much the same between women in the 2 groups. Baseline characteristics of women retained and those lost to follow-up at 12 months were similar between trial groups.

Number eligible: 731

Number ineligible: 5102

Number of eligible people consented: 272

Number of eligible people recruited: 386

Recruitment setting of participants (e.g. healthcare setting, community setting, shelter setting): healthcare setting (family doctors' clinics)

Sample power calculation: our calculated sample size was 136 women from 34 practices (4 women/ practitioner; appendix). This calculation was based on a 2-sample t test, allowing for a design effect of 1.08 due to clustering (ICC of 0.02 ) and variable cluster size.

Total number of participants approached: 20,100 women (attending 55 doctors) sent health and lifestyle survey

Total number of participants within all groups: 272

Number declined to participate: 39

\section{Intervention characteristics}

Experimental intervention

- Brief description (rationale, main components): the trial intervention consisted of the following: training of doctors, notification to doctors of women screening positive for fear of a partner, and invitation to women for brief counselling for relationship and emotional issues (appendix). The counselling intervention was based on the Psychosocial Readiness Model.

- Type of intervention: brief counselling intervention

- Mode of delivery (how): face-to-face individual counselling sessions

- Frequency and duration of delivery (when and how much): women attending the practices of doctors in the intervention group who were fearful of a partner were sent a letter from the doctor to invite them to attend between one and 6 counselling sessions (depending on women's needs) over a 6-month period at no cost to the patient.

- Level of mental health training of person delivering the intervention: doctors in the intervention group received the Healthy Relationships Training programme, designed to train them to respond to women and deliver a brief counselling intervention (appendix). Training consisted of a 6-h distance learning package and 21 -h interactive practice visits delivered by an academic clinician using simulated patient role plays. Training emphasised the importance of patient-centred care and promoted active listening, MI, and problem-solving techniques for validating women's experiences and feelings, assessing readiness for change, and supporting decisions.

- Fidelity assessment: NR

- Intervention model: brief counselling for relationship and emotional issues based on the Psychosocial Readiness Model

- Person delivering the intervention (who provided the intervention): therapist, self-directed or combined: family doctor (GP)

- Intervention location (where): family doctor clinic

- Tailoring of intervention (personalised, titrated or adapted): women attending the practices of doctors in the intervention group who were fearful of a partner were sent a letter from the doctor to invite them to attend between one and 6 counselling sessions (depending on women's needs) over a 6 month period at no cost to the patient.

- Intervention modifications (during the trial): NR 
- Main techniques of intervention: patient-centred care and active listening, MI, and problem-solving techniques for validating women's experiences and feelings, assessing readiness for change, and supporting decisions.

- Intervention manual: doctors in the intervention group received the Healthy Relationships Training programme, designed to train them to respond to women and deliver a brief counselling intervention (appendix)

- Intervention attendance: of the 137 women invited for counselling, 67 women (49\%) attended 160 appointments (median of 1 visit, range 1-6). 29 women (21\%)had not attended an appointment at 6 months despite 3 reminder calls.

- CCDAN psychological therapies: integrative therapies

- Intensity of intervention: $\geq 5$ sessions

- Person delivering the intervention: healthcare workers

- Gender of therapist: $62 \%$ female

Comparator intervention

- Brief description (rationale, main components): usual care

- Type of intervention: women in the control group received usual care if they presented to their doctor with concerns during the trial period

- Outcome type: dichotomous outcome

- Reporting: fully reported

- Scale: HADS

- Direction: lower is better

\section{Anxiety}

- Outcome type: dichotomous outcome

- Reporting: fully reported

- Scale: HADS

- Direction: lower is better

\section{Mental health}

- Outcome type: continuous outcome

- Reporting: fully reported

- Scale: Mental Health Status SF-12

- Direction: higher is better

Safety planning and/or safety behaviour

- Outcome type: dichotomous outcome

- Reporting: fully reported

- Scale: Safety Promoting Behaviour Checklist

- Direction: higher is better

\section{Quality of life}

- Outcome type: continuous outcome

- Reporting: fully reported

- Scale: WHO Quality of Life-BREF

- Direction: higher is better

\section{Dropouts from treatment}

- Outcome type: dichotomous outcome

- Reporting: fully reported 
Comments: approximately 500 women (16-50 years) seen by the GP in the previous year are mailed a short lifestyle survey containing an item to screen for IPV

Trialauthors: Kelsey Hegarty, Lorna O’Doherty, Angela Taft, Patty Chondros, Stephanie Brown, Jodie Valpied, Jill Astbury, Ann Taket, Lisa Gold, Gene Feder, Jane Gunn

Institution: General Practice and Primary Health Care Academic Centre, The University of Melbourne, VIC, Australia

Email: k.hegarty@unimelb.edu.au

Address: General Practice and Primary Health Care Academic Centre, The University of Melbourne, 200 Berkeley Street, Carlton, VIC 3053, Australia

Trial title (main outcome publication): Screening and counselling in the primary care setting for women who have experienced intimate partner violence (WEAVE): a cluster randomised controlled trial

Registered trial protocol ID: ACTRN12608000032358

Process evaluation: the published protocol has a detailed section about process evaluation. However, the outcome trial does not report any process evaluation.

Summary of participants' views about the intervention: most women agreed that they were glad they participated, and for half of them the quality of their life was somewhat better or better. Several women described negative and positive partner behaviours when their partner became aware they were in the trial, but we detected no between-group difference. No adverse events were reported and we detected no evidence of a difference in harm or abuse between groups. The harm reported was at a similar level (4\%) to the WHO multi-country trial with few women's partners being aware that they were involved in the trial.

Adverse events (harm) related to participation: no adverse events were reported and we detected no evidence of a difference in harm or abuse between groups.

\section{Risk of bias}

\begin{tabular}{lll}
\hline Bias & Authors' judgement & Support for judgement \\
\hline $\begin{array}{l}\text { Random sequence genera- } \\
\text { tion (selection bias) }\end{array}$ & Low risk & $\begin{array}{l}\text { Judgement comment: "a statistician who was otherwise not involved in the } \\
\text { trial follow-up generated a random allocation sequence in Stata, stratified } \\
\text { by location of each doctor's practice (urban vs rural), with random permuted } \\
\text { block sizes of } 2 \text { and 4 within each stratum". }\end{array}$ \\
\hline $\begin{array}{l}\text { Allocation concealment } \\
\text { (selection bias) }\end{array}$ & Low risk & $\begin{array}{l}\text { Judgement comment: "because of the nature of the intervention, neither } \\
\text { doctors nor patients could be masked to intervention, but trial investigators } \\
\text { and researchers following-up patients and entering and analysing data were } \\
\text { masked to allocation. A statistician who was otherwise not involved in the trial } \\
\text { follow-up generated a random allocation sequence in Stata (version 24) strat- } \\
\text { ified by location of each doctor's practice (urban vs rural), with random per- } \\
\text { muted block sizes of } 2 \text { and } 4 \text { within each stratum". }\end{array}$ \\
\hline
\end{tabular}


Hegarty 2013 (Continued)

Blinding of participants High risk Judgement comment: WEAVE is a pragmatic intervention trial. Due to the naand personnel (perforture of the intervention (professional training plus patient counselling) it is not mance bias)

All outcomes possible to blind the GPs to their status as intervention or control. Similarly, the immediate project team is not blind to GP participant status as much interaction between the team and the GPs must occur as part of the training and organising for women to attend their counselling appointments. In the same vein, women are not blinded in that they need to be aware that they may (intervention group) or may not (comparison group) be invited by the GP to discuss relationship issues as part of WEAVE.

\section{Blinding of outcome as- Low risk} sessment (detection bias)

Judgement comment: there is no blinding as regards to data collection based All outcomes on the CONSORT guidelines, as the women and GPs themselves complete the surveys (i.e. data were not collected by a research assistant blinded to the allocation). However the wider investigator team (and the statistician) remain blinded to the identity and allocation of GP participants and women.

\begin{tabular}{lll}
\hline $\begin{array}{l}\text { Incomplete outcome data } \\
\text { (attrition bias) } \\
\text { All outcomes }\end{array}$ & Low risk & $\begin{array}{l}\text { Judgement comment: attrition and exclusions adequately reported, with doc- } \\
\text { umentation about reasons for dropouts }\end{array}$ \\
\hline $\begin{array}{l}\text { Selective reporting (re- } \\
\text { porting bias) }\end{array}$ & Low risk & $\begin{array}{l}\text { Judgement comment: primary outcomes were quality of life (WHO Quali- } \\
\text { ty of Life-BREF), safety planning and behaviour, mental health (SF-12) at } 12 \\
\text { months. Secondary outcomes included depression and anxiety (HADS Scale; } \\
\text { cut-off } \geq 8 \text { 8). These were fully reported, consistent with published trial protocol. }\end{array}$ \\
\hline Other bias & Low risk & $\begin{array}{l}\text { Judgement comment: the trial was conducted according to the published pro- } \\
\text { tocol, with various strategies to minimise bias. }\end{array}$
\end{tabular}

\section{Study characteristics}

Methods

Trial design: $\mathrm{RCT}$

Trial grouping: parallel group

Conflict of interest of trial authors: none declared

Funding for trial: Australian Research Council (grant no DP130102799)

Primary outcomes: self-efficacy and depression

Randomisation method: once women were enrolled in the trial, they were randomly assigned by computer to the intervention or control group. An automated, computerised algorithm for simple 1:1 randomisation was used, with no stratification.

Secondary outcomes: fear of partner, number of helpful behaviours for safety and well being, and cost-effectiveness

Timing of outcome measurements: baseline, immediately after completion of the website, at 6 months, and 12 months

Total duration of trial: 1 year

Types of participants: women who had screened positive for any form of intimate partner violence or fear of a partner in the 6 months before recruitment.

ITT analyses: multiple imputation were used to estimate the values of missing outcome data 
Hegarty 2019 (Continued)

Participants

\section{Baseline characteristics}

Experimental intervention

- Age: $M=34.6$ years, $S D=8.1$

- Ethnicity: NR

- Employment: 45 (21\%) unemployed

- Education: NR

- Relationship status: currently in a relationship with perpetrator of violence (100, 44\%)

- Gender (\% women): 100

- Dependent children: 107 (48\%)

- Number analysed (data analysis at post-treatment stage): 178

- Number of participants eligible assigned: 227

- Number of participants dropped out (by final follow-up): 48

- Comorbidities: NR

- Experience of IPV: all participating women had screened positive for any form of IPV or fear of a partner in the 6 months before recruitment

- Experience of other types of violence and abuse: NR

- Remuneration: participants gave informed consent for participation via an online form and were compensated for their time up to AUS 150 depending on how many surveys they answered.

- Financial dependence (on partner): NR (although $84,41 \%$ received government income support)

Comparator intervention

- Age: $\mathrm{M}=32.8$ years, $\mathrm{SD}=8.8$

- Ethnicity: NR

- Employment: 37 (21\%) unemployed

- Education: NR

- Relationship status: currently in a relationship with perpetrator of violence $(93,48 \%)$

- Gender (\% women): 100

- Dependent children: $80(42 \%)$

- Number analysed (data analysis at post-treatment stage): 162

- Number of participants eligible assigned: 195

- Number of participants dropped out (by final follow-up): 39

- Comorbidities: NR

- Experience of IPV: all participating women had screened positive for any form of IPV or fear of a partner in the 6 months before recruitment

- Experience of other types of violence and abuse: NR

- Remuneration: participants gave informed consent for participation via an online form and were compensated for their time up to AUS 150 depending on how many surveys they answered.

- Financial dependence (on partner): NR (although $75,44 \%$ received government income support)

Total sample

- Age: $\mathrm{M}=33.7$ years, $\mathrm{SD}=8.48$

- Ethnicity: NR

- Employment: 82 (21\%) unemployed

- Education: NR

- Relationship status: currently in a relationship with perpetrator of violence $(193,46 \%)$

- Gender (\% women): 100

- Dependent children: 187 (45\%)

- Number analysed (data analysis at post-treatment stage): 340

- Number of participants eligible assigned: 422

- Number of participants dropped out (by final follow-up): 87 
- Comorbidities: NR

- Experience of IPV: all participating women had screened positive for any form of IPV or fear of a partner in the 6 months before recruitment

- Experience of other types of violence and abuse: NR

- Remuneration: participants gave informed consent for participation via an online form and were compensated for their time up to AUS 150 depending on how many surveys they answered.

- Financial dependence (on partner): NR (although 159, 42\% received government income support)

Included criteria: women aged 16-50 years currently residing in Australia, who had safe access to a computer and an internet connection, and who answered positively to one of the screening questions in English were eligible for inclusion. The screening questions asked whether in the last 6 months a woman's partner or ex-partner had made her feel afraid or unsafe; followed her or harassed her over the telephone or online; called her names, humiliated, bullied, or criticised her, or threatened her in any way; isolated her from her family and friends or restricted her behaviour in any way; physically harmed her in any way; or forced her to do sexual things she did not want to do.

Excluded criteria: women were excluded if in a follow-up phone call they identified that they had not been in an unhealthy or abusive relationship or experienced fear of partner in the past 6 months

Pretreatment: baseline characteristics of participants were similar between the intervention and control groups

Number of eligible people consented: 422

Number of eligible people recruited: 584

Sample power calculation: yes

Number ineligible: 162

Number eligible: 422

Recruitment setting of participants (e.g. healthcare setting, community setting, shelter setting): community (online intervention)

Total number of participants approached: 584

Total number of participants within all groups: 422

Number declined to participate: NR

Experimental intervention

- Brief description (rationale, main components): the Psychosocial Readiness Model underpinning the I-DECIDE intervention, describes the interplay of factors that may motivate a woman experiencing domestic violence to engage in positive action for safety and healing. The intervention website consisted of modules on healthy relationships, abuse and safety, and relationship priority setting, and a tailored action plan.

- Type of intervention: healthy relationship tool and safety decision aid

- Mode of delivery (how): online (website)

- Frequency and duration of delivery (when and how much): completion of the baseline trial measures is estimated to take up to $20 \mathrm{~min}$. For women who then proceeded to the intervention, the overall time commitment could be up to $60 \mathrm{~min}$, and for the comparison group up to $40 \mathrm{~min}$. Women were not required to complete their visit all in one sitting, but could log out and back in at another time. It was expected that 6 and 12 month visits would take approximately $15 \mathrm{~min}$.

- Level of mental health training of person delivering the intervention: N/A

- Fidelity assessment: N/A (delivered via website)

- Intervention model: healthy relationship tool with a focus on MI

- Person delivering the intervention (who provided the intervention): therapist, self-directed or combined: self-directed through website 
- Intervention location (where): online

- Tailoring of intervention (personalised, titrated or adapted): the intervention provided tailored feedback and messaging to women at various stages so that they felt listened to, rather than being 'just another user'.

- Intervention modification (during the trial): NR

- Main techniques of intervention: the I-DECIDE website commences with 3 modules: healthy relationships, safety, and priorities. The Psychosocial Readiness Model underpin the I-DECIDE intervention and includes MI.

- Intervention manual: NR

- Intervention attendance: this was a web-based intervention. Completion rate for the intervention was $79 \%$.

- CCDAN psychological therapies: integrative therapies

- Intensity of intervention: up to 4 sessions

- Person delivering the intervention: web-based

- Gender of therapist: N/A (web-based)

Comparator intervention

- Brief description (rationale, main components): women in the control group received a static website (5-min duration) developed for this project, which contained brief information about domestic violence (appendix p 2) and a standard emergency safety plan as per standard practice in the IPV sector in Australia. The emergency safety plan was in the same format as that delivered to women in the intervention group who scored highly on the Danger Assessment or the CAS.

- Type of intervention: website

- Outcome type: continuous outcome

- Reporting: fully reported

- Scale: Generalized Self-Efficacy Scale

- Direction: higher is better

\section{Depression}

- Outcome type: continuous outcome

- Reporting: fully reported

- Scale: the Center for Epidemiologic Studies Depression Scale, Revised

- Direction: lower is better

\section{Fear of partner or ex-partner}

- Outcome type: continuous outcome

- Reporting: fully reported

- Scale: Visual Analogue scale 0-10

- Direction: lower is better

Number of helpful actions taken

- Outcome type: continuous outcome

- Reporting: fully reported

- Scale: purposefully developed questions

- Direction: higher is better

\section{Dropouts from treatment}

- Outcome type: dichotomous outcome

- Reporting: fully reported

- Direction: lower is better 
Hegarty 2019 (Continued)

Identification
Sponsorship source: the I-DECIDE project was funded by a Discovery Project grant from the Australian Research Council (grant no DP130102799).

Country: Australia

Setting: community

Comments: women who had screened positive for any form of IPV or fear of a partner in the 6 months before recruitment

Trialauthors: Kelsey Hegarty, Laura Tarzia, Jodie Valpied, Elizabeth Murray, Cathy Humphreys, Angela Taft, Kitty Novy, Lisa Gold, Nancy Glass

Institution: the University of Melbourne

Email: k.hegarty@unimelb.edu.au

Address: Department of General Practice, The University of Melbourne, Melbourne 3010, VIC, Australia

Trial title (main outcome publication): An online healthy relationship tool and safety decision aid for women experiencing intimate partner violence (I-DECIDE): a randomised controlled trial

Registered trial protocol ID: ACTRN12614001306606
Analysis of any cost/benefit measures: "Detailed cost-effectiveness data are not presented in this Article". Other publications related to this trial had not reported analysis of any cost/benefit measures.

Process evaluation: 32 women agreed to take part in a process evaluation interview $(13(41 \%)$ from the intervention group and 19 (59\%) from the control group). Women's recollections were hampered by a delay of over 14 months between viewing the original website and being interviewed. This was a particular issue for women in the control group, who had received elements of the intervention (CAS and Danger Assessment) during 12-month follow-up data collection. This affected their memory of what the original comparator website contained, despite receiving reminder screen shots of the content before being interviewed. We recorded key themes with example quotes from participants in the intervention and control arms (appendix pp 7-8). Findings indicated that both websites affected awareness, self-efficacy, getting thoughts straight, pushing for action, having a plan, and perceptions of support. The main differences between the intervention and control groups were around how well the websites linked women with face-to- face support and the amount of tailoring offered to participants. Women in the intervention group also articulated a stronger and more specific sense of the website forcing them to confront the reality of the abuse in their relationship, whereas women in the control group merely talked about becoming more informed about different types of IPV.

Summary of participants' views about the intervention: most women in both groups in the trial agreed that they were glad they participated, and around two-thirds agreed that the quality of their life was somewhat better or better (table 3 )

Adverse events (harm) related to participation: improvement on all outcomes over time, with no clinically meaningful differences between intervention and control, suggests that the intervention website was not harmful compared with a standard website.

\section{Risk of bias}

\begin{tabular}{lll}
\hline Bias & Authors' judgement & Support for judgement \\
\hline $\begin{array}{l}\text { Random sequence genera- } \\
\text { tion (selection bias) }\end{array}$ & Low risk & $\begin{array}{l}\text { Judgement comment: "participants were computer-randomised automati- } \\
\text { cally using simple randomisation, with no stratification, as the sample size is } \\
\text { large enough that the groups should be balanced in terms of participant num- } \\
\text { bers and demographics. Participants were randomly assigned (1:1) by comput- } \\
\text { er to receive either the intervention or control website". }\end{array}$ \\
& & \\
\hline
\end{tabular}


Hegarty 2019 (Continued)

Allocation concealment Low risk Judgement comment: "all I-DECIDE investigators and members of the re(selection bias) search team were blinded to the allocation of participants, until after collection and analysis of the 12-month data".

\begin{tabular}{|c|c|c|}
\hline $\begin{array}{l}\text { Blinding of participants } \\
\text { and personnel (perfor- } \\
\text { mance bias) } \\
\text { All outcomes }\end{array}$ & Low risk & $\begin{array}{l}\text { Judgement comment: "as the initial portion of the website containing the } \\
\text { baseline questions was identical for both groups, there was no way for women } \\
\text { to tell which group they had been allocated to, and the research team were al- } \\
\text { so masked to participant allocation until after analysis of the 12-month data". }\end{array}$ \\
\hline
\end{tabular}

Blinding of outcome as- Low risk sessment (detection bias) All outcomes
Judgement comment: "all data were collected online. Follow-up by the research assistant by phone for those who failed to log on and complete baseline, 6-month or 12-month measures is by an administrative assistant not connected with any of the data management or analyses".
Incomplete outcome data Low risk (attrition bias)

All outcomes

\section{Judgement comment: attrition and exclusions adequately reported, with doc-} umentation about reasons for dropouts.

\begin{tabular}{|c|c|c|}
\hline $\begin{array}{l}\text { Selective reporting (re- } \\
\text { porting bias) }\end{array}$ & Unclear risk & $\begin{array}{l}\text { Judgement comment: the protocol include measures not reported in the main } \\
\text { outcome publication. These included "women's use of services, cost effective- } \\
\text { ness using the COST questionnaire." }\end{array}$ \\
\hline
\end{tabular}

Other bias Low risk Judgement comment: none

Hernandez-Ruiz 2005

\section{Study characteristics}

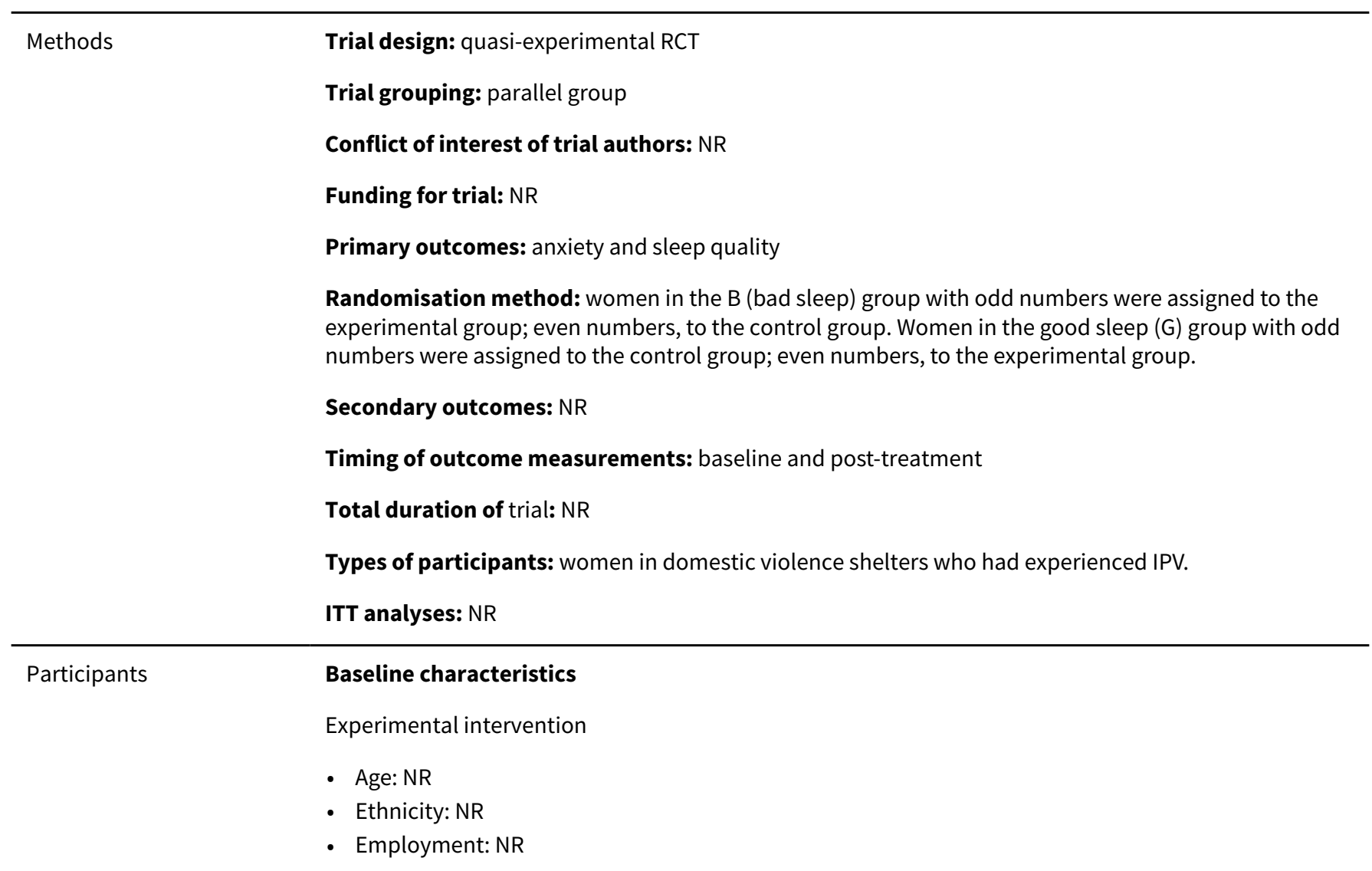




\section{- Education: NR}

- Relationship status: NR

- Gender (\% women): 100

- Dependent children: NR

- Number analysed (data analysis at post-treatment stage): 14

- Number of participants eligible assigned: 14

- Number of participants dropped out (by final follow-up): NR

- Comorbidities: NR

- Experience of IPV: NR

- Experience of other types of violence and abuse: NR

- Remuneration: the researcher provided a copy of the CD with her preferred music and progressive muscle relaxation procedure to every participant, in both the experimental and control groups, as a compensation for her collaboration.

- Financial dependence (on partner): NR

Comparator intervention

- Age: NR

- Ethnicity: NR

- Employment: NR

- Education: NR

- Relationship status: NR

- Gender (\% women): 100

- Dependent children: NR

- Number analysed (data analysis at post-treatment stage): 14

- Number of participants eligible assigned: 14

- Number of participants dropped out (by final follow-up): NR

- Comorbidities: NR

- Experience of IPV: NR

- Experience of other types of violence and abuse: NR

- Remuneration: the researcher provided a copy of the CD with her preferred music and progressive muscle relaxation procedure to every participant, in both the experimental and control groups, as a compensation for her collaboration.

- Financial dependence (on partner): NR

Total sample

- Age: $M=35.36$ years

- Ethnicity: NR

- Employment: NR

- Education: NR

- Relationship status: NR

- Gender (\% women): 100

- Dependent children: average of 2 children (range = 0-5),

- Number analysed (data analysis at post-treatment stage): 28

- Number of participants eligible assigned: 28

- Number of participants dropped out (by final follow-up): NR

- Comorbidities: NR

- Experience of IPV: from the total sample of 28 women, 26 participants reported verbal abuse and 23 also reported physical abuse. The women had been an average of 7.94 years in the last abusive relationship, and the abuse had lasted an average of 4.23 years (range from 1 day to 34 years).

- Experience of other types of violence and abuse: NR

- Remuneration: the researcher provided a copy of the CD with her preferred music and progressive muscle relaxation procedure to every participant, in both the experimental and control groups, as a compensation for her collaboration. 
Included criteria: the women were referred to the trial by the shelter staff if they had been in the shelter for at least 2 days and no more than 1 week

Excluded criteria: NR

Pretreatment: NR

Number of eligible people consented: NR

Number of eligible people recruited: NR

Sample power calculation: NR

Number ineligible: NR

Number eligible: NR

Recruitment setting of participants (e.g. healthcare setting, community setting, shelter setting): Shelter

Total number of participants approached: NR

Total number of participants within all groups: 28

Number declined to participate: NR

Interventions Intervention characteristics

Experimental intervention

- Brief description (rationale, main components): participant-selected music paired with progressive muscle relaxation for $20 \mathrm{~min}$.

- Type of intervention: music therapy and muscle relaxation

- Mode of delivery (how): face-to-face

- Frequency and duration of delivery (when and how much): each participant had contact with the researcher for 5 consecutive days for approximately 30 min each day, in individual sessions.

- Level of mental health training of person delivering the intervention: NR

- Fidelity assessment: NR

- Intervention model: music therapy (participant-selected music)

- Person delivering the intervention (who provided the intervention): therapist, self-directed or combined: therapist (researcher)

- Intervention location (where): NR

- Tailoring of intervention (personalised, titrated or adapted): NR

- Intervention modification (during the trial): NR

- Main techniques of intervention: participant-selected music paired with progressive muscle relaxation

- Intervention manual: NR

- Intervention attendance: NR

- CCDAN psychological therapies: other psychologically-orientated interventions

- Intensity of intervention: $\geq 5$ sessions

- Person delivering the intervention: non-healthcare workers

- Gender of therapist: female

Comparator intervention

- Brief description (rationale, main components): the control group were instructed to "lie down quietly for $20 \mathrm{~min}$," but they did not listen to the music/progressive muscle relaxation intervention.

- Type of intervention: quiet time 
Hernandez-Ruiz 2005 (Continued)

Anxiety
- Outcomes
- Reporting: fully reported
- Scale: State Trait Anxiety Inventory
- Direction: lower is better
Dropouts from treatment
- Outcome type: dichotomous outcome
- Reporting: fully reported
- Direction: lower is better

Identification

Sponsorship source: NR

Country: USA

Setting: domestic violence shelters

Comments: abused women in shelters

Trialauthors: Eugenia Hernandez-Ruiz

Institution: University of Kansas

Email: NR

Address: University of Kansas

Trial title (main outcome publication): Effect of music therapy on the anxiety levels and sleep patterns of abused women in shelters

Registered trial protocol ID: NR

Process evaluation: NR

Summary of participants' views about the intervention: NR

Adverse events (harm) related to participation: NR

\section{Risk of bias}

\begin{tabular}{lll}
\hline Bias & Authors' judgement & Support for judgement \\
\hline $\begin{array}{l}\text { Random sequence genera- } \\
\text { tion (selection bias) }\end{array}$ & High risk & $\begin{array}{l}\text { Judgement comment: "a pre-test-post-test design with control and experi- } \\
\text { mental groups was used. The women were referred to the trial by the shel- } \\
\text { ter staff if they had been in the shelter for at least } 2 \text { days and no more than } 1 \\
\text { week". There is no evidence of sequence generation. }\end{array}$ \\
\hline $\begin{array}{ll}\text { Allocation concealment } \\
\text { (selection bias) }\end{array}$ & High risk & $\begin{array}{l}\text { Judgement comment: "after scoring the first pretest questionnaire, the re- } \\
\text { searcher assigned a code number to each participant: B1, B2, G1, G2, and } \\
\text { so on. The letter (B/G) stood for bad-sleep group or good sleep group. Since } \\
\text { not all women participated in the trial simultaneously, the number was as- } \\
\text { signed as they were interviewed (i.e. the first woman interviewed with a bad } \\
\text { sleep score received a B1, the second woman with bad sleep, a B2 etc.; the } \\
\text { first woman in the good-sleep group received a G1, etc.). After receiving a code } \\
\text { number, each woman was assigned to a group in the following way: women in } \\
\text { the B group with odd numbers were assigned to the experimental group; even } \\
\text { numbers, to the control group. Women in the G group with odd numbers were } \\
\text { assigned to the control group; even numbers, to the experimental group. In }\end{array}$ \\
\hline
\end{tabular}


this way, an even number of participants for both conditions was ensured in all cases $(n=14)$. This method for distribution was defined a priori to avoid researcher bias. Other randomisation methods were not possible since the participants did not participate nor sign up for the trial simultaneously".

\begin{tabular}{|c|c|c|}
\hline $\begin{array}{l}\text { Blinding of participants } \\
\text { and personnel (perfor- } \\
\text { mance bias) }\end{array}$ & High risk & $\begin{array}{l}\text { Judgement comment: "all women (both experimental and control group) were } \\
\text { residing within the same domestic violence shelter, and the assessment and } \\
\text { intervention was conducted by the same researcher". }\end{array}$ \\
\hline
\end{tabular}

Blinding of outcome as- High risk Judgement comment: the same researcher, knowing participant group allocasessment (detection bias) tion, conducted all outcome measurements.

All outcomes

\begin{tabular}{|c|c|c|}
\hline $\begin{array}{l}\text { Incomplete outcome data } \\
\text { (attrition bias) } \\
\text { All outcomes }\end{array}$ & High risk & $\begin{array}{l}\text { Judgement comment: there is no information about participant attrition and } \\
\text { exclusions. It is not clear as to how many participants were approached (or re- } \\
\text { ferred by shelter staff), and if any were ineligible or refused to participate. }\end{array}$ \\
\hline
\end{tabular}

\begin{tabular}{ll}
\hline $\begin{array}{l}\text { Selective reporting (re- } \\
\text { porting bias) }\end{array}$ & Unclear risk \\
& $\begin{array}{l}\text { Judgement comment: all outcomes described in the methods section were ad- } \\
\text { equately collected, analysed and reported in the results section of the main } \\
\text { published trial. However, there was insufficient or unclear evidence from ei- } \\
\text { ther the trial protocol or trial registry sites to assess this domain. }\end{array}$
\end{tabular}

\begin{tabular}{ll}
\hline Other bias & High risk \\
& $\begin{array}{l}\text { Judgement comment: there are various methodological issues about this trial. } \\
\text { For example, there is no mention of ethics approval, neither any information } \\
\text { about group differences at baseline. This was a pre-test-post-test design with } \\
\text { control and experimental groups, however with very small sample size. }\end{array}$
\end{tabular}

Hirani 2010

\section{Study characteristics}

Methods

Trial design: cluster-RCT

Trial grouping: parallel group

Assessment modality (e.g. face-to-face, telephone, online): women received the outcome instruments 2 weeks following the final intervention class.

\section{Conflict of interest of trial authors: NR}

Funding for trial: this research is funded through a grant from The Aga Khan University Research Council.

Primary outcomes: outcome measures included depression, measured by the BDI-II, self reports of abuse, self-efficacy as measured on the General Self-Efficacy Scale, and self-reported employment status.

Randomisation method: with a large diverse population, a 3-arm RCT with cluster-randomisation sampling was followed, whereby blocks of similar ethnic, language, and cultural affiliated families were randomised to an intervention

\section{Secondary outcomes: NR}

Timing of outcome measurements: 2 weeks following the final intervention class

Total duration of trial: 8-week community-derived intervention

Type of analysis: analysis of Variance; $\mathrm{Chi}^{2}$ test 
Hirani 2010 (Continued)

Types of participants: women in adult literacy programmes

ITT analysis: NR

\section{Baseline characteristics}

Experimental intervention

- Age: NR

- Ethnicity: NR

- Employment: NR

- Education: NR

- Relationship status: NR

- Gender (\% women): 100

- Dependent children: NR

- Number analysed (data analysis at post-treatment stage): 7

- Number of participants eligible assigned: 7

- Number of participants dropped out (by final follow-up): NR

- Comorbidities: NR

- Experience of IPV: $57.1 \%$ (at follow-up)

- Experience of other types of violence and abuse: NR

- Remuneration: NR

- Financial dependence (on partner): NR

Comparator intervention

- Age: NR

- Ethnicity: NR

- Employment: NR

- Education: NR

- Relationship status: NR

- Gender (\% women): 100

- Dependent children: NR

- Number analysed (data analysis at post-treatment stage): 8

- Number of participants eligible assigned: 8

- Number of participants dropped out (by final follow-up): NR

- Comorbidities: NR

- Experience of IPV: $66.66 \%$ (at follow-up)

- Experience of other types of violence and abuse: NR

- Remuneration: NR

- Financial dependence (on partner): NR

Total sample

- Age: most of the women were between 25-35 years of age

- Ethnicity: NR

- Employment: most women were not employed; monthly household income averaged USD 55.00

- Education: most women reported $<4$ years of formal education

- Relationship status: NR

- Gender (\% women): 100

- Dependent children: household size was between 6-10 people for most women

- Number analysed (data analysis at post-treatment stage): 24

- Number of participants eligible assigned: 24

- Number of participants dropped out (by final follow-up): NR

- Comorbidities: NR 
Hirani 2010 (Continued)

- Experience of IPV: NR

- Experience of other types of violence and abuse: NR

- Remuneration: NR

- Financial dependence (on partner): NR

Included criteria: NR in details. Following IRB approval, women in adult literacy programmes in each of the randomly chosen clusters were recruited into the trial. Following the signing of informed consent, women received 8 weeks of counselling or economic skill-building

Excluded criteria: NR

Pretreatment: no significant differences existed in demographic characteristics between the groups

Number eligible: NR

Number ineligible: NR

Number of eligible people consented: NR

Number of eligible people recruited: 24

Recruitment setting of participants (e.g. healthcare setting, community setting, shelter setting): adult literacy centres

\section{Sample power calculation: NR}

Total number of participants approached: NR

Total number of participants within all groups: 24

Number declining to participate: NR

Experimental intervention

- Brief description (rationale, main components): the empirically tested counselling module was delivered weekly for 8 weeks. The key components of the module included stress and anger management, effective communication, active listening and supportive problem-solving.

- Type of intervention: group counselling

- Mode of delivery (how): group session

- Frequency and duration of delivery (when and how much): 8 weeks, 1 session/week

- Level of mental health training of person delivering the intervention: NR

- Fidelity assessment: NR

- Intervention model: counselling

- Person delivering the intervention (who provided the intervention): therapist, self-directed or combined: CHWs

- Intervention location (where): adult literacy centres

- Tailoring of intervention (personalised, titrated or adapted): NR

- Intervention modifications (during the trial): NR

- Main techniques of intervention: the key components of the module included stress and anger management, effective communication, active listening and supportive problem-solving

- Intervention manual: NR

- Intervention attendance: NR

- CCDAN psychological therapies: integrative therapies

- Intensity of intervention: $\geq 5$ sessions

- Person delivering the intervention: healthcare workers

- Gender of therapist: NR

Comparator intervention 
- Type of intervention: no treatment

- Outcome type: continuous outcome

- Reporting: fully reported

- Scale: BDI II

- Range: 0-63

- Direction: lower is better

\section{Re-exposure to IPV}

- Outcome type: adverse event (dichotomous)

- Reporting: fully reported

- Scale: non-standardised questionnaire

- Direction: lower is better

\section{Dropouts from treatment}

- Outcome type: dichotomous outcome

- Reporting: fully reported

- Direction: lower is better

\section{Self-efficacy}

- Outcome type: continuous outcome

- Reporting: partially reported

- Scale: General Self-Efficacy Scale

- Range: 10-40

- Direction: higher is better

Identification

Sponsorship source: this research is funded through a grant from The Aga Khan University Research Council.

\section{Country: Pakistan}

Setting: community

Comments: urban economically disadvantaged women in Pakistan attending adult literacy programmes

Trialauthors: Hirani SS; Karmaliani R; McFarlane J; Asad N; Madhani F; Shehzad S

Institution: Aga Khan University, Karachi

Email: NR

Address: Aga Khan University, Karachi

Title (main 'outcome' publication): Testing a community derived intervention to promote women's health: preliminary results of a 3-arm randomized controlled trial in Karachi, Pakistan

Registered trial protocol ID: NR 
Hirani 2010 (Continued)

Adverse events (harm) related to participation: NR

\section{Risk of bias}

\begin{tabular}{lll}
\hline Bias & Authors' judgement & Support for judgement \\
\hline $\begin{array}{l}\text { Random sequence genera- } \\
\text { tion (selection bias) }\end{array}$ & High risk & $\begin{array}{l}\text { Judgement comment: "with a large diverse population, a 3-arm RCT with clus- } \\
\text { ter-randomisation sampling was followed, whereby blocks of similar ethnic, } \\
\text { language, and cultural affiliated families were randomised to an intervention. } \\
\end{array}$ \\
$\begin{array}{l}\text { The randomisation took place maintaining the community based participatory } \\
\text { approach and the internal validity of the research remained strong". No further } \\
\text { information mentioned about randomisation method and/or sequence gener- } \\
\text { ation. }\end{array}$
\end{tabular}

\begin{tabular}{ll}
\hline $\begin{array}{l}\text { Allocation concealment } \\
\text { (selection bias) }\end{array}$ & Unclear risk \\
& selling were delivered through the trained CHWs for 8 weeks, 1 session/week \\
& at the adult literacy centres randomly selected for these interventions". No \\
& further information mentioned regarding participant assignment and alloca- \\
& tion concealment.
\end{tabular}

$\begin{array}{ll}\begin{array}{l}\text { Blinding of participants } \\ \text { and personnel (perfor- }\end{array} & \text { High risk } \\ \begin{array}{l}\text { mance bias) } \\ \text { All outcomes }\end{array} & \begin{array}{l}\text { Judgement comment: "following the signing of informed consent, women re- } \\ \text { outcome instruments } 2 \text { weeks following the final intervention class". There is } \\ \text { no further information about blinding of participants and/or personnel. }\end{array}\end{array}$

\begin{tabular}{|c|c|c|}
\hline $\begin{array}{l}\text { Blinding of outcome as- } \\
\text { sessment (detection bias) } \\
\text { All outcomes }\end{array}$ & High risk & $\begin{array}{l}\text { Judgement comment: "women received the outcome instruments } 2 \text { weeks } \\
\text { following the final intervention class". There is no further information about } \\
\text { blinding of outcome assessors. }\end{array}$ \\
\hline $\begin{array}{l}\text { Incomplete outcome data } \\
\text { (attrition bias) } \\
\text { All outcomes }\end{array}$ & High risk & $\begin{array}{l}\text { Judgement comment: attrition and exclusions were not sufficiently described. } \\
\text { Further, women only received the outcome instruments } 2 \text { weeks following } \\
\text { the final intervention class. There was no information about the participants' } \\
\text { baseline characteristics. }\end{array}$ \\
\hline $\begin{array}{l}\text { Selective reporting (re- } \\
\text { porting bias) }\end{array}$ & Unclear risk & $\begin{array}{l}\text { Judgement comment: although the measures mentioned in the methods sec- } \\
\text { tion are presented in the results section, the outcome measures of depression, } \\
\text { partner violence, and self-efficacy appear to be only collected } 2 \text { weeks follow- } \\
\text { ing the final intervention class. The trial lacks an assessment of change over } \\
\text { time. In addition there was insufficient or unclear evidence from either the trial } \\
\text { protocol or trial registry sites to assess this domain. }\end{array}$ \\
\hline Other bias & High risk & $\begin{array}{l}\text { Judgement comment: this trial has a number of methodological issues that } \\
\text { need to be considered when interpreting the findings (e.g. lack of baseline da- } \\
\text { ta, insufficient information about randomisation method, lack of fidelity as- } \\
\text { sessment and very small sample size). }\end{array}$ \\
\hline
\end{tabular}

\section{Study characteristics}

Trial design: feasibility RCT
Trial grouping: parallel group
$\begin{aligned} & \text { Assessment modality (e.g. face-to-face, telephone, online): pre-treatment assessment was com- } \\ & \text { pleted at a university-based laboratory (face-to-face), other assessments were completed from partici- } \\ & \text { pants' homes or a location of their choosing }\end{aligned}$




\section{Conflict of interest of trial authors: NR}

Funding for trial: NR

Primary outcomes: dating violence; attitudes about violence; alcohol-aggression beliefs

Randomisation method: there were 3823 undergraduate students attending a large Midwestern university who were randomly emailed information about the project (described as a skills-based "relationship enhancement workshop") along with a link to a survey containing screening measures (listed earlier). Of the 130 students who completed the screener survey, 57 (43.8\%) met inclusion criteria; 32 of these individuals (56.1\%) enrolled in the trial, with 16 participants randomly assigned to either the treatment or control condition

\section{Secondary outcomes: NR}

Timing of outcome measurements: baseline, post-treatment, and 1-month follow-up

Total duration of trial: NR

Type of analysis: generalised linear mixed models

Types of participants: university students

ITT analyses: NR

\section{Participants}

\section{Baseline characteristics}

Experimental intervention

- Age: NR

- Ethnicity: NR

- Employment: NR

- Education: NR

- Relationship status: NR

- Gender (\% women): NR

- Dependent children: no participants reported having children

- Number analysed (data analysis at post-treatment stage): 10

- Number of participants eligible assigned: 16

- Number of participants dropped out (by final follow-up): 6

- Comorbidities: NR

- Experience of IPV: NR

- Experience of other types of violence and abuse: NR

- Remuneration: participants were paid USD 10/h or received course credit as incentive for completing each component

- Financial dependence (on partner): NR

Comparator intervention

- Age: NR

- Ethnicity: NR

- Employment: NR

- Education: NR

- Relationship status: NR

- Gender (\% women): NR

- Dependent children: no participants reported having children

- Number analysed (data analysis at post-treatment stage): 9

- Number of participants eligible assigned: 16

- Number of participants dropped out (by final follow-up): 7

- Comorbidities: NR 
- Experience of IPV: NR

- Experience of other types of violence and abuse: NR

- Remuneration: participants were paid USD $10 / \mathrm{h}$ or received course credit as incentive for completing each component

- Financial dependence (on partner): NR

Total sample

- Age: ranged from 19-29 years ( $M=20.59$ years, $S D=2.08$ years)

- Ethnicity: $93.8 \%$ identified as white, with the other 6.2\% identifying as Asian/Pacific Islander

- Employment: NR

- Education: NR

- Relationship status: 1 participant (3.0\%) reported being in a same-sex relationship

- Gender (\% women): $71.9 \%$ were female

- Dependent children: no participants reported having children

- Number analysed (data analysis at post-treatment stage): 19

- Number of participants eligible assigned: 32

- Number of participants dropped out (by final follow-up): 13

- Comorbidities: NR

- Experience of IPV: on the screener survey, 30 participants (93.8\% of the current sample) endorsed at least 1 act of psychological or physical dating violence in the past year, including 23 participants (71.9\%) who reported physical dating violence in the past year

- Experience of other types of violence and abuse: NR

- Remuneration: participants were paid USD 10/h or received course credit as incentive for completing each component

- Financial dependence (on partner): NR

Included criteria: to be eligible for Project PEACE, individuals were required to be in a romantic relationship for at least 3 months and endorse risk for mild to moderate dating violence on an online screening survey, as evidenced by (a) past physical or psychological aggression toward a dating partner, or (b) the presence of significant risk factors for such violence. Past aggression was indicated by the endorsement of at least 2 acts of psychological aggression or 1 act of physical assault toward a partner in the past year on the Revised CTS. As an alternative to actually experiencing past aggression, participants who reported no past aggression but were deemed to be at risk for dating violence were also eligible.

Excluded criteria: Project PEACE is a time-limited, skills-based intervention designed to address risk for mild to moderate dating violence. Thus, individuals who reported severe dating violence (e.g. any use of a knife or gun against a partner; punched, choked, or burned partner more than 10 times in the past year were considered inappropriate for the current intervention because of the likelihood of needing more intensive services. Students were also excluded if they were $<19$ years of age (the local age of majority), were married, or were not available during scheduled group times.

Pretreatment: NR

Number eligible: 32

Number ineligible: 73

Number of eligible people consented: 32

Number of eligible people recruited: 57

Recruitment setting of participants (e.g. healthcare setting, community setting, shelter setting): university setting

\section{Sample power calculation: NR}

Total number of participants approached: 3823 invitations; 130 completed screener 


\section{Total number of participants within all groups: 32}

Number declined to participate: 25

Interventions

\section{Intervention characteristics}

Experimental intervention

- Brief description (rationale, main components): Project PEACE (Partner Enrichment to Address Conflict Effectively) seeks to reduce physical and psychological dating violence in both men and women by promoting skills in mindfulness, emotion regulation, distress tolerance, cognitive restructuring, and interpersonal communication

- Type of intervention: psychoeducation

- Mode of delivery (how): group-based

- Frequency and duration of delivery (when and how much): mixed-gender groups of 6-8 individuals, each involved in a romantic relationship, met for $4 \times 90$-min sessions

- Level of mental health training of person delivering the intervention: each group had 2 facilitators; the lead facilitator of each group had a master's degree in clinical psychology

- Fidelity assessment: facilitators met weekly with the third author (DD), a licensed psychologist, for supervision and to review group content (to ensure fidelity).

- Intervention model: This model is highly influenced by behavior analytic, social learning, and situational theories of partner violence and, as such, highlights specific, process-orientated factors that can be targeted through skills-based intervention and are amenable to change through well-supported cognitive and behavioral techniques.

- Person delivering the intervention (who provided the intervention): therapist, self-directed or combined: each group had 2 facilitators; the lead facilitator of each group had a master's degree in clinical psychology

- Intervention location (where): university-based rooms

- Tailoring of intervention (personalised, titrated or adapted): NR

- Intervention modifications (during the trial): NR

- Main techniques of intervention: psycho-education on dating violence; mindfulness; emotion identification and regulation; effective communication; cognitive restructuring; distress tolerance

- Intervention manual: Project PEACE is a manualised intervention designed to prevent physical and psychological dating violence in at-risk college students

- Intervention attendance: 11 of the 16 participants in the intervention group completed $\geq 3$ of the 4 sessions. Most (68.8\%) participants attended at least 3 of the 4 treatment sessions, indicating use of the intervention

- CCDAN psychological therapies: third-wave CBTs

- Intensity of intervention: up to 4 sessions

- Person delivering the intervention: healthcare workers

- Gender of therapist: NR

Comparator intervention

- Brief description (rationale, main components): no-treatment control

- Type of intervention: no treatment

\section{Re-exposure to IPV}

- Outcome type: continuous outcome

- Reporting: fully reported

- Scale: Revised CTS

- Direction: lower is better

\section{Dropouts from treatment}

- Outcome type: dichotomous outcome

- Reporting: fully reported 
Jaffe 2017 (Continued)

- Direction: lower is better

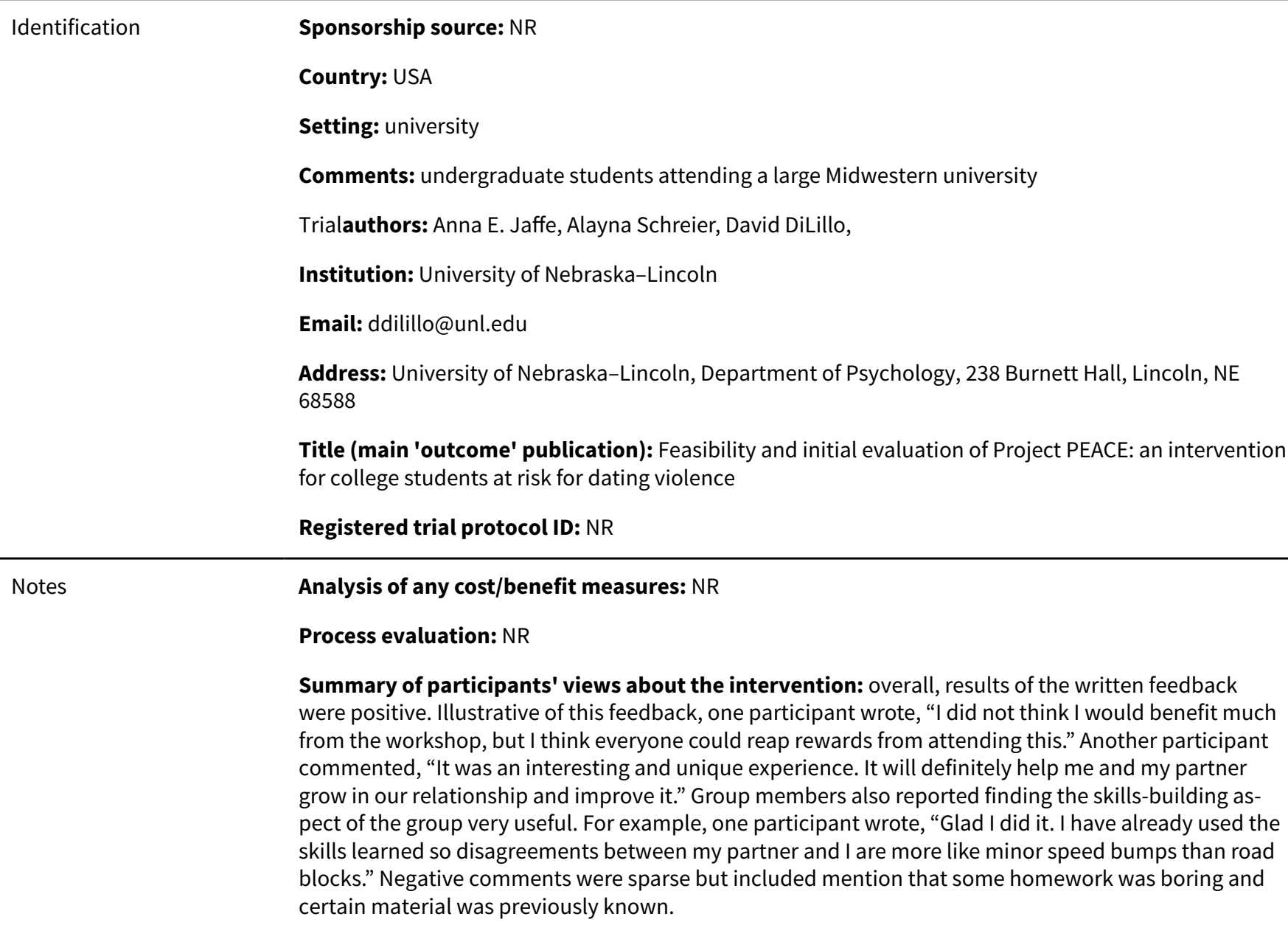

Adverse events (harm) related to participation: NR

\section{Risk of bias}

\section{Bias \\ Authors' judgement Support for judgement}

Random sequence genera- Unclear risk tion (selection bias)
Judgement comment: "there were 3823 undergraduate students attending a large Midwestern university who were randomly emailed information about the project (described as a skills-based "relationship enhancement workshop") along with a link to a survey containing screening measures (listed earlier). Of the 130 students who completed the screener survey, 57 (43.8\%) met inclusion criteria; 32 of these individuals (56.1\%) enrolled in the trial, with 16 participants randomly assigned to either the treatment or control condition". However, no further information mentioned about sequence generation.

Allocation concealment $\quad$ Unclear risk
(selection bias)
Judgement comment: "methodological strengths of the trial included random recruitment of participants university-wide, the inclusion of a no-treatment control group, and randomisation of participants to conditions". However, no further information mentioned about allocation concealment.

\begin{tabular}{|c|c|c|}
\hline $\begin{array}{l}\text { Blinding of participants } \\
\text { and personnel (perfor- } \\
\text { mance bias) } \\
\text { All outcomes }\end{array}$ & Unclear risk & $\begin{array}{l}\text { Judgement comment: "individuals who met inclusion criteria (described ear- } \\
\text { lier) and expressed interest in the group provided informed consent and com- } \\
\text { pleted outcome measures online at pre- and post-treatment, as well as at a } \\
\text { 1-month follow-up. The pretreatment assessment was completed in a lab af- }\end{array}$ \\
\hline
\end{tabular}

Psychological therapies for women who experience intimate partner violence (Review) 
Jaffe 2017 (Continued)

ter providing informed consent in person; other assessments were completed from participants' homes or a location of their choosing. However, no further information mentioned about the blinding of participants and personnel. Further, there were 2 facilitators for the treatment groups; the lead facilitator of each group had a master's degree in clinical psychology. Facilitators met weekly with the third author (DD), a licensed psychologist, for supervision and to review group content (to ensure fidelity)". However, no further information mentioned about blinding of trial personnel.

Blinding of outcome as- Unclear risk sessment (detection bias) All outcomes
Judgement comment: "the pretreatment assessment was completed in a lab after providing informed consent in person; other assessments were completed from participants' homes or a location of their choosing". However, no further information mentioned about how and who administered and collected the measures/outcomes.
Incomplete outcome data Low risk (attrition bias)

All outcomes
Judgement comment: retention at follow-up assessment for the control group was 10/16 (63\%) and 9/16 (56\%) for the treatment group. The sample was relatively small, which likely resulted in low statistical power to detect significant effects. The participants' flow chart diagram mentions exclusions and attrition rates.

Selective reporting (re- Unclear risk
porting bias)

\begin{abstract}
Judgement comment: all clinical measures mentioned in the methods section were fully collected, analysed and reported in the results section. However, differences between control and interventions at different time points are non-interpretable and are therefore not indicated. No further information mentioned as to why this is the case. In addition, there was insufficient or unclear evidence from either the trial protocol or trial registry sites to assess this domain.
\end{abstract}

\begin{tabular}{ll}
\hline Other bias & High risk \\
& $\begin{array}{l}\text { Judgement comment: several limitations of this trial should be noted and con- } \\
\text { sidered when interpreting the findings (e.g. small sample size, insufficient in- } \\
\text { formation about randomisation method, and lack of reporting of change over } \\
\text { time on key measures). Collectively, these may contribute to various forms of } \\
\text { risk of bias. }\end{array}$ \\
\hline
\end{tabular}

Johnson 2011

\section{Study characteristics}

\section{Methods}

\section{Trial design: $\mathrm{RCT}$}

Trial grouping: parallel group

Assessment modality (e.g. face-to-face, telephone, online): face-to-face and phone interviews

Conflict of interest of trial authors: "For one of my studies, treatment of postpartum depression, Pfizer provides the medication for the trial"

Funding for trial: this work was supported by NIMH grant 1 R34MH080786-01. ClinicalTrials.gov Identifier: NCT00602069

Primary outcomes: PTSD, IPV, comorbidity, trauma history

Randomisation method: 1 week after baseline, the first author randomly assigned participants to 1 of the conditions using an urn randomisation procedure stratifying participants according to PTSD status (i.e. PTSD and subthreshold PTSD) and medication status (i.e. on psychotropic medications or not)

Secondary outcomes: depression, empowerment, resource loss, social support, treatment credibility, client satisfaction, adherence and competence, 
Timing of outcome measurements: baseline, 1-week post-shelter, 3 months post-shelter, 6 months post-shelter

Total duration of trial: recruitment occurred from 2004-2007

Type of analysis: hierarchical linear modelling

Types of participants: participants were residents of 1 of 2 inner-city battered women's shelters within the same shelter system serving a mid-sized Midwestern city

ITT analyses: yes

Participants

\section{Baseline characteristics}

Experimental intervention

- Age: $M=31.74$ years, $S D=9.12$

- Ethnicity: African American 17 (48.6\%); white 17 (48.6\%); other race 1 (2.9\%); Hispanic 1 (2.9\%)

- Employment: employed 13 (37.1\%)

- Education: < high school 9 (25.7\%); high school 7 (20.0\%); completed some college 14 (40.0\%); graduated from college 5 (14.3\%)

- Relationship status: cohabitated/married to abuser 25 (71.4\%)

- Gender (\% women): 100

- Dependent children: have children 30 (85.7\%)

- Number analysed (data analysis at post-treatment stage): 32

- Number of participants eligible assigned: 35

- Number of participants dropped out (by final follow-up): 3

- Comorbidities: major depression 48.6\% (17); substance use disorder 5.7\% (2); other anxiety disorder $16(45.7 \%)$

- Experience of IPV: IPV in month prior to shelter: psychological 35 (100\%); physical 31 (88.6\%); sexual $23(65.7 \%)$

- Experience of other types of violence and abuse: number of types of prior lifetime trauma other than index IPV: 6.86

- Remuneration: participants were paid USD 50 for each assessment.

- Financial dependence (on partner): NR

Comparator intervention

- Age: $\mathrm{M}=33.34$ years, $\mathrm{SD}=6.74$

- Ethnicity: African American 18 (51.4\%); white 13 (37.1\%); other race 4 (11.4\%); Hispanic 3 (5.7\%)

- Employment: employed 6 (17.1\%)

- Education: < high school 10 (28.6\%); high school/GED 9 (25.7\%); completed some college 16 (45.7\%); graduated from college $0(0)$

- Relationship status: cohabitated/married to abuser 28 (80.0\%)

- Gender (\% women): 100

- Dependent children: have children 33 (94.3\%)

- Number analysed (data analysis at post-treatment stage): 34

- Number of participants eligible assigned: 35

- Number of participants dropped out (by final follow-up): 1

- Comorbidities: major depression $85.7 \%$ (30); substance use disorder $17.1 \%$ (6); other anxiety disorder $34(48.6 \%)$

- Experience of IPV: IPV in month prior to shelter: psychological 35 (100\%); physical 34(97.1\%); sexual $24(68.6 \%)$

- Experience of other types of violence and abuse: Number of types of prior lifetime trauma other than index IPV: 5.77

- Remuneration: participants were paid USD 50 for each assessment.

- Financial dependence (on partner): NR 
- Age: $\mathrm{M}=32.55$ years, $\mathrm{SD}=8.00$

- Ethnicity: African American 35 (50.0\%); white 30 (42.9\%); other race 5 (7.1\%); Hispanic 3 (4.3\%)

- Employment: employed 19 (27.1\%)

- Education: < high school 19 (27.1\%); high school/GED 16 (22.9\%); completed some college 30 (42.9\%); graduated from college $5(7.1 \%)$

- Relationship status: cohabitated/married to abuser 53 (75.7\%)

- Gender (\% women): 100

- Dependent children: have children 63 (90.0\%)

- Number analysed (data analysis at post-treatment stage): 66

- Number of participants eligible assigned: 70

- Number of participants dropped out (by final follow-up): 4

- Comorbidities: major depression $67.1 \%$ (47); substance use disorder $11.4 \%$ (8); other anxiety disorder $18(51.4 \%)$

- Experience of IPV: IPV in month prior to shelter: psychological 70 (100\%); physical 65 (92.9\%); sexual $47(67.1 \%)$

- Experience of other types of violence and abuse: Number of types of prior lifetime trauma other than index IPV: 6.31

- Remuneration: participants were paid USD 50 for each assessment.

- Financial dependence (on partner): NR

Included criteria: to be eligible, participants had to experience an incident of IPV on the CTS-Revised the month prior to shelter admission and meet diagnostic criteria for IPV-related PTSD or subthreshold PTSD according to the Clinician-Administered PTSD Scale. To meet criteria for subthreshold PTSD, participants had to meet Criteria A (exposure to IPV), Criteria B (re-experiencing symptoms), Criteria $E$ (symptom duration of at least 1 month), and Criteria $F$ (significant distress or impairment of functioning) as well as either Criteria C (avoidance symptoms) or Criteria D (increased arousal). Subthreshold PTSD was chosen as the inclusion criteria in an effort to increase the generalisability of findings. Furthermore, subthreshold PTSD is associated with significant impairment and morbidity (Stein et al., 1997) and is frequently used as inclusion criteria in RCTs of treatments that target PTSD symptoms.

Excluded criteria: participants were excluded from the trial if they (a) had symptoms of psychosis on the psychotic screen of the Structured Clinical Interview for Axis I disorders, (b) met diagnostic criteria for lifetime bipolar disorder on the SCID-I/P, (c) endorsed significant suicidal ideation with intent and plan, (d) if on psychotropic medications, have had any change in medication dose or type in the last month, or (e) were in concurrent individual therapy.

Pretreatment: No other significant differences emerged between the 2 treatment groups on any demographic, diagnostic, trauma/IPV characteristics at baseline, or on the length of shelter stay, occurrence of contact with abuser, or receipt of therapy over the follow-up period (all ps.05).

Number eligible: 70

Number ineligible: 205

Number of eligible people consented: 70

Number of eligible people recruited: 70

Recruitment setting of participants (e.g. healthcare setting, community setting, shelter setting): women's shelter

Sample power calculation: a power analysis was completed a priori to assure ability to detect large effects (Cohen's d 0.80), with the overall aim of this trial to assess acceptability and feasibility, and to determine estimates of ranges of effect sizes for future larger-scale trials.

Total number of participants approached: 281

Total number of participants within all groups: 70 


\section{Number declined to participate: 7}

Interventions

\section{Intervention characteristics}

Experimental intervention

- Brief description (rationale, main components): core modules cover engagement and goal setting, psycho education about abuse and PTSD, safety planning, empowerment, establishing trust, cognitive restructuring, managing triggers, self-soothing, establishing boundaries, anger management, and establishing long-term support

- Type of intervention: CBT

- Mode of delivery (how): individual face-to-face sessions

- Frequency and duration of delivery (when and how much): participants received a maximum of 12 sessions of HOPE while in shelter over a maximum of 8 weeks. Sessions were offered biweekly and lasted approximately $1-1.5 \mathrm{~h}$.

- Level of mental health training of person delivering the intervention: the first author, as well as 5 additional therapists who held a minimum of a master's degree in psychology or counselling and had at least 1 year of prior experience in working with traumatised populations, conducted HOPE. HOPE training included a 12-h workshop with the first author that provided an overview of the HOPE theoretical approach and specific instructions on how to deliver each module. All therapists were supervised weekly by the first author. Supervision by the first author included discussion of specific HOPE cases, audio tape review, role-plays, and discussion of adherence and competence ratings.

- Fidelity assessment: adherence and competence was rated by trial therapists who did not conduct the session being rated. The HOPE Adherence and Competence Scales (HOPEACS) contained 5 items assessing therapist general skillfulness (e.g. empathy, efficient use of time) on a 6-point scale ranging from 0 (not present) to 5 (excellent). Additionally, for 15 general (e.g. adherence to HOPE hierarchy, discussion of accomplishments) and 18 module-specific (i.e. psycho education regarding abuse and PTSD, education and application of cognitive restructuring techniques) HOPE strategies, the presence or absence of each strategy, protocol adherence (i.e. not enough (1) to too much (1)), and quality (i.e. poor (1) to excellent (5)) were rated. Finally, therapist overall adherence and competence were rated on 5-point scales ranging from 1 (poor) to 5 (excellent). Internal consistency of both the HOPE Adherence Scale ( .95$)$ and HOPE Competence Scale ( .80$)$ were excellent. Of the 27 sessions rated for adherence and competence, 13 were rated by a second rater, in order to assess inter-rater reliability. Average measure reliability (ICC) was also good for adherence (.92) and adequate for competence (.75) ratings.

- Intervention model: CBT

- Person delivering the intervention (who provided the intervention): therapist, self-directed or combined: therapist

- Intervention location (where): all sessions occurred at shelter, and childcare was provided.

- Tailoring of intervention (personalised, titrated or adapted): throughout HOPE, clients were encouraged to identify the aspects of any threats to their physical and emotional safety that were within their control and to use the empowerment toolbox to manage these threats. Participants identified and prioritised their personal goals during the first 2 sessions, and these goals were used in conjunction with the HOPE hierarchy to individualise treatment and to further engage and empower women in the treatment.

- Intervention modifications (during the trial): NR

- Main techniques of intervention: HOPE session structure was the same for each session: (a) checking in regarding safety and progress on accomplishments (i.e. homework), (b) agenda setting, (c) discussion of module-specific information, and (d) negotiating accomplishments for the next session. A hierarchy of target behaviours (i.e. the HOPE hierarchy) was developed to guide the content of treatment within and across sessions: (a) immediate physical and emotional risks; (b) PTSD symptoms, behaviours, and cognitions that interfere with (b1) achieving shelter and treatment goals and (b2) quality of life; and (c) post-shelter goals and safety. This hierarchy determined the order of specific modules over the course of therapy (e.g. safety discussed before skill-specific modules), as well as in prioritising safety issues within each session (e.g. new material is not presented until safety issues have been addressed).

- Intervention manual: HOPE was manualised and measures were included to assure that the treatment protocol was delivered in an adherent and competent manner

- Intervention attendance: participants in HOPE attended $0-12$ sessions ( $M=6.8, S D=4.3)$. All but 1 participant (97.1\%) attended at least 1 session of HOPE, with $22(62.9 \%)$ attending 5 sessions, and 9 (25.7\%) attending all 12 sessions. 
- Intensity of intervention: $\geq 5$ sessions

- Person delivering the intervention: healthcare workers

- Gender of therapist: NR

Comparator intervention

- Brief description (rationale, main components): all participants received standard shelter services. Standard shelter services included case management, a supportive milieu environment, and attendance of educational groups offered through the shelter (i.e. parenting and support groups). No therapy was offered through standard shelter services.

- Type of intervention: standard shelter services

Outcomes

\section{Depression}

- Outcome type: continuous outcome

- Reporting: fully reported

- Scale: BDI

- Direction: lower is better

PTSD

- Outcome type: continuous outcome

- Reporting: fully reported

- Scale: Clinician-Administered PTSD Scale

- Direction: lower is better

Social support

- Outcome type: continuous outcome

- Reporting: fully reported

- Scale: Inventory of Socially Supportive Behaviors

- Direction: higher is better

\section{Dropouts from treatment}

- Outcome type: dichotomous outcome

- Reporting: fully reported

- Direction: lower is better

Setting: women's shelter

Comments: participants were residents of 1 of 2 inner-city battered women's shelters within the same shelter system serving a mid-sized Midwestern city

Trialauthors: Dawn M. Johnson, Caron Zlotnick, Sara Perez

Institution: Department of Psychology, University of Akron

Email: johnsod@uakron.edu

Address: Department of Psychology, University of Akron, Akron, $\mathrm{OH} 44325-4301$

Title (main 'outcome' publication): Cognitive behavioral treatment of PTSD in residents of battered women's shelters: results of a randomized clinical trial

Registered trial protocol ID: NCT02398227 
Johnson 2011 (Continued)

Notes

\section{Analysis of any cost/benefit measures: NR \\ Process evaluation: NR}

Summary of participants' views about the intervention: there was no report of qualitative/views of participants about the intervention. However, average credibility rating $(n=29)$ on a 9-point scale ranging from 0 (not at all) to 8 (extremely) was 6.78 (SD 1.42). Average satisfaction ratings on the 4-point scale were 3.4 at 1-week (n 32), 3.5 at 3-month PS (n 31), and 3.4 at 6-month (n 30).

Adverse events (harm) related to participation: there was no unanticipated research related to serious adverse events that required participants to be removed from the trial. There were 7 hospitalisations ( 5 medical, 2 substance-related) and 4 life-threatening traumatic experiences ( 2 abuse-related) reported over the course of the 6-month follow-up period.

\section{Risk of bias}

\begin{tabular}{lll}
\hline Bias & Authors' judgement & Support for judgement \\
\hline $\begin{array}{ll}\text { Random sequence genera- } \\
\text { tion (selection bias) }\end{array}$ & Low risk & $\begin{array}{l}\text { Judgement comment: "the project co-ordinator randomly assigned partici- } \\
\text { pants to HOPE + standard shelter services or standard shelter services using } \\
\text { urn randomisation stratifying participants according to PTSD diagnosis and } \\
\text { medication status". }\end{array}$ \\
& & \\
\hline
\end{tabular}

\begin{tabular}{ll}
\hline $\begin{array}{l}\text { Allocation concealment } \\
\text { (selection bias) }\end{array}$ & Unclear risk \\
& however there is insufficient information to assess allocation concealment. 1 \\
& week after baseline, the first author randomly assigned participants to 1 of the \\
& conditions using an urn randomisation procedure stratifying participants ac- \\
cording to PTSD status (i.e. PTSD and subthreshold PTSD) and medication sta- & tus (i.e. on psychotropic medications or not).
\end{tabular}

\begin{tabular}{|c|c|c|}
\hline $\begin{array}{l}\text { Blinding of participants } \\
\text { and personnel (perfor- } \\
\text { mance bias) }\end{array}$ & Unclear risk & $\begin{array}{l}\text { Judgement comment: this was an RCT design with blind assessors, yet there is } \\
\text { insufficient information provided to assess blinding of participants and other } \\
\text { personnel. }\end{array}$ \\
\hline
\end{tabular}

\begin{tabular}{|c|c|c|}
\hline $\begin{array}{l}\text { Blinding of outcome as- } \\
\text { sessment (detection bias) }\end{array}$ & Low risk & $\begin{array}{l}\text { Judgement comment: all assessments were conducted by trained and blinded } \\
\text { doctoral students in psychology. }\end{array}$ \\
\hline
\end{tabular}

All outcomes doctoral students in psychology.

\begin{tabular}{|c|c|c|}
\hline $\begin{array}{l}\text { Incomplete outcome data } \\
\text { (attrition bias) } \\
\text { All outcomes }\end{array}$ & Low risk & $\begin{array}{l}\text { Judgement comment: the ITT sample was adequately described as well as } \\
\text { minimum attendance sample, with complete outcome data. Attrition and ex- } \\
\text { clusions were adequately described. }\end{array}$ \\
\hline
\end{tabular}

\begin{tabular}{|c|c|c|}
\hline $\begin{array}{l}\text { Selective reporting (re- } \\
\text { porting bias) }\end{array}$ & Unclear risk & $\begin{array}{l}\text { Judgement comment: all outcomes described in the methods section were ad- } \\
\text { equately collected, analysed and reported in the results section of the main } \\
\text { published trial. However, there was insufficient or unclear evidence from ei- } \\
\text { ther the trial protocol or trial registry sites to assess this domain. }\end{array}$ \\
\hline Other bias & Low risk & Judgement comment: none \\
\hline
\end{tabular}

Kiely 2010

\section{Study characteristics}

\begin{tabular}{ll}
\hline Methods & Trial design: $\mathrm{RCT}$ \\
& Trial grouping: parallel group
\end{tabular}


Conflict of interest of trial authors: the author(s) declare that they have no competing interests.

Funding for trial: this trial was supported by grants no.3U18HD030445; 3U18HD030447; 5U18HD31206; 3U18HD031919;5U18HD036104, National Institute of Child Health and Human Development and the National Center on Minority Health and Health Disparities. The content of this work is solely the responsibility of the authors and does not necessarily represent the official views of the funding agencies.

Primary outcomes: the primary goal was to estimate whether a multi-modal, integrated counselling and educational intervention reduces smoking and environmental tobacco smoke exposure (ETSE), depression, and IPV (defined as being victimised) among pregnant African American and Latina women. Abuse Assessment Screen, a measure designed and validated for use in pregnancy if a woman reported physical or sexual abuse by a partner in the previous year. During the baseline and follow-up interviews, the frequency of physical assault and sexual coercion(partner to self) was measured by the CTS

Randomisation method: site- and risk-specific block randomisation to intervention or usual care was conducted. Investigators and field workers were blinded to the block size. A computer-generated randomisation scheme considered all possible risk combinations within each of the recruitment sites. Recruitment staff at each site called in the details of the risk profile for a new recruit, and the assignment was generated centrally by the data co-ordinating centre.

Secondary outcomes: a secondary goal was to estimate whether a clinic-based intervention reducing smoking, ETSE, depression, and IPV in pregnancy would reduce adverse pregnancy outcomes (e.g. prematurity and low birth weight) and lower infant morbidity and mortality.

Timing of outcome measurements: these assessments were obtained at baseline, 22-26 weeks and 34-38 weeks of gestation, and 8-10 weeks postpartum

Total duration of trial: July 2001-October 2003; women were screened at 6 community-based prenatal care sites serving mainly minority women in DC between July 2001 and October 2003. Intervention and follow-up activities continued until July 2004.

Type of analysis: ITT approach, Bivariate analyses, Multivariate logistic regression

Types of participants: women presenting to 6 community-based clinical sites serving minority women (African-American and Hispanic) in the District of Columbia

ITT analyses: yes

\section{Baseline characteristics}

Experimental intervention

- Age: $18-22$ years $(47.0 \%) ; 23-27(29.4 \%) ;>28(23.6 \%)$

- Ethnicity: African American women and Latinas

- Employment: working now (34.5\%); not working now, worked previous to pregnancy (37.0\%); not working now, did not work previous to pregnancy $(28.3 \%)$

- Education: < high school (28.3\%); high school graduate (50.1\%); at least some college $(21.6 \%)$

- Relationship status: single/separated/widowed/ divorced (75.9\%); married or living with partner (24.1\%)

- Gender (\% women): 100

- Dependent children: NR

- Number analysed (data analysis at post-treatment stage): 362

- Number of participants eligible assigned: 403

- Number of participants dropped out (at final follow-up): 41

- Comorbidities: depression (43.4\%)

- Experience of IPV: 132 (32.8\%)

- Experience of other types of violence and abuse: NR 
- Remuneration: women in the intervention group received USD 10 for each intervention session and additional USD 15 and USD 25 gift certificates for the first and second postpartum intervention sessions, respectively.

- Financial dependence (on partner): NR

Comparator intervention

- Age: $18-22$ years (39.8\%); 23-27 (32.8\%); > 28 (27.5\%)

- Ethnicity: African American women and Latinas

- Employment: working now (37.6\%); not working now, worked previous to pregnancy (38.1\%); not working now, did not work previous to pregnancy (23.6\%)

- Education: < high school (28.6\%); high school graduate (47.6\%); at least some college $(23.8 \%)$

- Relationship status: single/separated/widowed/ divorced (74.3\%); married or living with partner (25.7\%)

- Gender (\% women): 100

- Dependent children: NR

- Number analysed (data analysis at post-treatment stage): 374

- Number of participants eligible assigned: 416

- Number of participants dropped out (at final follow-up): 42

- Comorbidities: depression (44.2\%)

- Experience of IPV: 131 (31.5\%)

- Experience of other types of violence and abuse: NR

- Remuneration: NR

- Financial dependence (on partner): NR

Total sample

- Age: $18-22$ years (43.3\%); $23-27$ (31.1\%); > 28 (25.6\%)

- Ethnicity:

- Employment: working now (36.1\%); not working now, worked previous to pregnancy (37.5\%); not working now, did not work previous to pregnancy $(25.9 \%)$

- Education: < high school (28.4\%); high school graduate (48.8\%); at least some college $(22.7 \%)$

- Relationship status: single/separated/widowed/ divorced (75.1\%); married or living with partner (24.9\%)

- Gender (\% women): 100

- Dependent children:

- Number analysed (data analysis at post-treatment stage): 736

- Number of participants eligible assigned: 819

- Number of participants dropped out (at final follow-up): 83

- Comorbidities: at baseline, environmental tobacco smoke exposure was the most commonly reported risk (82.7\%), followed by depression (50.7\%) and IPV (36.8\%), among those with at least 1 risk. Outcomes with/for women with IPV were published in a separate paper.

- Experience of IPV: 263 (32.1\%)

- Experience of other types of violence and abuse: NR

- Remuneration: women in the intervention group received USD 10 for each intervention session and additional USD 15 and USD 25 gift certificates for the first and second postpartum intervention sessions, respectively.

- Financial dependence (on partner): NR

Included criteria: women were deemed demographically eligible if self-identified as belonging to a minority group, being $\geq 18$ years, $<29$ weeks pregnant, a DC resident and English-speaking. Verbal prescreening for other characteristics was discouraged by the trial protocol in order to avoid bias.

Excluded criteria: no risk factors, $<18$ years old, $>28$ weeks estimated gestational age, not race eligible, not DC residents, not pregnant, suicidal thoughts 
Kiely 2010 (Continued)

Pretreatment: for the total sample $(n=1044)$, socio-demographic, psycho- behavioural and reproductive characterisation at baseline showed that there were no significant differences between women randomised to the IG $(n=521)$ or UCG $(n=523)$, except for the number of prenatal care visits. In addition, there were no significant differences between women with a known infant outcome and those missing this information.

Number eligible: 1398

Number ineligible: 1515

Number of eligible people consented: 1070

Number of eligible people recruited: 1398

Recruitment setting of participants (e.g. healthcare setting, community setting, shelter set-

ting): women with pregnancy presenting to 6 community-based clinical sites serving minority women (African-Americans and Hispanics) in the District of Columbia

Sample power calculation: the sample size was determined to ensure adequate statistical power to test the hypotheses that our cognitive behavioral intervention would result in reductions in the targeted risks. Assuming a $5 \%$ level of significance, $80 \%$ power would allow the detection of $10 \%-20 \%$ reductions in risk-specific factors among women in the intervention group from a $100 \%$ prevalence at recruitment time, a sample of 1050 women needed to be retained at the end of the follow-up period.

Total number of participants approached: 2913

Total number of participants within all groups: 1070

Number declined to participate: 207

\section{Intervention characteristics}

Experimental intervention

- Brief description (rationale, main components): the intervention was evidence-based and specific to each of the designated psycho-behavioral risks. Intervention for IPV, interventions for smoking and environmental tobacco smoke exposure, depression intervention. A structured intervention was based on Dutton's Empowerment Theory which emphasised safety behaviours, was adapted for the IPV intervention. This brochure based intervention provided information about the types of abuse, the cycle of violence, a danger assessment component, in addition to the development of a safety plan. Individualised counselling sessions provided an integrated approach to multiple risks responsive to a woman's specific risk combination.

- Type of intervention: integrated behavioural intervention (individualised counselling targeting their area(s) of risk, e.g. IPV)

- Mode of delivery (how): face-to-face prenatal sessions and telephone interviews

- Frequency and duration of delivery (when and how much): the intervention occurred immediately before or after routine prenatal care, for an average of $35 \pm 15 \mathrm{~min}$. The intervention was designed to be delivered during prenatal care visits with 8 discrete sessions. Delivery in a minimum of 4 sessions, with 8 prenatal sessions required for a complete intervention. 2 additional postpartum booster sessions were provided to reinforce risk-specific intervention goal. Each session lasted for an average of $35 \pm$ 15 min.

- Level of mental health training of person delivering the intervention: the intervention specialists were trained to apply the content of these sessions during $\geq 1$ visits as needed. in a separate publication it was mentioned that master's level social workers or psychologists were trained specifically to deliver this intervention.

- Fidelity assessment: NR

- Intervention model: integrated behavioural intervention (individualised counselling); the intervention for IPV emphasised safety behaviours and was based on the structured intervention developed by Parker and colleagues and based on Dutton's Empowerment Theory. The interventions for smoking and environmental tobacco smoke exposure were combined and based on Smoking Cessation or Reduction in Program Treatment. The depression intervention was developed by Miranda and Munoz based on cognitive behavioral theory. 
Kiely 2010 (Continued)

Outcomes
- Person delivering the intervention (who provided the intervention): therapist, self-directed or combined: pregnancy advisors delivered the intervention

- Intervention location (where): intervention sessions were conducted privately in a room proximate to the prenatal care clinics

- Tailoring of intervention (personalised, titrated or adapted): individualised counselling; at each intervention session, the woman identified which of the 4 risks she was experiencing. The intervention was delivered by the interventionist and targeted to address all risks reported at each session, regardless of previously reported risks.

- Intervention modifications (during the trial): NR

- Main techniques of intervention: intervention provided information about the types of abuse (e.g. emotional, physical, and sexual) and the cycle of violence (e.g. escalating, IPV honeymoon period), a danger assessment component to assess risks, and preventive options women might consider (e.g. filing a protection order) as well as the development of a safety plan (e.g. leaving important documents and papers with others). In addition, a list of community resources with addresses and phone numbers was provided. Cognitive-behavioral. Individualised counselling sessions provided an integrated approach to multiple risks responsive to a woman's specific risk combination.

- Intervention manual: a structured intervention developed by Parker et al. (34) and based on Dutton's Empowerment Theory (35) which emphasised safety behaviours, was adapted for the IPV intervention (no further information)

- Intervention attendance: $51 \%$ of the women randomly assigned to the intervention group received $\geq$ 4 sessions; $1 / 4$ of the women attended no intervention sessions

- CCDAN psychological therapies: CBT

- Intensity of intervention: $\geq 5$ sessions

- Person delivering the intervention: healthcare workers

- Gender of therapist: NR

Comparator intervention

- Brief description (rationale, main components): usual care

- Type of intervention: women assigned to the usual care group met with their primary care providers as per standard clinic practice

\section{Depression}

- Outcome type: adverse event (dichotomous)

- Reporting: fully reported

- Scale: Johns Hopkins Depression Scale

- Direction: lower is better

\section{Re-exposure to IPV}

- Outcome type: adverse event (dichotomous)

- Reporting: fully reported

- Scale: CTS

- Direction: lower is better

\section{Dropouts from treatment}

- Outcome type: dichotomous outcome

- Reporting: fully reported

- Direction: lower is better
Sponsorship source: this trial was supported by grants no. 3U18HD030445; 3U18HD030447; 5U18HD31206; 3U18HD031919; 5U18HD036104, National Institute of Child Health and Human Development and the National Center on Minority Health and Health Disparities.

Country: District of Columbia, USA

Setting: community-based clinical sites 
Kiely 2010 (Continued)

Comments: prenatal care sites serving mainly minority women; women presenting to 6 community-based clinical sites serving minority women (African-American and Hispanic) in the District of Columbia

Trialauthors: Ayman A. E. El-Mohandes, Michele Kiely, Marie G. Gantz, M. Nabil El-Khorazaty

Institution: Eunice Kennedy Shriver National Institute of Child Health and Human Development

Email: aelmohandes@unmc.edu

Address: College of Public Health, University of Nebraska MedicalCenter, WH 5030, 984355, Omaha, NE 68198-4355, USA

Trial title (main outcome publication): an integrated intervention to reduce intimate partner violence in pregnancy: a randomised controlled trial

Registered trial protocol ID: NCT00381823

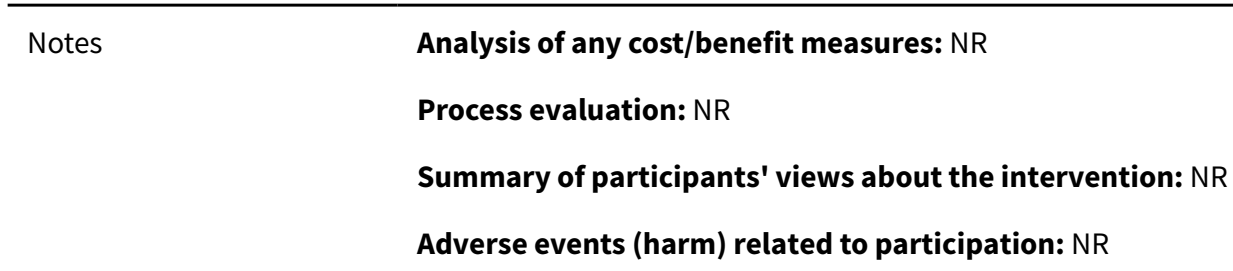

Risk of bias

\begin{tabular}{lll}
\hline Bias & Authors' judgement & Support for judgement \\
\hline $\begin{array}{ll}\text { Random sequence genera- } \\
\text { tion (selection bias) }\end{array}$ & Low risk & $\begin{array}{l}\text { Judgement comment: "site- and risk-specific block randomisation to interven- } \\
\text { tion or usual care was conducted. Investigators and field workers were blinded } \\
\text { to the block size". }\end{array}$ \\
\hline
\end{tabular}

Allocation concealment Low risk Judgement comment: "a computer-generated randomisation scheme consid(selection bias) ered all possible risk combinations within each of the recruitment sites. Recruitment staff at each site called in the details of the risk profile for a new recruit, and the assignment was generated centrally by the data co-ordinating centre. When a woman completed the baseline interview and was ready for randomisation, the recruitment staff would call the data co-ordinating centre, where the participant's assignment was determined".

\begin{tabular}{|c|c|c|}
\hline $\begin{array}{l}\text { Blinding of participants } \\
\text { and personnel (perfor- } \\
\text { mance bias) } \\
\text { All outcomes }\end{array}$ & Unclear risk & $\begin{array}{l}\text { Judgement comment: site- and risk-specific block randomisation to interven- } \\
\text { tion or usual care was conducted. Investigators and field workers were blinded } \\
\text { to the block size, yet it is not clear whether participants were blinded. }\end{array}$ \\
\hline $\begin{array}{l}\text { Blinding of outcome as- } \\
\text { sessment (detection bias) } \\
\text { All outcomes }\end{array}$ & Low risk & $\begin{array}{l}\text { Judgement comment: "telephone interviewers and their supervisors were } \\
\text { blinded to the participants' randomisation group. Research staff maintained } \\
\text { confidentiality when communicating with participants outside the clinic set- } \\
\text { ting". }\end{array}$ \\
\hline $\begin{array}{l}\text { Incomplete outcome data } \\
\text { (attrition bias) } \\
\text { All outcomes }\end{array}$ & Low risk & $\begin{array}{l}\text { Judgement comment: attrition and exclusions were adequately described. A } \\
\text { separate paper was published about recruitment and retention rates. }\end{array}$ \\
\hline $\begin{array}{l}\text { Selective reporting (re- } \\
\text { porting bias) }\end{array}$ & Unclear risk & $\begin{array}{l}\text { Judgement comment: all outcomes described in the methods section were ad- } \\
\text { equately collected, analysed and reported in the results section of the main } \\
\text { published trial. However, there was insufficient or unclear evidence from ei- } \\
\text { ther the trial protocol or trial registry sites to assess this domain. }\end{array}$ \\
\hline
\end{tabular}


Kiely 2010 (Continued)
Other bias
Low risk
Judgement comment: none

Kokka 2019

\section{Study characteristics}

Methods

Trial design: RCT

Trial grouping: parallel group

Assessment modality (e.g. face-to-face, telephone, online): face-to-face

Conflict of interest of trial authors: none declared

Funding for trial: the trial author(s) received no financial support for the research, authorship, and/or publication of this article.

Primary outcomes: Depression, Anxiety, and Stress Scale (DASS-21), Perceived Stress Scale 14 (PSS-14), self-esteem, Spiritual Well-Being Scale, Women Abuse Screening Tool (WAST), Self-Efficacy Scale, Coping Orientation to Problems Experienced (COPE),

Randomisation method: eligible women were randomly allocated in intervention and control group, using the random number generator (random.org). The trial complied fully with the Declaration of Helsinki guidelines.

\section{Secondary outcomes: NR}

Timing of outcome measurements: all women were assessed using self-reported questionnaires preand post-intervention by A.K. and M.M. researchers

Total duration of trial: January 2015-June 2015

Type of analysis: demographics and baseline group characteristics are presented as means, SD, and absolute and proportion values. Group comparisons were performed with Pearson's $\mathrm{Chi}^{2}$ and the Mann-Whitney U tests.

Types of participants: women currently being victims of physical, psychological, sexual, or combined abuse (present and past)

\section{ITT analyses: NR}

Baseline characteristics
Experimental intervention
- Age: $\mathrm{M}=47.23$ years, $\mathrm{SD}=9.96$
- Ethnicity: $\mathrm{NR}$
- Employment: $14(46.7 \%)$ unemployed
- Education: high school $15(50 \%)$
- Relationship status: 18 ( $60 \%)$ married
- Gender (\% women): 100
- Dependent children: NR
- Number analysed (data analysis at post-treatment stage): 30
- Number of participants eligible assigned: 30
- Number of participants dropped out (by final follow-up): 0
- Comorbidities: NR
- Experience of IPV: all included women were currently being victims of physical, psychological, sexual,

or combined abuse (present and past), mean abuse 18.5, SD (2)


- Experience of other types of violence and abuse: NR

- Remuneration: NR

- Financial dependence (on partner): NR

Comparator intervention

- Age: $\mathrm{M}=48.77$ years, $\mathrm{SD}=8.90$

- Ethnicity: NR

- Employment: 7 (23.3\%) unemployed

- Education: high school 18 (60\%)

- Relationship status: 20 (66.7\%) married

- Gender (\% women): 100

- Dependent children: NR

- Number analysed (data analysis at post-treatment stage): 30

- Number of participants eligible assigned: 30

- Number of participants dropped out (by final follow-up): 0

- Comorbidities: NR

- Experience of IPV: all included women were currently being victims of physical, psychological, sexual, or combined abuse (present and past), mean abuse 17.3, SD (3.2)

- Experience of other types of violence and abuse: NR

- Remuneration: NR

- Financial dependence (on partner): NR

Total sample

- Age: NR

- Ethnicity: NR

- Employment: NR

- Education: NR

- Relationship status: NR

- Gender (\% women): 100

- Dependent children: NR

- Number analysed (data analysis at post-treatment stage): 60

- Number of participants eligible assigned: 60

- Number of participants dropped out (by final follow-up): 0

- Comorbidities: NR

- Experience of IPV: all included women were currently being victims of physical, psychological, sexual, or combined abuse (present and past)

- Experience of other types of violence and abuse: NR

- Remuneration: NR

- Financial dependence (on partner): NR

Included criteria: (a) currently being victims of physical, psychological, sexual, or combined abuse (present and past); (b) age 18-70; and (c) literate in Greek

Excluded criteria: exclusion criteria were (a) substance abuse (as ascertained by the available official medical records), (b) use of tricyclic antidepressants or diagnosis with any major psychiatric disorder, and $(c)$ being under cortisone treatment for any reason.

Pretreatment: 3 significant differences were found between intervention and control group in terms of percentage of caregiver, working status and income satisfaction.

Number eligible: 60

Number ineligible: NR

Number of eligible people consented: 60 
Recruitment setting of participants (e.g. healthcare setting, community setting, shelter setting): community

Sample power calculation: NR

Total number of participants approached: NR

Total number of participants within all groups: 60

Number declined to participate: NR (although none withdrew from the trial)

\section{Intervention characteristics}

Experimental intervention

- Brief description (rationale, main components): stress management and health promotion programme with a focus on relaxation, cognitive reconstruction and gratitude techniques.

- Type of intervention: stress management

- Mode of delivery (how): 8 sessions. In the first session, the initial assessment took place: Questionnaires were distributed and completed, and the participants were given all the necessary information about the programme structure. During this session, an informative lecture on stress, lifestyle, and physical exercise was given. Women were also provided with details on where current literature was available and were given a practical guide and a pedometer to monitor their progress. During the second session, women were trained in biofeedback-assisted abdominal breathing (using the Nexus ${ }^{\circledR}$ system). They were encouraged to implement relaxation breathing (RB) twice/day (morning and evening). In the third session, the intervention group was introduced to another stress management technique, progressive muscular relaxation (PMR). Training was performed with the aid of a CD under the presence of the researchers. Women were also advised to implement PMR twice a day. During the next session, the intervention group was introduced to cognitive reconstruction and gratitude techniques. Besides information, women were provided with specific exercises (e.g. positive aspects of themselves). The next session included dietary counseling and encouragement to comply with the stress management programme. The 6th session was about training and implementation of guided imagery. Instructions were given via CD in the presence of the researchers. The forthcoming session goal was to discuss the progress as perceived by each participant regarding the relaxation techniques, to encourage compliance, and to solve possible questions. During the last session, participants completed the final measurements and returned their progress notes, where they recorded their physical activity (pedometer indications) and frequency of implementing the taught relaxation techniques (maximum 112).

- Frequency and duration of delivery (when and how much): $1 \mathrm{~h} /$ week for 8 weeks

- Level of mental health training of person delivering the intervention: NR

- Fidelity assessment: NR, although mentioned that "After the initiation of the trial, no change of the initial protocol took place".

- Intervention model: NR

- Person delivering the intervention (who provided the intervention): therapist, self-directed or combined: combined

- Intervention location (where): the interventions were conducted at the university establishments such as at the establishments of specialised centres.

- Tailoring of intervention (personalised, titrated or adapted): NR

- Intervention modifications (during the trial): after the initiation of the trial, no change of the initial protocol took place.

- Main techniques of intervention: stress management techniques, relaxation, cognitive reconstruction and gratitude techniques

- Intervention manual: NR

- Intervention attendance: NR

- CCDAN psychological therapies: other psychologically-orientated interventions

- Intensity of intervention: $\geq 5$ sessions

- Person delivering the intervention: non-healthcare workers 
Kokka 2019 (Continued)

- Gender of therapist: NR

Comparator intervention

- Brief description (rationale, main components): waiting list

- Type of intervention: waiting list (At the end of the intervention, the control group was provided with all the Compact Disks (CDs) of the relaxation techniques).

Outcomes

Perceived stress

- Outcome type: continuous outcome

- Reporting: fully reported

- Scale: Perceived Stress Scale 14 (PSS-14).

- Direction: lower is better

\section{Self-esteem}

- Outcome type: continuous outcome

- Reporting: fully reported

- Scale: Darviri and Varvogli "Self-Esteem Assessment Questionnaire."

- Direction: higher is better

\section{Women Abuse Screening Tool (WAST).}

- Outcome type: continuous outcome

- Reporting: fully reported

- Scale: Women Abuse Screening Tool (WAST)

- Direction: lower is better

\section{Self-efficacy}

- Outcome type: continuous outcome

- Reporting: fully reported

- Scale: Self-Efficacy Scale

- Direction: Higher is better

\section{Depression}

- Outcome type: continuous outcome

- Reporting: fully reported

- Scale: BDI

- Direction: lower is better

\section{Dropouts from treatment}

- Outcome type: dichotomous outcome

- Reporting: fully reported

- Direction: lower is better

Sponsorship source: NR

Country: Greece

Setting: community

Comments: the aim of the present trial was to investigate the effectiveness of a stress management programme regarding mitigating psychological repercussions in women who experience IPV

Trialauthors: Anastasia Kokka, Maria Mikelatou, Georgia Fouka, Liza Varvogli, George P. Chrousos, and Christina Darviri 
Kokka 2019 (Continued)

Institution: University of Athens

Email: cdarviri@yahoo.com

Address: School of Medicine, University of Athens, Soranou Ephessiou Str., 4, Athens GR-115-27, Greece.

Trial title (main outcome publication): Stress management and health promotion in a sample of women with intimate partner violence: a randomised controlled trial

Registered trial protocol ID: NR

Analysis of any cost/benefit measures: NR
Process evaluation: NR
Summary of participants' views about the intervention: NR
Adverse events (harm) related to participation: NR

Risk of bias

\begin{tabular}{lll}
\hline Bias & Authors' judgement & Support for judgement \\
\hline $\begin{array}{l}\text { Random sequence genera- } \\
\text { tion (selection bias) }\end{array}$ & Unclear risk & $\begin{array}{l}\text { Judgement comment: this was a 2-armed, parallel group, RCT with a 1:1 al- } \\
\text { location ratio of female violence survivor to intervention and control group. } \\
\text { However, there is no further information to assess random sequence genera- } \\
\text { tion. }\end{array}$ \\
\hline $\begin{array}{l}\text { Allocation concealment } \\
\text { (selection bias) }\end{array}$ & High risk & $\begin{array}{l}\text { Judgement comment: there is not sufficient evidence of allocation conceal- } \\
\text { ment. }\end{array}$ \\
\hline $\begin{array}{l}\text { Blinding of participants } \\
\text { and personnel (perfor- } \\
\text { mance bias) } \\
\begin{array}{l}\text { All outcomes } \\
\text { ill }\end{array}\end{array}$ & High risk & Judgement comment: the trial was not blind \\
\hline
\end{tabular}

\begin{tabular}{|c|c|c|}
\hline $\begin{array}{l}\text { Blinding of outcome as- } \\
\text { sessment (detection bias) }\end{array}$ & High risk & $\begin{array}{l}\text { Judgement comment: all women were assessed using self-reported question- } \\
\text { naires pre- and post-intervention by A.K. and M.M. researchers }\end{array}$ \\
\hline
\end{tabular}

All outcomes

\begin{tabular}{lll}
\hline $\begin{array}{l}\text { Incomplete outcome data } \\
\text { (attrition bias) } \\
\text { All outcomes }\end{array}$ & Low risk & Judgement comment: no dropout was recorded in either group. \\
\hline $\begin{array}{l}\text { Selective reporting (re- } \\
\text { porting bias) }\end{array}$ & Unclear risk & $\begin{array}{l}\text { Judgement comment: there was insufficient information to assess selective re- } \\
\text { porting. }\end{array}$ \\
\hline Other bias & High risk & $\begin{array}{l}\text { Judgement comment: this trial has a number of limitations regarding both tri- } \\
\text { al procedures and sample size, which was relatively small. }\end{array}$ \\
\hline
\end{tabular}

\section{Koopman 2005}

\section{Study characteristics}

Methods

Trial design: RCT

Trial grouping: parallel group 
Assessment modality (e.g. face-to-face, telephone, online): each potential participant initiated contact with the research assistant, who then conducted a brief screening interview by telephone to ensure that she met all of the inclusion criteria.

Conflict of interest of trial authors: none declared

Funding for trial: Drs Bala Manian and Tasneem Ismailji

Primary outcomes: Bodily Pain Scale of the SF-36 Health Survey, BDI, PTSD Checklist-Specific Version (PCL-S)

Randomisation method: each participant was randomly assigned to either the expressive writing condition in which she wrote about the most stressful event(s) of her life or to the neutral writing condition in which she wrote about her daily schedule.

\section{Secondary outcomes: NR}

Timing of outcome measurements: after each woman provided informed consent for her participation, she completed the questionnaires assessing demographic characteristics, bodily pain and depression and PTSD symptoms. Each woman completed 4 writing sessions, scheduled at weekly intervals. Follow-up assessment conducted 4 months following the conclusion of the writing intervention

Total duration of trial: NR

Type of analysis: descriptive statistics, multiple regression analysis

Types of participants: women who had survived IPV

ITT analyses: NR

Participants

\section{Baseline characteristics}

Experimental intervention

- Age: NR

- Ethnicity: NR

- Employment: NR

- Education: $\mathrm{M}=16.8$ years, $\mathrm{SD}=3.0$

- Relationship status: $28 \%$ married

- Gender (\% women): 100

- Dependent children: NR

- Number analysed (data analysis at post-treatment stage): 25

- Number of participants eligible assigned: NR

- Number of participants dropped out (by final follow-up): NR

- Comorbidities: NR

- Experience of IPV: NR

- Experience of other types of violence and abuse: NR

- Remuneration: Each participant received USD 50 compensation upon completion of the 4-month follow-up assessment.

- Financial dependence (on partner): NR

Comparator intervention

- Age: NR

- Ethnicity: NR

- Employment: NR

- Education: $\mathrm{M}=14.8$ years, $\mathrm{SD}=1.9$

- Relationship status: $9 \%$ married

- Gender (\% women): 100

- Dependent children: NR 
- Number analysed (data analysis at post-treatment stage): 22

- Number of participants eligible assigned:

- Number of participants dropped out (by final follow-up):

- Comorbidities: NR

- Experience of IPV: NR

- Experience of other types of violence and abuse: NR

- Remuneration: Each participant received USD 50 compensation upon completion of the 4-month follow-up assessment.

- Financial dependence (on partner): NR

Total sample

- Age: $\mathrm{M}=36.5$ years, $\mathrm{SD}=8.9$

- Ethnicity: white/European American (68\%), Latina/Hispanic (13\%), Middle Eastern (6\%), African American (6\%), Asian American (2\%) and other (4\%)

- Employment: full time: $43 \%$, part time: $21 \%$, unemployed: $36 \% .60 \%$ of the sample reported a household income under USD 40,000

- Education: education ranged from completing high school to completing graduate school, with a median of having completed some college

- Relationship status: only $19 \%$ of women were currently married or living with a new partner; whereas $38 \%$ were single, $15 \%$ separated, $17 \%$ divorced and $11 \%$ described their relationship status as 'other'.

- Gender (\% women): 100

- Dependent children: $60 \%$ did not have children, $32 \%$ had 1 or 2 children and $8 \%$ had $\geq 3$ children

- Number analysed (data analysis at post-treatment stage): 47

- Number of participants eligible assigned: 59

- Number of participants dropped out (by final follow-up): 12

- Comorbidities: NR

- Experience of IPV: the kinds of IPV that women reported were quite severe: $83 \%$ had been slapped, hit or punched, $79 \%$ had been pushed or shoved, $50 \%$ had been choked, $46 \%$ had been kicked, $46 \%$ had been raped and $16 \%$ had been threatened with a weapon

- Experience of other types of violence and abuse: NR

- Remuneration: each participant received USD 50 compensation upon completion of the 4-month follow-up assessment.

- Financial dependence (on partner): NR

Included criteria: inclusion criteria included having been a victim of IPV, being $>18$ years of age and the ability to converse and write in English. Thus, we included women who had been abused in dating relationships in which they were not living with the abusive partner as well as women who had lived with their partners. Also, each woman was required to be living in conditions that she judged as providing herself safety from the abuse, as this trial's intervention was not considered to be appropriate for addressing the safety issues of women who were currently involved in abusive relationships.

Excluded criteria: women were excluded from this trial if they had been romantically involved with their abusive partners within the previous 30 days or if they had lived with them within the previous 6 months.

Pretreatment: 2 significant differences were found, with $28 \%$ of the women in the expressive writing group married, compared to $9 \%$ of the neutral writing group, and with the women in the expressive writing group significantly higher $(P=0.01)$ in completed education (mean $=16.8$ years, $S D=3.0)$ compared with the control group $($ mean $=14.8$ years, $S D=1.9)$

Number eligible: NR

Number ineligible: NR

Number of eligible people consented: 59

Number of eligible people recruited: 59 
Recruitment setting of participants (e.g. healthcare setting, community setting, shelter setting): community

\section{Sample power calculation: NR}

Total number of participants approached: NR

Total number of participants within all groups: 47

Number declined to participate: NR

\section{Intervention characteristics}

Experimental intervention

- Brief description (rationale, main components): a number of studies have focused on the benefits of expressive writing in healthy populations. This approach of repeated narrative construction promotes cognitive processing of the event. Such cognitive processing should convert traumatic memories into normal memories, whose emotional intensity fades over time, resulting in less intrusive thoughts, increased emotion regulation and decreased autonomic arousal linked to activation of stressful thoughts and memories. For the expressive writing task, women were instructed to explore their deepest emotions and feelings

- Type of intervention: expressive writing

- Mode of delivery (how): these instructions were inserted within a journal that each woman used for all of her writing sessions. Each woman was asked to do the writing without discussing it with the research assistant

- Frequency and duration of delivery (when and how much): each writing session was 20 min in duration and each woman completed 4 writing sessions, scheduled at weekly intervals.

- Level of mental health training of person delivering the intervention: as a precaution, our research team included a clinical licensed psychologist (CC) who was available on a beeper for a preliminary evaluation and possible referral of any woman who was traumatised by the writing intervention

- Fidelity assessment: the writing sessions were strictly timed by the research assistant to standardise their length. All writing sessions occurred with the research assistant present, which assured adherence to the task of writing

- Intervention model: NR

- Person delivering the intervention (who provided the intervention): therapist, self-directed or combined: combined

- Intervention location (where): at the preference of each woman, these writing sessions were either done in a university office or at a coffee shop or restaurant near the participant's residence.

- Tailoring of intervention (personalised, titrated or adapted): NR

- Intervention modifications (during the trial): NR

- Main techniques of intervention: writing

- Intervention manual: NR

- Intervention attendance: NR

- CCDAN psychological therapies: humanistic therapies

- Intensity of intervention: up to 4 sessions

- Person delivering the intervention: healthcare workers

- Gender of therapist: NR

Comparator intervention

- Brief description (rationale, main components): the neutral writing task, women were asked to write about how they used their time and were further instructed: 'I am not interested in your emotions or opinions. Rather be as objective as possible.'

- Type of intervention: neutral writing

Outcomes

\section{Depression}

- Outcome type: continuous outcome 
Koopman 2005 (Continued)

- Reporting: fully reported

- Scale: BDI

- Direction: lower is better

PTSD

- Outcome type: continuous outcome

- Reporting: fully reported

- Scale: PTSD Checklist-Specific Version

- Direction: lower is better

\section{Dropouts from treatment}

- Outcome type: dichotomous outcome

- Reporting: fully reported

- Direction: lower is better

Identification

Sponsorship source: Drs Bala Manian and Tasneem Ismailji

Country: USA

Setting: community

Comments: San Francisco Bay Area of California

Trialauthors: Cheryl Koopman, Tasneem Ismailji, Danielle Holmes, Catherine C. Classen, Oxana Palesh, \& Talor Wales

Institution: Stanford University School of Medicine

Email:Koopman@stanford.edu

Address: Department of Psychiatry and Behavioral Sciences, Stanford University, Stanford, CA 943055718, USA

Trial title (main outcome publication): The effects of expressive writing on pain, depression and posttraumatic stress disorder symptoms in survivors of intimate partner violence

Registered trial protocol ID: NR

Notes

Analysis of any cost/benefit measures: NR

Process evaluation: NR

Summary of participants' views about the intervention: NR

Adverse events (harm) related to participation: as a precaution, our research team included a clinical licensed psychologist (CC) who was available on a beeper for a preliminary evaluation and possible referral of any woman who was traumatised by the writing intervention. Fortunately, this did not occur.

\section{Risk of bias}

\begin{tabular}{lll}
\hline Bias & Authors' judgement & Support for judgement \\
\hline $\begin{array}{l}\text { Random sequence genera- } \\
\text { tion (selection bias) }\end{array}$ & High risk & $\begin{array}{l}\text { Judgement comment: "59 women in the San Francisco Bay Area of California } \\
\text { were recruited through fliers, newspaper advertisements, and electronic bill- } \\
\text { board postings. Each participant was randomly assigned to either the expres- } \\
\text { sive writing condition in which she wrote about the most stressful event(s) of } \\
\text { her life or to the neutral writing condition in which she wrote about her daily } \\
\text { schedule". However, no further information mentioned about randomisation } \\
\text { method and sequence generation. }\end{array}$ \\
&
\end{tabular}


Koopman 2005 (Continued)

Allocation concealment Unclear risk Judgement comment: "47 women completed baseline and 4-month follow-up (selection bias) assessments and were randomly assigned to 4 writing sessions of either expressive writing focused on traumatic life events or writing about a neutral topic, which the participant completed individually". However, allocation concealment remains unclear.

Blinding of participants and personnel (performance bias)

All outcomes
Unclear risk

Judgement comment: "each woman was asked to do the writing without discussing it with the research assistant, to ensure that the intervention was restricted to the writing and also to keep the research assistant blind to the writing instructions condition to which each woman was assigned". However, there is no further information to assess the blinding of participants and personnel.
Blinding of outcome as- Unclear risk sessment (detection bias) All outcomes
Judgement comment: "after each woman provided informed consent for her participation, she completed the questionnaires assessing demographic characteristics, bodily pain and depression and PTSD symptoms". However, no further information mentioned about blinding outcome assessors.
Incomplete outcome data Low risk (attrition bias)

All outcomes
Judgement comment: attrition and exclusions were adequately described. At a follow-up assessment conducted 4 months following the conclusion of the writing intervention, 47 of the 59 women (an $80 \%$ follow-up rate) completed reassessments of their symptoms of pain, depression, and PTSD. All measures mentioned in the method section were collected, analysed and reported in the results section. Although, there was no published trial protocol, this risk appears to be low.

Selective reporting (re- Unclear risk
porting bias)

Judgement comment: all outcomes described in the methods section were adequately collected, analysed and reported in the results section of the main published trial. However, there was insufficient or unclear evidence from either the trial protocol or trial registry sites to assess this domain.

Other bias Low risk Judgement comment: no other biases detected

Kubany 2004

\section{Study characteristics}

Methods

Trial design: randomised treatment-outcome experiment

Trial grouping: parallel group

Assessment modality (e.g. face-to-face, telephone, online): face-to-face and telephone

Conflict of interest of trial authors: none declared

Funding for trial: this trial was support by Grant N980207 from the TriService Nursing Research Group, Department of Defense

ITT analyses: yes

Primary outcomes: Clinician-Administered PTSD Scale; Distressing Event Questionnaire; Beck Depression Inventory; Rosenberg Self-Esteem Scale;

Randomisation method: every 2 consecutive women determined to be eligible were randomly assigned either to an immediate CTT-BW condition or to a delayed CTT-BW condition

Secondary outcomes: TSources of Trauma-Related Guilt Survey-Partner Abuse Version; Personal Feelings Questionnaire; Client Satisfaction Questionnaire 
Timing of outcome measurements: baseline, post-treatment, 3-months' follow-up and 6 months' follow-up

Total duration of trial: NR

Type of analysis: Descriptivr statistics, ANOVA, MANOVA,

Types of participants: participants included 125 formerly battered women, most of whom were referred by victim services agencies that serve battered women in Hawaii

Participants

Baseline characteristics

Experimental intervention

- Age: NR

- Ethnicity: NR

- Employment: NR

- Education: NR

- Relationship status: NR

- Gender (\% women): 100

- Dependent children: NR

- Number analysed (data analysis at post-treatment stage): 45

- Number of participants eligible assigned: 46

- Number of participants dropped out (by final follow-up): 14

- Comorbidities: NR

- Experience of IPV: NR

- Experience of other types of violence and abuse: NR

- Remuneration: NR

- Financial dependence (on partner): NR

Comparator intervention

- Age: NR

- Ethnicity: NR

- Employment: NR

- Education: NR

- Relationship status: NR

- Gender (\% women): 100

- Dependent children: NR

- Number analysed (data analysis at post-treatment stage): 39

- Number of participants eligible assigned: 40

- Number of participants dropped out (by final follow-up): 10

- Comorbidities: NR

- Experience of IPV: NR

- Experience of other types of violence and abuse: NR

- Remuneration: NR

- Financial dependence (on partner): NR

Total sample

- Age: $\mathrm{M}=42.2$ years, $\mathrm{SD}=10.1$

- Ethnicity: participants' ethnic backgrounds were diverse and included white $(n=66)$, Native Hawaiian ( $n=11$ ), Filipino ( $n=9)$, Japanese ( $(n)$, Black $(n=6)$, Samoan $(n=6)$, American Indian ( $n=2)$, and other or mixed ethnicity $(n=17)$

- Employment: NR

- Education: participants' levels of education ranged from 5th grade to a doctorate, with a mean of 13.5 years $(S D=2.4)$ 
- Relationship status: NR

- Gender (\% women): 100

- Dependent children: NR

- Number analysed (data analysis at post-treatment stage): 84

- Number of participants eligible assigned: 86

- Number of participants dropped out (by final follow-up): 24

- Comorbidities: NR (although descriptives provided about specific types of traumatic events)

- Experience of IPV: all participants had been physically, sexually, and/or psychologically abused (e.g. threatened, stalked, badgered, humiliated) by an intimate or romantic partner. $68 \%$ of the sample (n 85 ) reported having been physically hurt by intimate partners $>5$ times, and $51 \%$ ( $n 64$ ) had been physically hurt by $>1$ intimate partner

- Experience of other types of violence and abuse: most participants reported histories of multiple traumatizations in addition to partner abuse. Participants reported experiencing intense fear, helplessness, or horror in response to a mean 9.0 (SD $=4.2)$ types of events listed on the Traumatic Life Events Questionnaire

- Remuneration: NR

- Financial dependence (on partner): NR

Included criteria: women qualified for participation if they (a) had been out of an abusive relationship for at least 30 days with no intention of reconciling, (b) had not been physically or sexually abused or stalked by anyone for at least 30 days, (c) met diagnostic criteria for partner abuse-related PTSD, (d) obtained a score on the Global Guilt Scale of the TRGI reflecting at least moderate abuse-related guilt, (e) were not currently abusing alcohol or drugs, and (f) did not have schizophrenia or bipolar disorder

Excluded criteria: NR

Pretreatment: there were no significant differences on any of the comparisons, suggesting that random assignment was effective in cancelling out error related to relevant measured variables.

Number declined to participate: NR

Number eligible: NR

Number ineligible: NR

Number of eligible people consented: 125

Number of eligible people recruited: NR

Recruitment setting of participants (e.g. healthcare setting, community setting, shelter setting): community (most of whom were referred by victim services agencies that serve battered women in Hawaii)

\section{Sample power calculation: NR}

Total number of participants approached: NR

Total number of participants within all groups: 125

Experimental intervention

- Brief description (rationale, main components): cognitive trauma therapy for battered women with PTSD, a psycho-educational, multi-component, cognitive-behavioral intervention aimed at alleviating PTSD, depression, guilt, shame, and negative self-esteem in formerly battered women

- Type of intervention: cognitive trauma therapy

- Mode of delivery (how): individual-therapy format

- Frequency and duration of delivery (when and how much): cognitive trauma therapy for battered women (CTT-BW) was conducted in a 2-session/week, individual-therapy format-designed for implementation in $8-11$ sessions of $1.5 \mathrm{~h}$ for most clients 
- Level of mental health training of person delivering the intervention: CTT-BW was conducted by Edward S. Kubany and 6 other individuals - 1 man and 5 women - who were trained to conduct CTT-BW by him. The man is a clinical psychologist with postdoctoral training in PTSD. Among the women, 2 have advanced degrees in nursing, one has a master's degree in counselling psychology and also works as a victim witness advocate, and 2 have baccalaureate degrees and several years of experience in the field of domestic violence as counsellors and educators. All the CTT-BW therapists had completed multi-day workshops on domestic violence

- Fidelity assessment: all therapy sessions were audio-taped. Using CTT-BW therapist-adherence rating scales, therapist adherence ratings were obtained for 60 therapy sessions (approximately $7.5 \%$ of all sessions)

- Intervention model: cognitive-behavioral model

- Person delivering the intervention (who provided the intervention): therapist, self-directed or combined: therapist

- Intervention location (where): NR

- Tailoring of intervention (personalised, titrated or adapted): self-monitoring recording form used to address negative self-talk each day

- Intervention modifications (during the trial): NR

- Main techniques of intervention: the main treatment components in CTT-BW include (1) exploration of partner abuse history and exposure to other trauma; (2) psycho education on PTSD; (3) negotiation of imaginal and invivo exposure homework; (4) psycho-education on maladaptive self-talk; (5) stress management and relaxation training; (6) cognitive therapy for trauma-related guilt; (7) psycho-education on assertiveness and responses to verbal aggression; (8) managing unwanted contacts with former partners; (9) learning to identify potential perpetrators and avoid re-victimisation; and (10) psycho-education on positive coping strategies that focus on self-advocacy and self-empowerment (e.g. placing oneself first, decision-making that promotes self interest

- Intervention manual: CTT-BW was conducted following a preliminary 55-page procedural manual and 30-page therapist-client workbook

- CCDAN psychological therapies: third-wave CBTs

- Intervention attendance: the 46 women who completed immediate CTT-BW received between 8 and 17 therapy sessions, with a mean of 9.5 sessions $(\mathrm{SD}=1.6)$ and a mode of 9 sessions

- Intensity of intervention: $\geq 5$ sessions

- Person delivering the intervention: healthcare workers

- Gender of therapist: $80 \%$ female

Comparator intervention

- Brief description (rationale, main components): delayed treatment

- Type of intervention: waiting list

\section{Depression}

- Outcome type: continuous outcome

- Reporting: fully reported

- Scale: BDI

- Direction: lower is better

\section{PTSD}

- Outcome type: continuous outcome

- Reporting: fully reported

- Scale: Clinician Administered PTSD Scale

- Direction: lower is better

\section{Dropouts from treatment}

- Outcome type: dichotomous outcome

- Reporting: fully reported 
Kubany 2004 (Continued)

$$
\text { - Direction: lower is better }
$$

Identification

Sponsorship source: this trial was supported by Grant N980207 from the TriService Nursing Research Group, Department of Defense. The trial is also based on work supported in part by the Office of Research and Development, Department of Veterans Affairs, Honolulu, Hawaii

Country: USA

Setting: community

Comments: participants included 125 formerly battered women, most of whom were referred by victim services agencies that serve battered women in Hawaii

Trialauthors: Kubany ES, Hill EE, Owens JA, Iannce-Spencer C, McCaig MA, Tremayne KJ, \& Williams PL

Institution: National Center for Posttraumatic Stress Disorder (PTSD), Department of Veterans Affairs, Honolulu, Hawaii

Email: kubany@hawaii.rr.com

Address: National Center for PTSD, Pacific Islands Division, Department of Veterans Affairs, 1132 Bishop Street, Suite 307, Honolulu, HI 96813.

Title (main 'outcome' publication): Cognitive trauma therapy for battered women with PTSD (CTTBW)

Registered trial protocol ID: NR

Notes Analysis of any cost/benefit measures: NR

Process evaluation: therapist adherence to the CTT-BW protocol was assessed using CTT-BW therapist-adherence rating scales. In addition, the Client Satisfaction Questionnaire assessed post-service client satisfaction. However, there was insufficient information to assess how the researchers assessed processes related to how the intervention was implemented and the observed treatment effect.

Summary of participants' views about the intervention: the percentage of participants who were maximally satisfied with services received (on the basis of CSQ-8 scores of 32 ) was $64 \%, 67 \%$, and $72 \%$ at post-test, 3-month follow-up, and 6-month follow-up, respectively. However, there was insufficient information about participants' qualitative comments and views about the intervention.

Adverse events (harm) related to participation: NR

\section{Risk of bias}

\begin{tabular}{lll}
\hline Bias & Authors' judgement & Support for judgement \\
\hline $\begin{array}{l}\text { Random sequence genera- } \\
\text { tion (selection bias) }\end{array}$ & High risk & $\begin{array}{l}\text { Judgement comment: "participants included } 125 \text { formerly battered women, } \\
\text { most of whom were referred by victim services agencies that serve battered } \\
\text { women in Hawaii". There is no evidence of sequence generation. }\end{array}$ \\
\hline
\end{tabular}

Allocation concealment High risk
(selection bias)

Judgement comment: "every 2 consecutive women determined to be eligible were randomly assigned either to an immediate CTT-BW condition or to a delayed CTT-BW condition". No further information provided about allocation concealment.

\begin{tabular}{|c|c|c|}
\hline $\begin{array}{l}\text { Blinding of participants } \\
\text { and personnel (perfor- } \\
\text { mance bias) } \\
\text { All outcomes }\end{array}$ & Unclear risk & $\begin{array}{l}\text { Judgement comment: there is insufficient information about the blinding of } \\
\text { participants and personnel. }\end{array}$ \\
\hline $\begin{array}{l}\text { Blinding of outcome as- } \\
\text { sessment (detection bias) }\end{array}$ & Low risk & $\begin{array}{l}\text { Judgement comment: the assessors were blind to participants' condition as- } \\
\text { signments, and none served as therapists in the trial. }\end{array}$ \\
\hline
\end{tabular}


Kubany 2004 (Continued)

All outcomes

Incomplete outcome data Low risk (attrition bias)

All outcomes
Judgement comment: attrition and exclusions were reasonably described, however, reasons for dropouts were not adequately described. Of 63 women assigned to the immediate CTT-BW condition, 4 did not start treatments, and 13 did not complete treatment. Of 62 women assigned to the delayed CTTBW condition, 14 did not start treatment (10 dropped out before their second pre-therapy assessment) and 13 did not complete treatment. Neverthelss, ITT analysis was conducted to examine effects of attrition.

Selective reporting (re- Unclear risk
porting bias)

Judgement comment: all outcomes described in the methods section were adequately collected, analysed and reported in the results section of the main published trial. However, there was insufficient or unclear evidence from either the trial protocol or trial registry sites to assess this domain.

\begin{tabular}{ll}
\hline Other bias $\quad$ Low risk Judgement comment: none \\
\hline
\end{tabular}

Michalopoulou 2015

\section{Study characteristics}

\section{Methods}

Trial design: $\mathrm{RCT}$

Trial grouping: parallel group

Assessment modality (e.g. face-to-face, telephone, online): face-to-face

\section{Conflict of interest of trial authors: NR}

Funding for trial: NR

Primary outcomes: Perceived Stress Scale, Health Locus of Control, BDI, Ways of Coping Checklist

Randomisation method: participants were randomised to the intervention or control group according to a list of random permutations prepared by computer-generated blocked randomisation (www.random.org). Randomisation, baseline, and final measurements were not blinded.

\section{Secondary outcomes: NR}

Timing of outcome measurements: after obtaining written consent, the research assistant administered the baseline survey. The last measurement assessment after the 8-week follow-up period took place in July 2013 (recruitment stopped in May 2013 after reaching a total of 34 eligible patients)

Total duration of trial: September 2012-July 2013

Type of analysis: for group comparisons, Pearson's Chi2 , student's t-test, or the Mann-Whitney U-test were used according to normality

Types of participants: most of the participants were middle-aged and married with children. Furthermore, most of them completed secondary education, were employed, and were smokers.

ITT analysis: NR 
- Education: primary, 1 (6.3\%); secondary, 13 (81.2\%); tertiary, 2 (12.5\%)

- Relationship status: 9 (56.3\%) married

- Gender (\% women): 100

- Dependent children: 13 (81.3\%) reported having children

- Number analysed (data analysis at post-treatment stage): 16

- Number of participants eligible assigned: 17

- Number of participants dropped out (by final follow-up): 1

- Comorbidities: NR

- Experience of IPV: NR

- Experience of other types of violence and abuse: Perceived Stress Scale Score was 29.50 (21-42); no other types of violence and abuse reported

- Remuneration: relaxation CD

- Financial dependence (on partner): NR

Comparator intervention

- Age (median, min-max): 38 years (24-66)

- Ethnicity: NR

- Employment: employed 12,66.7\%

- Education: primary, 11 (11.1\%); secondary, 9 (50\%), tertiary, 7 (38.9\%)

- Relationship status: 6 (33.3\%) married

- Gender (\% women): 100

- Dependent children: 12 (66.7\%) reported having children

- Number analysed (data analysis at post-treatment stage): 18

- Number of participants eligible assigned: 18

- Number of participants dropped out (by final follow-up): 0

- Comorbidities:

- Experience of IPV: NR

- Experience of other types of violence and abuse: Perceived Stress Scale Score was 25 (17-40); no other types of violence and abuse reported

- Remuneration: relaxation CD

- Financial dependence (on partner): NR

Total sample

- Age (median, min-max): most of the participants were middle-aged

- Ethnicity:

- Employment:

- Education: most of participants completed secondary education

- Relationship status: most of the participants were married with children

- Gender (\% women):

- Dependent children:

- Number analysed (data analysis at post-treatment stage): 34

- Number of participants eligible assigned: 35

- Number of participants dropped out (by final follow-up): 1

- Comorbidities:

- Experience of IPV: all participants who self-identified as verbally, emotionally, physically, and/or sexually abused by a man were invited to participate

- Experience of other types of violence and abuse:

- Remuneration: relaxation CD

- Financial dependence (on partner): NR

Included criteria: all participants who self-identified as verbally, emotionally, physically, and/or sexually abused by a man were invited to participate. Other inclusion criteria included being $>18$ years of 

30 days with no intention of reconciling.

Excluded criteria: potential participants were excluded if they were using psychotropic drugs (e.g. antidepressants, benzodiazepines, antipsychotics, cannabis, or other stimulants) or if they were practicing other relaxation techniques (e.g. yoga, pilates, meditation, psychotherapy).

Pretreatment: there were no significant baseline differences between the 2 trial groups

Number eligible: 35

Number ineligible: 1

Number of eligible people consented: 35

Number of eligible people recruited: 35

Recruitment setting of participants (e.g. healthcare setting, community setting, shelter setting): healthcare/community

Sample power calculation: NR

Total number of participants approached: 36

Total number of participants within all groups: 34

Number declined to participate: 0

\section{Intervention characteristics}

Experimental intervention

- Brief description (rationale, main components): relaxation breathing (RB) and progressive muscle relaxation (PMR)

- Type of intervention: stress management

- Mode of delivery (how): women who were randomly assigned into the intervention group were trained in RB and PMR. Both RB and PMR were administered in the form of an audio CD, consisting of $10 \mathrm{~min}$ of RB and 15 min of PMR. Moreover, counselling in the intervention group was provided as soon as a problem occurred. Counselling interventions were brief and often problem-specific, mainly aimed to prevent women from IPV.

- Frequency and duration of delivery (when and how much): abused women were instructed to practice the guided RB-PMR CD twice a day for 8 weeks at home

- Level of mental health training of person delivering the intervention: Not applicable: audio-CD stress management

- Fidelity assessment: NR

- Intervention model: NR

- Person delivering the intervention (who provided the intervention): therapist, self-directed or combined: combined

- Intervention location (where): the trial was conducted at a non-government organisation called Frontida, a family violence unit of Athens. Each woman consulted in a private room with the researcher

- Tailoring of intervention (personalised, titrated or adapted): NR

- Intervention modifications (during the trial): NR

- Main techniques of intervention: Relaxation Breathing (RB) and Progressive Muscle Relaxation (PMR)

- Intervention manual: NR

- Intervention attendance: NR

- CCDAN psychological therapies: CBT

- Intensity of intervention: $\geq 5$ sessions

- Person delivering the intervention: unclear

- Gender of therapist: NR 
Michalopoulou 2015 (Continued)

\section{Comparator intervention}

- Brief description (rationale, main components): the women in the control group continued to receive the standard shelter services, including meals, shelter, job searching, legal issues surrounding divorce, and social services appointments

- Type of intervention: standard usual care

\begin{tabular}{ll}
\hline Outcomes & Dropouts from treatment \\
- Outcome type: dichotomous outcome \\
- Reporting: fully reported \\
- Direction: lower is better
\end{tabular}

Identification

Sponsorship source: NR

Country: Greece

Setting: Shelter and family violence unit

Comments: the trial was conducted at a non-government organization called "Frontida", a family violence unit of Athens and also with the referral of a church located in Loutraki, Korinthos,between September 2012 and May 2013

Trialauthors: Eleni Michalopoulou, Georgia Tzamalouka, George P. Chrousos, Christina Darviri

Institution: School of Medicine, University of Athens

Email: stressmasterssubmissions@yahoo.gr

Address: School of Medicine, University of Athens, Soranou Ephessiou Str., 4,GR-115-27 Athens, Greece

Trial title (main outcome publication): Stress Management and Intimate Partner Violence: a Randomized Controlled Trial

Registered trial protocol ID: NR

Notes

Analysis of any cost/benefit measures: NR

Process evaluation: NR

Summary of participants' views about the intervention: NR

Adverse events (harm) related to participation: Despite this, RB-PMR is a simple relaxation technique for abused women. It should be deemed as highly cost-effective with practice feasibility and no harm or adverse effects. However the trial did not report adverse events (harm) related to participation.

\section{Risk of bias}

\begin{tabular}{lll}
\hline Bias & Authors' judgement & Support for judgement \\
\hline $\begin{array}{l}\text { Random sequence genera- } \\
\text { tion (selection bias) }\end{array}$ & Low risk & $\begin{array}{l}\text { Judgement comment: "participants were randomised to the intervention or } \\
\text { control group according to a list of random permutations prepared by com- } \\
\text { puter-generated blocked randomisation (www.random.org)". }\end{array}$ \\
\hline $\begin{array}{l}\text { Allocation concealment } \\
\text { (selection bias) }\end{array}$ & High risk & $\begin{array}{l}\text { Judgement comment: randomisation, baseline, and final measurements were } \\
\text { not blinded. Further, there is no evidence to suggest adequate allocation con- } \\
\text { cealment. }\end{array}$ \\
\hline $\begin{array}{l}\text { Blinding of participants } \\
\text { and personnel (perfor- } \\
\text { mance bias) }\end{array}$ & High risk & $\begin{array}{l}\text { Judgement comment: randomisation, baseline, and final measurements were } \\
\text { not blinded. There is no evidence about the blinding of participants and per- } \\
\text { sonnel. }\end{array}$ \\
\hline
\end{tabular}


Michalopoulou 2015 (Continued)

All outcomes

\begin{tabular}{|c|c|c|}
\hline $\begin{array}{l}\text { Blinding of outcome as- } \\
\text { sessment (detection bias) }\end{array}$ & High risk & $\begin{array}{l}\text { Judgement comment: randomisation, baseline, and final measurements were } \\
\text { not blinded. There is no evidence about the blinding of outcome assessors. }\end{array}$ \\
\hline
\end{tabular}

All outcomes

$\begin{array}{ll}\begin{array}{l}\text { Incomplete outcome data } \\ \text { (attrition bias) }\end{array} & \text { Low risk } \\ \text { All outcomes } & \begin{array}{l}\text { Judgement comment: attrition and exclusions were adequately reported } \\ \text { and all outcome measures mentioned in the method sections were collected, } \\ \text { analysed and fully reported in the results section. }\end{array}\end{array}$

All outcomes analysed and fully reported in the results section.

Selective reporting (re- Unclear risk porting bias)

Judgement comment: all outcomes described in the methods section were adequately collected, analysed and reported in the results section of the main published trial. However, there was insufficient or unclear evidence from either the trial protocol or trial registry sites to assess this domain.

Other bias Low risk Judgement comment: none

\section{Myers 2015}

\section{Study characteristics}

Methods
Trial design: RCT

Trial grouping: parallel group

Assessment modality (e.g. face-to-face, telephone, online): face-to-face

Conflict of interest of trial authors: the trial authors report no financial relationships with commercial interests.

Funding for trial: this trial was funded by K23AA015707 (SN), T32AA013525 (KB, UM), and F31AA018909 (KB). NIAAA (AA015707)

\section{Primary outcomes: CAPS}

Randomisation method: NR

Secondary outcomes: NR

Timing of outcome measurements: baseline, mid-treatment (6-weeks), post-treatment, and 3- and 6month follow-up

Total duration of trial: NR

Type of analysis: t-tests and $\mathrm{Chi}^{2}$ tests, logistic regression, multivariate model

Types of participants: female survivors of IPV

ITT analysis: NR

\section{Participants}

\section{Baseline characteristics}

Experimental intervention

- Age: NR

- Ethnicity: NR

- Employment: NR

- Education: NR

- Relationship status: NR 
- Gender (\% women): 100

- Dependent children: NR

- Number analysed (data analysis at post-treatment stage): 14

- Number of participants eligible assigned: 31

- Number of participants dropped out (by final follow-up): 17

- Comorbidities: NR

- Experience of IPV: past experience

- Experience of other types of violence and abuse:

- Remuneration: 25 USD

- Financial dependence (on partner): NR

Comparator intervention

- Age: NR

- Ethnicity: NR

- Employment: NR

- Education: NR

- Relationship status: NR

- Gender (\% women): 100

- Dependent children: NR

- Number analysed (data analysis at post-treatment stage): 4

- Number of participants eligible assigned: 9

- Number of participants dropped out (by final follow-up): 5

- Comorbidities: NR

- Experience of IPV: past experience

- Experience of other types of violence and abuse:

- Remuneration: 25 USD

- Financial dependence (on partner): NR

Total sample

- Age: $M=42.23$ years, $S D=10.48$

- Ethnicity: white: 24 (55.8\%); African American: 5 (11.6\%); Hispanic: 10 (27.5\%);

- Employment: NR

- Education: 12.31 years, $\mathrm{SD}=2.81$

- Relationship status: NR

- Gender (\% women): 100

- Dependent children: 13 (32.5\%)

- Number analysed (data analysis at post-treatment stage): 40

- Number of participants eligible assigned: 40

- Number of participants dropped out (by final follow-up): 22

- Comorbidities: NR

- Experience of IPV: NR

- Experience of other types of violence and abuse: NR

- Remuneration: 25 USD

- Financial dependence (on partner): NR

Included criteria: participants were: (a) women $>18$ years old, (b) at least 1 month out of the abusive relationship, (c) currently meeting DSM-IV criteria for PTSD, and (d) English-speaking

Excluded criteria: exclusion criteria for women were: (a) moderate or severe cognitive impairment (mini-mental state examination score of $\leq 18$ ), and (b) history of psychosis or mania not well managed by pharmacotherapy for the most recent 6 -month period. 
Pretreatment: there was no significant difference between Seeking Safety and facilitated twelve-step in treatment engagement ( 14 of $31,45.2 \%$ vs 4 of $9,44.4 \%$, respectively; Chi $2=0.28, d f=1, P>0.05$ ). There were also no significant differences between treatment conditions in post-treatment PTSD symptoms based on the CAPS ( $M=50, S D=26.7$ in Seeking Safety; $M=45.5, S D=37.5$ in facilitated twelvestep); $t(16)=0.27, P>.05$, or in post-treatment percentage of days drinking in the past month $(M=$ 13.23, $S D=21.25$ in Seeking Safety; $M=13.5, S D=4.95$ in facilitated twelve-step); $t(16)=0.025, P>0.05$. With regard to therapy condition, compared to women who did not engage in Seeking Safety, engagers were significantly older $(P=.012)$; there were no significant differences in age or other demographics among women who engaged in facilitated twelve-step compared to dropouts. None of the other demographics differed significantly by treatment condition.

Number eligible: 40

Number ineligible: 27

Number of eligible people consented: 40

Number of eligible people recruited: 40

Recruitment setting of participants (e.g. healthcare setting, community setting, shelter setting): community, healthcare

\section{Sample power calculation: NR}

Total number of participants approached: 78

Total number of participants within all groups: 40

Number declined to participate: 2

\section{Intervention characteristics}

Experimental intervention

- Brief description (rationale, main components): Seeking Safety is a present-centred therapy that focuses on current PTSD and alcohol use symptoms. The treatment has 25 sessions with topics related to its 5 principles: (1) the first priority is safety; (2) treatment for both disorders is integrated; (3) focus on ideals; (4) cognitive, behavioral, interpersonal, and case management content areas; and (5) attention to therapist processes.

- Type of intervention: group therapy (Seeking Safety), individual therapy

- Mode of delivery (how): face-to-face

- Frequency and duration of delivery (when and how much): the 25 therapy sessions were conducted twice a week over a 12-week period. 90-minute sessions

- Level of mental health training of person delivering the intervention: individual therapists included 2 postdoctoral level psychologists. Group therapy was co-led by a psychologist and a doctoral student in clinical psychology. Interventions were co-delivered by senior clinicians (e.g. clinical staff or postdoctoral fellows) and doctoral students

- Fidelity assessment: monitoring occurred via manual review, direct observation, review of audio recordings, and weekly supervision by the trial principal investigator (S.B.) and co-investigators

- Intervention model: Seeking Safety

- Person delivering the intervention (who provided the intervention): therapist, self-directed or combined: therapist

- Intervention location (where): community-based outpatient clinic

- Tailoring of intervention (personalised, titrated or adapted): NR

- Intervention modifications (during the trial): at the start of the trial, both treatments were delivered in a group format with cohort admission; however, due to scheduling and recruitment difficulties, halfway through the trial the format was changed to individual treatment

- Main techniques of intervention:

- Intervention manual: Seeking Safety is a present-centred therapy that focuses on current PTSD and alcohol use symptoms (Najavits et al., 1998) 
- Intervention attendance: this trial examined differences in baseline characteristics between women who engaged in treatment (i.e. attended $\geq 25 \%$ of 25 psychotherapy sessions)

- CCDAN psychological therapies: third-wave CBTs

- Intensity of intervention: $\geq 5$ sessions

- Person delivering the intervention: healthcare workers

- Gender of therapist: NR

Comparator intervention

- Brief description (rationale, main components): the control condition was a manualised facilitated twelve-step group treatment, modified from Project MATCH. Facilitated twelve-step is a therapist-led supportive group using a 12-step model, which contains 3 modules designed to cover specific steps (e.g. step 1-3) over multiple sessions. Participants were encouraged to discuss issues related to abstinence from alcohol without any discussion of trauma.

- Type of intervention: minimum intervention

Dropouts from treatment
- Outcome type: dichotomous outcome
- Reporting: fully reported
- Direction: lower is better
- Data value: endpoint

Identification Sponsorship source: this trial was funded by K23AA015707 (SN), T32AA013525 (KB, UM), and F31AA018909 (KB). NIAAA (AA015707)

Country: USA

Setting: community clinics and agencies

Comments: participants were recruited from University of California, San Diego Outpatient Psychiatry Service, community clinics and agencies providing services related to intimate partner violence, and newspaper advertisements.

Trial authors: Ursula S. Myers, M.S., Dr. Kendall C. Browne, Ph.D., and Dr. Sonya B. Norman, Ph.D.

Institution: San Diego State University/University of California, San Diego Joint Doctoral Program in Clinical Psychology

Email: umyers@ucsd.edu

Address: VA San Diego Healthcare System, 3350 La Jolla Village Drive (116B), San Diego, CA 92161.

Title (main 'outcome' publication): Treatment engagement: female survivors of intimate partner violence in treatment for PTSD and alcohol use disorder

Registered trial protocol ID: NCT00607412

Notes Analysis of any cost/benefit measures: NR

Process evaluation: NR

Summary of participants' views about the intervention: NR

Adverse events (harm) related to participation: NR

\section{Risk of bias}

Bias Authors' judgement Support for judgement


Myers 2015 (Continued)

Random sequence genera- Unclear risk Judgement comment: women were randomly assigned to 25, 90-min sessions tion (selection bias) of either Seeking Safety or facilitated twelve-step. However, no further information provided about randomisation methods and sequence generation.

$\begin{array}{ll}\begin{array}{l}\text { Allocation concealment } \\ \text { (selection bias) }\end{array} & \text { Unclear risk }\end{array}$

Blinding of participants Unclear risk
and personnel (perforJudgement comment: there is insufficient information to assess the blinding mance bias) of participants and personnel. The trial mentions that the baseline assessment All outcomes consisted of both structured interviews and self-report measures (detailed below) before randomisation to Seeking Safety or facilitated twelve-step. Further, at the start of the trial, both treatments were delivered in a group format with cohort admission; however, due to scheduling and recruitment difficulties, halfway through the trial the format was changed to individual treatment.

Blinding of outcome as-
sessment (detection bias)

Judgement comment: "the baseline assessment consisted of both structured sessment (detection bias) interviews and self-report measures before randomisation to Seeking Safety or facilitated twelve-step. Participants were then assessed at mid-treatment (6-weeks), post-treatment, and 3- and 6-month follow-up". However, there is insufficient information to assess the blinding of outcome assessors.

\begin{tabular}{lll}
\hline $\begin{array}{l}\text { Incomplete outcome data } \\
\text { (attrition bias) } \\
\text { All outcomes }\end{array}$ & Low risk & $\begin{array}{l}\text { Judgement comment: attrition and exclusion were adequately reported. This } \\
\text { paper reports about women who engaged in therapy (treatment engagement), } \\
\text { versus those who did not. }\end{array}$ \\
\hline $\begin{array}{l}\text { Selective reporting (re- } \\
\text { porting bias) }\end{array}$ & Unclear risk & $\begin{array}{l}\text { Judgement comment: all outcomes described in the methods section were ad- } \\
\text { equately collected, analysed and reported in the results section of the main } \\
\text { published trial. However, there was insufficient or unclear evidence from ei- } \\
\text { ther the trial protocol or trial registry sites to assess this domain. }\end{array}$ \\
\hline Other bias & Unclear risk & $\begin{array}{l}\text { Judgement comment: there is insufficient information about various aspects } \\
\text { of the trial. Overall, this trial is about the treatment engagement of female sur- } \\
\text { vivors of IPV in treatment for PTSD and alcohol use disorder. Outcome data are } \\
\text { not presented. }\end{array}$ \\
\hline
\end{tabular}

Orang 2017

\section{Study characteristics}

Methods

Trial design: RCT

Trial grouping: parallel group

Assessment modality (e.g. face-to-face, telephone, online): telephone, face-to-face

Conflict of interest of trial authors: the trial authors report no financial relationships with commercial interests

Funding for trial: this trial was funded by K23AA015707 (SN), T32AA013525 (KB, UM), and F31AA018909 (KB). NIAAA(AA015707).

Primary outcomes: Post-traumatic Stress Symptom Scale-Interview, PHQ-9, Perceived Stress Scale-4

Randomisation method: randomisation was implemented with the psych package in R statistical software

Secondary outcomes: CAS, LEC Modified Adverse Childhood Experiences, Work and Social Adjustment Scale, Borderline Symptom List-23 
Total duration of trial: each therapy period lasted between 3 and 6 months, due to various cancellations and interruptions, such as IPV occurrence during the therapy, everyday problems, or unexpected journeys or illness

Type of analysis: the trial hypotheses were examined through the use of mixed designs, with symptom change as a 3-level repeated measures variable and treatment group as a 2-level between-subject variable for each outcome measure. We tested interactions between within-subject and between-subject variables to examine different effects of treatment groups over time. Mauchly's tests of sphericity were calculated for the repeated measures analysis of variance

Types of participants: IPV-affected women with a diagnosis of PTSD

\section{ITT analysis: NR}

Participants

\section{Baseline characteristics}

Experimental intervention

- Age: $M=38.04$ years, $S D=9.69$

- Ethnicity: Iranian women

- Employment: working (full-or part-time job) 8, 33.3\%

- Education: under diploma: 10 (41.7\%); diploma and above: 14 (58.7\%)

- Relationship status: $100 \%$ married

- Gender (\% women): 100

- Children: number of children: 0: $16.7 \%$; 1 or 2: $58.4 \% ; 3-5: 25 \%$

- Number analysed (data analysis at post-treatment stage): 17

- Number of participants eligible assigned: 24

- Number of participants dropped out (by final follow-up): 7

- Comorbidities: use of physical health medicine (14, 58.3\%); use of mental health medicine $(5,20.8 \%)$

- Experience of IPV: $M=50.20, S D=18.00$, past IPV

- Experience of other types of violence and abuse: number of traumatic event type: M (SD) 10.70 (3.36); childhood abuse intensity M (SD): 13.95 (10.11)

- Remuneration: NR

- Financial dependence (on partner): NR

Comparator intervention

- Age: $\mathrm{M}=37.28$ years, $\mathrm{SD}=7.92$

- Ethnicity: Iranian women

- Employment: working (full- or part-time job) 9, $42.9 \%$

- Education: under diploma: 10 (47.6\%); diploma and above: 11 (52.4\%)

- Relationship status: $100 \%$ married

- Gender (\% women): 100

- Children: number of children: 0: $4.8 \%$; 1 or 2: $66.7 \%$; 3-5: $28.6 \%$

- Number analysed (data analysis at post-treatment stage): 17

- Number of participants eligible assigned: 21

- Number of participants dropped out (by final follow-up): 4

- Comorbidities: use of physical health medicine (8, 38.1\%); use of mental health medicine $(8,38.1 \%)$

- Experience of IPV: $M=49.00, S D=16.18$, past IPV

- Experience of other types of violence and abuse: Number of traumatic event type: M (SD)10.85 (3.51); childhood abuse intensity M (SD): 14.71 (7.22)

- Remuneration: NR

- Financial dependence (on partner): NR

Total sample 


\section{- Age: NR}

- Ethnicity: the present trial was conducted in the city of Tehran, Iran. We recruited women currently living in a context of continuous IPV through health professionals, social activists, and other relevant people/institutions working in roles involving abused women

- Employment: NR

- Education: NR

- Relationship status: $100 \%$ married

- Gender (\% women): 100

- Children: NR

- Number analysed (data analysis at post-treatment stage): 34

- Number of participants eligible assigned: 45

- Number of participants dropped out (by final follow-up): 11

- Comorbidities: NR

- Experience of IPV: NR

- Experience of other types of violence and abuse: NR

- Remuneration: NR

- Financial dependence (on partner): NR

Included criteria: inclusion criteria were having experienced IPV during the past year, the diagnosis of PTSD with at least the minimum cut-off point of 15 on the Post-traumatic Stress Symptom Scale-Interview (PSS-I), age between 16-60, and a married status of living with a violent partner at the time of the interview.

Excluded criteria: exclusion criteria, which were substance abuse and suffering from schizophrenia, epilepsy, or intellectual disabilities

Pretreatment: there were no significant differences between the 2 groups in terms of socio-demographic variables and violence/traumatic experiences at baseline.

\section{Number eligible: 47}

Number ineligible: 16

Number of eligible people consented: 45

Number of eligible people recruited: 45

Recruitment setting of participants (e.g. healthcare setting, community setting, shelter setting): healthcare, community

Sample power calculation: power was limited

Total number of participants approached: 63

Total number of participants within all groups: 45

Number declined to participate: 2

\section{Intervention characteristics}

Experimental intervention

- Brief description (rationale, main components): In NET, the patient describes their autobiography, in the process of talking about their traumatic life experiences in detail. In the process of this, they intensely re-experience the emotions, thoughts, and even physical sensations associated with this event. The patient, with the help of the therapist, then shapes this into a written narrative of their life. The fragmented and intense sensory elements of the traumatic experiences are translated into an ordered verbal story and integrated into the broad narrative of the patient's life.

- Type of intervention: narrative exposure therapy (NET)

- Mode of delivery (how): face-to-face 
- Frequency and duration of delivery (when and how much): the number of completed therapy sessions ranged from 8-15, independent of the treatment group, with a mean of 11.38 and SD of 1.55. Each NET session lasted approximately 120-150 min

- Level of mental health training of person delivering the intervention: the therapy sessions were conducted by another group of 3 local counsellors (all women, with master's level psychology qualifications). We assigned therapists to one of the treatment groups, depending upon availability of therapists and the proximity to therapy settings. All 3 counsellors participated in treatment-as-usual group, but only 2 of them conducted NET. These had received training in NET through workshops held by experienced NET trainers.

- Fidelity assessment: the first sessions of NET were supervised by 1 clinical psychologist (S. A.) with expertise in NET and a local NET expert (T. O.) to ensure that the therapy sessions were in accordance with the NET manual guidelines.

- Intervention model: NET

- Person delivering the intervention (who provided the intervention): therapist, self-directed or combined: therapist

- Intervention location (where): a variety of places such as day clinics, community centres, or private institutes were chosen as locations for the interview and the treatment sessions

- Tailoring of intervention (personalised, titrated or adapted): NR

- Intervention modifications (during the trial): NR

- Main techniques of intervention: the first NET session always began with psycho-education about PTSD symptoms and explanation of the goal and procedure of treatment. Shortly afterwards, the patient, with the help of the therapist, completed the 'lifeline' exercise. This begins with a piece of thin rope that represents the course of life. The patient then places small stones of varying sizes, and flowers along this line: The stones represent violence or traumatic experiences, and flowers depict joyful events and successes. The lifeline aims to chronologically reconstruct the patient's biography, from her birth up to the present, plus their hopes or wishes for the future. It represents all the violence and traumatic experiences throughout childhood and adulthood. This helps therapist and patient to recognise the most detrimental events and determine which events will be the focus of the following 9-10 exposure sessions. Subsequent exposure sessions are devoted to detailed accounts of the most traumatic and violent experiences, which in turn activate the whole fear/trauma network and cause the patient to re-experience the same feelings, sensations, thoughts, and physiological responses that they went through during the traumatic events.

- Intervention manual: Narrative exposure therapy

- Intervention attendance: NR

- CCDAN psychological therapies: humanistic therapies

- Intensity of intervention: $\geq 5$ sessions

- Person delivering the intervention: healthcare workers

- Gender of therapist: female

Comparator intervention

- Brief description (rationale, main components): treatment as usual was defined as the commonly used psychotherapy for abused women in Iran, including life skill training and supportive counselling. The methods were chosen on the basis of the specific characteristics of the clients, and their own wishes, and ranged widely from joint sessions with an abusive husband to cognitive-behavioral techniques

- Type of intervention: treatment as usual

Outcomes

\section{Depression}

- Outcome type: continuous outcome

- Reporting: fully reported

- Scale: PHQ

- Direction: lower is better

\section{PTSD}

- Outcome type: continuous outcome 
Orang 2017 (Continued)

- Reporting: fully reported

- Scale: Posttraumatic Stress Symptoms-Interview

- Direction: lower is better

\section{Mental health}

- Outcome type: continuous outcome

- Reporting: fully reported

- Scale: Perceived Stress Scale

- Direction: lower is better

\section{Dropouts from treatment}

- Outcome type: dichotomous outcome

- Reporting: fully reported

- Direction: lower is better

Identification

Sponsorship source: this trial was funded by K23AA015707 (SN), T32AA013525 (KB, UM), and F31AA018909 (KB). NIAAA(AA015707).

Country: Iran

Setting: community

Comments: the present trial was conducted in the city of Tehran, Iran. We recruited women currently living in a context of continuous IPV through health professionals, social activists, and other relevant persons/institutions working in roles involving abused women. Consequently, a variety of places such as day clinics, community centres, or private institutes were chosen as locations for the interview and the treatment sessions

Trialauthors: Tahereh Orang, Sarah Ayoughi, James K. Moran, Hakimeh Ghaffari, Saeedeh Mostafavi, Maryam Rasoulian, Thomas Elbert

Institution: Department of Psychology, University of Konstanz, Konstanz, Germany

Email: tahereh.orang@uni-konstanz.de

Address: Department of Psychology,University of Konstanz, 78457 Konstanz,Germany

Title (main 'outcome' publication): The efficacy of narrative exposure therapy in a sample of Iranian women exposed to ongoing intimate partner violence- a randomised controlled trial

Registered trial protocol ID: NCT01731418

Notes Analysis of any cost/benefit measures: NR

Process evaluation: NR

Summary of participants' views about the intervention: NR

Adverse events (harm) related to participation: NR

\section{Risk of bias}

\begin{tabular}{lll}
\hline Bias & Authors' judgement & Support for judgement \\
\hline $\begin{array}{l}\text { Random sequence genera- } \\
\text { tion (selection bias) }\end{array}$ & Low risk & $\begin{array}{l}\text { Judgement comment: The 45 IPV-affected women were randomised to each of } \\
\text { 2 treatment arms. Randomisation was implemented with the psych package in } \\
\text { R statistical software. }\end{array}$ \\
\hline $\begin{array}{l}\text { Allocation concealment } \\
\text { (selection bias) }\end{array}$ & Unclear risk & $\begin{array}{l}\text { Judgement comment: 45 IPV-affected women with a diagnosis of PTSD were } \\
\text { randomised to } 10-12 \text { sessions of either NET }(n=24) \text { or treatment as usual }(n=\end{array}$ \\
\hline
\end{tabular}


21). Participants were randomly allocated to different conditions: 24 participants were allocated to the NET group, and 21 were offered treatment as usual. However, there is insufficient information to assess allocation concealment, hence this domain appears to be unclear.

\begin{tabular}{|c|c|c|}
\hline $\begin{array}{l}\text { Blinding of participants } \\
\text { and personnel (perfor- } \\
\text { nance bias) }\end{array}$ & Unclear risk & $\begin{array}{l}\text { Judgement comment: the interviewers were blind with respect to the treat- } \\
\text { ment. However, it is unclear whether participants and other personnel were } \\
\text { blinded. }\end{array}$ \\
\hline
\end{tabular}

All outcomes

$\begin{array}{ll}\begin{array}{l}\text { Blinding of outcome as- } \\ \text { sessment (detection bias) }\end{array} & \text { Low risk } \\ \text { All outcomes } & \text { Judgement comment: the interviewers were blind with respect to the treat- } \\ \end{array}$

All outcomes

$\begin{array}{ll}\begin{array}{l}\text { Incomplete outcome data } \\ \text { (attrition bias) }\end{array} & \begin{array}{l}\text { Judgement comment: attrition and exclusion adequately described. } 10 \text { partic- } \\ \text { ipants did not begin or complete the treatment for various reasons: } 3 \text { clients }\end{array} \\ \text { All outcomes } & \text { (2 NET and } 1 \text { treatment as usual) dropped out during the course of therapy } \\ & \text { due to extremely high IPV occurrences. } 3 \text { participants ( } 2 \text { NET and } 1 \text { treatment } \\ & \text { as usual) discontinued therapy, as they could not tolerate the overwhelming } \\ & \text { emotions evoked through talking about IPV experiences. } 2 \text { NET participants } \\ \text { discontinued therapy as they had to move to another city to live, and the oth- } & \text { er } 2 \text { participants (1 NET and } 1 \text { treatment as usual) did not continue therapy be- } \\ \text { cause they reported that their lives had become too busy. Figure } 1 \text { presents } & \text { the flow chart of the trial protocol. }\end{array}$

\begin{tabular}{lll}
$\begin{array}{l}\text { Selective reporting (re- } \\
\text { porting bias) }\end{array}$ & Unclear risk & $\begin{array}{l}\text { Judgement comment: all outcome measures mentioned in the method sec- } \\
\text { tion were collected, analysed and fully reported in the results section. Fur- } \\
\text { ther, the measures mentioned in the trial registration ClinicalTrials.gov ID: } \\
\text { NCT01731418 were adequately presented in the published paper. }\end{array}$ \\
\hline Other bias & Low risk & Judgement comment: none. \\
\hline
\end{tabular}

Rhodes 2015

\section{Study characteristics}

Methods

Trial design: $\mathrm{RCT}$

Trial grouping: parallel group

Assessment modality (e.g. face-to-face, telephone, online): face-to-face, telephone, IVRS.

Conflict of interest of trial authors: the trial authors have completed and submitted the ICMJE Form for Disclosure of Potential Conflicts of Interest. Dr Sommers reported receiving book royalties from FA Davis. No other disclosures were reported.

Funding for trial: this project was funded by award R01-AA018705 from the National Institute on Alcohol Abuse and Alcoholism.

Primary outcomes: alcohol; days of heavy drinking (AUDIT/AUDIT-C). IPV: number of IPV events (CTS2S)

Randomisation method: participants were block-randomised in groups of 20 using a 2:2:1 distribution to the brief intervention group, the assessed control group, or the no-contact control group.

Secondary outcomes: secondary drinking outcomes included changes in the full AUDIT28 score and both quantity and frequency of drinking using the Timeline Follow-Back technique. 32 secondary IPV outcomes included frequency and severity of experiencing or perpetrating IPV (16 items) from the full CTS2S and changes in the CAS. Other health-related outcomes included measures of self-rated health, 
Rhodes 2015 (Continued)

depression, sleep, social support, quality of life, satisfaction with relationship, and engagement with treatment

Timing of outcome measurements: Baseline and 3, 6, and 12 months

Total duration of trial: January 2011-December 2014

Type of analysis: ITT analysis; hierarchical generalised linear model

Types of participants: IPV-involved female ED patients

ITT analysis: yes

Participants

\section{Baseline characteristics}

Experimental intervention

- Age: $\mathrm{M}=32.3$ years $(95 \% \mathrm{Cl} 30.9$ to 33.6$)$

- Ethnicity: Black 178 (74.8\%); white 53 (22.3\%); Native American 11 (4.6\%); Hispanic 13 (5.5\%); Pacific Islander 2 (0.8\%); Asian 4 (1.7\%); other 14 (5.9\%)

- Employment: yes employed 120 (50.2\%)

- Education: some high school 49 (20.5\%); high school degree 61 (25.5\%); some college or degree 107 (44.8\%); some postgraduate or degree $22(9.2 \%)$

- Relationship status: single 184 (77.0\%); married 37 (15.5\%); divorced, widowed, or separated 18 (7.5\%)

- Gender (\% women): 100

- Dependent children: 0: 116 (48.5\%); 1: 49 (20.5\%); > 2: 65 (27.2\%)

- Number analysed (data analysis at post-treatment stage): 165

- Number of participants eligible assigned: 242

- Number of participants dropped out (by final follow-up): 77

- Comorbidities: PTSD 228 (40.4\%)

- Experience of IPV: 19.7 (17.8-21.4)

- Experience of other types of violence and abuse: childhood sexual abuse 100 (41.8\%)

- Remuneration: NR

- Financial dependence (on partner): NR

Comparator intervention

- Age: $\mathrm{M}=32.1$ (95\% Cl 30.6 to 33.6$)$

- Ethnicity: Black 187 (81.3\%); white 38 (16.5\%); Native American 5 (2.2\%); Hispanic 12 (5.2\%); Pacific Islander 0; Asian 3 (1.3\%); Other 17 (7.4\%)

- Employment: yes employed 117 (50.4\%)

- Education: some high school 48 (20.7\%); high school degree 65 (28.0\%); some college or degree 106 (45.7\%); some postgraduate or degree 12 (5.2\%)

- Relationship status: single 181 (78.0\%); married 31 (13.4\%); divorced, widowed, or separated 19 (8.2\%)

- Gender (\% women): 100

- Dependent children: 0: 96 (41.4\%); 1: 47 (20.3\%); >2: 81 (34.9\%)

- Number analysed (data analysis at post-treatment stage): 165

- Number of participants eligible assigned: 237

- Number of participants dropped out (by final follow-up): 72

- Comorbidities: PTSD

- Experience of IPV: 20.5 (18.6-22.3)

- Experience of other types of violence and abuse: childhood sexual abuse 98 (42.8\%)

- Remuneration: NR

- Financial dependence (on partner): NR

Total sample

- Age: $\mathrm{M}=32.1$ years, $(95 \% \mathrm{Cl} 31.3$ to 33.0$)$ 
- Ethnicity: Black 471 (80.1\%); white 108 (18.4\%); Native American 20 (3.4\%); Hispanic 28 (4.8\%); Pacific Islander 3 (0.5\%); Asian 8 (1.4\%); Other 35 (6.0\%)

- Employment: yes employed 291 (49.2\%)

- Education: some high school 116 (19.6\%); high school degree 164 (27.7\%); some college or degree 273 (46.1\%); some postgraduate or degree $37(6.3 \%)$

- Relationship status: single 459 (77.5\%); married 86 (14.5\%); divorced, widowed, or separated 46 (7.8\%)

- Gender (\% women): 100

- Dependent children: 0: 261 (44.1\%); 1: 124 (20.9\%); $\geq 2$ : 186 (31.4\%)

- Number analysed (data analysis at post-treatment stage): ITT analysis

- Number of participants eligible assigned: 600

- Number of participants dropped out (by final follow-up): 175

- Comorbidities: comorbid risk factors were prominent: $60 \%$ smoked cigarettes, $17 \%$ disclosed using illicit drugs (46\% including marijuana), 12\% were positive for non-medical use of prescription drugs, $43 \%$ disclosed a history of child sexual abuse, $40 \%$ screened positive for PTSD, and $86 \%$ screened positive for depression

- Experience of IPV: mean IPV levels were severe on the Women's Experience with Battering Scale (score range: 10-40; 20 indicates higher severity) and Danger Assessment (score range: 0-39; $\geq 18$ indicates extreme danger)

- Experience of other types of violence and abuse: childhood sexual abuse 243 (43.2\%)

- Remuneration: learning from the successes and failures of previous research, we utilise a variety of safety measures, strategic retention procedures, and innovative technologies for incentive payments and remote data collection.

- Financial dependence (on partner): NR

Included criteria: patients must meet the following criteria to qualify for trial participation at the time of assessment: female patients ages 18-64; able to participate verbally and cognitively in an English language interview; heavy drinking ( $\geq 4$ drinks on at least 1 day; $\geq 7$ drinks in 1 week); and a positive screen for IPV by a current or former partner in the past 3 months

Excluded criteria: intoxication at the time of screening; cognitive impairment or psychosis identified on physical examination or chart review; serious current medical illness or injury, defined as respiratory distress, haemodynamic instability, active vomiting, bleeding, labour, severe pain, or acute need for hospital admission; suicidal or homicidal ideation by chart review; no identifiable residence or contact phone number; under arrest at the time of ED visit; non-English speaking; previously enrolled in the trial

Pretreatment: baseline demographics and risk factors were fairly well balanced across the groups (Table 1). Exceptions were slightly more white participants in the intervention group, the no-contact control group had higher rates of IPV at baseline, and more women in the assessed control group had previously used community-based IPV services compared with the intervention group (10\% vs $4 \%$, respectively).

\section{Number eligible: 1245}

Number ineligible: 5523

Number of eligible people consented: 600

Number of eligible people recruited: 600

Recruitment setting of participants (e.g. healthcare setting, community setting, shelter setting): female patients approached at EDs

Sample power calculation: sample size estimation for analyses comparing all 3 groups. A priori power analysis was based on a 2-sided 0.03 significance level to accommodate 2 primary outcomes: heavy drinking days and IPV incidents, with significance on either outcome providing evidence of a positive trial. Based on prior studies, 199 participants/group were required to achieve at least $80 \%$ power to detect 2 days of heavy drinking/month. Without prior effect sizes for IPV interventions, trial designed to have $94 \%$ power to detect a $20 \%$ difference between groups in the incidence rate of IPV (assuming $30 \%$ attrition) during the first 12 weeks of treatment. 
Total number of participants within all groups: 600

Number declined to participate: 302

Interventions

\section{Intervention characteristics}

Experimental intervention

- Brief description (rationale, main components): the Motivational Enhancement Therapy (MET) intervention is a short, $20-25$ min counselling session that incorporates brief feedback and guidance with motivational enhancement techniques to assist patients in increasing their safety. This intervention includes a (non-recorded) phone follow-up booster component, which is intended to consolidate and reinforce the MET session

- Type of intervention: MI

- Mode of delivery (how): face-to-face followed with telephone booster

- Frequency and duration of delivery (when and how much): the intervention group received a 20- to 30-min manual guided motivational intervention and a telephone booster at 10 days.

- Level of mental health training of person delivering the intervention: all therapists had training and experience working with abused women before the initiation of this trial. They received extensive additional training and supervision by MI experts through biweekly review of recorded sessions

- Fidelity assessment: the intervention, which was delivered by master's-level therapists during the ED visit, was recorded and monitored for fidelity. An independent MI consultant reviewed and rated $10 \%$ of randomly sampled recordings with ongoing feedback to therapists

- Intervention model: MI

- Person delivering the intervention (who provided the intervention): therapist, self-directed or combined: master's level therapists

- Intervention location (where): ED visit

- Tailoring of intervention (personalised, titrated or adapted): for many, the outcomes of the MET intervention will be an agreement to reduce either alcohol use or its ability to cause harm (medical problems or trauma), to identify and agree to implement effective coping strategies for situations that are high-risk for IPV, and/or a connection with informal or community-based supports via therapist referral. The practitioner and patient come to this agreement through a process of negotiation. If the patient expresses an unwillingness or inability to consider change, the MET therapist's primary role is to encourage the patient to explore any existing ambivalence and to support the patient's autonomy and personal agency, even if she makes a decision not to change. In MET, the spirit of preserving and supporting the client autonomy and personal choice is paramount

- Intervention modifications (during the trial): NR

- Main techniques of intervention: the goal of the intervention was to elicit the patient's self identified reasons for change and personal goals. During the intervention sessions, which were recorded and analysed for fidelity, the therapists encouraged participants to identify any linkages between their drinking and IPV, and helped them to resolve ambivalence regarding behavioural change, while also supporting the patient's autonomy and personal choice. An unrecorded telephone follow-up booster with the same therapist occurred approximately 10 days later to consolidate and reinforce the MI session.

- Intervention manual: prior to project funding, the research team developed, piloted, and revised a brief MET manual. The manual derives from previous motivational intervention manuals targeting drinking and risky driving, adapted for ED settings (26-29). Our manual was developed with input from MI expert, Theresa Moyers, developer of the Motivational Interviewing Treatment Integrity (MITI-3.1.1 rating scale) (30), which is the adherence measure used in our trial. The manual is available on request from the principal investigator, Karin Rhodes, MD MS (Contact email: Karin.Rhodes@uphs.upenn.edu).

- Intervention attendance: NR

- CCDAN psychological therapies: integrative therapies

- Intensity of intervention: up to 4 sessions

- Person delivering the intervention: healthcare workers

- Gender of therapist: NR 
Rhodes 2015 (Continued)

\section{Comparator intervention}

- Brief description (rationale, main components): completion of primary and secondary outcome measures at baseline and 3, 6, and 12 months

- Type of intervention: no treatment

Outcomes

\section{Re-exposure to IPV}

- Outcome type: continuous outcome

- Reporting: fully reported

- Scale: Revised CTS

- Direction: lower is better

\section{Use of healthcare and IPV services}

- Outcome type: dichotomous outcome

- Reporting: fully reported

- Scale: Engagement With Services Domestic Violence

- Direction: lower is better

\section{Social support}

- Outcome type: dichotomous outcome

- Reporting: fully reported

- Scale: do you have someone to talk to about any problem?

- Direction: higher is better

\section{Safety planning and/or safety behaviour}

- Outcome type: continuous outcome

- Reporting: fully reported

- Scale: how safe do you feel in your daily life?

- Range: 1-5

- Direction: lower is better

\section{Quality of life}

- Outcome type: continuous outcome

- Reporting: fully reported

- Scale: QOL Scale

- Range: 1-5

- Direction: lower is better

\section{Dropouts from treatment}

- Outcome type: dichotomous outcome

- Reporting: fully reported

- Direction: lower is better

Identification

Sponsorship source: this project was funded by award R01-AA018705 from the National Institute on Alcohol Abuse and Alcoholism.

Country: USA

Setting: ED

Comments: women attending ED who disclosed IPV and problem drinking in previous 3 months

Trialauthors: Karin V. Rhodes, MD, MS; Melissa Rodgers, BA; Marilyn Sommers, PhD; Alexandra Hanlon, PhD; Jesse Chittams, MS; Andrea Doyle, PhD; Elizabeth Datner, MD; Paul Crits-Christoph, PhD 
Email: karin.rhodes@uphs.upenn.edu

Address: Center for Emergency Care Policy and Research, Department of Emergency Medicine, Perelman School of Medicine,University of Pennsylvania, 34th and Spruce Streets, First Floor Ravidin, HUP, Philadelphia, PA 19104

Title (main 'outcome' publication): Brief motivational intervention for intimate partner violence and heavy drinking in the emergency department a randomized clinical trial

Registered trial protocol ID: NCT01207258

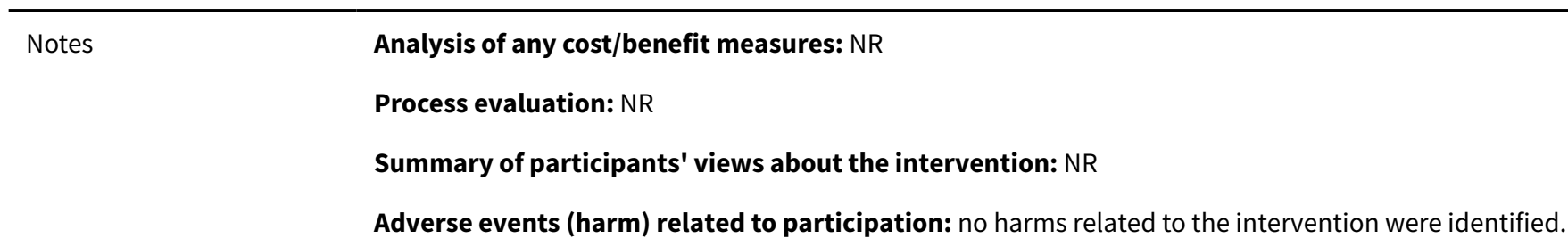

\section{Risk of bias}

\begin{tabular}{lll}
\hline Bias & Authors' judgement & Support for judgement \\
\hline $\begin{array}{l}\text { Random sequence genera- } \\
\text { tion (selection bias) }\end{array}$ & Low risk & $\begin{array}{l}\text { Judgement comment: "using a statistical software pseudo-random num- } \\
\text { ber generator, the trial statistician created a block-randomisation scheme, } \\
\text { in groups of } 20 \text {, to determine trial assignment. The project manager used the } \\
\text { scheme to prepare } 2 \text { sets of opaque envelopes }-1 \text { for each ED - that are indis- } \\
\text { tinguishable from each other and thick enough so that their contents are not } \\
\text { legible from the outside. All patients who give consent for participation are } \\
\text { randomised by pulling the next sequential envelope". }\end{array}$
\end{tabular}

\begin{tabular}{ll}
\hline $\begin{array}{l}\text { Allocation concealment } \\
\text { (selection bias) }\end{array}$ & Jow risk \\
& $\begin{array}{l}\text { Judgement comment: "all patients who give consent for participation are ran- } \\
\text { those who enroll participants are unaware of group assignment until after } \\
\text { they are consented to be in the trial; only the project manager and statistician } \\
\text { are aware of the randomisation scheme". }\end{array}$
\end{tabular}

\begin{tabular}{|c|c|c|}
\hline $\begin{array}{l}\text { Blinding of participants } \\
\text { and personnel (perfor- } \\
\text { mance bias) }\end{array}$ & Unclear risk & $\begin{array}{l}\text { Judgement comment: all protocols and blinded data were reviewed at inter- } \\
\text { vals by a data and safety monitoring board. However, there is insufficient in- } \\
\text { formation about the blinding of participants and personnel. }\end{array}$ \\
\hline
\end{tabular}

All outcomes

\begin{tabular}{lll}
\hline Blinding of outcome as- & Low risk & Judgement comment: "all non-IVRS post-baseline assessments were conduct- \\
sessment (detection bias) & & ed by trained interviewers blinded to treatment assignment. RAs conducting \\
fll outcomes & follow-up interviews are blinded to the participant's group assignment during \\
& data collection".
\end{tabular}

\begin{tabular}{|c|c|c|}
\hline $\begin{array}{l}\text { Incomplete outcome data } \\
\text { (attrition bias) } \\
\text { All outcomes }\end{array}$ & Low risk & $\begin{array}{l}\text { Judgement comment: all demographic, baseline and primary and secondary } \\
\text { outcome measures were collected from participants via self-report. All } \\
\text { dropouts and withdrawal from the trial were presented, and appropriate sta- } \\
\text { tistical analysis was used (e.g. ITT analysis). Attrition and exclusion adequately } \\
\text { described. }\end{array}$ \\
\hline
\end{tabular}

\begin{tabular}{ll}
\hline $\begin{array}{l}\text { Selective reporting (re- } \\
\text { porting bias) }\end{array}$ & Low risk
\end{tabular}

Other bias Low risk




\section{Study characteristics}

Methods

Trial design: $\mathrm{RCT}$

Trial grouping: parallel group

Conflict of interest of trial authors: none reported

Funding for trial: funding was provided through a grant from the Centers for Disease Control and Prevention: R49 CE000924

Primary outcomes: self-efficacy

Randomisation method: the field co-ordinator randomised the subjects in a 1:1 allocation to the intervention or control group using a simple random sampling program available in SAS software (Cary, NC)

Secondary outcomes: depression, state-of-readiness-to-change

Timing of outcome measurements: baseline and 6-months' follow-up

Total duration of trial: December 2007-December 2010

Types of participants: women had to screen positive for IPV by a current partner within the past year and had to be aged $\geq 18$ years, English-speaking, and neither currently pregnant nor incarcerated.

ITT analyses: yes

Participants

\section{Baseline characteristics}

Experimental intervention

- Age: $18-19$ years (24, 22.6\%); 20-24 (43, 40.6\%); 25-29 (29, 27.4\%); $30-39$ (3, 2.8\%); 40+ (6, 5.7\%)

- Ethnicity: Hispanic 7 (7.1\%); non-Hispanic 90 (91.8\%)

- Employment: employed at time of baseline: yes 62 (63.3\%); No 36 (36.7\%)

- Education: $\leq$ high school 40 (40.8\%); some college 42 (42.9\%); college/graduate degree 15 (15.3\%)

- Relationship status: cohabitation status: living together 49 (50.0\%); not living together 48 (49.0\%)

- Experience of IPV: positive for battering 77 (78.6\%)

- Gender (\% women): 100

- Financial dependence (on partner): NR

- Dependent children: NR

- Number analysed (data analysis at post-treatment stage): 98

- Number of participants eligible assigned: 155

- Number of participants dropped out (by final follow-up): 57

- Comorbidities: NR

- Experience of other types of violence and abuse: NR

- Remuneration: participants received the trial's toll-free number and compensation for travel expenses and completed surveys

Comparator intervention

- Age: 18-19 years (21, 21.4\%); 20-24 (38, 38.8\%); $25-29$ (17, 17.4\%); $30-39$ (16, 16.3\%); 40+ (6, 6.1\%)

- Ethnicity: Hispanic 16 (15.1\%); non-Hispanic 89 (84.0\%)

- Employment: employed at time of baseline: yes 65 (61.3\%); No 35 (33.0\%) 
- Education: $\leq$ high school 47 (44.3\%); some college 46 (43.4\%); college/graduate degree 13 (12.3\%)

- Relationship status: cohabitation status: living together 54 (50.9\%); not living together 50 (47.2\%)

- Experience of IPV: positive for battering (WEB) 83 (78.3\%)

- Gender (\% women): 100

- Financial dependence (on partner): NR

- Dependent children: NR

- Number analysed (data analysis at post-treatment stage): 106

- Number of participants eligible assigned: 151

- Number of participants dropped out (by final follow-up): 45

- Comorbidities: NR

- Experience of other types of violence and abuse: NR

- Remuneration: participants received the trial's toll-free number and compensation for travel expenses and completed surveys

Total sample

- Age: NR

- Ethnicity: NR

- Employment: NR

- Education: NR

- Relationship status: NR

- Experience of IPV: NR

- Gender (\% women): 100

- Financial dependence (on partner): NR

- Dependent children: NR

- Number analysed (data analysis at post-treatment stage): 204

- Number of participants eligible assigned: 306

- Number of participants dropped out (by final follow-up): 99

- Comorbidities: NR

- Experience of other types of violence and abuse: the Abuse Assessment Screen (15) to ascertain physical, sexual, and threats of IPV and identify the frequency, severity, and perpetrator of the abuse. However, these were core aspects of IPV.

- Remuneration: participants received the trial's toll-free number and compensation for travel expenses and completed surveys

Included criteria: to be eligible for the trial, women had to screen positive for IPV by a current partner within the past year and had to be aged $\geq 18$ years, English-speaking, and neither currently pregnant nor incarcerated.

Excluded criteria: $<18$ years of age; pregnant at time of screening and consent

Pretreatment: baseline demographic characteristics did not differ significantly by group, indicating randomisation was successful

Number of eligible people consented: 306

Number of eligible people recruited: 478

Sample power calculation: prior analysis of this clinic system administrative data suggested that given historical clinic volume and assuming $80 \%$ participation and $80 \%$ follow-up, we could retain a final sample of 250 .

Total number of participants within all groups: 306

Number declined to participate: 172

Number ineligible: 3732

Number eligible: 478 
Recruitment setting of participants (e.g. healthcare setting, community setting, shelter setting): healthcare setting (family planning clinics)

Total number of participants approached: 5211

Interventions

\section{Intervention characteristics}

Experimental intervention

- Brief description (rationale, main components): MI was implemented to guide women in identifying feasible individual goals and small steps that they could safely take to increase their self-efficacy and feelings of control. MI is a 'client-centred' counselling approach developed to deliver stage-tailored intervention messages using non-confrontational, supportive communication strategies. The client is encouraged to explore the barriers and facilitating factors associated with the behaviour change. The interviewer's role is to guide the conversation and avoid use of conventional directive approaches (e.g. persuasion, providing information).

- Type of intervention: MI

- Mode of delivery: face-to-face and telephone

- Frequency and duration of delivery: 1-h face-to-face educational session at baseline, followed by 3 $\mathrm{x} 10$ - to 15 -min MI telephone sessions conducted 1, 2, and 4 months post-enrolment. Women who participated in the full protocol received approximately $90 \mathrm{~min}$ of MI counselling

- Level of mental health training of person delivering the intervention: the field co-ordinators were trained during 2 half-day interactive sessions held at the University of lowa by MI experts

- Fidelity assessment: no formal assessment, however periodic face-to-face meetings with the trial team and the Ml experts were conducted to support intervention delivery. Field issues and problems were discussed and addressed through role-playing exercises. Actual taped MI sessions conducted by the field co-ordinators with consenting subjects were also played and reviewed for training purposes.

- Intervention model: MI

- Intervention attendance: 204 women completed the trial (referral: $n=108 ; \mathrm{MI}: \mathrm{n}=98$ ) for a completion rate of $66.6 \%$. Nearly two-thirds of participants who completed the MI arm participated in all 4 scheduled $\mathrm{MI}$ sessions. $11 \%$ of women randomised to intervention had no $\mathrm{MI}$ sessions.

- CCDAN psychological therapies: integrative therapies

- Person delivering the intervention (who provided the intervention): therapist, self-directed or combined: therapist

- Intervention location (where): telephone sessions but unclear for face-to-face session,

- Tailoring of intervention (personalised, titrated or adapted): women could self-select areas and were not required to focus on the abusive relationship

- Intervention modification (during the trial): NR

- Main techniques of intervention: at each session, the field co-ordinators used MI techniques to help women identify small steps that they could take to improve their physical health, emotional health, social support, quality of work or home life, or their relationship. Women could self-select areas and were not required to focus on the abusive relationship

- Intervention manual: NR

- Intensity of intervention: up to 4 sessions

- Person delivering the intervention: unclear

- Gender of therapist: NR

Comparator intervention

- Brief description (rationale, main components): women randomised into the "control" or referral arm of the trial met with the field coordinator or an on-site, certified domestic abuse advocate who provided written materials and referrals to community-based resources.

- Type of intervention: referrals to community-based resources

\section{Depression}

- Outcome type: continuous outcome

- Reporting: fully reported 
Saftlas 2014 (Continued)

- Scale: Center for Epidemiologic Studies Short Depression Scale

- Direction: lower is better

\section{Self-efficacy}

- Outcome type: continuous outcome

- Reporting: fully reported

- Scale: Domestic Violence Coping Self-Efficacy Measure (DV-CSE)

- Range: 0-100

- Direction: higher is better

\section{Safety planning and/or safety behaviour}

- Outcome type: dichotomous outcome

- Reporting: fully reported

- Scale: stage-of-readiness-to-change

- Direction: higher is better

\section{Dropouts from treatment}

- Outcome type: dichotomous outcome

- Reporting: fully reported

- Direction: lower is better

Identification

Sponsorship source: Funding was provided through a grant from the Centers for Disease Control and Prevention: R49 CE000924.

\section{Country: USA}

Setting: family planning clinics

Comments: RCT among women who experienced IPV in a current relationship over the past 12 months. Participants were recruited from 2 family planning clinics (December 2007-May 2010).

Trialauthors: Audrey F. Saftlas, Karisa K. Harland, Anne B. Wallis, Joseph Cavanaugh, Penny Dickey, Corinne Peek-Asa

Institution: Department of Epidemiology, University of lowa, lowa City, IA

Email: audrey-saftlas@uiowa.edu

Address: Department of Epidemiology, University of lowa College of Public Health, 105 River St., CPHB S427, lowa City, IA 52242. Tel.: p1 3193845013

Trial title (main outcome publication): Motivational interviewing and intimate partner violence: a randomised trial

Registered trial protocol ID: NCT0141066

Notes

Analysis of any cost/benefit measures: NR

Process evaluation: NR

Summary of participants' views about the intervention: NR

Adverse events (harm) related to participation: no harms attributable to the intervention were recorded.

\section{Risk of bias}

\section{Bias}

Authors' judgement Support for judgement 
Saftlas 2014 (Continued)

Random sequence genera- Low risk tion (selection bias)
Judgement comment: "all participants completed a 20-min baseline questionnaire that was self-administered and computer-based to collect information on the trial outcomes. During this time, the field co-ordinator randomised the subjects in a 1:1 allocation to the intervention or control group using a simple random sampling program available in SAS software (Cary, NC)".
Allocation concealment High risk

(selection bias)
Judgement comment: "the field co-ordinator randomised the participants in a 1:1 allocation to the intervention or control group using a simple random sampling program available in SAS software (Cary, NC). However, blinding of the participants and staff delivering the intervention would have been ideal, logistical constraints related to limited clinic space and staffing made this unfeasible".
Blinding of participants and personnel (perforHigh risk mance bias)

All outcomes
Judgement comment: "blinding of the participants and staff delivering the intervention was also not feasible".
Blinding of outcome assessment (detection bias) All outcomes
High risk

Judgement comment: " 1 field co-ordinator at each site administered the screening, consent, intervention, and follow-up procedures. Although blinding of the field co-ordinator would have been ideal, logistical constraints related to limited clinic space and staffing made this unfeasible".

\begin{tabular}{|c|c|c|}
\hline $\begin{array}{l}\text { Incomplete outcome data } \\
\text { (attrition bias) } \\
\text { All outcomes }\end{array}$ & Low risk & $\begin{array}{l}\text { Judgement comment: outcome measures completion rate was } 204 / 306= \\
66.6 \% \text {. Both attrition and exclusions were adequately reported and modified } \\
\text { ITT analyses of completed participants were conducted using multivariate } \\
\text { analysis of variance for continuous outcomes and polytomous logistic regres- } \\
\text { sion for dichotomous outcomes. }\end{array}$ \\
\hline
\end{tabular}

Selective reporting (re- Unclear risk porting bias) Judgement comment: all outcomes described in the methods section were adequately collected, analysed and reported in the results section of the main published trial. However, there was insufficient or unclear evidence from either the trial protocol or trial registry sites to assess this domain.

Other bias Low risk Judgement comment: none.

\section{Saggurti 2014}

\section{Study characteristics}

\section{Methods}

Trial design: cluster-RCT

Trial grouping: parallel group

Assessment modality (e.g. face-to-face, telephone, online): face-to-face

Conflict of interest of trial authors: the trial authors have no conflicts of interest

Funding for trial: the trial was funded via grant from the Indian Council of Medical Research and the National Institutes of Health through the Indo-US Joint Working Group on Prevention of STIs and HIV/ AIDS during 2009-2011 (R21MH85614 \& Indo-US/38/2007-ECD-II)

Primary outcomes: the outcome measures used for the trial were single questions in the survey instrument, including the following: 'Did you and your husband have any argument in the past 3 months?' (marital conflict); 'Have you and your husband had an argument or fight where he physically or sexually hurt you in the past 3 months?' (physical/sexual violence); and 'Was there any coercion or pressure on you to have sex the last time you had sex with your husband?' (sexual coercion). 
Randomisation method: a 2-armed cluster-RCT. Clusterswere selected via mapping of the trial area by geographic boundaries (e.g. a hill or a street) and population density, such that each geographic cluster included approximately 300 households. This approach resulted in 22 clusters, 13 of which were selected for trial inclusion based on indications of large numbers of alcohol venues within them. One cluster was utilised for piloting but retained in the trial, as no changes were made to the programme. The remaining 12 clusters were randomised to intervention or control conditions.

\section{Secondary outcomes: NR}

Timing of outcome measurements: baseline and 4.5-month follow-up

Total duration of trial: 4 June 2010-30 June 2011

Type of analysis: $\mathrm{Chi}^{2}$ analyses, logistic generalised linear mixed models post-hoc treatment groupstratified analyses

Types of participants: women contending with a history of IPV and/or husband's drunken behaviour

ITT analysis: yes

\section{Participants}

\section{Baseline characteristics}

Experimental intervention

- Age: $M=28.9$ years, $S D=6.0$

- Ethnicity: NR

- Employment: NR

- Education: formal education 91 (77.1\%); no formal education 27 (22.9\%)

- Relationship status: NR

- Gender (\% women): 100

- Dependent children: number of children 2.3 (1.1)

- Number analysed (data analysis at post-treatment stage): 118

- Number of participants eligible assigned: 143

- Number of participants dropped out (by final follow-up): 25

- Comorbidities: NR

- Experience of IPV (past 90 days): IPV (physical or sexual) in past 90 days 45 (38.1\%)

- Experience of other types of violence and abuse: marital conflict (past 3 months) 75 (63.6\%); sexual coercion $36(3 . .7 \%)$

- Remuneration: no monetary incentive was provided for trial or programme participation.

- Financial dependence (on partner): NR

Comparator intervention

- Age: $M=29.9$ years, $S D=5.5$

- Ethnicity: NR

- Employment: NR

- Education: formal education 82 (80.4\%); no formal education 20 (19.6\%)

- Relationship status: NR

- Gender (\% women): 100

- Dependent children: number of dependent children 2.4 (1.4)

- Number analysed (data analysis at post-treatment stage): 102

- Number of participants eligible assigned: 120

- Number of participants dropped out (by final follow-up): 18

- Comorbidities: NR

- Experience of IPV (past 90 days): IPV (physical or sexual) in past 90 days 32 (31.4\%)

- Experience of other types of violence and abuse: marital conflict (past 3 months) 53 (51.9\%); sexual coercion $19(18.8 \%)$

- Remuneration: no monetary incentive was provided for trial or programme participation. 
Total sample

- Age: $M=29$ years, $S D=5.8$

- Ethnicity: NR

- Employment: NR

- Education: formal education 173 (78.6\%); no formal education 47 (21.4\%)

- Relationship status: $100 \%$ married

- Gender (\% women): 100

- Dependent children: number of dependent children 2.4 (1.2)

- Number analysed (data analysis at post-treatment stage): 220

- Number of participants eligible assigned: 220

- Number of participants dropped out (by final follow-up): 43

- Comorbidities: NR

- Experience of IPV (past 90 days): IPV (physical or sexual) in past 90 days 77 (35\%)

- Experience of other types of violence and abuse: NR

- Remuneration: no monetary incentive was provided for trial or programme participation.

- Financial dependence (on partner): NR

Included criteria: the woman was identified as eligible if she satisfied the following criteria: 18-40 years of age; fluent in Hindi or Marathi; resided with her husband in the area of trial for a period of $\geq 2$ months; reported that her husband engaged in heavy drinking (past 30-day drunken behaviour or 3 drinking days in past 7 days) or that she had experienced lifetime physical or sexual spousal violence perpetration; and had no plans to relocate from the area in the next year.

Excluded criteria: NR

Pretreatment: only religion differed significantly between groups at baseline $(P=0.03)$

Number eligible: 285

Number ineligible: 2125

Number of eligible people consented: 220

Number of eligible people recruited: 220

Recruitment setting of participants (e.g. healthcare setting, community setting, shelter setting): community setting

Sample power calculation: NR

Total number of participants approached: 2410

Total number of participants within all groups: 220

Number declined to participate: 65

\section{Intervention characteristics}

Experimental intervention

- Brief description (rationale, main components): the RHANI Wives intervention was based on social cognitive theory and the theory of gender and power (TGP). Social cognitive theory application supported focus on HIV/STI knowledge and condom skills building, as well as safer-sex social norms and motivation. TGP guided the intervention focus on problem-solving and skills-building toward marital communication and conflict; embedded in this was gender-equity counselling and support. The TGP approach supported women to take a more active and assertive stance with husbands.

- Type of intervention: RHANI wives

- Mode of delivery (how): face-to-face 
- Frequency and duration of delivery (when and how much): the RHANI Wives intervention included 4 individual sessions in the household and 2 group sessions in the community delivered over 6-9 weeks

- Level of mental health training of person delivering the intervention: trained master's-level counsellor. Prior to the start of the programme, intervention counsellors were trained on the safety protocol for survivors of IPV, as recommended by WHO

- Fidelity assessment: NR

- Intervention model: RHANI Wives

- Person delivering the intervention (who provided the intervention): therapist, self-directed or combined: therapist

- Intervention location (where): NR

- Tailoring of intervention (personalised, titrated or adapted): NR

- Intervention modifications (during the trial): NR

- Main techniques of intervention: the first individual session was conducted in week 1 (post-baseline survey) and was aimed at introductions and discussion regarding financial stresses and women's health using tools created for the intervention, such as a modified thermometer and various scales to measure levels of stress and burden. The second individual session was conducted during weeks 1-2, depending on convenience for the participant. The second session focused on issues around alcohol, violence, financial stress, and poor health of family using cyclical figures designed for the project to illustrate the relationship between these areas. Subsequent to the 2 individual sessions, the first group session was conducted in weeks 2-4; a counsellor used reconstructed stories documenting women's marital issues (alcohol, violence, sexual infidelity) to stimulate group discussion on problem-solving issues related to alcohol, violence, and sexual infidelity through marital communication and negotiation. The third individual session was conducted in weeks 3-6 and focused on implementation of problem-solving and action plans discussed during the previous group session. Subsequent to this, the second group session was conducted using games created to share stories documenting women's marital issues (alcohol, violence, and sexual infidelity) and to facilitate discussion on ways to reduce harms associated with these issues via acquisition of local services specific to each topic. The last individual session was conducted during weeks 5-9; it focused on implementation of problem-solving and action plans discussed during the second group session and to check on and update the problem-solving and action plans (if the participant could not attend group session) to reduce risk/stress

- Intervention manual: NR

- Intervention attendance: NR

- CCDAN psychological therapies: humanistic therapies

- Intensity of intervention: $\geq 5$ sessions

- Person delivering the intervention: healthcare workers

- Gender of therapist: NR

Comparator intervention

- Brief description (rationale, main components): the control condition involved referral to local social services for survivors of violence, and local urban health centres for HIV and STI testing and treatment.

- Type of intervention: no treatment

Outcomes

\section{Re-exposure to IPV}

- Outcome type: adverse event (dichotomous)

- Reporting: fully reported

- Scale: single-item question

- Direction: lower is better

\section{Dropouts from treatment}

- Outcome type: dichotomous outcome

- Reporting: fully reported

- Direction: lower is better 
Saggurti 2014 (Continued)

Identification
Sponsorship source: the trial was funded via grant from the Indian Council of Medical Research and the National Institutes of Health through the Indo-US Joint Working Group on Prevention of STIs and HIV/AIDS during 2009-2011 (R21MH85614 \& Indo-US/38/2007-ECD-II)

Country: India

Setting: community

Comments: RHANI Wives intervention was conducted with 220 women contending with a history of IPV and/or husband's drunken behavior

Trialauthors: Niranjan Saggurti, Saritha Nair, Jay G. Silverman, Dattaram D. Naik, Madhusudana Battala, Anindita Dasgupta, Donta Balaiah, AnitaRaj

Institution: Population Council, New Delhi, India

Email: nsaggurti@popcouncil.org

Address: IV and AIDS Program, Population Council, New Delhi 110003, India

Title (main 'outcome' publication): Impact of the RHANI Wives intervention on marital conflict and sexual coercion

Registered trial protocol ID: NCT01592994

Notes

\section{Analysis of any cost/benefit measures: NR}

Process evaluation: a number of procedures were conducted to ensure high-quality implementation of programme and adherence to curriculum, and to track participant response to and engagement with the programme. Attendance records with dates and length of sessions were maintained by the RHANI Wives counselor. The RHANI Wives counselor also maintained case notes detailing information about activities or strategies used in each session, as well as type and nature of social and health service referrals provided at each contact. Counselor notes and records were reviewed periodically and feedback was provided as necessary. Weekly meetings with the RHANI Wives counselor were held to help problem-solve any difficulties with any clients. Finally, a brief participant satisfaction survey was conducted with participants at the 4.5 month follow-up, to obtain their feedback on the counsellor and their perceptions of the utility of the programme in terms of supporting the participant to be safe and healthy in their marriage. Summary of participants' views about the intervention: finally, a brief participant satisfaction survey was conducted with participants at the 4.5 month follow-up, to obtain their feedback on the counsellor and their perceptions of the utility of the programme in terms of supporting the participant to be safe and healthy in their marriage.

Adverse events (harm) related to participation: NR

\section{Risk of bias}

\begin{tabular}{lll}
\hline Bias & Authors' judgement & Support for judgement \\
\hline $\begin{array}{l}\text { Random sequence genera- } \\
\text { tion (selection bias) }\end{array}$ & Unclear risk & $\begin{array}{l}\text { Judgement comment: "the trial community was further divided into differ- } \\
\text { ent geographic clusters for randomisation by the research team prior to tri- } \\
\text { al implementation. Clusters were selected via mapping of the trial area by } \\
\text { geographic boundaries (e.g. a hill or a street) and population density, such } \\
\text { that each geographic cluster included approximately } 300 \text { households. This } \\
\text { approach resulted in } 22 \text { clusters, } 13 \text { of which were selected for trial inclusion } \\
\text { based on indications of large numbers of alcohol venues within them. } 1 \text { cluster } \\
\text { was utilised for piloting but retained in the trial, as no changes were made to } \\
\text { the programme. The remaining } 12 \text { clusters were randomised to intervention or } \\
\text { control conditions". However, the approach of sequence generation remains } \\
\text { unclear. }\end{array}$ \\
\hline
\end{tabular}

$\begin{array}{ll}\begin{array}{l}\text { Allocation concealment } \\ \text { (selection bias) }\end{array} & \text { High risk } \\ \end{array}$


form clarified that participants would participate in 2 rounds of survey assessment and would receive either the multi-session intervention or a brief single-session intervention. The participants were informed after consent whether they were in the intervention or the control group. Thus, neither participants nor research staff members were blinded to treatment condition".

\begin{tabular}{|c|c|c|}
\hline $\begin{array}{l}\text { linding of participants } \\
\text { nd personnel (perfor- } \\
\text { nance bias) }\end{array}$ & High risk & $\begin{array}{l}\text { Judgement comment: the participants were informed after consent whether } \\
\text { they were in the intervention or the control group. Thus, neither participants } \\
\text { nor research staff members were blinded to treatment condition. }\end{array}$ \\
\hline
\end{tabular}

All outcomes

$\begin{array}{lll}\begin{array}{l}\text { Blinding of outcome as- } \\ \text { sessment (detection bias) }\end{array} & \text { Unclear risk } & \begin{array}{l}\text { Judgement comment: neither participants nor research staff members were } \\ \text { blinded to treatment condition. However, there is insufficient information } \\ \text { All outcomes }\end{array} \\ \text { about the blinding of outcome assessors. }\end{array}$

All outcomes

\section{Incomplete outcome data Low risk}

(attrition bias)

All outcomes

$\begin{array}{ll}\begin{array}{l}\text { Selective reporting (re- } \\ \text { porting bias) }\end{array} & \text { Unclear risk } \\ & \begin{array}{l}\text { Judgement comment: all outcomes described in the methods section were ad- } \\ \text { equately collected, analysed and reported in the results section of the main } \\ \text { published trial. However, there was insufficient or unclear evidence from ei- } \\ \text { ther the trial protocol or trial registry sites to assess this domain. }\end{array}\end{array}$

Other bias Low risk Judgement comment: none

\section{Stevens 2015}

\section{Study characteristics}

Trial grouping: parallel group

Assessment modality (e.g. face-to-face, telephone, online): telephone

Conflict of interest of trial authors: the trial author(s) declared no potential conflicts of interest with respect to the research, authorship, and/or publication of this article.

Funding for trial: the trial author(s) disclosed receipt of the following financial support for the research, authorship, and/or publication of this article: this research was supported through a grant (R01CE001196-03) from the Centers for Disease Control and Prevention.

Primary outcomes: IPV

Randomisation method: assignment to condition was based on a computer-generated random number table.

Secondary outcomes: depression, PTSD, physical health, resources use, perceived social support 
Total duration of trial: September 2008-July 2010

Type of analysis: unclear - $\mathrm{Chi}^{2}$ and independent $\mathrm{t}$ tests

Types of participants: women ( $\geq 18$ years) who had reported IPV within the past year during a visit to a paediatric ED

\section{ITT analysis: NR}

Participants

\section{Baseline characteristics}

Experimental intervention

- Age: $\mathrm{M}=28.8$ years, $\mathrm{SD}=8.3$

- Ethnicity: African American 45.0\%; white 46.5\%; Hispanic 1.6\%; Asian American 1.6\%; other/multiracial $5.4 \%$

- Employment: NR

- Education: middle school or less 3.2\%; some high school $18.9 \%$; high school graduate $26.8 \%$; some college $36.2 \%$; college graduate $9.4 \%$; more than college $5.5 \%$

- Relationship status: single $55.1 \%$; married $13.4 \%$; divorced $17.3 \%$; separated $11.8 \%$; widowed $2.4 \%$

- Gender (\% women): 100

- Dependent children: child average age 5.1 years (SD = 5.4)

- Number analysed (data analysis at post-treatment stage): 90

- Number of participants eligible assigned: 129

- Number of participants dropped out (by final follow-up): 39

- Comorbidities: NR

- Experience of IPV: 100

- Experience of other types of violence and abuse: NR

- Remuneration: USD 20, USD 30, and USD 40 for completing measures at the 3 time points

- Financial dependence (on partner): NR

Comparator intervention

- Age: $\mathrm{M}=29.5$ years, $\mathrm{SD}=8.5$

- Ethnicity: African American 51.6\%; white 37.1\%; Hispanic 1.6\%; Asian American 0.8\%; other/multiracial $8.9 \%$

- Employment: NR

- Education: middle school or less 3.3\%; some high school $21.7 \%$; high school graduate $27.5 \%$; some college $33.3 \%$; college graduate $10.0 \%$; more than college $4.2 \%$

- Relationship status: single 60.3\%; married $13.2 \%$; divorced $13.2 \%$; separated $10.7 \%$; widowed $2.5 \%$

- Gender (\% women): 100

- Dependent children: child average age 6.4 years (SD = 6.1)

- Number analysed (data analysis at post-treatment stage): 93

- Number of participants eligible assigned: 124

- Number of participants dropped out (by final follow-up): 31

- Comorbidities: NR

- Experience of IPV: 100

- Experience of other types of violence and abuse: NR

- Remuneration: USD 20, USD 30, and USD 40 for completing measures at the 3 time points

- Financial dependence (on partner): NR

Total sample:

- Age: NR

- Ethnicity: NR 
- Employment: NR

- Education: NR

- Relationship status: NR

- Gender (\% women): 100

- Dependent children: NR

- Number analysed (data analysis at post-treatment stage): 183

- Number of participants eligible assigned: 253

- Number of participants dropped out (by final follow-up): 70

- Comorbidities: NR

- Experience of IPV: considering that roughly half the sample had police involvement for IPV victimisation and that our average CAS scores in the 30 s were $>4$ times higher than the recommended cut-off score of 7, our sample had experienced a substantial level of IPV victimisation

- Experience of other types of violence and abuse:

- Remuneration: USD 20, USD 30, and USD 40 for completing measures at the 3 time points

- Financial dependence (on partner): NR

Included criteria: women aged $\geq 18$ years were recruited from a Midwestern paediatric ED

Excluded criteria: not specified

Pretreatment: at baseline, no significant differences between the 2 groups, with 1 exception. Participants in the intervention condition were more likely to have experienced police involvement for IPV victimisation by the perpetrator relative to participants in the control condition.

Number eligible: 380

Number ineligible: unclear

Number of eligible people consented: 300

Number of eligible people recruited: 253

Recruitment setting of participants (e.g. healthcare setting, community setting, shelter setting): paediatric ED

Sample power calculation: none reported

Total number of participants approached: total number approached unclear. However, 4104 caregivers screened positive for IPV in the ED. Apparently 380 were eligible which doesn't add up. No flow of participants provided so it is difficult to understand the gap between those screened or screening positive and those ultimately randomised.

Total number of participants within all groups: 253 enrolled and randomised

Number declined to participate: 80

\section{Intervention characteristics}

Experimental intervention

- Brief description (rationale, main components): telephone support services' (TSS) nurse interventionists identified appropriate referrals to community programmes, helped participants by problem-solving barriers to obtaining these local services, and provided social support. Throughout all phases, the interventionist relied heavily on $\mathrm{MI}$ - a non-confrontational, non-judgmental style of communication.

- Type of intervention: $\mathrm{MI}$ and increasing access to resources/referrals

- Mode of delivery (how): telephone

- Frequency and duration of delivery (when and how much): 12 phone calls with a total duration of 360-720 min over 6 months

- Level of mental health training of person delivering the intervention: 2 registered nurses, 1 with a master's degree and 1 with an associate's degree, served as the trial interventionists. Each nurse had several years of nursing and clinical research experience and was selected for her non-confrontation- 
al, empathetic interpersonal style. Each nurse completed $32 \mathrm{~h}$ of initial training. $16 \mathrm{~h}$ consisted of a 2day workshop on MI. The remaining $16 \mathrm{~h}$ consisted of reviewing the local community resource manual and multiple role-plays with the principal investigator and a licensed psychologist who himself had completed a 2-day MI training session and had 2-years experience providing MI consultation to other healthcare providers.

- Fidelity assessment: 1 psychology postdoctoral fellow and 2 bachelor's-level research assistants with undergraduate psychology degrees served as fidelity raters for audio recordings of these calls. They completed their fidelity coding independent of one another on the same participants and then came together to obtain consensus ratings. Every 4th participant for each nurse since the beginning of the trial was randomly selected for review.

- Intervention model: MI

- Person delivering the intervention (who provided the intervention): therapist, self-directed or combined: nurses

- Intervention location (where): telephone

- Tailoring of intervention (personalised, titrated or adapted): to an extent - nature of resources provided and other guidance dependent on woman's circumstances

- Intervention modifications (during the trial): no

- Main techniques of intervention: advocacy, information provision, support and MI

- Intervention manual: no

- Intervention attendance: NR

- CCDAN psychological therapies: integrative therapies

- Intensity of intervention: $\geq 5$ sessions

- Person delivering the intervention: healthcare workers

- Gender of therapist: female

Comparator intervention

- Brief description (rationale, main components): The interventionist followed the same first 2 steps from initial TSS call-inquiring briefly about the child's recent visit to the ED and following up on any non-IPV injury concerns endorsed by the woman through the ED computerised screening. However, the interventionist politely ended the EUC call without following the 5 advocacy phases described above. In other words, the interventionist was not permitted to initiate discussions about IPV or community resources.

- Type of intervention: a telephone call not related to IPV

\section{Depression}

- Outcome type: continuous outcome

- Reporting: fully reported

- Scale: Center for Epidemiological Studies Depression Scale

- Direction: lower is better

\section{PTSD}

- Outcome type: continuous outcome

- Reporting: fully reported

- Scale: PCL

- Direction: lower is better

\section{Re-exposure to IPV}

- Outcome type: continuous outcome

- Reporting: fully reported

- Scale: CAS

- Direction: lower is better

\section{Social support}


Stevens 2015 (Continued)

- Outcome type: continuous outcome

- Reporting: fully reported

- Scale: Social Provisions Scale

- Direction: higher is better

\section{Dropouts from treatment}

- Outcome type: dichotomous outcome

- Reporting: fully reported

- Direction: lower is better

Identification

Sponsorship source: this research was supported through a grant (R01CE001196-03) from the Centers for Disease Control and Prevention

Country: USA

Setting: Pediatric ED

Comments: women who had reported IPV within the past year during a visit to a paediatric ED

Trialauthors: Jack Stevens, Philip V. Scribano, Jessica Marshall, Radha Nadkarni, John Hayes, and Kelly J. Kelleher

Institution: Nationwide Children's Hospital, Columbus, OH, USA

Email: Jack.Stevens@nationwidechildrens.org

Address: Jack Stevens, Nationwide Children's Hospital, 700 Children's Drive, JWest 4th Floor, Columbus, OH 43205, USA.

Title (main 'outcome' publication): A trial of telephone support services to prevent further intimate partner violence

Registered trial protocol ID: NR

Notes

Analysis of any cost/benefit measures: NR

Process evaluation: NR

Summary of participants' views about the intervention: NR

Adverse events (harm) related to participation: there were no reported adverse effects from TSS or EUC.

\section{Risk of bias}

\begin{tabular}{|c|c|c|}
\hline Bias & Authors' judgement & Support for judgement \\
\hline $\begin{array}{l}\text { Random sequence genera- } \\
\text { tion (selection bias) }\end{array}$ & Low risk & $\begin{array}{l}\text { Judgement comment: "assignment to condition was based on a comput- } \\
\text { er-generated random number table. } 129 \text { participants were randomly assigned } \\
\text { to the intervention condition (TSS), and } 124 \text { participants were randomly as- } \\
\text { signed to the control condition (EUC)". }\end{array}$ \\
\hline $\begin{array}{l}\text { Allocation concealment } \\
\text { (selection bias) }\end{array}$ & Unclear risk & $\begin{array}{l}\text { Judgement comment: there is insufficient information about concealing the } \\
\text { allocation or how this was achieved. }\end{array}$ \\
\hline $\begin{array}{l}\text { Blinding of participants } \\
\text { and personnel (perfor- } \\
\text { mance bias) } \\
\text { All outcomes }\end{array}$ & Unclear risk & $\begin{array}{l}\text { Judgement comment: relative to other studies in this area, this would seem } \\
\text { low risk. The personnel/staff involved in delivering the intervention knew that } \\
\text { they were in the intervention group and this was also the case for the control } \\
\text { arm, however, there was strong fidelity assessment built in which would have } \\
\text { reduced deviations from the protocol. For example, "There was strong agree- } \\
\text { ment between the two fidelity raters and between their consensus ratings and }\end{array}$ \\
\hline
\end{tabular}


the nurses' self-ratings on structural aspects of the call, including number of complete calls, duration of the calls, and number of referrals made (Pearson correlation coefficients ranged from .87-.99). There was good to strong agreement regarding whether specific types of referral were made (Pearson correlation coefficients ranged from.46-1.00)." However, it is not clear whether participants were blinded.

\begin{tabular}{|c|c|c|}
\hline $\begin{array}{l}\text { Blinding of outcome as- } \\
\text { sessment (detection bias) } \\
\text { All outcomes }\end{array}$ & Low risk & $\begin{array}{l}\text { Judgement comment: "bachelor's-level research assistants served as our in- } \\
\text { dependent evaluators by contacting both groups for measurement. Numerous } \\
\text { steps were taken to keep the research assistants unaware of trial condition. } \\
\text { First, the interventionists made calls in separate offices from the research as- } \\
\text { sistants. Second, the research assistants were not given access to files contain- } \\
\text { ing condition assignment until the end of data collection from participants. } \\
\text { Third, the interventionists called participants in both trial conditions so that a } \\
\text { participant's infrequent mention to a research assistant of a conversation with } \\
\text { a trial nurse would not automatically reveal trial condition". }\end{array}$ \\
\hline
\end{tabular}

Incomplete outcome data Low risk
(attrition bias)

Judgement comment: attrition and exclusions were adequately described. All outcomes "There was a $76 \%$ retention rate for the intervention group from baseline to 3 months $(n=98)$, and a $77 \%$ retention rate for the control group from baseline to 3 months $(n=95)$. There was a $70 \%$ retention rate for the intervention group at 6 months $(n=90)$, and a $72 \%$ retention rate for the control group at 6 months $(n=93)$. There was no significant difference in retention between the 2 conditions ( $\left.\mathrm{Chi}^{2}=0.155, \mathrm{P}=0.92\right)$ ". Although data are not indicated on the exact numbers per outcome thus we have had to assume that all participants at each time point completed all measures.

Selective reporting (re- Unclear risk
porting bias)
Judgement comment: all outcomes described in the methods section were adequately collected, analysed and reported in the results section of the main published trial. However, there was insufficient or unclear evidence from either the trial protocol or trial registry sites to assess this domain.

Other bias Low risk Judgement comment: none

Taghizadeh 2018

\section{Study characteristics}

Methods

Trial design: quasi-experimental RCT

Trial grouping: parallel group

Assessment modality (e.g. face-to-face, telephone, online): face-to-face

Conflict of interest of trial authors: the trial authors declare no competing interests

Funding for trial: funded and supported by Tehran University of Medical Science

Primary outcomes: CTS2

Randomisation method: the present quasi-experimental trial was conducted in Tehran Province over a period of 8 months on samples selected through random stratified cluster sampling. The researcher randomly selected 2 health networks affiliated with Tehran University of Medical Sciences (lot drawing) in Shahr-e-Rey and southern Tehran, as the intervention and the control groups, respectively. Then 8 centres from each of the southern Tehran network and Shahr-e-Rey network were randomly selected (using the table of random numbers). The assigned centres to the intervention group and the control groups had a good dispersion and were not affiliated with each other to prevent participants from running into each other. 


\section{Secondary outcomes: NR}

Timing of outcome measurements: eligible pregnant women were contacted and briefed on the trial objectives and invited to participate in the trial by visiting the health centre. The revised CTS2 was completed by all the pregnant women that attended. The control group was requested to visit the centre 3 months later for completing the violence screening form. (Intervention group): at the end of the 4th session, participants were asked to visit the health centres 2 months later to complete the violence screening questionnaire again.

Total duration of trial: trial was conducted in Tehran Province over a period of 8 months

Type of analysis: for calculating the prevalence of each type of violence, first, the score of each type of violence was divided into 2 states of 0 and $>1 ; 0$ meant not having experienced violence and $>1$ meant, having experienced violence during the past year.

Types of participants: pregnant women visiting the health centres of Tehran

ITT analysis: NR

\begin{tabular}{|c|c|}
\hline \multirow[t]{2}{*}{ nts } & 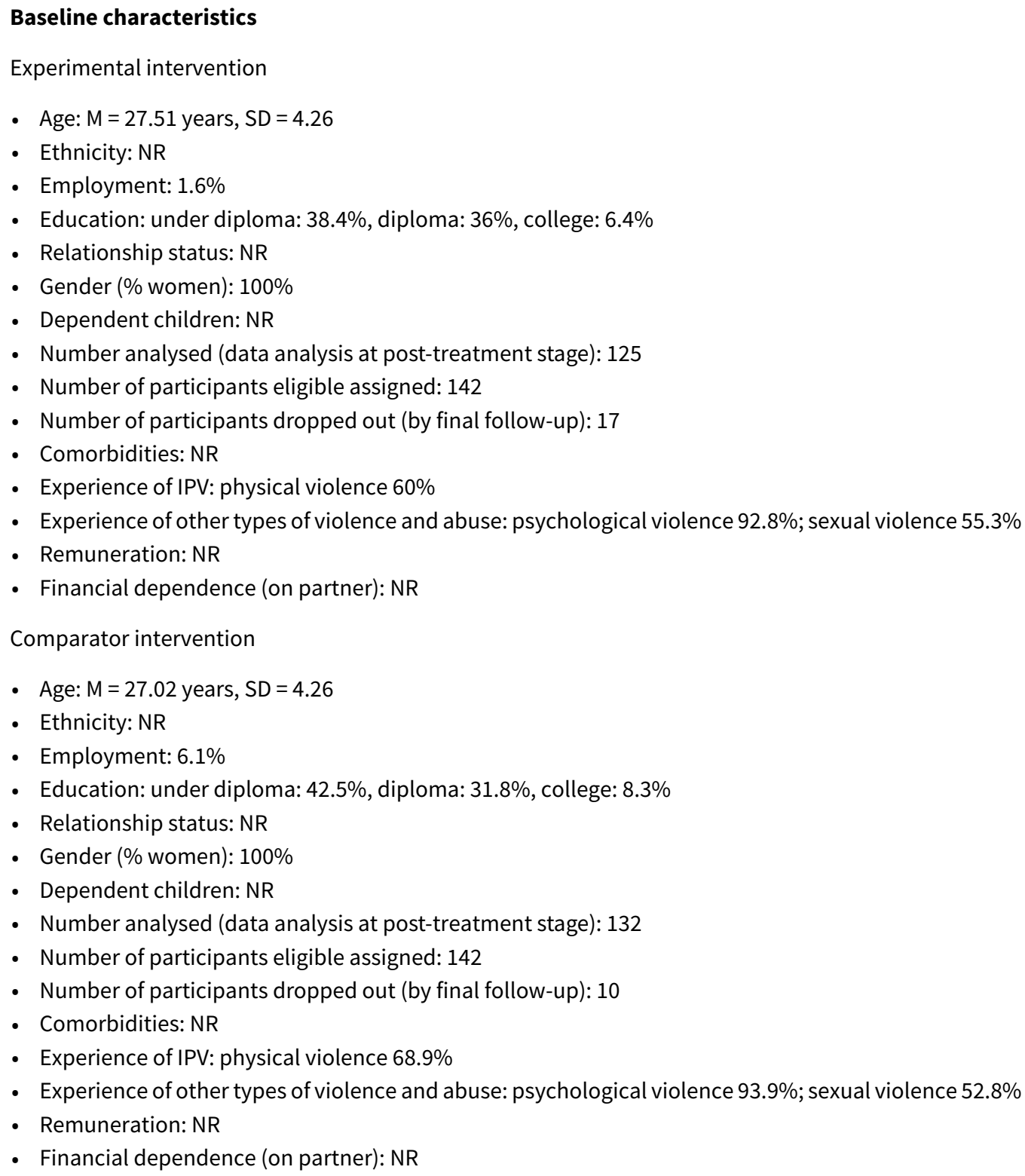 \\
\hline & \\
\hline
\end{tabular}


- Age: NR

- Ethnicity: NR

- Employment: NR

- Education: NR

- Relationship status: NR

- Gender (\% women): 100\%

- Dependent children: NR

- Number analysed (data analysis at post-treatment stage): 257

- Number of participants eligible assigned: 284

- Number of participants dropped out (by final follow-up): 27

- Comorbidities: NR

- Experience of IPV: NR

- Experience of other types of violence and abuse: NR

- Remuneration: NR

- Financial dependence (on partner): NR

Included criteria: being pregnant in the first half of their pregnancy, having reading and writing literacy and having no previous participation in similar studies

Excluded criteria: occurrence of a stressful event such as death and serious illness of a family member, unwillingness to co-operate, and missing > 1 session.

Pretreatment: there were no significant differences between demographic characteristics of the control and the intervention groups. In addition, there were no significant differences between the types of experienced IPV before the intervention, in the 2 groups

Number eligible: 284

Number ineligible: 135

Number of eligible people consented: 284

Number of eligible people recruited: 284

Recruitment setting of participants (e.g. healthcare setting, community setting, shelter setting): healthcare

Sample power calculation: NR

Total number of participants approached: 429

Total number of participants within all groups: 257

Number declined to participate: 10

\section{Intervention characteristics}

Experimental intervention

- Brief description (rationale, main components): problem-orientated coping is a coping strategy adopted in stressful conditions that focuses on the existing problem, plans for solving it and pursues help in others. The problem-orientated coping strategy is closely connected to problem-solving skills. Various studies have shown that, for many different reasons, pregnancy is the best time for performing interventions in health centres in order to reduce and prevent the risk of IPV against them

- Type of intervention: problem-solving skills training

- Mode of delivery (how): face-to-face, group

- Frequency and duration of delivery (when and how much): 4 problem-solving skills training sessions were scheduled with the intervention group and according to their preferences (one session/week, lasting $90 \mathrm{~min}$ ) 
- Level of mental health training of person delivering the intervention: trained midwife (researcher) with qualification in problem-solving teaching.

- Fidelity assessment: not reported

- Intervention model: not reported

- Person delivering the intervention (who provided the intervention): therapist, self-directed or combined: trained midwife (researcher) with qualification in problem-solving teaching.

- Intervention location (where): a separate room in each health centre to the research to enable holding a private session between the midwife (researcher) and each participant.

- Tailoring of intervention (personalised, titrated or adapted): NR

- Intervention modifications (during the trial): NR

- Main techniques of intervention: the used techniques in the training sessions included class activity, role play and home assignments. women of both groups that needed counselling or help were confidentially referred to the relevant organizations

- Intervention manual: NR

- Intervention attendance: sample loss occurred for different reasons, including not visiting the centre for the second time as advised $(n=5)$, concerns about answering the questions on violence $(n=5)$, failure to attend all the sessions of training $(n=10)$

- CCDAN psychological therapies: CBT

- Intensity of intervention: $\geq 5$ sessions

- Person delivering the intervention: healthcare workers

- Gender of therapist: female

Comparator intervention

- Brief description (rationale, main components): after completing the demographic details and the obstetrics history forms, the control group was requested to visit the centre 3 months later for completing the violence screening form

- Type of intervention: no treatment

\section{Re-exposure to IPV}

- Outcome type: dichotomous outcome

- Reporting: fully reported

- Scale: CTS

- Direction: lower is better

\section{Dropouts from treatment}

- Outcome type: dichotomous outcome

- Reporting: fully reported

- Direction: lower is better

Setting: Health networks and centres

Comments: women who had experienced IPV in Tehran Province

Trialauthors: Ziba Taghizadeh, Maryam Poorbakhtiar, Sogand Ghasemzadeh, Khadijeh Azimi, Abbas Mehran

Institution: Nursing and Midwifery Care Research Center and School of Nursing and Midwifery, Tehran University of Medical Sciences

Email: NR

Address: School of Nursing and Midwifery, Tehran University of Medical Sciences, Tehran, Iran 
Trial title (main outcome publication): The effect of training problem-solving skills for pregnant women experiencing intimate partner violence: a randomised control trial

Registered trial protocol ID: IRCT2013041713046N1

Notes

\author{
Analysis of any cost/benefit measures: NR
}

Process evaluation: NR

Summary of participants' views about the intervention: NR

Adverse events (harm) related to participation: NR

\title{
Risk of bias
}

\begin{tabular}{lll}
\hline Bias & Authors' judgement & Support for judgement \\
\hline $\begin{array}{l}\text { Random sequence genera- } \\
\text { tion (selection bias) }\end{array}$ & Low risk & $\begin{array}{l}\text { Judgement comment: "the present quasi-experimental trial was conducted in } \\
\text { Tehran Province over a period of } 8 \text { months on samples selected through ran- } \\
\text { dom stratified cluster sampling. The researcher randomly selected } 2 \text { health } \\
\text { networks affiliated with Tehran University of Medical Sciences (lot drawing) in } \\
\text { Shahr-e-Rey and southern Tehran, as the intervention and the control groups, } \\
\text { respectively. Then 8 centres from each of the southern Tehran network and } \\
\text { Shahr-e-Rey network were randomly selected (using the table of random num- } \\
\text { bers)". }\end{array}$ \\
\hline
\end{tabular}

\begin{tabular}{ll}
\hline $\begin{array}{l}\text { Allocation concealment } \\
\text { (selection bias) }\end{array}$ & Unclear risk \\
& $\begin{array}{l}\text { Judgement comment: } 142 \text { women were randomly assigned to } 2 \text { groups of con- } \\
\text { trol and intervention. However, there is insufficient information about alloca- } \\
\text { tion concealment. }\end{array}$
\end{tabular}

\begin{tabular}{|c|c|c|}
\hline $\begin{array}{l}\text { Blinding of participants } \\
\text { and personnel (perfor- }\end{array}$ & Unclear risk & $\begin{array}{l}\text { Judgement comment: there is insufficient information to assess and deter- } \\
\text { mine blinding of participants and personnel. }\end{array}$ \\
\hline
\end{tabular}

mance bias)

All outcomes

\begin{tabular}{|c|c|c|}
\hline $\begin{array}{l}\text { Blinding of outcome as- } \\
\text { sessment (detection bias) } \\
\text { All outcomes }\end{array}$ & Unclear risk & $\begin{array}{l}\text { Judgement comment: explanations on how to complete the scales were pro- } \\
\text { vided by the midwife (researcher). However, no further information provided } \\
\text { about blinding of outcome assessors. }\end{array}$ \\
\hline
\end{tabular}

Incomplete outcome data Low risk
(attrition bias)

All outcomes
Judgement comment: sample loss and attrition were adequately reported and all outcome measures mentioned in the method section were collected, analysed and fully reported in the results section.

Selective reporting (re- Unclear risk
porting bias) Judgement comment: all outcomes described in the methods section were adequately collected, analysed and reported in the results section of the main published trial. However, there was insufficient or unclear evidence from either the trial protocol or trial registry sites to assess this domain.

Other bias Low risk Judgement comment: none

Tirado-Muñoz 2015

\section{Study characteristics}

\begin{tabular}{ll}
\hline Methods & Trial design: $\mathrm{RCT}$ \\
& Trial grouping: parallel group
\end{tabular}


Assessment modality (e.g. face-to-face, telephone, online): combined face-to-face and telephone

Conflict of interest of trial authors: all trial authors declare that they have no conflicts of interest.

Funding for trial: this work was supported by grants: RD12/0028/009 from Fondo de Investigaciones Sanitarias, Instituto Carlos III-FEDER and a co-operation agreement between Departament de Salut and Parc de Salut Mar de Barcelona to develop and implement a group intervention to reduce IPV among female drug users.

Primary outcomes: IPV and depression

Randomisation method: participants were randomly assigned to receive either the experimental or control condition. Participants were assigned a random number generated using SPSS from 1-14. Numbers 1-7 were assigned to (a) the treatment condition (10 session IPaViT-CBT group intervention) and numbers 8-14 were assigned to (b) control condition.

Secondary outcomes: QoL and substance misuse

Timing of outcome measurements: 1,3 and 12 months post-intervention

Total duration of trial: March 2011-June 2012

Type of analysis: ITT analysis; Fisher's exact tests were used to compare differences in outcomes between groups for dichotomous variables, and Mann-Whitney tests were used to compare differences in outcomes between groups for continuous variables. Prepost-differences between baseline and follow-ups were calculated.

Types of participants: female drug abusers were eligible for the trial if they a) were aged $\geq 18$; b) were currently receiving substance abuse treatment in a outpatient drug treatment centre; $c$ ) were currently in a relationship with a male partner and d) reported IPV in the past month using an adapted version of the CAS and the Psychological Maltreatment of Women Inventory; e) could communicate in Spanish.

ITT analysis: ITT analyses were conducted to avoid loss to follow-up using baseline scores or last follow-up scores.

\section{Participants}

\section{Baseline characteristics}

Experimental intervention

- Age: $M=42.0$ years, $S D=5.56$

- Ethnicity: NR

- Employment: 2 employed

- Education: NR

- Relationship status: 7 partnered

- Gender (\% women): 100

- Dependent children: NR

- Number analysed (data analysis at post-treatment stage): 5

- Number of participants eligible assigned: 7

- Number of participants dropped out (by final follow-up): 0

- Comorbidities: NR

- Experience of IPV: $100 \%$

- Experience of other types of violence and abuse: NR

- Remuneration: none of the participants received financial incentive to respond the follow-up assessments

- Financial dependence (on partner): NR

Comparator intervention

- Age: $\mathrm{M}=39.8$ years, $\mathrm{SD}=11.61$

- Ethnicity: NR

- Employment: 1 employed 
- Education: NR

- Relationship status: 7 partnered

- Gender (\% women): 100

- Dependent children: NR

- Number analysed (data analysis at post-treatment stage): 5

- Number of participants eligible assigned: 7

- Number of participants dropped out (by final follow-up): 5

- Comorbidities: NR

- Experience of IPV: $100 \%$

- Experience of other types of violence and abuse: NR

- Remuneration: none of the participants received financial incentive to respond the follow-up assessments

- Financial dependence (on partner): NR

Total sample

- Age: $M=40$ years, $S D=8.81$

- Ethnicity: NR

- Employment: 3/14 employed

- Education: NR

- Relationship status: 14 partnered

- Gender (\% women): 100

- Dependent children: NR

- Number analysed (data analysis at post-treatment stage): 10

- Number of participants eligible assigned: 14

- Number of participants dropped out (by final follow-up): 5

- Comorbidities: NR

- Experience of IPV: $100 \%$

- Experience of other types of violence and abuse: NR

- Remuneration: none of the participants received financial incentive to respond the follow-up assessments

- Financial dependence (on partner): NR

Included criteria: female drug abusers were eligible for the trial if they a) were aged $\geq 18$; b) were currently receiving substance abuse treatment in a outpatient drug treatment centre; $c$ ) were currently in a relationship with a male partner and d) reported IPV in the past month using an adapted version of the CAS (Hegarty, Sheehan, \& Sconfeld, 1999) and the Psychological Maltreatment of Women Inventory (Tolman, 1999) e) could communicate in Spanish.

Excluded criteria: none indicated

Pretreatment: regarding baseline outcome variables, the intervention and control groups were comparable on almost all characteristics with the exception of self-perceived health status. At baseline, all 14 participants reported any IPV (cut-off score of $\geq 7$ ) but those allocated to the intervention group described slightly more psychological maltreatment than those allocated to the control group. Baseline differences in the outcomes variables could not be statistically controlled due to the small sample size.

\section{Number eligible: 15}

Number ineligible: 55

Number of eligible people consented: 14

Number of eligible people recruited: 14

Recruitment setting of participants (e.g. healthcare setting, community setting, shelter setting): outpatient treatment for a drug use 
Tirado-Muñoz 2015 (Continued)

Sample power calculation: none available

Total number of participants approached: 70

Total number of participants within all groups: 14

Number declined to participate: 1

Interventions

\section{Intervention characteristics}

Experimental intervention

- Brief description (rationale, main components): 10 sessions CBT (Intimate Partner Violence Therapy-Cognitive Behavioral Therapy (IPaViTCBT)) group intervention

- Type of intervention: the final (adapted) intervention IPaViT-CBT was a manualised small-group, cognitive behavioural intervention, designed to reduce IPV and improve depressive symptoms in female drug users

- Mode of delivery (how): face-to-face, group

- Frequency and duration of delivery (when and how much): women randomised to the intervention condition received $10 \times 2$-h sessions over 5 weeks.

- Level of mental health training of person delivering the intervention: high

- Fidelity assessment: participants in the intervention arm of the trial completed an evaluation form at the end of each of the 10 group sessions to determine whether the content and information gained in each session met the objectives of each session with responses ranging from 1 (strongly disagree) to 5 (totally agree). Furthermore, 3 questions were asked at the end of each session about: 1) how comfortable the participant felt during the session, ranging from 1 (very uncomfortable) to 5 (very comfortable); 2) the skill/ability of the therapist, ranging from 1 (excellent) to 5 (poor) and 3) their satisfaction with the session, ranging from 1 (not at all satisfied) to 5 (very satisfied).

- Intervention model: IPaViTCBT

- Person delivering the intervention (who provided the intervention): therapist, self-directed or combined: therapist

- Intervention location (where): outpatients drug clinic

- Tailoring of intervention (personalised, titrated or adapted): Cultural adaptation of the intervention content and activities was conducted taking into account the following aspects: 1 ) language: some concepts or words that could not be literally translated were replaced in order to make more sense to participants; and 2) resources: some exercises, quotes and resources information were replaced to be more culturally meaningful to the target population.

- Intervention modifications (during the trial):

- Main techniques of intervention: the format of the intervention across sessions consisted of a sequence of 5 steps. Each session started with: (1) an inspirational opening (quote from a female artist or writer), which served to inspire and motivate participants in their recovery, and tied into the context of the session; (2) a recovery and relationship check-in was completed for each participant thereafter to detect and discuss any incident occurring between sessions related to participants' drug use or relationship conflicts, identifying triggers that would help women generate safety plans and provides new skills to reduce IPV; (3) raising awareness, through discussion, about the relationship between drug-related behaviours and different forms of IPV; (4) skills-building and group discussion; and (5) a check-in of the participants' outstanding needs/problems and how to address these. At the end of each session, participants were encouraged to practice 'homework' exercises between sessions (e.g. positive self talk, behavioural activation)

- Intervention manual: the intervention was manualised, therefore, instructions were contained in the manual. However, JT met with the clinical psychologist to discuss the delivery of the manualised therapy before each session was delivered.

- Intervention attendance: participation in the group intervention was variable (average 5 sessions attended, range 0-9 sessions)

- CCDAN psychological therapies: CBT

- Intensity of intervention: $\geq 5$ sessions

- Person delivering the intervention: healthcare workers

- Gender of therapist: NR 
Tirado-Muñoz 2015 (Continued)

\section{Comparator intervention}

- Brief description (rationale, main components): the control condition received treatment as usual provided by the outpatient drug treatment centre.

- Type of intervention: usual consisted of fortnightly monitoring of their substance use and psychiatric comorbidity by relevant professionals (psychiatrists, psychologists, and social workers) and monitoring medication if it was prescribed. Treatment as usual included MI, relapse prevention and counselling. While addressing IPV was not part of usual treatment, if participants in the control group disclosed IPV during a routine visit with a professional, the usual referral/treatment pathways were followed to ensure the participant was not in immediate danger

\section{Outcomes}

\section{Depression}

- Outcome type: continuous outcome

- Reporting: fully reported

- Scale: BDI-II

- Range: 0-63

- Direction: lower is better

\section{Quality of life}

- Outcome type: continuous outcome

- Reporting: fully reported

- Scale: visual analogue scale

- Range: 0-100

- Direction: higher is better

\section{Re-exposure to IPV}

- Outcome type: continuous outcome

- Reporting: fully reported

- Scale: Psych Maltreat of Women Inventory Emot/verbal

- Direction: lower is better

\section{Dropouts from treatment}

- Outcome type: dichotomous outcome

- Reporting: fully reported

- Direction: lower is better

Sponsorship source: this work was supported by grants: RD12/0028/009 from Fondo de Investigaciones Sanitarias, Instituto Carlos III--FEDER and a co-operation agreement between "Departament de Salut" and "Parc de Salut Mar de Barcelona"

Country: Spain

Setting: community substance abuse centre

Comments: participants were recruited from the waiting rooms of 2 outpatient drug treatment centres in Barcelona

Trial authors: Judit Tirado-Muñoz, Gail Gilchrist, Eva Lligoña, Louisa Gilbert, Marta Torrens

Institution: Addiction Research Group, IMIM-Institut Hospital del Mar d'Investigacions Mèdiques; Universitat Autónoma de Barcelona, Barcelona, Spain

Email: mtorrens@parcdesalutmar.cat

Address: Marta Torrens MD, PhD. Addiction Unit. Institute of Neuropsychiatry and Addictions, Parc de Salut Mar, Passeig Marítim, 25-29, 08003 Barcelona, Spain 
Tirado-Muñoz 2015 (Continued)

Title (main 'outcome' publication): A group intervention to reduce intimate partner violence among female drug users: results from a randomised controlled pilot trial in a community substance abuse centre

Registered trial protocol ID: NR

Notes

Analysis of any cost/benefit measures: NR

Process evaluation: NR

Summary of participants' views about the intervention: an assessment of the quality of the session was completed by participants at the end of each session. Participants reported that the content knowledge acquired during the sessions was high, with a mean score of 4.5 (SD: 0.50) - the maximum score possible was 5 . In relation to feeling comfortable during sessions, $83 \%$ of participants reported fee-ling "very comfortable" during the sessions, $90 \%$ considered the therapist's performance was "excellent" and $80 \%$ evaluated sessions overall, as "excellent". Trial strengths: This pilot randomised control trial shows encouraging results in terms of feasibility and initial effectiveness of the intervention studied.

Adverse events (harm) related to participation: NR

\section{Risk of bias}

Bias Authors' judgement Support for judgement

Random sequence genera- Low risk

tion (selection bias)

Judgement comment: "the 14 participants were randomly assigned to receive either the experimental or control condition. Participants were assigned a random number generated using SPSS from 1-14. Numbers 1-7 were assigned to (a) the treatment condition (10 session IPaViT-CBT group intervention) and numbers 8-14 were assigned to (b) control condition".

$\begin{array}{ll}\begin{array}{l}\text { Allocation concealment } \\ \text { (selection bias) }\end{array} & \text { Unclear risk } \\ \end{array}$

Blinding of participants and personnel (performance bias)

All outcomes
Judgement comment: there is insufficient information about allocation conJudgement comment: due to the nature of the trial, blinding of the participants was not feasible. Assessors were not blinded to the trial condition. Further, at recruitment, potential participants were informed that they would be randomised to receive either the integrated intervention or to treatment as usual and that if the intervention significantly reduced IPV, those randomised to the control group would be offered the intervention in the future. This was explained to participants prior to their consenting to be randomised.
Blinding of outcome as-
sessment (detection bias)

All outcomes
Judgement comment: "assessors were not blinded to the trial condition. A pre-experimental evaluation was not conducted; the researcher (JT) was responsible for collecting baseline and follow-up data from participants in both the intervention and control conditions".

Judgement comment: attrition rates were moderate but equivalent at 12 months $-2 / 7$ in each group, and no missing data were reported.

\begin{tabular}{lll}
\hline $\begin{array}{l}\text { Incomplete outcome data } \\
\text { (attrition bias) } \\
\text { All outcomes }\end{array}$ & Low risk & $\begin{array}{l}\text { Judgement comment: attrition rates were moderate but equivalent at } 12 \\
\text { months - } 2 / 7 \text { in each group, and no missing data were reported. }\end{array}$ \\
\hline $\begin{array}{l}\text { Selective reporting (re- } \\
\text { porting bias) }\end{array}$ & Unclear risk & $\begin{array}{l}\text { Judgement comment: all outcomes described in the methods section were ad- } \\
\text { equately collected, analysed and reported in the results section of the main } \\
\text { published trial. However, there was insufficient or unclear evidence from ei- } \\
\text { ther the trial protocol or trial registry sites to assess this domain. }\end{array}$ \\
\hline Other bias & High risk & $\begin{array}{l}\text { Judgement comment: there is a high risk of type Il error, as well as issues relat- } \\
\text { ed to the imbalance at baseline that could not be accommodated due to low } \\
\text { sample size. }\end{array}$ \\
\hline
\end{tabular}


Zlotnick 2011

\section{Study characteristics}

Methods
Trial design: $\mathrm{RCT}$ (pilot trial)

Trial grouping: parallel group

Assessment modality (e.g. face-to-face, telephone, online): face-to-face by the therapists is an assumption, because it is very unclear who administered the assessment. It could have been the principal investigator.

Conflict of interest of trial authors: trial author is the principal investigator and also person who supervised intervention therapists. They may have been her students, although it is never clearly stated.

Funding for trial: US National Institute of Mental Health

Primary outcomes: effects of an intervention in reducing PTSD and depressive symptoms from pregnancy until 3 months postpartum in a sample of low-income, pregnant women with IPV within the last year.

Randomisation method: women were randomly assigned to either the intervention or standard care condition. The randomisation allocation schedule was generated by computer and concealed in consecutively numbered, sealed envelopes by the principal investigator who was masked to the women's intake assessments.

\section{Secondary outcomes: none}

Timing of outcome measurements: the following measures were administered at intake, 5-6 weeks after intake, 2 weeks after delivery, and 3-month postpartum:

\section{Total duration of trial: NR}

Type of analysis: all analyses were conducted on the ITT sample. Summary statistics were compiled for the intervention and control groups on rates of IPV victimisation and demographic characteristics. Group differences in baseline characteristics were examined using $\mathrm{Chi}^{2}$ tests for dichotomous variables and one way ANOVAs for continuous variables. In our primary analyses we assessed for a major depressive disorder and PTSD using repeated-measures analysis of variance. Given that all of our dependent measures were assessed at multiple time points, we used the repeated-measures approach in our analyses. In secondary analyses we controlled for the presence of childhood sexual trauma.

Types of participants: pregnant women, between 18-40 years of age, who were attending their prenatal care visit, were approached to participate in the trial.

ITT analysis: all analyses were conducted on the ITT sample

Participants

\section{Baseline characteristics}

Experimental intervention

- Age: $M=24.2$ years, $S D=4.4$

- Ethnicity (\%): $35.7 \%$ (white), $42.9 \%$ (Hispanic), $14.3 \%$ Black; $7.1 \%$ other

- Employment: NR

- Education: $10.7 \%$ (college graduate), $64.3 \%$ (high school graduate), $17.9 \%$ (some high school)

- Relationship status: $39.3 \%$ (single), $25.0 \%$ (married), $21.4 \%$ (cohabiting). $10.7 \%$ (divorced/separated)

- Gender (\% women): 100

- Dependent children: NR

- Number analysed (data analysis at post-treatment stage): 28

- Number of participants eligible assigned: 28

- Number of participants dropped out (by final follow-up): 3

- Comorbidities: NR 
- Experience of IPV: CTS2 mean $=33.4$ (28.4)

- Experience of other types of violence and abuse: NR

- Remuneration: USD 15 (baseline), 20 (6weeks after intake) and 30.00 (4 weeks and 3 months postpartum

- Financial dependence (on partner): NR

Comparator intervention

- Age: $\mathrm{M}=23.5$ years, $\mathrm{SD}=4.7$

- Ethnicity (\%): $42.3 \%$ (white), $42.3 \%$ (Hispanic), $7.7 \%$ (Black), $7.7 \%$ (other)

- Employment: NR

- Education: $15.4 \%$ (college graduate), $50.0 \%$ high school graduate, $34.6 \%$ (some high school)

- Relationship status: $50.0 \%$ (single), $23.1 \%$ (married), 15.4\% (cohabiting) $11.5 \%$ (divorced/separated)

- Gender (\% women): 100

- Dependent children: NR

- Number analysed (data analysis at post-treatment stage): 26

- Number of participants eligible assigned: 26

- Number of participants dropped out (by final follow-up): 5

- Comorbidities: NR

- Experience of IPV: CTS2 mean = 38.7 (39)

- Experience of other types of violence and abuse: NR

- Remuneration: USD 15 (baseline), 20 (6weeks after intake) and 30.00 (4 weeks and 3 months postpartum

- Financial dependence (on partner): NR

Total sample

- Age: $M=23.8$ years, $S D=4.6$

- Ethnicity (\%): 38.9\% (white), $42.6 \%$ (Hispanic), $11.1 \%$ (Black), $7.4 \%$ (other)

- Employment: $33.3 \%$ employed full time and $33.3 \%$ unemployed

- Education: 13.0 (college graduate), 57.4\% (high school graduate), $25.9 \%$ (some high school)

- Relationship status: $44.4 \%$ (single), $24.1 \%$ (married), 18.5\% (cohabiting), $11.1 \%$ (divorced/separated)

- Gender (\% women): 100

- Dependent children: NR

- Number analysed (data analysis at post-treatment stage): 54

- Number of participants eligible assigned: 54

- Number of participants dropped out (by final follow-up): 8

- Comorbidities: NR

- Experience of IPV: 54 pregnant women in abusive relationships reported on average 35.9 acts of physical, sexual, and/or psychological abuse in the past year (intervention group, $\mathrm{M}=33.4$; control group, $M=38.7 ; p=0.57)$. In terms of severity of abuse, 38 (70.4\%) participants reported at least 1 act of severe abuse in the past year with the majority of severe abuse being acts of psychological abuse (average of 4.7 severe psychological acts; 2.4 severe physical and 0.6 severe sexual).

- Experience of other types of violence and abuse: not fully reported. Secondary analyses found that there was a moderate effect for women with histories of childhood sexual abuse and rape compared to women in the control condition in reducing level of depressive symptoms and a large effect in reducing level of PTSD symptoms from intake to 3-months postpartum.

- Remuneration: USD 15 (baseline), 20 ( 6 weeks after intake) and 30 (4 weeks and 3 months postpartum

- Financial dependence (on partner): NR

Included criteria: pregnant women, between 18-40 years of age, who were attending their prenatal care visit, were approached to participate in the trial. Those women who screened positive for recent (past year) IPV, based on their CTS2 responses, were invited to participate in the next phase of the trial.

Excluded criteria: for safety reasons, we did not approach women who were with their partners. At a separate meeting, women were assessed for current affective disorders, PTSD, and substance use as 
determined by the relevant modules of the Structured Clinical Interview for the DSM-IV Axis I Disorders Nonpatient Version (SCID-NP). Women who met criteria for any one of these disorders were excluded from the trial and referred for appropriate treatment.

Pretreatment: no significant socio-demographic differences. However, the intervention group and the control group differed on number of lifetime traumas. The intervention group reported significantly more traumas than the control group $\left(\mathrm{Chi}^{2}=3.80, \mathrm{P}=0.051\right)$. In particular, rape and child molestation were more prevalent in the intervention group $(\mathrm{N}=12 ; 42.9 \%)$ compared to the control group $(\mathrm{N}=4$; $15.4 \%)$.

Number eligible: unclear. No flowchart. Of the 1633 women approached to be in the trial, $317(19.4 \%)$ women denied the presence of IPV, 106 (6.5\%) were already in some form of mental health treatment, $421(25.8 \%)$ said they were too busy or gave no reason, and $578(35.4 \%)$ were with their male partners. For safety reasons, we did not approach women who were with their partners.

Number ineligible: 99

Number of eligible people consented: 211

Number of eligible people recruited: 80

Recruitment setting of participants (e.g. healthcare setting, community setting, shelter setting): ANCs

Sample power calculation: To achieve $80 \%$ power (alpha $=0.05)$, the sample size in each group would need to be 50 participants.

Total number of participants approached: 1633

Total number of participants within all groups: 54

Number declined to participate: 1475

Interventions

\section{Intervention characteristics}

Experimental intervention

- Brief description (rationale, main components): the intervention was designed to help participants improve their significant interpersonal relationships, change their expectations about them, assist them in building, or improving their social support networks, and master their role transition to motherhood since deficits in these areas appear to play a salient role in the onset of perinatal depression and PTSD.

- Type of intervention: the intervention was based primarily on the principles of Interpersonal Psychotherapy, a pertinent theoretical framework. The IPT approach, with its emphasis on the enhancement of social support, is especially relevant for women with IPV because research has found social support to be protective against the negative effects of partner violence on women's mental health

- Mode of delivery (how): face-to-face

- Frequency and duration of delivery (when and how much): the IPT-based intervention involved $4 \times 60$ min individual sessions over a 4-week period before delivery and followed by 1 × 60-min individual 'booster' session within 2 weeks of delivery

- Level of mental health training of person delivering the intervention: NR

- Fidelity assessment: the first author (Dr. Zlotnick) provided training on how to deliver the highly scripted intervention and provided weekly supervision to the 2 trial interventionists.

- Intervention model: interpersonal psychotherapy

- Person delivering the intervention (who provided the intervention): therapist, self-directed or combined: therapist

- Intervention location (where): ANCs

- Tailoring of intervention (personalised, titrated or adapted): NR

- Intervention modifications (during the trial): NR

- Main techniques of intervention: briefly, the content of the intervention sessions consisted of the following: the first session focused on topics that included a rationale for the programme, review of the 
course outline, evaluation of healthy relationships, types of interpersonal disputes, and abusive relationships. Topics for session 2 included stress management skills, consequences of abuse, cycle of abuse, and making a safety plan. Topics for session 3 included emotional risks of abuse-signs and symptoms of 'baby blues' and postpartum depression, PTSD and substance use, and the management of role transitions with an emphasis on transition to motherhood and self-care. Topicsfor session 4 included the development of a support system,techniques for asking for support, resolving interpersonal conflicts, and goal-setting. The last session (within 2 weeks of delivery) provided an opportunity to review and reinforce the content of the previous sessions ('booster' session) and address any new issues related to the birth of the infant.

- Intervention manual: NR, however, the article states that "The first author (Dr. Zlotnick) provided training on how to deliver the highly scripted intervention and provided weekly supervision to the 2 trial interventionists."

- Intervention attendance: 28 women in the intervention attended on average, 3 out of the 5 available intervention sessions.

- CCDAN psychological therapies: integrative therapies

- Intensity of intervention: $\geq 5$ sessions

- Person delivering the intervention: healthcare workers

- Gender of therapist: NR

Comparator intervention

- Brief description (rationale, main components): women in the standard care condition received the usual medical care provided for pregnant women at their clinic as well as the educational material and a listing of resources for IPV.

- Type of intervention: usual care

\section{Depression}

- Outcome type: continuous outcome

- Reporting: fully reported

- Scale: Edinburgh Postnatal Depression Scale

- Direction: lower is better

PTSD

- Outcome type: continuous outcome

- Reporting: fully reported

- Scale: Longitudinal Interval Follow-up Examination

- Range: 1-6

- Direction: lower is better

\section{Re-exposure to IPV}

- Outcome type: continuous outcome

- Reporting: fully reported

- Scale: revised CTS

- Direction: lower is better

\section{Dropouts from treatment}

- Outcome type: dichotomous outcome

- Reporting: fully reported

- Direction: lower is better

Identification
Sponsorship source: this trial was supported by a grant to Caron Zlotnick from the National Institute of Mental Health (R34MH075013-01).

Country: USA 
Setting: primary care clinics

Comments: participants for the trial were recruited from 3 Rhode Island sites: 2 primary care clinics and one private obstetrics and gynaecology clinic.

Trial authors: Caron Zlotnick

Institution: Warren Alpert School of Medicine, Brown University

Email: caron_zlotnick@brown.edu

Address: Warren Alpert School of Medicine, Brown University, Providence, Rhode Island, USA

Title (main 'outcome' publication): An interpersonally based intervention for low-income pregnant women with intimate partner violence: a pilot trial

Registered trial protocol ID: NCT00602732

Notes Analysis of any cost/benefit measures: NR

Process evaluation: NR

Summary of participants' views about the intervention: NR

Adverse events (harm) related to participation: NR

\section{Risk of bias}

\begin{tabular}{lll}
\hline Bias & Authors' judgement & Support for judgement \\
\hline $\begin{array}{l}\text { Random sequence genera- } \\
\text { tion (selection bias) }\end{array}$ & Low risk & $\begin{array}{l}\text { Judgement comment: "computer randomisation was put into sealed en- } \\
\text { velopes by lead researcher who was blinded to allocation". }\end{array}$ \\
\hline $\begin{array}{l}\text { Allocation concealment } \\
\text { (selection bias) }\end{array}$ & Low risk & $\begin{array}{l}\text { Judgement comment: "the randomisation allocation schedule was generat- } \\
\text { ed by computer and concealed in consecutively numbered, sealed envelopes } \\
\text { by the principal investigator who was masked to the women's intake assess- } \\
\text { ments". }\end{array}$
\end{tabular}

\begin{tabular}{|c|c|c|}
\hline $\begin{array}{l}\text { Blinding of participants } \\
\text { and personnel (perfor- } \\
\text { mance bias) }\end{array}$ & High risk & $\begin{array}{l}\text { Judgement comment: it is very difficult in a pragmatic trial (and this is a feasi- } \\
\text { bility/pilot trial) to blind staff delivering the intervention and even more when } \\
\text { the Chief Investigator is supervising the staff delivering the intervention. }\end{array}$ \\
\hline
\end{tabular}

All outcomes the Chief Investigator is supervising the staff delivering the intervention.

\begin{tabular}{|c|c|c|}
\hline $\begin{array}{l}\text { Blinding of outcome as- } \\
\text { sessment (detection bias) }\end{array}$ & Unclear risk & $\begin{array}{l}\text { Judgement comment: this is a relatively well-conducted pilot trial. However, } \\
\text { there is insufficient information that assessment was blinded. }\end{array}$ \\
\hline
\end{tabular}
All outcomes

\begin{tabular}{|c|c|c|}
\hline $\begin{array}{l}\text { Incomplete outcome data } \\
\text { (attrition bias) } \\
\text { All outcomes }\end{array}$ & Unclear risk & $\begin{array}{l}\text { Judgement comment: attrition and exclusions of participants were adequate- } \\
\text { ly described. However, this is a feasibility trial with a very low recruitment suc- } \\
\text { cess rate for those eligible. Of the } 1633 \text { women approached to be in the trial, } \\
\text { the final sample was } 54 \text { pregnant women. }\end{array}$ \\
\hline
\end{tabular}

\begin{tabular}{ll}
\hline $\begin{array}{l}\text { Selective reporting (re- } \\
\text { porting bias) }\end{array}$ & Unclear risk \\
& $\begin{array}{l}\text { Judgement comment: all outcomes described in the methods section were ad- } \\
\text { equately collected, analysed and reported in the results section of the main } \\
\text { published trial. However, there was insufficient or unclear evidence from ei- } \\
\text { ther the trial protocol or trial registry sites to assess this domain. }\end{array}$
\end{tabular}

Other bias High risk
Judgement comment: "results of this trial must be viewed in light of the con-
straints of this investigation. Since the trial did not recruit women whose part-
ners were present, the intervention was tested with a select group of women.
Perhaps a percentage of the women with partners present for their prenatal
visit may have had partners who were particularly controlling and abusive. It 
is therefore unknown how these women may have responded to the intervention. Also, at least $54 \%$ of women approached to be in the trial denied any IPV or refused to participate in the trial. It is therefore unknown as to what percentage of these women may have had IPV experiences and if participants in the trial were representative of women with IPV. overall, it is highly likely that the small sample and the un-representativeness of the small sample used, even for a pilot will bias assumptions about lack of success. It may work differently with differently abused women".

\section{Study characteristics}

Methods

\author{
Trial design: $\mathrm{RCT}$ \\ Trial grouping: parallel group
}

Assessment modality (e.g. face-to-face, telephone, online): computer and face-to-face

Conflict of interest of trial authors: no competing financial interests exist for authors Golfo Tzilos Wernette and Christina Raker. Caron Zlotnick's husband is a consultant for Soberlink.

Funding for trial: this research was supported by the Eunice Kennedy Shriver National Institute of Child Health and Human Development (NICHD) HD077358.

Primary outcomes: these are as stated in the Clinical trials register. There is no mention in the article of any outcome measures, other than IPV (CAS). CAS Victimization Total Score, assessed at baseline, and again 3 months later

A widely used self-report of behaviours that includes a 36-item scale - only the CAS Victimization Total score was calculated. Items were scored between 0 and 5 , with Never $=0$ and Daily $=5$. Scale range is from 0-180. Mean score was used to calculate differences between baseline and follow-up and between groups. The lower the score, the better or less victimisation. Safety Behavior Checklist (SBC), assessed at baseline, and again 3 months later, Includes 15 items that assess the use of strategies suggested to keep victim safe (e.g. hiding money and extra clothing). Items were scored as Yes $=1$ or No $=0$ or Not applicable. Scale range is from 0-15. Mean score was used to calculate differences between baseline and follow-up and between groups. The higher the score, the better or more use of safety behaviours.

Effectiveness in Obtaining Resources Scale (EOR), assessed at baseline, and again 3 months later. Assesses the women's effectiveness in obtaining resources from 11 different types of community resources including mental health treatment, church or clergy, health care, legal services, police, or social services. Items were scored as Yes $=1$ or $\mathrm{No}=0$. Scale range is from $0-11$. Mean score was used to calculate differences between baseline and follow-up and between groups. The higher the score, the better or more they were effective in obtaining resources.

Randomisation method: computer based; after completion of the baseline assessment, the (computer) narrator "flipped a coin" and participants were randomised into the control or SURE intervention

Secondary outcomes: Motivation Scale, assessed at baseline, and again 3 months later. A 1-item measure, was modified to assess how ready they are to use treatment, resources, and/or support for any partner abuse. This item was scored with a range from $0-10.0=$ not ready at all to $10=$ completely ready. Mean score was used to calculate differences between baseline and follow-up and between groups. The higher the score, the better or more ready to use treatment, resources, or support for any partner abuse. The Readiness to Change Contemplation Ladder, assessed at baseline, and again 3 months later. 1-item measure to assess readiness to make changes to increase safety. This item was scored with a range from $0-10.0=$ not prepared to change to $10=$ already changing. Mean score was used to calculate differences between baseline and follow-up and between groups. The higher the score, the better or more ready to make changes to increase safety.

Timing of outcome measurements: baseline and 4-month follow-up (CAS) 


\section{Total duration of trial: NR}

Type of analysis: descriptive statistics, paired t-test, Wilcoxon signed rank

Types of participants: perinatal women who were $\geq 18$ years of age. English-speaking, and reported experiencing IPV in the past 12 months were eligible and recruited into the trial. Perinatal women (currently pregnant or within 6-months postpartum) seeking mental health treatment at an urban hospital-based behavioral health clinic for perinatal women were invited to a Health Survey, a survey to help mothers have healthier pregnancies and be healthy during the postpartum period

ITT analyses: NR

Participants

\section{Baseline characteristics}

Experimental intervention

- Age: $M=27.04, S D=5.76$

- Ethnicity: white (60.71\%); Black (7.14\%); bi-racial (3.57\%); other (28.57\%)

- Employment: full-time (25.00\%); part-time (21.43\%); student (0\%); housewife (21.43\%); unemployed (32.14\%)

- Education: did not graduate high school (14.29\%); high school graduate (32.14\%); technical/trade school (7.14\%); some college (21.43\%); college graduate (21.43\%); postgraduate $(3.57 \%)$

- Relationship status: married (17.86\%); separated (7.14\%); divorced (3.57\%); single, no relationship (28.57\%); single, in a relationship (35.71\%); single, same-sex partner $(7.14 \%)$

- Gender (\% women): 100

- Dependent children: NR

- Number analysed (data analysis at post-treatment stage): 26

- Number of participants eligible assigned: 28

- Number of participants dropped out (by final follow-up): 3

- Comorbidities: NR

- Experience of IPV: $\mathrm{M}=6.18, \mathrm{SD}=1.79$

- Experience of other types of violence and abuse: NR

- Remuneration: participants were given gift certificates for USD 5.00 for participation in the screening survey and financial incentive USD 30 for the baseline and 4-month follow-up assessment.

- Financial dependence (on partner): NR

Comparator intervention

- Age: $\mathrm{M}=28.32, \mathrm{SD}=4.75$

- Ethnicity: white (40.0\%\%); Black (16.0\%\%); bi-racial (16.0\%); other (28.0\%)

- Employment: full-time (32.00\%); part-time (12.00\%); student (12.00\%); housewife (16.00\%); unemployed (28.00\%)

- Education: did not graduate high school (8.00\%); high school graduate (32.00\%); technical/trade school (4.00\%); some college $(44.00 \%)$; college graduate $(8.00 \%)$; postgraduate $(4.00 \%)$

- Relationship status: married (12.0\%\%); separated (12.0\%); divorced (4.0\%); single, no relationship $(28.0 \%)$; single, in a relationship (44.0\%); single, same-sex partner $(0 \%)$

- Gender (\% women): 100

- Dependent children: NR

- Number analysed (data analysis at post-treatment stage): 23

- Number of participants eligible assigned: 25

- Number of participants dropped out (by final follow-up): 2

- Comorbidities: NR

- Experience of IPV: 6.04 (SD = 2.05)

- Experience of other types of violence and abuse: NR

- Remuneration: participants were given gift certificates for USD 5.00 for participation in the screening survey and financial incentive USD 30 for the baseline and 4-month follow-up assessment.

- Financial dependence (on partner): NR 
Total sample

- Age: $M=27.64, S D=5.30$

- Ethnicity: white (50.94\%); Black (11.32\%); bi-racial (9.43\%); other (28.30\%)

- Employment: full-time (28.30\%); part-time (16.98\%); student (5.66\%); housewife (18.87\%); unemployed (30.19\%)

- Education: did not graduate high school (11.32\%); high school graduate (32.08\%); technical/trade school (5.66\%); some college (32.08\%); college graduate (15.09\%); postgraduate $(3.77 \%)$

- Relationship status: married (15.09\%); separated (9.43\%); divorced (3.77\%); single, no relationship (28.30\%); single, in a relationship (39.62\%); single, same-sex partner (3.77\%)

- Gender: 100

- Dependent children: NR

- Number analysed (data analysis at post-treatment stage): 49

- Number of participants eligible assigned: 53

- Number of participants dropped out (by final follow-up): 5

- Comorbidities: perinatal women (currently pregnant or within 6-months postpartum) seeking mental health treatment at an urban hospital based hospital based behavioral health clinic for perinatal women

- Experience of IPV: there were no significant differences between the 2 conditions on the IPV at risk scores (i.e. WAST scores) at screening with SURE participants obtaining a mean of $6.18(S D=1.79)$ and the control a mean score of $6.04(S D=2.05)$ (t-test $p=0.794)$.

- Experience of other types of violence and abuse: NR

- Remuneration: participants were given gift certificates for USD 5.00 for participation in the screening survey and financial incentive USD 30 for the baseline and 4-month follow-up assessment.

- Financial dependence (on partner): NR

Included criteria: For safety reasons, only women who were unaccompanied while waiting for their appointment were approached by the research assistant to complete the health survey. Perinatal women who were 18 years of age or older, English-speaking, and reported experiencing IPV in the past 12 months were eligible and recruited into the trial

\section{Excluded criteria: NR}

Pretreatment: there were no significant differences between the 2 conditions on the IPV at risk scores (i.e. WAST scores) at screening with SURE participants obtaining a mean of $6.18(S D=1.79)$ and the control a mean score of $6.04(S D=2.05)$ (t-test $p=0.794)$. However, while demographic details was reported for both groups, there was no indication about any group differences.

Number eligible: 53

Number ineligible: 248

Number of eligible people consented: 53

Number of eligible people recruited: 53

Recruitment setting of participants (e.g. healthcare setting, community setting, shelter setting): perinatal women (currently pregnant or within 6-months postpartum) seeking mental health treatment at an urban hospital-based behavioral health clinic for perinatal women were invited to a 'Health Survey', a survey to help mothers have healthier pregnancies and be healthy during the postpartum period

Sample power calculation: the power was too low in the current sample to detect differences between the 2 conditions

Total number of participants approached: 443

Total number of participants within all groups: 53

Number declined to participate: 142 
- Brief description (rationale, main components): computer program based on MI principles, voice-led for low literacy and emphasises increasing participants' awareness to successful steps towards their own well-being in line with the empowerment model of care for IPV. Followed after 1 month with inperson booster session to bolster intervention effects

- Type of intervention: MI

- Mode of delivery (how): computer for $40 \mathrm{~min}$ and $15 \mathrm{~min}$ face-to-face

- Frequency and duration of delivery (when and how much): once for computer intervention (30-40 min) and 10-15 min booster session in person or phone

- Level of mental health training of person delivering the intervention: unclear for booster session

- Fidelity assessment: none mentioned for booster but computer unnecessary

- Intervention model: MI

- Person delivering the intervention (who provided the intervention): therapist, self-directed or combined: combined computer self-directed and face-to-face or phone booster

- Intervention location (where): unclear but the computer in the mental health clinic: participants completed the intervention in a private and confidential setting

- Tailoring of intervention (personalised, titrated or adapted): not mentioned. Computer is segmented so participants can choose sessions, but all could see same segments. unclear how flexible booster session was.

- Intervention modifications (during the trial): none mentioned

- Main techniques of intervention: computer self-completion

- Intervention manual: computer program, so software program would be 'manualised' in code

- Intervention attendance: all 28 participants completed computer program, but only 26 received booster

- CCDAN psychological therapies: integrative therapies

- Intensity of intervention: up to 4 sessions

- Person delivering the intervention: unclear

- Gender of therapist: NR

Comparator intervention

- Brief description (rationale, main components): the content of the control condition included watching brief segments of popular television shows and following up with questions for ratings of their preference.

- Type of intervention: watching brief segments of popular television shows and following up with questions for ratings of their preference

Outcomes

\section{Re-exposure to IPV}

- Outcome type: continuous outcome

- Reporting: fully reported

- Scale: the CAS

- Direction: lower is better

\section{Dropouts from treatment}

- Outcome type: dichotomous outcome

- Reporting: fully reported

- Direction: lower is better

Identification Sponsorship source: this research was supported by the Eunice Kennedy Shriver National Institute of Child Health and Human Development (NICHD) HD077358.

Country: USA

Setting: hospital-based behavioural health clinic 
Comments: currently pregnant or within 6-months postpartum women seeking mental health treatment at a large urban hospital-based behavioural health clinic for perinatal women

Trialauthors: Caron Zlotnick \& Golfo Tzilos Wernette \& Christina A. Raker

Institution: Department of Psychiatry and Human Behaviour, Brown University

Email: czlotnick@butler.org

Address: Department of Psychiatry and Human Behavior, Brown University, Providence, RI, USA

Title (main 'outcome' publication): A randomised controlled trial of a computer-based brief intervention for victimized perinatal women seeking mental health treatment

Registered trial protocol ID: NCT02370394

Notes Analysis of any cost/benefit measures: NR

\section{Process evaluation: NR}

Summary of participants' views about the intervention: participant self-reported ratings of both the computer software as well as aspects of the intervention were consistently high. With regard to the overall utility of the intervention, $100 \%$ of the women reported that the information and resources presented were helpful. Mean ratings of individual questions tapping satisfaction with the computer software ranged from a low of 3.2 (out of 5) to a high of 3.9. Mean ratings of the specific components of the SURE content ranged from a low of 5.7 (out of 7 ) (for the parrot avatar) to a high of 6.8 for the component that provided information on partner abuse and a high of 6.4 for the component on steps to increase your safety. During the in-person/telephone booster session, 22 women (out of 26 who completed a booster) noted that they used techniques mentioned in the intervention in working on making changes and found these techniques to be helpful (e.g. reminding themselves of their reasons for change, speaking to counsellor or relative/close friend about their safety plan, and reminding themselves that change is difficult but possible). 23 of the 26 women reported in the booster session that they were still in counselling and 18 of those reported that they had shared aspects of the SURE intervention and their IPV experiences with their counsellor.

Adverse events (harm) related to participation: there were a total of 22 serious adverse events that were classified as unrelated to the trial.

\section{Risk of bias}

\begin{tabular}{lll}
\hline Bias & Authors' judgement & Support for judgement \\
\hline $\begin{array}{ll}\text { Random sequence genera- } \\
\text { tion (selection bias) }\end{array}$ & Low risk & $\begin{array}{l}\text { Judgement comment: "after completion of the baseline assessment, the } \\
\text { (computer) narrator "flipped a coin" and participants were randomised into } \\
\text { the control or SURE intervention. Although the phrase 'flipped a coin' is un- } \\
\text { clear, this appears to mean random computer-generated selection". However, } \\
\text { flipping a coin creates a high risk of bias, yet given this was computer-generat- } \\
\text { ed, the risk is low. }\end{array}$ \\
\hline
\end{tabular}

$\begin{array}{ll}\begin{array}{l}\text { Allocation concealment } \\ \text { (selection bias) }\end{array} & \begin{array}{l}\text { Judgement comment: as the process of assessment, randomisation and inter- } \\ \text { vention or comparison program was all completed on a computer/tablet, it } \\ \text { appears as though the allocation is concealed. There could be no disruption } \\ \text { unless the Research Assistants took part or were asked to clarify aspects of } \\ \text { the trial and then it might be revealed. However, this was not sufficiently dis- } \\ \text { cussed. }\end{array}\end{array}$

Blinding of participants and personnel (performance bias)

All outcomes

\section{High risk}

Judgement comment: all assessments consisted of self-report measures, which were computer-delivered and this is a feasibility trial. But given that safety planning and effectiveness at gaining resources were primary outcomes (information derived from trial registration) and that during the inperson/telephone booster session, 22 women (out of 26 who completed a booster) noted that they used techniques mentioned in the intervention in 
Zlotnick 2019 (Continued)

working on making changes and found these techniques to be helpful (e.g. reminding themselves of their reasons for change, speaking to counsellor or relative/close friend about their safety plan, and reminding themselves that change is difficult but possible). Blinding of the booster interventionist and her assessment of 2 key outcomes is a problem. This is noted as a limitation of blinding of personnel and for the impact of the intervention.

\begin{tabular}{lll}
\hline Blinding of outcome as- & High risk & Judgement comment: in the reporting of this pilot trial, it is unclear as to how \\
sessment (detection bias) & personnel interacts with the participants at the 4-month follow-up. Further, \\
All outcomes & the person conducting the booster session with the women in the intervention \\
& arm also conducted the assessment and this could lead to a high risk of bias.
\end{tabular}

Incomplete outcome data Unclear risk (attrition bias)

Judgement comment: this is a feasibility trial, so this effect is disputable with All outcomes such a small number, but while the flowchart indicates that all women were involved in the ITT analysis, there were small dropouts, 2 from either arm. However, this is not sufficiently referred to in the article.

Selective reporting (re- Unclear risk

Judgement comment: during the in-person/telephone booster session, 22 porting bias) women (out of 26 who completed a booster) noted that they used techniques mentioned in the intervention in working on making changes and found these techniques to be helpful (e.g. reminding themselves of their reasons for change, speaking to counsellor or relative/close friend about their safety plan, and reminding themselves that change is difficult but possible). This is the only mention of the other 2 primary outcomes noted in the trial register. These outcomes are not reported at all for women in the comparison arm. The IPV measures are very detailed (almost too much and there is no hypothesis about how this intervention could feasibly limit partner's use of violence). All outcomes described in the methods section were adequately collected, analysed and reported in the results section of the main published trial. However, there was insufficient or unclear evidence from either the trial protocol or trial registry sites to assess this domain.

Other bias High risk

\begin{abstract}
Judgement comment: this is a feasibility trial and there is a high risk of Type I and II errors. Further authors state that "We also acknowledge the limitations of the trial. Our sample size is small $(N=53)$ yet adequate for a pilot feasibility trial. Women in our sample were recruited from a hospital in the Northeast and our results may not be generalizable to all perinatal women. Our sample was also a select sample of perinatal women in that the majority of women who were not approached for the trial were accompanied. It is possible that these women were with a partner who was particularly controlling and abusive. Further, $58 \%$ of the women screened reported no IPV experiences. It is unknown as to what \% of these women may have had IPV experiences and if participants in the trial were representatives of women with IPV seeking mental health treatment. Another limitation was that our booster session was conducted either by telephone or in person, rather than completed on the computer, which may have led to biased responses."
\end{abstract}

AAS: Abuse Assessment Scale; ANC: antenatal clinic; BAI: Beck Anxiety Inventory; BDI: Beck Depression Inventory; BSI: Brief Symptom Inventory; CAS: Composite Abuse Scale; CBT: cognitive behavioural therapy; CCDAN: Cochrane Collaboration Depression, Anxiety and Neurosis Group; CHW: community health worker; Cl: confidence interval; Col: conflict of interest; CORE-OM: Clinical Outcomes in Routine Evaluation- Outcome Measure; CSQ: Client Satisfaction Questionnaire; CTS: Conflict Tactics Scale; DSM IV: Diagnostic and Statistical Manual of Mental Disorders, volume 4; DVA: domestic violence and abuse; ED: Emergency Department; GAD: Generalized Anxiety Disorder questionnaire; GDS: Geriatric Depression Scale; GHQ-12: 12-item General Health Questionnaire; GP: general practitioner; HADS: Hospital Anxiety and Depression Scale; ICC: intra-class correlation; IPV: intimate partner violence; IRB: Institutional Review Board; ITT: intentionto-treat; IVRS: interactive voice response system; LEC: Life Events Checklist; M: mean; MANOVA: Multivariate analyses of variance; MI: Motivational Interviewing; N/A: not applicable; NIDA: National institute on Drug Abuse; NIH: National Institutes of Health; NIHR: National Institute for Health Research; NR: not reported; PCL: Post-traumatic Stress Disorder Checklist; PCL-C: Post-traumatic Stress Disorder Checklist - Civilian; PHQ: Patient Health Questionnaire; PSS: Post-traumatic Stress Disorder Symptom Scale; PTSD: post-traumatic stress disorder; QOL: quality of life; RCT: randomised controlled trial; SAE: serious adverse event; SCORE: systemic clinical outcome and routine 
evaluation; SD: standard deviation; SF-12: Short form-12; STI: sexually transmitted infection; WHO: World Health Organization; WHODAS: World Health Organization Disability Adjustment Schedule

Characteristics of excluded studies [ordered by study ID]

\begin{tabular}{|c|c|}
\hline Study & Reason for exclusion \\
\hline Anderson 2010 & Wrong participant (history of sexual assault not related to IPV) \\
\hline Arinero 2004 & Wrong study design (no randomisation) \\
\hline Bahadir Yilmaz 2018 & Wrong intervention (empowerment programme, non-psychological intervention) \\
\hline Bahia 2018 & Wrong intervention (couple-based therapy) \\
\hline Bass 2013 & Wrong participant (sexual violence not related to IPV) \\
\hline Braithwaite 2014 & Wrong intervention (non-psychological) \\
\hline Brannen 1996 & Wrong intervention (couple-based therapy) \\
\hline Calderón 2008 & Wrong intervention (non-psychological) \\
\hline Carlson 2012 & Wrong intervention (HIV/STI risk reduction intervention with women who exchange sex) \\
\hline Chermack 2017 & Wrong participant (women participants were only $30 \%$ of total sample) \\
\hline Chronister 2006 & Wrong intervention (career-focused intervention) \\
\hline Clark 2014 & $\begin{array}{l}\text { Wrong study design (availability sampling was used in order to place participants in either the trau- } \\
\text { ma sensitive yoga group therapy or the traditional group therapy conditions (no randomisation) }\end{array}$ \\
\hline Cogan 2003 & Wrong study design (no control comparison) \\
\hline Cohen 2006 & $\begin{array}{l}\text { Wrong participant (majority of participating women had traumatic interpersonal trauma before the } \\
\text { age of 16) }\end{array}$ \\
\hline Colosetti 2000 & Wrong study design (single-subject research design) \\
\hline Constantino 2005 & Wrong intervention (social support intervention, information/empowerment intervention) \\
\hline Cort 2014 & Wrong design (single group pre-post design) \\
\hline Crespo 2010 & $\begin{array}{l}\text { Wrong intervention (two intervention programmes 'exposure techniques' and communication } \\
\text { skills training, with no comparison/control group) }\end{array}$ \\
\hline Cruz-AlmanzaMa 2006 & Wrong study design \\
\hline Dutton 2013 & Conceptual and preliminary pilot trial \\
\hline Echeburúa 1996 & Wrong participant (rape and sexual aggression not related to IPV) \\
\hline Echeburúa 1997 & Wrong participant (rape and sexual aggression not related to IPV) \\
\hline Echeburúa 2014 & Wrong study design \\
\hline Eden 2015 & Wrong intervention (safety Decision Aid tool, non-psychological intervention) \\
\hline
\end{tabular}




\section{Study}

Faker 2016

Fallot 2011

\section{Reason for exclusion}

Wrong study design (comparing the personality characteristics and the rate of spouse abuse in spouse abused)

Wrong participant (heterogeneous population of women with various forms of traumatic experiences)

\begin{tabular}{|c|c|}
\hline Fals-Stewart 2002 & Wrong intervention (couple-based therapy) \\
\hline Fals-Stewart 2006 & Wrong study design \\
\hline Feinberg 2016 & Wrong intervention (non-IPV-related couple-therapy) \\
\hline Florsheim 2011 & Wrong intervention (parenthood programme) \\
\hline Foa 1995 & Wrong participant (rape and sexual assault not related to IPV) \\
\hline Foa 2005 & Wrong participant (rape and sexual assault not related to IPV) \\
\hline Foshee 2000 & Wrong participant (adolescent participants only and non-psychological intervention) \\
\hline Foshee 2016 & Wrong participant (average age of the participating teens was 13.6 years) \\
\hline Gallas 2009 & Wrong study design (pilot trial with no randomisation) \\
\hline Galovski 2009 & $\begin{array}{l}\text { Wrong participant (experience of rape (oral, anal, or vaginal) in childhood or adulthood, diverse } \\
\text { population) }\end{array}$ \\
\hline Gonzalez Guarda 2015 & Wrong participant (9th-grade students) \\
\hline Green 2006 & Wrong intervention (non IPV-related psychological trial) \\
\hline Grip 2011 & Wrong study design (single group evaluation (pre-post) with no comparison group) \\
\hline Hansen 2014 & Wrong study design (non comparison group) \\
\hline Hanson 1993 & $\begin{array}{l}\text { Wrong intervention (sexual assault prevention programme with college student women, psych stu- } \\
\text { dents) }\end{array}$ \\
\hline Harper 2014 & $\begin{array}{l}\text { Wrong intervention (REACH Forgiveness intervention; non-psychological and non-IPV-focused in- } \\
\text { tervention) }\end{array}$ \\
\hline Hayes 2015 & $\begin{array}{l}\text { Wrong study design (no comparison group; results cannot be evaluated against a comparison } \\
\text { group that did not receive therapy) }\end{array}$ \\
\hline Hembree 2004 & $\begin{array}{l}\text { Wrong participant (women who were assaulted by an intimate partner with whom they had an on- } \\
\text { going relationship and those with concurrent diagnoses of organic mental disorder, schizophrenia, } \\
\text { bipolar disorder, or active alcohol/drug dependence were excluded from the study) }\end{array}$ \\
\hline Heshmati 2016 & Wrong intervention (couple therapy) \\
\hline Hesser 2017 & Wrong participant (focus is on perpetrators of IPV for both women and men) \\
\hline Hoekenga 2010 & Wrong participant (non-IPV-related PTSD) \\
\hline Howard 2003 & Wrong study design (participatory evaluation study no control or comparison group) \\
\hline
\end{tabular}

Psychological therapies for women who experience intimate partner violence (Review)

Copyright $\odot 2020$ The Cochrane Collaboration. Published by John Wiley \& Sons, Ltd. 


\begin{tabular}{|c|c|}
\hline Study & Reason for exclusion \\
\hline Hughes 2010 & Wrong study design \\
\hline Hyman 2002 & Wrong intervention (advocacy intervention) \\
\hline Ironson 2002 & Wrong participant (non-IPV-focused) \\
\hline Iverson 2011 & $\begin{array}{l}\text { Wrong participant (women who had experienced sexual or physical assault in childhood and/or } \\
\text { adulthood) }\end{array}$ \\
\hline Jack 2012 & Wrong intervention (non-psychological therapy) \\
\hline Jones 2013 & Wrong intervention (non-psychological therapy) \\
\hline Kaslow 2010 & Wrong intervention (empowerment-focused group intervention) \\
\hline Katz 2008 & Wrong intervention \\
\hline Kelly 2016 & $\begin{array}{l}\text { Wrong participant (IPV was defined as physical or sexual abuse by a family member or intimate } \\
\text { partner during the life course) }\end{array}$ \\
\hline Kenyon 2016 & Wrong participant (only $2 \%$ of women with IPV) \\
\hline Kingston 2013 & Wrong participant (non-IPV-focused) \\
\hline Kraanen 2013 & Wrong participant (IPV perpetrators' treatment) \\
\hline Krupnick 2008 & $\begin{array}{l}\text { Wrong participant (study participants had experienced multiple episodes of trauma, usually begin- } \\
\text { ning in childhood) }\end{array}$ \\
\hline Labrador 2006 & Wrong study design (no randomisation) \\
\hline Latif 2017 & $\begin{array}{l}\text { Wrong study design (CBT-based self-help comparator intervention, includes active control condi- } \\
\text { tion) }\end{array}$ \\
\hline Launius 1987 & $\begin{array}{l}\text { Wrong participant ( } 19 \text { women were assigned to the Counseling group on the basis of a "Yes" re- } \\
\text { sponse to a question asking if they were currently receiving counselling for any personal or mar- } \\
\text { riage-family problems, and a "No" response to the abuse question described above) }\end{array}$ \\
\hline Lee 2015 & $\begin{array}{l}\text { Wrong participant (interpersonal trauma includes both childhood and adulthood and not separat- } \\
\text { ed in analysis) }\end{array}$ \\
\hline Leiner 2012 & Wrong participant (rape-related PTSD not specific to IPV) \\
\hline Liberman 2005 & $\begin{array}{l}\text { Wrong study design (child-parent psychotherapy compared with case management plus individual } \\
\text { psychotherapy) }\end{array}$ \\
\hline Liu 2013 & $\begin{array}{l}\text { Wrong study design (uncontrolled, non-randomised, retrospective examination of data on inter- } \\
\text { vention outcomes) }\end{array}$ \\
\hline Lynch 2012 & Wrong participant (interpersonal trauma includes various forms of trauma, not specific to IPV) \\
\hline Mancoske 1994 & Wrong study design (comparison of 2 types of social work counselling services) \\
\hline Matud 2016 & Wrong study design (no randomisation) \\
\hline
\end{tabular}




\begin{tabular}{|c|c|}
\hline Study & Reason for exclusion \\
\hline McBride 2002 & $\begin{array}{l}\text { Wrong study design (neither a control group nor the use of random assignment for the groups were } \\
\text { considered for this project) }\end{array}$ \\
\hline McWhirter 2011 & $\begin{array}{l}\text { Wrong study design (comparing } 2 \text { community-based group therapies, emotion-focused versus } \\
\text { goal-oriented) }\end{array}$ \\
\hline Mejdoubi 2013 & Wrong intervention (prevention trial, not psychological intervention for IPV) \\
\hline Melendez 2003 & Wrong intervention (gender-specific HIV-STI prevention intervention) \\
\hline Miller 2011 & Wrong intervention (family-planning-clinic-based intervention, non-psychological intervention) \\
\hline Miller 2016 & Wrong intervention (non-psychological intervention) \\
\hline Morrissey 2005 & Wrong participant (trauma includes childhood, adulthood and non-IPV-related) \\
\hline Ngo 2018 & Wrong intervention (non-psychological intervention, and sample not specifically women with IPV) \\
\hline Nguyen Feng 2016 & Wrong participant (history of interpersonal violence includes child abuse) \\
\hline Nishith 2005 & Wrong participant (sexual abuse in childhood) \\
\hline Norton 1997 & Wrong study design (no randomisation) \\
\hline O'Leary 1999 & Wrong intervention (couple therapy, and no randomisation) \\
\hline Orengo Aguayo 2017 & Wrong participant (incarcerated domestic violence offenders, and no randomisation) \\
\hline Overbeek 2013 & Wrong participant (paediatric population) \\
\hline Pallitto 2016 & Wrong intervention (nurse-led empowerment intervention) \\
\hline Parcesepe 2016 & Wrong participant (non-IPV-related, sex workers and interpersonal violence) \\
\hline Parker 1999 & Wrong study design \\
\hline Rasmussen 2008 & Wrong study design (no randomisation) \\
\hline Reed 2006 & $\begin{array}{l}\text { Wrong study design (comparison of two interventions, forgiveness therapy and alternative treat- } \\
\text { ment, anger validation, assertiveness, interpersonal skill building) }\end{array}$ \\
\hline Resick 2008 & $\begin{array}{l}\text { Wrong participant (participants were included if they had experienced sexual or physical assault in } \\
\text { childhood or adulthood and analysis were not IPV-focused) }\end{array}$ \\
\hline Resick 2012 & $\begin{array}{l}\text { Wrong participant ( } 41 \% \text { of the sample had been sexually abused (genital contact) as children and } \\
\text { the rest with diverse forms of trauma) }\end{array}$ \\
\hline Resnick 2007 & Wrong participant (rape not specifically within IPV context) \\
\hline Roemer 2004 & Wrong participant (rape not specific to IPV) \\
\hline Rosmalen-Nooijens 2017 & $\begin{array}{l}\text { Wrong intervention ( } 15-25 \text { years old impacted by family violence and non-psychological interven- } \\
\text { tion) }\end{array}$ \\
\hline Ross 2013 & Wrong intervention (advocacy and empowerment intervention, non-psychological) \\
\hline
\end{tabular}




\begin{tabular}{|c|c|}
\hline Study & Reason for exclusion \\
\hline Rothbaum 1997 & Wrong participant (sexual assault victims not specific to IPV) \\
\hline Rothbaum 2001 & $\begin{array}{l}\text { Wrong participant (adult female rape victims not clear if within IPV, also includes child sexual } \\
\text { abuse) }\end{array}$ \\
\hline Rotheram-Borus 2015 & Wrong intervention (non-psychological intervention) \\
\hline Rychtarik 2005 & Wrong participant (the sample included women from both non-violent and violent relationships) \\
\hline Sanci 2015 & Wrong intervention (clinician training intervention) \\
\hline Santandreu 2014 & Wrong study design (no control group) \\
\hline Schumm 2018 & Wrong intervention (couple therapy) \\
\hline Sharps 2008 & Wrong intervention (home visitation, non-psychological intervention) \\
\hline Sharps 2016 & Wrong intervention (IPV empowerment intervention - information and referral to services only) \\
\hline Shirk 2014 & Wrong participant (adolescent participants ages of 13-17) \\
\hline Stith 2004 & Wrong intervention (couple therapy) \\
\hline Taft 2009 & Wrong intervention (advocacy/empowerment intervention) \\
\hline Taft 2016 & Wrong study design (commentary paper) \\
\hline Taft 2017 & Wrong intervention (couple therapy) \\
\hline Tarquinio 2012 & $\begin{array}{l}\text { Wrong study design (no randomisation; the participants were offered the option of following eye } \\
\text { movement desensitisation and reprocessing or eclectic therapy) }\end{array}$ \\
\hline Tiwari 2010 & Wrong intervention (empowerment intervention, non-psychological) \\
\hline Tiwari 2012 & Wrong intervention (advocacy and empowerment intervention) \\
\hline Uchendu 2017 & Wrong participant \\
\hline Wagman 2015 & Wrong intervention (HIV prevention intervention) \\
\hline Walls 1985 & Wrong participant (rape survivors not related to IPV) \\
\hline Weir 2009 & Wrong participant \\
\hline Zarling 2015 & Wrong participant (IPV perpetration trial with $68 \%$ female sample) \\
\hline Zou 2010 & Wrong intervention (couple therapy) \\
\hline Zust 2000 & Wrong study design (no control group) \\
\hline
\end{tabular}

CTS: Conflict Tactics Scale; EMDR: eye movement desensitisation and reprocessing; IPV: intimate partner violence; PTSD: post-traumatic stress disorder; STI: sexually transmitted infection 
Characteristics of studies awaiting classification [ordered by study ID]

\begin{tabular}{ll}
\hline Akhtari $\mathbf{2 0 1 9}$ & RCT \\
\hline Pethods & Pregnant women exposed to domestic violence \\
& From 15-49 years old \\
& Absence of known psychological diseases (according to participants' self-declaration) \\
& Lack of underlying diseases \\
\hline Interventions & $\begin{array}{l}\text { Individual religious and spiritual counselling within } 8 \text { sessions of } 60-45 \text { min and control group will } \\
\text { not receive any advice }\end{array}$ \\
\hline Outcomes & Spiritual wellbeing; domestic violence \\
\hline Notes & $\begin{array}{l}\text { The registered protocol of this trial was first classified as ongoing, yet the outcome paper was pub- } \\
\text { lished after our search date. Hence this trial will be considered with the next update of this review. } \\
\text { Contact details: Assistant Professor Forouzan Olfati, Qazvin University of Medical Sciences, Qazvin, } \\
\text { Iran. Emails: folfati@ qums.ac.ir and papoy6olfati@yahoo.com }\end{array}$
\end{tabular}

Characteristics of ongoing studies [ordered by study ID]

\section{CTRI/2019/01/017009}

\begin{tabular}{ll} 
Study name & $\begin{array}{l}\text { A randomised controlled trial of the impact of behavioral intervention package on the health status } \\
\text { of married abused pregnant women aged 15-45 years attending antenatal clinic of LNJP hospital, } \\
\text { New Delhi }\end{array}$ \\
\hline Rethods & - All married pregnant women (first contact of confirmation of pregnancy, preferably i.e. $18-20$ \\
Participants & - Beeks) attending Obstetrics OPD of MAMC, LN hospital for antenatal registration \\
- Consented for participation in the trial by signing the informed consent form & - Screened positive for domestic violence during last year using Abuse Screening Tool (AST). \\
- Staying with the husband/in-laws (family) for at least 2 years \\
- Likely to stay in the area till delivery and attend LNJP hospital OPD for ANC \\
- Willing to come to hospital for follow-up as per schedule \\
- Willing to allow trial staff visiting her home for contact, if needed
\end{tabular}

The intervention package has 5 components: 1 . information sharing, awareness generation and 2 . counselling of the women respondent 3. advice (safety plan, choice making and problem solving) 4. skill development (assertiveness, communication skill, self-care etc) 5. body- mind relaxation and stress management. The intervention period will be for 7 months from date of recruitment to 6 weeks postnatal. A total of 14 sessions with the women will be done. Each one-to-one session for administration of the intervention package will last about 30-45 min, in a private room without the male partner or other individuals being present.

Outcomes

Change in health domain scale, the composite measures of physical and mental component score as assessed through survey scale SF-36 before and after intervention 
CTRI/2019/01/017009 (Continued)

Contact information@Meerambika Mahapatro, meerambika.mahapatro@gmail.com

Notes National Institute of Health and Family Welfare Baba Gangnath Marg New Delhi-67 Lok Nayak Hospital, Jawaharlal Nehru Road New ITO New Delhi South DELHI 110067 India

\section{IRCT20151103024866N11}

\begin{tabular}{ll}
\hline Study name & $\begin{array}{l}\text { Investigating the effectiveness of Gestalt counselling on the self-esteem and domestic violence } \\
\text { against pregnant women refereed to Kerman health centres }\end{array}$ \\
\hline Methods & RCT \\
\hline Participants & Pregnant women experienced domestic violence \\
\hline Interventions & Gestalt group counselling; control group will receive no consultation or intervention \\
\hline Outcomes & Reduction in domestic violence \\
\hline Starting date & 20 August 2018 \\
\hline Contact information & Ali Doosti, alidoosti@kmu.ac.ir \\
\hline Notes & Kerman University of Medical Sciences, Nursing and Midwifery College \\
\hline
\end{tabular}

IRCT2017040628352N4

\begin{tabular}{ll}
\hline Study name & $\begin{array}{l}\text { The effect of solution focused counselling on violence rate and quality of life of pregnant women at } \\
\text { risk of domestic violence }\end{array}$ \\
\hline Methods & RCT \\
\hline Participants & $\begin{array}{l}\text { Women experienced any kind of mild or average physical, psychological, or sexual violence based } \\
\text { on CTS-2 questionnaire; mothers > 18 years of age; the gestational age of } \leq 27 \text { weeks based on } \\
\text { sonography; the ability of reading and writing; living in Zanjan; having the minimum of } 1 \text { year mar- } \\
\text { ried life with her husband; not participating in other classes and counselling courses at the same } \\
\text { time; having a cell phone; lack of underlying diseases; lack of the known psychological disease; } \\
\text { not using psychological drugs; no substance abuse in women and their husbands; not having the } \\
\text { stressful events in the past year either for her or their husband; not having any pregnancy side ef- } \\
\text { fects such as pre-eclampsia or vaginal bleeding or etc; tendency in regular participation in coun- } \\
\text { selling sessions; married life with her husband during the trial period }\end{array}$ \\
\hline Interventions & $\begin{array}{l}\text { Solution focused counselling during } 6 \text { sessions of } 90 \text { min and control group will receive no coun- } \\
\text { selling }\end{array}$ \\
\hline Outcomes & Sexual, psychological, physical violence by an intimate partner; quality of life \\
\hline Starting date & 07 August 2017 \\
\hline Contact information & Roghieh Kharaghani, r.kharaghani@zums.ac.ir \\
\hline School of Nursing and Midwifery, Zanjan University of Medical Sciences, Mahdavi Blvd, Zanjan
\end{tabular}




\begin{tabular}{ll}
\hline Study name & $\begin{array}{l}\text { Effect of group-based problem solving on domestic violence in patients with bipolar disorder in } \\
\text { type I in Ebn'e Sina Hospital in Mashhad }\end{array}$ \\
\hline Methods & RCT \\
\hline Participants & Gomen aged 18-60 years who experience domestic violence and present with bipolar disorder \\
\hline Interventions & Domestic violence Score in the ISA Questionnaire \\
\hline Outcomes & 01 June 2018 (expected recruitment start date) \\
\hline Starting date & Maryam Seyyedi Nasooh Abad, vaghees@mums.ac.ir, +985138597313 \\
\hline Contact information & Mashhad University of Medical Sciences, College of Nursing \\
\hline Notes &
\end{tabular}

\section{IRCT20180204038609N1}

\begin{tabular}{ll}
\hline Study name & $\begin{array}{l}\text { The effect of group-based cognitive-behavioral therapy on self-esteem of women with domestic vi- } \\
\text { olence }\end{array}$ \\
\hline Methods & RCT \\
\hline Participants & $\begin{array}{l}\text { Willingness to participate in the trial, being married, having at least } 1 \text { child, Iranian nationality, age } \\
\text { naire, and }<15 \text { in self-esteem questionnaire. }\end{array}$ \\
& $\begin{array}{l}\text { Exclusion criteria: people with a positive history of CBT, severe physical or mental illness, unpleas- } \\
\text { ant events during the previous } 6 \text { months, and use of antipsychotics }\end{array}$ \\
\hline Interventions & $\begin{array}{l}\text { 8 sessions of } 90-\text { min CBT and control group who will receive an educational CD containing Cogni- } \\
\text { tive Behavioral Counselling at the end of the trial }\end{array}$ \\
\hline Outcomes & Self-esteem \\
\hline Starting date & 06 August 2018 (expected start date) \\
\hline Contact information & Esmat Zarifinejad, zarifinejad@stu.ajums.ac.ir, +98613373 8619 \\
\hline Notes &
\end{tabular}

\section{NCT03484390}

Study name
stress disorder related to intimate partner violence

Methods RCT

Participants Female 18-64 years of age, victim of IPV and meet diagnostic criteria for PTSD 
NCT03484390 (Continued)

Interventions

Behavioral: mindfulness-based stress reduction

Behavioral: wellness Group

\begin{tabular}{ll}
\hline Outcomes & $\begin{array}{l}\text { PTSD Symptoms Checklist, Difficulties in Emotion Regulation Scale, heart rate variability, Useful } \\
\text { Field of View Test, Five-Facet Mindfulness Questionnaire }\end{array}$ \\
\hline Starting date & 15 August 2016 (expected start date) and 31 May 2018 (expected completion date) \\
\hline Contact information & Autumn Gallegos, University of Rochester \\
\hline Notes & \\
\hline
\end{tabular}

\section{NCT03813901}

Study name
(IPV)
(IPV)

\begin{tabular}{ll}
\hline Methods & RCT \\
\hline Participants & $\begin{array}{l}\text { Women who have experienced IPV (physical, emotional, psychological, and/or sexual), including } \\
\text { coercive control from a current or former partner in the previous } 12 \text { months and no later than } 3 \\
\text { years }\end{array}$
\end{tabular}

\begin{tabular}{ll}
\hline Interventions & Samalochana Counselling and Wait list control \\
\hline Outcomes & Quality of life; anxiety \\
\hline Starting date & 21 June 2018 \\
\hline Contact information & Neha Sharma \\
\hline Notes & Warwick Research Services \\
\hline
\end{tabular}

\section{NCT04068662}

\begin{tabular}{ll}
\hline Study name & Intervention for IPV-exposed pregnant women \\
\hline Methods & RCT \\
\hline Participants & $\begin{array}{l}\text { Women currently pregnant (primi or multiparous); experienced IPV within the past year; Eng- } \\
\text { lish-speaking; age } \geq 16 ; \text { between 10-30 weeks pregnant }\end{array}$ \\
\hline Interventions & $\begin{array}{l}\text { The Pregnant Moms' Empowerment Program is a 5-session group therapy programme designed for } \\
\text { pregnant women with a recent history of exposure to IPV. 1 session is administered each week with } \\
\text { a duration of } 2 \text { h each, for a total of } 10 \text { contact hours across the course of the intervention. Inter- } \\
\text { vention topics include safety planning, social support, resilience, psycho-education about IPV, cog- } \\
\text { nitive and behavioural strategies for remediating distress, infant sleep and breastfeeding, respon- } \\
\text { sive parenting, and co-parenting }\end{array}$ \\
\hline Outcomes & PTSD; depression; resilience; IPV; social support \\
\hline Starting date & August 2019 \\
\hline \hline
\end{tabular}

Psychological therapies for women who experience intimate partner violence (Review) 
NCT04068662 (Continued)

Contact information

Laura Miller-Graff, Imiller8@nd.edu

\section{Notes}

\section{NL6450}

Study name

The effectiveness of "De Nieuwe Toekomst", a Dutch intervention for victims of intimate partner violence

\begin{tabular}{ll}
\hline Methods & RCT \\
\hline Participants & $\begin{array}{l}\text { Women who have been victims of IPV and are economically dependent (on their partners, receiv- } \\
\text { ing welfare or otherwise). There is no longer a crisis situation or are not receiving professional care } \\
\text { and/or assistance at the moment of inclusion. Understanding the Dutch language. }\end{array}$ \\
\hline
\end{tabular}

$\begin{array}{ll}\text { Interventions } & \begin{array}{l}\text { The intervention group receives "De Nieuwe Toekomst" (The New Future). The control group re- } \\ \text { ceives the usual care }\end{array}\end{array}$

\begin{tabular}{ll}
\hline Outcomes & $\begin{array}{l}\text { Employment rate, IPV, self-reported physical and mental health, use of health services and use of } \\
\text { welfare services }\end{array}$ \\
\hline Starting date & 01 October 2018 (expected start date) and 01 October 2022 (expected completion date) \\
\hline Contact information & Suzanne Bouma, s.bouma@atria.nl, +3120 3031533 \\
\hline Notes & $\begin{array}{l}\text { There is insufficient information to assess whether this is a culturally-adapted psychological thera- } \\
\text { py, correspondence with trial author(s) is required for further information and clarification }\end{array}$ \\
\hline
\end{tabular}

\section{Sapkota 2019}

\section{Study name}

Psychosocial intervention to improve mental health, social support, and help-seeking behaviours among abused pregnant women: a mixed method trial from Nepal

\begin{tabular}{ll}
\hline Methods & RCT \\
\hline Participants & $\begin{array}{l}\text { Women will be included in the trial if they i) are aged } 18 \text { years or older; ii) are 24-34 weeks pregnant; } \\
\text { iii) have an identified history of ever experiencing domestic violence in their life using the Abuse As- } \\
\text { sessment Screening (AAS) tool (at least one affirmative response in AAS); iv) can read and under- } \\
\text { stand Nepali language; and v) have access to a telephone. }\end{array}$
\end{tabular}

\begin{tabular}{ll}
\hline Interventions & A counselling and education session and standard care group \\
\hline Outcomes & Mental health (anxiety, depression); self-efficacy; safety behaviours; help-seeking behaviours \\
\hline Starting date & 6 June 2018 \\
\hline Contact information & Diksha Sapkota, diksha.sapkota@griffithuni.edu.au \\
\hline Notes & Logan campus Griffith University 68 University Drive MEADOWBROOK QLD 4131 \\
\hline
\end{tabular}


Tol 2017

\begin{tabular}{ll}
\hline Study name & $\begin{array}{l}\text { Nguvu: evaluating an integrated approach to reduce intimate partner violence and psychological } \\
\text { distress in refugees in Tanzania }\end{array}$ \\
\hline Methods & RCT \\
\hline Participants & $\begin{array}{l}\text { Women who are refugees from the Democratic Republic of the Congo (DRC) currently living in } \\
\text { of IPV and are experiencing psychological distress. }\end{array}$ \\
\hline Interventions & $\begin{array}{l}\text { Intervention (Nguvu) arm: participants will have access to the Nguvu intervention, which is an 8- } \\
\text { week programme that integrates advocacy and empowerment counselling with cognitive process- } \\
\text { ing therapy. }\end{array}$ \\
\hline Outcomes & Recurrence of IPV, psychological distress symptoms \\
\hline Starting date & 01 October 2017 \\
\hline Contact information & Wietse A Tol; wtol@jhu.edu \\
\hline Notes &
\end{tabular}

CBT: cognitive behavioural therapy; CTS: Conflict Tactics Scale; RCT: randomised controlled trial; PTSD: post-traumatic stress disorder; SF-36: Short Form 36 health survey

\section{DATA AND ANALYSES}

\section{Comparison 1. Intervention vs Control}

\begin{tabular}{|c|c|c|c|c|}
\hline Outcome or subgroup title & $\begin{array}{l}\text { No. of } \\
\text { studies }\end{array}$ & $\begin{array}{l}\text { No. of } \\
\text { partici- } \\
\text { pants }\end{array}$ & Statistical method & Effect size \\
\hline 1.1 Depression (continuous) & 17 & & $\begin{array}{l}\text { Std. Mean Difference (IV, Random, } \\
95 \% \mathrm{Cl} \text { ) }\end{array}$ & Subtotals only \\
\hline 1.1.1 Short-term FU (under 6 months) & 15 & 1247 & $\begin{array}{l}\text { Std. Mean Difference (IV, Random, } \\
95 \% \mathrm{Cl} \text { ) }\end{array}$ & $-0.45[-0.67,-0.22]$ \\
\hline $\begin{array}{l}\text { 1.1.2 Medium-term FU ( } 6 \text { to under } 12 \\
\text { months) }\end{array}$ & 4 & 600 & $\begin{array}{l}\text { Std. Mean Difference (IV, Random, } \\
95 \% \mathrm{Cl} \text { ) }\end{array}$ & $-0.24[-0.47,-0.01]$ \\
\hline 1.1.3 Long-term FU (12 months and above) & 3 & 503 & $\begin{array}{l}\text { Std. Mean Difference (IV, Random, } \\
95 \% \mathrm{Cl})\end{array}$ & $-0.08[-0.30,0.14]$ \\
\hline 1.2 Depression (categorical) & 2 & & Odds Ratio (IV, Fixed, 95\% CI) & Subtotals only \\
\hline $\begin{array}{l}\text { 1.2.1 Medium-term FU ( } 6 \text { to under } 12 \\
\text { months) }\end{array}$ & 2 & 528 & Odds Ratio (IV, Fixed, 95\% CI) & $0.68[0.47,0.98]$ \\
\hline 1.2.2 Long-term FU (12 months and above) & 1 & 195 & Odds Ratio (IV, Fixed, 95\% CI) & $0.50[0.29,0.89]$ \\
\hline $\begin{array}{l}\text { 1.3 Depression (recruitment setting of par- } \\
\text { ticipants) }\end{array}$ & 17 & 1729 & $\begin{array}{l}\text { Std. Mean Difference (IV, Random, } \\
95 \% \mathrm{Cl})\end{array}$ & $-0.36[-0.55,-0.17]$ \\
\hline
\end{tabular}




\begin{tabular}{|c|c|c|c|c|}
\hline Outcome or subgroup title & $\begin{array}{l}\text { No. of } \\
\text { studies }\end{array}$ & $\begin{array}{l}\text { No. of } \\
\text { partici- } \\
\text { pants }\end{array}$ & Statistical method & Effect size \\
\hline 1.3.1 Healthcare & 6 & 519 & $\begin{array}{l}\text { Std. Mean Difference (IV, Random, } \\
95 \% \mathrm{CI})\end{array}$ & $-0.18[-0.38,0.01]$ \\
\hline 1.3.2 Community & 5 & 469 & $\begin{array}{l}\text { Std. Mean Difference (IV, Random, } \\
95 \% \mathrm{Cl})\end{array}$ & $-0.24[-0.62,0.15]$ \\
\hline 1.3.3 Shelter / refuge & 1 & 52 & $\begin{array}{l}\text { Std. Mean Difference (IV, Random, } \\
95 \% \mathrm{Cl})\end{array}$ & $-0.55[-1.13,0.02]$ \\
\hline 1.3.4 Various & 5 & 689 & $\begin{array}{l}\text { Std. Mean Difference (IV, Random, } \\
95 \% \mathrm{Cl} \text { ) }\end{array}$ & $-0.55[-0.96,-0.13]$ \\
\hline 1.4 Depression (type of intervention) & 16 & 1604 & $\begin{array}{l}\text { Std. Mean Difference (IV, Random, } \\
95 \% \mathrm{CI} \text { ) }\end{array}$ & $-0.24[-0.37,-0.11]$ \\
\hline 1.4.1 Cognitive behavioral therapy & 4 & 262 & $\begin{array}{l}\text { Std. Mean Difference (IV, Random, } \\
95 \% \mathrm{CI} \text { ) }\end{array}$ & $-0.31[-0.55,-0.06]$ \\
\hline 1.4.2 Humanistic therapies & 4 & 161 & $\begin{array}{l}\text { Std. Mean Difference (IV, Random, } \\
95 \% \mathrm{CI})\end{array}$ & $-0.47[-0.91,-0.03]$ \\
\hline 1.4.3 Integrative therapies & 6 & 892 & $\begin{array}{l}\text { Std. Mean Difference (IV, Random, } \\
95 \% \mathrm{CI} \text { ) }\end{array}$ & $-0.09[-0.23,0.04]$ \\
\hline $\begin{array}{l}\text { 1.4.4 Other psychologically-oriented inter- } \\
\text { ventions }\end{array}$ & 2 & 289 & $\begin{array}{l}\text { Std. Mean Difference (IV, Random, } \\
95 \% \mathrm{Cl} \text { ) }\end{array}$ & $-0.41[-0.99,0.16]$ \\
\hline 1.5 Depression (intensity of intervention) & 17 & 1729 & $\begin{array}{l}\text { Std. Mean Difference (IV, Random, } \\
95 \% \mathrm{Cl})\end{array}$ & $-0.36[-0.55,-0.17]$ \\
\hline 1.5.1 Up to four sessions & 5 & 644 & $\begin{array}{l}\text { Std. Mean Difference (IV, Random, } \\
95 \% \mathrm{CI} \text { ) }\end{array}$ & $-0.03[-0.18,0.13]$ \\
\hline 1.5.2 Five or more sessions & 12 & 1085 & $\begin{array}{l}\text { Std. Mean Difference (IV, Random, } \\
95 \% \mathrm{Cl} \text { ) }\end{array}$ & $-0.49[-0.73,-0.25]$ \\
\hline $\begin{array}{l}\text { 1.6 Depression (person delivering the inter- } \\
\text { vention) }\end{array}$ & 15 & 1342 & $\begin{array}{l}\text { Std. Mean Difference (IV, Random, } \\
95 \% \mathrm{CI} \text { ) }\end{array}$ & $-0.36[-0.57,-0.16]$ \\
\hline 1.6.1 healthcare workers & 10 & 649 & $\begin{array}{l}\text { Std. Mean Difference (IV, Random, } \\
95 \% \mathrm{CI})\end{array}$ & $-0.42[-0.73,-0.11]$ \\
\hline 1.6.2 non-healthcare workers & 5 & 693 & $\begin{array}{l}\text { Std. Mean Difference (IV, Random, } \\
95 \% \mathrm{CI})\end{array}$ & $-0.21[-0.39,-0.03]$ \\
\hline 1.7 Self-efficacy & 4 & & $\begin{array}{l}\text { Std. Mean Difference (IV, Random, } \\
95 \% \mathrm{CI} \text { ) }\end{array}$ & Subtotals only \\
\hline 1.7.1 Short-term FU (under 6 months) & 3 & 279 & $\begin{array}{l}\text { Std. Mean Difference (IV, Random, } \\
95 \% \mathrm{CI} \text { ) }\end{array}$ & $0.20[-0.22,0.63]$ \\
\hline $\begin{array}{l}\text { 1.7.2 Medium-term FU ( } 6 \text { to under } 12 \\
\text { months) }\end{array}$ & 1 & 346 & $\begin{array}{l}\text { Std. Mean Difference (IV, Random, } \\
95 \% \mathrm{CI} \text { ) }\end{array}$ & $-0.12[-0.33,0.09]$ \\
\hline
\end{tabular}




\begin{tabular}{|c|c|c|c|c|}
\hline Outcome or subgroup title & $\begin{array}{l}\text { No. of } \\
\text { studies }\end{array}$ & $\begin{array}{l}\text { No. of } \\
\text { partici- } \\
\text { pants }\end{array}$ & Statistical method & Effect size \\
\hline 1.7.3 Long-term FU (12 months and above) & 1 & 331 & $\begin{array}{l}\text { Std. Mean Difference (IV, Random, } \\
95 \% \mathrm{CI})\end{array}$ & $-0.23[-0.45,-0.01]$ \\
\hline 1.8 Dropouts from treatment & 33 & & Odds Ratio (IV, Random, 95\% CI) & Subtotals only \\
\hline 1.8.1 Short-term FU (up to 6 months) & 22 & 3022 & Odds Ratio (IV, Random, 95\% CI) & $1.21[0.98,1.48]$ \\
\hline 1.8.2 Medium-term FU ( 6 to 12 months) & 5 & 840 & Odds Ratio (IV, Random, 95\% CI) & $1.04[0.75,1.44]$ \\
\hline 1.8.3 Long-term FU (greater than 12 months) & 6 & 1655 & Odds Ratio (IV, Random, 95\% CI) & $1.08[0.85,1.38]$ \\
\hline 1.9 Mental health & 4 & & $\begin{array}{l}\text { Std. Mean Difference (IV, Random, } \\
95 \% \mathrm{CI} \text { ) }\end{array}$ & Subtotals only \\
\hline 1.9.1 Short-term FU (under 6 months) & 2 & 353 & $\begin{array}{l}\text { Std. Mean Difference (IV, Random, } \\
95 \% \mathrm{CI})\end{array}$ & $-0.34[-0.55,-0.13]$ \\
\hline $\begin{array}{l}\text { 1.9.2 Medium-term FU ( } 6 \text { to under } 12 \\
\text { months) }\end{array}$ & 2 & 219 & $\begin{array}{l}\text { Std. Mean Difference (IV, Random, } \\
95 \% \mathrm{CI})\end{array}$ & $-0.11[-0.38,0.16]$ \\
\hline 1.9.3 Long-term FU (12 months and above) & 2 & 355 & $\begin{array}{l}\text { Std. Mean Difference (IV, Random, } \\
95 \% \mathrm{Cl})\end{array}$ & $-0.27[-0.48,-0.06]$ \\
\hline 1.10 Anxiety (continuous) & 5 & & $\begin{array}{l}\text { Std. Mean Difference (IV, Random, } \\
95 \% \mathrm{CI})\end{array}$ & Subtotals only \\
\hline 1.10.1 Short-term FU (under 6 months) & 4 & 158 & $\begin{array}{l}\text { Std. Mean Difference (IV, Random, } \\
95 \% \mathrm{CI})\end{array}$ & $-0.96[-1.29,-0.63]$ \\
\hline 1.10.2 Long-term FU (12 months and above) & 1 & 166 & $\begin{array}{l}\text { Std. Mean Difference (IV, Random, } \\
95 \% \mathrm{CI})\end{array}$ & $-0.20[-0.51,0.10]$ \\
\hline 1.11 Anxiety (categorical) & 1 & & Odds Ratio (IV, Random, 95\% CI) & Subtotals only \\
\hline $\begin{array}{l}\text { 1.11.1 Medium-term FU ( } 6 \text { to under } 12 \\
\text { months) }\end{array}$ & 1 & 192 & Odds Ratio (IV, Random, 95\% CI) & $0.82[0.45,1.49]$ \\
\hline 1.11.2 Long-term FU (12 months and above) & 1 & 195 & Odds Ratio (IV, Random, 95\% CI) & $0.87[0.48,1.57]$ \\
\hline $\begin{array}{l}1.12 \text { Post-traumatic stress disorder (continu- } \\
\text { ous) }\end{array}$ & 10 & & $\begin{array}{l}\text { Std. Mean Difference (IV, Random, } \\
95 \% \mathrm{CI} \text { ) }\end{array}$ & Subtotals only \\
\hline 1.12.1 Short-term FU (under 6 months) & 9 & 1150 & $\begin{array}{l}\text { Std. Mean Difference (IV, Random, } \\
95 \% \mathrm{CI})\end{array}$ & $-0.48[-0.80,-0.16]$ \\
\hline $\begin{array}{l}\text { 1.12.2 Medium-term FU ( } 6 \text { to under } 12 \\
\text { months) }\end{array}$ & 4 & 484 & $\begin{array}{l}\text { Std. Mean Difference (IV, Random, } \\
95 \% \mathrm{CI})\end{array}$ & $-0.24[-0.54,0.06]$ \\
\hline 1.12.3 Long-term FU ( 12 months and above) & 1 & 170 & $\begin{array}{l}\text { Std. Mean Difference (IV, Random, } \\
95 \% \mathrm{Cl})\end{array}$ & $-0.27[-0.57,0.04]$ \\
\hline 1.13 Quality of life & 4 & & $\begin{array}{l}\text { Std. Mean Difference (IV, Random, } \\
95 \% \mathrm{CI})\end{array}$ & Subtotals only \\
\hline
\end{tabular}




\begin{tabular}{|c|c|c|c|c|}
\hline Outcome or subgroup title & $\begin{array}{l}\text { No. of } \\
\text { studies }\end{array}$ & $\begin{array}{l}\text { No. of } \\
\text { partici- } \\
\text { pants }\end{array}$ & Statistical method & Effect size \\
\hline 1.13.1 Short-term FU (under 6 months) & 2 & 382 & $\begin{array}{l}\text { Std. Mean Difference (IV, Random, } \\
95 \% \mathrm{Cl} \text { ) }\end{array}$ & $0.16[-0.04,0.36]$ \\
\hline $\begin{array}{l}\text { 1.13.2 Medium-term FU ( } 6 \text { to under } 12 \\
\text { months) }\end{array}$ & 2 & 557 & $\begin{array}{l}\text { Std. Mean Difference (IV, Random, } \\
95 \% \mathrm{Cl} \text { ) }\end{array}$ & $0.10[-0.07,0.27]$ \\
\hline 1.13.3 Long-term FU (12 months and above) & 4 & 699 & $\begin{array}{l}\text { Std. Mean Difference (IV, Random, } \\
95 \% \mathrm{CI} \text { ) }\end{array}$ & $0.11[-0.04,0.25]$ \\
\hline 1.14 Re-exposure to IPV (continuous) & 9 & & $\begin{array}{l}\text { Std. Mean Difference (IV, Random, } \\
95 \% \mathrm{CI} \text { ) }\end{array}$ & Subtotals only \\
\hline 1.14.1 Short-term FU (under 6 months) & 7 & 749 & $\begin{array}{l}\text { Std. Mean Difference (IV, Random, } \\
95 \% \mathrm{CI})\end{array}$ & $-0.00[-0.29,0.29]$ \\
\hline $\begin{array}{l}\text { 1.14.2 Medium-term FU ( } 6 \text { to under } 12 \\
\text { months) }\end{array}$ & 2 & 547 & $\begin{array}{l}\text { Std. Mean Difference (IV, Random, } \\
95 \% \mathrm{CI} \text { ) }\end{array}$ & $0.03[-0.14,0.20]$ \\
\hline 1.14.3 Long-term FU (12 months and above) & 4 & 837 & $\begin{array}{l}\text { Std. Mean Difference (IV, Random, } \\
95 \% \mathrm{Cl})\end{array}$ & $-0.07[-0.20,0.07]$ \\
\hline 1.15 Re-exposure to IPV (categorical) & 7 & & Odds Ratio (IV, Random, 95\% CI) & Subtotals only \\
\hline 1.15.1 Short-term FU (under 6 months) & 5 & 797 & Odds Ratio (IV, Random, 95\% CI) & $0.45[0.21,0.96]$ \\
\hline $\begin{array}{l}\text { 1.15.2 Medium-term FU ( } 6 \text { to under } 12 \\
\text { months) }\end{array}$ & 1 & 186 & Odds Ratio (IV, Random, 95\% Cl) & $1.29[0.55,3.01]$ \\
\hline 1.15.3 Long-term FU (12 months and above) & 2 & 381 & Odds Ratio (IV, Random, 95\% CI) & $0.77[0.28,2.11]$ \\
\hline $\begin{array}{l}1.16 \text { Safety planning and/or safety behav- } \\
\text { iour (continuous) }\end{array}$ & 1 & & $\begin{array}{l}\text { Std. Mean Difference (IV, Random, } \\
95 \% \mathrm{CI})\end{array}$ & Subtotals only \\
\hline $\begin{array}{l}\text { 1.16.1 Medium-term FU ( } 6 \text { to under } 12 \\
\text { months) }\end{array}$ & 1 & 337 & $\begin{array}{l}\text { Std. Mean Difference (IV, Random, } \\
95 \% \mathrm{CI})\end{array}$ & $0.04[-0.18,0.25]$ \\
\hline 1.16.2 Long-term FU (12 months and above) & 1 & 318 & $\begin{array}{l}\text { Std. Mean Difference (IV, Random, } \\
95 \% \mathrm{CI} \text { ) }\end{array}$ & $0.00[-0.22,0.22]$ \\
\hline $\begin{array}{l}\text { 1.17 Safety planning and/or safety behav- } \\
\text { iour (categorical) }\end{array}$ & 2 & & Odds Ratio (IV, Random, 95\% Cl) & Subtotals only \\
\hline 1.17.1 Short-term FU (under 6 months) & 1 & 138 & Odds Ratio (IV, Random, 95\% CI) & $1.38[0.66,2.89]$ \\
\hline $\begin{array}{l}\text { 1.17.2 Medium-term FU ( } 6 \text { to under } 12 \\
\text { months) }\end{array}$ & 1 & 191 & Odds Ratio (IV, Random, 95\% CI) & $1.25[0.68,2.27]$ \\
\hline 1.17.3 Long-term FU (12 months and above) & 1 & 192 & Odds Ratio (IV, Random, 95\% Cl) & $2.14[1.18,3.91]$ \\
\hline 1.18 Use of healthcare and IPV services & 2 & & Odds Ratio (IV, Random, 95\% Cl) & Subtotals only \\
\hline 1.18.1 Short-term FU (under 6 months) & 1 & 371 & Odds Ratio (IV, Random, 95\% CI) & $1.60[0.68,3.76]$ \\
\hline
\end{tabular}




\begin{tabular}{|c|c|c|c|c|}
\hline Outcome or subgroup title & $\begin{array}{l}\text { No. of } \\
\text { studies }\end{array}$ & $\begin{array}{l}\text { No. of } \\
\text { partici- } \\
\text { pants }\end{array}$ & Statistical method & Effect size \\
\hline $\begin{array}{l}\text { 1.18.2 Medium-term FU ( } 6 \text { to under } 12 \\
\text { months) }\end{array}$ & 1 & 364 & Odds Ratio (IV, Random, 95\% Cl) & $1.21[0.44,3.33]$ \\
\hline 1.18.3 Long-term FU (12 months and above) & 2 & 526 & Odds Ratio (IV, Random, 95\% CI) & $0.79[0.21,2.97]$ \\
\hline 1.19 Social support (continuous) & 2 & & $\begin{array}{l}\text { Std. Mean Difference (IV, Random, } \\
95 \% \mathrm{CI})\end{array}$ & Subtotals only \\
\hline 1.19.1 Short-term FU (under 6 months) & 2 & 245 & $\begin{array}{l}\text { Std. Mean Difference (IV, Random, } \\
95 \% \mathrm{CI} \text { ) }\end{array}$ & $0.31[-0.38,1.00]$ \\
\hline $\begin{array}{l}\text { 1.19.2 Medium-term FU ( } 6 \text { to under } 12 \\
\text { months) }\end{array}$ & 2 & 235 & $\begin{array}{l}\text { Std. Mean Difference (IV, Random, } \\
95 \% \mathrm{CI} \text { ) }\end{array}$ & $0.05[-0.21,0.31]$ \\
\hline 1.20 Social support (categorical) & 1 & & Odds Ratio (IV, Random, 95\% Cl) & Totals not selected \\
\hline 1.20.1 Short-term FU (under 6 months) & 1 & & Odds Ratio (IV, Random, 95\% CI) & Totals not selected \\
\hline $\begin{array}{l}\text { 1.20.2 Medium-term FU ( } 6 \text { to under } 12 \\
\text { months) }\end{array}$ & 1 & & Odds Ratio (IV, Random, 95\% CI) & Totals not selected \\
\hline 1.20.3 Long-term FU (12 months and above) & 1 & & Odds Ratio (IV, Random, 95\% CI) & Totals not selected \\
\hline
\end{tabular}


Analysis 1.1. Comparison 1: Intervention vs Control, Outcome 1: Depression (continuous)

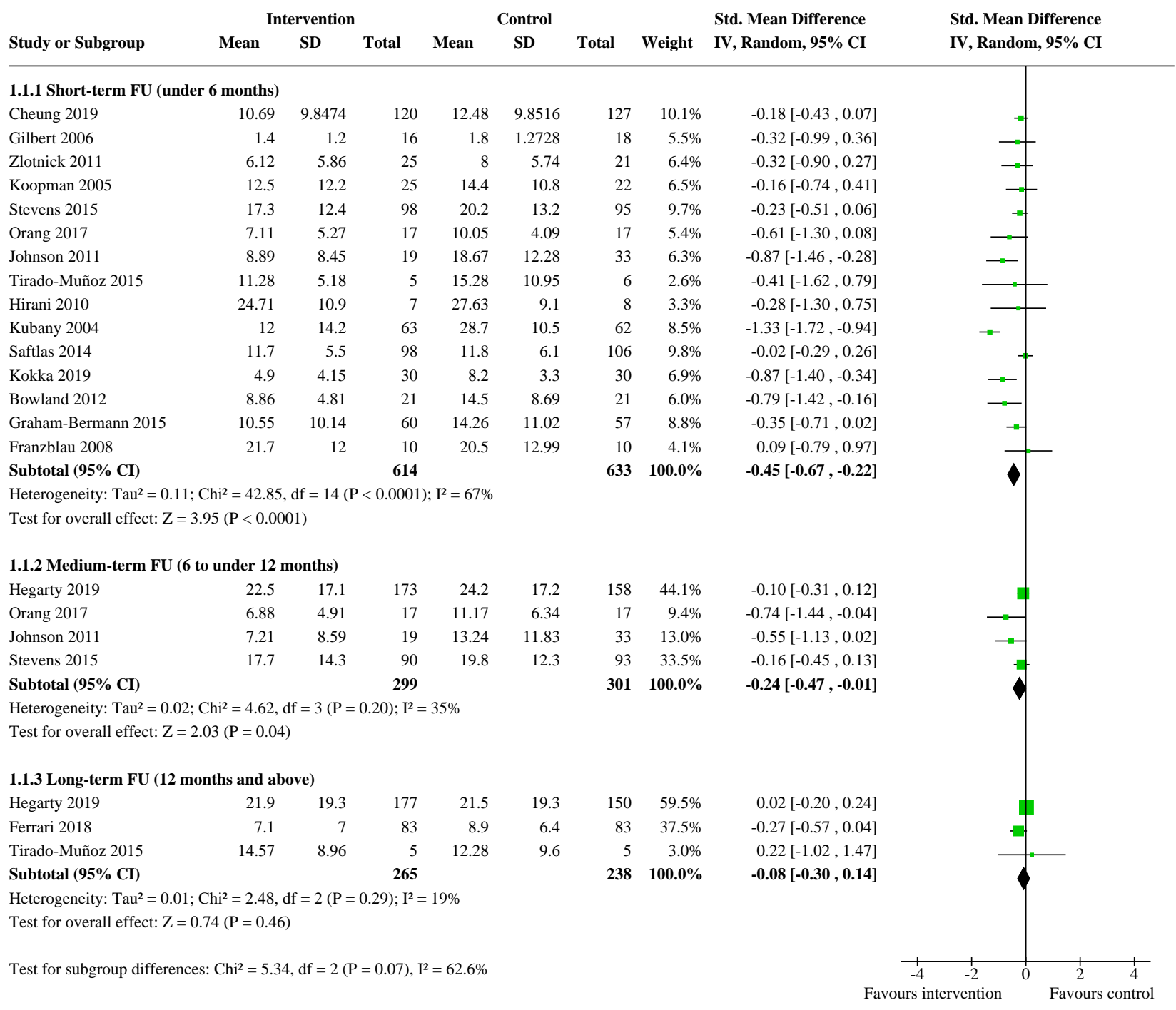


Analysis 1.2. Comparison 1: Intervention vs Control, Outcome 2: Depression (categorical)

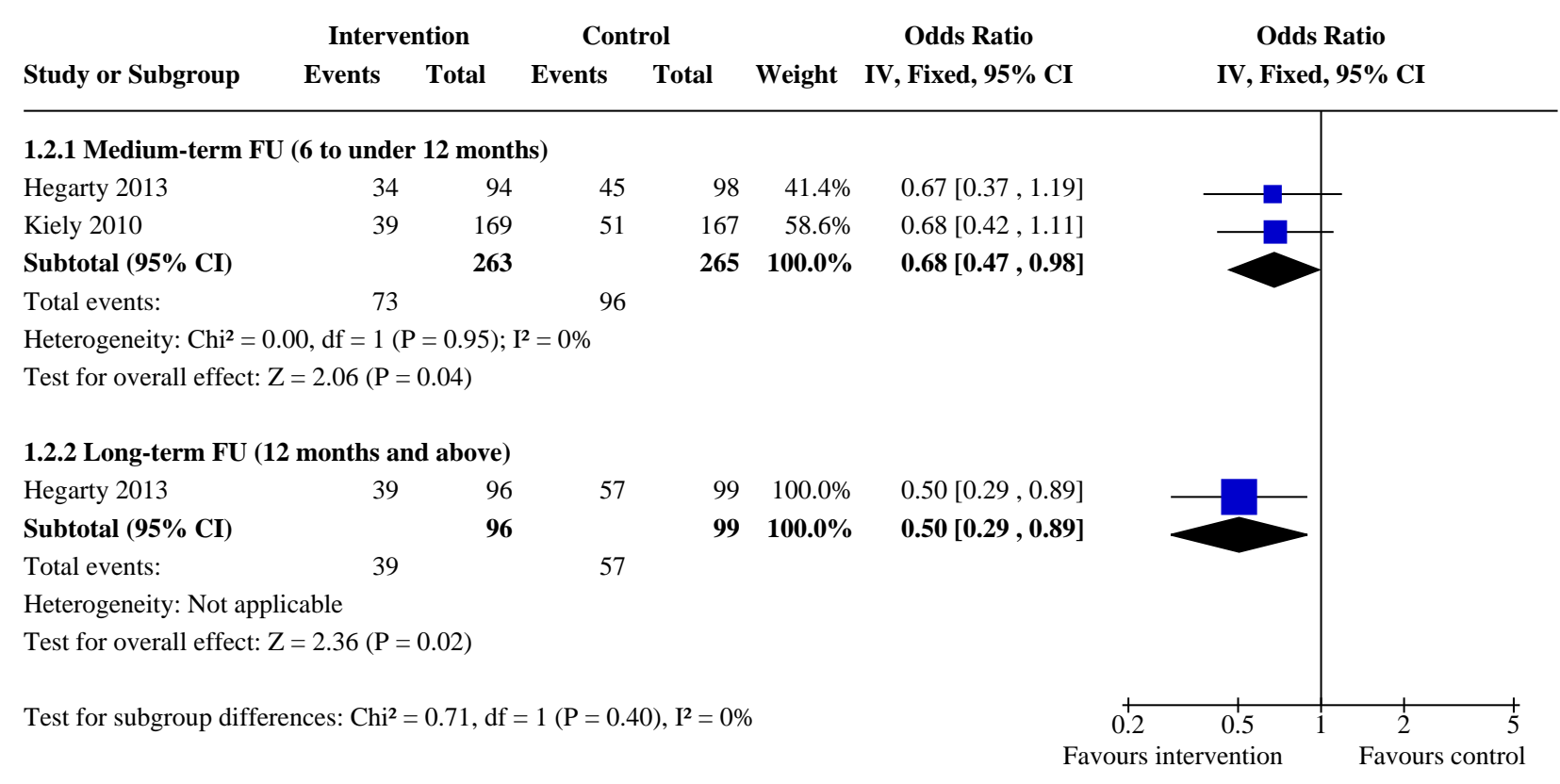


Analysis 1.3. Comparison 1: Intervention vs Control, Outcome 3: Depression (recruitment setting of participants)

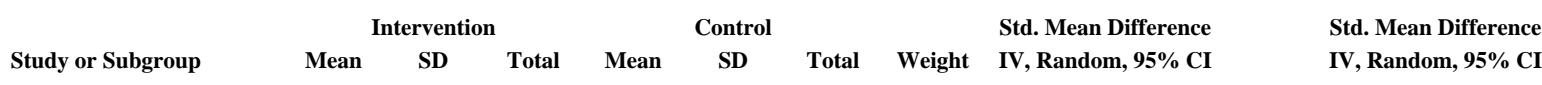

\subsubsection{Healthcare}

Zlotnick 2011

Gilbert 2006

Tirado-Muñoz 2015

Stevens 2015

Saftlas 2014

Bowland 2012

Subtotal (95\% CI)

Heterogeneity: $\mathrm{Tau}^{2}=0.01 ; \mathrm{Chi}^{2}=5.69, \mathrm{df}=5(\mathrm{P}=0.34) ; \mathrm{I}^{2}=12 \%$

Test for overall effect: $\mathrm{Z}=1.82(\mathrm{P}=0.07)$

\subsubsection{Community}

Hegarty 2019

Hirani 2010

Koopman 2005

Kokka 2019

Franzblau 2008

Subtotal (95\% CI)

$6.12 \quad 5.86$

\begin{tabular}{rr}
1.4 & 1.2 \\
\hline
\end{tabular}

$14.57 \quad 8.96$

14.57
17.7

$17.7 \quad 14.3$

14.3

5.5

25
16
5

5
90

$\begin{array}{rr}8 & 5.74 \\ 1.8 & 1.2728\end{array}$

$12.28 \quad 9.6$

19.8

11.8

14.5

12.3

6.1

8.69

21
255

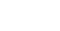

$\begin{array}{rr}21 & 5.2 \% \\ 18 & 4.5 \% \\ 5 & 1.9 \% \\ 93 & 8.2 \% \\ 106 & 8.4 \% \\ 21 & 4.8 \% \\ \mathbf{2 6 4} & \mathbf{3 3 . 1 \%}\end{array}$

Heterogeneity: $\mathrm{Tau}^{2}=0.10 ; \mathrm{Chi}^{2}=9.57, \mathrm{df}=4(\mathrm{P}=0.05) ; \mathrm{I}^{2}=58 \%$

Test for overall effect: $\mathrm{Z}=1.20(\mathrm{P}=0.23)$

\subsubsection{Shelter / refuge}

Johnson 2011

7.21

8.59

19

13.24

11.83

$33 \quad 5.3 \%$

Heterogeneity: Not applicable

Test for overall effect: $\mathrm{Z}=1.88(\mathrm{P}=0.06)$

\subsubsection{Various}

Cheung 2019

Ferrari 2018

Orang 2017

Graham-Bermann 2015

Kubany 2004

Subtotal (95\% CI)

$\begin{array}{rr}10.69 & 9.8474 \\ 7.1 & 7 \\ 6.88 & 4.91 \\ 10.55 & 10.14 \\ 12 & 14.2\end{array}$

$120 \quad 12.48$

$\begin{array}{rr}83 & 8.9 \\ 17 & 11.17 \\ 60 & 14.26\end{array}$

9.88916

6.4
6.34
11.02
10.5

343

14.26

$28.7 \quad 10.5$

Heterogeneity: $\mathrm{Tau}^{2}=0.18 ; \mathrm{Chi}^{2}=26.34, \mathrm{df}=4(\mathrm{P}<0.0001) ; \mathrm{I}^{2}=85 \%$

Test for overall effect: $\mathrm{Z}=2.58(\mathrm{P}=0.010)$

Total $(95 \%$ CI)

866

Heterogeneity: $\mathrm{Tau}^{2}=0.09 ; \mathrm{Chi}^{2}=51.39, \mathrm{df}=16(\mathrm{P}<0.0001) ; \mathrm{I}^{2}=69 \%$

Test for overall effect: $\mathrm{Z}=3.67(\mathrm{P}=0.0002)$

Test for subgroup differences: $\mathrm{Chi}^{2}=3.43, \mathrm{df}=3(\mathrm{P}=0.33), \mathrm{I}^{2}=12.5 \%$

$-0.18[-0.43,0.07]$
$26433.1 \%$

$-0.32[-0.90,0.27]$

$-0.32[-0.99,0.36]$

$0.22[-1.02,1.47]$

$-0.16[-0.45,0.13]$

$-0.02[-0.29,0.26]$

$-0.79[-1.42,-0.16]$

$-0.18[-0.38,0.01]$

$-0.55[-1.13,0.02]$

$-0.55[-1.13,0.02]$

IV, Random, 95\% CI

$0.02[-0.20,0.24]$

$-0.28[-1.30,0.75]$

$-0.16[-0.74,0.41]$

$-0.87[-1.40,-0.34]$

$0.09[-0.79,0.97]$

$\mathbf{- 0 . 2 4}[-0.62,0.15]$

|

$-0.27[-0.57,0.04]$

$-0.74[-1.44,-0.04]$

$-0.35[-0.71,0.02]$

$-1.33[-1.72,-0.94]$

$-0.55[-0.96,-0.13]$

$863100.0 \%$

$-0.36[-0.55,-0.17]$

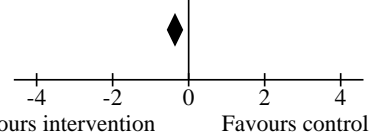


Analysis 1.4. Comparison 1: Intervention vs Control, Outcome 4: Depression (type of intervention)

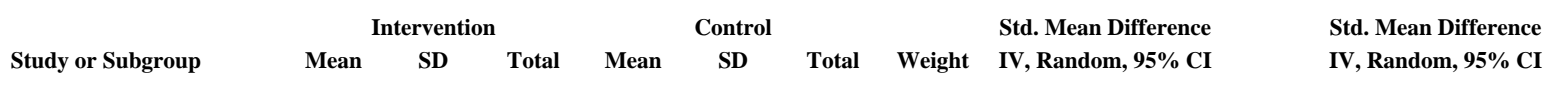

1.4.1 Cognitive behavioral therapy

Gilbert 2006

Ferrari 2018

1.4

1.2
7

16
83

Johnson 2011

7.1

8.59

83

1.8
8.9
13.24

$\begin{array}{rrr}1.2728 & 18 & 3.1 \% \\ 6.4 & 83 & 10.4 \% \\ 11.83 & 33 & 4.2 \% \\ 9.6 & 5 & 1.0 \% \\ & \mathbf{1 3 9} & \mathbf{1 8 . 7 \%}\end{array}$

Subtotal (95\% CI)

7.57
14.96

123

Heterogeneity: $\mathrm{Tau}^{2}=0.00 ; \mathrm{Chi}^{2}=1.45, \mathrm{df}=3(\mathrm{P}=0.69) ; \mathrm{I}^{2}=0 \%$

Test for overall effect: $Z=2.44(P=0.01)$

1.4.2 Humanistic therapies

Orang 2017

Koopman 2005

Kokka 2019

Franzblau 2008

$6.88 \quad 4.91$

$12.5 \quad 12.2$

17

11.17

6.34

Subtotal (95\% CI)

Heterogeneity: $\mathrm{Tau}^{2}=0.09 ; \mathrm{Chi}^{2}=5.39, \mathrm{df}=3(\mathrm{P}=0.15) ; \mathrm{I}^{2}=44 \%$

Test for overall effect: $\mathrm{Z}=2.11(\mathrm{P}=0.03)$

1.4.3 Integrative therapies

$\begin{array}{lrrrrrrr}\text { Hegarty 2019 } & 21.9 & 19.3 & 177 & 21.5 & 19.3 & 150 & 14.6 \% \\ \text { Zlotnick 2011 } & 6.12 & 5.86 & 25 & 8 & 5.74 & 21 & 4.0 \% \\ \text { Stevens 2015 } & 17.7 & 14.3 & 90 & 19.8 & 12.3 & 93 & 11.0 \% \\ \text { Hirani 2010 } & 24.71 & 10.9 & 7 & 27.63 & 9.1 & 8 & 1.5 \% \\ \text { Graham-Bermann 2015 } & 10.55 & 10.14 & 60 & 14.26 & 11.02 & 57 & 8.3 \% \\ \text { Saftlas 2014 } & 11.7 & 5.5 & 98 & 11.8 & 6.1 & 106 & 11.7 \%\end{array}$

Saftlas 2014

$$
457
$$

Heterogeneity: $\mathrm{Tau}^{2}=0.00 ; \mathrm{Chi}^{2}=4.10, \mathrm{df}=5(\mathrm{P}=0.54) ; \mathrm{I}^{2}=0 \%$

Test for overall effect: $\mathrm{Z}=1.41(\mathrm{P}=0.16)$

1.4.4 Other psychologically-oriented interventions

$\begin{array}{lrrrrrrr}\text { Cheung } 2019 & 10.69 & 9.8474 & 120 & 12.48 & 9.8516 & 127 & 12.9 \% \\ \text { Bowland 2012 } & 8.86 & 4.81 & 21 & 14.5 & 8.69 & 21 & 3.6 \% \\ \text { Subtotal (95\% CI) } & & & \mathbf{1 4 1} & & & \mathbf{1 4 8} & \mathbf{1 6 . 4 \%}\end{array}$

Subtotal (95\% CI)

$8) ; I^{2}=67 \%$

Test for overall effect: $\mathrm{Z}=1.40(\mathrm{P}=0.16)$

Total (95\% CI)

803

Heterogeneity: $\mathrm{Tau}^{2}=0.02 ; \mathrm{Chi}^{2}=20.91, \mathrm{df}=15(\mathrm{P}=0.14) ; \mathrm{I}^{2}=28 \%$

Test for overall effect: $\mathrm{Z}=3.66(\mathrm{P}=0.0003)$

Test for subgroup differences: $\mathrm{Chi}^{2}=4.93, \mathrm{df}=3(\mathrm{P}=0.18), \mathrm{I}^{2}=39.1 \%$

$17 \quad 3.0 \%$

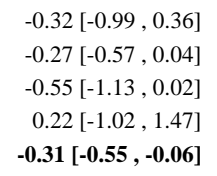

$-0.32[-0.99,0.36]$ $-0.27[-0.57,0.04]$ $-0.55[-1.13,0.02]$ $0.22[-1.02,1.47]$ $-0.31[-0.55,-0.06]$

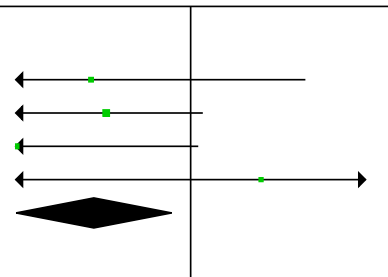

$-0.74[-1.44,-0.04]$ $-0.16[-0.74,0.41]$ $-0.87[-1.40,-0.34]$ $0.09[-0.79,0.97]$ $\mathbf{- 0 . 4 7}[-0.91,-0.03]$

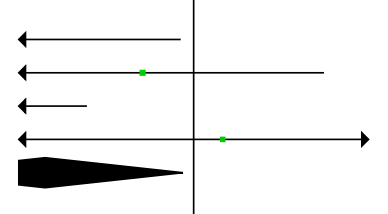

$0.02[-0.20,0.24]$ $-0.32[-0.90,0.27]$ $-0.16[-0.45,0.13]$ $-0.28[-1.30,0.75]$ $-0.35[-0.71,0.02]$ $-0.02[-0.29,0.26]$ $-0.09[-0.23,0.04]$

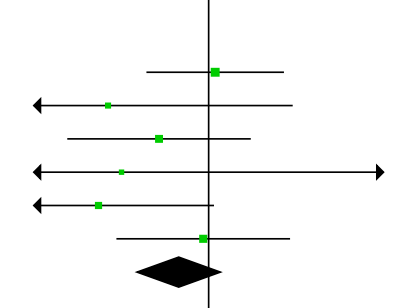

$-0.18[-0.43,0.07]$ $-0.79[-1.42,-0.16]$ $-0.41[-0.99,0.16]$

$-0.24[-0.37,-0.11]$

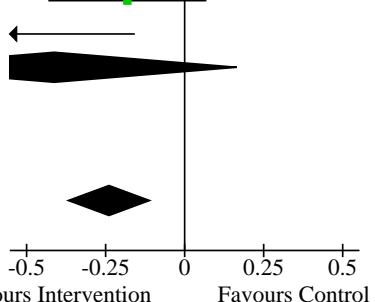


Analysis 1.5. Comparison 1: Intervention vs Control, Outcome 5: Depression (intensity of intervention)

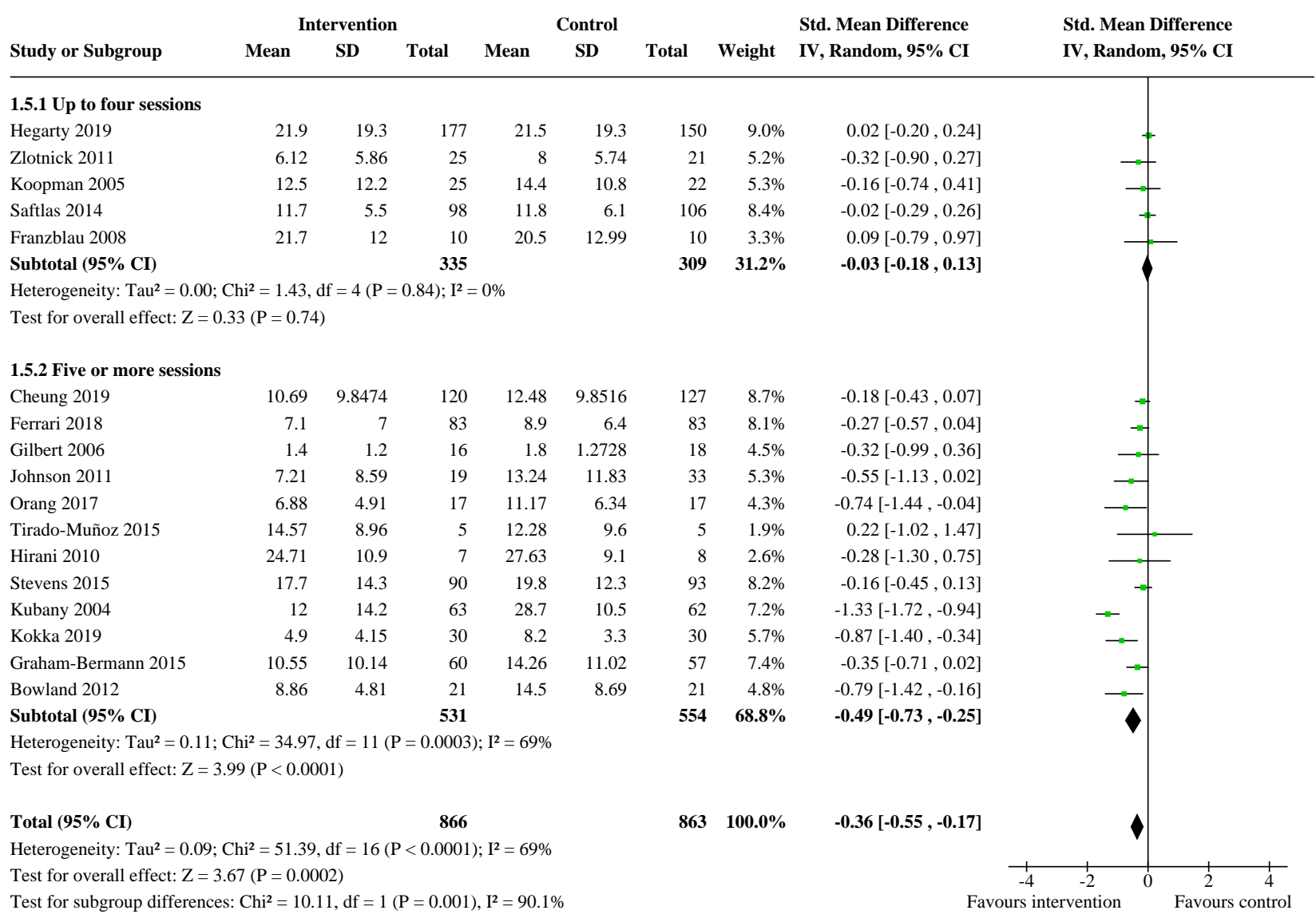


Analysis 1.6. Comparison 1: Intervention vs Control, Outcome 6: Depression (person delivering the intervention)

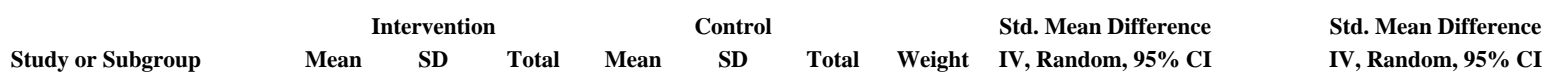

1.6.1 healthcare workers

Johnson 2011

$6.12 \quad 5.86$

Stevens 2015

$\begin{array}{ll}7.21 & 8.59\end{array}$

8.59
4.3

$\begin{array}{rrr}25 & 8 & 5.74 \\ 19 & 13.24 & 11.83\end{array}$

Hirani 2010

17.7

14.3

19
90
7

13.24

Orang 2017

$\begin{array}{rr}24.71 & 10.9 \\ 6.88 & 4.91\end{array}$

19.8
27.63

Tirado-Muñoz 2015

$14.57 \quad 8.96$

$\begin{array}{ll}77 & 11.17\end{array}$

Koopman 2005

$\begin{array}{ll}12.5 & 12.2\end{array}$

$\begin{array}{ll}5 & 12.28\end{array}$

Graham-Bermann 2015

$10.55 \quad 10.14$

$25 \quad 14.4$

Kubany 2004

Franzblau 2008

$\begin{array}{rr}12 & 14.2 \\ 21.7 & 12\end{array}$

$\begin{array}{rr}60 & 14.26 \\ 63 & 28.7\end{array}$

Subtotal (95\% CI)

$\begin{array}{rrr}63 & 28.7 & 10.5 \\ 10 & 20.5 & 12.99\end{array}$

321

12.3

9.1

6.34

9.6

10.8

11.02

Heterogeneity: $\mathrm{Tau}^{2}=0.15 ; \mathrm{Chi}^{2}=28.46, \mathrm{df}=9(\mathrm{P}=0.0008) ; \mathrm{I}^{2}=68 \%$

Test for overall effect: $\mathrm{Z}=2.64(\mathrm{P}=0.008)$

1.6.2 non-healthcare workers

Cheung 2019

Ferrari 2018

Gilbert 2006

Saftlas 2014

Bowland 2012

$\begin{array}{rrr}10.69 & 9.8474 & 12 \\ 7.1 & 7 & 8 \\ 1.4 & 1.2 & 1 \\ 11.7 & 5.5 & 98 \\ 8.86 & 4.81 & 2\end{array}$

120
83
16
98
21

12.48

9.8516
6.4

$127 \quad 10.3 \%$

$83 \quad 9.6 \%$

$-0.18[-0.43,0.07]$

$-0.27[-0.57,0.04]$

$-0.32[-0.99,0.36]$

$-0.02[-0.29,0.26]$

$-0.79[-1.42,-0.16]$

$\mathbf{- 0 . 2 1}[-0.39,-0.03]$

Heterogeneity: $\mathrm{Tau}^{2}=0.01 ; \mathrm{Chi}^{2}=5.36, \mathrm{df}=4(\mathrm{P}=0.25) ; \mathrm{I}^{2}=25 \%$

Test for overall effect: $\mathrm{Z}=2.25(\mathrm{P}=0.02)$

Total $(95 \%$ CI)

659

$\begin{array}{rrrr}1.8 & 1.2728 & 18 & 5.2 \% \\ 11.8 & 6.1 & 106 & 10.0 \%\end{array}$

$\begin{array}{llll}14.5 & 8.69 & 21 & 5.6 \%\end{array}$

$355 \quad 40.7 \%$

Heterogeneity: $\mathrm{Tau}^{2}=0.09 ; \mathrm{Chi}^{2}=39.03, \mathrm{df}=14(\mathrm{P}=0.0004) ; \mathrm{I}^{2}=64 \%$

$683 \quad 100.0 \%$

$-0.36[-0.57,-0.16]$

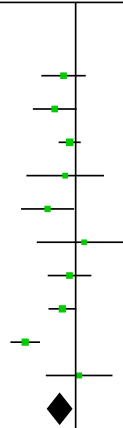

Test for overall effect: $\mathrm{Z}=3.53(\mathrm{P}=0.0004)$

Test for subgroup differences: $\mathrm{Chi}^{2}=1.30, \mathrm{df}=1(\mathrm{P}=0.25), \mathrm{I}^{2}=23.0 \%$

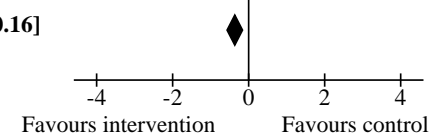

Analysis 1.7. Comparison 1: Intervention vs Control, Outcome 7: Self-efficacy

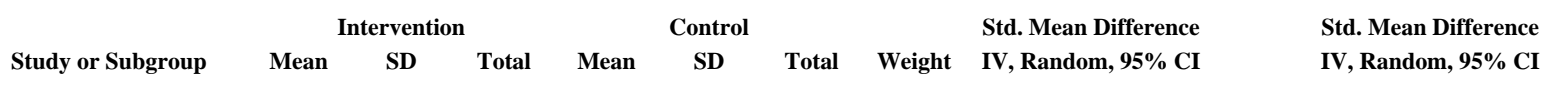

1.7.1 Short-term FU (under 6 months)

$\begin{array}{lrrrrrrrr}\text { Hirani } 2010 & 19 & 9.3 & 7 & 21.63 & 3.8 & 8 & 13.7 \% & -0.36[-1.38,0.67] \\ \text { Kokka 2019 } & 27.3 & 2.8 & 30 & 25.5 & 3.1 & 30 & 33.7 \% & 0.60[0.08,1.12] \\ \text { Saftlas 2014 } & 82.1 & 15.2 & 98 & 80.7 & 13.9 & 106 & 52.6 \% & 0.10[-0.18,0.37] \\ \text { Subtotal (95\% CI) } & & & \mathbf{1 3 5} & & & \mathbf{1 4 4} & \mathbf{1 0 0 . 0 \%} & \mathbf{0 . 2 0}[-\mathbf{0 . 2 2}, \mathbf{0 . 6 3}]\end{array}$

Heterogeneity: $\mathrm{Tau}^{2}=0.07 ; \mathrm{Chi}^{2}=3.96, \mathrm{df}=2(\mathrm{P}=0.14) ; \mathrm{I}^{2}=49 \%$

Test for overall effect: $\mathrm{Z}=0.94(\mathrm{P}=0.35)$

1.7.2 Medium-term FU (6 to under 12 months)

$\begin{array}{lllllllll}\text { Hegarty } 2019 & 27.5 & 5.2 & 181 & 28.1 & 4.4 & 165 & 100.0 \% & -0.12[-0.33,0.09] \\ \text { Subtotal }(\mathbf{9 5 \%} \text { CI) } & & & \mathbf{1 8 1} & & & \mathbf{1 6 5} & \mathbf{1 0 0 . 0 \%} & \mathbf{- 0 . 1 2}[-\mathbf{0 . 3 3}, \mathbf{0 . 0 9}]\end{array}$

Heterogeneity: Not applicable

Test for overall effect: $\mathrm{Z}=1.15(\mathrm{P}=0.25)$

1.7.3 Long-term FU (12 months and above)

$\begin{array}{lllllllll}\text { Hegarty } 2019 & 27.8 & 5.4 & 176 & 29 & 5 & 155 & 100.0 \% & -0.23[-0.45,-0.01] \\ \text { Subtotal }(\mathbf{9 5 \%} \text { CI) } & & & \mathbf{1 7 6} & & & \mathbf{1 5 5} & \mathbf{1 0 0 . 0 \%} & \mathbf{- 0 . 2 3}[-\mathbf{- 0 . 4 5}, \mathbf{- 0 . 0 1}]\end{array}$

Heterogeneity: Not applicable

Test for overall effect: $\mathrm{Z}=2.08(\mathrm{P}=0.04)$

Test for subgroup differences: $\mathrm{Chi}^{2}=3.18, \mathrm{df}=2(\mathrm{P}=0.20), \mathrm{I}^{2}=37.2 \%$

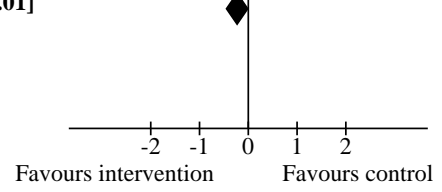


Analysis 1.8. Comparison 1: Intervention vs Control, Outcome 8: Dropouts from treatment

\begin{tabular}{|c|c|c|c|c|c|c|c|}
\hline & Inter & tion & Cor & & & Odds Ratio & Odds Ratio \\
\hline Study or Subgroup & Events & Total & Events & Total & Weight & IV, Random, 95\% CI & IV, Random, $95 \%$ CI \\
\hline
\end{tabular}

1.8.1 Short-term FU (up to 6 months)

Cheung 2019

Bryant 2017

Kiely 2010

Choo 2016

Gilbert 2006

Zlotnick 2011

Hirani 2010

Koopman 2005

Zlotnick 2019

Myers 2015

Jaffe 2017

Taghizadeh 2018

Hernandez-Ruiz 2005

Kubany 2004

Akor 2019

Bowland 2012

Saggurti 2014

Michalopoulou 2015

Kokka 2019

Saftlas 2014

Franzblau 2008

Ghahari 2017

Subtotal (95\% CI)

Total events:

Heterogeneity: $\mathrm{Tau}^{2}=0.00 ; \mathrm{Chi}^{2}=7.28, \mathrm{df}=16(\mathrm{P}=0.97) ; \mathrm{I}^{2}=0 \%$

Test for overall effect: $\mathrm{Z}=1.81(\mathrm{P}=0.07)$

1.8.2 Medium-term FU (6 to 12 months)

Cohen 2013

Orang 2017

Stevens 2015

Johnson 2011

Graham-Bermann 2015

Subtotal $(95 \%$ CI)

Total events:

$\begin{array}{rr}65 & 176 \\ 7 & 24 \\ 39 & 129 \\ 3 & 35 \\ 1 & 58 \\ & \mathbf{4 2 2}\end{array}$

Heterogeneity: $\mathrm{Tau}^{2}=0.00 ; \mathrm{Chi}^{2}=3.07, \mathrm{df}=4(\mathrm{P}=0.55) ; \mathrm{I}^{2}=0 \%$

Test for overall effect: $\mathrm{Z}=0.24(\mathrm{P}=0.81)$

1.8.3 Long-term FU (greater than 12 months)

$\begin{array}{lrrrrr}\text { Hegarty 2019 } & 48 & 227 & 39 & 195 & 22.6 \% \\ \text { Gilbert 2016 } & 12 & 103 & 8 & 102 & 6.5 \% \\ \text { Ferrari 2018 } & 46 & 131 & 47 & 132 & 20.2 \% \\ \text { Rhodes 2015 } & 77 & 242 & 72 & 237 & 31.5 \% \\ \text { Hegarty 2013 } & 41 & 137 & 35 & 135 & 18.6 \% \\ \text { Tirado-Muñoz 2015 } & 0 & 7 & 5 & 7 & 0.6 \% \\ \text { Subtotal (95\% CI) } & & \mathbf{8 4 7} & & \mathbf{8 0 8} & \mathbf{1 0 0 . 0 \%} \\ \text { Total events: } & 224 & & 206 & & \end{array}$

Total events:

224

206

Heterogeneity: $\mathrm{Tau}^{2}=0.01 ; \mathrm{Chi}^{2}=5.62, \mathrm{df}=5(\mathrm{P}=0.34) ; \mathrm{I}^{2}=11 \%$

Test for overall effect: $\mathrm{Z}=0.62(\mathrm{P}=0.53)$

Test for subgroup differences: $\mathrm{Chi}^{2}=0.79, \mathrm{df}=2(\mathrm{P}=0.67), \mathrm{I}^{2}=0 \%$
$2.12[0.87,5.13]$

$1.13[0.72,1.77]$

$1.01[0.64,1.59]$

$1.88[0.45,7.82]$

$0.53[0.04,6.51]$

$0.50[0.11,2.36]$

Not estimable $0.63[0.17,2.27]$

$1.38[0.21,9.01]$

$0.97[0.22,4.32]$

$1.30[0.32,5.33]$

1.80 [0.79, 4.07]

Not estimable

$1.31[0.51,3.40]$

Not estimable

$0.75[0.15,3.84]$

$1.25[0.64,2.46]$

3.36 [0.13, 88.39]

Not estimable

1.37 [0.85, 2.21]

Not estimable

$1.00[0.06,17.62]$

$1.21[0.98,1.48]$ 
Analysis 1.8. (Continued)

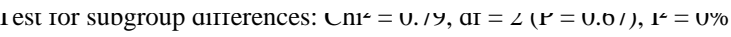

$\begin{array}{lllll}0.01 & 0.1 & 1 & 10 & 100\end{array}$

Favours intervention Favours control

\section{Analysis 1.9. Comparison 1: Intervention vs Control, Outcome 9: Mental health}

\begin{tabular}{|c|c|c|c|c|c|c|c|c|c|}
\hline \multirow[b]{2}{*}{ Study or Subgroup } & \multicolumn{3}{|c|}{ Intervention } & \multicolumn{3}{|c|}{ Control } & \multicolumn{2}{|r|}{ Std. Mean Difference } & \multirow{2}{*}{$\begin{array}{l}\text { Std. Mean Difference } \\
\text { IV, Random, } 95 \% \text { CI }\end{array}$} \\
\hline & Mean & SD & Total & Mean & SD & Total & Weight & IV, Random, $95 \%$ CI & \\
\hline
\end{tabular}

1.9.1 Short-term FU (under 6 months)

$\begin{array}{lrrrrrrr}\text { Bryant } 2017 & 8.7 & 6.3228 & 156 & 11 & 7.1118 & 163 & 90.4 \%\end{array}$

$\begin{array}{lrrrrrrr}\text { Orang } 2017 & 7.05 & 3.52 & 17 & 8.29 & 3.15 & 17 & 9.6 \%\end{array}$

Subtotal $(95 \% \mathrm{CI})$

Heterogeneity: $\mathrm{Tau}^{2}=0.00 ; \mathrm{Chi}^{2}=0.00, \mathrm{df}=1(\mathrm{P}=0.95) ; \mathrm{I}^{2}=0 \%$

Test for overall effect: $\mathrm{Z}=3.19(\mathrm{P}=0.001)$

1.9.2 Medium-term FU (6 to under 12 months)

$\begin{array}{lrrrrrrrr}\text { Hegarty } 2013 & 37.4 & 12.1 & 93 & 38.6 & 11.6 & 92 & 84.5 \% & -0.10[-0.39,0.19] \\ \text { Orang 2017 } & 7.29 & 3.09 & 17 & 7.76 & 2.63 & 17 & 15.5 \% & -0.16[-0.83,0.51] \\ \text { Subtotal (95\% CI) } & & & \mathbf{1 1 0} & & & \mathbf{1 0 9} & \mathbf{1 0 0 . 0 \%} & \mathbf{- 0 . 1 1}[\mathbf{- 0 . 3 8 , 0 . 1 6}]\end{array}$

Subtotal $(\mathbf{9 5 \%}$ CI $)$
Heterogeneity: $\mathrm{Tau}^{2}=0.00 ; \mathrm{Chi}^{2}=0.03, \mathrm{df}=1(\mathrm{P}=0.87) ; \mathrm{I}^{2}=0 \%$

$-0.11[-0.38,0.16]$

Test for overall effect: $\mathrm{Z}=0.81(\mathrm{P}=0.42$

1.9.3 Long-term FU (12 months and above)

$\begin{array}{lrrrrrrrr}\text { Ferrari } 2018 & 11.3 & 8.6 & 84 & 14.2 & 7.9 & 83 & 46.8 \% & -0.35[-0.66,-0.04] \\ \text { Hegarty } 2013 & 38.4 & 13 & 94 & 41 & 12.2 & 94 & 53.2 \% & -0.21[-0.49,0.08] \\ \text { Subtotal (95\% CI) } & & & \mathbf{1 7 8} & & & \mathbf{1 7 7} & \mathbf{1 0 0 . 0 \%} & \mathbf{- 0 . 2 7}[\mathbf{- 0 . 4 8 , - 0 . 0 6 ]}\end{array}$

Heterogeneity: $\mathrm{Tau}^{2}=0.00 ; \mathrm{Chi}^{2}=0.45, \mathrm{df}=1(\mathrm{P}=0.50) ; \mathrm{I}^{2}=0 \%$

Test for overall effect: $\mathrm{Z}=2.56(\mathrm{P}=0.01)$

Test for subgroup differences: $\mathrm{Chi}^{2}=1.84, \mathrm{df}=2(\mathrm{P}=0.40), \mathrm{I}^{2}=0 \%$

\section{Analysis 1.10. Comparison 1: Intervention vs Control, Outcome 10: Anxiety (continuous)}

\begin{tabular}{|c|c|c|c|c|c|c|c|c|c|}
\hline \multirow[b]{2}{*}{ Study or Subgroup } & \multicolumn{3}{|c|}{ Intervention } & \multicolumn{3}{|c|}{ Control } & \multicolumn{2}{|r|}{ Std. Mean Difference } & \multirow{2}{*}{$\begin{array}{l}\text { Std. Mean Difference } \\
\text { IV, Random, } 95 \% \text { CI }\end{array}$} \\
\hline & Mean & SD & Total & Mean & SD & Total & Weight & IV, Random, $95 \%$ CI & \\
\hline
\end{tabular}

1.10.1 Short-term FU (under 6 months)

$\begin{array}{lrrrrrrrr}\text { 1.10.1 Short-term FU (under 6 months) } & & & & & & \\ \text { Hernandez-Ruiz 2005 } & 32.86 & 11.88 & 14 & 45.21 & 10.15 & 14 & 17.1 \% & -1.09[-1.89,-0.28] \\ \text { Bowland 2012 } & 8.86 & 4.81 & 21 & 14.5 & 8.69 & 21 & 27.7 \% & -0.79[-1.42,-0.16] \\ \text { Kokka 2019 } & 4.8 & 3.8 & 30 & 8 & 2.9 & 30 & 38.5 \% & -0.93[-1.47,-0.40] \\ \text { Ghahari 2017 } & 47.6 & 4.2 & 14 & 53.12 & 4.9 & 14 & 16.7 \% & -1.17[-1.99,-0.36] \\ \text { Subtotal (95\% CI) } & & & \mathbf{7 9} & & & \mathbf{7 9} & \mathbf{1 0 0 . 0 \%} & \mathbf{- 0 . 9 6 [ - 1 . 2 9 , - 0 . 6 3}]\end{array}$

Heterogeneity: $\mathrm{Tau}^{2}=0.00 ; \mathrm{Chi}^{2}=0.66, \mathrm{df}=3(\mathrm{P}=0.88) ; \mathrm{I}^{2}=0 \%$

Test for overall effect: $\mathrm{Z}=5.67(\mathrm{P}<0.00001)$

1.10.2 Long-term FU (12 months and above)

$\begin{array}{lllllllll}\text { Ferrari } 2018 & 6.2 & 6.1 & 83 & 7.4 & 5.7 & 83 & 100.0 \% & -0.20[-0.51,0.10] \\ \text { Subtotal }(\mathbf{9 5 \%} \text { CI) } & & & \mathbf{8 3} & & & \mathbf{8 3} & \mathbf{1 0 0 . 0 \%} & \mathbf{- 0 . 2 0}[-\mathbf{0 . 5 1}, \mathbf{0 . 1 0}]\end{array}$

Heterogeneity: Not applicable

Test for overall effect: $\mathrm{Z}=1.30(\mathrm{P}=0.19)$

Test for subgroup differences: $\mathrm{Chi}^{2}=10.85, \mathrm{df}=1(\mathrm{P}=0.0010), \mathrm{I}^{2}=90.8 \%$ 95\% CI 
Analysis 1.11. Comparison 1: Intervention vs Control, Outcome 11: Anxiety (categorical)

\begin{tabular}{|c|c|c|c|c|c|c|c|}
\hline \multirow[b]{2}{*}{ Study or Subgroup } & \multicolumn{2}{|c|}{ Intervention } & \multicolumn{2}{|c|}{ Control } & \multicolumn{2}{|r|}{ Odds Ratio } & Odds Ratio \\
\hline & Events & Total & Events & Total & Weight & IV, Random, $95 \%$ CI & IV, Random, $95 \%$ CI \\
\hline
\end{tabular}

1.11.1 Medium-term FU (6 to under 12 months)

$\begin{array}{lllllll}\text { Hegarty } 2013 & 61 & 94 & 68 & 98 & 100.0 \% & 0.82[0.45,1.49]\end{array}$

$\begin{array}{lllll}\text { Subtotal }(95 \% \text { CI }) & 94 & 98 & 100.0 \% & 0.82[0.45,1.49]\end{array}$

Total events: $\quad 61 \quad 68$

Heterogeneity: Not applicable

Test for overall effect: $\mathrm{Z}=0.66(\mathrm{P}=0.51)$

1.11.2 Long-term FU (12 months and above)

$\begin{array}{lllllll}\text { Hegarty } 2013 & 61 & 96 & 66 & 99 & 100.0 \% & 0.87[0.48,1.57] \\ \text { Subtotal }(\mathbf{9 5 \%} \text { CI) } & & \mathbf{9 6} & & \mathbf{9 9} & \mathbf{1 0 0 . 0 \%} & \mathbf{0 . 8 7}[\mathbf{0 . 4 8 , \mathbf { 1 . 5 7 }}]\end{array}$

Total events: $\quad 61 \quad 66$

Heterogeneity: Not applicable

Test for overall effect: $\mathrm{Z}=0.46(\mathrm{P}=0.65)$

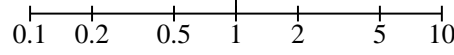

Favours intervention Favours control

Analysis 1.12. Comparison 1: Intervention vs Control, Outcome 12: Post-traumatic stress disorder (continuous)

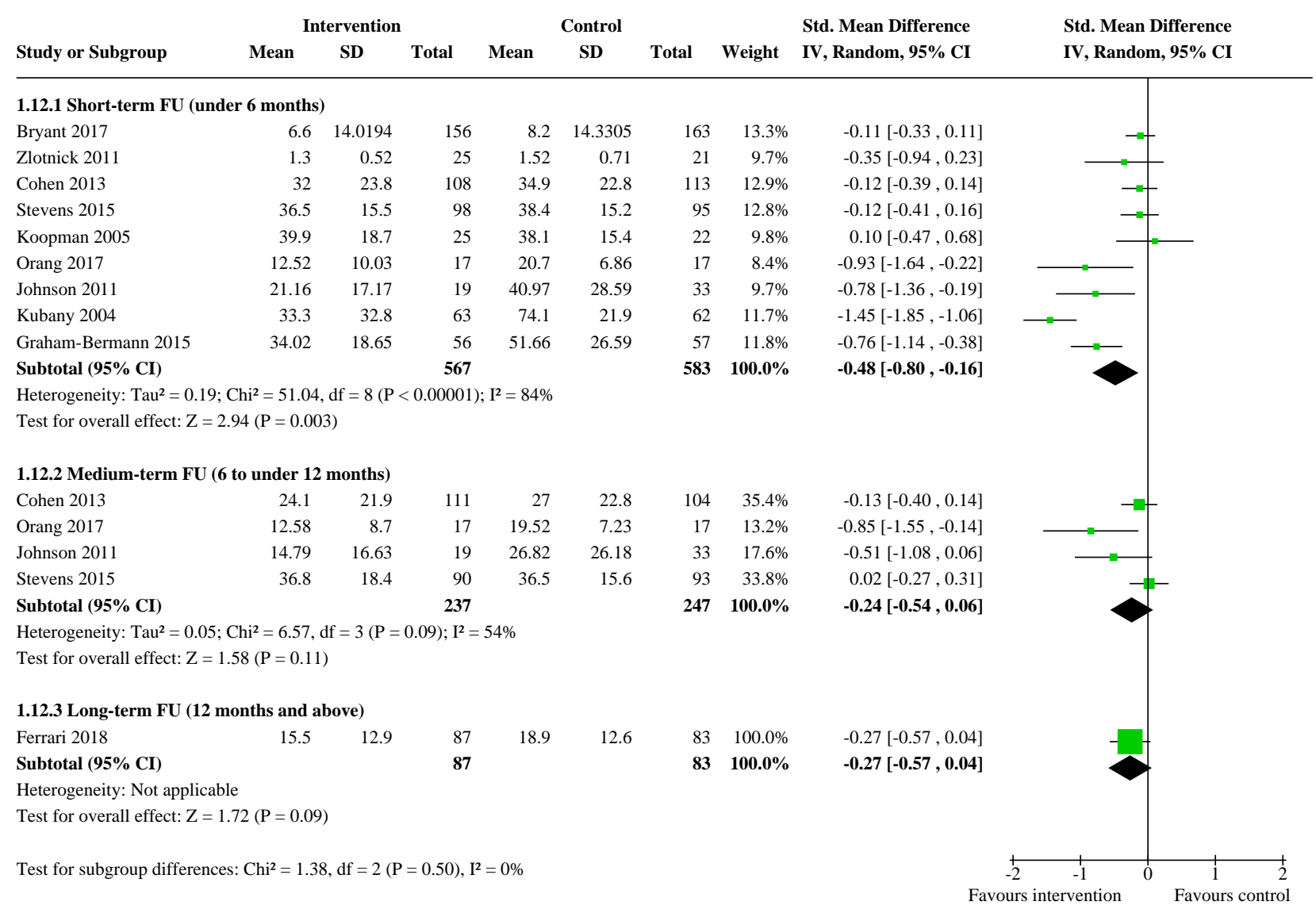


Analysis 1.13. Comparison 1: Intervention vs Control, Outcome 13: Quality of life

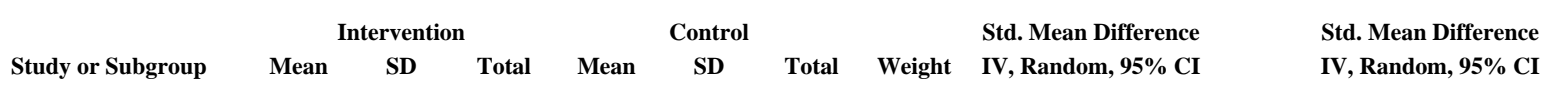

1.13.1 Short-term FU (under 6 months)

Rhodes $2015 \quad 2.4 \quad 1.405$

Tirado-Muñoz 2015

$55.71 \quad 17.18$

$192 \quad 2.2$

1.017

$179 \quad 97.1 \%$

$0.16[-0.04,0.37]$

Subtotal (95\% CI)

197

$185100.0 \%$

$0.14[-1.05,1.33]$

$0.16[-0.04,0.36]$

Heterogeneity: $\mathrm{Tau}^{2}=0.00 ; \mathrm{Chi}^{2}=0.00, \mathrm{df}=1(\mathrm{P}=0.98) ; \mathrm{I}^{2}=0 \%$

Test for overall effect: $\mathrm{Z}=1.57(\mathrm{P}=0.12)$

1.13.2 Medium-term FU (6 to under 12 months)

\begin{tabular}{|c|c|c|c|c|c|c|c|c|}
\hline Hegarty 2013 & 54.3 & 19.9 & 94 & 52.1 & 17.6 & 99 & $34.6 \%$ & $0.12[-0.17,0.40]$ \\
\hline Rhodes 2015 & 2.2 & 1.3901 & 188 & 2.1 & 0.6722 & 176 & $65.4 \%$ & $0.09[-0.12,0.30]$ \\
\hline Subtotal $(95 \%$ CI $)$ & & & 282 & & & 275 & $100.0 \%$ & $0.10[-0.07,0.27]$ \\
\hline \multicolumn{9}{|c|}{ Heterogeneity: $\mathrm{Tau}^{2}=0.00 ; \mathrm{Chi}^{2}=0.02, \mathrm{df}=1(\mathrm{P}=0.88) ; \mathrm{I}^{2}=0 \%$} \\
\hline \multicolumn{9}{|c|}{ Test for overall effect: $\mathrm{Z}=1.17(\mathrm{P}=0.24)$} \\
\hline \multicolumn{9}{|c|}{ 1.13.3 Long-term FU (12 months and above) } \\
\hline Ferrari 2018 & 40.5 & 15.6 & 82 & 36.2 & 14 & 81 & $23.1 \%$ & $0.29[-0.02,0.60]$ \\
\hline Rhodes 2015 & 2.2 & 0.6505 & 165 & 2.2 & 0.6505 & 165 & $47.4 \%$ & $0.00[-0.22,0.22]$ \\
\hline Hegarty 2013 & 55.4 & 20.4 & 96 & 53 & 17.3 & 100 & $28.1 \%$ & $0.13[-0.15,0.41]$ \\
\hline Tirado-Muñoz 2015 & 59.28 & 20 & 5 & 54.28 & 19.02 & 5 & $1.4 \%$ & $0.23[-1.02,1.48]$ \\
\hline Subtotal $(95 \%$ CI) & & & 348 & & & 351 & $100.0 \%$ & $0.11[-0.04,0.25]$ \\
\hline
\end{tabular}

Heterogeneity: $\mathrm{Tau}^{2}=0.00 ; \mathrm{Chi}^{2}=2.33, \mathrm{df}=3(\mathrm{P}=0.51) ; \mathrm{I}^{2}=0 \%$

Test for overall effect: $\mathrm{Z}=1.39(\mathrm{P}=0.16)$

Test for subgroup differences: $\mathrm{Chi}^{2}=0.25, \mathrm{df}=2(\mathrm{P}=0.88), \mathrm{I}^{2}=0 \%$

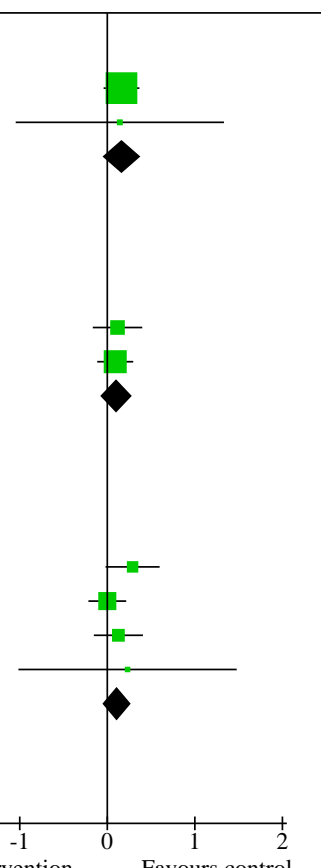

Favours intervention Favours control 
Analysis 1.14. Comparison 1: Intervention vs Control, Outcome 14: Re-exposure to IPV (continuous)

\begin{tabular}{|c|c|c|c|c|c|c|c|c|c|}
\hline \multirow[b]{2}{*}{ Study or Subgroup } & \multicolumn{3}{|c|}{ Intervention } & \multicolumn{3}{|c|}{ Control } & \multirow[b]{2}{*}{ Weight } & \multirow{2}{*}{$\begin{array}{l}\text { Std. Mean Difference } \\
\text { IV, Random, 95\% CI }\end{array}$} & \multirow{2}{*}{$\begin{array}{l}\text { Std. Mean Difference } \\
\text { IV, Random, } 95 \% \text { Cl }\end{array}$} \\
\hline & Mean & SD & Total & Mean & SD & Total & & & \\
\hline \multicolumn{10}{|c|}{ 1.14.1 Short-term FU (under 6 months) } \\
\hline Zlotnick 2011 & 16.3 & 28.6 & 25 & 12.1 & 23.1 & 21 & $13.5 \%$ & $0.16[-0.42,0.74]$ & - \\
\hline Rhodes 2015 & 8.5 & 10.5374 & 192 & 8.5 & 10.1697 & 179 & $25.2 \%$ & $0.00[-0.20,0.20]$ & $\phi$ \\
\hline Tirado-Muñoz 2015 & 10.6 & 1.34 & 5 & 17.16 & 2.31 & 6 & $2.0 \%$ & $-3.09[-5.09,-1.09]$ & $=$ \\
\hline Jaffe 2017 & 0.14 & 0.6 & 9 & 0.02 & 0.1581 & 10 & $7.7 \%$ & $0.27[-0.64,1.17]$ & - \\
\hline Zlotnick 2019 & 12.4 & 12.4 & 26 & 16.5 & 20.2 & 23 & $13.9 \%$ & $-0.24[-0.81,0.32]$ & - \\
\hline Stevens 2015 & 12 & 22.6 & 98 & 14.7 & 19.4 & 95 & $22.6 \%$ & $-0.13[-0.41,0.15]$ & $=$ \\
\hline Kokka 2019 & 17.7 & 1.5 & 30 & 17 & 1 & 30 & $15.2 \%$ & $0.54[0.03,1.06]$ & - \\
\hline Subtotal $(95 \%$ CI $)$ & & & 385 & & & 364 & $100.0 \%$ & $-0.00[-0.29,0.29]$ & \\
\hline \multicolumn{10}{|c|}{ Heterogeneity: $\mathrm{Tau}^{2}=0.08 ; \mathrm{Chi}^{2}=15.52, \mathrm{df}=6(\mathrm{P}=0.02) ; \mathrm{I}^{2}=61 \%$} \\
\hline \multicolumn{10}{|c|}{ Test for overall effect: $Z=0.00(P=1.00)$} \\
\hline \multicolumn{10}{|c|}{ 1.14.2 Medium-term FU ( 6 to under 12 months) } \\
\hline Rhodes 2015 & 6.2 & 7.6455 & 188 & 6.1 & 8.7385 & 176 & $66.5 \%$ & $0.01[-0.19,0.22]$ & \\
\hline Stevens 2015 & 9.8 & 21.8 & 90 & 8.7 & 13.7 & 93 & $33.5 \%$ & $0.06[-0.23,0.35]$ & + \\
\hline Subtotal $(95 \%$ CI $)$ & & & 278 & & & 269 & $100.0 \%$ & $0.03[-0.14,0.20]$ & $T$ \\
\hline \multicolumn{10}{|c|}{ Heterogeneity: $\mathrm{Tau}^{2}=0.00 ; \mathrm{Chi}^{2}=0.07, \mathrm{df}=1(\mathrm{P}=0.79) ; \mathrm{I}^{2}=0 \%$} \\
\hline \multicolumn{10}{|c|}{ Test for overall effect: $\mathrm{Z}=0.33(\mathrm{P}=0.74)$} \\
\hline \multicolumn{10}{|c|}{ 1.14.3 Long-term FU (12 months and above) } \\
\hline Hegarty 2019 & 17.1 & 20.5 & 178 & 17 & 19.5 & 155 & $39.7 \%$ & $0.00[-0.21,0.22]$ & 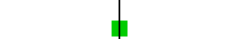 \\
\hline Ferrari 2018 & 16.5 & 28.9 & 81 & 21.9 & 29.7 & 82 & $19.5 \%$ & $-0.18[-0.49,0.12]$ & +7 \\
\hline Rhodes 2015 & 5.6 & 7.156 & 165 & 6.2 & 9.7582 & 165 & $39.5 \%$ & $-0.07[-0.29,0.15]$ & 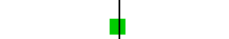 \\
\hline Tirado-Muñoz 2015 & 11.6 & 1.67 & 5 & 12.8 & 4.76 & 6 & $1.3 \%$ & $-0.30[-1.49,0.90]$ & - \\
\hline Subtotal (95\% CI) & & & 429 & & & 408 & $100.0 \%$ & $-0.07[-0.20,0.07]$ & 1 \\
\hline \multirow{2}{*}{\multicolumn{10}{|c|}{$\begin{array}{l}\text { Heterogeneity: } \mathrm{Tau}^{2}=0.00 ; \mathrm{Chi}^{2}=1.12, \mathrm{df}=3(\mathrm{P}=0.77) ; \mathrm{I}^{2}=0 \% \\
\text { Test for overall effect: } \mathrm{Z}=0.94(\mathrm{P}=0.35)\end{array}$}} \\
\hline & & & & & & & & & \\
\hline \multicolumn{9}{|c|}{ Test for subgroup differences: $\mathrm{Chi}^{2}=0.75, \mathrm{df}=2(\mathrm{P}=0.69), \mathrm{I}^{2}=0 \%$} & $\begin{array}{cc}1 & 1 \\
-4 & -2\end{array}$ \\
\hline
\end{tabular}


Analysis 1.15. Comparison 1: Intervention vs Control, Outcome 15: Re-exposure to IPV (categorical)

\begin{tabular}{|c|c|c|c|c|c|c|c|}
\hline \multirow[b]{2}{*}{ Study or Subgroup } & \multicolumn{2}{|c|}{ Intervention } & \multicolumn{2}{|c|}{ Control } & \multicolumn{2}{|r|}{ Odds Ratio } & Odds Ratio \\
\hline & Events & Total & Events & Total & Weight & IV, Random, $95 \%$ CI & IV, Random, $95 \%$ CI \\
\hline
\end{tabular}

1.15.1 Short-term FU (under 6 months)

$\begin{array}{lrrrrrr}\text { Gilbert 2006 } & 2 & 16 & 7 & 18 & 11.8 \% & 0.22[0.04,1.30] \\ \text { Kiely 2010 } & 17 & 134 & 29 & 137 & 27.0 \% & 0.54[0.28,1.04] \\ \text { Hirani 2010 } & 4 & 7 & 5 & 8 & 9.4 \% & 0.80[0.10,6.35] \\ \text { Taghizadeh 2018 } & 84 & 125 & 121 & 132 & 25.8 \% & 0.19[0.09,0.38] \\ \text { Saggurti 2014 } & 20 & 118 & 17 & 102 & 26.0 \% & 1.02[0.50,2.07] \\ \text { Subtotal (95\% CI) } & & \mathbf{4 0 0} & & \mathbf{3 9 7} & \mathbf{1 0 0 . 0 \%} & \mathbf{0 . 4 5}[\mathbf{0 . 2 1}, \mathbf{0 . 9 6}]\end{array}$

Total events: $\quad 127 \quad 179$

Heterogeneity: $\mathrm{Tau}^{2}=0.43 ; \mathrm{Chi}^{2}=12.03, \mathrm{df}=4(\mathrm{P}=0.02) ; \mathrm{I}^{2}=67 \%$

Test for overall effect: $\mathrm{Z}=2.07(\mathrm{P}=0.04)$

1.15.2 Medium-term FU (6 to under 12 months)

$\begin{array}{lllllll}\text { Gilbert } 2016 & 14 & 94 & 11 & 92 & 100.0 \% & 1.29[0.55,3.01] \\ \text { Subtotal }(\mathbf{9 5 \%} \text { CI) } & & \mathbf{9 4} & & \mathbf{9 2} & \mathbf{1 0 0 . 0 \%} & \mathbf{1 . 2 9}[\mathbf{0 . 5 5}, \mathbf{3 . 0 1}]\end{array}$

Total events:

14

Heterogeneity: Not applicable

Test for overall effect: $\mathrm{Z}=0.59(\mathrm{P}=0.56)$

1.15.3 Long-term FU (12 months and above)

Hegarty $2013 \quad 33 \quad 96$

Gilbert 2016

Subtotal (95\% CI)

$8 \quad 91$

96
91
187

Total events:

Heterogeneity: $\mathrm{Tau}^{2}=0.38 ; \mathrm{Chi}^{2}=3.50, \mathrm{df}=1(\mathrm{P}=0.06) ; \mathrm{I}^{2}=71 \%$

Test for overall effect: $Z=0.50(P=0.61)$

Test for subgroup differences: $\mathrm{Chi}^{2}=3.29, \mathrm{df}=2(\mathrm{P}=0.19), \mathrm{I}^{2}=39.3 \%$

$0 \mathrm{~m}, 95 \% \mathrm{CI}$

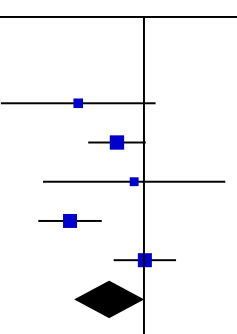

\section{Analysis 1.16. Comparison 1: Intervention vs Control, Outcome 16: Safety planning and/or safety behaviour (continuous)}

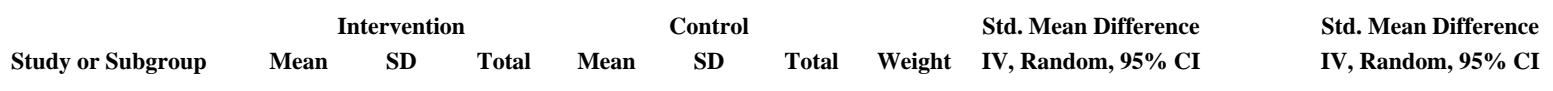

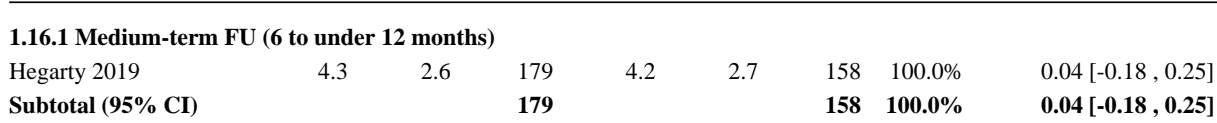

Heterogeneity: Not applicable

Test for overall effect: $\mathrm{Z}=0.35(\mathrm{P}=0.73)$

1.16.2 Long-term FU (12 months and above)

$\begin{array}{lllllllll}\text { Hegarty } 2019 & 4.2 & 2.8 & 171 & 4.2 & 2.6 & 147 & 100.0 \% & 0.00[-0.22,0.22] \\ \text { Subtotal }(\mathbf{9 5 \%} \text { CI) } & & & \mathbf{1 7 1} & & & \mathbf{1 4 7} & \mathbf{1 0 0 . 0 \%} & \mathbf{0 . 0 0}[-\mathbf{- 0 . 2 2}, \mathbf{0 . 2 2}]\end{array}$

Heterogeneity: Not applicable

Test for overall effect: $\mathrm{Z}=0.00(\mathrm{P}=1.00)$ 


\section{Analysis 1.17. Comparison 1: Intervention vs Control, Outcome} 17: Safety planning and/or safety behaviour (categorical)

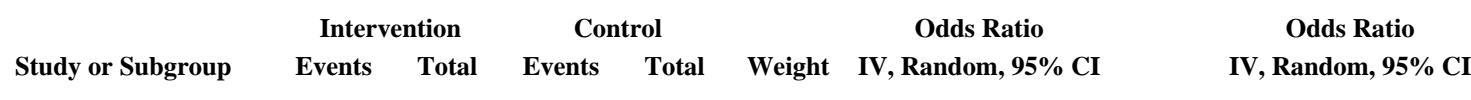

1.17.1 Short-term FU (under 6 months)

$\begin{array}{lcccccc}\text { Saftlas } 2014 & 52 & 70 & 46 & 68 & 100.0 \% & 1.38[0.66,2.89] \\ \text { Subtotal (95\% CI) } & & \mathbf{7 0} & & \mathbf{6 8} & \mathbf{1 0 0 . 0 \%} & \mathbf{1 . 3 8}[\mathbf{0 . 6 6}, \mathbf{2 . 8 9}] \\ \text { Total events: } & 52 & & 46 & & & \end{array}$

Heterogeneity: Not applicable

Test for overall effect: $\mathrm{Z}=0.86(\mathrm{P}=0.39)$

1.17.2 Medium-term FU ( 6 to under 12 months)

$\begin{array}{lcccccc}\text { Hegarty } 2013 & 34 & 93 & 31 & 98 & 100.0 \% & 1.25[0.68,2.27] \\ \text { Subtotal (95\% CI) } & & \mathbf{9 3} & & \mathbf{9 8} & \mathbf{1 0 0 . 0 \%} & \mathbf{1 . 2 5}[\mathbf{0 . 6 8 , 2 . 2 7}] \\ \text { Total events: } & 34 & & 31 & & & \end{array}$

Total events:

Heterogeneity: Not applicable

Test for overall effect: $\mathrm{Z}=0.72(\mathrm{P}=0.47)$

1.17.3 Long-term FU (12 months and above)

Hegarty $2013 \quad 43 \quad 95$

Subtotal (95\% CI)

Total events:

Heterogeneity: Not applicable

Test for overall effect: $\mathrm{Z}=2.49(\mathrm{P}=0.01)$

Test for subgroup differences: $\mathrm{Chi}^{2}=1.72, \mathrm{df}=2(\mathrm{P}=0.42), \mathrm{I}^{2}=0 \%$

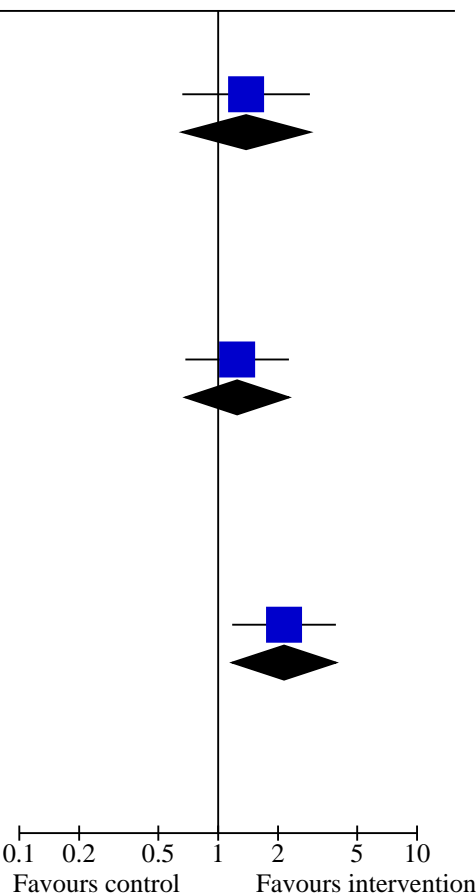


Analysis 1.18. Comparison 1: Intervention vs Control, Outcome 18: Use of healthcare and IPV services

\begin{tabular}{cccccccc} 
& \multicolumn{2}{c}{ Intervention } & \multicolumn{2}{c}{ Control } & \multicolumn{2}{c}{ Odds Ratio } & Odds Ratio \\
Study or Subgroup & Events & Total & Events & Total & Weight & IV, Random, 95\% CI & IV, Random, 95\% CI
\end{tabular}

1.18.1 Short-term FU (under 6 months)

Rhodes 2015

Subtotal (95\% CI)

$\begin{array}{lllll}9 & 179 & 100.0 \% & 1.60[0.68,3.76]\end{array}$

15

$179 \quad 100.0 \%$

$1.60[0.68,3.76]$

Heterogeneity: Not applicable

Test for overall effect: $\mathrm{Z}=1.08(\mathrm{P}=0.28)$

1.18.2 Medium-term FU (6 to under 12 months)

\begin{tabular}{|c|c|c|c|c|c|c|}
\hline Rhodes 2015 & 9 & 188 & 7 & 176 & $100.0 \%$ & $1.21[0.44,3.33]$ \\
\hline Subtotal $(95 \%$ CI) & & 188 & & 176 & $100.0 \%$ & $1.21[0.44,3.33]$ \\
\hline Total events: & 9 & & 7 & & & \\
\hline
\end{tabular}

Heterogeneity: Not applicable

Test for overall effect: $\mathrm{Z}=0.38(\mathrm{P}=0.71)$

1.18.3 Long-term FU (12 months and above)

Hegarty $2013 \quad 11 \quad 96$

Rhodes $2015 \quad 4 \quad 165$

Subtotal (95\% CI) $\quad 261$

Total events:

15

Heterogeneity: $\mathrm{Tau}^{2}=0.61 ; \mathrm{Chi}^{2}=3.04, \mathrm{df}=1(\mathrm{P}=0.08) ; \mathrm{I}^{2}=67 \%$

Test for overall effect: $\mathrm{Z}=0.34(\mathrm{P}=0.73)$

Test for subgroup differences: $\mathrm{Chi}^{2}=0.78, \mathrm{df}=2(\mathrm{P}=0.68), \mathrm{I}^{2}=0 \%$

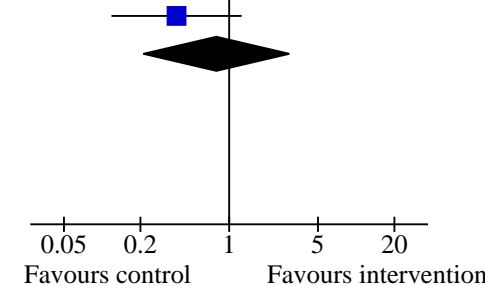

Analysis 1.19. Comparison 1: Intervention vs Control, Outcome 19: Social support (continuous)

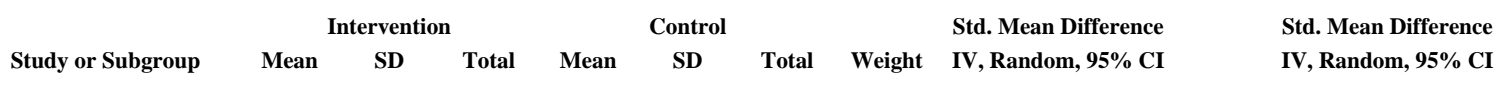

1.19.1 Short-term FU (under 6 months)

$\begin{array}{lrrrrrrrr}\text { Stevens 2015 } & 16 & 3.4 & 98 & 16 & 3.2 & 95 & 56.7 \% & 0.00[-0.28,0.28] \\ \text { Johnson 2011 } & 102.16 & 31.94 & 19 & 80.39 & 29.22 & 33 & 43.3 \% & 0.71[0.13,1.29] \\ \text { Subtotal (95\% CI) } & & & \mathbf{1 1 7} & & & \mathbf{1 2 8} & \mathbf{1 0 0 . 0 \%} & \mathbf{0 . 3 1}[-\mathbf{- 0 . 3 8 , \mathbf { 1 . 0 0 }}]\end{array}$

Heterogeneity: $\mathrm{Tau}^{2}=0.20 ; \mathrm{Chi}^{2}=4.62, \mathrm{df}=1(\mathrm{P}=0.03) ; \mathrm{I}^{2}=78 \%$

Test for overall effect: $\mathrm{Z}=0.87(\mathrm{P}=0.38)$

$\begin{array}{llrrrrrrr}\text { 1.19.2 Medium-term FU (6 to under } & \mathbf{1 2} \text { months) } \\ \text { Johnson 2011 } & 79.16 & 35.07 & 19 & 79.16 & 35.07 & 33 & 20.9 \% & 0.00[-0.56,0.56] \\ \text { Stevens 2015 } & 16.5 & 3.1 & 90 & 16.3 & 2.9 & 93 & 79.1 \% & 0.07[-0.22,0.36] \\ \text { Subtotal (95\% CI) } & & & \mathbf{1 0 9} & & & \mathbf{1 2 6} & \mathbf{1 0 0 . 0 \%} & \mathbf{0 . 0 5}[-\mathbf{0 . 2 1}, \mathbf{0 . 3 1}]\end{array}$

Heterogeneity: $\mathrm{Tau}^{2}=0.00 ; \mathrm{Chi}^{2}=0.04, \mathrm{df}=1(\mathrm{P}=0.84) ; \mathrm{I}^{2}=0 \%$

Test for overall effect: $\mathrm{Z}=0.40(\mathrm{P}=0.69)$

Test for subgroup differences: $\mathrm{Chi}^{2}=0.46, \mathrm{df}=1(\mathrm{P}=0.50), \mathrm{I}^{2}=0 \%$

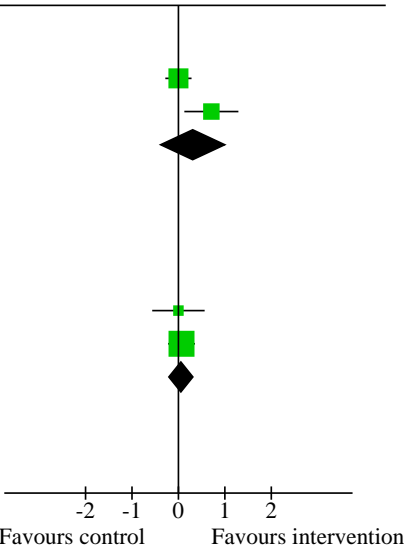


Analysis 1.20. Comparison 1: Intervention vs Control, Outcome 20: Social support (categorical)

\begin{tabular}{|c|c|c|c|c|c|c|}
\hline \multirow[b]{2}{*}{ Study or Subgroup } & \multicolumn{2}{|c|}{ Intervention } & \multicolumn{2}{|c|}{ Control } & Odds Ratio & Odds Ratio \\
\hline & Events & Total & Events & Total & IV, Random, 95\% CI & IV, Random, 95\% Cl \\
\hline
\end{tabular}

1.20.1 Short-term FU (under 6 months)

$\begin{array}{llllll}\text { Rhodes } 2015 & 170 & 192 & 154 & 179 & 1.25[0.68,2.32]\end{array}$

1.20.2 Medium-term FU ( 6 to under 12 months)

$\left.\begin{array}{llllll}\text { Rhodes } 2015 & 165 & 188 & 150 & 176 & 1.24[0.68,2.27\end{array}\right]$

1.20.3 Long-term FU (12 months and above)

Rhodes $2015 \quad 146 \quad 165$

$140 \quad 165 \quad 1.37[0.72,2.60]$

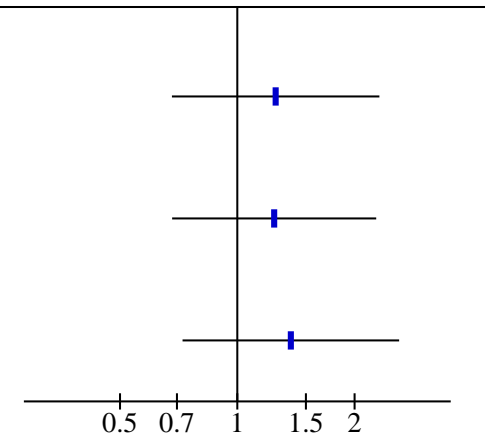

Favours control Favours intervention

\section{APPEN DICES}

\section{Appendix 1. CCMDCTR search}

\section{The Cochrane Common Mental Disorders' Controlled Trials Register (CCMDCTR)}

The Cochrane Common Mental Disorders Group (CCMD) maintains an archived clinical trials registers at their editorial base in York, UK. (cmd.cochrane.org/specialised-register). The CCMDCTR-References Register contains over 40,000 reports of RCTS for the treatment/ prevention of common mental disorders (depression, anxiety, eating disorders, self-harm). Approximately $50 \%$ of these reports have been tagged to individual, coded trials. The coded trials are held in the CCMDCTR-Studies Register and records are linked between the two registers through the use of unique trial ID tags. Coding of trials is based on the EU-Psi coding manual.

Reports of trials for inclusion in the Group's registers were collated from routine, generic searches of MEDLINE (1950-), Embase (1974-) and PsycINFO (1967-); quarterly searches of the Cochrane Central Register of Controlled Trials (CENTRAL) and review-specific searches of additional databases, to June 2016. Reports of trials were also sourced from international trials registers from the WHO's trials portal (ICTRP), ClinicalTrials.gov, drug companies, the handsearching of key journals, conference proceedings and other (non-Cochrane) systematic reviews and meta-analyses.

Details of CCMD's core search strategies (used to identify RCTs and inform the Group's specialised register) can be found on the Group's website with an example of the core MEDLINE search displayed below.

The CCMDCTR was searched (for this review) using the following terms to June 2016.

1. CCMDCTR-Studies Register: was searched using controlled vocabulary terms, Comorbid healthcare condition $=$ (domestic violence or rape or sexual abuse or spousal abuse)

2. CCMDCTR-References Register: was searched using a more sensitive set of free-text terms to identify additional untagged/uncoded reports of RCTs. Free-text $=\left(\left(\left(\right.\right.\right.$ home $^{*}$ or domestic ${ }^{*}$ or spous or partner $^{*}$ or women or woman or mother $\left.{ }^{*}\right)$ NEAR (abus ${ }^{*}$ or violen $\left.\left.{ }^{\star}\right)\right)$ or "battered women" or (violen* NEAR (date or dating)) or rape or ((sex* NEAR abuse*) not child*:ti

CCMD's core search strategy used to inform the Group's specialised register: OVID Medline A search alert based on condition + RCT filter only

1. [MeSH Headings]: eating disorders/ or anorexia nervosa/ or binge-eating disorder/ or bulimia nervosa/ or female athlete triad syndrome/ or pica/ or hyperphagia/ or bulimia/ or self-injurious behavior/ or self mutilation/ or suicide/ or suicidal ideation/ or suicide, attempted/ or mood disorders/ or affective disorders, psychotic/ or bipolar disorder/ or cyclothymic disorder/ or depressive disorder/ or depression, postpartum/ or depressive disorder, major/ or depressive disorder, treatment-resistant/ or dysthymic disorder/ or seasonal affective disorder/ or neurotic disorders/ or depression/ or adjustment disorders/ or exp antidepressive agents/ or anxiety disorders/ or agoraphobia/ or neurocirculatory asthenia/ or obsessive-compulsive disorder/ or obsessive hoarding/ or panic disorder/ or phobic disorders/ or stress disorders, traumatic/ or combat disorders/ or stress disorders, post-traumatic/ or stress disorders, traumatic, acute/ or anxiety/ or anxiety, castration/ or koro/ or anxiety, separation/ or panic/ or exp anti-anxiety agents/or somatoform disorders/ or body dysmorphic disorders/ or conversion disorder/ or hypochondriasis/ or neurasthenia/ or hysteria/ or munchausen syndrome by proxy/ or munchausen syndrome/ or fatigue syndrome, chronic/ or obsessive behavior/ or compulsive behavior/ or behavior, addictive/ or impulse 
control disorders/ or firesetting behavior/ or gambling/ or trichotillomania/ or stress, psychological/ or burnout, professional/ or sexual dysfunctions, psychological/ or vaginismus/ or Anhedonia/ or Affective Symptoms/ or *Mental Disorders/

2. [Title/ Author Keywords]: (eating disorder* or anorexia nervosa or bulimi* or binge eat ${ }^{\star}$ or (self adj (injur ${ }^{\star}$ or mutilat $^{\star}$ )) or suicide* or suicidal or parasuicid* or mood disorder* or affective disorder* or bipolar i or bipolar ii or (bipolar and (affective or disorder $\left.{ }^{\star}\right)$ ) or mania or manic or cyclothymic* or depression or depressive or dysthymi* or neurotic or neurosis or adjustment disorder* or antidepress* or anxiety disorder ${ }^{\star}$ or agoraphobia or obsess ${ }^{\star}$ or compulsi ${ }^{\star}$ or panic or phobi* or ptsd or posttrauma* or post trauma* or combat or somatoform or somati\#ation or medical ${ }^{\star}$ unexplained or body dysmorphi* or conversion disorder or hypochondria* or neurastheni* or hysteria or munchausen or chronic fatigue* or gambling or trichotillomania or vaginismus or anhedoni* or affective symptoms or mental disorder ${ }^{\star}$ or mental health).ti,kf.

3. [RCT filter]: (controlled clinical trial.pt. or randomised controlled trial.pt. or (randomi\#ed or randomi\#ation).ab,ti. or randomly.ab. or (random ${ }^{\star}$ adj3 (administ* or allocat $^{\star}$ or assign ${ }^{\star}$ or class $^{\star}$ or control ${ }^{\star}$ or determine or divide $^{\star}$ or distribut ${ }^{\star}$ or expose* or fashion or number ${ }^{\star}$ or place ${ }^{\star}$ or recruit* or subsitut ${ }^{\star}$ or treat $\left.{ }^{\star}\right)$ ).ab. or placebo* .ab,ti. or drug therapy.fs. or trial.ab,ti. or groups.ab. or (control* adj3 (trial ${ }^{\star}$ or trial or studies)).ab,ti. or ((singl ${ }^{\star}$ or doubl* or tripl* or trebl*) adj3 (blind ${ }^{\star}$ or mask ${ }^{\star}$ or dummy $\left.{ }^{\star}\right)$ ).mp. or clinical trial, phase ii/ or clinical trial, phase iii/ or clinical trial, phase iv/ or randomised controlled trial/ or pragmatic clinical trial/ or (quasi adj (experimental or random)).ti,ab. or ((waitlist* or wait* list* or treatment as usual or TAU) adj3 (control or group)).ab.)

4. (1 and 2 and 3)

Records are screened for reports of RCTs within the scope of the Cochrane Common Mental Disorders Group. Secondary reports of RCTs are tagged to the appropriate trial record.

Similar search alerts are also conducted on OVID EMBASE and PsycINFO, using relevant subject headings (controlled vocabularies) and search syntax, appropriate to each resource.

\section{Appendix 2. Other database search strategies (Search-1)}

Database: Cochrane Central Register of Controlled Trials (CENTRAL) and Database of Abstracts of Reviews for Effectiveness (DARE) Host: Wiley

Data Parameters: CENTRAL Issue 5 of 12, May 2018 and DARE (archived) Issue 2 of 4, April 2015

Date searched: Thursday June 28th 2018

\#1 MeSH descriptor: [Battered Women] this term only

\#2 MeSH descriptor: [Domestic Violence] this term only

\#3 MeSH descriptor: [Spouse Abuse] this term only

\#4 (abuse* near/3 wom?n)

\#5 (abuse* near/3 spous ${ }^{\star}$ )

\#6 (abuse ${ }^{\star}$ near/3 partner ${ }^{\star}$ )

\#7 ((wife or wives) near/3 abuse ${ }^{\star}$ )

\#8 ((wife or wives or wom?n) near/3 batter $\left.{ }^{\star}\right)$

\#9 (partner* near/3 violen ${ }^{\star}$ )

$\# 10$ (spous ${ }^{\star}$ near/3 violen ${ }^{\star}$ )

\#11 ((domestic ${ }^{\star}$ or home $\left.{ }^{\star}\right)$ near/3 violen $\left.{ }^{\star}\right)$

\#12 ((domestic ${ }^{\star}$ or home $\left.{ }^{\star}\right)$ near/3 abuse*)

$\# 13$ (violen ${ }^{\star}$ near/3 (date or dating))

\#14 (date near/3 rape)

\#15 (dating near/3 violen*)

\#16 (acquaintance near/3 (rape or violen $\left.{ }^{\star}\right)$ )

\#17 ((relation ${ }^{\star}$ or interperson $\left.{ }^{\star}\right)$ near/3 (abuse* or violen $\left.\left.^{\star}\right)\right)$

\#18 stalk*

\#19 ((domestic or marital or partner ${ }^{\star}$ or spous $\left.{ }^{\star}\right)$ near/3 (rape or $\left(\right.$ sex $^{\star}$ near/1 (abuse or assault $\left.\left.\left.\left.^{\star}\right)\right)\right)\right)$

\#20 ((abuse* or violen $\left.{ }^{\star}\right)$ near/3 (marital or marriage))

$\# 21$ (\#1 or \#2 or \#3 or \#4 or \#5 or \#6 or \#7 or \#8 or \#9 or \#10 or \#11 or \#12 or \#13 or \#14 or \#15 or \#16 or \#17 or \#18 or \#19 or \#20)

\#22 MeSH descriptor: [Psychotherapy] this term only

\#23 MeSH descriptor: [Psychotherapy, Group] this term only

\#24 (psychotherapy or (psychological near/1 (therap* or intervention)))

\#25 MeSH descriptor: [Cognitive Therapy] this term only

\#26 ((cogniti* near/3 behavio*) or CBT)

$\# 27$ (cognitive resructur* or cognitive processing)

\#28 (metacognitive or meta-cognitive)

\#29 CBASP

\#30 MeSH descriptor: [Behavior Therapy] this term only

Psychological therapies for women who experience intimate partner violence (Review) 
\#31 (behavio* near/3 (therap* or psychotherap* or intervention* or program* or training))

\#32 behavio* activat*

\#33 (psychobehavio* or psycho-behavio*)

\#34 MeSH descriptor: [Counseling] this term only

\#35 counsel* $^{*}$

\#36 MeSH descriptor: [Motivational Interviewing] this term only

\#37 MeSH descriptor: [Motivation] this term only

\#38 (motivational near/3 (interview* or intervention* or therap* or psychotherap* or program* or training))

\#39 MeSH descriptor: [Person-Centered Therapy] this term only

\#40 (non-directive or nondirective)

\#41 (analytic and (cogniti* or therap* or psychotherapy*))

\#42 (acceptance near/2 commitment)

\#43 compass $^{*}$ foc $^{*}$

\#44 (dialectic* or DBT)

\#45 diffusion

\#46 mind train*

\#47 (mindful* or meditation or relaxation)

\#48 problem sol*

\#49 rational emotive

\#50 (reality near/1 (therap* or psychotherap*))

$\# 51$ (\#22 or \#23 or \#24 or \#25 or \#26 or \#27 or \#28 or \#29 or \#30 or \#31 or \#32 or \#33 or \#34 or \#35 or \#36 or \#37 or \#38 or \#39 or \#40 or \#41

or \#42 or \#43 or \#44 or \#45 or \#46 or \#47 or \#48 or \#49 or \#50)

\#52 (\#21 and \#51)

Database: Ovid MEDLINE(R) Epub Ahead of Print, In-Process \& Other Non-Indexed Citations, Ovid MEDLINE(R) Daily and Ovid MEDLINE(R)

Host: Ovid

Data Parameters: 1946 to Present

Date searched: Thursday June 28th 2018

Search Strategy:

1 BATTERED WOMEN/

2 DOMESTIC VIOLENCE/

3 SPOUSE ABUSE/

4 (abuse $\$$ adj3 wom\#n).tw.

5 (abuse $\$$ adj3 spous\$).tw.

6 (abuse adj3 partner\$).tw.

7 ((wife or wives) adj3 abuse\$).tw.

8 ((wife or wives or wom\#n) adj3 batter\$).tw.

9 (partner\$ adj3 violen\$).tw.

10 (spous $\$$ adj3 violen\$).tw.

11 ((domestic ${ }^{\star}$ or home $\left.{ }^{\star}\right)$ adj3 violen $\left.{ }^{\star}\right)$.tw.

12 ((domestic ${ }^{\star}$ or home ${ }^{\star}$ ) adj3 abuse*).tw.

13 (violen* adj3 (date or dating)).tw.

14 (date adj3 rape).tw.

15 (dating adj3 violen\$).tw.

16 (acquaintance adj3 (rape or violen\$)).tw.

17 ((relation* or interperson $\left.{ }^{\star}\right)$ adj3 (abuse\$ or violen\$)).tw.

18 stalk*.tw.

$19\left(\left(\right.\right.$ domestic or marital or partner ${ }^{\star} 1$ or spous $\left.{ }^{\star}\right) \operatorname{adj} 3\left(\right.$ rape or $\left(\right.$ sex $^{\star} \operatorname{adj} 1\left(\right.$ abuse $^{\star}$ or assault $\left.\left.\left.\left.^{\star}\right)\right)\right)\right) . m p$.

20 ((abuse or violen $\left.^{\star}\right)$ adj3 (marital or marriage)).tw.

21 ( 1 or 2 or 3 or 4 or 5 or 6 or 7 or 8 or 9 or 10 or 11 or 12 or 13 or 14 or 15 or 16 or 17 or 18 or 19 or 20 )

22 PSYCHOTHERAPY/ Or PSYCHOTHERAPY, GROUP/

23 (psychotherapy or (psychological adj (therap* or intervention))).tw.

24 COGNITIVE THERAPY/

25 ((cogniti\$ adj3 behavio\$) or CBT).tw.

26 (cognitive resructur ${ }^{\star}$ or cognitive processing).tw.

27 (metacognitive or meta-cognitive).tw.

28 CBASP.tw.

29 BEHAVIOR THERAPY/

30 (behavio* adj3 (therap* or psychotherap* or intervention* or program* or training)).tw.

31 behavio* activat*.tw.

32 (psychobehavio* or psycho-behavio*).tw.

33 COUNSELING/

34 counsel $^{*}$.tw.

Psychological therapies for women who experience intimate partner violence (Review)

Copyright @ 2020 The Cochrane Collaboration. Published by John Wiley \& Sons, Ltd. 
35 MOTIVATIONAL INTERVIEWING/

36 MOTIVATION/

37 (motivational adj3 (interview* or intervention* or therap* or psychotherap* or program or training)).tw.

38 NONDIRECTIVE THERAPY/

39 (non-directive or nondirective).tw.

40 (analytic and (cogniti* or therap* or psychotherapy*)).tw.

41 (acceptance adj2 commitment).tw.

42 compass $^{\star}$ foc ${ }^{\star}$.tw.

43 (dialectic ${ }^{\star}$ or DBT).tw.

44 diffusion.tw.

45 mind train*.tw.

46 (mindful* ${ }^{\star}$ or meditation or relaxation).tw.

47 problem sol $^{\star}$.tw.

48 rational emotive.tw.

49 (reality adj (therap* or psychotherap $\left.\left.{ }^{\star}\right)\right) . t w$.

50 BATTERED WOMEN/th or DOMESTIC VIOLENCE/pc or VIOLENCE/pc or SPOUSE ABUSE/th, pc

51 or $/ 22-50$

52 randomized controlled trial.pt.

53 controlled clinical trial.pt.

54 randomi\#ed.ti,ab.

55 randomly.ab.

56 placebo.ab.

57 trial.ti,ab.

58 groups.ab.

59 (control\$ adj3 (trial or trial)).ab,ti.

60 ((singl\$ or doubl\$ or tripl\$ or trebl\$) adj3 (blind\$ or mask\$ or dummy)).mp.

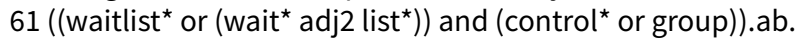

62 ((usual care or care as usual) and (control* or group)).ab.

63 (treatment as usual or TAU).ab.

64 or/52-63

65 ( 21 and 51 and 64)

Database: Embase

Host: Ovid

Data Parameters: 1974 to 2018 June 27

Date searched: Thursday June 28th 2018

Search Strategy:

1 BATTERED WOMEN/

2 DOMESTIC VIOLENCE/

3 partner violence/

4 (abuse adj3 wom\#n).tw.

5 (abuse $\$$ adj3 spous\$).tw.

6 (abuse $\$$ adj3 partner\$).tw.

7 ((wife or wives) adj3 abuse\$).tw.

8 ((wife or wives or wom\#n) adj3 batter\$).tw.

9 (partner\$ adj3 violen\$).tw.

10 (spous\$ adj3 violen\$).tw.

11 ((domestic ${ }^{\star}$ or home $\left.{ }^{\star}\right)$ adj3 violen $\left.{ }^{\star}\right)$.tw.

12 ((domestic ${ }^{\star}$ or home $\left.{ }^{\star}\right)$ adj3 abuse $\left.{ }^{\star}\right)$.tw.

13 (violen* adj3 (date or dating)).tw.

14 (date adj3 rape).tw.

15 (dating adj3 violen\$).tw.

16 (acquaintance adj3 (rape or violen\$)).tw.

17 ((relation* or interperson $\left.{ }^{\star}\right)$ adj3 (abuse\$ or violen\$)).tw.

18 stalk*.tw.

19 ((domestic or marital or partner ${ }^{\star} 1$ or spous $\left.{ }^{\star}\right) \operatorname{adj} 3\left(\right.$ rape or $\left(\right.$ sex $^{\star} \operatorname{adj1}\left(\right.$ abuse $^{\star}$ or assault $\left.\left.\left.\left.{ }^{\star}\right)\right)\right)\right)$.mp.

20 ((abuse ${ }^{\star}$ or violen $\left.{ }^{\star}\right)$ adj3 (marital or marriage)).tw.

21 or/1-20

22 PSYCHOTHERAPY/ or group therapy/

23 (psychotherapy or (psychological adj (therap* or intervention))).tw.

24 COGNITIVE THERAPY/

25 ((cogniti\$ adj3 behavio\$) or CBT).tw.

26 (cognitive resructur* or cognitive processing).tw.

Psychological therapies for women who experience intimate partner violence (Review) 
27 (metacognitive or meta-cognitive).tw.

\section{CBASP.tw.}

29 behavior therapy/

30 (behavio* adj3 (therap* or psychotherap* or intervention* or program* or training)).tw.

31 behavio* activat*.tw.

32 (psychobehavio* or psycho-behavio*).tw.

33 counseling/

34 counsel $^{\star}$.tw.

35 motivational interviewing/

36 motivation/

37 (motivational adj3 (interview* or intervention ${ }^{\star}$ or therap ${ }^{\star}$ or psychotherap ${ }^{\star}$ or program ${ }^{\star}$ or training)).tw.

38 client centered therapy/

39 (non-directive or nondirective).tw.

40 (analytic and (cogniti* or therap* or psychotherapy*)).tw.

41 (acceptance adj2 commitment).tw.

42 compass $^{\star}$ foc ${ }^{\star}$. tw.

43 (dialectic* or DBT).tw.

44 diffusion.tw.

45 mind train*.tw.

46 (mindful* ${ }^{\star}$ or meditation or relaxation).tw.

47 problem sol $^{*}$.tw.

48 rational emotive.tw.

49 (reality adj (therap* or psychotherap*)).tw.

50 BATTERED WOMEN/th or DOMESTIC VIOLENCE/pc or VIOLENCE/pc or partner violence/th, pc

51 or/22-50

52 random $\$ . t i, a b$.

53 placebo.ab.

54 trial.ti,ab.

55 groups.ab.

56 (control\$ adj3 (trial or trial)).ab,ti.

57 ((singl\$ or doubl\$ or tripl\$ or trebl\$) adj3 (blind\$ or mask\$ or dummy)).mp.

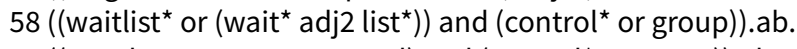

59 ((usual care or care as usual) and (control ${ }^{\star}$ or group)).ab.

60 (treatment as usual or TAU).ab.

61 or/52-60

62 (21 and 51 and 61)

Database: PsycINFO

Host: Ovid

Data Parameters: 1806 to June Week 32018

Date searched: Thursday June 28th 2018

Search Strategy:

1 Battered Females/

2 DOMESTIC VIOLENCE/

3 Partner Abuse/

4 (abuse adj3 wom\#n).tw.

5 (abuse $\$$ adj3 spous\$).tw.

6 (abuse adj3 partner\$).tw.

7 ((wife or wives) adj3 abuse\$).tw.

8 ((wife or wives or wom\#n) adj3 batter\$).tw.

9 (partner\$ adj3 violen\$).tw.

10 (spous\$ adj3 violen\$).tw.

11 ((domestic $^{\star}$ or home ${ }^{\star}$ ) adj3 violen $\left.{ }^{\star}\right)$.tw.

12 ((domestic ${ }^{\star}$ or home*) adj3 abuse*).tw.

13 (violen* adj3 (date or dating)).tw.

14 (date adj3 rape).tw.

15 (dating adj3 violen\$).tw.

16 (acquaintance adj3 (rape or violen\$)).tw.

17 ((relation* or interperson ${ }^{\star}$ ) adj3 (abuse\$ or violen\$)).tw.

18 stalk*.tw. $^{\star}$

19 ((domestic or marital or partner ${ }^{\star} 1$ or spous $\left.{ }^{\star}\right)$ adj3 (rape or (sex $\operatorname{adj}^{\star}\left(\right.$ abuse $^{\star}$ or assault $\left.\left.\left.\left.{ }^{\star}\right)\right)\right)\right) . m p$.

20 ((abuse* or violen $\left.{ }^{\star}\right)$ adj3 (marital or marriage)).tw.

21 or/1-20

Psychological therapies for women who experience intimate partner violence (Review) 
22 PSYCHOTHERAPY/ or Group Psychotherapy/

23 (psychotherapy or (psychological adj (therap* or intervention))).tw.

24 Cognitive Therapy/

25 ((cogniti\$ adj3 behavio\$) or CBT).tw.

26 (cognitive resructur ${ }^{\star}$ or cognitive processing).tw.

27 (metacognitive or meta-cognitive).tw.

28 CBASP.tw.

29 Behavior Therapy/

30 (behavio* adj3 (therap* or psychotherap* or intervention* or program* or training)).tw.

31 behavio* activat* ${ }^{\star}$.tw.

32 (psychobehavio* or psycho-behavio*).tw.

33 COUNSELING/

34 counsel $^{*}$.tw.

35 Motivational Interviewing/

36 MOTIVATION/

37 (motivational adj3 (interview* or intervention ${ }^{\star}$ or therap* or psychotherap ${ }^{\star}$ or program ${ }^{\star}$ or training)).tw.

38 Client Centered Therapy/

39 (non-directive or nondirective).tw.

40 (analytic and (cogniti* or therap* or psychotherapy*)).tw.

41 (acceptance adj2 commitment).tw.

42 compass $^{\star}$ foc $^{\star}$.tw.

43 (dialectic ${ }^{\star}$ or DBT).tw.

44 diffusion.tw.

45 mind train ${ }^{\star} . t w$.

46 (mindful ${ }^{\star}$ or meditation or relaxation).tw.

47 problem sol $^{\star}$.tw.

48 rational emotive.tw.

49 (reality adj (therap* or psychotherap*)).tw.

50 or/ $22-49$

51 randomi\#ed.ti,ab.

52 randomly.ab.

53 placebo.ab.

54 trial.ti,ab.

55 groups.ab.

56 (control\$ adj3 (trial or trial)).ab,ti.

57 ((singl\$ or doubl\$ or tripl\$ or trebl\$) adj3 (blind\$ or mask\$ or dummy)).ti,ab.

58 ((waitlist ${ }^{\star}$ or (wait* adj2 list $\left.\left.{ }^{\star}\right)\right)$ and (control ${ }^{\star}$ or group)).ab.

59 (("usual care" or "care as usual") and (control* or group)).ab.

60 ("treatment as usual" or TAU).ab.

61 ( 51 or 52 or 53 or 54 or 55 or 56 or 57 or 58 or 59 or 60 )

62 (21 and 50 and 61)

Database: CINAHL Complete

Host: EBSCOhost

Date searched: Thursday June 28th 2018

S63 (S21 AND S49 AND S6)

S62 (S50 OR S51 OR S52 OR S53 OR S54 OR S55 OR S56 OR S57 OR S58 OR S59 OR S60 OR S61)

S61 TI ( (treatment as usual or TAU) ) OR AB ( (treatment as usual or TAU) )

S60 TI ( ((usual care or care as usual) and (control* or group)) ) OR AB ( ((usual care or care as usual) and (control* or group)) )

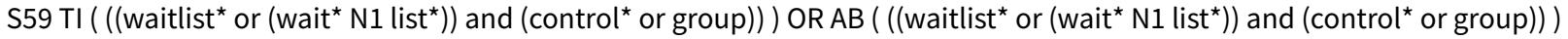

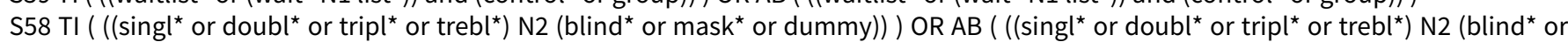
mask $^{\star}$ or dummy)) )

S57 TI ( (control* N2 (trial or trial)) ) OR AB ( (control* N2 (trial or trial)) )

S56 TI groups OR AB groups

S55 TI trial OR AB trial

S54 TI placebo OR AB placebo

S53 TI randomly OR AB randomly

S52 TI ( (randomised or randomized) ) OR AB ( (randomised or randomized) )

S51 (MM "Clinical Trials")

S50 (MM "Randomized Controlled Trials")

S49 (S22 OR S23 OR S24 OR S25 OR S26 OR S27 OR S28 OR S29 OR S30 OR S31 OR S32 OR S33 OR S34 OR S35 OR S36 OR S37 OR S38 OR

S39 OR S40 OR S41 OR S42 OR S43 OR S44 OR S45 OR S46 OR S47 OR S48)

S48 TI ( (reality N1 (therap* or psychotherap*)) ) OR AB ( (reality N1 (therap* or psychotherap*)) )

Psychological therapies for women who experience intimate partner violence (Review) 
S47 TI (rational emotive) OR AB (rational emotive)

S46 TI (problem sol*) OR AB (problem sol*)

S45 TI ( (mindful* or meditation or relaxation) ) OR AB ( (mindful* or meditation or relaxation) )

S44 TI (mind train*) OR AB (mind train*)

S43 TI diffusion OR AB diffusion

S42 TI ( (dialectic* or DBT) ) OR AB ( (dialectic* or DBT) )

S41 TI (compass* foc*) OR AB (compass* foc $\left.{ }^{\star}\right)$

S40 TI (acceptance N1 commitment) OR AB (acceptance N1 commitment)

S39 TI ( (analytic and (cogniti ${ }^{\star}$ or therap* or psychotherapy $\left.\left.{ }^{\star}\right)\right)$ ) OR AB ( (analytic and (cogniti ${ }^{\star}$ or therap* or psychotherapy $\left.\left.{ }^{\star}\right)\right)$ )

S38 TI ( (non-directive or nondirective) ) OR AB ( (non-directive or nondirective) )

S37 TI ( (motivational N2 (interview* or intervention* or therap* or psychotherap* or program* or training)) ) OR AB ( (motivational N2 (interview* ${ }^{\star}$ or intervention* or therap* or psychotherap* or program* or training)) )

S36 (MM "Motivation")

S35 (MM "Motivational Interviewing")

S34 TI counsel* OR AB counsel* $^{*}$

S33 (MM "Counseling")

S32 TI ( (psychobehavio* or psycho-behavio*) ) OR AB ( (psychobehavio* or psycho-behavio*))

S31 TI (behavio* activat*) OR AB (behavio* activat*)

S30 TI ( (behavio* N2 (therap* or psychotherap* or intervention* or program* or training)) ) OR AB ( (behavio* N2 (therap* or psychotherap* or intervention* or program* or training)) )

S29 (MM "Behavior Therapy")

S28 TI CBASP OR AB CBASP

S27 TI ( (metacognitive or meta-cognitive) ) OR AB ( (metacognitive or meta-cognitive) )

S26 TI ( (cognitive resructur* or cognitive processing) ) OR AB ( (cognitive resructur ${ }^{\star}$ or cognitive processing) )

S25 TI ( ((cogniti* N2 behavio*) or CBT) ) OR AB ( ((cogniti* N2 behavio*) or CBT) )

S24 (MM "Cognitive Therapy")

S23 TI ( (psychotherapy or (psychological N1 (therap* or intervention))) ) OR AB ( (psychotherapy or (psychological N1 (therap* or intervention))) )

S22 (MM "Psychotherapy") or (MM "Psychotherapy, Group")

S21 (S1 OR S2 OR S3 OR S4 OR S5 OR S6 OR S7 OR S8 OR S9 OR S10 OR S11 OR S12 OR S13 OR S14 OR S15 OR S16 OR S17 OR S18 OR

S19 OR S20)

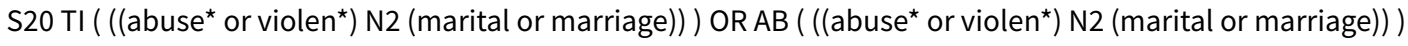

S19 TI ( ((domestic or marital or partner 1 or spous $\left.)^{\star}\right)$ 2 (rape or $\left(\right.$ sex $^{\star}$ N2 (abuse* or assault $\left.\left.\left.\left.{ }^{\star}\right)\right)\right)\right)$ ) OR AB ( ((domestic or marital or partner 1 or spous $\left.{ }^{\star}\right)$ N2 (rape or (sex ${ }^{\star}$ N2 (abuse* or assault $\left.\left.\left.\left.{ }^{\star}\right)\right)\right)\right)$ )

S18 TI stalk* OR AB stalk*

S17 TI ( ((relation* or interperson $\left.{ }^{\star}\right)$ N2 (abuse or violen $\left.\left.^{\star}\right)\right)$ ) OR AB ( ((relation ${ }^{\star}$ or interperson $\left.{ }^{\star}\right)$ N2 (abuse* or violen $\left.\left.{ }^{\star}\right)\right)$ )

S16 TI ( (acquaintance N2 (rape or violen*)) ) OR AB ( (acquaintance N2 (rape or violen $\left.\left.{ }^{\star}\right)\right)$ )

S15 TI (dating N2 violen*) OR AB (dating N2 violen*)

$\mathrm{S} 14 \mathrm{TI}$ (date $\mathrm{N} 2$ rape) OR AB (date N2 rape)

S13 TI ( (violen* N2 (date or dating)) ) OR AB ( (violen* N2 (date or dating)) )

S12 TI ( ((domestic ${ }^{\star}$ or home $\left.{ }^{\star}\right)$ N2 abuse $)$ ) OR AB ( ((domestic* or home $)$ N2 abuse $\left.\left.{ }^{\star}\right)\right)$

S11 TI ( ((domestic* or home*) N2 violen $\left.\left.{ }^{\star}\right)\right)$ OR AB ( ((domestic* or home*) N2 violen $\left.\left.{ }^{\star}\right)\right)$

S10 TI (spous ${ }^{\star}$ N2 violen*) OR AB (spous* N2 violen $\left.{ }^{\star}\right)$

S9 TI (partner* N2 violen*) OR AB (partner* N2 violen ${ }^{\star}$ )

S8 TI ( ((wife or wives or woman or women) N2 batter $\left.{ }^{\star}\right)$ ) OR AB ( ((wife or wives or woman or women) N2 batter $\left.{ }^{\star}\right)$ )

S7 TI ( ((wife or wives) N2 abuse $\left.{ }^{\star}\right)$ ) OR AB ( ((wife or wives) N2 abuse*) )

S6 TI (abuse* N2 partner ${ }^{\star}$ ) OR AB (abuse* N2 partner ${ }^{\star}$ )

S5 TI (abuse* N2 spous ${ }^{\star}$ ) OR AB (abuse* N2 spous ${ }^{\star}$ )

S4 TI ( ((abuse ${ }^{\star}$ 2 woman) or (abuse* N2 women)) ) OR AB ( ((abuse* N2 woman) or (abuse* N2 women)) )

S3 (MM "Intimate Partner Violence")

S2 (MM "Domestic Violence")

S1 (MM "Battered Women")

Database: Web of Science

Host: Clarivate AnalyticsIndexes=SCI-EXPANDED, SSCI, A\&HCI, CPCI-S, CPCI-SSH, ESCI Timespan=All yearsEdit

Date searched: Thursday June 28th 2018

\# 52 (\#51 AND \#41 AND \#19)

\# 51 (\#50 OR \#49 OR \#48 OR \#47 OR \#46 OR \#45 OR \#44 OR \#43 OR \#42)

\# 50 TOPIC: ((treatment as usual or TAU))

\# 49 TOPIC: (((usual care or care as usual) and (control* or group)))

\# 48 TOPIC: (((waitlist* or (wait* Near/1 list $\left.\left.{ }^{\star}\right)\right)$ and (control* or group)))

\# 47 TOPIC: (((singl ${ }^{\star}$ or doubl* or tripl ${ }^{\star}$ or trebl $\left.{ }^{\star}\right)$ Near/2 (blind ${ }^{\star}$ or mask ${ }^{\star}$ or dummy)))

\# 46 TOPIC: ((control* N2 (trial or trial))) 
\# 45 TOPIC: (groups)

\# 44 TOPIC: (trial)

\# 43 TOPIC: (Placebo)

\# 42 TOPIC: (Random*)

\# 41 (\#40 OR \#39 OR \#38 OR \#37 OR \#36 OR \#35 OR \#34 OR \#33 OR \#32 OR \#31 OR \#30 OR \#29 OR \#28 OR \#27 OR \#26 OR \#25 OR \#24 OR \#23 OR \#22 OR \#21 OR \#20)

\# 40 TOPIC: ((reality N1 (therap* or psychotherap*)))

\# 39 TOPIC: ((rational emotive))

\# 38 TOPIC: ((problem sol*))

\# 37 TOPIC: ((mindful* or meditation or relaxation))

\# 36 TOPIC: ((mind train $\left.\left.{ }^{\star}\right)\right)$

\# 35 TOPIC: (diffusion)

\# 34 TOPIC: ((dialectic ${ }^{\star}$ or DBT))

\# 33 TOPIC: ((compass ${ }^{\star}$ foc $\left.\left.^{\star}\right)\right)$

\# 32 TOPIC: ((acceptance Near/1 commitment))

\# 31 TOPIC: ((analytic and (cogniti* or therap* or psychotherapy*)))

\# 30 TOPIC: ((non-directive or nondirective))

\# 29 TOPIC: ((motivational Near/2 (interview* ${ }^{\star}$ or intervention* or therap* or psychotherap ${ }^{\star}$ or program ${ }^{\star}$ or training)))

\# 28 TOPIC: (counsel $\left.^{\star}\right)$

\# 27 TOPIC: ((psychobehavio* or psycho-behavio*))

\# 26 TOPIC: ((behavio* activat $\left.\left.{ }^{\star}\right)\right)$

\# 25 TOPIC: ((behavio ${ }^{\star}$ Near/2 (therap $^{\star}$ or psychotherap ${ }^{\star}$ or intervention ${ }^{\star}$ or program ${ }^{\star}$ or training)))

\# 24 TOPIC: (CBASP)

\# 23 TOPIC: ((metacognitive or meta-cognitive))

\# 22 TOPIC: ((cognitive resructur ${ }^{\star}$ or cognitive processing))

\# 21 TOPIC: (((cogniti* near/2 behavio*) or CBT))

\# 20 TOPIC: ((psychotherapy or (psychological near/1 (therap* or intervention))))

\# 19 (\#18 OR \#17 OR \#16 OR \#15 OR\#14 OR \#13 OR \#12 OR \#11 OR \#10 OR \#9 OR \#8 OR \#7 OR \#6 OR \#5 OR \#4 OR \#3 OR \#2 OR \#1)

\# 18 TOPIC: (((abuse* or violen*) near/2 (marital or marriage)))

\# 17 TOPIC: (((domestic or marital or partner ${ }^{\star}$ or spous $\left.{ }^{\star}\right)$ near/2 (rape or $\left(\right.$ sex $^{\star}$ near/1 (abuse* or assault $\left.\left.\left.\left.\left.{ }^{\star}\right)\right)\right)\right)\right)$

\# 16 TOPIC: $\left(\right.$ stalk $\left.^{\star}\right)$

\# 15 TOPIC: (((relation* or interperson $\left.{ }^{\star}\right)$ near/2 (abuse* or violen $\left.\left.\left.{ }^{\star}\right)\right)\right)$

\# 14 TOPIC: ((acquaintance near/2 (rape or violen*)))

\# 13 TOPIC: ((dating N2 violen $\left.\left.{ }^{\star}\right)\right)$

\# 12 TOPIC: ((date N2 rape))

\# 11 TOPIC: ((violen ${ }^{\star}$ N2 (date or dating)))

\# 10 TOPIC: (((domestic* or home $\left.{ }^{\star}\right)$ near/2 abuse $\left.\left.{ }^{\star}\right)\right)$

\# 9 TOPIC: (((domestic ${ }^{\star}$ or home $\left.{ }^{\star}\right)$ near $/ 2$ violen $\left.\left.{ }^{\star}\right)\right)$

\# 8 TOPIC: ((spous ${ }^{\star}$ near/2 violen $\left.\left.{ }^{\star}\right)\right)$

\# 7 TOPIC: ((partner ${ }^{\star}$ near $/ 2$ violen $\left.\left.{ }^{\star}\right)\right)$

\# 6 TOPIC: (((wife or wives or woman or women) near/2 batter $\left.\left.{ }^{\star}\right)\right)$

\# 5 TOPIC: (((wife or wives) near/2 abuse $\left.\left.{ }^{\star}\right)\right)$

\# 4 TOPIC: ((abuse* near/2 partner $\left.\left.{ }^{\star}\right)\right)$

\# 3 TOPIC: ((abuse* near/2 spous $\left.\left.{ }^{\star}\right)\right)$

\# 2 TOPIC: (((abuse* near/2 women) or (abuse ${ }^{\star}$ near/2 woman)))

\# 1 TOPIC: ((batter* near/2 (women or woman)))

Databases: PILOTS: Published International Literature On Traumatic Stress

Host: ProQuest

Data parameters: 1871-current

Date searched: Thursday June 28th 2018

Set\#: S1 Searched for: ti((batter* N/2 (women or woman))) OR ab((batter* N/2 (women or woman)))

Set\#: S2 Searched for: ti((domestic N/2 violence)) OR ab((domestic N/2 violence))

Set\#: S3 Searched for: ti((abuse* N/2 (woman or women))) OR ab((abuse* N/2 (woman or women)))

Set\#: S4 Searched for: ti ((abuse* N/2 spous $\left.\left.{ }^{\star}\right)\right)$ OR ab ((abuse ${ }^{\star} N / 2$ spous $\left.\left.^{\star}\right)\right)$

Set\#: S5 Searched for: ti ((abuse ${ }^{\star} N / 2$ partner $\left.\left.{ }^{\star}\right)\right)$ OR ab((abuse* N/2 partner $\left.\left.{ }^{\star}\right)\right)$

Set\#: S6 Searched for: ti(((wife or wives) N/2 abuse* $))$ OR ab(((wife or wives) N/2 abuse*))

Set\#: S7 Searched for: ti (((wife or wives or women or woman) N/2 batter $\left.\left.{ }^{\star}\right)\right)$ OR ab(((wife or wives or women or woman) N/2 batter $\left.)^{\star}\right)$

Set\#: S8 Searched for: ti((partner* N/2 violen $\left.\left.{ }^{\star}\right)\right)$ OR ab((partner* N/2 violen $\left.\left.{ }^{\star}\right)\right)$

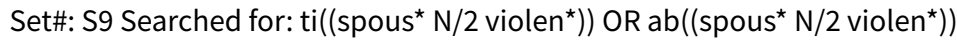

Set\#: S10 Searched for: ti (((domestic or home $\left.^{\star}\right)$ N/2 violen*) $)$ OR ab $\left(\left(\right.\right.$ domestic $^{\star}$ or home $\left.{ }^{\star}\right)$ N/2 violen $\left.\left.{ }^{\star}\right)\right)$

Set\#: S11 Searched for: ti (((domestic ${ }^{\star}$ or home* $)$ N/2 abuse* $\left.)\right)$ OR ab $\left(\left(\left(\right.\right.\right.$ domestic $^{\star}$ or home $\left.{ }^{\star}\right)$ N/2 abuse* $\left.)\right)$

Set\#: S12 Searched for: ti((violen* N/2 (date or dating))) OR ab((violen* N/2 (date or dating))) 
Set\#: S13 Searched for: ti((date N/2 rape)) OR ab((date $\mathrm{N} / 2$ rape))

Set\#: S14 Searched for: ti((dating N/2 violen*)) OR ab((dating N/2 violen*))

Set\#: S15 Searched for: ti((acquaintance N/2 (rape or violen*))) OR ab((acquaintance N/2 (rape or violen*)))

Set\#: S16 Searched for: ti (((relation* or interperson*) N2 (abuse* or violen*))) OR ab(((relation* or interperson*) N2 (abuse* or violen $\left.\left.\left.{ }^{\star}\right)\right)\right)$

Set\#: S17 Searched for: ti(stalk*) OR ab(stalk*)

Set\#: S18 Searched for: ti(((domestic or marital or partner* or spous*) N2 (rape or (sex* N1 (abuse* or assault*))))) OR ab(((domestic or marital or partner* or spous $\left.{ }^{\star}\right)$ N2 (rape or (sex* N1 (abuse* or assault*)))))

Set\#: S19 Searched for: ti(((abuse* or violen $\left.{ }^{\star}\right)$ N2 (marital or marriage))) OR ab(((abuse* or violen $\left.{ }^{\star}\right)$ N2 (marital or marriage)))

Set\#: S20 Searched for: s1 or s2 or s3 or s4 or s 5 or $\mathrm{s} 6$ or $\mathrm{s} 7$ or $\mathrm{s} 8$ or $\mathrm{s} 9$ or $\mathrm{s} 10$ or $\mathrm{s} 11$ or $\mathrm{s} 12$ or $\mathrm{s} 13$ or $\mathrm{s} 14$ or $\mathrm{s} 15$ or $\mathrm{s} 16$ or $\mathrm{s} 17$ or $\mathrm{s} 18$ or $\mathrm{s} 19$

Set\#: S21 Searched for: ti((psychotherapy or (psychological N1 (therap* or intervention)))) OR ab((psychotherapy or (psychological N1

(therap* or intervention))))

Set\#: S22 Searched for: ti(((cogniti* N2 behavio*) or CBT)) OR ab(((cogniti ${ }^{\star}$ N2 behavio*) or CBT))

Set\#: S23 Searched for: ti((cognitive resructur ${ }^{\star}$ or cognitive processing)) OR ab((cognitive resructur ${ }^{\star}$ or cognitive processing))

Set\#: S24 Searched for: ti((metacognitive or meta-cognitive)) OR ab((metacognitive or meta-cognitive))

Set\#: S25 Searched for: ti(CBASP) OR ab(CBASP)

Set\#: S26 Searched for: ti((behavio* N2 (therap* or psychotherap* or intervention* or program* or training))) OR ab((behavio* N2 (therap* or psychotherap* or intervention* or program* or training)))

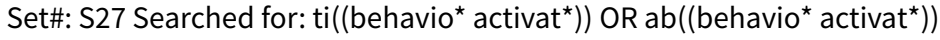

Set\#: S28 Searched for: ti((psychobehavio* or psycho-behavio*)) OR ab((psychobehavio* or psycho-behavio*))

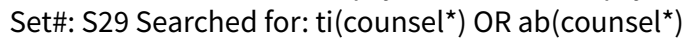

Set\#: S30 Searched for: ti((motivational N2 (interview* or intervention* or therap* or psychotherap* or program* or training))) OR $\mathrm{ab}\left(\left(\right.\right.$ motivational N2 (interview* $^{\star}$ or intervention* or therap* or psychotherap* or program* or training)))

Set\#: S31 Searched for: ti((non-directive or nondirective)) OR ab((non-directive or nondirective))

Set\#: S32 Searched for: $\mathrm{ti}\left(\left(\right.\right.$ analytic and $\left.\left.\left(\text { cogniti }^{\star} \text { or therap }{ }^{\star} \text { or psychotherapy }\right)^{\star}\right)\right)$ OR ab((analytic and (cogniti ${ }^{\star}$ or therap* or psychotherapy*)))

Set\#: S33 Searched for: ti((acceptance N1 commitment)) OR ab((acceptance N1 commitment))

Set\#: S34 Searched for: ti ((compass foc $\left.\left.^{\star}\right)\right)$ OR ab ((compass ${ }^{\star}$ foc $\left.\left.^{\star}\right)\right)$

Set\#: S35 Searched for: ti((dialectic* or DBT)) OR ab((dialectic* or DBT))

Set\#: S36 Searched for: ti(diffusion) OR ab(diffusion)

Set\#: S37 Searched for: ti ((mind train*)) OR ab((mind train*))

Set\#: S38 Searched for: ti((mindful* or meditation or relaxation)) OR ab((mindful ${ }^{\star}$ or meditation or relaxation))

Set\#: S39 Searched for: ti ((problem sol*)) OR ab((problem sol*))

Set\#: S40 Searched for: ti((rational emotive)) OR ab((rational emotive))

Set\#: S41 Searched for: ti((reality N1 (therap* or psychotherap $\left.\left.{ }^{\star}\right)\right)$ ) OR ab((reality N1 (therap* or psychotherap $\left.\left.{ }^{\star}\right)\right)$ )

Set\#: S42 Searched for: (s21 or s22 or s23 or s24 or s25 or s26 or s27 or s28 or s29 or or s30 or s31 or s32 or s33 or s34 or s35 or s36 or s37 or $\mathrm{s} 38$ or $\mathrm{s} 39$ or $\mathrm{s} 40$ or $\mathrm{s} 41$ )

Set\#: S43 Searched for: ti(random*) OR ab(random*)

Set\#: S44 Searched for: ti(placebo) OR ab(placebo)

Set\#: S45 Searched for: ti(trial) OR ab(trial)

Set\#: S46 Searched for: ti(groups) OR ab(groups)

Set\#: S47 Searched for: ti((control* N2 (trial or trial))) OR ab ((control* N2 (trial or trial)))

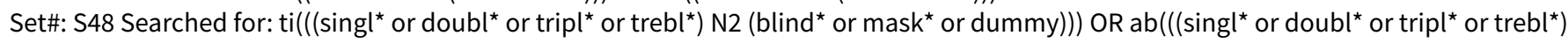
N2 (blind* or mask* or dummy)))

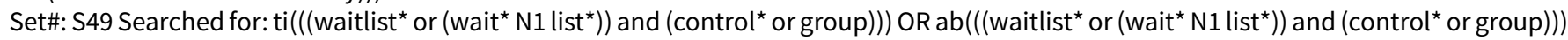
Set\#: S50 Searched for: ti (((usual care or care as usual) and (control* or group))) OR ab(((usual care or care as usual) and (control* or group))) Set\#: S51 Searched for: ti((treatment as usual or TAU)) OR ab((treatment as usual or TAU))

Set\#: S52 Searched for: $\mathrm{s} 43$ or $\mathrm{s} 44$ or $\mathrm{s} 45$ or $\mathrm{s} 46$ or $\mathrm{s} 47$ or $\mathrm{s} 48$ or $\mathrm{s} 49$ or $\mathrm{s} 50$ or $\mathrm{s} 51$

Set\#: S53 Searched for: s52 and s42 and s20

\section{ICTRP}

Searched via: http://apps.who.int/trialsearch/

Searched on: Thursday June 28th 2018

Search string used: (domestic violence or domestic abuse or partner abuse or partner violence or rape or sexual abuse or spousal abuse or battered)

Clinical Trials.gov

Searched via: https://www.clinicaltrials.gov/

Searched on: Thursday June 28th 2018

Search string used:

1. domestic violence

2. domestic abuse

3. partner abuse

4. partner violence 
5. rape

6. sexual abuse

7. spousal abuse

8. battered

\section{Appendix 3. Other database search strategies (Search-2)}

Database: Cochrane Central Register of Controlled Trials (CENTRAL)

Host: Wiley

Data Parameters: Issue 10 of 12, October 2019

\#1 MeSH descriptor: [Battered Women] this term only

\#2 MeSH descriptor: [Domestic Violence] this term only

\#3 MeSH descriptor: [Spouse Abuse] this term only

\#4 MeSH descriptor: [Intimate Partner Violence] this term only

\#5 MeSH descriptor: [Gender-Based Violence] this term only

\#6 (abus* near/3 women)

\#7 (abus ${ }^{\star}$ near $/ 3$ spous ${ }^{\star}$ )

\#8 (abus* near/3 partner ${ }^{\star}$ )

\#9 ((wife or wives) near/3 abus $\left.{ }^{\star}\right)$

$\# 10$ ((wife or wives or women) near/3 batter ${ }^{\star}$ )

\#11 (partner* near/3 violen*)

\#12 (spous ${ }^{\star}$ near/3 violen ${ }^{\star}$ )

\#13 ((domestic* or home*) near/3 violen $\left.{ }^{\star}\right)$

\#14 ((domestic* or home*) near/3 abus*)

\#15 (violen* near/3 (date or dating))

\#16 (date near/3 rape)

\#17 (dating near/3 violen ${ }^{\star}$ )

\#18 (acquaintance near/3 (rape or violen $\left.{ }^{\star}\right)$ )

\#19 ((relation* ${ }^{\star}$ or interperson $\left.{ }^{\star}\right)$ near/3 (abus ${ }^{\star}$ or violen $\left.^{\star}\right)$ )

\#20 stalk*

\#21 ((domestic or marital or partner ${ }^{\star}$ or spous $\left.{ }^{\star}\right)$ near/3 (rape or (sex* near/1 (abus or assault $\left.\left.\left.^{\star}\right)\right)\right)$ )

\#22 ((abus* or violen*) near/3 (marital or marriage))

\#23 ("coercive control" or "sexual coercion")

\#24 "interpersonal trauma"

\#25 (marital near/3 violen*)

\#26 ((marital or marriage) and sex ${ }^{\star}$ and coerci $\left.{ }^{\star}\right)$

\#27 ((women or womens) near/2 shelter $\left.{ }^{\star}\right)$

$\# 28$ (\#1 or \#2 or \#3 or \#4 or \#5 or \#6 or \#7 or \#8 or \#9 or \#10 or \#11 or \#12 or \#13 or \#14 or \#15 or \#16 or \#17 or \#18 or \#19 or \#20 or \#21 or \#22 or \#23 or \#24 or \#25 or \#26 or \#27)

Ovid MEDLINE(R) and Epub Ahead of Print, In-Process \& Other Non-Indexed Citations and Daily <1946 to October 25, 2019> Search Strategy:

[Lines 1-20 (population) as per 2018 search]

1 Battered Women/

2 Domestic Violence/

3 Souse Abuse/

4 (abuse* adj3 wom\#n).tw.

5 (abuse* adj3 spous\$).tw.

6 (abuse $\$$ adj3 partner\$).tw.

7 ((wife or wives) adj3 abuse\$).tw.

8 ((wife or wives or wom\#n) adj3 batter\$).tw.

9 (partner ${ }^{\star}$ adj3 violen\$).tw.

10 (spous $^{\star}$ adj3 violen\$).tw.

11 ((domestic $^{\star}$ or home*) adj3 violen $\left.{ }^{\star}\right)$.tw.

12 ((domestic ${ }^{\star}$ or home ${ }^{\star}$ ) adj3 abuse*).tw.

13 (violen* adj3 (date or dating)).tw.

14 (date adj3 rape).tw.

15 (dating adj3 violen\$).tw.

16 (acquaintance adj3 (rape or violen\$)).tw.

17 ((relation* or interperson $\left.{ }^{\star}\right)$ adj3 (abuse\$ or violen\$)).tw. 
18 stalk $^{*}$.tw.

19 ((domestic or marital or partner ${ }^{\star} 1$ or spous $\left.{ }^{\star}\right)$ adj3 (rape or (sex ${ }^{\star}$ adj1 (abuse* or assault $\left.\left.\left.{ }^{\star}\right)\right)\right)$ ).mp.

20 ((abuse ${ }^{\star}$ or violen $\left.{ }^{\star}\right)$ adj3 (marital or marriage)).tw.

21 Intimate Partner Violence/ [New MeSH Term 2016, previous indexing Spouse Abuse/]

22 Gender-Based Violence/ [New MeSH Term 2018]

23 or $/ 1-22$

[New RCT Filter]

24 controlled clinical trial.pt.

25 randomized controlled trial.pt.

26 clinical trials as topic/

27 (randomi\#ed or randomi\#ation or randomi\#ing).ti,ab,kf.

28 (RCT or "at random" or (random* adj3 (administ* or allocat* or assign* or class ${ }^{\star}$ or cluster or crossover or cross-over or control ${ }^{\star}$ or determine* or divide* or division or distribut ${ }^{\star}$ or expose $e^{\star}$ or fashion or number ${ }^{\star}$ or place* or pragmatic or quasi or recruit* or split or subsitut ${ }^{\star}$ or treat $\left.\left.\left.{ }^{\star}\right)\right)\right) . t i, a b, k f$.

29 placebo.ab,ti,kf.

30 trial.ti.

31 (control* $^{*}$ adj3 group $\left.{ }^{\star}\right)$.ab.

32 (control $^{\star}$ and (trial or trial or group ${ }^{\star}$ ) and (waitlist* or wait $^{\star}$ list ${ }^{\star}$ or ((treatment or care) adj2 usual))).ti,ab,kf,hw.

33 ((single or double or triple or treble) adj2 (blind* or mask* or dummy)).ti,ab,kf.

34 double-blind method/ or random allocation/ or single-blind method/

35 or/24-34

36 exp animals/ not humans.sh.

3735 not 36

$38\left(2018^{\star}\right.$ or $2019^{\star}$ or $\left.2020^{\star}\right) \cdot y r, d p, d t, e p, e z$.

39 (23 and 37 and 38)

PsycINFO <1806 to October Week $32019>$

Search Strategy:

1 Battered Females/

2 Domestic Violence/

3 Partner Abuse/

4 (abuse* adj3 wom\#n).tw.

5 (abuse* adj3 spous\$).tw.

6 (abuse* adj3 partner\$).tw.

7 ((wife or wives) adj3 abuse\$).tw.

8 ((wife or wives or wom\#n) adj3 batter\$).tw.

9 (partner\$ adj3 violen\$).tw.

10 (spous ${ }^{\star}$ adj3 violen\$).tw.

11 ((domestic ${ }^{\star}$ or home $\left.{ }^{\star}\right)$ adj3 violen $\left.{ }^{\star}\right)$.tw.

12 ((domestic ${ }^{\star}$ or home*) adj3 abuse $\left.{ }^{\star}\right)$. tw.

13 (violen* adj3 (date or dating)).tw.

14 (date adj3 rape).tw.

15 (dating adj3 violen\$).tw.

16 (acquaintance adj3 (rape or violen\$)).tw.

17 ((relation* or interperson $\left.{ }^{\star}\right)$ adj3 (abuse\$ or violen\$)).tw.

18 stalk $^{*}$.tw.

19 ((domestic or marital or partner ${ }^{\star} 1$ or spous $\left.{ }^{\star}\right) \operatorname{adj} 3\left(\right.$ rape or $\left(\operatorname{sex}^{\star} \operatorname{adj} 1\left(\right.\right.$ abuse or assault $\left.\left.\left.\left.^{\star}\right)\right)\right)\right) . m p$.

20 ((abuse* or violen $\left.{ }^{\star}\right)$ adj3 (marital or marriage)).tw.

21 Intimate Partner Violence/

22 or/1-21

23 clinical trials.sh.

24 (randomi\#ed or randomi\#ation or randomi\#ing).ti,ab,id.

25 (RCT or at random or (random* adj3 (administ ${ }^{\star}$ or allocat ${ }^{\star}$ or assign* or class ${ }^{\star}$ or control* or crossover or cross-over or determine* or

divide $^{\star}$ or division or distribut ${ }^{\star}$ or expose* or fashion or number ${ }^{\star}$ or place or recruit $^{\star}$ or split or subsitut ${ }^{\star}$ or treat $\left.\left.{ }^{\star}\right)\right)$ ).ti,ab,id.

26 (control $^{\star}$ and (trial or trial or group) and (placebo or waitlist* or wait $^{\star}$ list ${ }^{\star}$ or ((treatment or care) adj2 usual))).ti,ab,id,hw.

27 ((single or double or triple or treble) adj2 (blind* or mask* or dummy)).ti,ab,id.

28 trial.ti.

29 placebo.ti,ab,id,hw.

30 treatment outcome.md.

31 treatment effectiveness evaluation.sh.

32 mental health program evaluation.sh.

Psychological therapies for women who experience intimate partner violence (Review) 
33 or/34-43

3421 and 44

$35\left(2018^{\star}\right.$ or $2019^{\star}$ or $\left.2020^{\star}\right) \cdot y r$,an.

3634 and 35

37 dissertation abstract.pt.

38 *intimate partner violence/

39 (37 and 38)

40 (36 or 39$)$

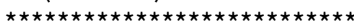

Web of Science

Indexes=SCI-EXPANDED, SSCI, A\&HCl, CPCI-S, CPCI-SSH, ESCI Timespan=All years

\#31 (\#30 AND \#27)

\#30 (\#29 OR \#28)

\#29 TOPIC: ((battered not (battery or batteries)))

\#28 TITLE: (violen* or abuse or trauma or "traumatic stress" or PTSD or batter* or "high risk" or IPV or coerci ${ }^{\star}$ )

\#27 (\#25 AND \#19) Refined by: PUBLICATION YEARS: ( 2019 OR 2018 )

\#26 (\#25 AND \#19)

\#25 (\#24 OR \#23 OR \#22 OR \#21 OR \#20)

\#24 TOPIC: ("quasi random*")

\#23 TOPIC: ((random* SAME control $\left.\left.{ }^{\star}\right)\right)$

\#22 TITLE: (trial)

\#21 TOPIC: (randomised or randomized or RCT or CRCT)

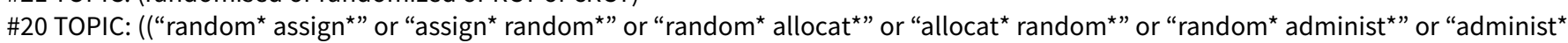
random" or "random* class" or "random* crossover" or "random* cross-over" " or "random" divi" or "divi" random" or "random* fashion" or "random* number" or "random* place*" or "random* recruit" or "recruit* random" or "random* split" or "split random " or "random* substitut" or "substitut" random" or "random* treat" or "treat* random" or "pragmatic random"))

\#19 (\#18 OR \#17 OR \#16 OR \#15 OR \#14 OR \#13 OR \#12 OR \#11 OR \#10 OR \#9 OR \#8 OR \#7 OR \#6 OR \#5 OR \#4 OR \#3 OR \#2 OR \#1)

\#18 TOPIC: ((((sex* and coer $\left.^{\star}\right)$ SAME (marital* or marriage $\left.\left.\left.\left.{ }^{\star}\right)\right)\right)\right)$

\#17 TOPIC: ("intimate partner violence")

\#16 TOPIC: (((abus* or violen*) SAME ("gender based")))

\#15 TOPIC: (((abus ${ }^{\star}$ or violen $\left.{ }^{\star}\right)$ SAME (marital* or marriage $\left.\left.\left.^{\star}\right)\right)\right)$

\#14 TOPIC: ((domestic or marital or partner* or spous ${ }^{\star}$ ) SAME (rape or "sex ${ }^{\star}$ abuse*" or "abuse* sex $^{\star}$ " or assault $)$ )

\#13 TOPIC: $\left(\right.$ stalk $\left.^{\star}\right)$

\#12 TOPIC: (("interperson* abus*" or "abusive interperson*" or "interperson* violen*" or "violen* interperson*"))

\#11 TOPIC: ((acquaintance* SAME (rape or raped or rapes or violen $\left.\left.{ }^{\star}\right)\right)$ )

\#10 TOPIC: ("relationship* abus" or "abusive relationship*" or "relationship* violen*" or "violen* relationship*")

\#9 TOPIC: ("dating violen*" or "date violen*" or "date rape*" or "violen* dating" or "violen* date*")

\#8 TOPIC: ("domestic* abus*" or "home abus*")

\#7 TOPIC: ("domestic* violen" or "home violen"

\#6 TOPIC: ("batter ${ }^{\star}$ women*" or "batter* woman*" or "batter* wife*" or "batter* wives"”)

\#5 TOPIC: ("violent partner" or "partner violence" or "violence against partner" or "violent spous" or "spous* violence" or "violence against spous")

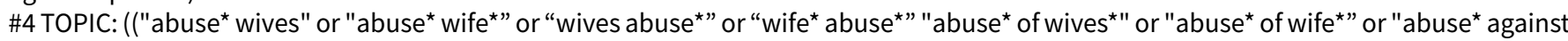
wives" or "abuse* against wife*”))

\#3 TOPIC: ((("abuse ${ }^{\star}$ women" or "women ${ }^{\star}$ abuse*" or "abuse* woman*" or "woman* abuse " or "abuse* of women*" or "abuse* against women*”)))

\#2 TOPIC: (("abuse* partner" or "partner* abuse*" or "abuse* of partner" or "abuse* against partner"”))

\#1 TOPIC: (("abuse* spous" or "spous* abuse*" or "abuse* of spous" or "abuse ${ }^{\star}$ against spous $\left.\left.{ }^{\star} "\right)\right)$

\section{Appendix 4. Data collection tool}

\section{Identification:}

- Sponsorship Source

- Country

- Setting

- Comments

- Author's contact details

- Author's name

- Institution 
- Email

- Address

- Trial title (main outcome publication)

- Registered trial protocol ID

\section{Methods:}

- Trial Design

- Assessment modality (e.g. face-to-face, telephone, online)

- Conflict of interest of trial authors

- Funding for trial

- Primary outcomes

- Randomisation method

- Secondary outcomes

- Timing of outcome measurements

- Total duration of trial

- Type of analysis

- Types of participants

- Intention-to-treat analyses

\section{Population:}

- Inclusion criteria

- Exclusion criteria

- Group differences at baseline

- Number ineligible

- Number eligible

- Number of eligible people consented

- Recruitment setting of participants (e.g. healthcare setting, community setting, shelter setting)

- Number of eligible people recruited

- Total number of participants approached

- Total number of participants within all groups

- Sample power calculation

- Number declined to participate

\section{Baseline characteristics (total sample, experimental group and comparator/control group):}

- Age

- Gender

- Ethnicity

- Employment

- Education

- Relationship status

- Dependent children

- Number analysed (data analysis at post-treatment stage)

- Number of participants eligible assigned

- Number of participants dropped out (by final follow-up)

- Comorbidities

- Experience of IPV

- Experience of other types of violence and abuse

- Remuneration

- Financial dependence (on partner)

\section{Intervention:}

- Brief description (rationale, main components) 
- Type of intervention

- Intervention model

- Mode of delivery (how)

- Frequency and duration of delivery (when and how much)

- Level of mental health training of person delivering the intervention

- Fidelity assessment

- Person delivering the intervention (who provided the intervention): Therapist, self-directed or combined

- Person delivering the intervention (healthcare workers, non healthcare workers)

- Gender of person delivering the intervention

- Intervention location (where)

- Tailoring of intervention (personalised, titrated or adapted)

- Intervention modification (during the trial)

- Main techniques of intervention

- Intervention manual

- Intervention attendance

- Intensity of intervention

- CCDAN psychological therapies

\section{Outcomes:}

- Primary outcomes (depression, self-efficacy, drop-outs from treatment)

- Secondary outcomes (mental health, anxiety, PTSD, quality of life, re-exposure to IPV, safety planning and/or safety behaviour, use of healthcare and IPV services, social support)

- Outcome type (continuous, dichotomous, adverse events)

- Outcome reporting (fully reported, partially reported)

- Scale (name, range, unit of measurement, direction of measurement, e.g. lower or higher better)

- Assessment time points

\section{Other information to aid understanding of the trials}

- Analysis of any cost/benefit measures

- Process evaluation

- Summary of participants views about the intervention

- Adverse events (harm) related to participation

\section{Appendix 5. Assessment of risk of bias in included trials}

\section{Random sequence generation}

- Description: Describe the method used to generate the allocation sequence in sufficient detail to allow an assessment of whether it should produce comparable groups.

- Ratings: "High" (high risk of bias); "Low" (low risk of bias); "Unclear" (uncertain risk of bias)

\section{Allocation concealment}

- Description: Describe the method used to conceal the allocation sequence in sufficient detail to determine whether intervention allocations could have been foreseen in advance of, or during, enrolment.

- Ratings: "High" (high risk of bias); "Low" (low risk of bias); "Unclear" (uncertain risk of bias)

\section{Blinding of participants and personnel}

- Description: Describe all measures used, if any, to blind trial participants and personnel from knowledge of which intervention a participant received. Provide any information relating to whether the intended blinding was effective.

- Ratings: "High" (high risk of bias); "Low" (low risk of bias); "Unclear" (uncertain risk of bias)

\section{Blinding of outcome assessment}

- Description: Describe all measures used, if any, to blind outcome assessors from knowledge of which intervention a participant received. Provide any information relating to whether the intended blinding was effective.

- Ratings: "High" (high risk of bias); "Low" (low risk of bias); "Unclear" (uncertain risk of bias) 


\section{Incomplete outcome data}

- Description: Describe the completeness of outcome data for each main outcome, including attrition and exclusions from the analysis. State whether attrition and exclusions were reported, the numbers in each intervention group (compared with total randomised participants), reasons for attrition/exclusions where reported, and any re-inclusions in analyses performed by the review authors.

- Ratings: "High" (high risk of bias); "Low" (low risk of bias); "Unclear" (uncertain risk of bias)

\section{Selective outcome reporting}

- Description: State how the possibility of selective outcome reporting was examined by the review authors, and what was found.

- Ratings: "High" (high risk of bias); "Low" (low risk of bias); "Unclear" (uncertain risk of bias)

\section{Other bias}

- Description: State any important concerns about bias not addressed in the other domains in the tool

- Ratings: "High" (high risk of bias); "Low" (low risk of bias); "Unclear" (uncertain risk of bias)

Additional factors that may impact outcomes:

\section{Therapist allegiance/conflict of interest}

- Description: whether the therapist has a vested interest in the provided therapies

- Ratings: "High" (high risk of bias); "Low" (low risk of bias); "Unclear" (uncertain risk of bias)

\section{Therapist qualifications and training}

- Description: whether the person delivering the intervention had appropriate training and/or qualifications

- Ratings: "High" (high risk of bias); "Low" (low risk of bias); "Unclear" (uncertain risk of bias)

\section{Researcher allegiance/conflict of interest}

- Description: whether the researcher has a vested interest in the provided therapies?

- Ratings: "High" (high risk of bias); "Low" (low risk of bias); "Unclear" (uncertain risk of bias)

\section{Protection against contamination}

- Description: Describe methods used to prevent or minimise the possibility that women in the comparator interventions might receive part, or all of the intervention

- Ratings: "High" (high risk of bias); "Low" (low risk of bias); "Unclear" (uncertain risk of bias)

\section{Reliability of outcome measures}

- Description: use of measures with appropriate psychometric properties and appropriately referenced

- Ratings: "High" (high risk of bias); "Low" (low risk of bias); "Unclear" (uncertain risk of bias)

\section{Treatment fidelity}

- Description: Whether the therapy was measured against a manual or scale? whether researchers / trialists used any methods to assess treatment fidelity?

- Ratings: "High" (high risk of bias); "Low" (low risk of bias); "Unclear" (uncertain risk of bias)

Assessment of Risk of Bias for cluster-randomised trials (in addition to above list):

\section{Identification and recruitment bias}

- Description: Describe strategies used to minimise identification and selection bias

- Ratings: "High" (high risk of bias); "Low" (low risk of bias); "Unclear" (uncertain risk of bias)

\section{Baseline imbalance between randomised groups}

- Description: Describe methods used to minimise imbalances between groups

- Ratings: "High" (high risk of bias); "Low" (low risk of bias); "Unclear" (uncertain risk of bias)

\section{Loss of clusters}

- Description: Adequately describe (with reasons) any loss of clusters

- Ratings: "High" (high risk of bias); "Low" (low risk of bias); "Unclear" (uncertain risk of bias) 


\section{Cluster Consideration in Analysis}

- Description: Describe adjusting results for cluster (e.g. intra-cluster correlation for baseline outcome)

- Ratings: "High" (high risk of bias); "Low" (low risk of bias); "Unclear" (uncertain risk of bias)

\section{H I S T O R Y}

Protocol first published: Issue 5, 2018

Review first published: Issue 7, 2020

\section{CONTRIBUTIONS OFAUTHORS}

Drafting of protocol: MT, KH, LOD, GF, GG, JT, AT, and PC.

Search strategy: $\mathrm{MT}$ and $\mathrm{KH}$.

Selection of trials: MH, LOD, JTM, GG and KH.

Extraction of data: MH, LOD, GG, JTM, AT.

Data entry into Review Manager 5.3: $\mathrm{MH}$.

Analysis and interpretation of analysis: $\mathrm{MH}, \mathrm{PC}$ and $\mathrm{KH}$.

Drafting of review: MH, KH, LOD, GF, GG, JTM, AT and PC.

Topic expertise and editing: MH, KH, LOD, GF, GG, JTM, AT, JR, and PC.

Updating of review: $\mathrm{MH}$ and $\mathrm{KH}$.

\section{DECLARATIONS OF INTEREST}

We acknowledge that the following authors were involved in the cited trials. These trials met the inclusion criteria for this review and were included. Authors' knowledge about these trials may represent a greater availability of knowledge about those specific trials. We ensured that no-one involved in a particular trial extracted data from their own trial or assessed that trial for risk of bias.

$M H:$ no conflicts of interest.

LOD: co-investigator of WEAVE trial (Hegarty 2013).

GG: co-investigator of an adapted version of the Women's Wellness Treatment trial (Tirado-Muñoz 2015).

JTM: lead investigator of an adapted version of the Women's Wellness Treatment trial (Tirado-Muñoz 2015).

AT: co-investigator of WEAVE (Hegarty 2013) and I-DECIDE trial (Hegarty 2019).

PC: co-investigator of WEAVE trial (Hegarty 2013).

GF: principal investigator of PATH trial (Ferrari 2018), co-investigator WEAVE trial (Hegarty 2013), chair of the WHO Intimate Partner Violence and Sexual Assault Guidelines Development Group and chair of the UK NICE Domestic Violence Programme Development Group.

MT: no conflicts of interest.

KH: lead investigator of WEAVE (Hegarty 2013) and I-DECIDE trial (Hegarty 2019).

\section{SOURCES OF SUPPORT}

\section{Internal sources}

- The University of Melbourne, Australia

Support for MT

\section{External sources}

- Australian General Practice Training, Australia

Funding support for MT

\section{DIFFERENCES BETWEEN PROTOCOLANDREVIEW}

In our protocol (Tan 2018), we stated that the timing of outcome assessment would include short-term time frames classified as zero to six months, medium-term greater than six months to 12 months, and long-term as periods greater than 12 months. As this did not distinguish which follow-up time point six months would fall into, we classified the timing of outcome assessments as short-term (up to 6 months), medium-term (six to under 12 months) and long-term (12 or more months).

A further difference relates to intensity of interventions. In the protocol we classified this as two to five sessions and five or more sessions. As it is unclear where five sessions should be included, in this review we changed intensity of interventions to include up to four sessions and five or more sessions.

For 'Summary of findings' table, our protocol did not specify whether we would present dichotomous or continuous outcomes. In this review, we decided to present continuous outcomes. 